\title{
Small-Scale Spray Releases: Additional Aerosol Test Results
}
PP Schonewill
LA Mahoney
PA Gauglitz
DN Tran
ML Kimura
CA Burns
GN Brown
DE Kurath

August 2013

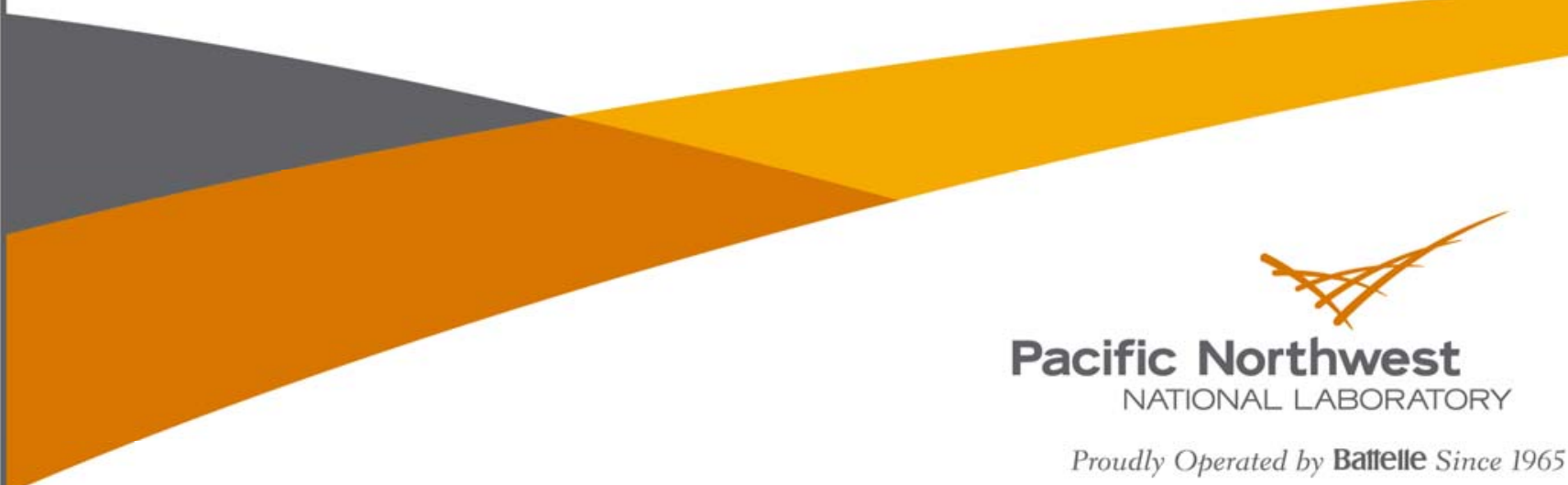


PNNL-22402

WTP-RPT-222, Rev. 0

\title{
Small-Scale Spray Releases: Additional Aerosol Test Results
}

\author{
PP Schonewill \\ LA Mahoney \\ PA Gauglitz \\ DN Tran \\ ML Kimura \\ CA Burns \\ GN Brown \\ DE Kurath
}

August 2013

Test Specification: None

Work Authorization: WA42AM01

Test Plan: TP-WTPSP-031, Rev. 1.0

Test Exceptions: None

Prepared for

the U.S. Department of Energy

under Contract DE-AC05-76RL01830

Pacific Northwest National Laboratory

Richland, Washington 99352 



\section{Completeness of Testing}

This report describes the results of work and testing specified by Test Plan TP-WTPSP-031, Rev. 1.0. The work followed the quality assurance requirements outlined in the test plan. The descriptions provided in this report are an accurate account of both the conduct of the work and the data collected. Test plan results are reported. Also reported are any unusual or anomalous occurrences that are different from expected results. The test results and this report have been reviewed and verified.

\section{Approved:}

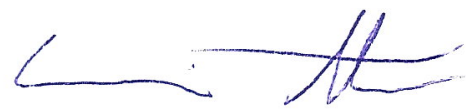

Reid A. Peterson, Manager WTP R\&T Support Project

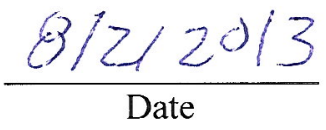





\section{Testing Summary}

One of the events postulated in the hazard analysis at the Waste Treatment and Immobilization Plant (WTP) and other U.S. Department of Energy (DOE) nuclear facilities is a breach in process piping that produces aerosols with droplet sizes in the respirable range. The current approach for predicting the size and concentration of aerosols produced in a spray leak involves extrapolating from correlations reported in the literature. These correlations are based on results obtained from small engineered spray nozzles using pure liquids with Newtonian fluid behavior. The narrow ranges of physical properties on which the correlations are based do not cover the wide range of slurries and viscous materials that will be processed in the WTP and across processing facilities in the DOE complex.

To expand the data set upon which the WTP accident and safety analyses were based, an aerosol spray leak testing program was conducted by Pacific Northwest National Laboratory (PNNL). PNNL's test program addressed two key technical areas to improve the WTP methodology (Larson and Allen 2010). The first technical area was to quantify the role of slurry particles in small breaches where slurry particles may plug the hole and prevent high-pressure sprays. The results from an effort to address this first technical area can be found in Mahoney et al. (2012). The second technical area was to determine aerosol droplet size distribution and total droplet volume from prototypic breaches and fluids, including sprays from larger breaches and sprays of slurries for which literature data are largely absent. To address the second technical area, the testing program collected aerosol generation data at two scales, commonly referred to as small-scale and large-scale. The small-scale testing and resultant data are described in Mahoney et al. (2013) and the large-scale testing and resultant data are presented in Schonewill et al. (2012). In tests at both scales, simulants were used to mimic the relevant physical properties projected for actual WTP process streams.

Examination of the results from the initial (subsequently referred to as Phase I) aerosol spray leak testing described in the preceding paragraph highlighted some uncertainties in evaluating aerosol generation that remained after the original scope of work was completed. The need for additional (subsequently referred to as Phase II) aerosol spray leak testing was identified. The purpose of the study described in this report is to provide experimental data and analysis to supplement the Phase I results and reduce uncertainty in the remaining technical area - determining aerosol droplet size distribution and total droplet volume from prototypic breaches and fluids - by performing small-scale tests with a range of orifice sizes and orientations representative of the WTP typical conditions. Specifically, there was

uncertainty with respect to aerosol behavior (namely aerosol release fraction and generation rates) for:

- simulant(s) near the WTP rheological boundaries of (6 Pa/6 mPa $\cdot \mathrm{s}$ and $30 \mathrm{~Pa} / 30 \mathrm{mPa} \cdot \mathrm{s})$

- $\operatorname{simulant(s)}$ with solids loadings greater than $20 \mathrm{wt} \%$

- simulant(s) with a small solids fraction of particles that have a relatively high density

- lower spray pressures (i.e., specifically 100 and 200 psig in the small-scale tests)

- in-spray measurements. 
As the list indicates, the testing and analysis in the Phase II small-scale study was primarily concerned with measuring sprays with simulants that were not used in Phase I. The companion study describing the Phase II large-scale testing, which also includes examinations of spray and chamber geometry, is available in Daniel et al. (2013).

\section{S.1 Objectives}

Table S.1 provides a summary of each small-scale aerosol test objective for the Phase II testing, whether the objective was met, and a discussion of the test results. Other objectives identified in Test Plan TP-WTPSP-031, Rev. 1.0 apply to either the Phase I test program, discussed in Mahoney et al. (2012), Mahoney et al. (2013), and Schonewill et al. (2012), respectively, or the Phase II large-scale aerosol testing presented in Daniel et al. (2013). 
Table S.1. Summary of Phase II Small-Scale Aerosol Test Objectives and Results

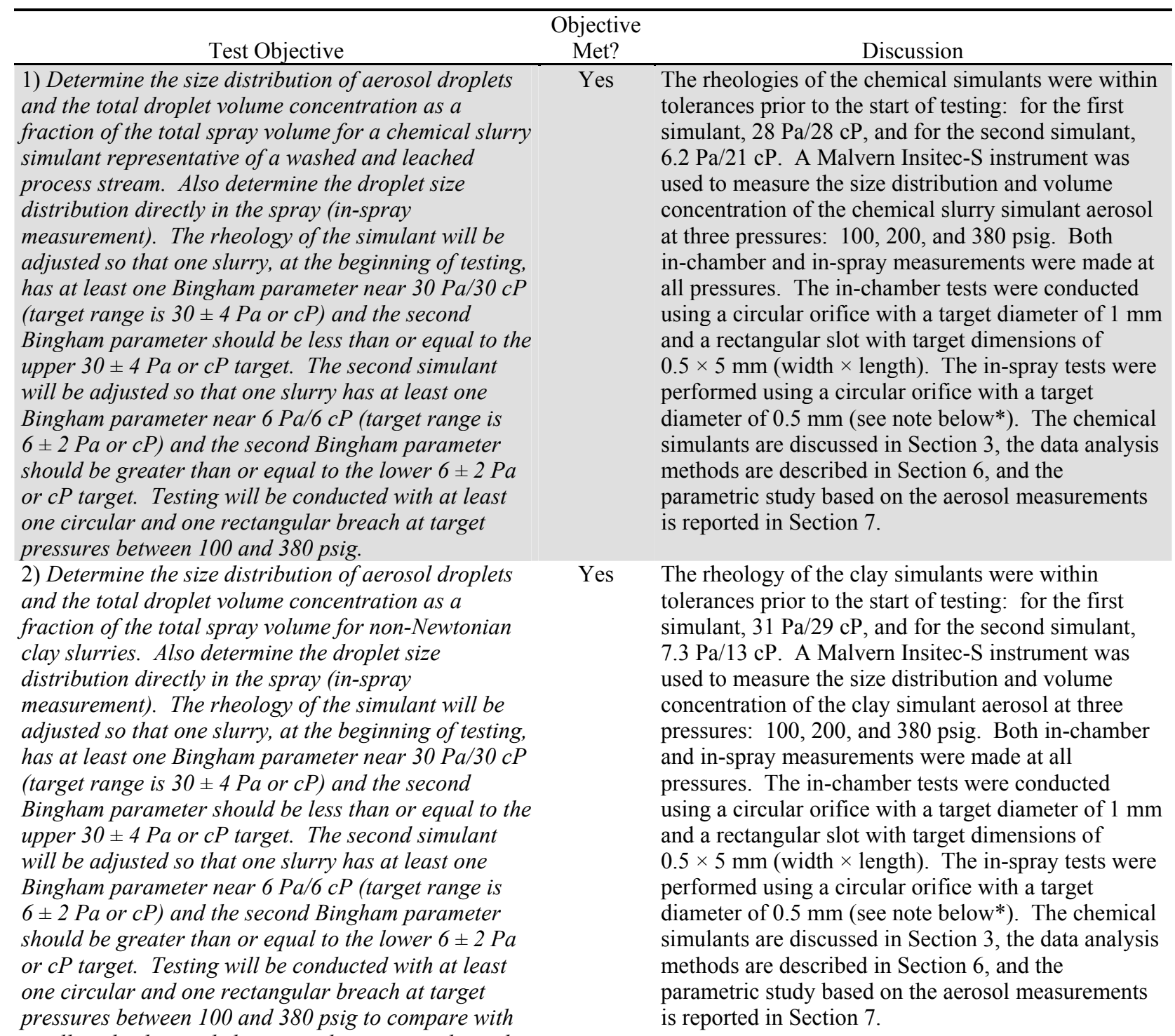

small-scale chemical slurry simulant test results and to support large-scale testing with equivalent clay slurry simulants.

3) Determine the size distribution of aerosol droplets and the total droplet volume concentration as a fraction of the total spray volume for a slurry simulant with 27 wt\% UDS. Also determine the droplet size distribution directly in the spray (in-spray measurement) for this simulant.

Yes

A Malvern Insitec-S instrument was used to measure the size distribution and volume concentration of the $27 \mathrm{wt} \%$ small treated (STR) simulant aerosol at three pressures: 100, 200, and 380 psig. Both in-chamber and in-spray measurements were made at all pressures. The in-chamber tests were conducted using a circular orifice with a target diameter of $1 \mathrm{~mm}$ and a rectangular slot with target dimensions of $0.5 \times 5 \mathrm{~mm}$ (width $\times$ length). The in-spray tests were performed using a circular orifice with a target diameter of $0.5 \mathrm{~mm}$ (see note below*). The STR simulant is discussed in Section 3, the data analysis methods are described in Section 6, and the parametric study based on the aerosol measurements is reported in Section 7. 
Table S.1. (contd)

\begin{tabular}{l} 
Test Objective \\
\hline 4) Determine the size distribution of aerosol droplets \\
and the total droplet volume concentration as a \\
fraction of the total spray volume for a slurry simulant \\
with 20 wt\% UDS including a target quantity of about \\
1 wt\% of very dense particles. Also determine the \\
droplet size distribution directly in the spray (in-spray \\
measurement) for this simulant.
\end{tabular}

5) Assess the capability of the Malvern Insitec-S in-process particle size analyzer, which is the instrument used in the aerosol testing, to measure accurately the concentration and size distribution of samples. This will be accomplished by measuring carefully controlled dilute aqueous slurries of known concentration and particle size distribution (PSD) and comparing the Malvern result to the known values. Testing will include mono- and poly-disperse suspensions and will evaluate all four Malvern configurations of 1) Phase I small-scale spacers and old lens, 2) Phase II small-scale spacers and new lens, 3) Phase I large-scale spacers and old lens, and 4) Phase II large-scale spacers and new lens (old lens $100 \mathrm{~mm}$, new lens $500 \mathrm{~mm}$ ).

6) Compare the aerosol results from the Malvern Insitec-S using the new 500-mm lens that has a nominal measurement range of $2.5-2500 \mu \mathrm{m}$ (Malvern Instruments, Ltd. 2010) to aerosol results using the 100-mm lens employed in Phase I (Mahoney et al. 2013) that provided a nominal range of 0.5 to $200 \mu \mathrm{m}$ (Malvern Instruments, Ltd. 2010). Tests will use one or more orifices.

* The in-spray measurements were conducted for all simulants as intended. However, as discussed in this report, meaningful in-spray data could not be acquired, i.e., the in-spray component of these objectives was not strictly met. Because the large-scale performed a series of successful in-spray measurements in Phase II (see Daniel et al. 2013), additional effort was not undertaken to obtain better small-scale in-spray data during the Phase II study.
Objective Met?

Discussion

Yes A Malvern Insitec-S instrument was used to measure the size distribution and volume concentration of the dense particle in small-treated (DST) simulant (19 wt\% STR and $1 \mathrm{wt} \%$ molybdenum [Mo] particles in the slurry) aerosol at 380 psig. Both in-chamber and in-spray measurements were made. The in-chamber tests were conducted using a circular orifice with a target diameter of $1 \mathrm{~mm}$ and a rectangular slot with target dimensions of $0.5 \times 5 \mathrm{~mm}$ (width $\times$ length). The in-spray tests were performed using a circular orifice with a target diameter of $0.5 \mathrm{~mm}$ (see note below*). The DST simulant is discussed in Section 3, the data analysis methods are reported in Section 6, and the parametric study based on the aerosol measurements is reported in Section 7.

Yes Validation testing was performed to assess the ability of the Malvern Insitec-S instrument to accurately measure concentration, PSD, and correct for attenuation of the laser over long path lengths. All configurations were tested, as well as some column dispersion tests, using a series of mono- and poly-disperse suspensions. The testing and results are summarized in Section 6.6 of this report and described extensively in Section 6 of Daniel et al. (2013).

\section{S.2 Results and Performance Against Success Criteria}

The success criteria for achieving the Phase II small-scale aerosol test objectives are discussed in Table S.2. Many of these are carried over from Phase I testing, but they are also specified in the Test Plan. 
Table S.2. Success Criteria for Phase II Small-Scale Aerosol Tests

\begin{tabular}{cc}
\hline & Objective \\
Success Criteria & Met? \\
\hline Objectives (1)-(4) [Note: Objectives 6-9 in the Test Plan]
\end{tabular}

Measure the droplet size distribution, total volume Yes concentration of droplets, and total volume sprayed for each of the breaches and simulants tested.

Measure the pressure and flow in the piping.

A Malvern Insitec-S instrument was used to measure the size distribution and volume concentration of the aerosol for each simulant. The total spray volume was calculated using mass measurements and time data recorded by a data logger.

Measure the pressure and flow in the piping.

Characterize the viscosity or rheology, PSD, bulk density, and surface tension of each simulant tested.

For in-spray measurements, measure the droplet size distribution for each of the breaches and simulants tested.

For the $30 \mathrm{~Pa} / 30 \mathrm{cP}$ simulant at the beginning of testing, at least one Bingham parameter is $30 \pm 4 \mathrm{~Pa}$ or $\mathrm{CP}$ and the second Bingham parameter should be less than or equal to this upper target of $30 \pm 4 \mathrm{~Pa}$ or $\mathrm{cP}$.

For the $6 \mathrm{~Pa} / 6 \mathrm{cP}$ simulant at the beginning of testing, at least one Bingham parameter is $6 \pm 2$ Pa or $c P$ and the second Bingham parameter should be greater than or equal to this lower target of $6 \pm 2 \mathrm{~Pa}$ or $\mathrm{cP}$.

\section{Objective (5) [Note: Objective 10 in the Test Plan]}

Measure the concentration and size distribution of Yes known aqueous suspensions with the Malvern used for aerosol measurements and quantitatively compare the Malvern results with the known values to estimate the Malvern accuracy for measuring aerosols.
Yes

The pressure and flow in the piping (i.e., the spray header pressure and flow rate conditions for the test sprays) were measured and recorded in a test instruction datasheet and with a data logger.

Yes The simulants tested were characterized prior to testing and, in some cases, after testing. The physical property measurements are in Section 3.

Yes A Malvern Insitec-S instrument was used to measure the size distribution and volume concentration of the in-spray aerosol for each simulant tested. Note that in-spray measurements were difficult to collect and the proximity of the Malvern Insitec-S to the orifice prevented the acquisition of meaningful in-spray data.

Yes At the start of testing, both of the $30 \mathrm{~Pa} / 30 \mathrm{cP}$ simulants were within specification (see Section 3):

- Chemical slurry simulant: $28 \mathrm{~Pa} / 28 \mathrm{cP}$

- Clay slurry simulant: $31 \mathrm{~Pa} / 29 \mathrm{cP}$

Yes At the start of testing, both of the $6 \mathrm{~Pa} / 6 \mathrm{cP}$ simulants were within specification (see Section 3):

- Chemical slurry simulant: $6.2 \mathrm{~Pa} / 21 \mathrm{cP}$

- Clay slurry simulant: $7.3 \mathrm{~Pa} / 13 \mathrm{cP}$

\section{Objective (6) [Note: Objective 11 in the Test Plan]}

Quantitative comparison demonstrates that the old and new lenses give equivalent results for aerosol conditions that have droplet sizes in a range appropriate for both lenses.
Yes The Malvern Insitec-S instrument was validated with a series of known mono- and poly-disperse particle dispersions to test its accuracy in measuring concentration, size distribution, and performing corrections for laser attenuation. The findings are presented in Section 6.6 of this report, with a full discussion in Section 6 of Daniel et al. (2013).
Yes
Old $(100 \mathrm{~mm})$ and new $(500 \mathrm{~mm})$ lenses were used to collect aerosol data for two simulants and three orifices. The $500 \mathrm{~mm}$ lens consistently measured release fractions greater than or equal to the $100 \mathrm{~mm}$ lens (and thus, the $500 \mathrm{~mm}$ lens will provide a bounding measurement of release fraction). However, between 10 and $100 \mu \mathrm{m}$, the release fraction measured by the $500 \mathrm{~mm}$ lens had the same dependence on size as that measured by $100 \mathrm{~mm}$ lens, with the measured concentration being larger by a factor of two to three. Thus, the comparison does not yield identical results, but the measured release fractions are considered similar, being generally within the range of uncertainty for the measurements. For additional discussion, see Section 6.4.1. 


\section{S.3 Quality Requirements}

The PNNL Quality Assurance (QA) program is based on requirements defined in DOE Order 414.1D, Quality Assurance, and Title 10 of the Code of Federal Regulations (CFR) Part 830, Energy/Nuclear Safety Management, and Subpart A-Quality Assurance Requirements (a.k.a., the Quality Rule). PNNL has chosen to implement the following consensus standards in a graded approach:

- ASME NQA-1 ${ }^{1}$-2000, Quality Assurance Requirements for Nuclear Facility Applications, Part 1, Requirements for Quality Assurance Programs for Nuclear Facilities

- ASME NQA-1-2000, Part II, Subpart 2.7, Quality Assurance Requirements for Computer Software for Nuclear Facility Applications

- ASME NQA-1-2000, Part IV, Subpart 4.2, Graded Approach Application of Quality Assurance Requirements for Research and Development.

The procedures necessary to implement the requirements are documented in PNNL's "How Do I...?" (HDI) system, which is a web-based system for managing delivery of PNNL policies, requirements, and procedures.

The Waste Treatment Plant Support Project (WTPSP) implements an NQA-1-2000 QA program, graded on the approach presented in NQA-1-2000, Part IV, Subpart 4.2. The WTPSP Quality Assurance Manual (QA-WTPSP-0002) describes the technology life-cycle stages under the WTPSP Quality Assurance Plan (QA-WTPSP-0001). The technology life cycle includes the progression of technology development, commercialization, and retirement in process phases of basic and applied research and development $(R \& D)$, engineering and production, and operation until process completion. The life cycle is characterized by flexible and informal QA activities in basic research, which becomes more structured and formalized through the applied R\&D stages.

The work described in this report has been completed under the QA technology level of Developmental Work. WTPSP addresses internal verification and validation activities by conducting an independent technical review of the final data report in accordance with the WTPSP procedure QA-WTPSP-601, Document Preparation and Change. This independent review verifies that the reported results are traceable, that inferences and conclusions are soundly based, and that the reported work satisfies the test plan objectives.

\section{S.4 Simulant Use}

Several simulants were developed and characterized for use in the small-scale aerosol tests. The simulants were selected to represent a range of relevant physical and rheological properties expected in the WTP (Table S.3 and Table S.4). The properties important to aerosol generation include PSD, viscosity, Bingham plastic rheological parameters (yield stress and plastic viscosity), bulk density, weight percent (wt $\%$ ) of undissolved solids (UDS), and surface tension. Actual simulant properties are reported in Section 3.

\footnotetext{
${ }^{1}$ NQA-1 is the abbreviation for Nuclear Quality Assurance-1, a regulatory standard created and maintained by the American Society of Mechanical Engineers.
} 
Table S.3. Phase II Target Simulants and the WTP Process Stream Categories

\begin{tabular}{|c|c|c|c|}
\hline $\begin{array}{l}\text { Simulant } \\
\text { Class }\end{array}$ & Material & Target Property Range & $\begin{array}{c}\text { WTP Process } \\
\text { Stream Categories }\end{array}$ \\
\hline Baseline & Water & $\begin{array}{l}\text { Viscosity of } 1 \mathrm{mPa} \cdot \mathrm{s}(1 \mathrm{cP}) \\
\text { Density of } 1000 \mathrm{~kg} / \mathrm{m}^{3} \\
\text { Surface tension of } 73 \mathrm{mN} / \mathrm{m}\end{array}$ & $\mathrm{N} / \mathrm{A}$ \\
\hline \multirow[t]{3}{*}{$\begin{array}{l}\text { Range of } \\
\text { slurries (non- } \\
\text { hazardous) }\end{array}$} & $\begin{array}{l}\text { Boehmite particulates } \\
\text { in water }\end{array}$ & $\begin{array}{l}\text { The PSDs of the slurries were selected to match } \\
\text { Hanford waste PSDs (see discussion in } \\
\text { Section } 3.1 \text { of Mahoney et al. 2013) at a } \\
\text { concentration of } 27 \mathrm{wt} \% \text { solids. }\end{array}$ & Newtonian slurries \\
\hline & $\begin{array}{l}\text { Small fraction of Mo } \\
\text { in water and a } \\
\text { boehmite-water slurry }\end{array}$ & $\begin{array}{l}1 \mathrm{wt} \% \text { (in the slurry) Mo particles included to } \\
\text { represent dense particles in the waste such as } \\
\text { plutonium oxides. The boehmite slurry tests had } \\
\text { a total solids loading of } 20 \mathrm{wt} \% \text { (i.e., } 19 \mathrm{wt} \% \\
\text { boehmite solids, } 1 \mathrm{wt} \% \text { Mo solids). }\end{array}$ & $\begin{array}{l}\text { Newtonian slurries } \\
\text { with a small } \\
\text { fraction being } \\
\text { dense particles }\end{array}$ \\
\hline & $\begin{array}{l}\text { Clay slurries } \\
\text { composed of a solids } \\
\text { phase with } 80 \mathrm{wt} \% \\
\text { kaolin and } 20 \mathrm{wt} \% \\
\text { bentonite in water }\end{array}$ & $\begin{array}{l}\text { The total solids loadings were adjusted, via } \\
\text { dilution, before testing began so that one } \\
\text { simulant had at least one Bingham parameter } \\
\text { near } 30 \mathrm{~Pa} / 30 \mathrm{cP} \text { (target range was } 30 \pm 4 \mathrm{~Pa} \text { or } \\
\mathrm{cP} \text { ) and the second Bingham parameter less than } \\
\text { or equal to the } 30 \pm 4 \mathrm{~Pa} \text { or } \mathrm{cP} \text { target. The } \\
\text { second simulant was adjusted so that at least one } \\
\text { Bingham parameter near } 6 \mathrm{~Pa} / 6 \mathrm{cP} \text { (target range } \\
\text { was } 6 \pm 2 \mathrm{~Pa} \text { or } \mathrm{cP} \text { ) and the second } \mathrm{Bingham} \\
\text { parameter greater than or equal to the } 6 \pm 2 \mathrm{~Pa} \text { or } \\
\text { cP target. }\end{array}$ & $\begin{array}{l}\text { Non-Newtonian } \\
\text { slurries }\end{array}$ \\
\hline $\begin{array}{l}\text { Washed and } \\
\text { leached } \\
\text { chemical } \\
\text { slurry } \\
\text { simulant }\end{array}$ & $\begin{array}{l}\text { Fe-rich solids similar } \\
\text { to the simulant used in } \\
\text { Pretreatment } \\
\text { Engineering Platform } \\
\text { (PEP) testing (Kurath } \\
\text { et al. 2009) with } \\
\text { gibbsite solids added }\end{array}$ & $\begin{array}{l}\text { The total solids loadings were adjusted, via } \\
\text { dilution, before testing began so that one } \\
\text { simulant had at least one Bingham parameter } \\
\text { near } 30 \mathrm{~Pa} / 30 \mathrm{cP} \text { (target range was } 30 \pm 4 \mathrm{~Pa} \text { or } \\
\mathrm{cP} \text { ) and the second Bingham parameter less than } \\
\text { or equal to the } 30 \pm 4 \mathrm{~Pa} \text { or } \mathrm{cP} \text { target. The } \\
\text { second simulant was adjusted so that at least one } \\
\text { Bingham parameter near } 6 \mathrm{~Pa} / 6 \mathrm{cP} \text { (target range } \\
\text { was } 6 \pm 2 \mathrm{~Pa} \text { or } \mathrm{cP} \text { ) and the second } \mathrm{Bingham} \\
\text { parameter greater than or equal to the } 6 \pm 2 \mathrm{~Pa} \text { or } \\
\mathrm{cP} \text { target. }\end{array}$ & $\begin{array}{l}\text { Non-Newtonian } \\
\text { slurries }\end{array}$ \\
\hline
\end{tabular}


Table S.4. Phase II Simulant Nomenclature

\begin{tabular}{|c|c|c|c|}
\hline Simulant Description & Alias & Component ${ }^{(\mathrm{a})}$ & Comments \\
\hline Small-treated simulant & STR & Boehmite & As in Phase I, only at higher solids loading \\
\hline Dense particle in water & DPW & Mo particles & Small fraction $(1 \mathrm{wt} \%)$ of dense particles (Mo) \\
\hline $\begin{array}{l}\text { Dense particle in } \\
\text { small-treated simulant }\end{array}$ & DST & $\begin{array}{l}\text { Mo particles } \\
\text { boehmite }\end{array}$ & $\begin{array}{l}\text { Small fraction }(1 \mathrm{wt} \%) \text { of dense particles }(\mathrm{Mo}) \\
\text { combined with boehmite solids ( } 20 \mathrm{wt} \% \text { in total) }\end{array}$ \\
\hline \multirow[t]{2}{*}{$\begin{array}{l}\text { Kaolin-bentonite clay slurry } \\
\text { (also simply clay slurry) }\end{array}$} & KBC-6 & $\begin{array}{l}80 / 20(\mathrm{wt} \%) \\
\text { kaolin/bentonite }\end{array}$ & $\begin{array}{l}\text { Target rheology of } 6 \text { Pa Bingham yield stress, } \\
6 \mathrm{mPa} \cdot \mathrm{s} \text { Bingham consistency }\end{array}$ \\
\hline & $\begin{array}{l}\text { KBC- } \\
30\end{array}$ & $\begin{array}{l}80 / 20(\mathrm{wt} \%) \\
\text { kaolin/bentonite }\end{array}$ & $\begin{array}{l}\text { Target rheology of } 30 \mathrm{~Pa} \text { Bingham yield stress, } \\
30 \mathrm{mPa} \cdot \mathrm{s} \text { Bingham consistency }\end{array}$ \\
\hline \multirow{2}{*}{$\begin{array}{l}\text { Washed and leached } \\
\text { iron-rich chemical slurry } \\
\text { simulant (also called Fe-rich) }\end{array}$} & FEG-6 & $\begin{array}{l}\text { Gibbsite } \\
\text { Fe-Rich }\end{array}$ & $\begin{array}{l}\text { Target rheology of } 6 \mathrm{~Pa} \text { Bingham yield stress, } \\
6 \mathrm{mPa} \cdot \mathrm{s} \text { Bingham consistency }\end{array}$ \\
\hline & FEG-30 & $\begin{array}{l}\text { Gibbsite } \\
\text { Fe-Rich }\end{array}$ & $\begin{array}{l}\text { Target rheology of } 30 \mathrm{~Pa} \text { Bingham yield stress, } \\
30 \mathrm{mPa} \cdot \mathrm{s} \text { Bingham consistency }\end{array}$ \\
\hline
\end{tabular}

(a) Balance of all of the simulants in this table is water.

\section{S.5 Summary of Results}

As in Phase I, the small-scale spray release test system consisted of a relatively small enclosure installed in a walk-in fume hood. This system was used for investigating aerosol formation from smaller breach sizes using hazardous and non-hazardous simulant slurries and water. A positive displacement pump recirculated simulant from a 40-gallon agitated feed vessel through the nominal 1-in.-diameter spray loop pipe at the target flow rate of 11.4 gallons per minute (gpm). This provided a line velocity of $6.1 \mathrm{ft} / \mathrm{s}$, which was chosen to provide approximately the same wall shear stress ${ }^{1}$ (within about 10 percent) that would exist in 3-in. schedule 40 pipe with a flow velocity of $6.5 \mathrm{ft} / \mathrm{s}$, a typical condition in the WTP system. A matched shear stress was desirable in order to obtain similar conditions at the entry to the spray orifice in the small- and large-scale systems, because entry shear could affect the behavior of solids in the simulant slurries.

A wide variety of orifice sizes and geometries could be inserted into the test section, but the number of orifices studied in Phase II was reduced by down-selecting to just three: round orifices with target diameters of 0.5 and $1 \mathrm{~mm}$, and a slot with the target dimensions $0.5 \times 5 \mathrm{~mm}$. The wall thicknesses of the orifices tested were equivalent to that of a 3 -in. schedule 40 stainless steel pipe, thus providing a leak-path length equal to much of the piping used in the WTP. For most of the simulants investigated, tests were conducted in triplicate at target test pressures of 100, 200, and 380 psig.

Aerosol measurements were obtained in real time using a single Malvern Insitec-S instrument. The instrument could be placed at most locations in the aerosol chamber; however, all tests were performed with the Malvern Insitec-S either in the top third of the chamber centered over the spray (an in-chamber test) or in the center of the chamber aligned with the spray (an in-spray test). A mixing fan placed near the bottom center of the chamber minimized heterogeneity in the aerosol concentrations. Additional

\footnotetext{
${ }^{1}$ Approximately the same wall shear stress would be achieved for all the Newtonian and $6 \mathrm{~Pa} / 6 \mathrm{cP}$ simulants used in spray release testing, which was the basis for the calculation. A line velocity of $6.1 \mathrm{ft} / \mathrm{s}$ results in a higher wall shear stress for $30 \mathrm{~Pa} / 30 \mathrm{cP}$ simulants than what would be experienced by $30 \mathrm{~Pa} / 30 \mathrm{cP}$ waste in the WTP.
} 
instruments provided real-time measurements of header flow rate, simulant density, tank mass, flow loop pressure, and temperature that were recorded with data acquisition systems. Approximately 300 separate spray release tests were conducted.

The tests were conducted using a single valve sequence to achieve the target pressure in a consistent manner. The target flow rate was met for all but one simulant (i.e., Fe-rich chemical slurry). In general, aerosol data were collected before the spray started, during the spray (typically lasting $2 \mathrm{~min}$ ), and after the spray was stopped. Still images of representative sprays were also collected. The system temperature was maintained between 65 and $85^{\circ} \mathrm{F}$ to minimize any effects that might be caused by condensation or evaporation. When possible, pre-sprays were conducted to humidify the aerosol chamber, thereby increasing the initial relative humidity and limiting evaporation. Samples were collected from the feed vessel to characterize the initial simulant. These samples were analyzed to determine the PSD, rheological parameters, bulk density, weight percent of UDS, and surface tension. For some simulants, other samples were taken to assess the evolution of a physical parameter of interest, e.g., rheology.

The experimental method focused on measuring the rate of increase in the aerosol concentration in the closed chamber of known volume. Because the chamber is essentially a closed system with no purge flow, the aerosol concentration is initially zero and builds up to a steady-state concentration at which point the net generation of aerosol (the generation by spray minus the capture by the splash wall) is equal to the aerosol losses. The aerosol losses can occur by any of several possible mechanisms (e.g., deposition on the walls, evaporation, and settling), as discussed in Section 3 of Schonewill et al. (2012). Aerosol could also be generated by spray impact with surfaces (splatter). Because the spray is aerosolized in a closed geometry, several of these mechanisms occur simultaneously and are not isolated from one another; however, the complex aerosol physics occurring in the chamber is expected to be representative of actual sprays. Based on a material balance, the initial rate of concentration increase (before losses are significant) gives the aerosol net generation rate from a spray. A key component of this approach is to have a concentration measurement for the chamber representative of the entire chamber, such that the time to achieve uniformity in the chamber (and obtain a representative measurement) is fast relative to the rise time of the concentration increase. Uniformity was demonstrated in Phase I by comparing release fraction data collected at different Malvern Insitec-S locations in the chamber (Mahoney et al. 2013).

A two-part approach was used to analyze data collected during small-scale spray release testing. The first part calculated the leak flow from data for feed tank weight versus time, and used data from the process instruments to determine the average pressure during each test. The average pressure, orifice dimensions, and simulant properties were used in calculating WTP model predictions for the test conditions. This WTP model calculates the aerosol size distribution and leak flow rate for a Newtonian fluid, based on fluid properties, system pressure, and the orifice dimensions and discharge coefficient. A comparison of this release-predicting model to data was one of the goals of the study.

The second part of the analysis used data from the Malvern Insitec-S aerosol instrument. Both differential and cumulative concentration data were analyzed by being fit to a model, allowing the determination of the initial rate of aerosol concentration increase. From this, the aerosol net generation rate was calculated. The net generation rate was divided by the spray leak flow rate to obtain estimates of the release fraction for the experiments. 
A series of shakedown tests were conducted to confirm that changes in the small-scale system configuration, procedure, and instrumentation implemented in Phase II resulted in aerosol data consistent with data collected in Phase I for the same experimental conditions. The shakedown tests occurred prior to the execution of any Phase II tests and repeated selected functional tests performed in Phase I. These tests included the following:

- Establishing that a Malvern Insitec-S aerosol analyzer data collection rate of $4 \mathrm{~Hz}$ did not impact the calculation of release fraction as compared to a data collection rate of $1 \mathrm{~Hz}$ used in Phase I testing.

- Verifying that the chamber mixing fan employed during testing promoted mixing and did not lead to additional heterogeneity in the chamber aerosol concentrations.

- Determining the optimum aerosol instrument configurations, including air purge flow rate of the Malvern Insitec-S for in-spray measurements.

- Examining the effect of initial chamber humidity (as measured by a meter that reported a For Information Only $[\mathrm{FIO}]$ relative humidity $[\mathrm{RH}]$ ) on the measured release fraction. Note that although the FIO humidity sensor was procured with a factory calibration, it was not sufficient for NQA-1 requirements.

- Comparing the aerosol data collected using different lenses installed in the Malvern Insitec-S.

- Performing head-to-head comparison of Phase I and II data collected at the same conditions.

Once the Phase II equipment configuration and operating test conditions were established, comparisons between small-scale test data (cumulative release fractions) collected for various simulants were made. The Phase II small-scale test program yielded the following conclusions:

- The orifice coefficients were determined using a method expected to be more accurate than the Phase I method. Many of the orifice coefficients, especially for the slurry simulants, are significantly greater than the coefficient of 0.62 used by the WTP model.

- The initial RH in the chamber (note: based on an FIO measurement) has an effect on the measured release fraction, in particular for RHs $<80$ percent. Using the data to extrapolate to 100 percent initial $\mathrm{RH}$ and interpolate to 80 percent initial $\mathrm{RH}$, the decrease in release fraction is approximately a factor of two across the range of typical initial RHs. The decrease in release fraction occurs across all droplet sizes. Consequently, release fractions measured at initial $\mathrm{RH}<80$ percent will be biased low by greater than a factor of two.

- In general, the Malvern Insitec-S focal length $(500 \mathrm{~mm})$ used in Phase II testing gave higher release fractions compared to the $100 \mathrm{~mm}$ focal length used in Phase I testing under the same conditions. The data from the $500 \mathrm{~mm}$ lens are higher because of the different measurable size ranges of the lens.

- Phase II release fraction measurements made using a $100 \mathrm{~mm}$ focal-length lens matched Phase I measurements for the same conditions within less than a factor of two.

- The cumulative release fractions did not have a monotonic relationship with pressure for the simulants tested in Phase II. Notably, there was no change or a small decrease in cumulative release fractions at $<10 \mu \mathrm{m},<32 \mu \mathrm{m}$, and $<102 \mu \mathrm{m}$ as pressure increased from 100 to $200 \mathrm{psig}$. Conversely, the cumulative release fractions had a similar increase compared to the rate of the WTP model as pressure increased from 200 to $380 \mathrm{psig}$. This suggests that release fractions at pressures $<100 \mathrm{psig}$ might be more likely to exceed the WTP model than they would be at pressures of $\geq 200$ psig; 
however, all of the apparent pressure trends are rendered ambiguous by the fact that the differences in release fraction from one pressure to another are of about the same size as the estimated uncertainty. Further, it is possible that this trend is dependent on chamber size (a similar trend was not observed in the large-scale chamber). In general, the cumulative release fraction at $<10 \mu \mathrm{m}$ was closer to the WTP model prediction than the release fraction at larger droplet sizes, particularly for the Fe-rich simulant.

- The $27 \mathrm{wt} \%$ small-treated (STR) slurry had cumulative release fractions that were very similar to, or at least indistinguishable from, both water and $20 \mathrm{wt} \%$ STR slurry (a Phase I measurement).

- The addition of a small fraction (nominally $1 \mathrm{wt} \%$ in the slurry) of dense particles (molybdenum with density $=10.2 \mathrm{~g} / \mathrm{cm}^{3}$ ) to water and $19 \mathrm{wt} \%$ STR simulant did not result in a significant effect on the measured release fractions when compared to test data collected when the simulants were devoid of dense particles.

- Comparison of clay slurry and water cumulative release fractions in the droplet size range of interest, i.e., 10 to $100 \mu \mathrm{m}$, showed that the clay release fractions at both 6 and 30 Pa yield stress are less than or equal to those of water.

- The cumulative release fractions of the chemical simulant (FEG) were consistently greater than those of water or the clay slurries. Generally, the $30 \mathrm{~Pa}$ chemical simulant release fractions were about equal to those of the $6 \mathrm{~Pa}$ chemical simulant. The chemical simulant release fractions were greater than those of water by as much as a factor of two to three.

- The PSD data collected for all simulants from in-spray measurements indicated that at least part of the liquid core of the spray was still intact at the point of measurement. Thus, the in-spray data could not be used as an estimate of an upper bound release fraction as it has been in the large-scale testing (see Daniel et al. 2013).

- Studies conducted to evaluate uncertainties surrounding the accuracy and performance of the Malvern Insitec-S aerosol instrument demonstrated that the concentration and particle size measurements of the instrument are not subject to any significant errors or biases and thus, do not require any adjustments. The Malvern Insitec-S accurately measures concentration and PSDs for spherical systems of droplets and applies appropriate corrections to account for attenuation.

The overall uncertainty for a given cumulative release fraction remains difficult to quantify. A number of factors affect the uncertainty in the measured aerosol net generation rates and release fractions. These may include bias in the method (or the exponential model used to calculate generation rate), aerosol measurement, and physical processes (such as evaporation) or randomness arising from variations in test conditions, turbulence of the spray jet, and instrument response. One way to assess the randomness in the experiments is to perform repeat tests. Repeated tests were found to have cumulative release fractions within a factor of two for all the Phase II simulants except for the chemical simulant, the variability of which is subject to a series of caveats. Some potential biases were assessed, including the effect of evaporation and the performance of the Malvern Insitec-S instrument, but a full analysis of uncertainty has not yet been completed. As such, the release fractions presented in this report are not adjusted to account for an estimate of overall uncertainty in the experiments. 


\section{S.6 Discrepancies and Follow-on Tests}

The following discrepancies and suggested follow-on tests associated with the small-scale tests are noted:

The intention of the Phase II small-scale testing was to conduct spray tests where the aerosol chamber was at or near saturation to limit the effect of evaporation. In practice, this was not always achieved due to the use of some challenging simulants. Tests probing the effect of the initial RH in the chamber demonstrated that lower initial humidity results in lower release fractions for test conditions that are otherwise the same. However, these tests were conducted with an FIO meter for the RH and thus, the data appearing in this report is not corrected for the effect of humidity. Performing the correction to 100 percent $\mathrm{RH}$, for example, would increase the release fractions presented herein. This effect was also observed in the large-scale test stand (see Daniel et al. 2013). Because the humidity was not corrected, direct comparison with the WTP model was typically not performed in this report.

The use of a different lens focal length in Phase II (i.e., a $500 \mathrm{~mm}$ focal length instead of the $100 \mathrm{~mm}$ focal length used in Phase I) was implemented to expand the range of droplet sizes that could be measured when in-spray measurements were being conducted. A consequence of that choice for the in-chamber measurements is the aerosol data are not identical (but similar, being typically different by less than or equal to a factor of two with the $500 \mathrm{~mm}$ lens data consistently larger) for the $500 \mathrm{~mm}$ and $100 \mathrm{~mm}$ focal lengths when they are measuring sprays generated at the same conditions. The differences are primarily a result of the different measurable size range. The $500 \mathrm{~mm}$ lens, which has a lower measurement limit of $2.5 \mu \mathrm{m}$, does not include droplets (or particulate) $<2.5 \mu \mathrm{m}$ in the PSD because the detector array is not configured to "see" droplets of this size. However, the laser is still obscured by these droplets and there is a resultant reduction in transmission. This reduction in transmission is interpreted by the instrument as a higher concentration, which is further amplified by an estimate of the Sauter mean diameter that is too large relative to the "true" PSD of the material. This complicated comparison with Phase I data, making some observed trends less certain.

Several attempts made with the small-scale test stand to obtain aerosol generation rates with a chemical slurry simulant representing WTP slurries have had limited success. The interpretation of data collected from tests with a chemical slurry simulant was challenging due to atypical behavior of the simulant, which exhibited a rheology that thickened with shear (dilatant) and was time dependent (rheopectic). The available literature and discussion with subject matter experts indicates that no other Hanford tank waste simulant or actual waste samples have exhibited this type of rheological behavior. Moreover, the UDS solids concentration, which ranged from 35 to $40 \mathrm{wt} \%$, exceeded the current WTP solids concentration upper limit of $27 \mathrm{wt} \%$ and thus was too concentrated to be truly representative. The unstable rheology of the simulant forced modifications to the test equipment and procedure to allow completion of the majority of the aerosol tests. Release fractions measured from chemical simulant tests were typically greater than the release fractions measured for water or clay. Due to the unusual rheology, the elevated UDS solids concentration, and the operational difficulties, direct comparison of the results between the chemical simulant and other simulants is questionable. If results using a chemical slurry simulant are needed, the cause of the unusual rheology should be identified and perhaps a new simulant formulation be developed. Further, use of the large-scale test stand should be considered for further testing of the chemical slurry simulant to allow a wider range of orifice sizes and in-spray measurements. 
In-spray measurements were collected for every condition where an in-chamber test was also performed. The in-spray data represents aerosol generation at the limiting condition of the absence of capture by the walls and other loss mechanisms caused by the geometry of the chamber. However, the proximity of the orifice to the Malvern Insitec-S measurement zone resulted in PSDs that were physically unrealistic. For example, PSDs frequently contained droplets greater than the orifice dimensions by as much as a factor of four and more than 10 percent of the PSD by volume appeared to be larger than the measurement range. It was concluded that the jet was not completely broken up at the location of the measurement. Thus, the in-spray data could not be used as an estimate of an upper bound release fraction as it has been in the large-scale testing (see Daniel et al. 2013). Furthermore, in future work, in-spray tests should be restricted to the large-scale system only or the small-scale chamber geometry will need to be modified to accommodate the acquisition of an accurate measurement (i.e., further downstream from the orifice).

As suggested in the Phase I report (Mahoney et al. 2013), method validation tests in which a well-characterized spray is introduced to the chamber should be considered. Determining whether the testing and analysis method used in both Phase I and II work contains any significant biases is the greatest remaining uncertainty in the aerosol data. These tests would apply the same measurement and analysis methods to sprays with known aerosol generation rate and size distribution to determine how well the estimates of aerosol generation rate match the expected values. Validation of the method would help improve some of the remaining uncertainties associated with the in-chamber measurements made at either scale.

The primary sources of uncertainty are test-to-test variation, bias in the methodology, accuracy of the aerosol measurements, the effect of chamber humidity (a bias), and randomness in the data. For a defensible estimate of uncertainty, the two biggest needs are 1) the method validation discussed in the previous paragraph, and 2) determination of the effect of humidity using a NQA-1 instrument measurement. In some respects, the need for a quantitative study of uncertainty in the small-scale test results is reduced by recent efforts to develop a reasonably conservative correlation based on the large-scale data (see Daniel et al. 2013). These results should allow a better assessment of the available margin in the WTP safety and accident analyses. 



\section{Acknowledgments}

The authors would like to thank Jesse Lang, Greg Boeringa, Susan Sande, Mac Zumhoff, Bill Buchmiller, Chen Song, Gourihar Kulkarni, and Jim Davis for their help in conducting all of the Phase II aerosol tests. We are grateful for the images of the orifices after testing were completed, which were collected by Justin Billing with the assistance of Jeremy Blanchard. Thanks are owed to Richard Daniel and Jagan Bontha for their thorough independent technical review. We also thank Kirsten Meier for her QA support. We are also indebted to Mike Parker, Cary Counts, and Kathy Neiderhiser for their editorial review of this report. Additionally, we greatly appreciate the data and calculation review efforts by Rich Pires, Sarah Suffield, Bill Buchmiller, Justin Billing, Amanda Casella, Christopher Strickland, Susan Sande, Lanée Snow, Marcia Kimura, Gourihar Kulkarni, Chen Song, and Ellen Baer.

We also sincerely appreciate the support, advice, and guidance from Mike Epstein (Fauske and Associates), Ameer Hassan (WTP Technical Lead), Susan Omberg-Carro (WTP), Chris Harrington

(DOE-ORP), Joel Fox (DOE-ORP), Ralph Crowe (Sludge Treatment Project - K-Basin) and Bruce Zimmerman (Hanford Tank Farms).

Funding for this effort was provided by the DOE Hanford Waste Treatment and Immobilization Plant Project. 



\section{Acronyms and Abbreviations}

APEL

AFA

ASME

BV

CFR

CSTR

DOE

DPW

DS

DST

FCV

FEG

FER

FIO

gpm

HDI

$\mathrm{KBC}$

LAW

LRB

Mo

OTP

$\mathrm{PC}$

$\mathrm{PCV}$

PEP

PNNL

ppmv

PSD

PTFE

QA

R\&D

$\mathrm{RCF}$

$\mathrm{RH}$

RI

SAR

SCFH

SMD
Applied Process Engineering Laboratory

anti-foam agent

American Society of Mechanical Engineers

ball valve

Code of Federal Regulations

continuously stirred tank reactor

U.S. Department of Energy

dense particle in water

dissolved solids

dense particle in small-treated

flow control valve

Fe-rich (iron-rich) with gibbsite

Fe-rich (Phase I simulant)

For Information Only

gallons per minute

"How Do I," the standards-based management system for PNNL

Kaolin-Bentonite clay

low-activity waste

Laboratory Record Book

molybdenum

orifice test piece

performance check

pressure control valve

Pretreatment Engineering Platform

Pacific Northwest National Laboratory

parts per million by volume

particle size distribution

polytetrafluoroethylene

quality assurance

research and development

relative centrifugal force

relative humidity

refractive index

small as-received (Phase I simulant)

standard cubic foot per hour

Sauter mean diameter 
SS

STR

TAR

TI

UDS

VFD

WTP

WTPSP small-scale

small treated

typical as-received (Phase I simulant)

test instruction

undissolved solid

variable frequency drive

Hanford Tank Waste Treatment and Immobilization Plant

Waste Treatment Plant Support Project 


\section{Contents}

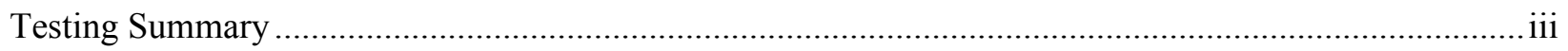

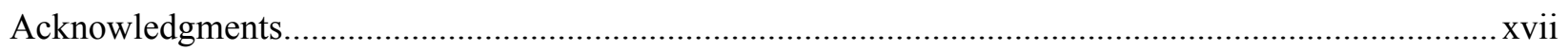

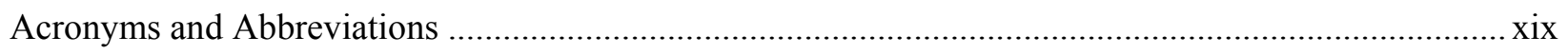

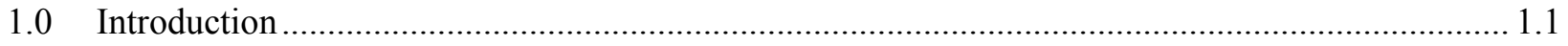

1.1 WTP Model for Estimating Aerosol Release Fraction and Generation ................................ 1.2

1.2 Technical Approach for Determining Aerosol Release Fraction ........................................ 1.4

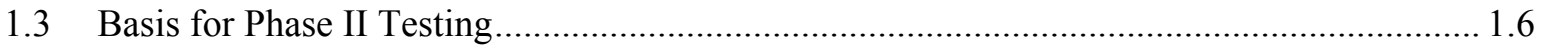

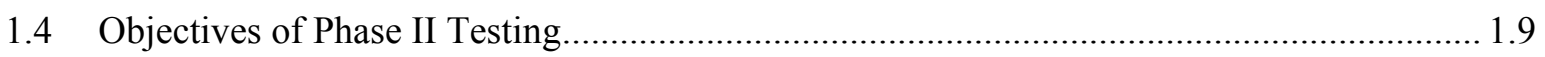

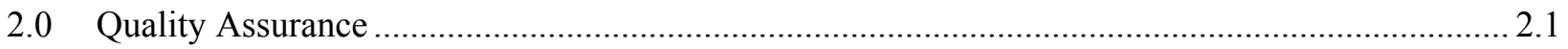

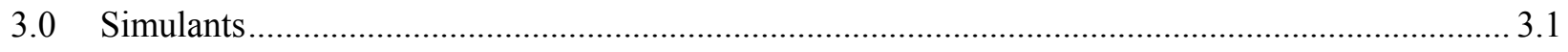

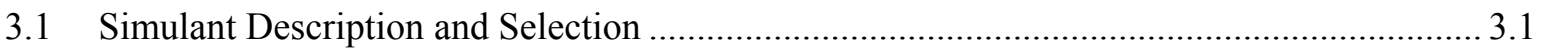

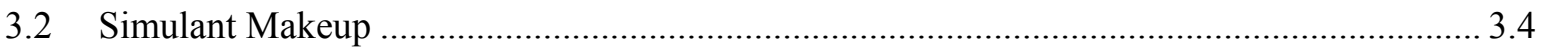

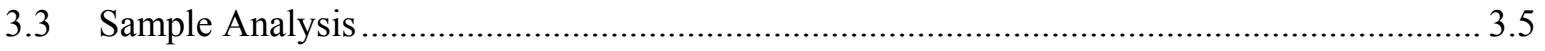

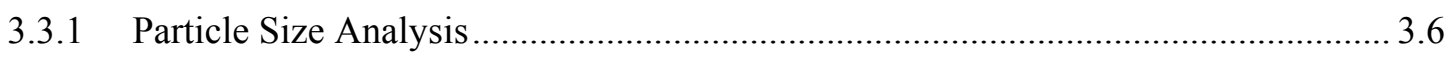

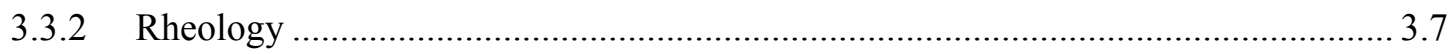

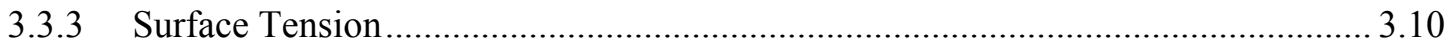

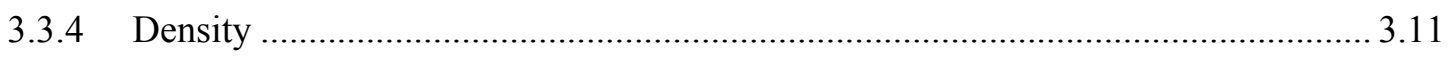

3.3.5 Total Solids Content and Undissolved Solids Content............................................ 3.11

3.3.6 Selection of Refractive Index for Laser Diffraction Analysis ................................. 3.12

3.4 Physical Properties of the Newtonian Simulants............................................................... 3.14

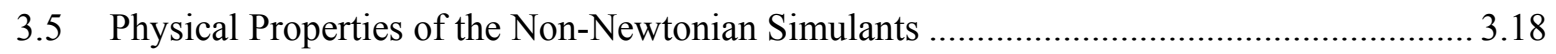

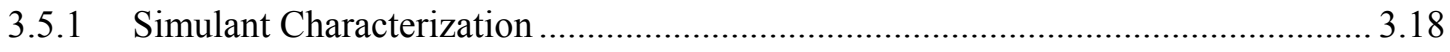

3.5.2 Variation of Non-Newtonian Rheology During Test Operations............................. 3.24

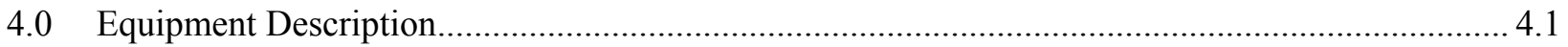

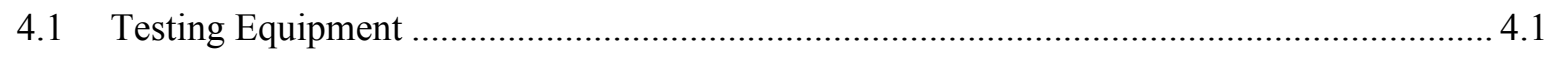

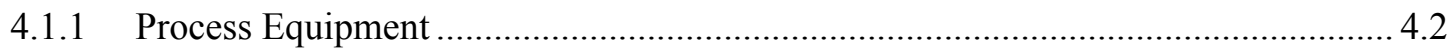

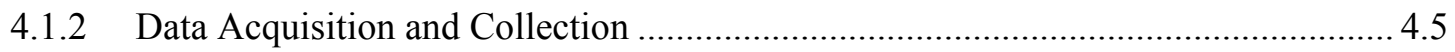

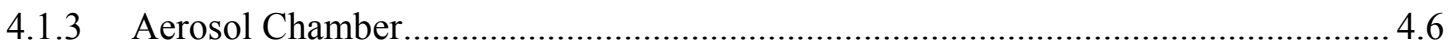

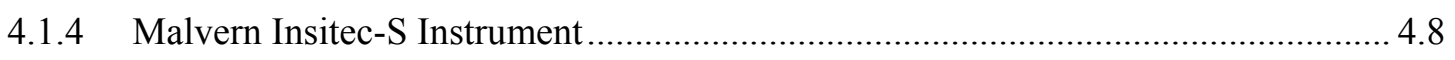

4.2 Modifications to Test System from Phase I .................................................................. 4.12

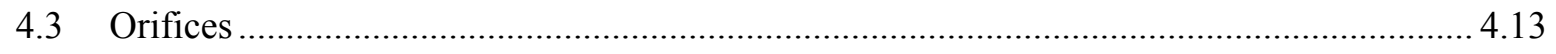

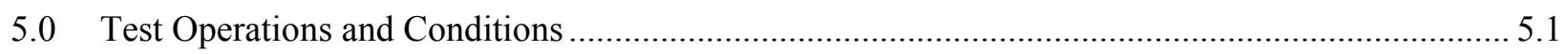

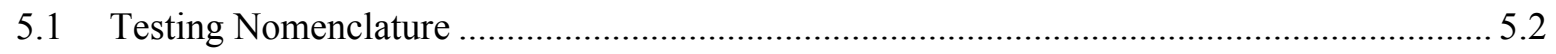

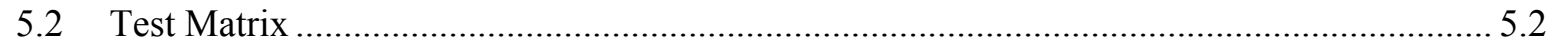

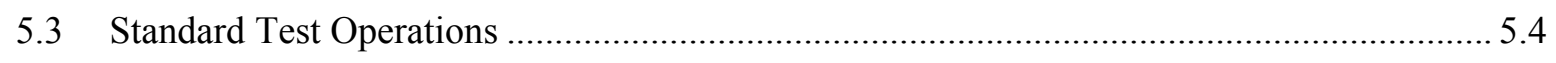

5.3.1 Daily Startup Checks and Preparation.............................................................. 5.4 


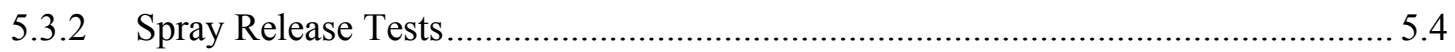

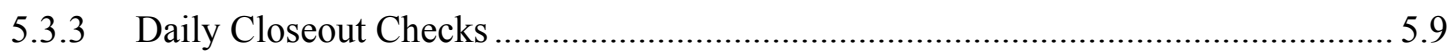

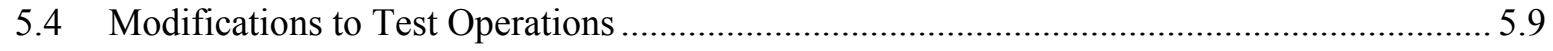

5.4.1 Differences from Phase I Testing ..................................................................... 5.9

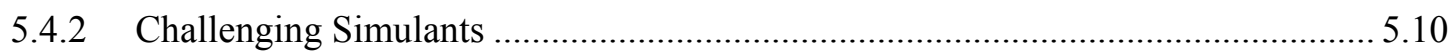

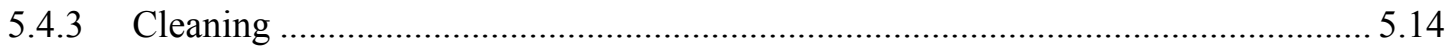

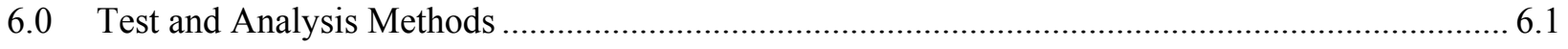

6.1 Analysis of Process Instrument Data...................................................................................... 6.2

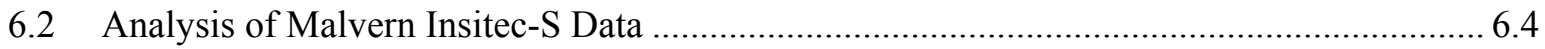

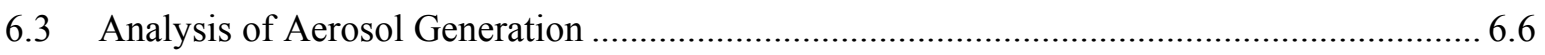

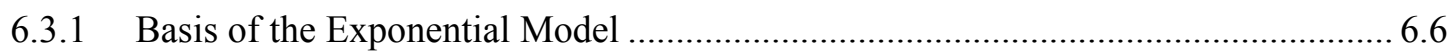

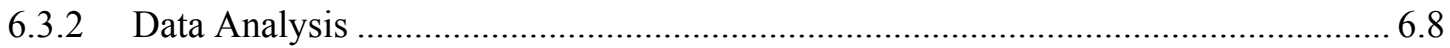

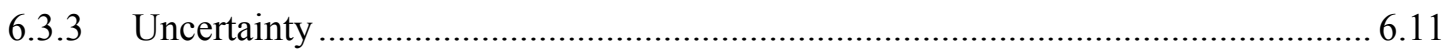

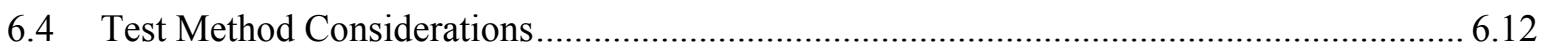

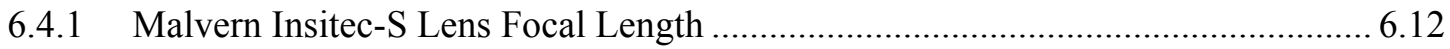

6.4.2 Malvern Insitec-S Data Collection Rate............................................................. 6.15

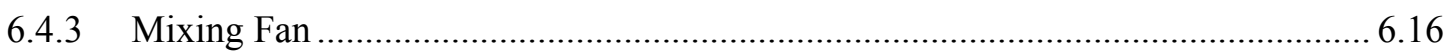

6.4.4 Malvern Insitec-S Instrument Purge Rate ....................................................... 6.18

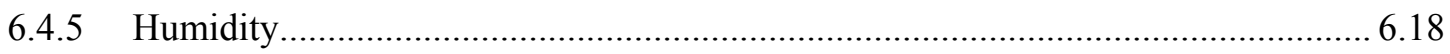

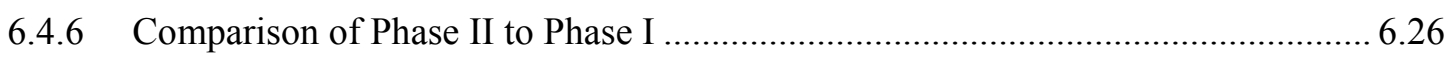

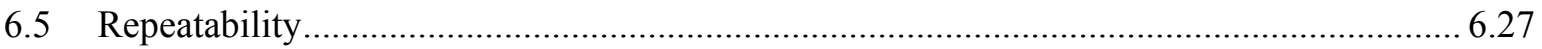

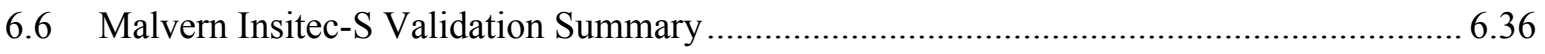

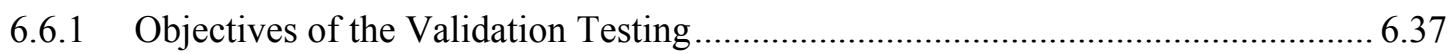

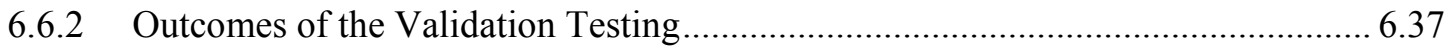

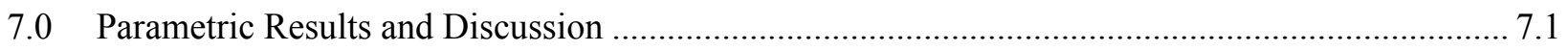

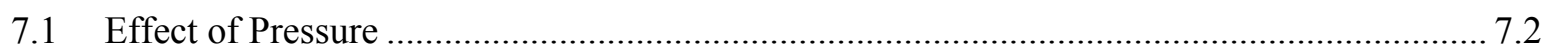

7.2 Effect of Solids Loading in Newtonian Slurry …........................................................ 7.15

7.3 Effect of Dense Particles ................................................................................................ 7.15

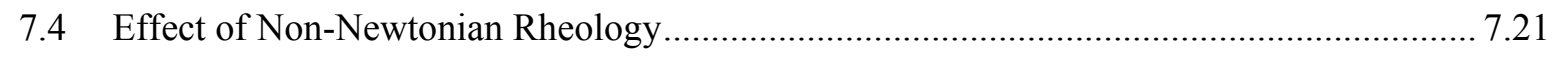

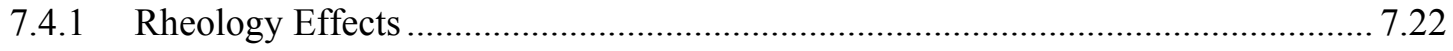

7.4.2 Comparison of Clay and FEG Non-Newtonian Slurries ....................................... 7.29

7.4.3 Discussion of Applicability of FEG Results ....................................................... 7.36

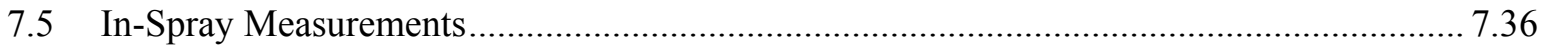

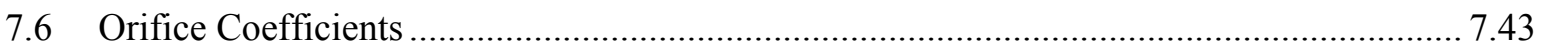

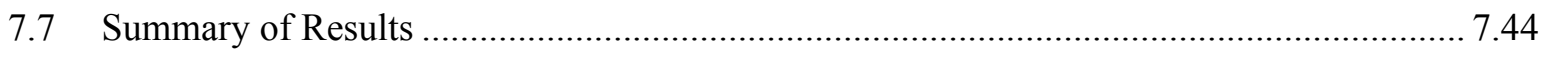

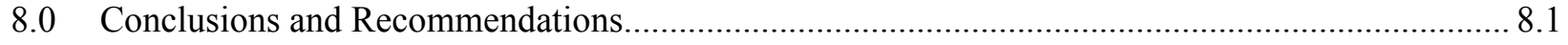

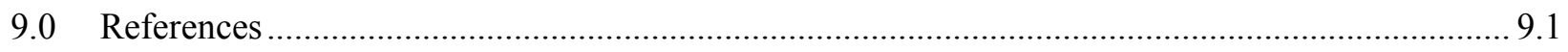

Appendix A - Run Log and Test Conditions................................................................................. A.1

Appendix B - Selected Release Fraction Plots ................................................................................. 


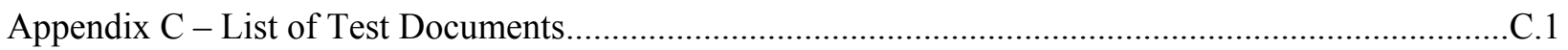

Appendix D - Cross-References for Parametric Plots in Sections 6 and 7 ........................................ D. 1

Appendix E - Selected Rheograms from Phase II Simulants .............................................................. 1

\section{Figures}

1.1. Conceptual Schematic of Aerosol Concentration Increasing with Time, Where the Aerosol Net Generation Rate is Calculated from the Initial Slope ....................................................... 1.5

3.1. Rotor and Cup Geometry Used in Rotational Viscometry Testing in Phase II.............................. 3.8

3.2. Summary of Flow Curve Behaviors Typically Observed for Concentrated Slurries ....................... 3.9

3.3. Cumulative Volume Percent Undersize for Unsonicated $27 \mathrm{wt} \%$ STR Simulant, $20 \mathrm{wt} \%$ DST Simulant, and Mo Powder ............................................................................................ 3.17

3.4. Volume Percent Differential PSDs for Unsonicated and Post-Sonication $27 \mathrm{wt} \%$ STR Simulant, $20 \mathrm{wt} \%$ DST Simulant, and Mo Powder.

3.5. Cumulative Volume Percent Undersize for Unsonicated and Post-Sonication $30 \mathrm{~Pa} / 30 \mathrm{cP}$

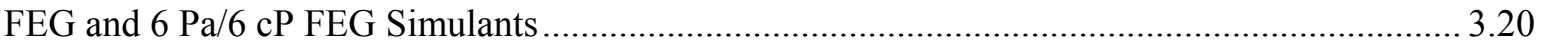

3.6. Volume Percent Differential PSDs for Unsonicated and Post-Sonication 30 Pa/30 cP FEG Simulant

3.7. Volume Percent Differential PSDs for Unsonicated and Post-Sonication 6 Pa/6 cP FEG Simulant....

3.8. Cumulative Volume Percent Undersize PSDs for Unsonicated and Post-Sonication $30 \mathrm{~Pa} / 30 \mathrm{cP} \mathrm{KBC}$ and $6 \mathrm{~Pa} / 6 \mathrm{cP} \mathrm{KBC}$ Simulants

3.9. Volume Percent Differential PSDs for Unsonicated $30 \mathrm{~Pa} / 30 \mathrm{cP} \mathrm{KBC}$ and $6 \mathrm{~Pa} / 6 \mathrm{cP} \mathrm{KBC}$

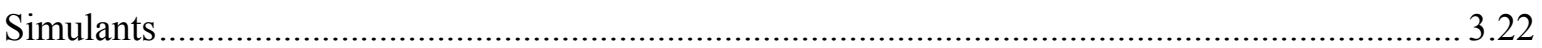

3.10. Bingham Yield Stress as a Function of Testing Time for Clay Slurry Simulants........................ 3.24

3.11. Bingham Consistency as a Function of Testing Time for Clay Slurry Simulants........................ 3.25

3.12. Rheogram for Clay Simulant at the Beginning of $30 \mathrm{~Pa} / 30 \mathrm{cP}$ Testing ...................................... 3.26

3.13. Rheogram for Clay Simulant at the End of $30 \mathrm{~Pa} / 30 \mathrm{cP}$ Testing ...............................................2.27

3.14. Rheogram for Clay Simulant at the Beginning of $6 \mathrm{~Pa} / 6 \mathrm{cP}$ Testing .............................................2.

3.15. Rheogram for Clay Simulant at the End of $6 \mathrm{~Pa} / 6 \mathrm{cP}$ Testing ................................................ 3.28

3.16. Bingham Yield Stress as a Function of Testing Time for Chemical Simulants ........................... 3.29

3.17. Bingham Consistency as a Function of Testing Time for Chemical Simulants ...........................3.29

3.18. Rheogram for Chemical Simulant at the Beginning of $30 \mathrm{~Pa} / 30 \mathrm{cP}$ Testing ............................... 3.30

3.19. Rheogram for Chemical Simulant at the End of $30 \mathrm{~Pa} / 30 \mathrm{cP}$ Testing; Measured Shear Rate Range of 0 to $1000 \mathrm{~s}^{-1}$

3.20. Rheogram for Chemical Simulant at the End of $30 \mathrm{~Pa} / 30 \mathrm{cP}$ Testing; Measured Shear Rate Range of 0 to $400 \mathrm{~s}^{-1}$

3.21. Rheogram for Chemical Simulant at the Beginning of $6 \mathrm{~Pa} / 6 \mathrm{cP}$ Testing; Measured Shear Rate Range of 0 to $400 \mathrm{~s}^{-1}$ 
3.22. Rheogram for Chemical Simulant at the End of $6 \mathrm{~Pa} / 6 \mathrm{cP}$ Testing; Measured Shear Rate Range of 0 to $400 \mathrm{~s}^{-1}$

4.1. Image of the Small-Scale Test System in the Fume Hood........................................................ 4.1

4.2. Example of a Spray from an Orifice Test Piece Installed in the Spray Header ............................. 4.2

4.3. Schematic of the Small-Scale Test Stand Used in Phase II .......................................................... 4.3

4.4. Isometric Drawing of the Aerosol Test Chamber ......................................................................... 4.6

4.5. Front View and Dimensions (in.) of the Small-Scale Chamber .................................................... 4.7

4.6. Basic Instrument Setup and Operation Principle of the Malvern Insitec-S Particle Size

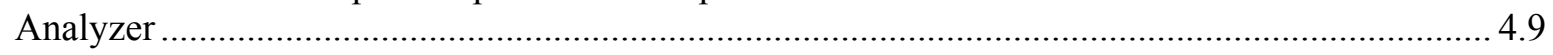

4.7. Conceptual Representation of the Malvern Insitec-S Locations Within the Test Chamber........... 4.10

4.8. Picture of an In-Spray Measurement Conducted in Phase II Testing ......................................... 4.10

5.1. Simplified Diagrams of Small-Scale Valve Protocol.................................................................... 5.8

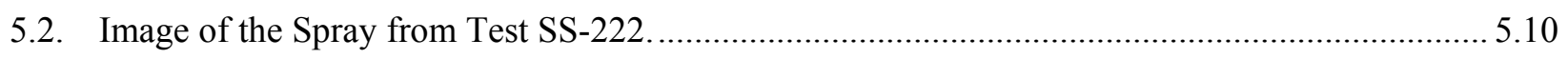

5.3. Comparison of Feed Tank Contents when Operating with FEG Simulant. ................................. 5.11

5.4. View Into the Inlet Line After Testing with the FEG Simulant................................................... 5.13

5.5. Picture of the "Spray Catcher" Used to Verify the Presence of Dense Mo Particles in the

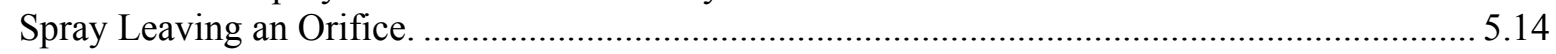

6.1. Effect of Lens Focal Length on Release Fractions for a $0.5 \mathrm{~mm}$ Hole, Water at $380 \mathrm{psig}$........... 6.13

6.2. Effect of Lens Focal Length on Release Fractions for a $1 \mathrm{~mm}$ Hole, $\sim 30 \mathrm{~Pa}$ Clay Slurry at 380 psig

6.3. Effect of Lens Focal Length on Release Fractions for a $0.5 \times 5 \mathrm{~mm}$ Slot, $\sim 30$ Pa Clay Slurry at $380 \mathrm{psig}$

6.4. Effect of Malvern Insitec-S Data Collection Rate on Release Fractions for a $0.5 \mathrm{~mm}$ Hole, Water at $380 \mathrm{psig}$

6.5. Effect of Malvern Insitec-S Data Collection Rate on Release Fractions for a $1 \mathrm{~mm}$ Hole, Water at 380 psig 6.16

6.6. Effect of Fan Speed on Release Fractions for a $0.5 \mathrm{~mm}$ Hole, Water at 380 psig.

6.7. Effect of Initial RH on Release Fractions for a $0.5 \mathrm{~mm}$ Hole, Water at $380 \mathrm{psig}$ 6.20

6.8. Effect of Initial RH on Release Fractions for a $0.5 \mathrm{~mm}$ Hole, Water at $100 \mathrm{psig}$..... 6.21

6.9. Effect of Initial RH on Concentrations of $10-\mu \mathrm{m}$ Droplets for a $0.5 \mathrm{~mm}$ Hole, Water at 380 psig.

6.10. Effect of Initial RH on Concentrations of $102-\mu \mathrm{m}$ Droplets for a $0.5 \mathrm{~mm}$ Hole, Water at 380 psig

6.11. Trends of Release Fraction with Initial Humidity for a $0.5 \mathrm{~mm}$ Hole, Water at $380 \mathrm{psig} . . . \ldots \ldots \ldots . . .6 .24$

6.12. Trends of Release Fraction with Initial Humidity for a $0.5 \mathrm{~mm}$ Hole, Water at $100 \mathrm{psig}$............ 6.25

6.13. Comparison of Phase I and Phase II Results for a $0.5 \mathrm{~mm}$ Round Hole, Water at $380 \mathrm{psig} . . . \ldots \ldots . .6 .27$

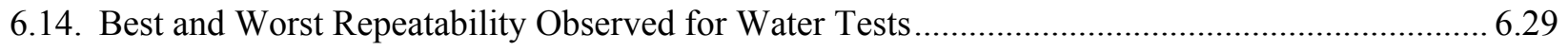

6.15. Best and Worst Repeatability Observed for $6 \mathrm{~Pa} / 6 \mathrm{mPa} \cdot \mathrm{s}$ Clay Tests ......................................... 6.30

6.16. Best and Worst Repeatability Observed for $30 \mathrm{~Pa} / 30 \mathrm{mPa} \cdot \mathrm{s}$ Clay Tests. .................................... 6.31

6.17. Best and Worst Repeatability Observed for $6 \mathrm{~Pa} / 6 \mathrm{mPa} \cdot \mathrm{s}$ FEG Tests ........................................ 6.32

6.18. Best and Worst Repeatability Observed for $30 \mathrm{~Pa} / 30 \mathrm{mPa} \cdot \mathrm{s}$ FEG Tests. ................................... 6.33 
6.19. Best and Worst Repeatability Observed for $27 \mathrm{wt} \%$ STR Tests................................................ 6.34

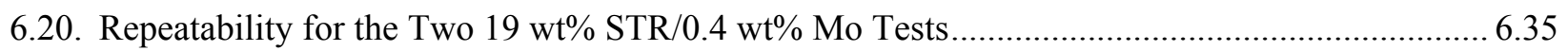

6.21. Repeatability for $0.4 \mathrm{wt} \%$ Mo in Water at $380 \mathrm{psig}, 0.5 \times 5 \mathrm{~mm}$ Slot............................................ 6.36

7.1. Effect of Pressure on Release Fractions for a Spray of Water from a Target $1 \mathrm{~mm}$ Hole. ............. 7.3

7.2. Effect of Pressure on Release Fractions for a Spray of Water from a Target $0.5 \times 5 \mathrm{~mm}$

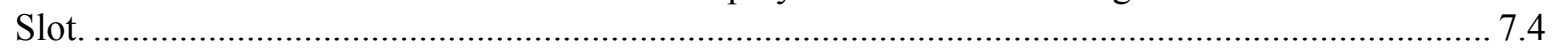

7.3. Effect of Pressure on Release Fractions for a Spray of 6 Pa Clay from a Target $1 \mathrm{~mm}$ Hole......... 7.7

7.4. Effect of Pressure on Release Fractions for a Spray of 6 Pa Clay from a Target $0.5 \times 5 \mathrm{~mm}$

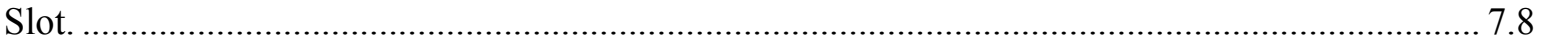

7.5. Effect of Pressure on Release Fractions for a Spray of 30 Pa Clay from a Target $1 \mathrm{~mm}$ Hole.

7.6. Effect of Pressure on Release Fractions for a Spray of 30 Pa Clay from a Target $0.5 \times 5 \mathrm{~mm}$ Slot.

7.7. Effect of Pressure on Release Fractions for a Spray of 6 Pa FEG from a Target $1 \mathrm{~mm}$ Hole........ 7.11

7.8. Effect of Pressure on Release Fractions for a Spray of 6 Pa FEG from a Target $0.5 \times 5 \mathrm{~mm}$ Slot.

7.9. Effect of Pressure on Release Fractions for a Spray of 30 Pa FEG from a Target $1 \mathrm{~mm}$ Hole.

7.10. Effect of Pressure on Release Fractions for a Spray of 30 Pa FEG from a Target $0.5 \times 5 \mathrm{~mm}$ Slot.

7.11. Effect of Pressure on Release Fractions for a Spray of $27 \mathrm{wt} \%$ STR from a Target $1 \mathrm{~mm}$ Hole.

7.12. Effect of Pressure on Release Fractions for a Spray of $27 \mathrm{wt} \%$ STR from a Target $0.5 \times 5 \mathrm{~mm}$ Slot.

7.13. Effect of STR Concentration for a $1 \mathrm{~mm}$ Round Hole at 380 psig. ............................................. 7.18

7.14. Effect of STR Concentration for a $0.5 \times 5 \mathrm{~mm}$ Slot at $380 \mathrm{psig}$............................................... 7.18

7.15. Effect of Dense Particle Concentration in Water for a $1 \mathrm{~mm}$ Round Hole at $380 \mathrm{psig}$................. 7.19

7.16. Effect of Dense Particle Concentration in Water for a $0.5 \times 5 \mathrm{~mm}$ Slot at 380 psig ................... 7.20

7.17. Effect of Dense Particle Concentration in $20 \mathrm{wt} \%$ STR Slurry for a $1 \mathrm{~mm}$ Round Hole at $380 \mathrm{psig}$

7.18. Effect of Dense Particle Concentration in $20 \mathrm{wt} \%$ STR Slurry for a $0.5 \times 5 \mathrm{~mm}$ Slot at 380 psig

7.19. Effect of Rheological Parameters in Clay Slurry for a $1 \mathrm{~mm}$ Round Hole at 100 psig................ 7.23

7.20. Effect of Rheological Parameters in Clay Slurry for a $1 \mathrm{~mm}$ Round Hole at 200 psig................ 7.23

7.21. Effect of Rheological Parameters in Clay Slurry for a $1 \mathrm{~mm}$ Round Hole at $380 \mathrm{psig}$................. 7.24

7.22. Effect of Rheological Parameters in Clay Slurry for a $0.5 \times 5 \mathrm{~mm}$ Slot at 100 psig. .................. 7.24

7.23. Effect of Rheological Parameters in Clay Slurry for a $0.5 \times 5 \mathrm{~mm}$ Slot at $200 \mathrm{psig}$................... 7.25

7.24. Effect of Rheological Parameters in Clay Slurry for a $0.5 \times 5 \mathrm{~mm}$ Slot at $380 \mathrm{psig}$................... 7.25

7.25. Effect of Rheological Parameters in FEG Slurry for a $1 \mathrm{~mm}$ Round Hole at $100 \mathrm{psig}$................. 7.26

7.26. Effect of Rheological Parameters in FEG Slurry for a $1 \mathrm{~mm}$ Round Hole at 200 psig................ 7.26

7.27. Effect of Rheological Parameters in FEG Slurry for a $1 \mathrm{~mm}$ Round Hole at 380 psig................ 7.27 
7.28. Effect of Rheological Parameters in FEG Slurry for a $0.5 \times 5 \mathrm{~mm}$ Slot at $100 \mathrm{psig}$................... 7.27

7.29. Effect of Rheological Parameters in FEG Slurry for a $0.5 \times 5 \mathrm{~mm}$ Slot at 200 psig ................... 7.28

7.30. Effect of Rheological Parameters in FEG Slurry for a $0.5 \times 5 \mathrm{~mm}$ Slot at 380 psig .................... 7.28

7.31. Clay and FEG Slurries at 30 Pa Target Yield Stress, Using a $1 \mathrm{~mm}$ Round Hole at 100 psig....... 7.30

7.32. Clay and FEG Slurries at 30 Pa Target Yield Stress, Using a $1 \mathrm{~mm}$ Round Hole at 200 psig....... 7.30

7.33. Clay and FEG Slurries at 30 Pa Target Yield Stress, Using a $1 \mathrm{~mm}$ Round Hole at 380 psig....... 7.31

7.34. Clay and FEG Slurries at $30 \mathrm{~Pa}$ Target Yield Stress, Using a $0.5 \times 5 \mathrm{~mm}$ Slot at 100 psig......... 7.31

7.35. Clay and FEG Slurries at 30 Pa Target Yield Stress, Using a $0.5 \times 5 \mathrm{~mm}$ Slot at $200 \mathrm{psig}$.......... 7.32

7.36. Clay and FEG Slurries at $30 \mathrm{~Pa}$ Target Yield Stress, Using a $0.5 \times 5 \mathrm{~mm}$ Slot at $380 \mathrm{psig}$......... 7.32

7.37. Clay and FEG Slurries at 6 Pa Target Yield Stress, Using a $1 \mathrm{~mm}$ Round Hole at 100 psig......... 7.33

7.38. Clay and FEG Slurries at 6 Pa Target Yield Stress, Using a $1 \mathrm{~mm}$ Round Hole at 200 psig......... 7.33

7.39. Clay and FEG Slurries at 6 Pa Target Yield Stress, Using a $1 \mathrm{~mm}$ Round Hole at 380 psig......... 7.34

7.40. Clay and FEG Slurries at 6 Pa Target Yield Stress, Using a $0.5 \times 5 \mathrm{~mm}$ Slot at 100 psig........... 7.34

7.41. Clay and FEG Slurries at 6 Pa Target Yield Stress, Using a $0.5 \times 5 \mathrm{~mm}$ Slot at 200 psig........... 7.35

7.42. Clay and FEG Slurries at 6 Pa Target Yield Stress, Using a $0.5 \times 5 \mathrm{~mm}$ Slot at 380 psig. ........... 7.35

7.43. Cumulative In-Spray Size Distribution for a 380 psig Jet of Water from a $0.5 \mathrm{~mm}$ Round Hole.

7.44. Differential In-Spray Size Distribution for a 380 psig Jet of Water from a $0.5 \mathrm{~mm}$ Round Hole.

7.45. Differential In-Spray Size Distribution for a 380 psig Jet of Water/Mo from a $0.5 \mathrm{~mm}$ Round Hole

7.46. Cumulative In-Spray Size Distribution for a 380 psig Jet of 30 Pa Clay from a $0.5 \mathrm{~mm}$ Round Hole

7.47. Differential In-Spray Size Distribution for a 380 psig Jet of 30 Pa Clay from a 0.5 mm Round Hole

\section{Tables}

S.1 Summary of Phase II Small-Scale Aerosol Test Objectives and Results................................... v

S.2 Success Criteria for Phase II Small-Scale Aeorsol Tests ............................................................ vii

S.3 Phase II Target Simulants and the WTP Process Stream Categories ........................................... ix

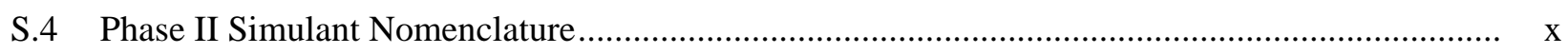

3.1. WTP Process Stream Categories and Representative Fluid Properties......................................... 3.1

3.2. Target Simulants for Phase II and the WTP Process Stream Categories ........................................ 3.2

3.3. Summary of Component Masses for Batches of Simulants Used for Phase II Small-Scale

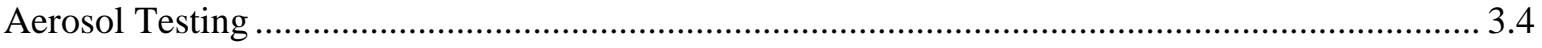

3.4. Summary of Malvern Mastersizer 2000 Instrument Information ................................................... 3.6

3.5. Refractive Indices for Simulants Used in Small-Scale Spray Leak Testing. ................................ 3.15

3.6. Newtonian Simulants Used in Phase II Testing ............................................................................ 3.16 
3.7. Summary of Properties for Newtonian Slurries Used for Aerosol Testing ................................. 3.16

3.8. Non-Newtonian Simulants and Parent Materials Used in Phase II Testing ................................. 3.18

3.9. Summary of Measured Properties for Non-Newtonian Simulants Used for Phase II Small-Scale Aerosol Testing

4.1. Instruments Used in Small-Scale Aerosol Tests and Data Analysis .............................................. 4.6

4.2. Dimensions of the Chamber Configuration During Phase II Testing .......................................... 4.8

4.3. Malvern Insitec-S Instrument Configuration and Software Parameters Used in Typical Measurements .....

4.4. Exit Dimensions for the Orifices for Which Release Fractions Were Determined in Phase II Testing

4.5. Comparison of Exit Dimensions Measured Before and After Aerosol Testing

5.1. Phase II Test Matrix: Planned and Actual Tests 5.3

7.1. Orifice Coefficients Measured in Round Holes in Phase I and Phase II 7.43

7.2. Orifice Coefficients Measured in the $0.5 \times 5 \mathrm{~mm}$ Slot in Phase I and Phase II 7.44 



\subsection{Introduction}

One of the events postulated in the hazard analyses for the Waste Treatment and Immobilization Plant (WTP) is a breach in process piping that produces a spray with aerosol droplet sizes in the respirable range. The postulated breach is expected to be rough and irregular, and could result from a number of causes (e.g., jumper connection misalignment, pipe erosion/corrosion, mechanical impact, seal/gasket failures).

In general, per Hanford practice, the generation rates and size distributions of aerosol droplets produced in spray leaks have been predicted using correlations published in the literature. These correlations are based on results obtained from small engineered spray nozzles using solids-free liquids. However, the fluids processed at the WTP include slurries and high-viscosity liquids with very different properties than those of the liquids used to develop the correlations currently used to evaluate spray leaks. Further, the range of geometries postulated for random breaches differs from the geometry of the engineered spray nozzles used to develop the correlation in terms of nozzle or orifice throat geometry, aspect ratio, and cross-sectional area. Therefore, the correlations used to model spray leaks from process piping may not accurately represent spray leak conditions at the WTP (or elsewhere on the Hanford Site).

In response to this concern, Pacific Northwest National Laboratory (PNNL) conducted aerosol spray leak testing to provide data to support WTP hazard analyses. PNNL's test program addressed two key technical areas to improve the WTP methodology (Larson and Allen 2010). The first technical area was to quantify the role of slurry particles in small breaches where slurry particles may plug the hole and prevent high-pressure sprays. The results from an effort to address the first technical area can be found in Mahoney et al. (2012). The second technical area was to determine aerosol droplet size distribution and total droplet volume from prototypic breaches and fluids, including sprays from larger breaches and sprays of slurries for which literature data are largely absent. To address the second technical area, the testing program collected aerosol generation data at two scales, commonly referred to as small-scale and large-scale. Small-scale testing and resultant data are described in Mahoney et al. (2013) and large-scale testing and resultant data are presented in Schonewill et al. (2012). In tests at both scales, simulants were used to mimic the relevant physical properties projected for actual WTP process streams.

Examination of the results from the initial aerosol spray leak testing (subsequently referred to as Phase I) described in the preceding paragraph highlighted some uncertainties in evaluating aerosol generation that remained after the original scope of work was completed. The need for additional aerosol spray leak testing (subsequently referred to as Phase II) was identified. The purpose of the study described in this report is to provide experimental data and analysis to supplement the Phase I results and reduce uncertainty in the remaining technical area (i.e., determining aerosol droplet size distribution and total droplet volume from prototypic breaches and fluids) by performing small-scale tests with a range of orifice sizes and orientations representative of typical WTP conditions. Specifically, there was uncertainty with respect to aerosol behavior (namely aerosol release fraction and generation rates) for:

- simulant(s) near the WTP rheological boundaries of (6 Pa/6 mPa $\mathrm{s}$ and $30 \mathrm{~Pa} / 30 \mathrm{mPa} \cdot \mathrm{s})$

- simulant(s) with solids loadings greater than $20 \mathrm{wt} \%$

- simulant(s) with a small solids fraction of particles that have a relatively high density 
- lower spray pressures (i.e., specifically 100 and 200 psig in the small-scale tests)

- in-spray measurements.

As the list indicates, the testing and analysis in the Phase II small-scale study was primarily concerned with measuring sprays with simulants that were not used in Phase I. The companion study describing the Phase II large-scale testing is available in Daniel et al. (2013).

Section 1.1 presents the WTP model for estimating aerosol release fraction and generation. The model predictions outlined therein are used to compare expected values with the data collected during testing. Section 1.2 provides an overview of the technical approach used to obtain release fraction data. Section 1.3 summarizes selected findings of the initial aerosol testing (Phase I) and describes the need for additional aerosol testing (Phase II). Finally, Section 1.4 presents the objectives of the Phase II small-scale testing.

Additional sections of this report present the details of the testing and the data collected. The rest of the report proceeds as follows:

- Section 2 details the basis of the PNNL Quality Assurance (QA) program that governs the work described in this report

- Section 3 describes the liquid and slurry simulants used in testing

- Section 4 provides a description of the equipment and instruments used to perform Phase II testing

- Section 5 summarizes the test operations as conducted in Phase II and observed test behavior

- Section 6 provides the analysis methodology and other data analysis considerations

- Section 7 discusses the Phase II data and results

- Section 8 contains the conclusions of the study

- Section 9 compiles the references used in the report.

The appendices provide a table of test metadata and conditions (Appendix A), release fraction plots for completed tests (Appendix B), a list of the technical documents governing this work (Appendix C), a cross-reference table for parametric plots in Sections 6 and 7 (Appendix D), and selected rheograms for the simulants used in testing (Appendix E).

\subsection{WTP Model for Estimating Aerosol Release Fraction and Generation}

Larson and Allen (2010) summarize the methodology used by the WTP for estimating the aerosol release fraction and generation rate of spray releases and McAllister (2010) provides additional details on the equations and method. The method uses the theoretically based correlation by Dombrowski and Johns (1963) for estimating the Sauter mean diameter (SMD) and then the Rosin and Rammler (1933)

distribution for estimating the aerosol droplet size distributions. A primary objective of the current study is to collect data to determine the range of applicability of the method. The Dombrowski and Johns (1963) equations used in the WTP methodology for estimating the SMD are 


$$
\begin{gathered}
d_{l}=0.9614\left(\frac{\mathrm{K}^{2} \sigma^{2}}{\rho_{l} \rho_{a} V^{4}}\right)^{1 / 6}\left[1+2.6 \mu\left(\frac{\mathrm{K} \rho_{a}^{4} V^{7}}{72 \rho_{l}^{2} \sigma^{5}}\right)^{1 / 3}\right]^{1 / 5} \\
d_{d}=1.882 d_{l}\left[1+\frac{3 \mu}{\left(\rho_{l} d_{l} \sigma\right)^{1 / 2}}\right]^{1 / 6} \\
S M D=0.63 d_{d}
\end{gathered}
$$

where $\quad d_{l}=$ the theoretical ligament diameter $(\mathrm{m})$

$d_{d}=$ the theoretical droplet diameter $(\mathrm{m})$

$\mu=$ the liquid viscosity $\left(\mathrm{Pa}^{*} \mathrm{~s}\right)^{1}$

$\rho_{l}=$ the liquid density $\left(\mathrm{kg} / \mathrm{m}^{3}\right)$

$\rho_{a}=$ the air density $\left(\mathrm{kg} / \mathrm{m}^{3}\right)$

$\sigma=$ the surface tension of the liquid $(\mathrm{N} / \mathrm{m})$

$\mathrm{V}=$ the fluid velocity at the breach $(\mathrm{m} / \mathrm{s})$

$\mathrm{K}=$ the spray nozzle parameter $\left(\mathrm{m}^{2}\right)$.

The K parameter is determined with the (McAllister 2010) relationship

$$
\mathrm{K}=\frac{0.5 \mathrm{~A}}{\sin (\theta / 2)}
$$

where $\mathrm{A}$ is the area of the breach for all shapes and $\theta$ is the full spray angle, assumed to be the maximum value of $150^{\circ}$ for a fan spray. Using the assumed maximum value of the spray angle, $\sin (\theta / 2)$ is practically unity (0.97) and $K$ is approximately $A / 2$.

Other applications of the Dombrowski and Johns (1963) model for spray release evaluations have used models for the $\mathrm{K}$ parameter that distinguish between breaches with different shapes, rather than just using the area for all breaches (Crowe 2010; Williams 2000).

The SMD for a particular spray can be determined using Equations (1.1) to (1.4). To determine the fraction of a spray contained in droplets below any particular size for a spray release accident analysis, a relationship is needed for the droplet size distribution. For the WTP methodology, Larson and Allen (2010) use the Rosin and Rammler (1933) distribution and further assume that the release fraction of a spray is equal to the droplet size distribution. The following equations give the release fraction for sprays used in the WTP methodology:

$$
\begin{gathered}
R F=1-\exp \left[-\left(\frac{S M D}{X} \frac{D}{S M D}\right)^{q}\right] \\
\frac{S M D}{X}=[\Gamma(1-1 / q)]^{-1}
\end{gathered}
$$

where $\quad D=$ droplet size

$q=$ a fitting constant that provides a measure of the spread in the droplet size distribution

$R F=$ fraction of the total spray volume contained in drops of diameter less than $\mathrm{D}$

\footnotetext{
${ }^{1}$ A typographical error in McAllister (2010) shows incorrect units for viscosity (uses kinematic viscosity units), but uses the correct viscosity and units in the example calculation.
} 


$$
\begin{aligned}
S M D & =\text { Sauter mean diameter } \\
X & =\text { a characteristic diameter } \\
\Gamma & =\text { gamma function }
\end{aligned}
$$

Larson and Allen (2010) evaluated the value of $q$ and chose $q=2.4$, which gives a constant value of the ratio $\mathrm{SMD} / \mathrm{X}=0.65415$.

To determine the aerosol generation rate from a spray, the flow rate of the spray is needed in addition to the release fraction given by Equation (1.5). The generation rate $\mathrm{G}$ is given by

$$
\mathrm{G}=\mathrm{Q} * \mathrm{RF}
$$

where $Q$ is the volumetric flow of the spray $\left(\mathrm{m}^{3} / \mathrm{s}\right)$ and $\mathrm{G}$ is the generation rate $\left(\mathrm{m}^{3} / \mathrm{s}\right)$ of droplets less than diameter $\mathrm{D}$ (not accounting for any losses in the system).

For use in Equation (1.1), the velocity of the liquid leaving the orifice can be determined from the pressure difference with an orifice flow equation (e.g., see Denn 1980). The WTP model (as described in McAllister (2010)) uses the following orifice flow equation with a typical value of 0.62 for the orifice coefficient:

$$
\mathrm{V}=0.62\left(\frac{2 \Delta P}{\rho_{l}}\right)^{1 / 2}
$$

Here $\Delta P$ is the difference between the pressure in the pipe and the discharge pressure (i.e., atmospheric pressure).

In the WTP model, the volumetric flow for calculating the total release with Equation (1.7) is simply the spray velocity times the area of the orifice.

$$
\mathrm{Q}=\mathrm{V} * \mathrm{~A}
$$

The equations presented above represent the WTP model. Predictions from this model are compared with some of the experimental results shown in Sections 6 and 7. The model predictions presented in these sections will show the quantitative dependence on the various parameters in the Dombrowski and Johns (1963) correlation.

\subsection{Technical Approach for Determining Aerosol Release Fraction}

The technical approach used in Phase I aerosol testing remained the same for Phase II testing, with some minor changes. The constancy in approach permits comparison (subject to the caveat that there were differences in equipment, e.g., Malvern Insitec-S lens with different working droplet size ranges) between Phase I and Phase II data sets, where applicable. Section 6 presents further specifics of the aerosol measurement and calculation methodology.

The primary experimental method used in Phase II consists of measuring the rate of increase in aerosol concentration in a closed chamber of known volume. Using a simple material balance (i.e., a model based on assumed aerosol behavior) the initial rate of concentration increase gives the aerosol net generation rate from a spray. Figure 1.1 shows a conceptual example of concentration increase with time, for different cumulative droplet volumes, which show an initial rapid and linear increase in aerosol 
concentration with time. Eventually, the concentrations no longer increase linearly with time and approach steady-state values. This behavior results from aerosol losses in the chamber. Section 6 includes an analysis of this behavior and discusses the method used to determine the initial slope from the collected data. In this report, the experimental method is often referred to as the in-chamber measurement and, for all intents and purposes, is interchangeable with measurements of the rate of concentration increase with time.

A key component of the experimental approach is to have a concentration measurement in the chamber that is representative of the entire chamber, such that the time to achieve uniformity in the chamber (and obtain a representative measurement) is fast relative to the time of the concentration increase. In Phase I, tests were done with the Malvern Insitec-S in various locations in the chamber and the release fraction data suggested the chamber was well-mixed.

An advantage of using a chamber is that it allows for isolation of the spray, providing a safe testing platform for spraying simulants that are chemically hazardous. In addition, this methodology allows testing in different-sized chambers (e.g., a larger chamber could accommodate larger sprays but the overall experimental and data analysis approach would be the same). Further, creating sprays inside a chamber allows the spray to impact the walls of the chamber and influence aerosol generation (by splatter) and loss (by wall capture, which was observed in Phase I testing to be a dominant loss mechanism). These additional mechanisms of droplet physics are likely to occur in spray leak accidents and help provide a more physically representative estimate of the net aerosol generation in a closed system. The approach of measuring the concentration increase in a closed chamber also allows the role of changing orifice-to-wall distances to be determined. Because of the size and configuration of WTP piping, sprays could impact internal systems (e.g., walls, pipes, valves) at distances ranging from inches to hundreds of feet. In addition, because the same experimental method was used in both the small- and large-scale tests, results can be compared to assess the effect of chamber geometry or perform extrapolations to longer distances with more confidence than was previously possible.

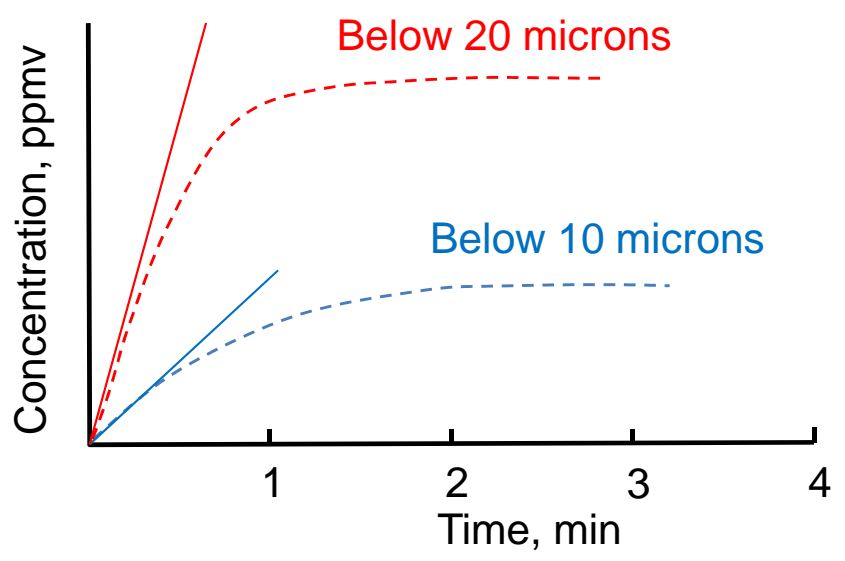

Figure 1.1. Conceptual Schematic of Aerosol Concentration Increasing with Time, Where the Aerosol Net Generation Rate is Calculated from the Initial Slope (solid lines)

One outcome of assessing the data collected during Phase I testing was the observation that the in-spray measurements represented an upper bound on the release fraction for a particular spray (i.e., orifice, pressure, simulant, and chamber configuration) and may be useful for estimating worst cases 
(Schonewill et al. 2012). That is, an in-chamber measurement could approach, but not exceed, the in-spray measurement. However, only a limited number of in-spray measurements were made in the Phase I small-scale testing (Mahoney at al. 2013). Because of the limited number of data sets, Phase II testing focused on collecting a greater number of in-spray measurements.

In-spray measurements, as the name suggests, measure the aerosol directly in the downstream path of the spray, positioned vertically with the jet centerline. Measurements of this type provide a measurement of the aerosol droplet size distribution produced by the spray before loss mechanisms affect the size distribution. The release fraction for any given size of droplet is equal to its volume fraction in the spray, as given by the droplet size distribution. Though in-spray measurements are useful as worst-case estimates, they are difficult to make and the droplet size distribution is dependent on the measurement position. For instance, Epstein and Plys (2006) showed that in-spray measurements at a reasonable distance $(0.5$ to $1 \mathrm{~m})$ become difficult for larger orifices because the liquid core of the jet remained intact. In particular, they had to measure the droplet size distribution a short distance from the center of the spray for the largest slot they tested $(1.2 \times 10 \mathrm{~mm})$ to avoid the liquid core. In addition, the measured in-spray droplet size distribution does not account for the droplets in the spray moving at different velocities. This affects how long droplets of different sizes remain in the instrument measuring zone and results in the presence of an unknown spatial variation of droplet concentrations within the spray. This effect introduces uncertainty in equating the spray release fraction with the measured droplet size distribution. The literature gives methods for determining the spatial variation in droplet concentration (Boyaval and Dumouchel 2001) and for determining the velocity distribution across a spray (e.g., see Levy et al. 1997); however, these studies indicate that determining spatial variation in the concentration and velocity is quite challenging.

\subsection{Basis for Phase II Testing}

The Phase II testing was executed in response to gaps (or remaining areas of uncertainty) identified after analysis of the initial (Phase I) test data had been completed. Since one of the findings in the Phase I testing was that the WTP model did not always bound the measured release fractions, filling these gaps and reducing some of the uncertainty in the data was needed to assess the ability of the model to bound aerosol behavior across the expected range of parameters in the WTP. In general, the small-scale Phase II testing concentrated on assessing the aerosol generation of previously unstudied simulants and the large-scale testing was concerned with collecting data to improve the extrapolation of the results to larger scales. The particular simulants of interest were:

- simulant(s) near the WTP rheological boundaries, which previously had not been explored

- simulant(s) with solids loadings greater than $20 \mathrm{wt} \%$ to test at or near the bounding UDS for WTP slurries

- simulant(s) with a small solids fraction of particles that have a relatively high density.

In the course of testing these new simulants, there were opportunities to expand the measurement data base by conducting additional tests at lower pressures and collecting in-spray data. The basis for conducting the Phase II tests for each of these simulants is outlined below, as each addresses a different technical gap identified during Phase I testing. 
As mentioned previously, Phase I small-scale testing was performed with several different simulants chosen to imitate WTP process streams. For some of the tested slurry simulants, the measured release fraction exceeded the measured release fraction for water (for part or all of the droplet size range) when sprayed under the same conditions. In particular, the chemical simulant (also referred to as Fe-rich or FER in Phase I) tested in Phase I had the largest divergence from the water data, especially at droplet sizes $\leq 10 \mu \mathrm{m}$. The intent during testing with the chemical simulant was to control the rheology at the current rheological limits ${ }^{1}$ for non-Newtonian slurries in the WTP, which are $30 \mathrm{~Pa} / 30 \mathrm{cP}$ for the upper limit and $6 \mathrm{~Pa} / 6 \mathrm{cP}$ for the lower limit (Gimpel 2010). In practice, the rheology of the chemical simulant (which had anti-foam agent [AFA] added to it) was closer to $15 \mathrm{~Pa} / 16 \mathrm{cP}$ for the target $30 \mathrm{~Pa} / 30 \mathrm{cP}$ FER+AFA and $10 \mathrm{~Pa} / 14 \mathrm{cP}$ for the target $6 \mathrm{~Pa} / 6 \mathrm{cP}$ FER+AFA (see Table 3.8 in Mahoney et al. 2013). Note that in Phase I, the rheology of the chemical simulant was not intended to be a formally controlled parameter (i.e., it was not an objective of the test plan).

As stated in the preceding paragraph, Phase I testing indicated that some slurries, particularly those with non-Newtonian rheology, were measured to have in-chamber release fractions greater than that of water over a portion of the droplet size range of interest (10 to $100 \mu \mathrm{m})$ and would be the bounding materials for worst-case WTP safety analyses. However, the full extent of the effect of rheology had not been probed because the Phase I FER simulant was not at the rheological limits defined for WTP operation. Small-scale testing with a chemical simulant at the $30 \mathrm{~Pa} / 30 \mathrm{cP}$ and $6 \mathrm{~Pa} / 6 \mathrm{cP}$ rheological limits, where the rheology is controlled as tightly as possible, could assist in assessing whether a non-Newtonian slurry is the bounding case. Very few lower pressure (100 and $200 \mathrm{psig}$ ) spray tests were conducted with the chemical simulant in Phase I; thus the effect of pressure on the aerosol behavior of non-Newtonian slurries in the small-scale system was largely unknown and additional tests using chemical simulant were needed. The data from the literature is very sparse (see Nasr et al. 2002), but results from literature studies suggest that high-pressure sprays of non-Newtonian slurries, generally those with small particles, should behave similarly to Newtonian fluids with viscosities that match the high shear rate viscosities of the slurries. However, a recent study by Zhao et al. (2011), based on using coal water slurries with yield stresses of 1 to $8 \mathrm{~Pa}$, suggests that a different breakup mechanism governs non-Newtonian slurries as the yield stress becomes non-negligible.

Furthermore, one significant discovery of the Phase I large-scale testing was that when testing orifices with the largest cross-sectional areas release fractions approached or exceeded the WTP model predictions for release fractions (Schonewill et al. 2012). During Phase I large-scale testing, the largest orifices were only tested with water; however, because slurry release fractions were observed to be higher than those for water in some of the Phase I small-scale testing, Phase II large-scale testing included non-Newtonian slurries (see Daniel et al. 2013). Ideally, a chemical simulant would have been tested in the large-scale system as a potential worst-case spray. However, the large-scale system was not designed to operate with hazardous simulants. Thus, a non-hazardous rheological simulant (clay slurry) was used. In addition, to permit better extrapolation to the larger orifice sizes, the non-hazardous rheological simulant could be tested in the small-scale system. This would allow both a hazardous (Fe-rich) simulant and non-hazardous (clay slurry) simulant with similar rheological properties (at or near the WTP rheological boundaries) to be tested at the same scale. Assuming that the relationship between the release fractions of the two rheological simulants determined in small-scale testing was similar at larger scale, the

\footnotetext{
${ }^{1}$ In this report, the rheological properties are referred to as, for example, $30 \mathrm{~Pa} / 30 \mathrm{cP}$ or $6 \mathrm{~Pa} / 6 \mathrm{cP}$. The first number is the Bingham yield stress and the second number is the Bingham consistency (sometimes called plastic viscosity). Two equivalent units ( $\mathrm{cP}$ and $\mathrm{mPa} \cdot \mathrm{s}$ ) for consistency are used interchangeably in this report.
} 
relationship could be used to predict aerosol behavior of hazardous chemical slurries at larger orifice sizes based on the clay simulant data collected in the large-scale system.

Two areas of uncertainty with Newtonian slurries were also identified after the completion of Phase I testing. One area involves the effect of solids loading on the measured release fraction. Several Newtonian slurries were tested in Phase I at nominal undissolved solids (UDS) loadings of 8 and $20 \mathrm{wt} \%$ ( $20 \mathrm{wt} \%$ being the upper bounding UDS value for the WTP). In general, the measured release fractions from these tests exhibited little or no difference from those of water tests at equivalent conditions (Mahoney et al. 2013). Recently, however, the WTP has increased the upper bounding UDS value to $27 \mathrm{wt} \%$ in the vessels commonly referred to as the non-Newtonian vessels. Though no significant effect on release fraction due to increased UDS was expected, small-scale tests with at least one Newtonian slurry at nominally $27 \mathrm{wt} \%$ UDS could assess whether the effect of solids loading found in Phase I was still applicable.

The second area of uncertainty for Newtonian slurries is related to the presence of a small fraction of dense particles in the slurry solids. It was unknown if differences in particle density in slurries at the same UDS would significantly affect release fractions. The dense particles are a proxy for heavy particles that will be found in the WTP, such as plutonium-containing solids. From the Phase I small-scale test effort, a database of Newtonian slurry aerosol data was already available. By selecting at least one of the previously tested slurries and substituting a dense particle for a small fraction of the UDS, the effect of the presence of dense particles could be evaluated. This is necessary because there are no data in the literature for slurries with dense particles; for a discussion of the effect on aerosol formation when slurries contain solid particles, refer to Section 1.3.4 in Mahoney et al. (2013). Resolving these two areas of uncertainty also necessitated the ability to compare easily across Phase I and Phase II data sets, an activity which required confirming that test configuration changes in Phase II yielded similar release fraction results to Phase I.

Lastly, the Phase I testing encompassed a broad range of variables and, due to the size of the parameter space being explored, had some data gaps. In particular, replicate test data was not always obtained and tests were not always conducted at lower pressures (100 or 200 psig). In addition, Phase I testing did not focus on collecting in-spray measurements. Phase II testing provided an opportunity to fill in some of these gaps with a more focused set of in-chamber tests (i.e., using a smaller set of simulants and orifice sizes), which could be performed at three target pressures in triplicate. In-spray measurements could also be collected at all the same conditions.

An unrelated, but important, uncertainty outstanding from the Phase I aerosol testing was the performance of the Malvern Insitec-S instrument. Early in the Phase I testing, the Malvern Insitec-S was identified as the primary aerosol instrument and all subsequent data analysis was derived from Malvern Insitec-S measurements. Because the experimental method depends on an accurate and well-resolved measure of droplet concentration and size distribution with time, understanding the performance of the Malvern Insitec-S is an issue that applies to both the small- and large-scale testing. Reticle studies presented in the Phase I reports suggested that the cumulative particle size distribution (by volume) measured by the Malvern Insitec-S instrument becomes less precise below volume fractions of approximately $5 \times 10^{-3}$. The error in the concentration measurement was estimated to be \pm 40 percent by performing an evaluation of the laser diffraction technique (see Section 5.3.2 in Schonewill et al. 2012). The measurement of precision in Phase I was based on a significant number of data sets, but the assessment of accuracy was anecdotal: it was based on a single data set and was not conducted using the 
Malvern Insitec-S, but a related instrument using the same analysis principle. A more rigorous study to validate the Malvern Insitec-S performance and assess its accuracy has been conducted in Phase II to address this uncertainty. The validation work is fully described in Daniel et al. (2013) and only the portions with direct bearing on the small-scale results are summarized in this report.

\subsection{Objectives of Phase II Testing}

The Phase II small-scale testing described in this report was designed to address the remaining areas of uncertainty described in the previous section. Phase II testing focused on using comparative studies (e.g., measuring the release fractions across simulant types) to observe the effects of various physical properties. The objectives of Phase II testing were less broad than those of Phase I and examined smaller sections of the available parameter space. The objectives governing the Phase II small-scale testing were defined as follows in the Test Plan: ${ }^{1}$

1. Aerosol Quantification of Small-Scale Spray Leaks with Chemical Slurry Simulant at 6 Pa/6 cP and $30 \mathrm{~Pa} / 30 \mathrm{cP}$

Determine the size distribution of aerosol droplets and the total droplet volume concentration as a fraction of the total spray volume for a chemical slurry simulant representative of a washed and leached process stream. Also determine the droplet size distribution directly in the spray (in-spray measurement). The rheology of the simulant will be adjusted so that one slurry, at the beginning of testing, has at least one Bingham parameter near $30 \mathrm{~Pa} / 30 \mathrm{cP}$ (target range is $30 \pm 4 \mathrm{~Pa}$ or cP) and the second Bingham parameter should be less than or equal to the upper $30 \pm 4 \mathrm{~Pa}$ or cP target. The second simulant will be adjusted so that one slurry has at least one Bingham parameter near $6 \mathrm{~Pa} / 6 \mathrm{cP}$ (target range is $6 \pm 2 \mathrm{~Pa}$ or $\mathrm{cP}$ ) and the second Bingham parameter should be greater than or equal to the lower $6 \pm 2 \mathrm{~Pa}$ or $c P$ target. Testing will be conducted with at least one circular and one rectangular breach at target pressures between 100 and 380 psig.

2. Aerosol Quantification of Small-Scale Spray Leaks with Clay Slurry Simulants Achieving Rheology Limits of $6 \mathrm{~Pa} / 6 \mathrm{cP}$ and $30 \mathrm{~Pa} / 30 \mathrm{cP}$

Determine the size distribution of aerosol droplets and the total droplet volume concentration as a fraction of the total spray volume for non-Newtonian clay slurries. Also determine the droplet size distribution directly in the spray (in-spray measurement). The rheology of the simulant will be adjusted so that one slurry, at the beginning of testing, has at least one Bingham parameter near $30 \mathrm{~Pa} / 30 \mathrm{cP}$ (target range is $30 \pm 4 \mathrm{~Pa}$ or $\mathrm{cP}$ ) and the second Bingham parameter should be less than or equal to the upper $30 \pm 4 \mathrm{~Pa}$ or $\mathrm{cP}$ target. The second simulant will be adjusted so that one slurry has at least one Bingham parameter near $6 \mathrm{~Pa} / 6 \mathrm{cP}$ (target range is $6 \pm 2 \mathrm{~Pa}$ or $c P$ ) and the second Bingham parameter should be greater than or equal to the lower $6 \pm 2 \mathrm{~Pa}$ or $\mathrm{cP}$ target. Testing will be conducted with at least one circular and one rectangular breach at target pressures between 100 and 380 psig to compare with small-scale the chemical slurry simulant test results and to support large-scale testing with equivalent clay slurry simulants.

3. Aerosol Quantification of Small-Scale Spray Leaks of a Non-Hazardous Slurry at $\mathbf{2 7} \mathbf{w t} \%$ UDS Determine the size distribution of aerosol droplets and the total droplet volume concentration as a fraction of the total spray volume for a slurry simulant with $27 \mathrm{wt} \%$ UDS. Also determine the droplet size distribution directly in the spray (in-spray measurement) for this simulant.

\section{Aerosol Quantification of Small-Scale Spray Leaks of a Non-Hazardous Slurry with a Small} Fraction of Very Dense Particles

\footnotetext{
${ }^{1}$ Gauglitz PA. 2012. Test Plan for Spray Leak Quantification to Support WTP Spray Release Methodology. TP-WTPSP-031 R1.0, Pacific Northwest National Laboratory, Richland, Washington.
} 
Determine the size distribution of aerosol droplets and the total droplet volume concentration as a fraction of the total spray volume for a slurry simulant with $20 \mathrm{wt} \%$ UDS including a target quantity of about $1 \mathrm{wt} \%$ of very dense particles. Also determine the droplet size distribution directly in the spray (in-spray measurement) for this simulant.

\section{Malvern Insitec-S Validation Testing}

Assess the capability of the Malvern Insitec-S in-process particle size analyzer, which is the instrument used in the aerosol testing, to measure accurately the concentration and size distribution of samples. This will be accomplished by measuring carefully controlled dilute aqueous slurries of known concentration and PSD and comparing the Malvern Insitec-S result to the known values. Testing will include mono- and poly-disperse suspensions and will evaluate all four Malvern Insitec-S configurations of 1) Phase I small-scale spacers and old lens, 2) Phase II small-scale spacers and new lens, 3) Phase I large-scale spacers and old lens, and 4) Phase II large-scale spacers and new lens (old lens $100 \mathrm{~mm}$, new lens $500 \mathrm{~mm}$ ).

\section{Compare Aerosol Measurements from Old and New Malvern Insitec-S Lenses}

Compare the aerosol results from the Malvern Insitec-S using the new 500-mm lens that has a nominal measurement range of $2.5-2500 \mu \mathrm{m}$ (Malvern Instruments, Ltd. 2010) to aerosol results using the 100-mm lens employed in Phase I (Mahoney et al. 2013) that provided a nominal range of 0.5 to $200 \mu \mathrm{m}$ (Malvern Instruments, Ltd. 2010). Tests will use one or more orifices.

The testing supporting these objectives is presented in the remainder of this report. 


\subsection{Quality Assurance}

The PNNL QA program is based upon the requirements defined in the U.S. Department of Energy (DOE) Order 414.1D, Quality Assurance, and 10 CFR 830, Energy/Nuclear Safety Management, and Subpart A-Quality Assurance Requirements (a.k.a., the Quality Rule). PNNL has chosen to implement the following consensus standards in a graded approach:

- ASME NQA-1 ${ }^{1}$-2000, Quality Assurance Requirements for Nuclear Facility Applications, Part 1, Requirements for Quality Assurance Programs for Nuclear Facilities

- ASME NQA-1-2000, Part II, Subpart 2.7, Quality Assurance Requirements for Computer Software for Nuclear Facility Applications

- ASME NQA-1-2000, Part IV, Subpart 4.2, Graded Approach Application of Quality Assurance Requirements for Research and Development.

The procedures necessary to implement the requirements are documented through PNNL's "How Do I...?” (HDI) system, which is a web-based system for managing delivery of PNNL policies, requirements, and procedures.

The Waste Treatment Plant Support Project (WTPSP) implements an NQA-1-2000 QA program, graded on the approach presented in NQA-1-2000, Part IV, Subpart 4.2. The WTPSP Quality Assurance Manual (QA-WTPSP-0002) describes the technology life-cycle stages under the WTPSP Quality Assurance Plan (QA-WTPSP-0001). The technology life cycle includes the progression of technology development, commercialization, and retirement in process phases of basic and applied research and development $(\mathrm{R} \& \mathrm{D})$, engineering and production, and operation until process completion. The life cycle is characterized by flexible and informal QA activities in basic research, which becomes more structured and formalized through the applied R\&D stages.

The work described in this report has been completed under the QA technology level of Developmental Work. WTPSP addresses internal verification and validation activities by conducting an independent technical review of the final data report in accordance with the WTPSP procedure QA-WTPSP-601, Document Preparation and Change. This independent review verifies that the reported results are traceable, that inferences and conclusions are soundly based, and that the reported work satisfies the test plan objectives.

\footnotetext{
${ }^{1}$ NQA-1 is the abbreviation for Nuclear Quality Assurance-1, a regulatory standard created and maintained by the American Society of Mechanical Engineers.
} 



\subsection{Simulants}

This chapter discusses the simulants used for Phase II small-scale aerosol tests. Section 3.1 provides simulant descriptions and the basis for their selection. Section 3.2 describes the preparation of simulants for testing in the small-scale system. Section 3.3 discusses simulant characterization methods. Sections 3.4 and 3.5 discuss the physical properties measured for Newtonian and non-Newtonian simulants, respectively.

\subsection{Simulant Description and Selection}

Table 3.1 lists the WTP process streams and typical ranges for important fluid properties. ${ }^{1}$ The ranges of properties and descriptions are only generalized representations; actual waste examples may vary. The process stream categories shown in Table 3.1 are those chosen to be simulated in the spray leak testing. The non-Newtonian simulants represent slurries expected to be in the vessels commonly referred to as the non-Newtonian vessels. These include the ultrafiltration feed vessels (UFP-VSL-00002 A/B) and the high-level waste lag storage and blend vessels (HLP-VSL-0027 A/B and HLP-VSL-0028). During some of the process steps the slurries in the ultrafiltration feed vessels are expected to exhibit a Newtonian rheology. Other vessels are expected to contain Newtonian slurries, including, but not limited to, the high-level waste receipt vessel (HLP-VSL-00022) and the ultrafiltration feed preparation vessels (UFP-VSL-0001 A/B).

Table 3.1. WTP Process Stream Categories and Representative Fluid Properties

\begin{tabular}{|c|c|c|c|c|}
\hline & $\begin{array}{c}\text { WTP Process } \\
\text { Stream Categories }\end{array}$ & Particles & Composition & Viscosity/Rheology \\
\hline & $\begin{array}{l}\text { Ultrafilter Permeate } \\
\text { Treated LAW }\end{array}$ & negligible & $\begin{array}{c}\text { Caustic solution } \\
5-10 \mathrm{M} \mathrm{Na}\end{array}$ & $\begin{array}{l}\text { Newtonian } \\
2-3 \mathrm{cP}\end{array}$ \\
\hline & Cs Ion Exchange Eluate & negligible & $\begin{array}{c}\mathrm{Na}, \mathrm{K} \text {, Cs ions with } \\
0.5 \mathrm{M} \text { Nitric Acid }\end{array}$ & $\begin{array}{c}\text { Newtonian } \\
0.5 \mathrm{cP} \text { and above }\end{array}$ \\
\hline & Recycle Streams & $<2 \mathrm{wt} \%$ & $0.2-2 \mathrm{M} \mathrm{Na}$ & $\begin{array}{c}\text { Newtonian } \\
0.5 \mathrm{cP} \text { and above }\end{array}$ \\
\hline & Newtonian Slurries & about $2-16$ wt $\%^{(a)}$ & up to $8 \mathrm{M} \mathrm{Na}$ & $\begin{array}{l}\text { Newtonian }{ }^{(b)} \\
\text { about } 1-3 \mathrm{cP}\end{array}$ \\
\hline & Non-Newtonian Slurries & up to $\sim 20 \mathrm{wt} \%$ & $0.2-2 \mathrm{M} \mathrm{Na}$ & $\begin{array}{c}\text { Non-Newtonian }{ }^{(\mathrm{c})} \\
6 \mathrm{cP} / 6 \mathrm{~Pa} \text { to } 30 \mathrm{cP} / 30 \mathrm{~Pa}\end{array}$ \\
\hline (a) & \multicolumn{4}{|c|}{$\begin{array}{l}\text { The upper limit of about } 16 \mathrm{wt} \% \text { corresponds to a limit of } 200 \mathrm{~g} / \mathrm{L} \text { in the waste acceptance criteria (ICD-19 } \\
\left.2011^{(\mathrm{d})}\right) \text {. A new upper limit of } 144 \mathrm{~g} / \mathrm{L} \text { in } 7 \mathrm{M} \text { Na feed, which corresponds to about } 10 \mathrm{wt} \% \text { solids, has been } \\
\text { recommended (Campbell et al. 2010). } \\
\text { This category could also be a weakly non-Newtonian fluid based on the feed acceptance criteria allowing up to } \\
\left.1 \text { Pa Bingham yield stress slurries to be delivered to the WTP (ICD- } 192011^{(\mathrm{d})}\right) \text {. } \\
\text { In this report the rheology limits are referred to as } 30 \mathrm{~Pa} / 30 \mathrm{cP} \text { and } 6 \mathrm{~Pa} / 6 \mathrm{cP} \text {. The first number is the Binghan } \\
\text { yield stress and the second number is the Bingham consistency (sometimes called plastic viscosity). } \\
\text { ICD 19. 2011. ICD } 19 \text { - Interface Control Document for Waste Feed. } 24590 \text { WTP ICD MG } 01 \text { 19, Rev. } 5 \text {, } \\
\text { River Protection Project, Richland, Washington. }\end{array}$} \\
\hline
\end{tabular}

\footnotetext{
${ }^{1}$ These categories and ranges of process parameters were provided by the WTP client as guidance for proposal preparation before the start of Phase I testing (see also TP-WTPSP-031, Rev. 1.0).
} 
Table 3.2 summarizes the four target simulant classes and materials chosen to represent the range of wastes shown in Table 3.1 for the spray leak testing effort. The final column of Table 3.2 matches each chosen simulant with a representative WTP process stream category. The primary process streams in the WTP represented by the selected Newtonian simulants include ultrafilter permeate, treated low-activity waste (LAW), Cs ion exchange eluate, and recycle streams. ${ }^{1}$ Tap water was a Newtonian simulant used for shakedown testing and to obtain baseline aerosol characterization data for both Phases I and II testing. Newtonian aqueous salt solution simulants were used for Phase I testing and are discussed in both Mahoney et al. (2013) and Schonewill et al. (2012).

Table 3.2. Target Simulants for Phase II and the WTP Process Stream Categories

\begin{tabular}{|c|c|c|c|}
\hline Simulant Class & Material [Acronyms] & Target Property Range & $\begin{array}{l}\text { WTP Process } \\
\text { Stream } \\
\text { Categories } \\
\end{array}$ \\
\hline Baseline & Water [W] & $\begin{array}{l}\text { Viscosity of } 1 \mathrm{mPa} \cdot \mathrm{s}(1 \mathrm{cP}) \\
\text { Density of } 1000 \mathrm{~kg} / \mathrm{m}^{3} \\
\text { Surface tension of } 73 \mathrm{mN} / \mathrm{m}\end{array}$ & $\mathrm{N} / \mathrm{A}$ \\
\hline $\begin{array}{l}\text { Range of } \\
\text { Newtonian } \\
\text { viscosity } \\
\text { [Phase I only] }\end{array}$ & $\begin{array}{l}\text { Solutions of water and } \\
\text { non-hazardous salts } \\
\text { (sodium nitrate and } \\
\text { sodium thiosulfate) }\end{array}$ & Viscosities of $\sim 1.5,2.5 \mathrm{cP}$ & $\begin{array}{l}\text { Ultrafilter } \\
\text { permeate/ } \\
\text { treated LAW } \\
\text { Cs ion } \\
\text { exchange eluate } \\
\text { and recycle } \\
\text { streams }\end{array}$ \\
\hline \multirow[t]{3}{*}{$\begin{array}{l}\text { Range of } \\
\text { slurries (non- } \\
\text { hazardous) }\end{array}$} & $\begin{array}{l}\text { Boehmite particulates in } \\
\text { water [STR] }\end{array}$ & $\begin{array}{l}\text { The particle size distribution (PSD) of the } \\
\text { particulates was selected to match that of } \\
\text { Hanford waste (see discussion in Section } 3.1 \text { of } \\
\text { Mahoney et al. 2013) at a concentration of } \\
27 \mathrm{wt} \% \text { solids [STR27] }\end{array}$ & $\begin{array}{l}\text { Newtonian } \\
\text { slurries }\end{array}$ \\
\hline & $\begin{array}{l}\text { Small fraction of } \\
\text { molybdenum (Mo) in } \\
\text { water [DPW] and a } \\
\text { boehmite-water slurry } \\
\text { [DST] }\end{array}$ & $\begin{array}{l}1 \mathrm{wt} \% \text { (in the slurry) Mo particles included to } \\
\text { represent dense particles in the waste (e.g., } \\
\text { plutonium oxides). The Mo/boehmite slurry } \\
\text { tests had a total solids loading of } 20 \mathrm{wt} \% \\
\text { (19 } \mathrm{wt} \% \text { boehmite solids, } 1 \mathrm{wt} \% \text { Mo solids). }\end{array}$ & $\begin{array}{l}\text { Newtonian } \\
\text { slurries with a } \\
\text { small fraction } \\
\text { being dense } \\
\text { particles }\end{array}$ \\
\hline & $\begin{array}{l}\text { Clay slurries composed } \\
\text { of a solids phase with } \\
80 \mathrm{wt} \% \text { kaolin and } \\
20 \mathrm{wt} \% \text { bentonite in } \\
\text { water }[\mathrm{KBC}]\end{array}$ & $\begin{array}{l}\text { The total solids loadings were adjusted, via } \\
\text { dilution, before testing began to meet target } \\
\text { Bingham parameters of } 30 \mathrm{~Pa} / 30 \mathrm{cP}[\mathrm{KBC}-30] \\
\text { and } 6 \mathrm{~Pa} / 6 \mathrm{cP}[\mathrm{KBC}-6]\end{array}$ & $\begin{array}{l}\text { Non-Newtonian } \\
\text { slurries }\end{array}$ \\
\hline $\begin{array}{l}\text { Washed and } \\
\text { leached } \\
\text { chemical slurry } \\
\text { simulant }\end{array}$ & $\begin{array}{l}\text { Fe-solids similar to the } \\
\text { simulant used in } \\
\text { Pretreatment } \\
\text { Engineering Platform } \\
\text { (PEP) testing (Kurath } \\
\text { et al. 2009) with gibbsite } \\
\text { solids added [FEG] }\end{array}$ & $\begin{array}{l}\text { The total solids loadings were adjusted, via } \\
\text { dilution, before testing began to meet target } \\
\text { Bingham parameters of } 30 \mathrm{~Pa} / 30 \mathrm{cP} \text { [FEG-30] } \\
\text { and } 6 \mathrm{~Pa} / 6 \mathrm{cP} \text { [FEG- } 6]\end{array}$ & $\begin{array}{l}\text { Non-Newtonian } \\
\text { slurries }\end{array}$ \\
\hline
\end{tabular}

${ }^{1}$ Gauglitz PA. 2012. Test Plan for Spray Leak Quantification to Support WTP Spray Release Methodology. TP WTPSP-031 R1.0, Pacific Northwest National Laboratory, Richland, Washington. 
Non-hazardous Newtonian slurries of gibbsite and boehmite in water with solids concentrations of 8 and $20 \mathrm{wt} \%$ were evaluated in Phase I testing by Mahoney et al. (2013). A non-hazardous Newtonian slurry simulant (boehmite particles in water) was further tested in Phase II but with higher solids concentration (i.e., $27 \mathrm{wt} \%$ ) because the WTP has increased the upper solids concentration limit from 20 to $27 \mathrm{wt} \%$. In addition, the same boehmite particles were tested with a small fraction ( $1 \mathrm{wt} \%)$ of very dense Mo particles that represent the more dense Hanford waste particles (e.g., plutonium oxides). As a reference, the Mo particles were also tested at $1 \mathrm{wt} \%$ in water.

Phase I testing used the chemical slurry simulant and showed higher than expected release fractions; however, the slurries tested had measured Bingham parameters that did not match the specified rheological limits of $30 \mathrm{~Pa} / 30 \mathrm{cP}$ and $6 \mathrm{~Pa} / 6 \mathrm{cP}$ for non-Newtonian slurries. Phase II testing of chemical slurry simulant was conducted to achieve the Bingham parameter boundaries of $30 \mathrm{~Pa} / 30 \mathrm{cP}$ and $6 \mathrm{~Pa} / 6 \mathrm{cP}$ to quantify the release fraction results at the rheology limits. The solids loading was adjusted by dilution of parent material (see Section 3.2) so that testing could be conducted with one batch of chemical slurry simulant. First, the undiluted chemical simulant, which contained approximately $42 \mathrm{wt} \%$ UDS, was diluted with bottled (distilled) water until at least one Bingham parameter was near $30 \mathrm{~Pa} / 30 \mathrm{cP}$ (target range was $30 \pm 4 \mathrm{~Pa}$ or $\mathrm{cP}$ ) and the second Bingham parameter was less than or equal to the upper $30 \pm 4 \mathrm{~Pa}$ or $\mathrm{cP}$ target (the upper rheological limit). After testing was completed with the simulant at the upper rheological limit, the chemical slurry simulant was adjusted by additional dilution with distilled water so that, at the beginning of testing, at least one Bingham parameter was near $6 \mathrm{~Pa} / 6 \mathrm{cP}$ (target range was $6 \pm 2 \mathrm{~Pa}$ or $\mathrm{cP}$ ) and the second Bingham parameter was greater than or equal to the lower $6 \pm 2 \mathrm{~Pa}$ or $\mathrm{cP}$ target (the lower rheological limit).

Chemical hazards and associated concerns (e.g., safety issues and the waste disposal costs) make chemical slurry simulant unsuitable for testing in the large-scale test system. Based on the results from previous simulant development studies, a slurry of mixed clay solids (80 wt $\%$ kaolin clay / $20 \mathrm{wt} \%$ bentonite clay) in water was selected as the first choice to meet the needs for a non-hazardous, non-Newtonian simulant for Phase II testing. ${ }^{1}$ The $80 \mathrm{wt} \%$ kaolin and $20 \mathrm{wt} \%$ bentonite blend clay simulant has been used in a variety of studies (e.g., Poloski et al. 2004, Bamberger et al. 2005, and Russell et al. 2005). A wide range of Bingham rheological parameters can be obtained by varying the total clay fraction in water. In the Phase II testing, clay and chemical slurry simulants were tested in the small-scale system to define the relationship in the aerosol generation behavior between the two simulants. The solids concentrations of the clay simulants were adjusted (i.e., by dilution with water) to meet the Bingham parameter targets as described above for the chemical slurry simulants, with the exception that dilution was performed with tap water for the clay slurries.

The non-Newtonian simulants were developed to bound the expected range of operating rheologies for the WTP. However, it is important to note that they do not have particle size distributions representative of actual waste in WTP process streams. In the specific case of the clay slurry simulants, the chemical constituents are not representative of those found in actual waste. Although the chemical simulant contains chemically representative components (or surrogate components), it was not intended to mimic specific WTP waste streams.

\footnotetext{
${ }^{1}$ Gauglitz PA. 2012. Test Plan for Spray Leak Quantification to Support WTP Spray Release Methodology. TP WTPSP-031 R1.0, Pacific Northwest National Laboratory, Richland, Washington.
} 


\subsection{Simulant Makeup}

The masses of components and tap water used to produce batches of simulants are given in Table 3.3. All simulants used in the Phase II tests were prepared using similar procedures. ${ }^{1}$ The materials were mixed in a separate mixing vessel and transferred to the small-scale test system. For the clay (35 wt $\%$ KBC) and STR simulants ( $27 \mathrm{wt} \%$ STR and $20 \mathrm{wt} \% \mathrm{DST}$ ), the required solid components and water were weighed out on calibrated scales and added to the mixing vessel. For the chemical simulant (42 wt $\%$ FEG), appropriate amounts of gibbsite and the Fe-rich sludge (approximately $22 \mathrm{wt} \%$ UDS and $13.6 \mathrm{wt} \%$ dissolved solids [DS]) were weighed using calibrated scales and added to the mixing vessel. After all components were added to the mixing vessel, the slurries were blended for a minimum of 30 min using an overhead agitator. At the completion of mixing, the slurry simulants were sampled for rheology and other physical property analyses, and then stored until needed for testing. When simulants were removed from storage, they were mechanically mixed before being transferred to the feed tank. The exception was simulants containing $1 \mathrm{wt} \% \mathrm{Mo}$, which were prepared by weighing out the appropriate mass of Mo powder and adding it directly into the feed tank. This exception was due to concerns with achieving suspension in the mixing vessels and losing Mo particles via deposition during the transfer. These simulants either contained tap water (in the case of the $1 \mathrm{wt} \%$ Mo in water) or $19 \mathrm{wt} \%$ STR slurry, which was prepared using the standard procedure and added to the test vessel prior to the Mo addition.

Table 3.3. Summary of Component Masses for Batches of Simulants Used for Phase II Small-Scale Aerosol Testing

\begin{tabular}{lccccc}
\hline & & & \\
\multicolumn{1}{c}{ Component } & $27 \mathrm{wt} \%$ & $1 \mathrm{wt} \%$ & $20 \mathrm{wt} \%$ & $35 \mathrm{wt} \%$ & $42 \mathrm{wt} \%$ \\
& STR & DPW & DST & KBC & FEG \\
\hline Tap water $(\mathrm{kg})$ & 134.611 & 150.965 & 139.984 & 125.470 & --- \\
NOAH R6000 boehmite $(\mathrm{kg})$ & 9.957 & --- & 6.646 & --- & --- \\
Nabaltec APYRAL AOH60 boehmite $(\mathrm{kg})$ & 39.832 & --- & 26.586 & --- & --- \\
American Elements Mo powder $(\mathrm{kg})$ & --- & 1.525 & 1.749 & --- & --- \\
NOAH Fe-rich Sludge at $\sim 22 \mathrm{wt} \% \mathrm{UDS}(\mathrm{kg}){ }^{(\mathrm{a})}$ & --- & --- & --- & --- & 159.400 \\
Nabaltec APYRAL 40CD gibbsite $(\mathrm{kg})$ & --- & --- & --- & --- & 54.055 \\
Sieved $(106 \mu \mathrm{m})$ kaolin clay $(\mathrm{kg})$ & --- & --- & --- & 54.049 & --- \\
Sieved $(106 \mu \mathrm{m})$ bentonite clay $(\mathrm{kg})$ & --- & --- & --- & 13.512 & --- \\
Mt. Hood 480 biocide $(5 \%$ active) $(\mathrm{kg})$ & --- & --- & --- & 0.019 & --- \\
\hline
\end{tabular}

--- = Material not used in the simulant.

(a) Fe-rich sludge is a slurry with a supernatant that contains $13.6 \mathrm{wt} \% \mathrm{DS}$.

In two of the Newtonian slurry simulants (i.e., $27 \mathrm{wt} \%$ STR and $20 \mathrm{wt} \%$ DST), a combination of boehmites was used to match the desired particle size distribution, following the same approach as in Phase I. The components were chosen to mimic the PSD of Group 1/2 waste (see Section 3.1 in Mahoney et al. 2013), which is the slurry simulant with the smallest PSD of any tested in Phase I. In contrast, the gibbsite component added to the chemical simulant slurry (42 wt \% FEG in Table 3.3) was primarily for the purpose of increasing the rheology of the simulant and not to produce any specific PSD in the material.

\footnotetext{
${ }^{1}$ The simulant makeup procedure for this purpose was governed by the following test instruction: TI-WTPSP-043, "Simulant Blending to Support Phase II Small-Scale Spray Testing," GN Brown.
} 
The non-Newtonian chemical and clay slurry simulants were prepared in the mixing vessel with solids concentrations higher than actually needed to facilitate in situ solids loading adjustments by dilution to achieve the rheology targets of $30 \mathrm{~Pa} / 30 \mathrm{cP}$ at the beginning of testing as shown in Table 3.3. The chemical slurry simulant was prepared by adding approximately one part (by weight) Nabaltec APYRAL 40CD gibbsite to three parts Fe-rich sludge, which was a precipitated slurry manufactured by NOAH Technologies. Additional information on the recipe and preparation procedure for the Fe-rich sludge is reported in Mahoney et al. (2013). In Phase I testing, the Fe-rich sludge was used as-received with a solids concentration of approximately $15 \mathrm{wt} \%$ UDS. However, in Phase II, to achieve the Bingham parameter upper limit of $30 \mathrm{~Pa} / 30 \mathrm{cP}$, the Fe-rich slurry was concentrated by settling and decanting to obtain a higher solids concentration of approximately $22 \mathrm{wt} \% \mathrm{UDS}$. The appropriate amount of APYRAL 40CD gibbsite was added to the concentrated Fe-rich sludge to prepare the chemical simulant with an initial target solids concentration of approximately $42 \mathrm{wt} \%$ UDS. The target concentration was chosen based on scoping studies, which indicated that a $42 \mathrm{wt} \%$ UDS FEG slurry had a rheology greater than the target of $30 \mathrm{~Pa} / 30 \mathrm{cP}$. It is important to note that the FEG slurries tested in Phase II were atypical of expected washed and leached wastes in the WTP in two areas. First, the UDS of the FEG simulants was much larger than those expected in the WTP $(>35 \mathrm{wt} \%$ versus a maximum of $27 \mathrm{wt} \%$ ). Furthermore, though the Fe-rich solids are similar to the solids found in expected washed and leached wastes in the WTP, the addition of significant amounts of gibbsite is not representative of washed and leached solids. Gibbsite was chosen because boehmite solids, which were used in the initial Phase I chemical simulant, resulted in anomalous rheological behavior (see Mahoney et al. 2013). At the start of testing, the simulant was transferred to the feed tank in the small-scale test system and in situ adjustments of the solids concentrations were made by diluting with bottled (distilled) water to achieve the target Bingham parameter range of $30 \pm 4 \mathrm{~Pa}$ or $\mathrm{cP}$.

The clay slurry simulant was blended with an initial target concentration of $35 \mathrm{wt} \%$ UDS, which had an estimated Bingham yield stress of $\sim 35 \mathrm{~Pa}$. Preliminary bench-scale tests noted a small amount of larger particles in both the kaolin and bentonite materials (i.e., $\sim 0.5 \mathrm{wt} \%$ of the bentonite material having particles larger than $425 \mu \mathrm{m}$, and $\sim 0.1 \mathrm{wt} \%$ of the kaolin material having agglomerated particles larger than $425 \mu \mathrm{m}$ ). Because of concerns that these larger particles could possibly plug the smallest orifice (with a nominal diameter of $\sim 500 \mu \mathrm{m}$ ) used in Phase II small-scale testing, the kaolin and bentonite materials used for preparing the clay simulant for the small-scale testing were sieved to $106 \mu \mathrm{m}$. The clay slurry simulant was prepared by adding the appropriate amount of sieved kaolin and bentonite powder to tap water in the mixing vessel, followed by thorough mixing. In addition, a small amount (nominal concentration of $5 \mathrm{ppm}$ ) of Mt. Hood 480 biocide was also added to the clay slurry to prevent the growth of bacteria and fungi in the clay while the clay simulant was stored and awaiting testing. Before the start of aerosol testing with clay, the clay simulant was transferred to the feed tank in the small-scale test system and in situ adjustments of the solids concentrations were made by diluting with tap water to achieve the target Bingham parameter range of $30 \pm 4 \mathrm{~Pa}$ or $\mathrm{cP}$ of at the beginning of testing.

\subsection{Sample Analysis}

Simulant samples were characterized for physical properties including PSD, rheology, surface tension, bulk density, and solids concentrations (UDS). PSD analyses were performed according to test procedure OP-WTPSP-003 Rev. $1.0^{1}$ The operating procedure that governed the rheology and bulk

${ }^{1}$ Daniel RC. 2011. Size Analysis Using Malvern MS2000 (re-issuance of TPR-RPP-WTP-626). OP-WTPSP-003, Rev. 1.0 (unpublished), Pacific Northwest National Laboratory, Richland, Washington. 
density analyses was RPL-COLLOID-02 Rev. 2. ${ }^{1}$ Surface tension measurements were conducted per operating procedure OP-WTPSP-035 Rev. $0 .^{2}$ Total solids content and UDS measurements were performed according to test procedure OP-WTPSP-004 R1.0. ${ }^{3}$

\subsubsection{Particle Size Analysis}

Particle size characterization was accomplished using a Mastersizer 2000 (Malvern Instruments, Inc., Southborough, Massachusetts) with a Hydro G wet dispersion accessory (equipped with a continuously variable and independent pump, stirrer, and ultrasound). The Mastersizer has a nominal size measurement range of 0.02 to $2000 \mu \mathrm{m}$. The actual range is dependent on the accessory used and the properties of the solids being analyzed. When coupled with the Hydro G wet dispersion accessory, the nominal measuring range is 0.02 to $2000 \mu \mathrm{m}$, dependent on material density. Table 3.4 provides a summary of basic information regarding the analyzer and accessory. A National Institute of Standards and Technology traceable particle size standard is used to evaluate the performance of the particle size analyzer as per OP-WTPSP-003, Rev. 1.0.

Table 3.4. Summary of Malvern Mastersizer 2000 Instrument Information

\begin{tabular}{ll}
\hline Analyzer: & Malvern Mastersizer 2000, Serial Number MAL 1019545 \\
Measurement principle: & Laser Diffraction (Mie Scattering) \\
Analyzer accessory: & Hydro G, 800-mL capacity \\
Measurement range: & $0.02-2000 \mu \mathrm{m}$ nominal \\
Type: & Flow cell system with continuously variable and independent pump, overhead stirrer, \\
& and ultrasound (20 watt full power) \\
\hline
\end{tabular}

Small aliquots of the simulant samples ( $<1 \mathrm{~mL}$ for slurries, $\sim 0.2-1 \mathrm{~g}$ for dry simulants) were diluted with de-ionized water in the Hydro $G$ dispersion unit with pump and stirrer speed set typically at 2000 and $800 \mathrm{rpm}$, respectively, for $60 \mathrm{~s}$ before making the particle size measurements. Due to the higher density of the Mo powder, it was analyzed at a pump speed of $2500 \mathrm{rpm}$ and a stirrer speed of $1000 \mathrm{rpm}$ to ensure all the material recirculated through the system. Appropriate dilutions were determined by the amount of light passing through the dispersed material (obscuration), which was measured by the particle size analyzer. Samples were analyzed on the same aliquot initially without sonication, then during sonication (at 100\% of the sonicator setting for all dispersions except the $1 \mathrm{wt} \%$ dense particle in $19 \mathrm{wt} \%$ small treated [STR] boehmite slurry [DST], which was sonicated at the $50 \%$ setting) after an initial sonication period of $60 \mathrm{~s}$, and also after sonication. Observing changes in the particle size distribution as a function of mixing time (shear) and with and without sonication can provide valuable information about particle-particle agglomeration, the presence of fine particles adhered to larger ones, and/or particle breakage due to sonication.

\footnotetext{
${ }^{1}$ Daniel RC. 2011. Measurement of Physical and Rheological Properties of Solutions, Slurries, and Sludges. RPL-COLLOID-02, Rev. 2 (unpublished), Pacific Northwest National Laboratory, Richland, Washington.

2 Tran DN. 2011. Measurement of Static Surface Tension of Liquids, Dispersions, and Slurries. OP-WTPSP-035, Rev. 0.0 (unpublished), Pacific Northwest National Laboratory, Richland, Washington.

${ }^{3}$ Burns CA. 2012. Operation of the Mettler Moisture Analyzer. OP-WTPSP-004, Rev. 1.0 (unpublished), Pacific Northwest National Laboratory, Richland, Washington.
} 
All simulant samples were well mixed before taking aliquots for PSD measurements. Duplicate samples were measured to confirm the mixing and subsampling technique. All PSD data reported in this report are the average of six measurements taken from two different aliquots (i.e., three measurements per aliquot).

\subsubsection{Rheology}

The rheology of all the non-Newtonian slurries and the STR and DST slurries (which were expected to be Newtonian) were measured. The DPW simulant was assumed to have the same rheology as water. Understanding and interpreting the measured flow curves is critical to assessing the rheology of the simulants (particularly those that were non-Newtonian) used in Phase II. For these measurements, non-elastic flow of non-Newtonian materials is characterized with rotational viscometry. The goal of rotational viscometry is measurement of a material's flow curve, which describes the shear stress response, $\tau$, as a function of applied shear rate, $\dot{\gamma}$ (also called the rate-of-strain). The result of a flow curve measurement is a set of $\tau$ versus $\dot{\gamma}$ measurements, which are called flow curve data. Flow curve data can be interpreted with several constitutive equations that relate viscous stress to shear rate. Such analysis allows the flow behavior over a broad range of conditions to be described with just a few rheological descriptors (e.g., viscosity, yield stress, consistency, and flow index).

Simulants were flow curve tested using a concentric cylinder rotational viscometer operated in controlled-rate mode. These types of viscometers operate by placing a given volume of test sample into a measurement cup of known geometry. A cylindrical rotor attached to a torque sensor is lowered into the sample until the slurry is even with, but does not cover, the top of the rotor. For a given concentric cylinder geometry, both the radius and height of the rotor are known such that the gap distance between cup and rotor and surface area of fluid contact can be determined. In addition, the top and bottom of the rotor have recessed surfaces such that the fluid only contacts the radial surfaces of the rotor. A filled rotor-in-cup test geometry is shown in Figure 3.1. Fluid flow properties of a sample are determined by spinning the rotor at a known rotational speed, $\Omega$, and measuring the resisting torque, $M$, acting on the rotor. Because fluid only contacts the rotor on the radial surfaces of rotation, all of the force resisting steady-state rotation can be ascribed to shearing of the fluid in the cup-rotor gap. Assuming an isotropic fluid and cup and rotor dimensions as shown in Figure 3.1, the torque acting on the rotor can be directly related to the shear stress at the rotor using the equation,

$$
\tau=\frac{M}{2 \pi H R_{I}^{2}}
$$




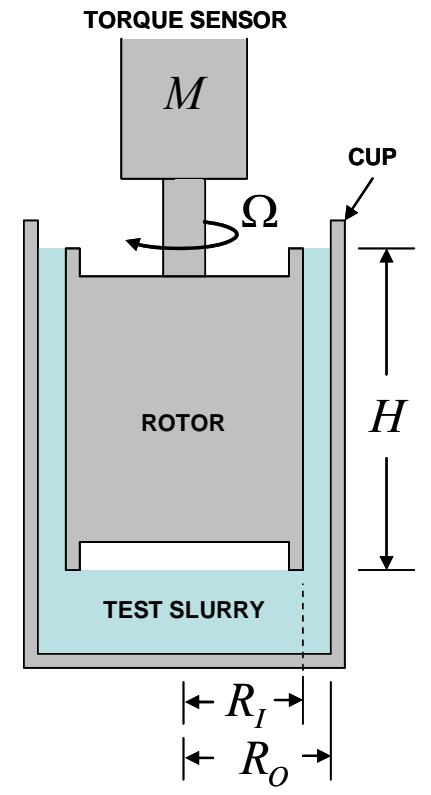

Figure 3.1. Rotor and Cup Geometry Used in Rotational Viscometry Testing in Phase II

Shear stress is measured in units of force per area $\left[\mathrm{N} / \mathrm{m}^{2}\right]$. Calculation of the fluid shear rate at the rotor is complicated by the fact that shear rate depends on both the measurement system geometry and the fluid rheological properties. For the simplest fluids (i.e., Newtonian fluids) the shear rate of the fluid at the rotor can be calculated given the geometry of the cup-rotor shear (see Figure 3.1) by using the equation,

$$
\dot{\gamma}=\left(\frac{2 R_{O}^{2}}{R_{O}^{2}-R_{I}^{2}}\right) \Omega
$$

Here, shear rate has units of inverse seconds [1/s]. Calculation of shear rate for materials showing more complex shear stress versus shear rate behavior (i.e., non-Newtonian fluids) requires input of flow curve parameters (e.g., yield stress and degree of shear thinning or shear thickening). Typically, because the required input parameters are not known prior to measurement, this requirement is circumvented by using a cup and rotor system with a small gap $(\sim 1 \mathrm{~mm})$ such that shear rate effects introduced by fluid properties are minimized. For these systems, Equation (3.2) provides an accurate determination of shear rate for non-Newtonian materials.

The resistance of a fluid to flow can also be described in terms of the fluid's apparent viscosity, $\eta_{a p p}$, which is defined as the ratio of the shear stress to shear rate:

$$
\eta_{a p p}=\frac{\tau}{\dot{\gamma}}
$$

The units of apparent viscosity are $\mathrm{Pa} \cdot \mathrm{s}$; however, viscosity is typically reported in units of centipoise (cP; where $1 \mathrm{cP}=1 \mathrm{mPa} \cdot \mathrm{s})$. 
The flow curve data provided in this section and in Appendix E are presented in plots of $\tau$ as a function of $\dot{\gamma}$. As stated above, flow curve data can be interpreted with several constitutive equations (i.e., flow curves), allowing characterization of that data with just a few rheological descriptors. The behaviors of the slurries have been described by the Bingham plastic flow curve equation for the simulants used in Phase II testing.

Bingham plastics are fluids that show finite yield points. This stress (i.e., the yield stress) must be exceeded before these types of materials flow. Once flow is initiated, the stress response of the material is linear over the rest of the shear rate range. Bingham plastics are described by the expression

$$
\tau=\tau_{o}^{B}+k_{B} \dot{\gamma}
$$

where $\tau_{o}^{B}$ is the Bingham yield index (or stress) and $k_{B}$ is the Bingham consistency index.

Concentrated slurries can show complex flow curve phenomena, including both time-independent and time-dependent behaviors. Figure 3.2 outlines flow behaviors typical of sludge materials. With respect to time-independent behaviors, yield stress materials can be classified by changes in the slope of the equilibrium flow curve after material yield (see Figure 3.2a). Materials that show increasing slope with applied shear rate are considered "shear-thickening" yield materials, whereas materials with decreasing slope are considered to be "shear thinning." Materials with constant slope after yield are referred to as Bingham plastics. These types of flow behavior are time-independent and do not depend on the direction the flow curve is being measured (i.e., the stress response is the same when measured with increasing shear rate or decreasing shear rate). As such, time-independent changes are reversible.

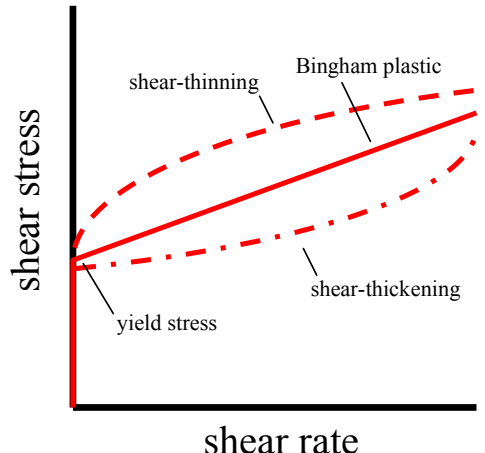

(a)

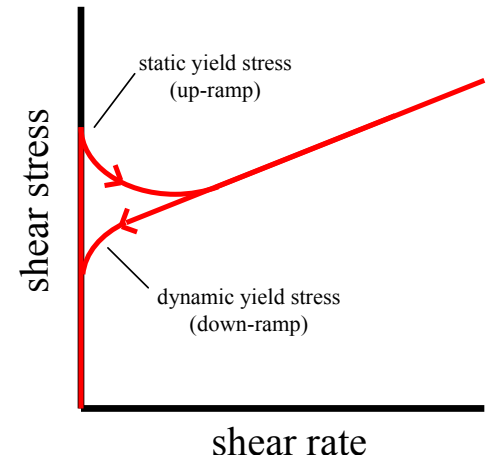

(b)

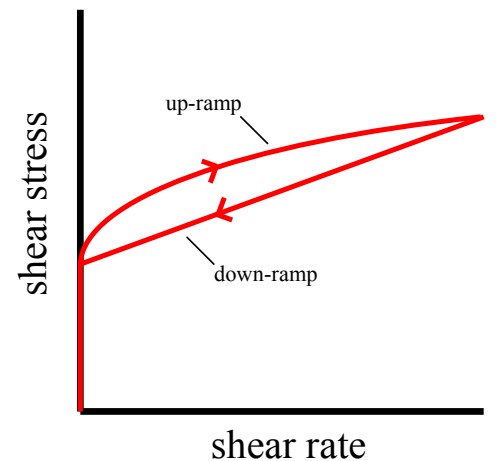

(c)

Figure 3.2. Summary of Flow Curve Behaviors Typically Observed for Concentrated Slurries, Including (a) Common Time-Independent Behaviors, (b) Static and Dynamic Yield Stress, and (c) Flow Curve Hysteresis

Time-dependent flow curve phenomena refer to immediately irreversible (i.e., either short-term or permanent) changes in the stress response of a material. These changes can be caused by the application of shear or may simply occur over time. Time-dependent phenomena can be attributed to breakage of slurry structure, settling of dispersed solids, or changes in the chemistry of slurry components. As shown in Figure 3.2b and Figure 3.2c, time-dependent phenomena can manifest as the different static and dynamic slurry yield stresses and as flow curve hysteresis. 
For Phase II small-scale testing, rheological characterizations were performed in accordance with RPL-COLLOID-02, Rev. 2.0 using a Haake RS600 rheometer operated with RheoWin Pro software Version 2.97 (Thermo Fisher Corporation). The RS600 rheometer was equipped with a low-inertia torque motor and coaxial cylinder measurement geometry. The drive shaft of the motor was centered by an air bearing, which ensures virtually frictionless transmission of the applied torque to the sample. Unless specified otherwise, all rheological analyses were conducted at $25^{\circ} \mathrm{C}$. Samples were gently shaken by hand to ensure complete mixing before introducing them into the measuring device. Before any flow curves were measured a pre-shear at a constant $250 \mathrm{~s}^{-1}$ rate for 3 min was performed on all simulant samples. Flow curves were obtained by shearing the sample at a controlled rate from zero to $1000 \mathrm{~s}^{-1}$ for $5 \mathrm{~min}$, holding constant at $1000 \mathrm{~s}^{-1}$ for $1 \mathrm{~min}$, and then shearing at a controlled rate from $1000 \mathrm{~s}^{-1}$ to zero for $5 \mathrm{~min}$. This flow curve routine was performed twice for each sample aliquot, and the down ramp measured on the second flow curve routine was used for determination of fluid rheology (i.e., viscosity or Bingham yield stress and consistency). Where possible the down ramp between 800 and $250 \mathrm{~s}^{-1}$ was used to evaluate the flow curves. Unless specified otherwise, each sample was measured with duplicate aliquots. The averages of the Bingham yield stress and Bingham consistency values measured for both aliquots are reported as the Bingham yield stress and Bingham consistency measurements for the respective sample.

\subsubsection{Surface Tension}

Only slurry samples were tested for surface tension in Phase II. Surface tension analysis was performed on supernatants of the slurry samples. Supernatant samples were obtained from the simulant slurries for surface tension analysis by centrifugation at 3345 relative centrifugal force (RCF) for $4 \mathrm{hr}$. It is worth noting that after $4 \mathrm{hr}$ of centrifugation at $3345 \mathrm{RCF}$, the liquids and solids of the chemical slurry samples were very well separated. The supernatants of chemical simulant samples appeared to be slightly greenish brown and clear of solids. The $27 \mathrm{wt} \%$ STR and $20 \mathrm{wt} \%$ DST samples were also well separated after $4 \mathrm{hr}$ of centrifugation. The resultant supernatants appeared cloudy. The clay slurry samples were very difficult to separate. After $4 \mathrm{hr}$ of centrifugation, a thin layer of emulsion (gel-like material) remained between the liquid and solid layers. However, the resultant supernatants of the clay slurry samples appeared to be light brown and clear of solids. The resultant supernatants were collected for surface tension analysis. Because the supernatants of the $27 \mathrm{wt} \%$ STR and $20 \mathrm{wt} \%$ DST appeared cloudy, it was suspected that the supernatant may have contained some fine solids particles. Therefore, a portion of the supernatants were filtered through a $0.2 \mu \mathrm{m}$ polytetrafluoroethylene (PTFE) disposable filter to remove possible submicron solid particles from the supernatants. These filtered supernatants still appeared cloudy; however additional filtration was not performed because the $0.2 \mu \mathrm{m}$ PTFE filters were the smallest filters on hand at the time. The filtered supernatants were also analyzed for surface tension.

Surface tension measurements were performed in accordance with OP-WTPSP-035, Rev. 0 using a commercial force-balance K-12 MK6 Tensiometer (Kruss USA). The tensiometer consisted of a K-12 MK6 Tensiometer processor unit, a force measuring unit (the balance), a Wilhelmy platinum plate, and a quartz sample vessel. The static surface tension of each sample was measured using the plate method (a.k.a., the Wilhelmy method), which is based on a force measurement. A platinum plate with exactly known geometry was hung vertically above the sample liquid. Then, the lower edge of the plate was brought into contact with the sample liquid surface. The sample liquid wetted the plate and pulled it into the liquid. The pull from the sample liquid due to wetting is known as the Wilhelmy force. The Wilhelmy force was measured by moving up the plate to the level of the sample liquid surface. The 
resulting force was determined from the weight measured by the balance. Surface tension measurements for all samples were carried out at room temperature and the temperature associated with each measurement was measured and recorded.

\subsubsection{Density}

Bulk densities of the selected simulant samples were measured in accordance with RPL-COLLOID-02, Rev. 2.0 using certified glass pycnometers (Wilmad LabGlass) and a calibrated balance. A pycnometer is a volumetric flask with a known volume specifically designed for density measurements. To start a bulk density measurement, the tare weight of the pycnometer to be used was obtained and recorded on the density measurement bench sheet. The pycnometer was then filled with the simulant fluid to be measured. The gross weight of the pycnometer containing the simulant fluid was obtained and recorded on the density measurement bench sheet. The net weight of the simulant fluid was calculated by subtracting the pycnometer tare weight from the gross weight of the pycnometer containing the simulant. The bulk density of the simulant fluid was calculated using Equation (3.5). Unless otherwise specified, all density measurements were carried out at room temperature. In addition, room temperature associated with each density measurement was measured using a calibrated thermocouple and thermocouple readout and recorded on the density measurement bench sheet.

$$
\rho=\frac{M}{V}
$$

where $\quad \rho=$ bulk density in $\mathrm{g} / \mathrm{mL}$

$\mathrm{M}=$ net weight of the simulant fluid in $\mathrm{g}$

$\mathrm{V}=$ volume of the simulant fluid in $\mathrm{mL}$.

\subsubsection{Total Solids Content and Undissolved Solids Content}

Total solids content consists of two components: 1) dissolved (soluble) and 2) undissolved (insoluble) solids (UDS). For samples that do not contain DS, the total solids and the UDS are equivalent. In such cases, the total solids content and the UDS of the sample may be obtained using the following single-step measurement process. The total solids content and/or the UDS of slurry samples by weight were determined using a Mettler-Toledo Halogen Moisture analyzer, Model HR83, in accordance with OP-WTPSP-004, Rev. 1. Approximately 5 to $10 \mathrm{~g}$ of slurry material was introduced into the moisture analyzer and subjected to a preprogrammed drying program. The program consisted of an initial hold period of $30 \mathrm{~min}$ at $95^{\circ} \mathrm{C}$, followed by a period of $105^{\circ} \mathrm{C}$ used to complete the drying process. The end criterion was set to a mean weight loss of $1 \mathrm{mg}$ (or less) over a 140-s period. The results were recorded as dry content (total solids) by the moisture analyzer; the definition of this result is given by Equation (3.6).

$$
\text { Dry Content }=\frac{d r y \text { weight }}{\text { wet weight }} \times 100 \%
$$

For samples containing both soluble and insoluble solids, the UDS of the samples were measured using the following two-step measurement process. First, the total solids content of the slurry sample was obtained as described above. Second, the DS content of the sample was measured from the liquid portion of the sample. The sample liquid (supernatant) was obtained by centrifugation of the slurry sample 
(typically at $3345 \mathrm{RCF}$ for $15 \mathrm{~min}$ ), followed by filtration of the resultant separated liquid through a $0.45-\mu \mathrm{m}$ PTFE filter. The DS content of the supernates was then determined and the UDS of the sample was calculated as given in Equation (3.7).

$$
W t \% \text { Undissolved Solids }=\left(1-\frac{100-W t \% \text { Total Solids }}{100-W t \% \text { Dissolved Solids }}\right) \times 100
$$

\subsubsection{Selection of Refractive Index for Laser Diffraction Analysis}

The aerosol systems tested in the current application consist of both pure liquids or solutions and suspensions of solid particles in pure liquids or solutions. For the majority of aerosols generated from pure liquids or suspension, determination of aerosol refractive index (RI) is straightforward, as RIs for the test solutions are available in the literature. In particular, the RI of water can be readily found in general chemistry references.

While RIs are generally available for the liquids and supernates tested, assignment of appropriate values for RI for aerosols generated during spray of slurries is not straightforward. Difficulties in assigning a RI for slurry systems result from the fact that 1) aerosols generated by slurry sprays are heterogeneous composite particles of solids either fully or partially encapsulated in liquid, and 2) the composition of the composite particles is not necessarily equal to that of the test slurry as confinement and jamming of particles at the point of release may alter the composition and in-flight drag or evaporation may strip the aerosol of water content or particles. For the purpose of estimating release fractions, a single RI may be assigned to the system with the understanding that the concentration and size distribution derived from the laser diffraction aerosol instrumentation used in the current testing may not be accurate. One of three basic RIs can be assigned: 1) that of the solid component, 2) that of the liquid component, or 3) an effective RI for a particular concentration of solid in liquid. For simple simulants, solid and liquid RIs are generally available in the literature. These values can be used to bound the RI of aerosol composites and are necessary inputs for estimating the effective RI of the composite liquid-solid aerosol.

Estimates of RI for composite solids or aerosols can be made by using one of several mixing rules available in the literature. These rules are derived for binary mixtures where optical properties of the pure components are known. These rules are:

- Bruggeman Rule (Bruggeman, 1935)

$$
f \frac{\epsilon_{1}-\epsilon_{e f f}}{\epsilon_{1}+2 \epsilon_{e f f}}+(1-f) \frac{\epsilon_{2}-\epsilon_{e f f}}{\epsilon_{2}+2 \epsilon_{e f f}}=0
$$

- Maxwell-Garnett Rule (Garnett 1904)

$$
\frac{\epsilon_{e f f}-\epsilon_{2}}{\epsilon_{e f f}+2 \epsilon_{2}}=f \frac{\epsilon_{1}-\epsilon_{2}}{\epsilon_{1}+2 \epsilon_{2}}
$$


- Looyenga Rule (Looyenga 1965)

$$
\epsilon_{e f f}^{1 / 3}=f \epsilon_{1}^{1 / 3}+(1-f) \epsilon_{2}^{1 / 3}
$$

- Monecke Rule (Monecke 1994)

$$
\epsilon_{e f f}=\frac{2\left\{f \epsilon_{1}+(1-f) \epsilon_{2}\right\}^{2}+\epsilon_{1} \epsilon_{2}}{(1+f) \epsilon_{1}+(2-f) \epsilon_{2}}
$$

- Hollow sphere equivalent (Bohren and Huffman 1983)

$$
\epsilon_{e f f}=\epsilon_{1} \frac{(3-2 f) \epsilon_{2}+2 f \epsilon_{1}}{f \epsilon_{2}+(3-f) \epsilon_{1}}
$$

Here, $f$ is the volume fraction of component $1, \varepsilon_{\text {eff }}$ is the effective dielectric constant on the medium, and $\varepsilon_{1}$ and $\varepsilon_{2}$ are the dielectric constants of component 1 and 2, respectively. The dielectric constant is related to the complex RI (denoted by $n$ ) by

$$
\epsilon=n^{2}
$$

Of these five mixing rules, two appear amenable to extension beyond binary systems. These are the Bruggeman and Looyenga mixing rules. From these rules, the following extensions to multicomponent systems are proposed:

- Extended Bruggeman Rule

$$
\sum_{i} f_{i} \frac{\epsilon_{i}-\epsilon_{e f f}}{\epsilon_{i}+2 \epsilon_{e f f}}=0
$$

- Extended Looyenga Rule

$$
\epsilon_{e f f}^{1 / 3}=\sum_{i} f_{i} \epsilon_{i}^{1 / 3}
$$

Here, $f_{i}$ and $\varepsilon_{i}$ are the volume fraction and dielectric constant of component $i$, respectively. These multicomponent rules produce complex RIs that are in reasonable agreement with results produced by nesting the binary rules to produce RI estimates for complex mixtures of particles.

The test matrix for the small-scale Phase II effort includes water, two double-component solid slurries and two multicomponent solids slurries. To determine RIs for this diverse array of test mixtures, the approach used was to:

1. Determine, using standard chemical references, the RI for all solid components, pure liquids, and salt solutions. For the latter, the dissolved component concentration was measured directly or determined by mass balance.

2. Determine the composition of all test mixtures in terms of volume fraction by mass balance or available documentation of mixture chemical makeup. 
3. Based on the composition determined in step 2 and the pure component and suspending phase RIs assigned in step 1, calculate the mixture RI using the appropriate set of mixing rules (i.e., Equations (3.8) to (3.15)). The general approach used was to apply all of the applicable mixing rules (either all five for binary systems or just two for multicomponent mixtures) to evaluate composite RI for a given system. It should be noted that each mixing rule produces a slightly different RI result; however, differences in the RI produced by application of different mixing rules to the same chemical mixture typically occur in the third decimal place, and as such, analysis of Malvern Insitec-S data using the different RI will not produce a noticeable difference in final aerosol concentration or PSD results. To produce a single RI result for analysis, the final composite RI for the mixture was calculated as the arithmetic average of the RI results produced by the individual mixing rules.

RIs for all pure liquid and solid components and test slurries are presented in Table 3.5. In general, the absorption index (i.e., the imaginary component) of pure solids is not available in the literature. However, unless the solid particles are spherical, the imaginary component may be generally taken to be non-zero. Likewise, even spherical aerosol droplets of supernatant that contain non-spherical insoluble particles will have a non-zero absorption index. Because the exact value of solid particle absorption index is not known, it has been set to 1.000 for all solid particles, such that they are represented (in terms of Mie theory) as being opaque. Proper assignment of solid particle absorption index is not expected to strongly impact the size distribution result, as the diffraction result above $1 \mu \mathrm{m}$ is expected to be robust with respect to order-of-magnitude changes in absorption index, so long as the RI is reasonable for non-spherical particles (i.e., non-zero and on the order of 0.01 to 1 ).

\subsection{Physical Properties of the Newtonian Simulants}

The four Newtonian simulants used for Phase II small-scale testing and their target compositions are listed in Table 3.6. Table 3.7 summarizes the actual compositions and measured properties of the Newtonian simulants. Details of simulant preparation methods are described in Section 3.2. Table 3.7 provides select particle size percentiles for the unsonicated $27 \mathrm{wt} \%$ STR and $20 \mathrm{wt} \%$ DST simulants and for the unsonicated Mo powder. The corresponding cumulative percent undersize and differential PSD plots of these simulants and the Mo powder are shown in Figure 3.3 and Figure 3.4. Particle size was measured 1) without sonication, 2) undergoing sonication after an initial period of sonication of approximately $60 \mathrm{~s}$, and finally 3 ) after sonication (i.e., post-sonication). Figure 3.3 shows only the unsonicated data; Figure 3.4 presents the differential distribution of sonication and post-sonication data.

From Figure 3.4 we can see that both the $27 \mathrm{wt} \%$ STR and the $20 \mathrm{wt} \%$ DST simulants exhibit a slight increase in particle volume around the $10-\mu \mathrm{m}$ particle size post-sonication, which is due to sonication-induced particle agglomeration that occurs frequently when analyzing very fine powders (e.g., $<10 \mu \mathrm{m})$. In addition, the Mo powder developed a slight increase in the same size range, which is indicative of the presence of a fine fraction of material (dust) that was previously adhered to the larger

particles. If the volume contribution of this region had continuously increased as a function of time with applied sonication it would have implied that the particle was undergoing a physical change such a particle fracturing, or deagglomeration of hard agglomerates; neither of which were observed for this material. The unsonicated data are considered more representative of the simulant in its natural state within the mixing tank while the sonicated particle size more closely represents the primary particles, hence only the unsonicated data are given in Table 3.7 and Figure 3.3. The small amount of Mo powder, $1 \mathrm{wt} \%$, added into the $20 \mathrm{wt} \%$ DST simulant was not detectable in the measured PSD. It is possible that the subsample taken from the tank and submitted for particle size analysis was not representative of the 
tank inventory due to subsampling and mixing challenges associated with dense particles. Even if a representative sample of the $20 \mathrm{wt} \%$ DST simulant was obtained, the volume contribution of the Mo powder in the mix was $\sim 1.5$ volume $\%$ which is near the 98 th percentile by volume. It is, therefore, not surprising that the Mo powder in the $20 \mathrm{wt} \%$ DST simulant was not detected.

Table 3.5. Refractive Indices for Simulants Used in Small-Scale Spray Leak Testing. The RI of pure components has been taken from reference sources available in the literature. Refractive indices for mixtures have been calculated using both binary and multicomponent mixing rules. Both the real (RI) and the imaginary (i.e., the absorption index) components of the complex RI are given. All RIs are listed to three decimal places to demonstrate the value of RIs provided to the Malvern Insitec-S software. Real RI values are typically significant to two figures, whereas imaginary RI values are significant to one figure.

\begin{tabular}{|c|c|c|c|}
\hline Component & Method $^{(a)}$ & RI (Real) & $\begin{array}{c}\text { Absorption Index } \\
\text { (Imaginary RI } \\
\text { Component) }\end{array}$ \\
\hline \multicolumn{4}{|c|}{ Pure Liquids and Solutions } \\
\hline Water & Reference & 1.330 & 0.000 \\
\hline \multicolumn{4}{|c|}{ Pure and Mixed Solids } \\
\hline Mo powder & Reference & 3.740 & 3.560 \\
\hline Gibbsite & Reference & 1.580 & 1.000 \\
\hline Boehmite & Reference & 1.690 & 1.000 \\
\hline FEG & Calculated & 1.723 & 1.002 \\
\hline DST & Calculated & 1.716 & 1.030 \\
\hline Kaolin-bentonite Solids & Calculated & 1.592 & 1.000 \\
\hline \multicolumn{4}{|c|}{ Slurry Systems } \\
\hline $27 \mathrm{wt} \%$ STR & Calculated & 1.378 & 0.096 \\
\hline $20 \mathrm{wt} \% \mathrm{DST}$ & Calculated & 1.367 & 0.066 \\
\hline $1 \mathrm{wt} \% \mathrm{DPW}$ & Calculated & 1.332 & 0.002 \\
\hline $30 \mathrm{~Pa} / 30$ cP FEG & Calculated & 1.442 & 0.190 \\
\hline $6 \mathrm{~Pa} / 6 \mathrm{cP}$ FEG & Calculated & 1.425 & 0.157 \\
\hline $30 \mathrm{~Pa} / 30 \mathrm{cP} \mathrm{KBC}$ & Calculated & 1.389 & 0.149 \\
\hline $6 \mathrm{~Pa} / 6 \mathrm{cP} \mathrm{KBC}$ & Calculated & 1.377 & 0.116 \\
\hline \multicolumn{4}{|c|}{ Slurry System Suspending Phase } \\
\hline STR, DPW, DST & Reference & 1.330 & 0.000 \\
\hline $30 \mathrm{~Pa} / 30 \mathrm{cP}$ FEG & Calculated & 1.348 & 0.000 \\
\hline $6 \mathrm{~Pa} / 6 \mathrm{cP}$ FEG & Calculated & 1.345 & 0.000 \\
\hline $30 \mathrm{~Pa} / 30 \mathrm{cP} \mathrm{KBC}$ & Reference & 1.330 & 0.000 \\
\hline $6 \mathrm{~Pa} / 6 \mathrm{cP} \mathrm{KBC}$ & Reference & 1.330 & 0.000 \\
\hline
\end{tabular}

(a) Method: 1) Reference - taken from a reference chemical handbook or literature source, and 2) Calculated - determined by known component indices and averaging of all mixing rules (two for binary systems and five for multicomponent systems). 
Representative rheograms for the $27 \mathrm{wt} \%$ STR and $20 \mathrm{wt} \%$ DST simulants performed at the beginning of testing are given in Appendix E. Methods and procedures used for obtaining the simulant rheology and physical properties are discussed in Section 3.3. The physical properties of the $1 \mathrm{wt} \%$ Mo in water simulant were not analyzed because they are expected to be the same or similar to those of water. All Newtonian simulants were made up in City of Richland, Washington tap water. Both the $27 \mathrm{wt} \%$ STR and $20 \mathrm{wt} \%$ DST simulants were determined to have zero Bingham yield stress, which confirmed that they were Newtonian simulants. In addition, the viscosity value for each simulant is $\sim 2 \mathrm{cP}$ (i.e., in the specified range of 1 to $3 \mathrm{cP}$ for Newtonian slurries as listed in Table 3.1).

Table 3.6. Newtonian Simulants Used in Phase II Testing

\begin{tabular}{|c|c|c|}
\hline $\begin{array}{l}\text { Simulant } \\
\text { Name }\end{array}$ & Simulant Description & Target Simulant Composition \\
\hline Water & $\begin{array}{l}\text { City of Richland, Washington } \\
\text { tap water }\end{array}$ & Tap water \\
\hline $27 \mathrm{wt} \%$ STR & $\begin{array}{l}27 \mathrm{wt} \% \text { of small-treated } \\
\text { boehmite particulates in tap } \\
\text { water }\end{array}$ & $\begin{array}{l}27 \mathrm{wt} \% \text { of small-treated boehmite particulates in tap water } \\
\text { Small-treated boehmite material consists of } 80 \mathrm{wt} \% \text { of AOH } 60 \\
\text { boehmite and } 20 \mathrm{wt} \% \text { of NOAH R } 6000 \text { boehmite. }\end{array}$ \\
\hline $1 \mathrm{wt} \% \mathrm{DPW}$ & $1 \mathrm{wt} \%$ Mo in tap water & $1 \mathrm{wt} \%$ Mo particulates in tap water \\
\hline $20 \mathrm{wt} \% \mathrm{DST}$ & $\begin{array}{l}19 \mathrm{wt} \% \mathrm{STR} \text { and } 1 \mathrm{wt} \% \text { Mo in } \\
\text { tap water }\end{array}$ & $\begin{array}{l}19 \mathrm{wt} \% \text { of small-treated boehmite particulates and } 1 \mathrm{wt} \% \mathrm{Mo} \\
\text { particulates in tap water } \\
\text { Small-treated boehmite material consists of } 80 \mathrm{wt} \% \text { of AOH } 60 \\
\text { boehmite and } 20 \mathrm{wt} \% \text { of NOAH R } 6000 \text { boehmite. }\end{array}$ \\
\hline
\end{tabular}

Table 3.7. Summary of Properties for Newtonian Slurries Used for Aerosol Testing

\begin{tabular}{|c|c|c|c|}
\hline Component/Property & $27 \mathrm{wt} \%$ STR & $1 \mathrm{wt} \% \mathrm{DPW}$ & $20 \mathrm{wt} \% \mathrm{DST}$ \\
\hline Measured UDS (wt $\%$ ) & 26.3 & $\mathrm{NM}$ & 19.6 \\
\hline Slurry density $(\mathrm{kg} / \mathrm{L})$ & 1.21 & NM & 1.15 \\
\hline Surface tension $(\mathrm{mN} / \mathrm{m})^{(\mathrm{a})}$ & $69.4 \pm 0.1$ & NM & $71.1 \pm 0.2$ \\
\hline Viscosity (cP) & 2.0 & NM & 1.8 \\
\hline Percentile & \multicolumn{3}{|c|}{ Unsonicated Particle Diameter, $\mu \mathrm{m}$} \\
\hline $\mathrm{d}_{01}$ & 0.27 & 10.9 & 0.27 \\
\hline $\mathrm{d}_{05}$ & 0.33 & 13.6 & 0.34 \\
\hline $\mathrm{d}_{10}$ & 0.39 & 15.5 & 0.40 \\
\hline$d_{20}$ & 0.48 & 18.3 & 0.50 \\
\hline$d_{25}$ & 0.53 & 19.6 & 0.54 \\
\hline$d_{30}$ & 0.57 & 20.8 & 0.59 \\
\hline $\mathrm{d}_{40}$ & 0.67 & 23.2 & 0.69 \\
\hline $\mathrm{d}_{50}$ & 0.78 & 25.6 & 0.79 \\
\hline$d_{60}$ & 0.90 & 28.3 & 0.92 \\
\hline $\mathrm{d}_{70}$ & 1.06 & 31.4 & 1.08 \\
\hline $\mathrm{d}_{75}$ & 1.16 & 33.2 & 1.18 \\
\hline $\mathrm{d}_{80}$ & 1.28 & 35.3 & 1.30 \\
\hline $\mathrm{d}_{90}$ & 1.70 & 41.1 & 1.70 \\
\hline $\mathrm{d}_{95}$ & 2.35 & 45.9 & 2.21 \\
\hline $\mathrm{d}_{99}$ & 5.84 & 53.6 & 7.47 \\
\hline
\end{tabular}

(a) The error ( \pm ) is the standard deviation for three consecutive measurements on the same sample aliquot. $\mathrm{NM}=$ Not measured. 


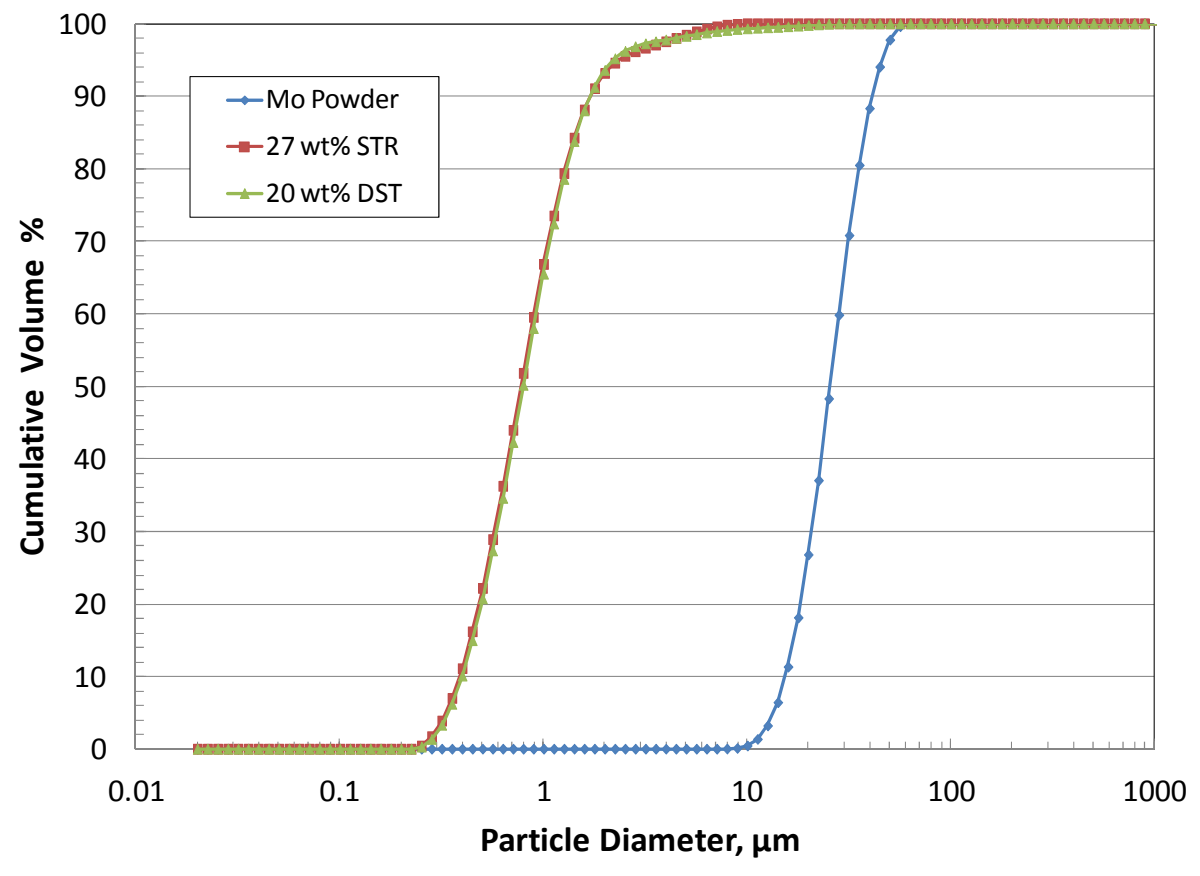

Figure 3.3. Cumulative Volume Percent Undersize for Unsonicated $27 \mathrm{wt} \%$ STR Simulant, $20 \mathrm{wt} \%$ DST Simulant, and Mo Powder

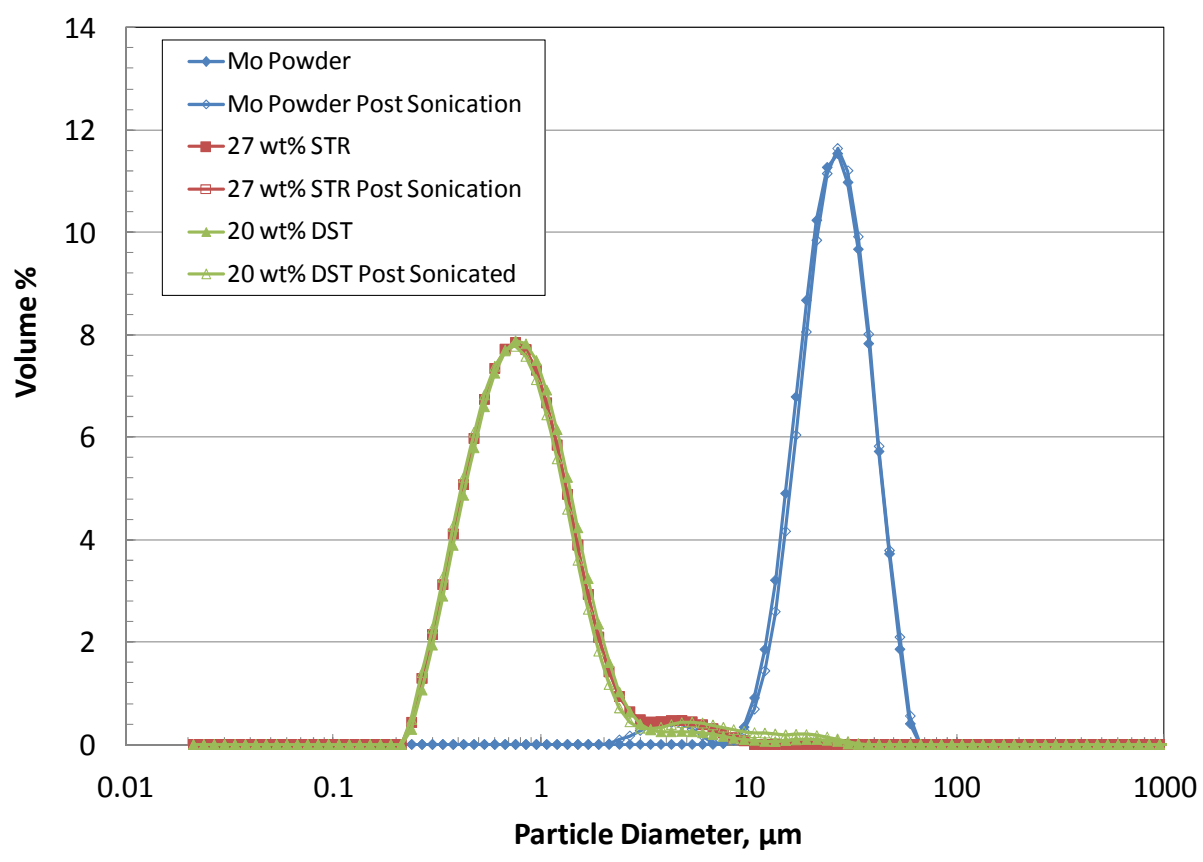

Figure 3.4. Volume Percent Differential PSDs for Unsonicated and Post-Sonication $27 \mathrm{wt} \%$ STR Simulant, 20 wt\% DST Simulant, and Mo Powder 
The $\mathrm{wt} \%$ UDS measurements for both of these Newtonian simulants are slightly lower than the respective targets (26.3 vs. $27 \mathrm{wt} \%$ for STR and 19.6 vs. $20 \mathrm{wt} \%$ for DST). These small discrepancies are likely due to a small percentage of hydration in the boehmite material used to make up these Newtonian simulants. The slurry density values for both of these Newtonian simulants agree well with the calculated value of $1.2 \mathrm{~kg} / \mathrm{L}$. The surface tensions of the centrifuged, filtered $(0.2 \mu \mathrm{m}$ PTFE) supernatants for the $27 \mathrm{wt} \%$ STR and $20 \mathrm{wt} \%$ DST simulants are $69.4 \pm 0.1$ and $71.1 \pm 0.2 \mathrm{mN} / \mathrm{m}$ at $25^{\circ} \mathrm{C}$, respectively, which is slightly lower than value of $72.0 \mathrm{mN} / \mathrm{m}$ for tap water at $25^{\circ} \mathrm{C}$ (measured in Phase I testing [Mahoney et al. 2013]). These small discrepancies may be due to suspensions of submicron particulates (i.e., $<0.2 \mu \mathrm{m}$ ).

\subsection{Physical Properties of the Non-Newtonian Simulants}

\subsubsection{Simulant Characterization}

The four non-Newtonian simulants (and the parent materials from which they are derived) used in Phase II small-scale testing and their target compositions are listed in Table 3.8. The chemicals and materials used for fabrication of these non-Newtonian simulants are given in Table 3.3. The compositions and properties of the non-Newtonian simulants used for Phase II small-scale testing are summarized in Table 3.9. In addition Table 3.9 presents select particle size percentiles for the unsonicated $30 \mathrm{~Pa} / 30 \mathrm{cP}$ and $6 \mathrm{~Pa} / 6 \mathrm{cP}$ chemical simulants and the unsonicated $30 \mathrm{~Pa} / 30 \mathrm{cP}$ and $6 \mathrm{~Pa} / 6 \mathrm{cP}$ clay simulants. Cumulative percent undersize and differential PSD plots for both the Fe-rich with gibbsite (FEG) and clay simulants are shown in Figure 3.5 through Figure 3.9. Particle size was measured 1) without sonication, 2) during sonication after an initial period of sonication of $\sim 60 \mathrm{~s}$, and finally 3) after sonication (i.e., post-sonication). Both the unsonicated and post-sonication data are shown for the FEG simulants (Figure 3.5 through Figure 3.7) and cumulative clay PSD (Figure 3.8), whereas only the unsonicated data are shown for the differential clay PSD in Figure 3.9.

Table 3.8. Non-Newtonian Simulants and Parent Materials Used in Phase II Testing

\begin{tabular}{|c|c|c|}
\hline $\begin{array}{l}\text { Simulant } \\
\text { Name }\end{array}$ & Simulant Description & Target Simulant Composition \\
\hline $\begin{array}{l}42 \mathrm{wt}^{\%} \% \\
\text { FEG }\end{array}$ & $\begin{array}{l}\text { Fe-rich slurry and gibbsite, parent } \\
\text { slurry for dilution }\end{array}$ & $\begin{array}{l}\text { Gibbsite was added to the Fe-rich slurry consisting of } \sim 22 \mathrm{wt} \% \text { UDS to } \\
\text { achieve a } 42 \mathrm{wt} \% \text { UDS chemical slurry simulant. This resulted in } \\
\text { weight fractions in solids phase of } 0.39 \text { (Fe-rich solids) and } 0.61 \\
\text { (gibbsite) for the parent slurry. }\end{array}$ \\
\hline $\begin{array}{l}30 \mathrm{~Pa} / 30 \mathrm{cP} \\
\text { FEG }\end{array}$ & Fe-rich slurry and gibbsite & $\begin{array}{l}\text { In situ dilution of the } 42 \mathrm{wt} \% \text { FEG slurry simulant with distilled bottled } \\
\text { water to achieve the Bingham parameter target of } 30 \mathrm{~Pa} / 30 \mathrm{cP} \text { at the } \\
\text { beginning of testing. }\end{array}$ \\
\hline $\begin{array}{l}6 \mathrm{~Pa} / 6 \mathrm{cP} \\
\mathrm{FEG}\end{array}$ & Fe-rich slurry and gibbsite & $\begin{array}{l}\text { In situ dilution of the } 30 \mathrm{~Pa} / 30 \mathrm{cP} \text { FEG slurry simulant with bottled } \\
\text { water to achieve the Bingham parameter target of } 6 \mathrm{~Pa} / 6 \mathrm{cP} \text { at the } \\
\text { beginning of testing. }\end{array}$ \\
\hline $\begin{array}{l}35 \mathrm{wt}^{\%} \% \\
\mathrm{KBC}\end{array}$ & $\begin{array}{l}\text { Clay slurry of } 80 \mathrm{wt} \% \text { kaolin and } \\
20 \mathrm{wt} \% \text { bentonite blend in tap water, } \\
\text { parent slurry for dilution }\end{array}$ & $\begin{array}{l}35 \mathrm{wt} \% \text { UDS clay slurry of } 80 \mathrm{wt} \% \text { kaolin and } 20 \mathrm{wt} \% \text { bentonite blend } \\
\text { in tap water. }\end{array}$ \\
\hline $\begin{array}{l}30 \mathrm{~Pa} / \\
30 \mathrm{cP} \mathrm{KBC}\end{array}$ & $\begin{array}{l}\text { Clay slurry of } 80 \mathrm{wt} \% \text { kaolin and } \\
20 \mathrm{wt} \% \text { bentonite blend in tap water }\end{array}$ & $\begin{array}{l}\text { In situ dilution of the } 35 \mathrm{wt} \% \mathrm{KBC} \text { clay slurry simulant with tap water to } \\
\text { achieve the Bingham parameter target of } 30 \mathrm{~Pa} / 30 \mathrm{cP} \text { at the beginning of } \\
\text { testing. }\end{array}$ \\
\hline $\begin{array}{l}6 \mathrm{~Pa} / 6 \mathrm{cP} \\
\mathrm{KBC}\end{array}$ & $\begin{array}{l}\text { Clay slurry of } 80 \mathrm{wt} \% \text { kaolin and } \\
20 \mathrm{wt} \% \text { bentonite blend in tap water }\end{array}$ & $\begin{array}{l}\text { In situ dilution of the } 30 \mathrm{~Pa} / 30 \mathrm{cP} \mathrm{KBC} \text { clay slurry simulant with tap } \\
\text { water to achieve the Bingham parameter target of } 6 \mathrm{~Pa} / 6 \mathrm{cP} \text { at the } \\
\text { beginning of testing. }\end{array}$ \\
\hline
\end{tabular}


Table 3.9. Summary of Measured Properties for Non-Newtonian Simulants Used for Phase II Small-Scale Aerosol Testing

\begin{tabular}{|c|c|c|c|c|}
\hline Property & $\begin{array}{c}30 \mathrm{~Pa} / 30 \mathrm{cP} \\
\text { FEG }\end{array}$ & $\begin{array}{c}6 \mathrm{~Pa} / 6 \mathrm{cP} \\
\mathrm{FEG}\end{array}$ & $\begin{array}{c}30 \mathrm{~Pa} / \\
30 \mathrm{cP} \mathrm{KBC}\end{array}$ & $\begin{array}{c}6 \mathrm{~Pa} / 6 \mathrm{cP} \\
\mathrm{KBC}\end{array}$ \\
\hline Measured UDS (wt $\%)$ & 39.7 & 34.8 & 32.5 & 26.5 \\
\hline Measured DS (wt\%) & 12.9 & 10.5 & --- & --- \\
\hline Slurry density $(\mathrm{kg} / \mathrm{L})$ & 1.43 & 1.36 & 1.24 & 1.19 \\
\hline Surface tension $(\mathrm{mN} / \mathrm{m})$ & $\begin{array}{l}\text { Varying from } \\
56.2 \text { to } 62.5^{\text {(c) }}\end{array}$ & $\begin{array}{l}\text { Varying from } \\
60.2 \text { to } 64.8^{\text {(c) }}\end{array}$ & $71.9 \pm 0.1^{(\mathrm{a})}$ & $71.77 \pm 0.00^{(\mathrm{b})}$ \\
\hline Bingham yield stress $(\mathrm{Pa})^{(\mathrm{d})}$ & 28.4 & 6.18 & 30.6 & 7.34 \\
\hline Bingham consistency $(\mathrm{cP})^{(\mathrm{e})}$ & 28.1 & 21.1 & 29.2 & 12.5 \\
\hline Percentile & \multicolumn{4}{|c|}{ Unsonicated Particle Diameter, $\mu \mathrm{m}$} \\
\hline $\mathrm{d}_{01}$ & 0.40 & 0.43 & 0.36 & 0.35 \\
\hline$d_{05}$ & 0.70 & 0.77 & 0.60 & 0.60 \\
\hline $\mathrm{d}_{10}$ & 1.01 & 1.09 & 0.92 & 0.91 \\
\hline$d_{20}$ & 1.57 & 1.70 & 1.63 & 1.63 \\
\hline$d_{25}$ & 1.87 & 2.04 & 2.03 & 2.03 \\
\hline$d_{30}$ & 2.20 & 2.45 & 2.46 & 2.47 \\
\hline $\mathrm{d}_{40}$ & 3.05 & 3.80 & 3.50 & 3.53 \\
\hline$d_{50}$ & 4.65 & 23.6 & 4.91 & 4.93 \\
\hline $\mathrm{d}_{60}$ & 10.2 & 46.7 & 6.95 & 6.87 \\
\hline $\mathrm{d}_{70}$ & 22.6 & 61.7 & 10.1 & 9.66 \\
\hline $\mathrm{d}_{75}$ & 30.0 & 70.0 & 12.3 & 11.6 \\
\hline $\mathrm{d}_{80}$ & 37.8 & 79.5 & 15.3 & 14.0 \\
\hline $\mathrm{d}_{90}$ & 58.1 & 108 & 26.2 & 22.4 \\
\hline $\mathrm{d}_{95}$ & 77.3 & 137 & 40.0 & 31.9 \\
\hline $\mathrm{d}_{99}$ & 191 & 381 & 74.8 & 55.5 \\
\hline
\end{tabular}

(a) The error $( \pm)$ is the standard deviation for three consecutive measurements on the same sample.

(b) The error $( \pm)$ is the standard deviation for two consecutive measurements on the same sample.

(c) The surface tension measurements for the FEG simulants had unusual behavior. The accuracy of the measurement is not known and the actual surface tension may fall outside the range given here.

(d) Bingham yield stress of the non-Newtonian simulants at the beginning of the respective tests.

(e) Bingham consistency of the non-Newtonian simulants at the beginning of the respective tests.

$--\quad=$ Material not used in simulant formulation.

$\mathrm{NM}=$ Not measured.

UDS $=$ Undissolved solid. 


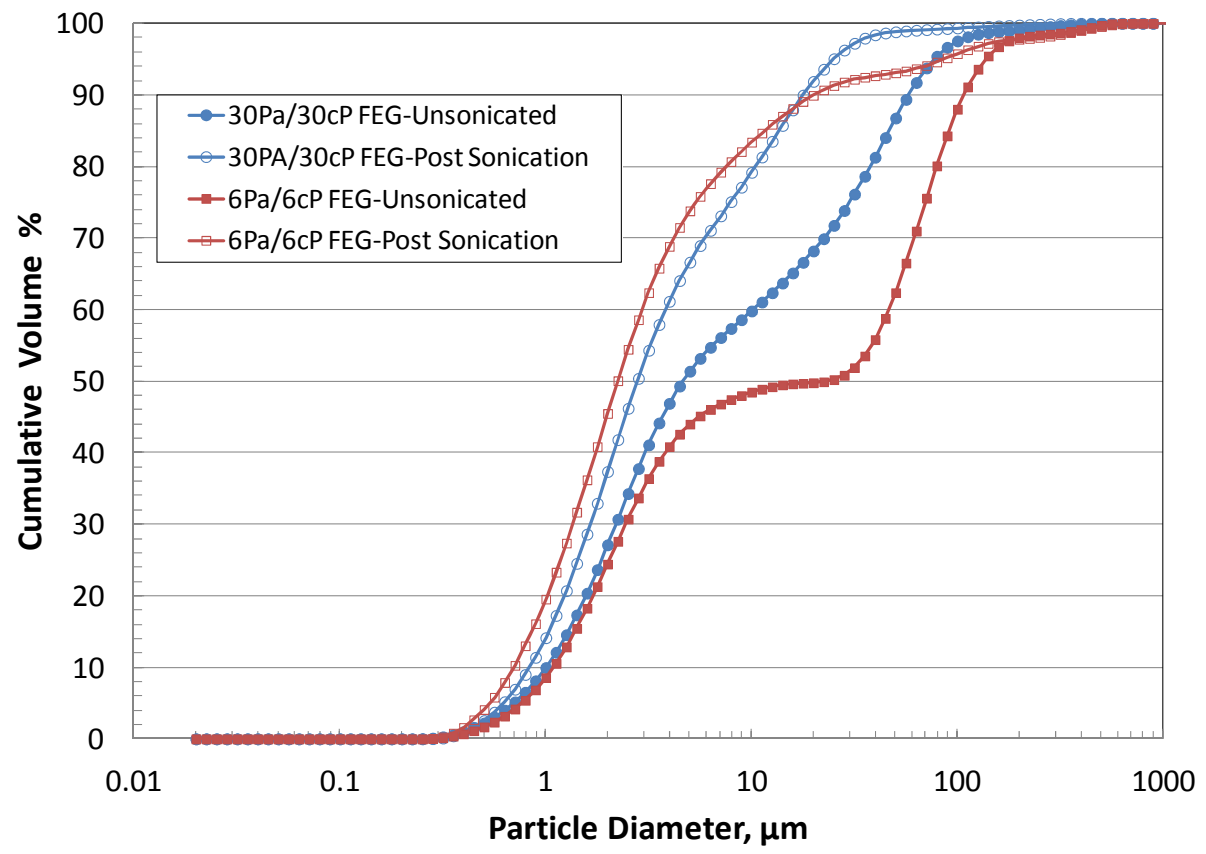

Figure 3.5. Cumulative Volume Percent Undersize for Unsonicated and Post-Sonication $30 \mathrm{~Pa} / 30 \mathrm{cP}$ FEG and 6 Pa/ 6 cP FEG Simulants

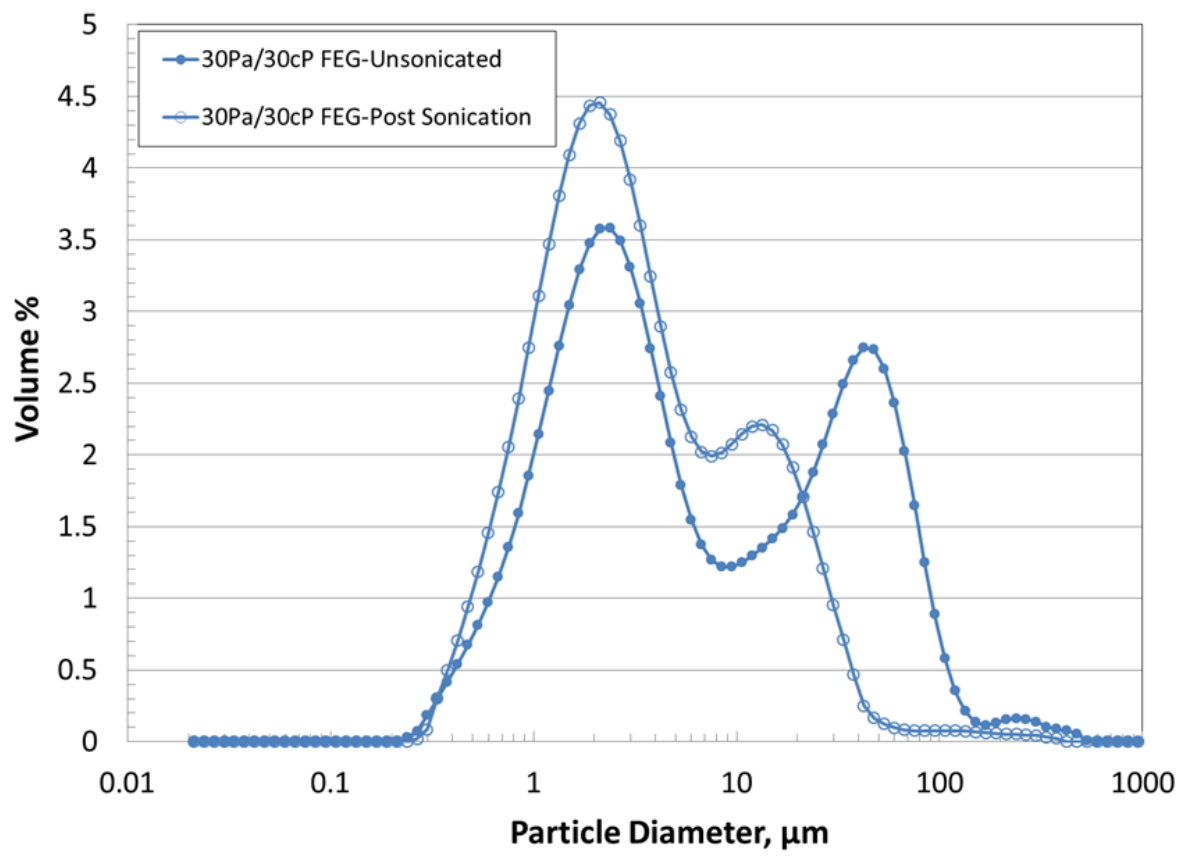

Figure 3.6. Volume Percent Differential PSDs for Unsonicated and Post-Sonication $30 \mathrm{~Pa} / 30 \mathrm{cP}$ FEG Simulant 


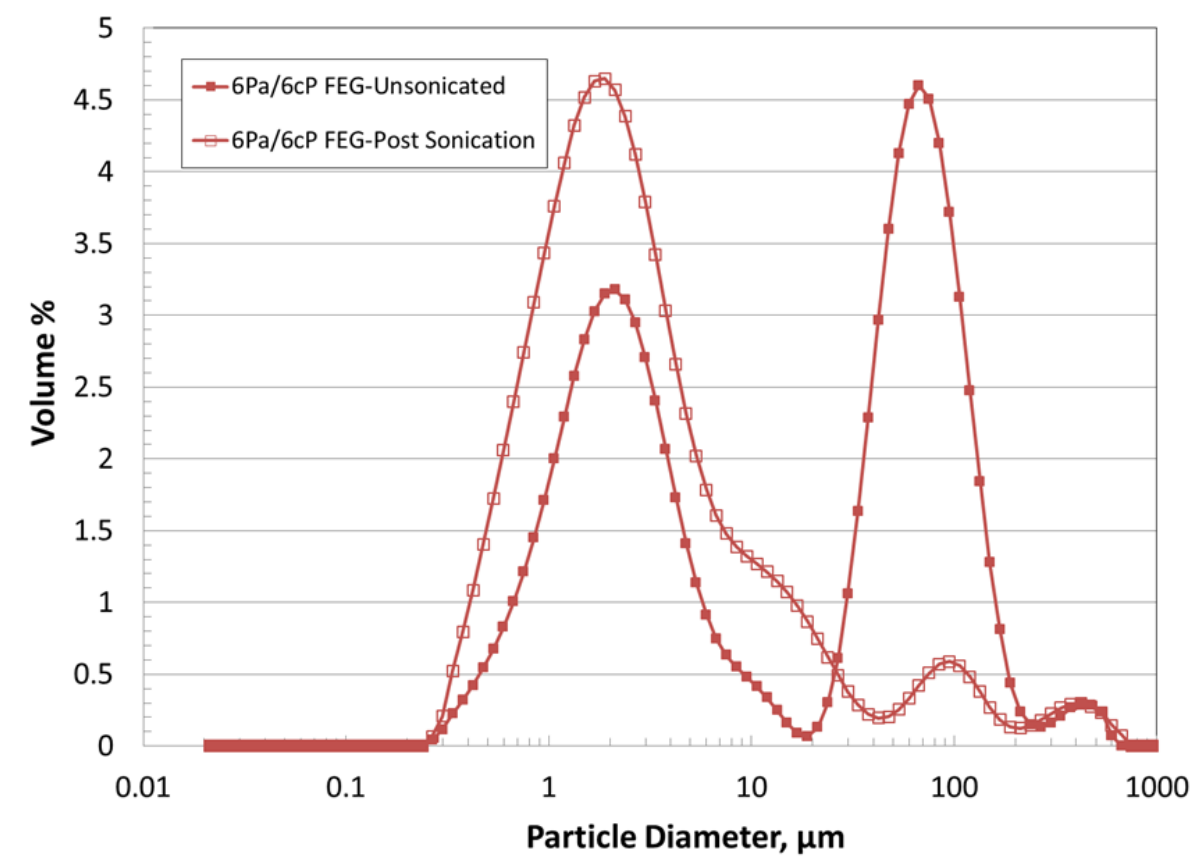

Figure 3.7. Volume Percent Differential PSDs for Unsonicated and Post-Sonication $6 \mathrm{~Pa} / 6 \mathrm{cP}$ FEG Simulant

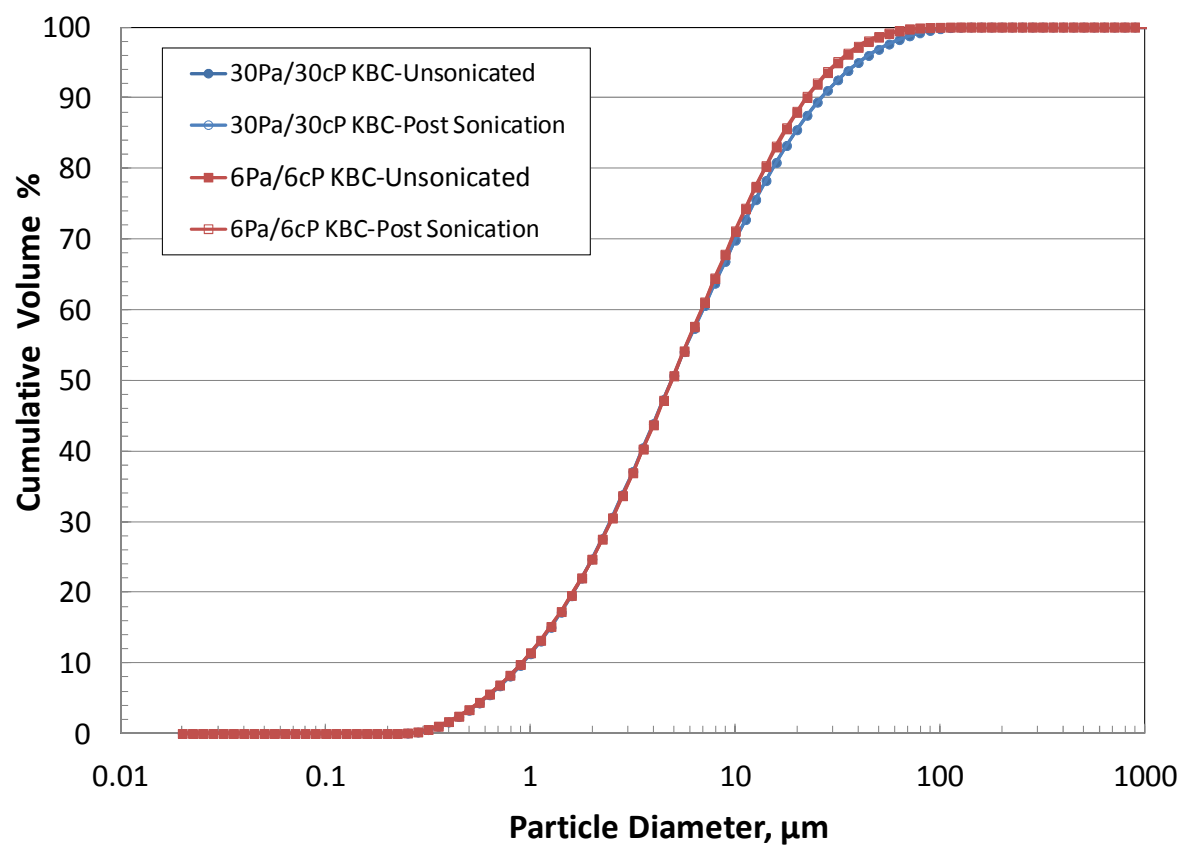

Figure 3.8. Cumulative Volume Percent Undersize PSDs for Unsonicated and Post-Sonication $30 \mathrm{~Pa} / 30 \mathrm{cP} \mathrm{KBC}$ and $6 \mathrm{~Pa} / 6 \mathrm{cP}$ KBC Simulants 


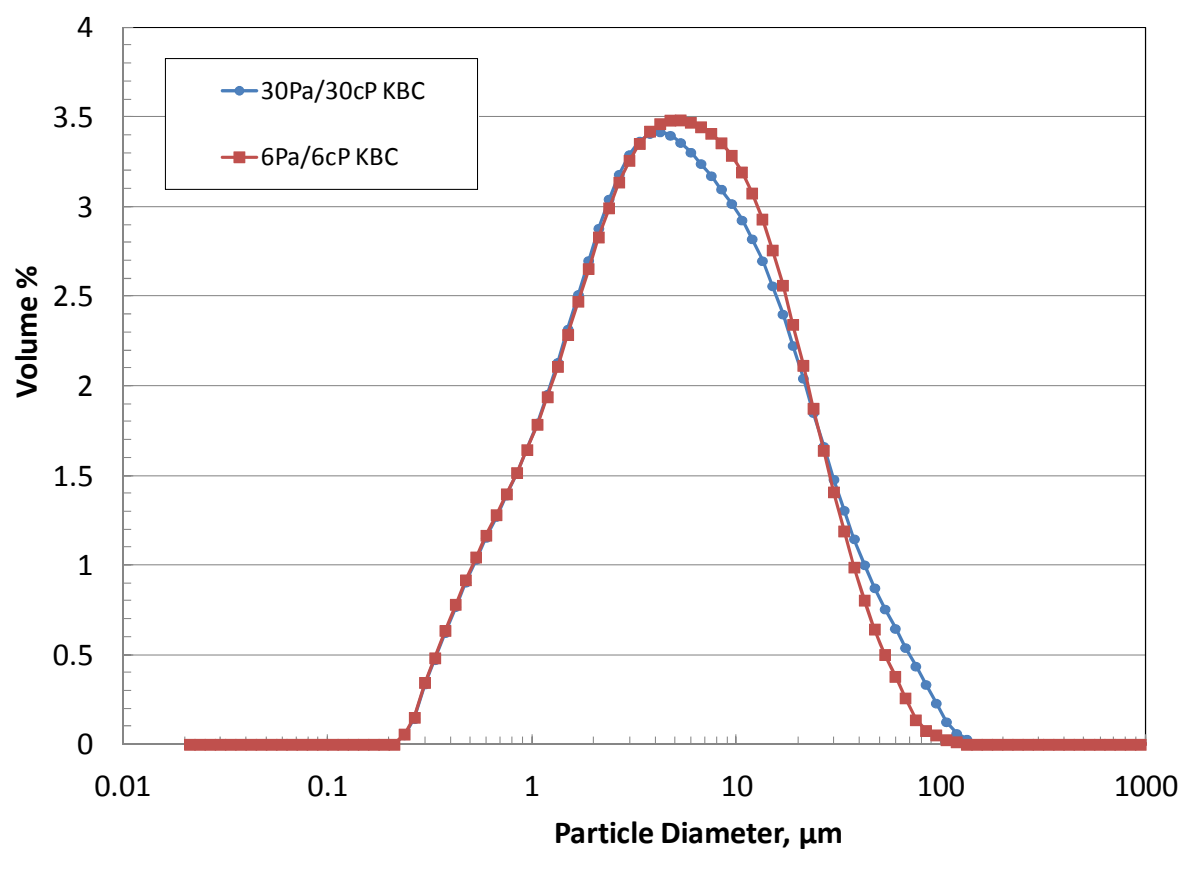

Figure 3.9. Volume Percent Differential PSDs for Unsonicated $30 \mathrm{~Pa} / 30 \mathrm{cP} \mathrm{KBC}$ and $6 \mathrm{~Pa} / 6 \mathrm{cP} \mathrm{KBC}$ Simulants

The unsonicated $30 \mathrm{~Pa} / 30 \mathrm{cP}$ FEG simulant exhibited a bimodal PSD (see Figure 3.6) with two maxima, the first at $\sim 2 \mu \mathrm{m}$ and the second at $\sim 40 \mu \mathrm{m}$. The observed change in particle size after applying sonication is indicative of the breakup of weak particle agglomerates which caused a shift of the second maxima from $\sim 40$ to $\sim 13 \mu \mathrm{m}$. Even after a sonication period of $\sim 2 \mathrm{~min}$ at an applied sonication power of $20 \mathrm{~W}$, the $30 \mathrm{~Pa} / 30 \mathrm{cP}$ FEG simulant remained bimodal, though the second maximum was less distinct and appeared as a sharp shoulder peak. The PSD of the APYRAL 40CD gibbsite (one of the two major components of the FEG simulant) has been measured previously. The volume contribution from the gibbsite added to the Fe-rich simulant overlaps the first maxima at around $\sim 2 \mu \mathrm{m}$. The volume contribution of the second maxima appears to originate from the Fe-rich component of the chemical simulant. Note that the MS2000 only applies gentle in situ sonication to avoid fracturing or breaking fragile particles, and consequently only weakly agglomerated particles are resolved during sonication at $20 \mathrm{~W}$. Stronger agglomerates in the FEG simulant, which are probable given that the material was produced from the co-precipitation of salt solutions, would require more aggressive sonication. In addition, sonication could accelerate dissolution of precipitated salts suspended in lower ionic strength solutions, as is the case for the FEG simulant (which has approximately $13 \mathrm{wt} \%$ DS) because all PSD measurements were performed in distilled water. However, this is less likely to be the case given that the simulant was washed to decrease the salt content of the aqueous phase.

The differential PSD of the $6 \mathrm{~Pa} / 6 \mathrm{cP}$ FEG simulant is given in Figure 3.7. The $6 \mathrm{~Pa} / 6 \mathrm{cP}$ FEG simulant was obtained by diluting the $30 \mathrm{~Pa} / 30 \mathrm{cP}$ FEG simulant after the completion of aerosol testing. As before, the PSD is bimodal with maxima at $\sim 2$ and $67 \mu \mathrm{m}$ and the volume contribution of the weak agglomerates appear to be greater in the diluted simulant. After sonication, the weak agglomerates break up, causing a shift in the PSD and rendering a post-sonication distribution with a continuous broad distribution and a maxima just below $2 \mu \mathrm{m}$. As observed for the $30 \mathrm{~Pa} / 30 \mathrm{cP}$ FEG simulant, the 
broadened tail observed at $\sim 10$ through $40 \mu \mathrm{m}$ is attributed to the Fe-rich sludge. The $6 \mathrm{~Pa} / 6 \mathrm{cP}$ FEG simulant appears to have undergone attrition compared with the $30 \mathrm{~Pa} / 30 \mathrm{cP}$ FEG simulant as shown in Figure 3.6. This may be caused by mechanical shear as the slurry is recirculated around the test flow loop or from the spray itself (because the simulant was recycled during testing).

To validate this hypothesis, the PSD of the $30 \mathrm{~Pa} / 30 \mathrm{cP}$ FEG simulant was measured again after sonication with a $50 \mathrm{~W}$ sonic bath for $4 \mathrm{~min}$; the data obtained (not shown) suggested that the more powerful applied sonication resulted in the breakdown of harder agglomerates and a resulting PSD very similar to that obtained for the $6 \mathrm{~Pa} / 6 \mathrm{cP}$ FEG slurry. Small residual volume contributions above $40 \mu \mathrm{m}$ remain even after sonication for the $6 \mathrm{~Pa} / 6 \mathrm{cP}$ FEG slurry, but are not present in the post-sonication $30 \mathrm{~Pa} / 30 \mathrm{cP}$ FEG slurry PSD. This could be due to the formation of harder agglomerates either formed in the mixing tank as the thick slurry dried around the edges of the tank and slurry surface or from the introduction of contaminants from the simulant recycling efforts. The FEG simulant appears to have a particle system that tends to form agglomerates and sticks together even when gentle sonication $(20 \mathrm{~W})$ is applied. More aggressive sonication was required to break the agglomerates and overcome the particle-particle interactions $(50 \mathrm{~W})$; similar behavior was also observed when measuring the rheological properties of the simulant and is discussed in detail below.

The cumulative volume percent undersize PSD for the $30 \mathrm{~Pa} / 30 \mathrm{cP}$ clay and $6 \mathrm{~Pa} / 6 \mathrm{cP}$ clay simulants, both unsonicated and post-sonication are shown in Figure 3.8 and the differential PSDs are given in Figure 3.9. Sonication did not appear to have any impact on the PSD of either of the clay simulants, suggesting that both simulants were well hydrated and the slurry contained representative primary particles. As was observed for the chemical simulant, some particle attrition appeared to result from mechanical shear as the slurry was recirculated around the test flow loop or from the spray itself, as evidenced by the small change in the $30 \mathrm{~Pa} / 30 \mathrm{cP}$ and $6 \mathrm{~Pa} / 6 \mathrm{cP}$ clay simulant PSDs.

At the beginning of testing, both Bingham parameters (Bingham yield stress and Bingham consistency) for the $30 \mathrm{~Pa} / 30 \mathrm{cP}$ clay and $30 \mathrm{~Pa} / 30 \mathrm{cP}$ chemical slurry simulants were within the target range of $30 \pm 4 \mathrm{~Pa}$ or $\mathrm{cP}$, although only one Bingham parameter was required to meet the target range as stated in Table 3.2. Furthermore, the Bingham yield stress measurements for both the $6 \mathrm{~Pa} / 6 \mathrm{cP}$ clay and $6 \mathrm{~Pa} / 6 \mathrm{cP}$ chemical slurry simulants were also within the target range of $6 \pm 2 \mathrm{~Pa}$ or $\mathrm{cP}$ and the Bingham consistency measurements were both greater than the lower value of the target range at the beginning of testing, as specified in Table 3.2. The rheology is discussed in more detail in Section 3.5.2.

The surface tension values measured for the $30 \mathrm{~Pa} / 30 \mathrm{cP}$ and $6 \mathrm{~Pa} / 6 \mathrm{cP}$ clay simulants were $71.9 \pm 0.1$ and $71.8 \pm 0.0 \mathrm{mN} / \mathrm{m}$, respectively, at $25^{\circ} \mathrm{C}$, consistent with the value of $72.0 \mathrm{mN} / \mathrm{m}$ for tap water at $25^{\circ} \mathrm{C}$ (measured in Phase I testing [Mahoney et al. 2013]). The supernatant obtained from the clay simulants was visibly yellow, suggesting the presence of soluble organic components. The presence of soluble material from the clay did not impact the surface tension values measured for the clay simulants.

In contrast, the surface tension values vary from 56.2 to $62.5 \mathrm{mN} / \mathrm{m}$ and from 60.2 to $64.8 \mathrm{mN} / \mathrm{m}$ for four consecutive measurements at $25^{\circ} \mathrm{C}$ for the $30 \mathrm{~Pa} / 30 \mathrm{cP}$ and $6 \mathrm{~Pa} / 6 \mathrm{cP}$ chemical slurry simulants, respectively. The monotonic increase in surface tension with consecutive measurements is unusual behavior; hence the accuracy of the measurement is difficult to assess and the actual surface tension may fall outside the measured range. The surface tension values obtained from the chemical simulant supernate are lower than water. The observed decrease in surface tension is inconsistent with the typical behavior of surface tension at high ionic strengths $(10-13 \mathrm{wt} \% \mathrm{DS})$ in the presence of anions such as 
hydroxides, nitrates, and the sodium cation. The reason for these low surface tension values (with respect to water) is unclear; however, the values were similar to those measured for the Phase I FER simulant (see Table 3.8 in Mahoney et al. 2013).

\subsubsection{Variation of Non-Newtonian Rheology During Test Operations}

This section focuses on how the rheology of the non-Newtonian simulants varied throughout the Phase II testing. For a more specific discussion of how the rheology affected test operations, see Section 5.4.2. The values for Bingham yield stress and Bingham consistency as a function of testing time (measured in hours) for the clay simulants are presented in Figure 3.10 and Figure 3.11, respectively. Note that, in general, these figures include measurements at the start and end of each test day. Thus, the measurements give an idea of how the rheological parameters evolved during the testing but do not necessarily describe the rheological parameters that were present for any particular set of tests. The data points at $\mathrm{t}=0 \mathrm{hr}$ (represented by the filled purple circles) are from samples of the original parent clay slurry. There are two measurements: one sample was collected directly from the parent clay before transfer to the small-scale feed tank, and the other sample was from the small-scale feed tank after circulating for $1 \mathrm{hr}$. The red data in these figures show the Bingham yield stress and Bingham consistency measurements for the $30 \mathrm{~Pa} / 30 \mathrm{cP}$ clay; samples were typically collected the beginning and the end of each day of testing. The data represent 10 days of non-consecutive testing of the $30 \mathrm{~Pa} / 30 \mathrm{cP}$ clay.

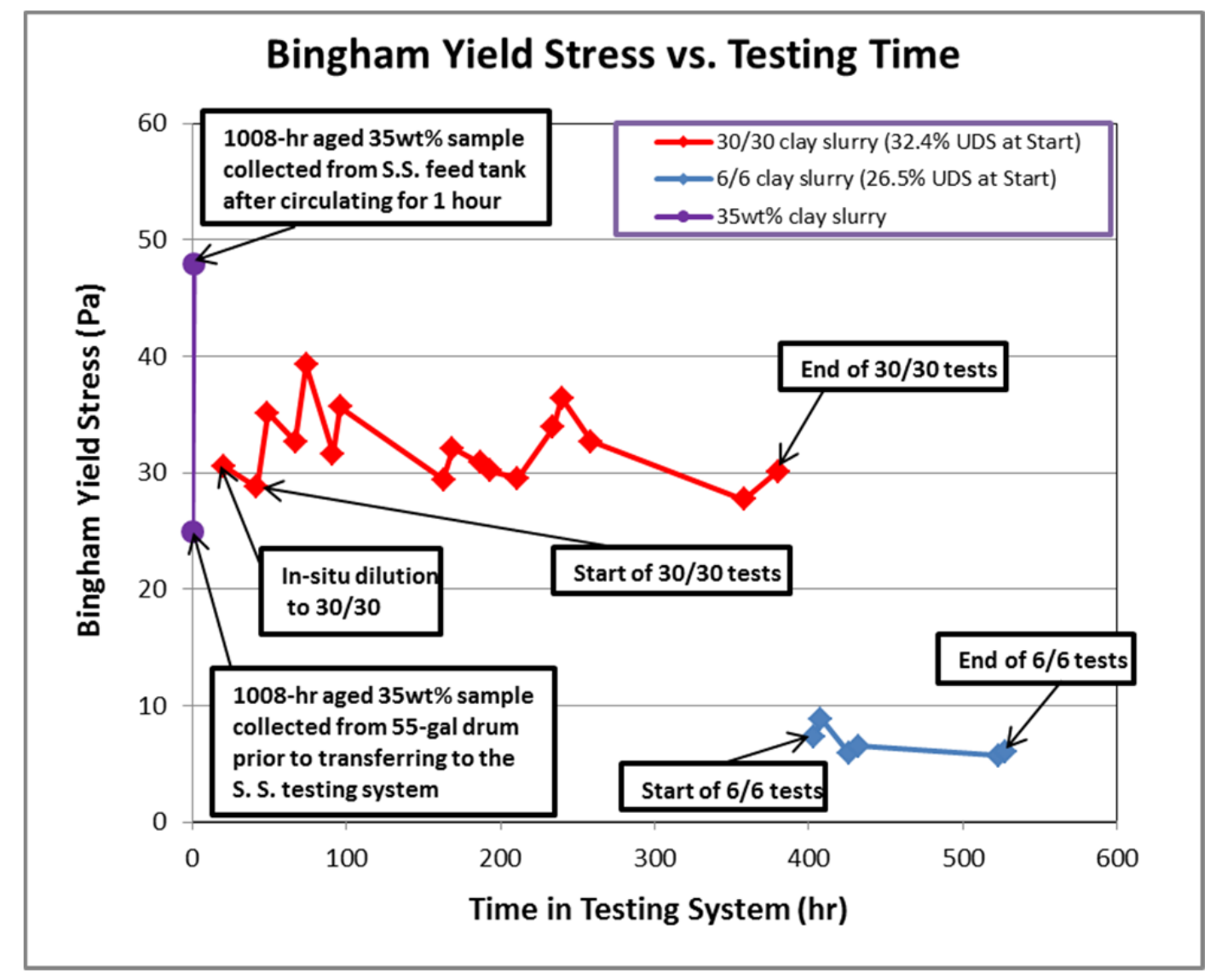

Figure 3.10. Bingham Yield Stress as a Function of Testing Time for Clay Slurry Simulants 
The parent clay was prepared to be more concentrated than the target concentration to allow for in situ concentration adjustments by dilution with City of Richland tap water to achieve the target Bingham parameter for the $30 \mathrm{~Pa} / 30 \mathrm{cP}$ simulant at the beginning of testing. The Bingham yield stress and Bingham consistency measurements for the original simulant samples increased from approximately 25 to $48 \mathrm{~Pa}$ and 35 to $41 \mathrm{cP}$, respectively, after circulating for $1 \mathrm{hr}$ in the small-scale system. These increases were expected and are attributed to the shear the clay slurry experienced in the small-scale system. Simulant rheology was monitored daily in an attempt to keep the simulant within the rheology targets at the beginning of each day of testing. If simulant rheology fell outside of the target range of $30 \pm 4 \mathrm{~Pa}$ or $\mathrm{cP}$, concentration adjustments (by dilution with City of Richland tap water) were made to bring the simulant rheology back to the target values. The cause of the increase was primarily due to evaporative losses over a 24 -hr period. The losses occurred both during storage, when the tank was covered but not airtight, and during testing, as clay that was sprayed was periodically recycled from the chamber back to the mixing tank. As shown in Figure 3.11, the Bingham consistency of the $30 \mathrm{~Pa} / 30 \mathrm{cP}$ clay simulant was within the rheology target range throughout the entire course of testing. The Bingham yield stress of the simulant was between 28 and $39 \mathrm{~Pa}$ over the course of testing and exceeded $34 \mathrm{~Pa}$ in four instances.

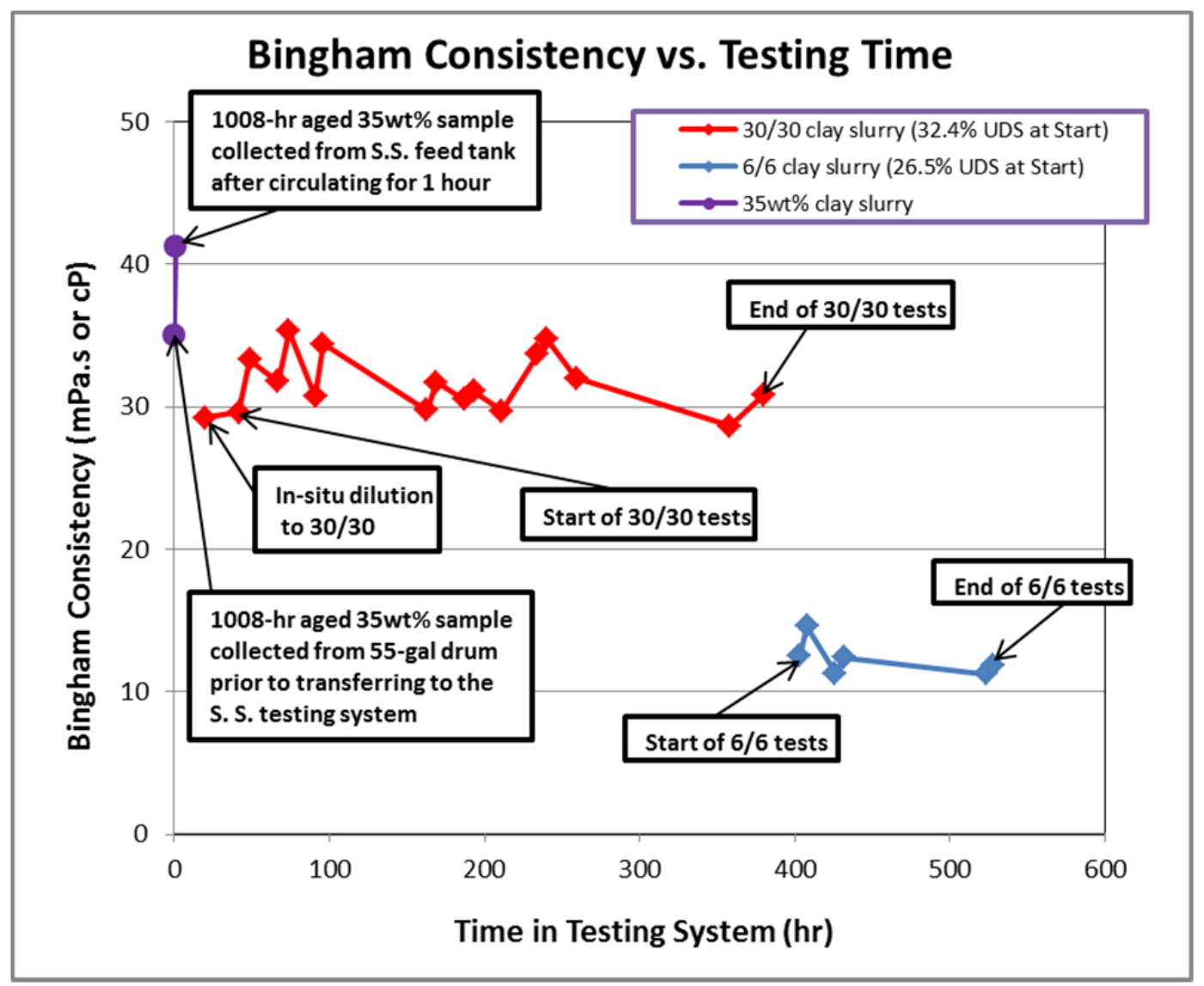

Figure 3.11. Bingham Consistency as a Function of Testing Time for Clay Slurry Simulants

At the completion of the $30 \mathrm{~Pa} / 30 \mathrm{cP}$ testing, the clay simulant was diluted with City of Richland tap water to reach the target range for the $6 \mathrm{~Pa} / 6 \mathrm{cP}$ simulant (blue data in the figures). As with the $30 \mathrm{~Pa} / 30 \mathrm{cP}$ clay simulant, the $6 \mathrm{~Pa} / 6 \mathrm{cP}$ clay simulant rheology was monitored daily and off-target rheology was adjusted by dilution. As shown in Figure 3.10, the Bingham yield stress of the $6 \mathrm{~Pa} / 6 \mathrm{cP}$ 
clay simulant was within the target range with the exception of the sample taken at the end of the second day of testing. The consistency was stable but was above $6 \mathrm{cP}$, generally between 11 and $15 \mathrm{cP}$. Overall, the rheology of the clay simulants was fairly stable and well behaved. In addition, as discussed previously, the stability of the clay simulants was observed in the relatively stable PSDs of the clay materials.

Overall, Figure 3.10 and Figure 3.11 indicate the stability of the rheology of the $30 \mathrm{~Pa} / 30 \mathrm{cP}$ and $6 \mathrm{~Pa} / 6 \mathrm{cP}$ clay simulants throughout the course of testing.

Representative rheograms for the $30 \mathrm{~Pa} / 30 \mathrm{cP}$ clay simulant at the beginning and the end of testing are shown in Figure 3.12 and Figure 3.13. These figures show similar flow curves for the $30 \mathrm{~Pa} / 30 \mathrm{cP}$ clay simulant at the beginning and the end of the 10-day testing. Figure 3.14 and Figure 3.15 show representative rheograms for the $6 \mathrm{~Pa} / 6 \mathrm{cP}$ clay simulant at the beginning and the end of testing, respectively. Similar to the case of the $30 \mathrm{~Pa} / 30 \mathrm{cP}$ clay simulant, these figures show the rheological stability of the $6 \mathrm{~Pa} / 6 \mathrm{cP}$ clay simulant.

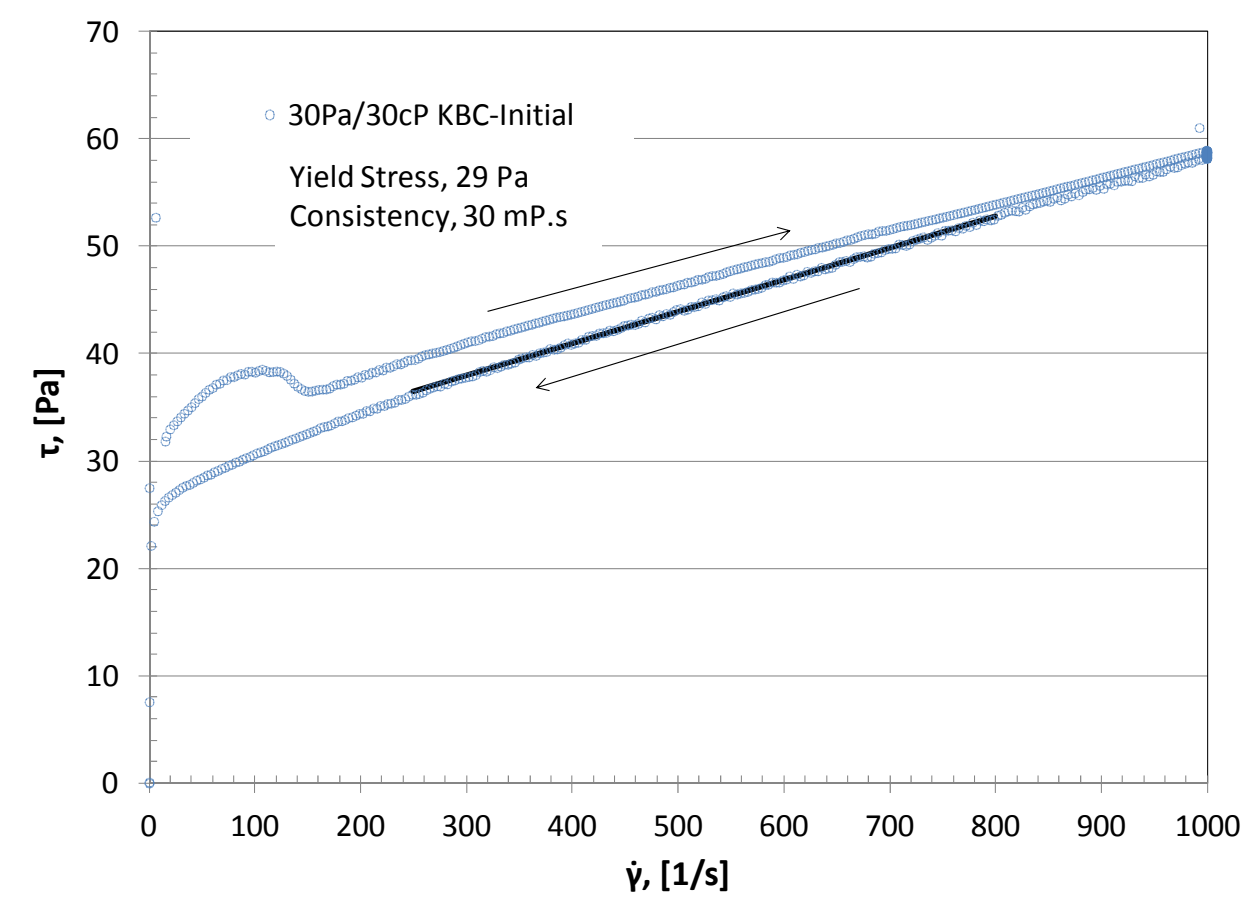

Figure 3.12. Rheogram for Clay Simulant at the Beginning of $30 \mathrm{~Pa} / 30 \mathrm{cP}$ Testing 


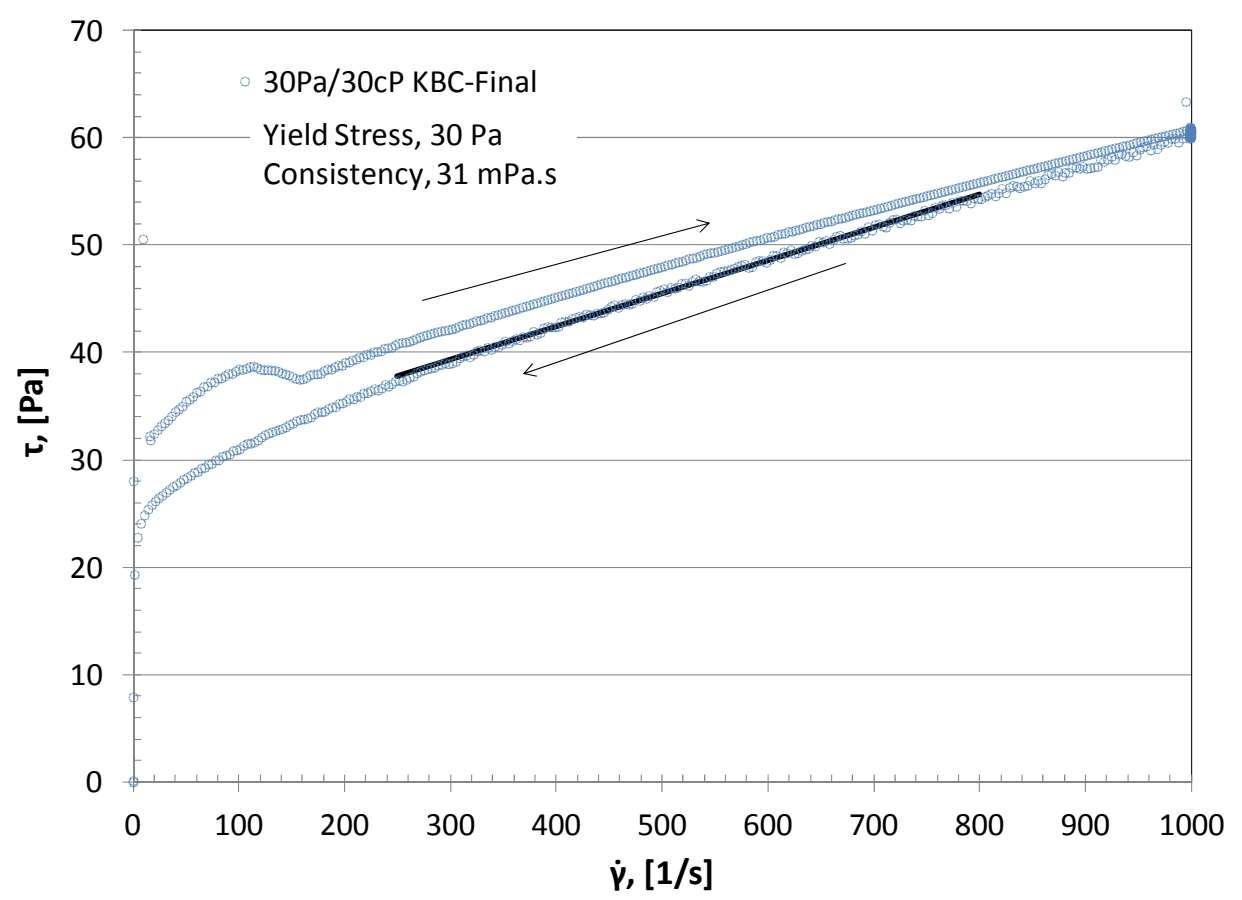

Figure 3.13. Rheogram for Clay Simulant at the End of $30 \mathrm{~Pa} / 30 \mathrm{cP}$ Testing

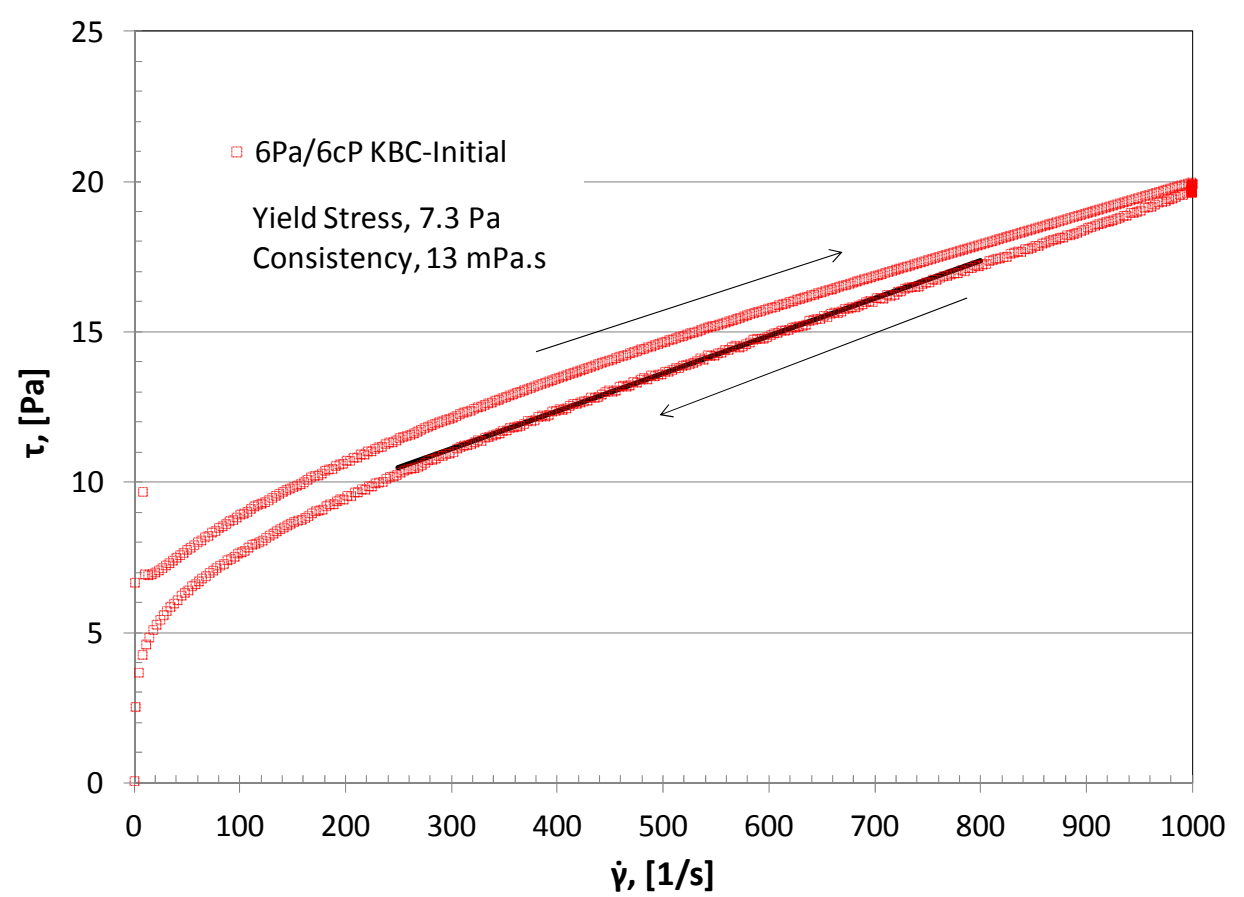

Figure 3.14. Rheogram for Clay Simulant at the Beginning of $6 \mathrm{~Pa} / 6 \mathrm{cP}$ Testing 


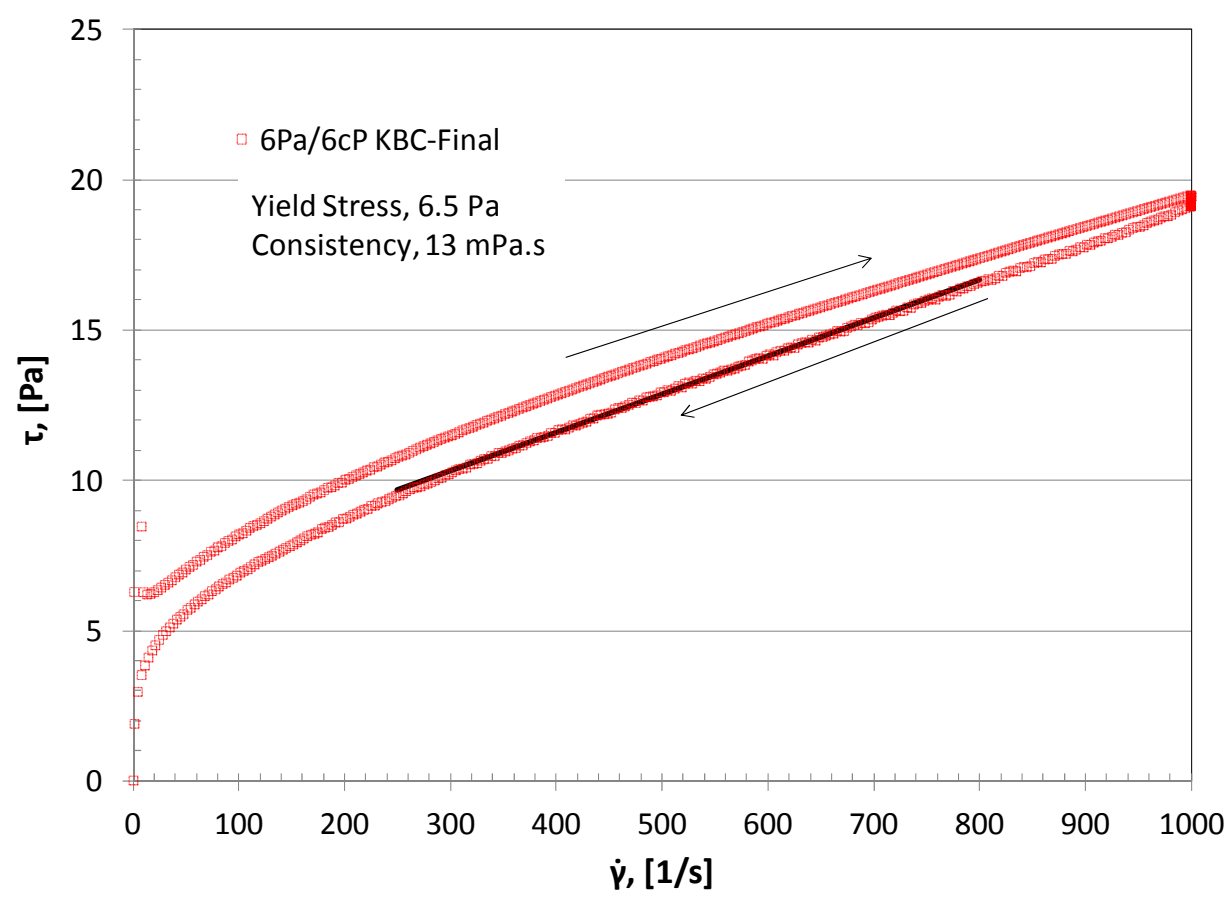

Figure 3.15. Rheogram for Clay Simulant at the End of $6 \mathrm{~Pa} / 6 \mathrm{cP}$ Testing

Figure 3.16 and Figure 3.17 show the Bingham yield stress and Bingham consistency as a function of testing time for the chemical simulants. As with the companion clay figures discussed previously, the measurements give an idea of how the rheological parameters evolved during the testing but do not necessarily describe the rheological parameters that were present for any particular set of tests. The figures for the FEG simulant follow the same convention as for the clay simulant: the purple data point is a sample of the parent FEG slurry, the red data points for samples collected over the 7-day testing of the $30 \mathrm{~Pa} / 30 \mathrm{cP}$ FEG simulant, and the blue data points for samples collected over the 7-day testing of the $6 \mathrm{~Pa} / 6 \mathrm{cP}$ FEG simulant. Daily monitoring of the simulant rheology was performed as previously discussed.

Unlike the stable, well-behaved $30 \mathrm{~Pa} / 30 \mathrm{cP}$ clay simulant, the $30 \mathrm{~Pa} / 30 \mathrm{cP}$ FEG simulant behaved anomalously, which made keeping the simulant within the rheology targets difficult. Beginning at the end of the fourth day of testing (approximately $336 \mathrm{hr}$ ), the simulant started to exhibit noticeable increases in the measured Bingham parameters. In particular, the consistency for the simulant continued to increase over time despite efforts made to bring the simulant rheology back to the target values by concentration adjustments. These unexpected rheological behaviors of the FEG simulant were suspected to be caused by thickening with the application of shear, which is evident from the representative rheograms provided in this section. This thickening was time-dependent but reversible (rheopecty). The underlying cause for this observed behavior was not identified, but could include air entrainment, deposition of solids, or particle agglomeration. One of these phenomena, particle agglomeration, was observed in the PSD results, but may not be the sole cause. 


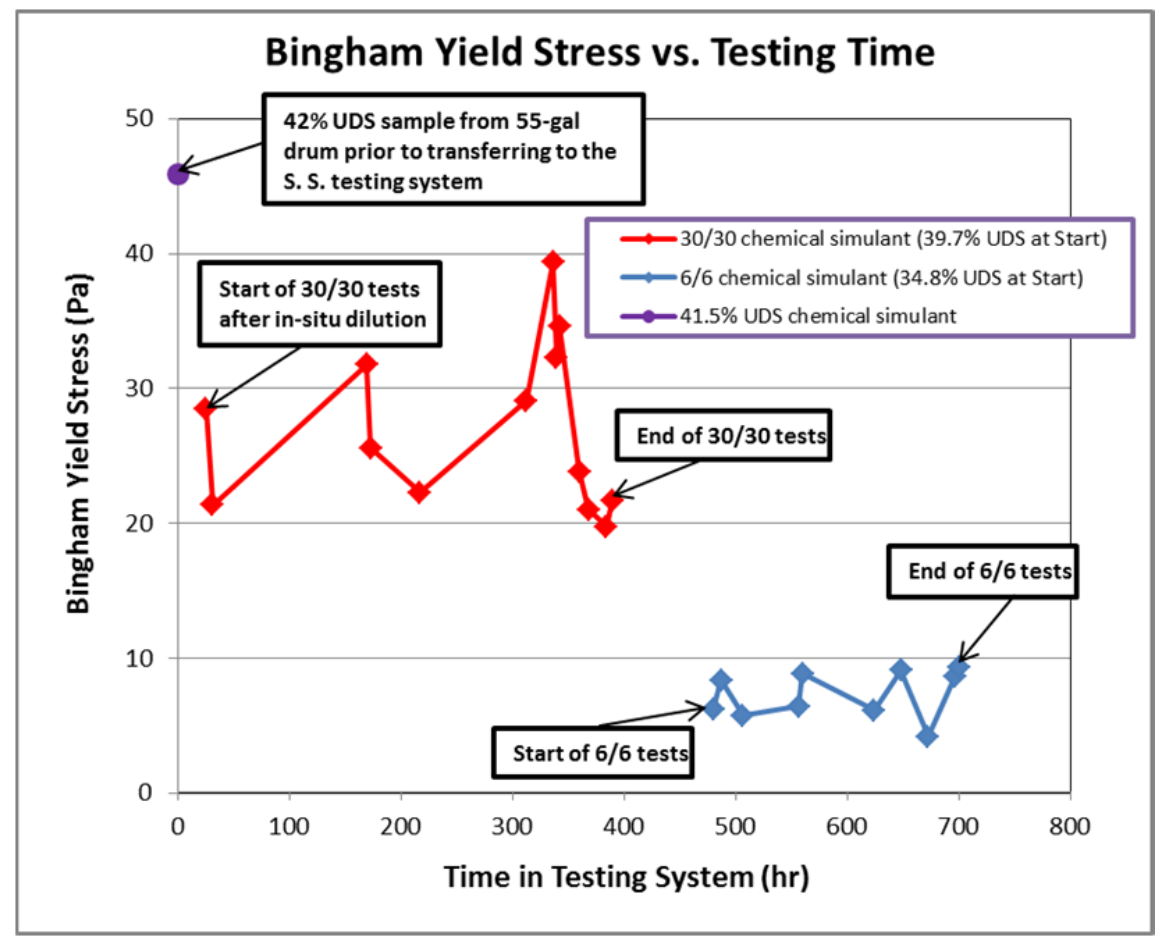

Figure 3.16. Bingham Yield Stress as a Function of Testing Time for Chemical Simulants

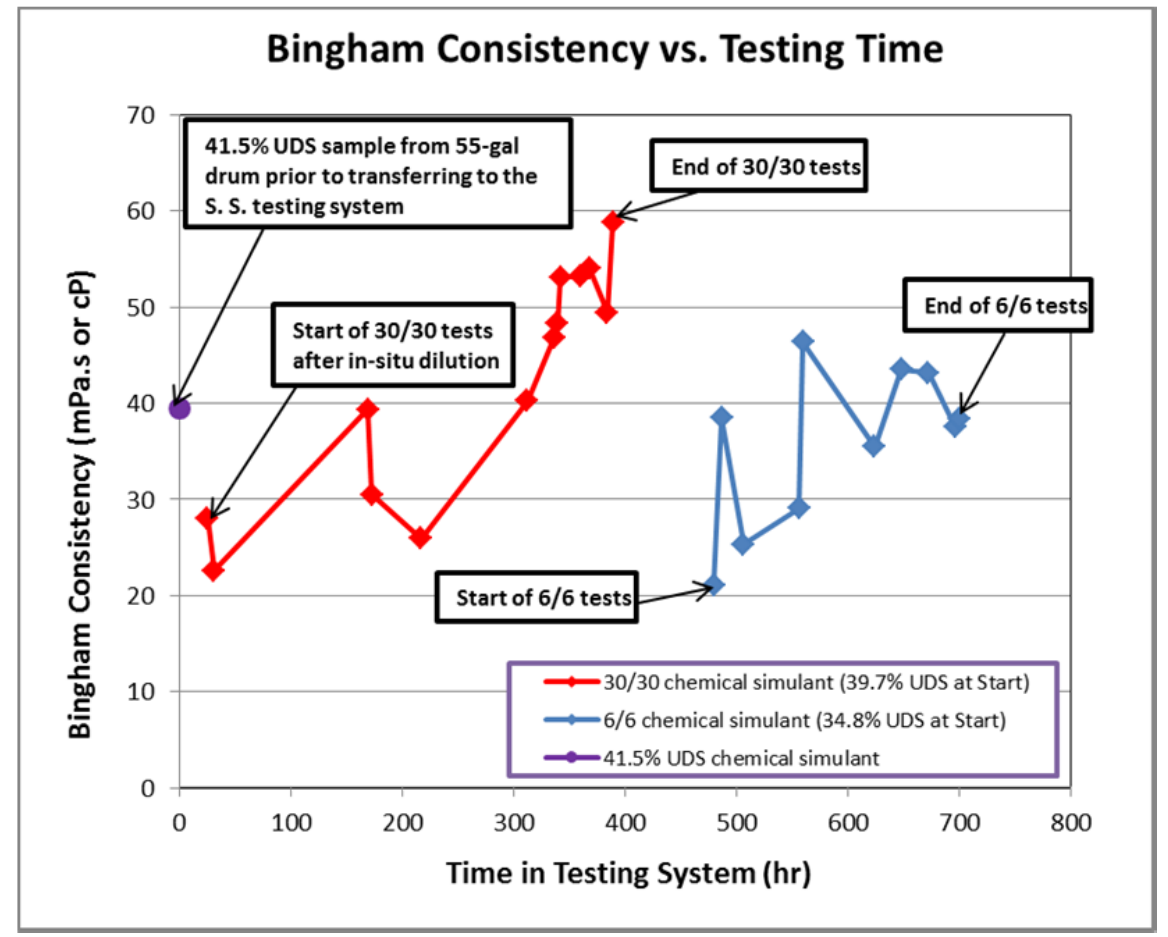

Figure 3.17. Bingham Consistency as a Function of Testing Time for Chemical Simulants 
At the completion of the $30 \mathrm{~Pa} / 30 \mathrm{cP}$ testing, the FEG simulant was diluted with bottled distilled water to achieve the Bingham parameter target for the $6 \mathrm{~Pa} / 6 \mathrm{cP}$ simulant. The $6 \mathrm{~Pa} / 6 \mathrm{cP}$ FEG simulant also exhibited anomalous rheological behavior suspected to be due to time-dependent thickening and particle agglomeration. Particularly, Figure 3.17 shows an upward trend in the Bingham consistency of the $6 \mathrm{~Pa} / 6 \mathrm{cP}$ chemical simulant ranging from 20 to $46 \mathrm{cP}$ over the course of 7 days of testing. Overall, in contrast to the rheology of the clay simulants, the rheology of the chemical slurry simulants was anomalous and difficult to control by using the simple concentration adjustments. Even the sample data provided in the figures does not reflect how rapidly the rheology was observed to change with time when circulated in the small-scale system.

Representative rheograms for the $30 \mathrm{~Pa} / 30 \mathrm{cP}$ FEG simulant at the beginning and the end of testing are given in Figure 3.18 and Figure 3.19, respectively. These figures show noticeable changes in the rheology of the $30 \mathrm{~Pa} / 30 \mathrm{cP}$ chemical simulant at the beginning and the end of the 7 -day testing. Due to the observed rheopectic behavior, a lower shear rate range $\left(0\right.$ to $\left.400 \mathrm{~s}^{-1}\right)$ was used for measuring the flow curve of the simulant samples. Note that shear rates in this range are considerably lower than shear rates experienced by the simulant in the small-scale system, where small clearances in needle valves and at the spray orifice itself lead to much larger shear. The apparent viscosity of the FEG simulant may be much greater when it is being sprayed than the values that were measured from samples. This adds to the difficulty in assessing the rheology of the FEG simulants.

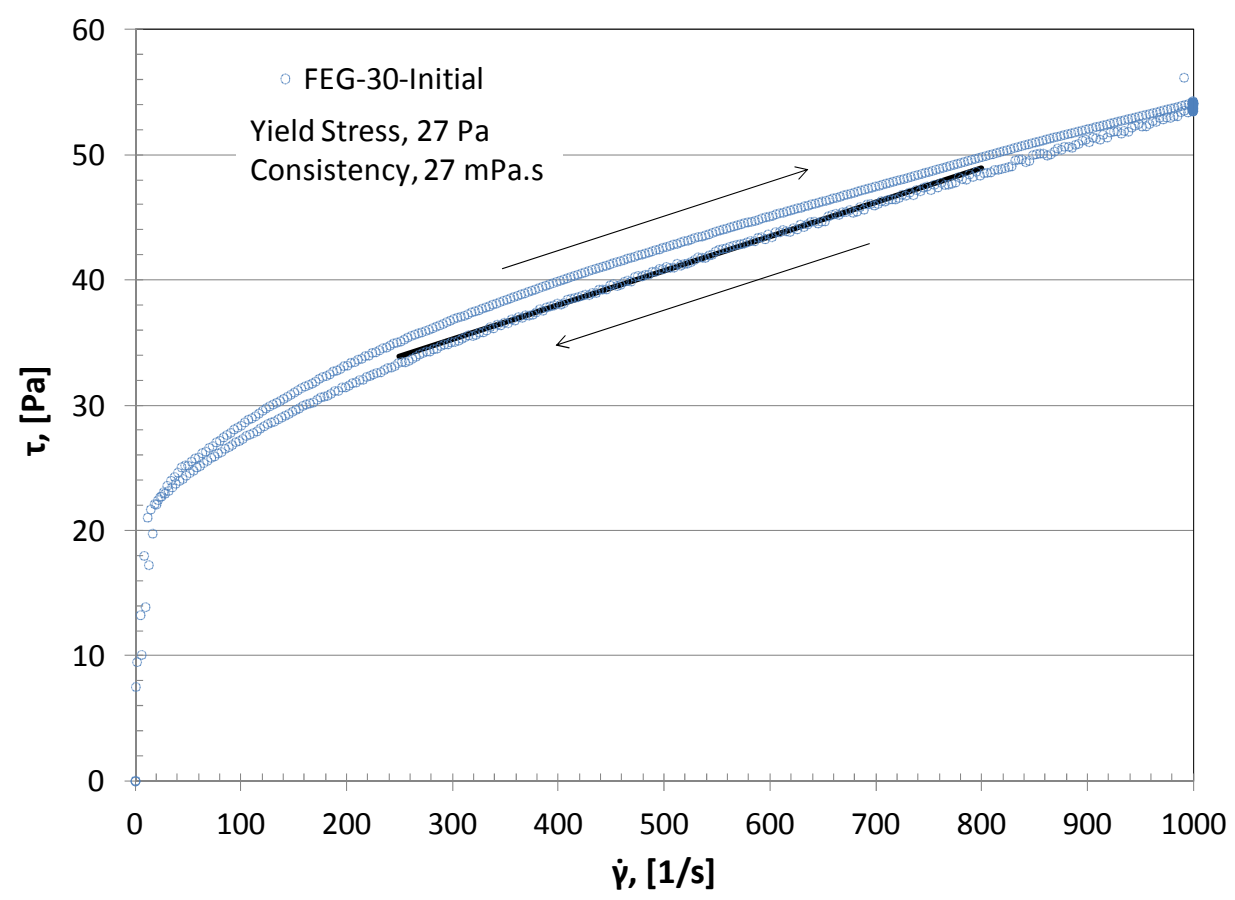

Figure 3.18. Rheogram for Chemical Simulant at the Beginning of $30 \mathrm{~Pa} / 30 \mathrm{cP}$ Testing

Figure 3.20 shows a rheogram of the same $30 \mathrm{~Pa} / 30 \mathrm{cP}$ chemical simulant sample that was collected at the end of testing but was measured over a lower shear rate range of 0 to $400 \mathrm{~s}^{-1}$ as opposed to the typical range of 0 to $1000 \mathrm{~s}^{-1}$. Figure 3.21 and Figure 3.22 display representative rheograms for the $6 \mathrm{~Pa} / 6 \mathrm{cP}$ chemical simulant at the beginning and the end of testing, respectively. These rheograms were measured over the lower shear rate range, as the dramatic thickening exhibited in Figure 3.19 continued to 
be observed if the typical range was used. Additional rheograms for chemical simulant samples collected at other times of testing are provided in Appendix E.

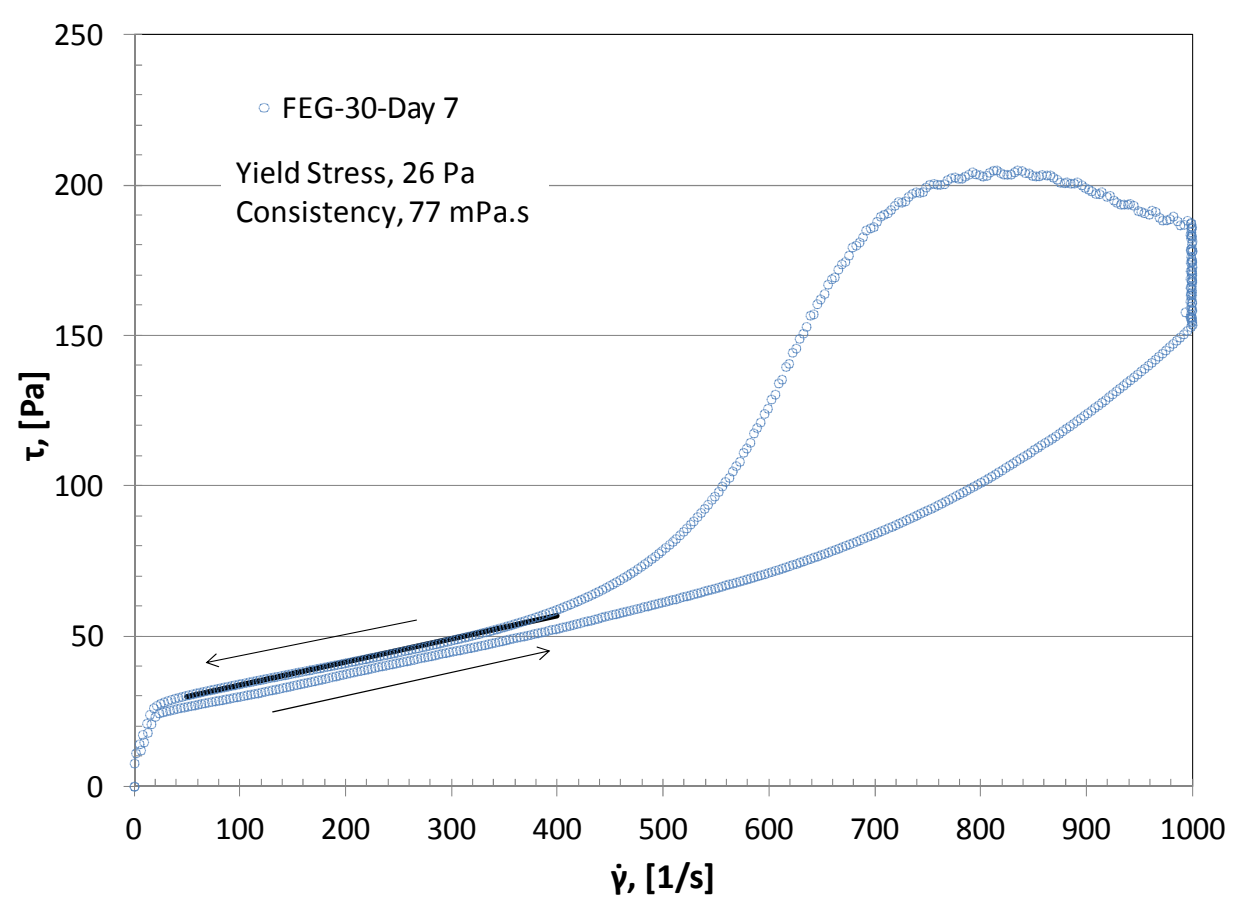

Figure 3.19. Rheogram for Chemical Simulant at the End of $30 \mathrm{~Pa} / 30 \mathrm{cP}$ Testing; Measured Shear Rate Range of 0 to $1000 \mathrm{~s}^{-1}$

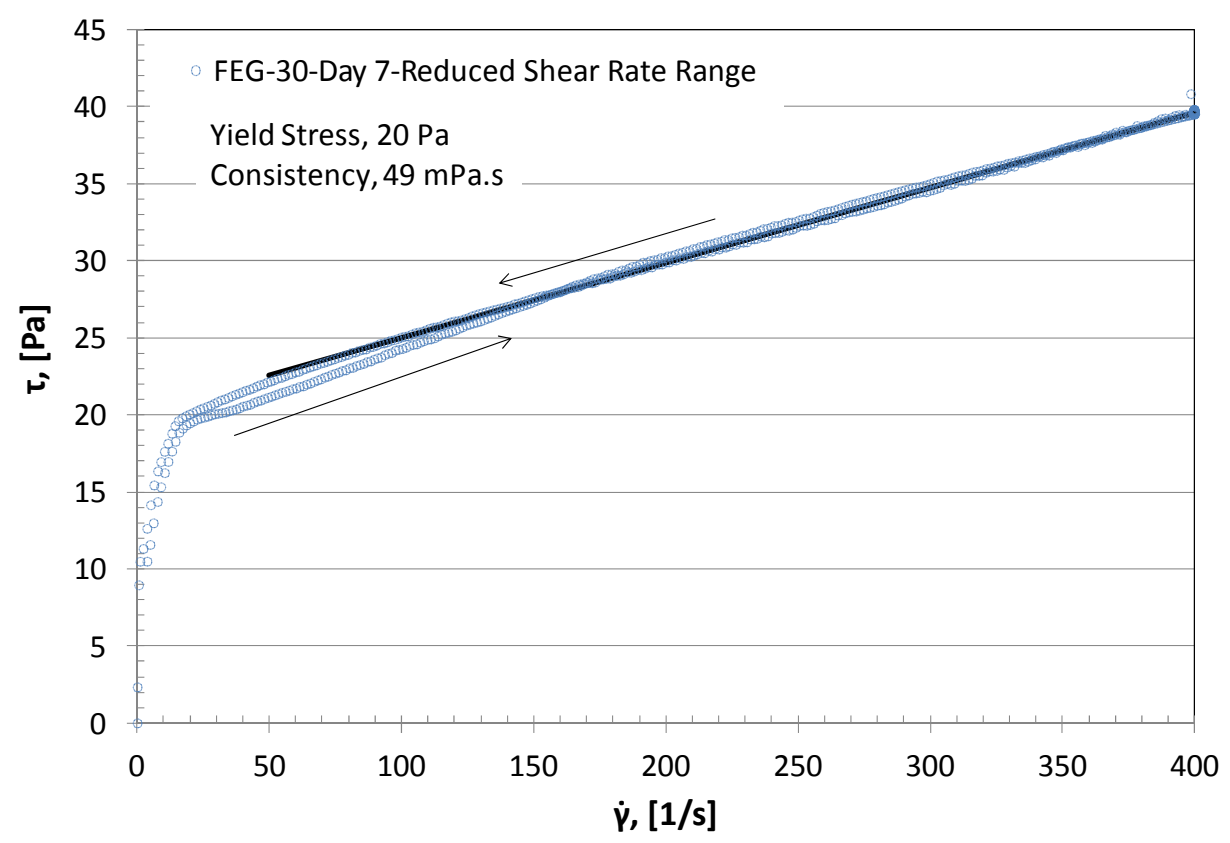

Figure 3.20. Rheogram for Chemical Simulant at the End of $30 \mathrm{~Pa} / 30 \mathrm{cP}$ Testing; Measured Shear Rate Range of 0 to $400 \mathrm{~s}^{-1}$ 


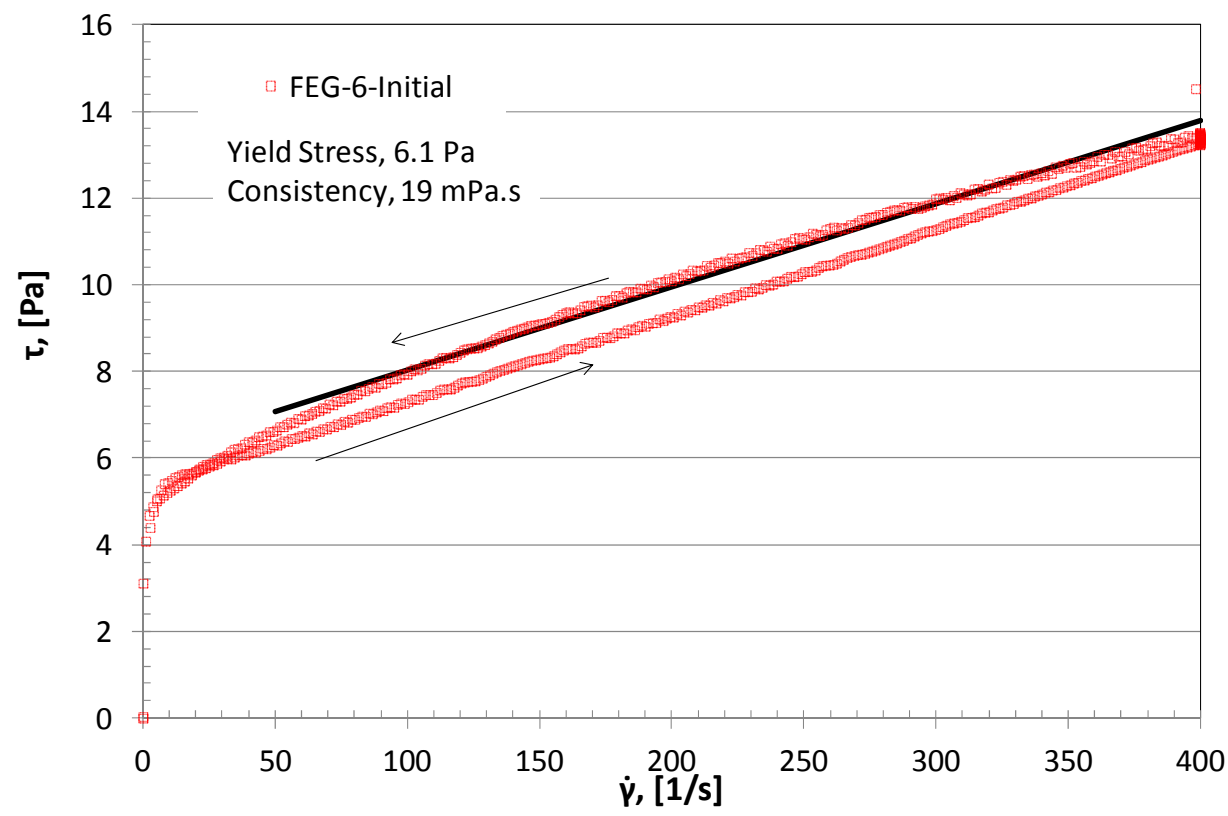

Figure 3.21. Rheogram for Chemical Simulant at the Beginning of $6 \mathrm{~Pa} / 6 \mathrm{cP}$ Testing; Measured Shear Rate Range of 0 to $400 \mathrm{~s}^{-1}$

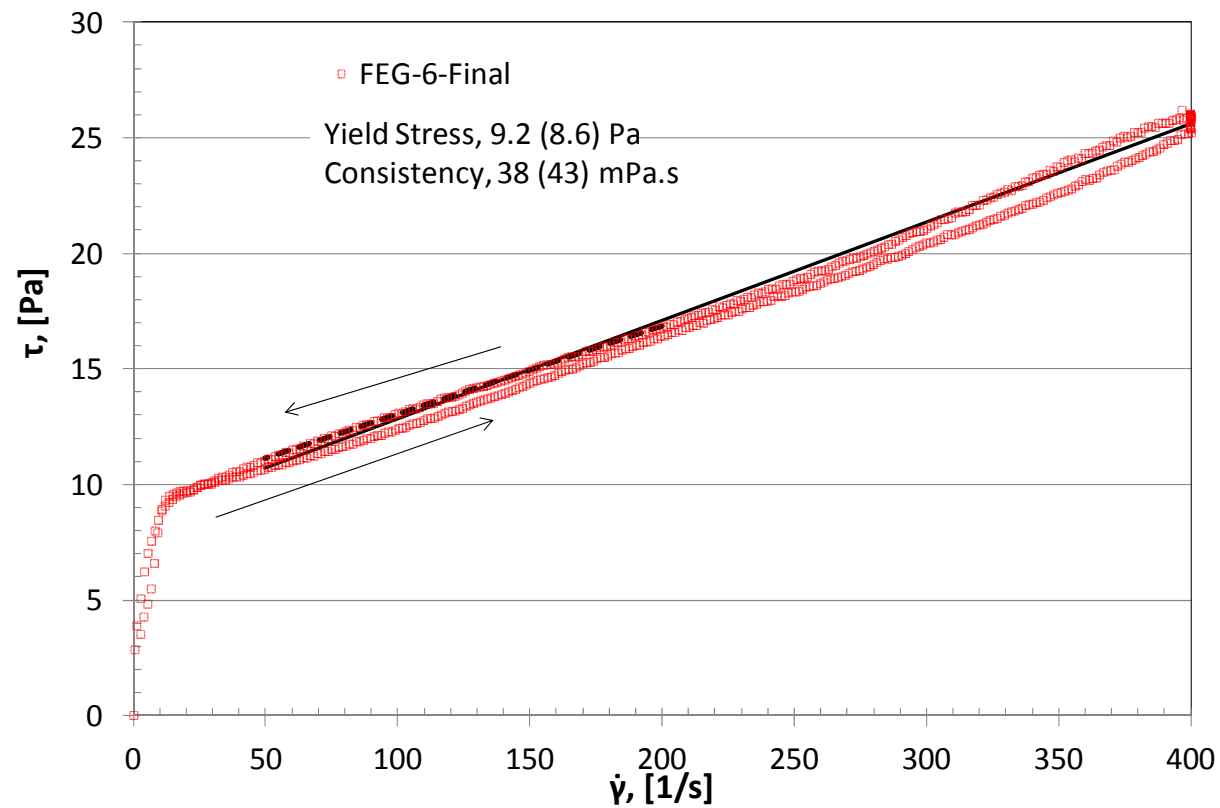

Figure 3.22. Rheogram for Chemical Simulant at the End of $6 \mathrm{~Pa} / 6 \mathrm{cP}$ Testing; Measured Shear Rate Range of 0 to $400 \mathrm{~s}^{-1}$. Bingham fit analysis given from $250-800 \mathrm{~s}^{-1}$ and $\left(50-200 \mathrm{~s}^{-1}\right)$. 


\subsection{Equipment Description}

The Phase II small-scale aerosol testing was performed using the Phase I equipment and instrumentation with a few modifications. As before, the test system is capable of circulating liquids and slurries at constant flow rates and pressures to test a range of orifice sizes, orifice configurations, and chamber configurations. For completeness, the system will be described in Section 4.1. Section 4.2 delineates modifications to the test system that resulted in differences from the Phase I system. Note that the potential impacts of these modifications on system operation (and differences from Phase I operations) will not be discussed until Section 5. Some Phase II modifications required additional shakedown testing to verify that the collected aerosol data was similar to Phase I data at the same conditions. These comparison test results are described in Section 6. Finally, Section 4.3 identifies and describes the orifices used in testing.

\subsection{Testing Equipment}

The small-scale test stand was comprised of four major components: the process equipment, the data acquisition system, the aerosol chamber, and the Malvern Insitec-S (the aerosol instrument). These components are described in the following subsections. For additional information about some non-standard testing configurations, see Section 5.4.2. The entire system is located in a walk-in fume hood in Laboratory 107 of the Applied Process Engineering Laboratory (APEL). A representative photograph of the system is shown in Figure 4.1. Note that this is an image from the Phase I configuration, but all components of the Phase II system are similar. All other images in this section are from the Phase II configuration.

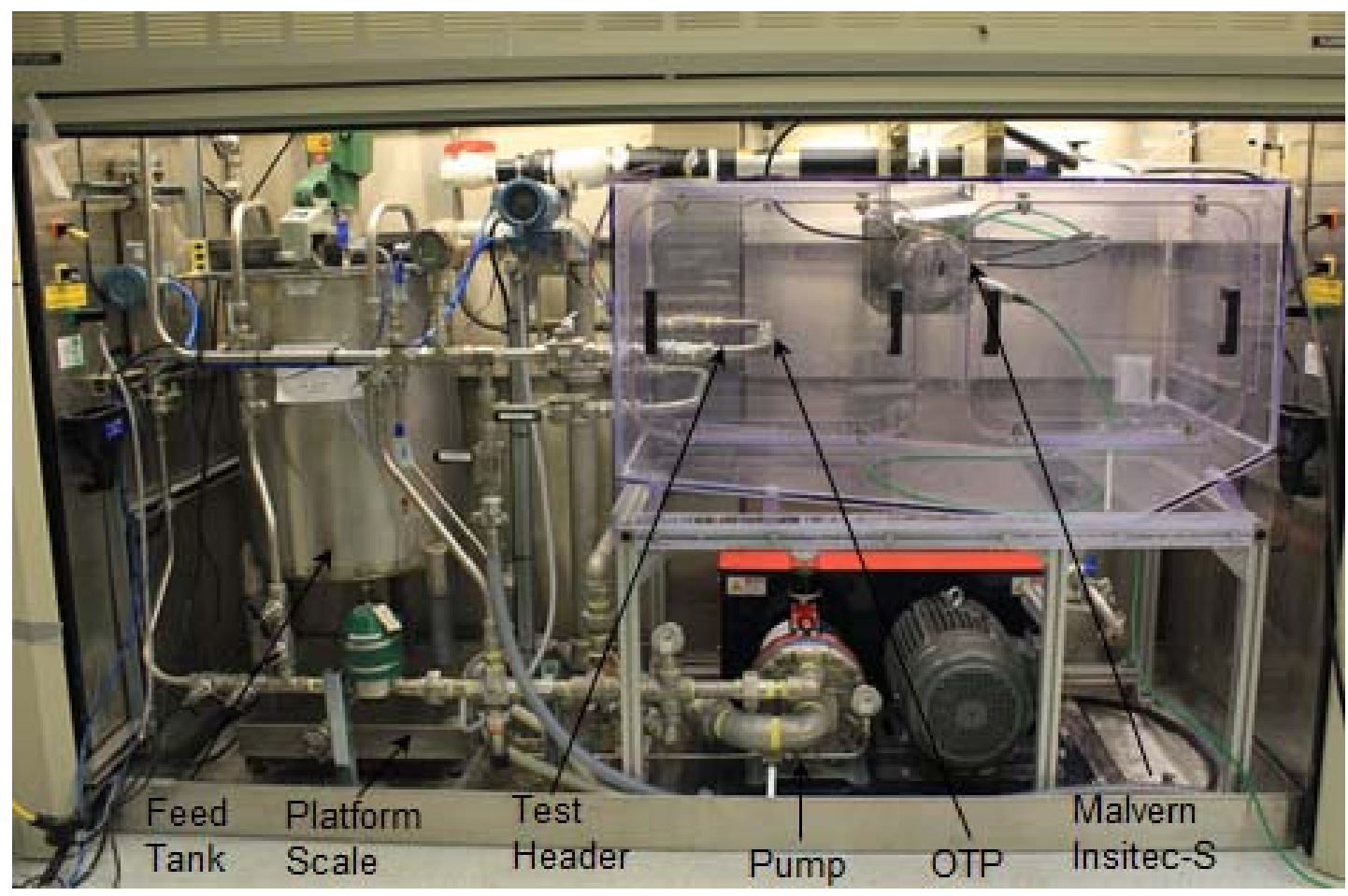

Figure 4.1. Image of the Small-Scale Test System in the Fume Hood. The term OTP in the image refers to the orifice test piece where the spray originates during a test. 


\subsubsection{Process Equipment}

The small-scale spray release system circulates simulant solutions using a positive displacement pump so they can be sprayed in a controlled manner into the aerosol chamber. An example of a spray is shown in Figure 4.2. The schematic of the system when it is in its standard configuration is presented in Figure 4.3. A simulant batch was typically prepared in another vessel or drum and then transferred into the feed tank. The standard simulant batch was approximately 40 gallons when first loaded into the feed tank. The tank was equipped with a Lightnin Model X5P100 1-HP clamp mount mixer with two impellers, which were attached approximately $9 \mathrm{in}$. apart, with the lower blade on the bottom of the 33-in. shaft. The feed tank was free-standing on a platform scale, with all process piping suspended independently of the tank. Six process lines were suspended into the tank: the inlet line, the bypass line, the purge line, the return line, the pressure bypass line, and a pressure relief line. Nearly all of the fittings and line connections were Swagelok-type to accommodate disassembly and cleaning.

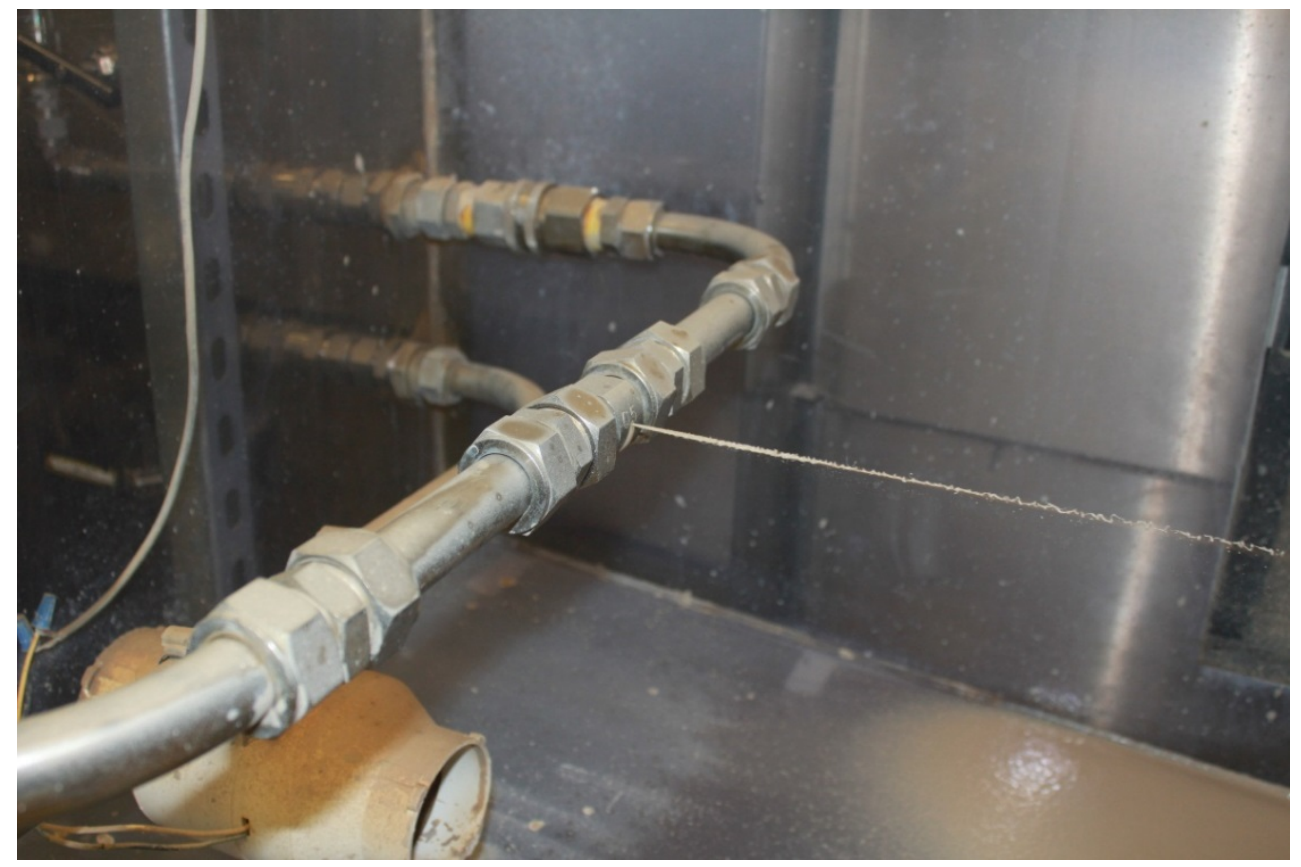

Figure 4.2. Example of a Spray from an Orifice Test Piece Installed in the Spray Header

The inlet line on the suction side of the pump was 2.5-in.-diameter stainless steel piping with flanged connections. A ball valve was located at the highest point of the inlet line to assist in priming the pump using a disengagement pot and house vacuum. The pump, a Hydra-Cell D/G-35-X diaphragm feed pump controlled by a Honeywell variable frequency drive (VFD), was capable of delivering constant flow rates of up to 30 gallons per minute (gpm). The pump had a 0.5 -in.-diameter stainless steel tube purge line installed with a pressure control valve that returned to the feed tank. The discharge side of the pump had 1.25-in.-diameter stainless steel tubing with two pressure relief devices that diverted flow back to the tank in the event of excessive pressures. The first was a pressure relief valve rated to $600 \mathrm{psig}$ with a 1-in.-diameter stainless steel tube (the pressure bypass line), and the second was a pressure relief valve that was manually set to $500 \mathrm{psig}$, also with a 1-in.-diameter stainless steel tube (the pressure relief line). Downstream of the pressure relief valves was a pulse dampener to mitigate any pulsation produced by the positive displacement pump. 


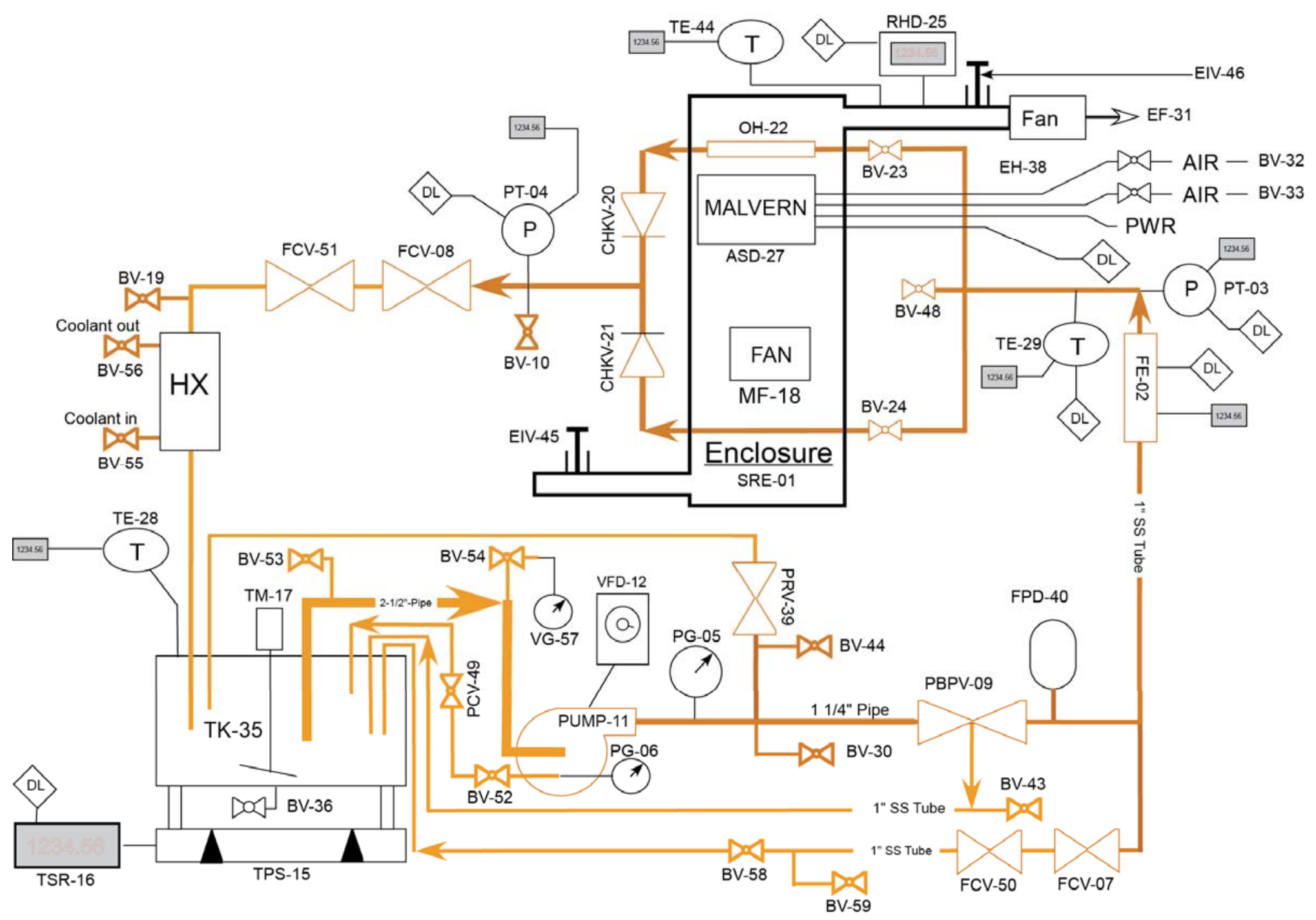

Figure 4.3. Schematic of the Small-Scale Test Stand Used in Phase II 
The pump discharge was then split between the test line and a bypass line (both were 1-in.-diameter stainless steel tube). The bypass line used two needle valves in series to control the ratio of flow between the two flow paths. Simulant traveling through the bypass line returned to the tank, while simulant in the test line traveled through some instrumentation before taking one of three paths:

1. Simulant was circulated back to the tank using auxiliary flexible hose to purge air from the system or perform other maintenance tasks. This path bypassed the aerosol chamber entirely (the hose was connected at BV-48, see Figure 4.3).

2. Simulant traveled inside the aerosol chamber through the bypass header (BV-24 was open, BV-23 was closed) and returned to the tank. After leaving the chamber, the simulant passed through two more needle valves which were throttled to set the pressure in the system. After the needle valves, the simulant was cooled using a stainless steel spiral tube-in-tube heat exchanger interfaced with a chiller and returned to the tank via a 1-in diameter stainless steel tube. The Exergy spiral heat exchanger had a 1.25-in.-diameter outer tube (coolant side) and 0.75-in.-diameter inner tube (simulant side). The Dimplex Thermal Solutions chiller was a SV Series 2-ton air-cooled unit designed for indoor use. The coolant was a $30 \mathrm{wt} \%$ propylene glycol solution in water.

3. Simulant traveled inside the aerosol chamber through the spray header (BV-23 was open, BV-24 was closed) and sprayed out of the orifice installed in the header. The spray header is described in more detail in the following paragraph. Simulant which was not sprayed out the orifice returned to the tank by the same route as simulant that went through the bypass header as described in item $\# 2$.

The spray header could be removed from the enclosure if required. Swappable orifice test pieces (OTPs) were positioned in an interchangeable portion of the spray header within the aerosol test enclosure to facilitate various sprays (recall that an example of a spray is shown in Figure 4.2). The wall thickness of each OTP was equivalent to that in a 3 -in. schedule 40 stainless steel pipe, thus providing a leak-path length equal to the large-scale breaches and much of the piping used in the WTP. The inner surface of each OTP was flush with the inner wall of the stainless steel tubing. The tubing had a nominal outer diameter of 1-in. and a nominal wall thickness of 0.065 in. The fluid velocity at the target flow rate of $11.4 \mathrm{gpm}$ was $6.1 \mathrm{ft} / \mathrm{s}^{1}{ }^{1}$ The velocity was calculated using the nominal outer diameter of 1 -in. tubing with a wall thickness of 0.065 in. A flow rate of approximately 11.4 gpm through the test header was calculated to provide the same wall shear stress as would exist in 3-in. schedule 40 pipes with a flow velocity of $6.5 \mathrm{ft} / \mathrm{s}$. This flow velocity and pipe size are typical of the smaller lines in the WTP equipment, and the velocity in the spray header was established at $6.5 \mathrm{ft} / \mathrm{s}$ or greater for the large-scale tests; therefore, the approximate matching of wall shear stress provided consistent conditions for the orifice entry point between the two test stands. The matched-shear-stress criterion was met by being within about 10 percent of the target flow rate for all simulants except the $30 \mathrm{~Pa}$ and $6 \mathrm{~Pa}$ non-Newtonian FEG slurries. For these simulants, flow rates were seldom as high as $10 \mathrm{gpm}$ and often were in the 3 to 9 gpm range due to the difficulty of pumping these rheopectic fluids. The OTPs used in the Phase II testing are discussed further in Section 4.3.

\footnotetext{
${ }^{1}$ Note this value is higher than the target velocity of $5.4 \mathrm{ft} / \mathrm{s}$ used in earlier testing (see Mahoney et al. 2013). At this flow rate, approximately the same wall shear stress would be achieved for all the Newtonian and $6 \mathrm{~Pa} / 6 \mathrm{cP}$ simulants used in spray release testing, which was the basis for the calculation. A line velocity of $6.1 \mathrm{ft} / \mathrm{s}$ results in a higher wall shear stress for $30 \mathrm{~Pa} / 30 \mathrm{cP}$ simulants than what would be experienced by $30 \mathrm{~Pa} / 30 \mathrm{cP}$ waste in the WTP.
} 
As Phase II testing proceeded, some adjustment or modifications to the standard configuration were made in order to manage the challenges various simulants presented. Significant configuration changes included:

- Removing the heat exchanger from its location on the return line to a loop off of the bypass line (inlet upstream of FCV-07, outlet at BV-59) where it could be isolated from the rest of the system.

- Adding a portable pneumatic diaphragm pump as a priming pump to assist PUMP-11. The diaphragm pump suction was from the bottom of the feed tank (BV-36) and discharged to the PUMP-11 inlet via BV-30.

- Swapping out the existing 2.5-in. stainless steel piping on the inlet line for 1.5-in. clear flexible hose when simulants with dense particles were being tested. This change was made to reduce the possibility that the dense particles would settle out in the inlet piping before reaching the pump inlet and thus not be represented in the spray out of the orifice.

Of these significant configuration changes, only the third one was planned. The first two changes were in response to difficulties experienced during testing with the FEG simulant and are described in more detail in Section 5.4.2.

\subsubsection{Data Acquisition and Collection}

The instruments that were part of the small-scale test stand monitored operating parameters to assist operators in performing tests in a controlled and repeatable manner. Data from several of these instruments was recorded for each test. A calibrated Omega data logger, connected to a laptop computer in the laboratory, was used to collect temperature data and raw voltages (from instruments that did not measure temperature) that could be converted, using the instrument calibration data, into the appropriate engineering units. The data logger collected instrument data at $2 \mathrm{~Hz}$ resolution. Time, temperature, and voltage data were saved as comma-separated value files derived from the data logger. Inventory changes in the loop were accounted for using a RS232 digital interface of the platform scale display with Microsoft Excel ${ }^{\mathrm{TM}}$ that recorded the mass at $1 \mathrm{~Hz}$ resolution.

Table 4.1 lists instruments used to collect data to support data analysis for the small-scale aerosol tests. Key instrument data was also manually recorded as part of the test procedure. Note that some instruments were For Information Only (FIO) as they were not calibrated. Table 4.1 does not include the Malvern Insitec-S, which has its own data acquisition system and is described in Section 4.1.4. The Malvern Insitec-S recorded aerosol data at $4 \mathrm{~Hz}$, so accurate resolution of spray events in time was critical. This was achieved by performing all three data acquisition tasks (process instruments via the data logger, scale data via an Excel digital interface, and the proprietary Malvern Insitec-S data acquisition software) on the same computer to force synchronization of time series data. It was standard to collect at least 1 min of data before the spray was initiated and 2 min of data after the spray was discontinued.

As can be seen in Figure 4.3, there were other instruments (primarily pressure gauges) that were FIO operational aids but were not recorded by any of the data acquisition systems. 
Table 4.1. Instruments Used in Small-Scale Aerosol Tests and Data Analysis

\begin{tabular}{|c|c|c|}
\hline Instrument Name & Measurement & $\begin{array}{l}\text { Calibrated } \\
\text { Range }\end{array}$ \\
\hline \multirow[t]{2}{*}{ Micro Motion Coriolis mass flow sensor } & Flow rate in test header ${ }^{(a, b)}$ & $1-35 \mathrm{gpm}$ \\
\hline & Density of the simulant & FIO \\
\hline Honeywell pressure transmitter & Pressure in test header upstream of the OTP ${ }^{(a, b)}$ & $0-500$ psig \\
\hline Honeywell pressure transmitter & Pressure in test header downstream of the $\mathrm{OTP}^{(\mathrm{a}, \mathrm{b})}$ & $0-500$ psig \\
\hline Thermocouple (Type T) & Temperature upstream of test header ${ }^{(a, b)}$ & $32-120^{\circ} \mathrm{F}$ \\
\hline Thermocouple (Type T) & Feed tank temperature ${ }^{(b)}$ & $32-120^{\circ} \mathrm{F}$ \\
\hline \multirow[t]{2}{*}{ Omega temperature transmitter } & Temperature in aerosol chamber ${ }^{(b)}$ & $39-167^{\circ} \mathrm{F}$ \\
\hline & Relative humidity (RH) in aerosol chamber & FIO \\
\hline & Mass in the feed $\operatorname{tank} \mathrm{k}^{(\mathrm{c})}$ & $0-600 \mathrm{lb}$ \\
\hline Dwyer VFA-3 rotameter & Purge air flow to Malvern Insitec-S ${ }^{(b)}$ & $0-5 \mathrm{SCFH}$ \\
\hline Dwyer RMB-55-SSV rotameter & Purge air flow to Malvern Insitec-S ${ }^{(b)}$ & 40-400 SCFH \\
\hline Valve switches & Open/close state for BV-23 and BV-24 & on/off (d) $^{(1)}$ \\
\hline \multicolumn{3}{|c|}{$\begin{array}{l}\text { (a) Connected to data logger OMB DAQ 2416-4AO, Serial No. 29991; Software: TracerDAQ Pro Version 2.1.6.1. } \\
\text { Calibrated by the PNNL standards laboratory. }\end{array}$} \\
\hline \multicolumn{3}{|l|}{ (b) Calibrated to local display. } \\
\hline \multicolumn{3}{|c|}{ (c) Local display output to Excel spreadsheet using a RS232 digital interface. } \\
\hline \multicolumn{3}{|c|}{ (d) On/off refers to the voltage: open was high voltage, closed was low voltage. } \\
\hline
\end{tabular}

\subsubsection{Aerosol Chamber}

During small-scale spray tests, the spray leaving the orifice was contained in a chamber. The chamber confined all the droplets emanating from the jet and other droplet formation mechanisms to provide a fixed volume, of which it was assumed the Malvern Insitec-S instrument took a representative sample. An isometric representation of the aerosol chamber is shown in Figure 4.4. The chamber had two removable windows on the front side to permit routine operations and installation or maintenance of equipment.

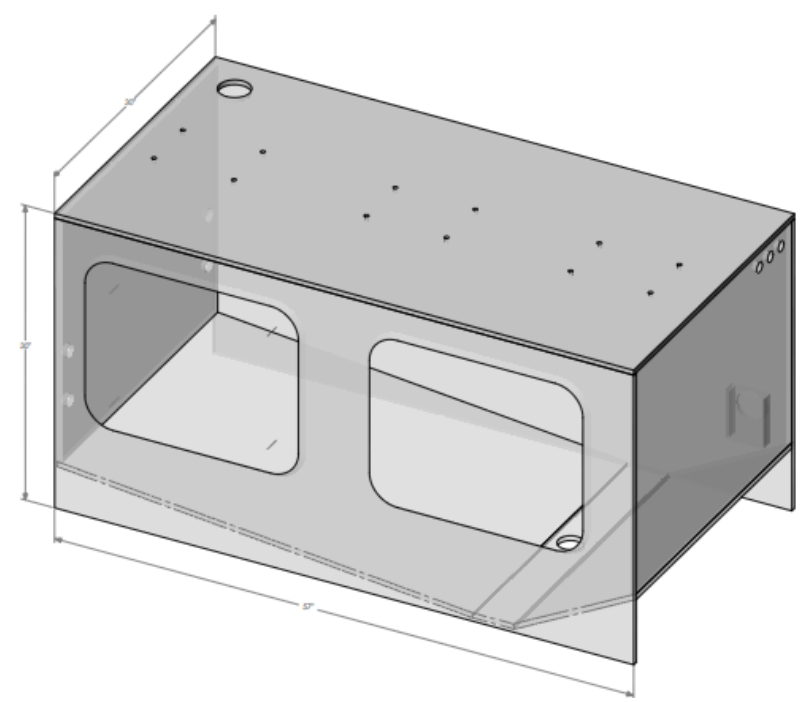

Figure 4.4. Isometric Drawing of the Aerosol Test Chamber 
For reference, the spray header was located at the left end of the chamber as shown in Figure 4.4. The spray header elevation was halfway between the floor and the greatest height of the enclosure. The sloped bottom directed the portion of the spray that did not remain aloft to the drain near the right end. The overall dimensions of the aerosol chamber were approximately $30 \mathrm{in}$. wide $\times 30 \mathrm{in}$. high $\times 57 \mathrm{in}$. long with an internal volume of $24.8 \mathrm{ft}^{3}$. More exact dimensions and equipment locations are given in Figure 4.5 and Table 4.2. To accommodate a new lens assembly (explained in more detail in Section 4.1.4) installed on the Malvern Insitec-S, a portion of the chamber had to be cut out. The "lens cut-out" is shaded grey in Figure 4.5 and its location along the back wall of the chamber is noted. The portion of the chamber that was cut out was covered with a box constructed out of sheet metal that had the same interior dimensions ( 8 in. $\times 19.1$ in.) as the cut-out and a depth of 4 in. The sheet metal box was open to the chamber and thus, increased the total internal volume. Therefore, to keep the internal volume consistent with that of Phase I testing, when the Malvern Insitec-S was installed, the cut-out/box opening was covered using plastic panels attached to the back wall using Velcro.

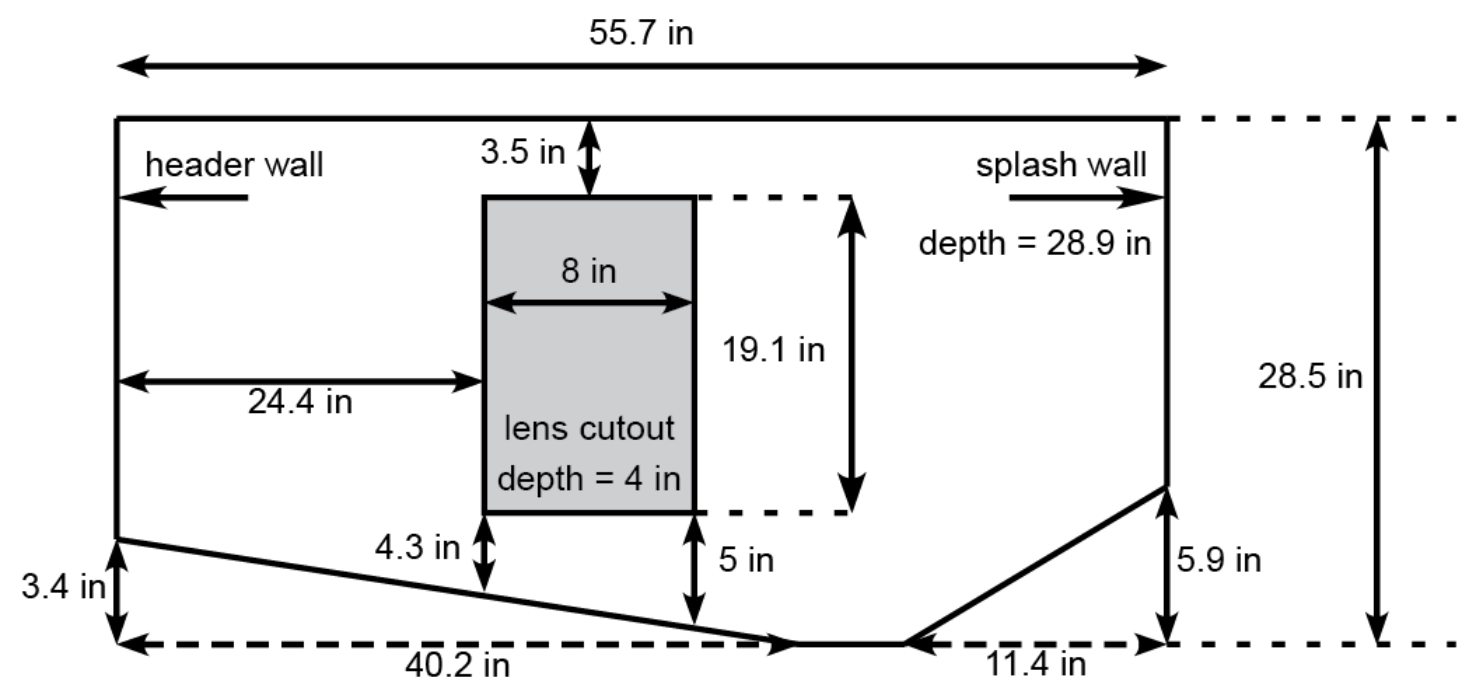

Figure 4.5. Front View and Dimensions (in.) of the Small-Scale Chamber. This diagram includes the lens cut-out locations and dimensions. Note the location of the spray header is at the left of the figure and that the depths of the cut-out and chamber are provided.

The aerosol chamber was also equipped with additional equipment to assist in conducting tests. A blower (Detmar Model 754R, with a capacity of $170 \mathrm{ft}^{3} / \mathrm{min}$ at $13.6 \mathrm{~V}$ ) installed under the bypass header was employed to improve mixing and provide a more homogeneous aerosol concentration in the aerosol chamber. The fan setting that provided adequate aerosol mixing within the chamber was determined to be $6 \mathrm{~V}$ (for more details see Section 6.4.3). In addition, the chamber had a second, external blower (same model as in-chamber blower) used to draw air out of the chamber after an aerosol test and expel it into the hood space. Purge air lines and a cable were introduced through the back-right corner on the top of the chamber to provide air and power to the Malvern Insitec-S, which was mounted using four rods of all-thread suspended from the chamber ceiling. 
Table 4.2. Dimensions of the Chamber Configuration During Phase II Testing

\begin{tabular}{|c|c|c|c|}
\hline & \multicolumn{3}{|c|}{ Distance (in.) From } \\
\hline & Ceiling & Left (Upstream) Wall & Front Wall \\
\hline $\begin{array}{l}\text { Centerline of orifice in its } \\
\text { in-chamber location (Position 2) }\end{array}$ & 14.4 & $\begin{array}{c}13.1 \\
\text { (42.6 in. from splash wall) }\end{array}$ & 14.4 \\
\hline $\begin{array}{l}\text { Centerline of orifice in its } \\
\text { in-spray location (Position 4) }\end{array}$ & 14.4 & $\begin{array}{c}19.1 \\
\text { (36.6 in. from splash wall) }\end{array}$ & 14.4 \\
\hline $\begin{array}{l}\text { Top left front corner of Malvern } \\
\text { Insitec-S when in Position } 2 \\
\text { (default in-chamber) }\end{array}$ & $2.6 \pm 0.3$ & $23.5 \pm 0.3$ & $10.8 \pm 0.3$ \\
\hline $\begin{array}{l}\text { Top left front corner of Malvern } \\
\text { Insitec-S when in Position } 4 \\
\text { (in-spray) }\end{array}$ & $9.6 \pm 0.3$ & $23.5 \pm 0.3$ & $10.8 \pm 0.3$ \\
\hline $\begin{array}{l}\text { Top of front edge of blower when } \\
\text { Malvern Insitec-S is in Position } 2 \\
\text { or } 4\end{array}$ & $20.9 \pm 0.1$ & $10.2 \pm 0.3$ & $12 \pm 1$ \\
\hline \multicolumn{4}{|c|}{$\begin{array}{l}\text { Malvern Insitec-S framework is } 9.6 \text {-in. top to bottom, } 9.6 \text {-in. left to right, } 7.0 \text {-in. front to bacl } \\
\text { is centered both vertically and left-to-right within the framework. } \\
\text { Fan has a } 3 \text {-in. outer diameter at its exhaust and is } 8.1 \text {-in. long. } \\
\text { Positions } 2 \text { and } 4 \text { are shown schematically in Figure } 4.7 \text {. }\end{array}$} \\
\hline
\end{tabular}

\subsubsection{Malvern Insitec-S Instrument}

The size distribution and concentration of aerosol droplets in the small-scale tests were measured using the Malvern Insitec-S particle size analyzer. The Malvern Insitec-S is an open frame aerosol size analyzer that uses laser diffraction to determine aerosol size and concentration. The basic instrument setup and operation principle are illustrated in Figure 4.6. The laser module, housed in the transmitter module, produces a collimated beam that is $10 \mathrm{~mm}$ in diameter and has a wavelength of $670 \mathrm{~nm}$. The receiver module houses the lens and detector assemblies. In Phase II, lenses of different focal lengths, either $100 \mathrm{~mm}$ (Phase I focal length) or $500 \mathrm{~mm}$ (default Phase II focal length), were used. The lens focuses the scattered light onto the detector held within the receiver module. Two glass windows, which are located at the interfaces with the transmitter module and the receiver module, respectively, separate the laser, lens, and detectors from the humid wet chamber environment. The measurement volume is the volume swept out by the laser beam as it passes from the laser source to the detector assembly, with the condition that only the portion that does not restrict free-moving aerosol droplets is considered the measurement volume. The distance between the laser source and detector assembly is set by a $150 \mathrm{~mm}$ spacer bar. To minimize direct contamination from water and slurry, two spray shrouds were installed on top of the glass windows. The shrouds were approximately $1.5 \mathrm{in}$. in diameter and $1.7 \mathrm{in}$. in height, where the diameter refers to the diameter on the outside of the conical shrouds. The measurement volume was calculated from the spacer bar length (subtracting the shroud height on both sides, because the shrouds restrict free motion of aerosol) and beam diameter, resulting in a volume of $5.5 \mathrm{~cm}^{3}$. A purge gas system was used to separate the droplets in the small-scale testing chamber from the windows, thus keeping the windows clean. The purge gas was generated by an air compressor and was particle free. The purge gas flow was variable depending on the type of measurement: for the in-chamber measurements, a flow rate of 1.2 standard cubic foot per hour (SCFH) was used for each glass window, and for the in-spray measurements, a flow rate of approximately 35 to $40 \mathrm{SCFH}$ was used for each 
window. The increased purge air flow rate for the in-spray measurements was used because the droplets were present in higher concentrations and contained larger droplets with sufficient inertia to overcome lower purge flows; thus, there was a higher probability of contamination, but a reduced potential for diluting the local concentration.

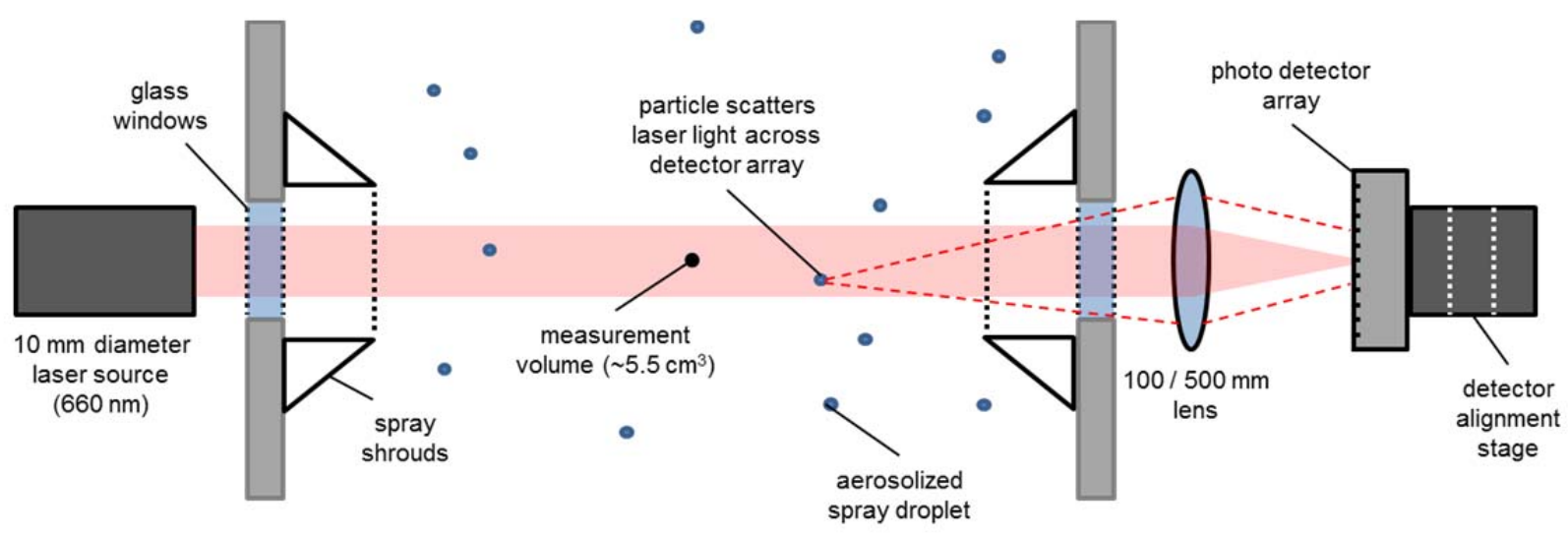

Figure 4.6. Basic Instrument Setup and Operation Principle of the Malvern Insitec-S Particle Size Analyzer

The Malvern Insitec-S detector assembly consists of the receiver electronics and the optical detector array. The optical detector array is made up of 32 individual detectors, each of which collects the light scattered by a particular range of angles. Light from the laser beam is scattered by the particles within the measurement volume. This scattered light is focused by the lens and picked up by the detector array. Unscattered light is focused by the lens so that it passes through the pinhole at the center of the detector array and is subsequently measured by the beam power detector to give the light transmission. The angle at which a particle scatters light is inversely proportional to the size of the particle. By measuring the angle of scattering, the size of the particle is determined. In cases where the particle loading is high, the measurement process is complicated by scattered light being re-scattered by other droplets before it reaches the detector. A 'multiple scattering' algorithm can be applied to correct for these cases. The decrease of the light transmission relative to that measured when no aerosol is present (termed the background light transmission), along with the size distribution estimated from the light scattering pattern, can be used to determine the volume concentration of droplets.

The nominal size range measured by the Malvern Insitec-S is dictated by the focal length of the lens. For small-scale testing, the $100 \mathrm{~mm}$ lens yielded a nominal measuring range of 0.5 to $200 \mu \mathrm{m}$ and the $500 \mathrm{~mm}$ lens had a nominal measuring range of 2.5 to $2500 \mu \mathrm{m}$. The primary reason for using the $500 \mathrm{~mm}$ lens over the $100 \mathrm{~mm}$ lens was to increase the droplet size range when in-spray measurements, which have larger droplets, were collected. In general, the Malvern Insitec-S can measure aerosols in the range of 0.01 to 1000 parts per million by volume (ppmv), which is determined primarily by the length of the spacer bars and the geometry of the spray. For small-scale testing, the Malvern Insitec-S can detect particle concentrations above a lower threshold of $\sim 0.1 \mathrm{ppmv}$.

During Phase II small-scale testing, the Malvern Insitec-S was located in one of two positions for all testing: either at the middle of the small-scale testing chamber, vertically positioned above the core of the spray (Position 2 in Figure 4.7), or in the middle of the chamber and in-line with the spray (Position 4). In this report, a measurement in Position 2 and an in-chamber measurement are synonymous. In addition, 
Position 4 and an in-spray measurement are synonymous. For reference, Figure 4.7 also shows other available positions that were used in Phase I testing in the small-scale testing chamber. Note that with the $500 \mathrm{~mm}$ lens installed, only Positions 2 and 4 are accessible. Figure 4.8 shows an example of the configuration used to perform the in-spray (Position 4) measurement.

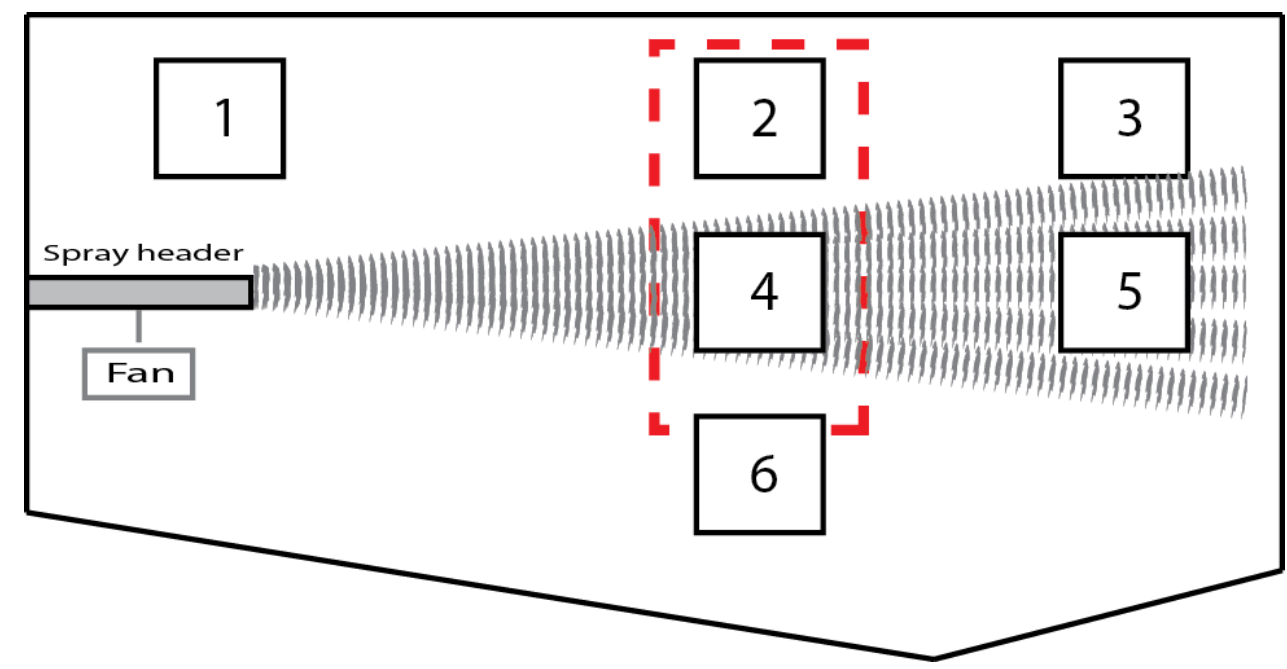

Figure 4.7. Conceptual Representation of the Malvern Insitec-S (numbered) Locations Within the Test Chamber. The approximate location of the cut-out area required for the $500 \mathrm{~mm}$ lens is noted in red.

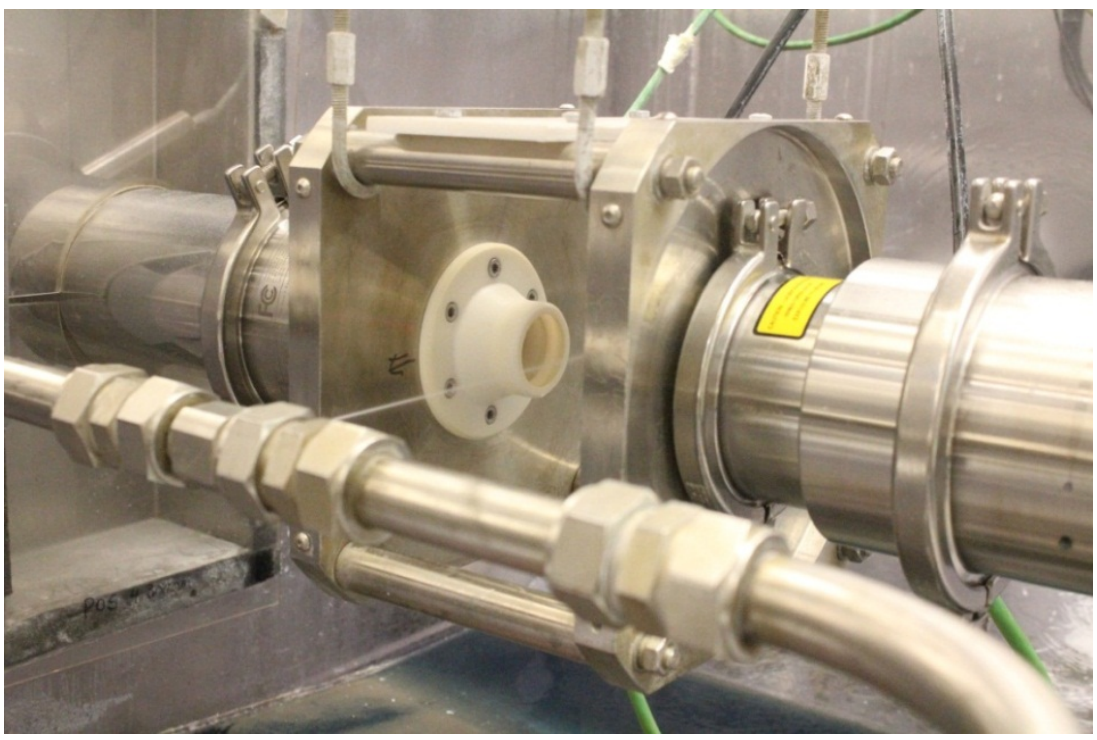

Figure 4.8. Picture of an In-Spray (Position 4) Measurement Conducted in Phase II Testing

The Malvern Insitec-S was interfaced to a single control computer through a local communications interface box (Part\# MPS2092). Malvern Instruments Limited's RTSizer software (Version 7.40, Copyright 2010) was used to collect, analyze, and report the aerosol data sampled by the Malvern Insitec-S. The primary program outputs were aerosol size and concentration. Other measurement parameters included raw data such as the raw light scattering signal, laser transmission, and background; calculated parameters included SMD. The typical instrument configuration and software settings used for 
analysis are listed in Table 4.3. A complete description of parameters is given in the RTSizer user manual (Malvern Instruments Ltd. 2010). The first three parameters, the lens, gain, and update period, must be set prior to data collection because they cannot be changed using post-analysis properties. The lens parameter must be set to match the focal length of the lens installed in the instrument. The gain is a photodiode multiplier that determines the instrument response to scattered light. The highest gain setting of $2 \times$ was employed because aerosol concentrations were expected to be low ( $\leq 10 \mathrm{ppmv}$ based on initial estimates). The update period (or accumulation period) determines the time period over which results are integrated. Longer update periods tend to smooth variations in aerosol concentration with time, yielding smoother data, but may also time-average over periods during which the transient aerosol concentration is of interest (e.g., the initial increase in concentration from which release fraction estimates are made). Shorter updated periods can be selected to capture fast transients; however, they tend to yield an increase in noise-to-signal ratio. For Phase II small-scale testing, the update period was set to $0.25 \mathrm{~s}(4 \mathrm{~Hz})$.

Table 4.3. Malvern Insitec-S Instrument Configuration and Software Parameters Used in Typical Measurements

\begin{tabular}{ll}
\hline \multicolumn{1}{c}{ Parameter } & \multicolumn{1}{c}{ Setting } \\
\hline Lens & $100 \mathrm{~mm}$ or $500 \mathrm{~mm}$ \\
Gain & $2 \times$ \\
Update period & $0.25 \mathrm{~s}$ \\
Particulate RI & Varies depending on test slurry \\
Media RI & Air: $1.00+0.00 \mathrm{i}$ \\
Particle Density & 1.00 gm/cc (may also be set to slurry density without affecting the measurement, as \\
& this parameter is only used in specific surface area calculations $)$ \\
Scattering Threshold & 2 \\
Minimum Size & $0.10 \mu \mathrm{m}(100 \mathrm{~mm}$ lens $) / 2.5 \mu \mathrm{m}(500 \mathrm{~mm}$ lens $)$ \\
Maximum Size & $1500 \mu \mathrm{m}(100 \mathrm{~mm}$ lens $) / 2500 \mu \mathrm{m}(500 \mathrm{~mm}$ lens $)$ \\
First Scattering Start & 1 (default value $)$ \\
Multiple Scatter & On \\
Spray Properties & Checked: Uniform spray concentration in measurement volume \\
\hline
\end{tabular}

Users can change some parameters (e.g., the particulate and media RI and scattering threshold) for post-analysis processing undertaken to evaluate the effect of instrument results. RI and spray properties are two such parameters. The RI (see Section 3.3.6 for the simulant RI values) is a complex number that specifies how light refracts through a material (real component) and how the material attenuates or absorbs light (imaginary component). Because all aerosols are tested in air, the media RI is always set to that of air $(1.00+0 \mathrm{i})$. The particulate RI depends on the material being tested and is discussed in greater detail in Section 3.3.6 and Schonewill et al. (2012). The spray properties parameter allows for definition of the shape, size, and concentration profile of the spray as it passes through the measurement volume. 


\subsection{Modifications to Test System from Phase I}

As mentioned at the beginning of this section, the Phase II test equipment was very similar to that used in Phase I (see Section 4 in Mahoney et al. 2013) with some modifications. For clarity, the modifications and equipment updates will be delineated below. The significant modifications were as follows:

- A large portion of the Phase I process lines, which were built using threaded connections, were rebuilt in Phase II using either flanged piping (the inlet line) or tubing with Swagelok connections (most other process lines). This was for convenience only and not expected to have an impact on the test results.

- A heat exchanger was added in-line with the return line to remove mechanical heat from the circulating simulants. Mechanical heat removal was a problem in Phase I that was mitigated in Phase II. The addition of a heat exchanger also required installation of a chiller and coolant lines. For part of the Phase II testing program, the heat exchanger was removed from the return line and installed on the bypass line.

- The spray chamber was modified to accommodate the $500 \mathrm{~mm}$ lens used in Phase II by cutting out a small area on the back wall of the chamber and covering it with a sheet metal box. The box was covered when the Malvern Insitec-S was in place to keep the Phase II chamber volume consistent with the Phase I volume. No effect on test results was anticipated because of this change. The cut-out only permitted testing in a small range of locations with the $500 \mathrm{~mm}$ lens.

- The capacity of the purge air lines for the Malvern Insitec-S was increased by installing higher flow rotameters (up to a maximum of $80 \mathrm{SCFH}$ was achieved through a single rotameter). The higher flow rate was used for in-spray measurements. This capability was not available in Phase I for in-spray measurements.

- The platform scale was interfaced with the data acquisition computer to provide mass data as a function of time. In Phase I, mass data was only available at discrete points before and after a spray.

- Switches were added to the valves that initiated the spray to assist in determining the start and stop times of the spray events. These switches gave on/off indications and were recorded by the data acquisition system. No such information was available in Phase I.

- All data acquisition was performed on a single computer to avoid the time synchronization problems that could result from using multiple computers. Two computers were used for data acquisition in Phase I.

- The Process Particle Counter, an aerosol instrument used sparingly in Phase I, was never installed or used in Phase II.

- The following modifications were specific to the Malvern Insitec-S:

- A $500 \mathrm{~mm}$ lens was used for the majority of testing in Phase II. The $500 \mathrm{~mm}$ lens has a different nominal droplet size range than the $100 \mathrm{~mm}$ lens used in Phase I.

- Purge air flow rates were available up to 40 SCFH per glass window.

- Data was collected at $4 \mathrm{~Hz}(0.25 \mathrm{~s}$ update rate) instead of $1 \mathrm{~Hz}(1 \mathrm{~s}$ update rate), which was the standard Phase I collection rate. 
- The splash guard was not used in any Phase II testing.

- Testing was only performed in Positions 2 and 4 in Phase II (see Figure 4.7), which were the only accessible locations with the $500 \mathrm{~mm}$ lens installed.

For additional details on these changes, refer to other sections in this report against similarly named sections in Mahoney et al. (2013). Some of the changes are evident when comparing Figure 4.3 of this report with Figure 4.5 in Mahoney et al. (2013). The list given above does not include modifications to the system that were made in response to the behavior of certain simulants used in Phase II. These are listed in Section 4.1.1 and described in detail in Section 5.4.2.

\subsection{Orifices}

As mentioned previously, orifices of various size and dimension can be installed in the spray header. These orifices are also referred to as OTPs. Due to the focused objectives of the Phase II testing, the number of orifices used in testing was greatly reduced. In Phase I, release fractions were determined for 15 different orifices. In the Phase II, only two orifices were tested to obtain in-chamber measurements, and one additional orifice was tested to obtain in-spray measurements. These OTPs were selected because they were observed to result in consistent aerosol behavior during Phase I testing. They are presented in Table 4.4 along with their dimensions. The dimensions were determined for the OTP at the outer diameter of the pipe (the surface where the simulant exits the orifice). Note that the orifices are suspected to have smaller dimensions at the inner diameter of the pipe and have a (slight) diverging geometry; however, quantitative measurements using imaging techniques have not been successful. Accurate measurement of the inner dimensions can be achieved via destructive examination but had not been pursued as of this writing.

Table 4.4. Exit Dimensions for the Orifices for Which Release Fractions Were Determined in Phase II Testing

\begin{tabular}{ccccc}
\hline $\begin{array}{c}\text { Orifice } \\
\text { Designation }\end{array}$ & Type & $\begin{array}{c}\text { Target Orifice } \\
\text { Dimensions } \\
(\mathrm{mm})\end{array}$ & $\begin{array}{c}\text { Exit Diameter or } \\
\text { Length } \times \text { Width } \\
(\mathrm{mm})\end{array}$ & $\begin{array}{c}\text { Cross-Sectional } \\
\text { Area at Exit } \\
\left(\mathrm{mm}^{2}\right)\end{array}$ \\
\hline OTP-03 & Round & 0.5 & 0.534 & 0.22 \\
OTP-05 & Round & 1.0 & 0.975 & 0.75 \\
OTP-16 & Slot $^{(\mathrm{a})}$ & $0.5 \times 5.0$ & $0.534 \times 4.886$ & 2.6 \\
\hline
\end{tabular}

(a) This slot was orientated axially, i.e., the longest dimension was orientated with the direction of flow in the spray header.

The dimensions were measured prior to Phase I testing and were assumed to remain the same for the Phase II testing. After testing was completed, the dimensions were measured again to assess whether the orifices had changed significantly over the course of the Phase I and Phase II testing programs. The measurement of the orifice dimensions after completion of the Phase II aerosol tests were conducted using an imaging system composed of a digital camera, lens, lens spacer, and a micro-ruler. The camera used was an Edmund Optics EO-1918C, with image size of $1600 \times 1200$ pixels (horizontal $\times$ vertical). An InfiniGage CW lens and lens spacers, also from Edmund Optics, were attached to the camera, and images were captured with StreamPix software, version 5.3.0. An MR-1 Micro-Ruler, supplied by Geller 
MicroAnalytical Laboratory Inc. and calibrated per QA requirements, was used as a calibration standard. Images were collected per OP-WTPSP-055. ${ }^{1}$ Analysis of the images was performed using MATLAB ${ }^{\circledR}$ (The MathWorks, Inc.). The comparison of the dimensions determined before Phase I and after Phase II testing is shown in Table 4.5.

Table 4.5. Comparison of Exit Dimensions Measured Before and After Aerosol Testing

\begin{tabular}{ccccc}
\hline & $\begin{array}{c}\text { Target } \\
\text { Orifice } \\
\text { Orifice } \\
\text { Dimensions } \\
(\mathrm{mm})\end{array}$ & $\begin{array}{c}\text { Exit Diameter or } \\
\text { Length } \times \text { Width } \\
\text { Measured Prior to } \\
\text { Phase I Testing } \\
(\mathrm{mm})\end{array}$ & $\begin{array}{c}\text { Exit Diameter or } \\
\text { Length } \times \text { Width Measured } \\
\text { Upon Completion of } \\
\text { Phase II Testing } \\
(\mathrm{mm})\end{array}$ & $\begin{array}{c}\text { Change Over } \\
\text { Testing } \\
(\%)\end{array}$ \\
\hline OTP-03 & 0.5 & 0.534 & 0.505 & -5.4 \\
OTP-05 & 1.0 & 0.975 & 0.995 & 2.0 \\
OTP-16 & $0.5 \times 5.0$ & $0.534 \times 4.886$ & $0.588 \times 4.845$ & 10.1 (length) \\
& & & & -0.8 (width) \\
\hline (a) Refers to the percent difference of the post-testing dimensions to the pre-testing dimensions.
\end{tabular}

The comparison between orifice dimensions in Table 4.5 does not reveal any clear trends in orifice size resulting from the several hundred tests conducted over both phases of testing. The dimensions for the round orifices (OTP-03, OTP-05) were determined to be very near their nominal dimensions after Phase II testing, but OTP-03 was measured to be smaller and OTP-05 larger when compared to the measurements before Phase I testing. The only slot used in Phase II testing (OTP-16) was determined to have $>50 \mu \mathrm{m}$ increase in length, suggesting that the orifice may have been eroded by repeated aerosol testing. However, a corresponding increase was not observed in the width. It is possible that this observation is due to the sensitivity of the size measurement to the image collection process. The images collected after Phase II testing are noticeably better resolved and their analysis is less complicated. There is not strong enough evidence, based on these factors, to suggest that OTP-16 was being eroded by slurry particulate. In addition, visual inspection of the before and after images does not indicate that erosion was taking place. In the absence of any trends in orifice dimensions, the dimensions measured prior to testing were used in all the analyses in this report.

\footnotetext{
${ }^{1}$ Billing JM. 2012. Imaging Techniques for the Measurement of Spool Piece Orifice Dimensions for Large Scale Spray Release Testing. OP-WTPSP-055, Rev 0.0 (unpublished), Pacific Northwest National Laboratory, Richland, Washington.
} 


\subsection{Test Operations and Conditions}

Aerosol tests were performed using the small-scale system described in Section 4 to measure aerosol droplet size distributions and concentration produced from prototypic breaches and fluids with physically prototypic WTP slurries and liquid solutions. The Phase II tests had objectives that were narrower in scope and focused primarily on comparing release fractions across simulant types to elucidate the effect of various physical properties (e.g., rheology, solids loading, presence of dense particles). Consequently, the parameter space that was investigated was less broad; for example, the Phase II tests used only three orifices whereas Phase I tests used 15.

The success criteria for the Phase II aerosol testing can be summarized as follows:

- Measure the droplet size distribution, total volume concentration of droplets, and total volume flow rate sprayed for each breach and simulant tested.

- For in-spray measurements, measure the droplet size distribution for each breach and simulant tested.

- Characterize the viscosity or rheology, PSD, bulk density, and surface tension for each simulant tested.

- For the $30 \mathrm{~Pa} / 30 \mathrm{cP}$ simulants at the beginning of testing, at least one Bingham parameter is $30 \pm 4 \mathrm{~Pa}$ or $\mathrm{cP}$ and the second Bingham parameter should be less than or equal to this upper target of $30 \pm 4 \mathrm{~Pa}$ or $\mathrm{cP}$.

- For the $6 \mathrm{~Pa} / 6 \mathrm{cP}$ simulants at the beginning of testing, at least one Bingham parameter is $6 \pm 2 \mathrm{~Pa}$ or $\mathrm{cP}$ and the second Bingham parameter should be greater than or equal to this lower target of $6 \pm 2 \mathrm{~Pa}$ or $\mathrm{cP}$.

- Measure the pressure and flow in the piping.

- Quantitatively compare old $(100 \mathrm{~mm})$ and new $(500 \mathrm{~mm})$ lenses to demonstrate that the old and new lenses give equivalent results for aerosol conditions that have droplet sizes in a range appropriate for both lenses.

- Measure the concentration and size distribution of known aqueous suspensions with the Malvern Insitec-S used for aerosol measurements and quantitatively compare the Malvern Insitec-S results with the known values to estimate the Malvern Insitec-S accuracy for measuring aerosols.

The following subsections discuss the test operations performed to meet the success criteria listed above. Section 5.1 presents the test nomenclature and how it relates to the testing. Section 5.2 presents the matrix of tests performed in Phase II. Section 5.3 discusses typical (or standard) test operations. Finally, Section 5.4 deals with how test operations in Phase II differed from those of Phase I and describes some modifications to the approach that were required when dealing with certain simulants. 


\subsection{Testing Nomenclature}

The reduction in parameters that were varied in Phase II testing suggested that a simpler test nomenclature could be used to describe the tests that were performed. Each test was described using the following test naming convention:

\section{Test ID: SS-\#\#\#-SIMxx-J}

where

$\begin{array}{ll}\text { SS } & \text { Small-Scale } \\ \text { \#\#\# } & \text { Unique Test Number, beginning with 091 } \\ \text { SIM } & \text { Simulant tested (as presented in Section 3, or see Table 3.2) } \\ & \text { - } \mathrm{W}=\text { Water } \\ & \text { - } \mathrm{KBC}=\text { Kaolin-Bentonite Clay (also referred to simply as clay) } \\ & \text { - } \mathrm{FEG}=\text { Iron-Rich/Iron-Rich Gibbsite (also referred to as chemical simulant) } \\ & \text { - } \mathrm{DPW}=\text { Denall-Treated Simulant } \\ & \text { - } \mathrm{DST}=\text { Dense Particle (Mo) in Water } \\ & \text { Specifies a property of interest, e.g., FEG-30 indicates the simulant had a target } \\ & \text { rheology of } 30 \text { Pa/30 cP. This is left blank for some simulants. } \\ \text { Ox } & \text { Optional indicator to specify if aerosol measurement was in-spray. The letter "J" } \\ & \text { was used to indicate in-spray measurement; the absence of the letter "J" indicates } \\ & \text { an in-chamber measurement. }\end{array}$

Note that referring to a test using the shorthand notation SS-\#\#\# still specifies a unique aerosol test. This shorthand notation is called the test number to distinguish it from the full Test ID. Test numbers begin at SS-091 to distinguish them from Phase I numerical identifiers. To simplify the presentation of results in this report, all Phase II tests are referred to by only their test numbers. To link a test number to other test information or metadata, refer to Appendix A. All data streams (e.g., sample identification numbers, image file names, Malvern Insitec-S files, test documentation) are linked to the test number.

In Phase I test nomenclature, repeat or replicate tests were referred to using the same base Test ID (e.g., SS-001-A, SS-001-B, SS-001-C). However, in Phase II test nomenclature, if three spray tests were conducted consecutively using a particular orifice, pressure, and simulant, the test numbers are also consecutive (e.g., SS-001, SS-002, SS-003). ${ }^{1}$

\subsection{Test Matrix}

The planned tests to meet the Phase II objectives were based on examining the simulants of interest at three pressures of interest (i.e., 100, 200, and 380 psig) using two orifices, a round hole (OTP-05, nominally $1 \mathrm{~mm}$ in diameter) and a slot (OTP-16, nominally $0.5 \times 5 \mathrm{~mm}$ ). The exception was the dense particle simulants, which would only be tested at $380 \mathrm{psig}$. The planned number of tests is presented in Table 5.1 along with the number of tests actually performed. For reference, Table 5.1 also provides the range of test numbers for each simulant.

\footnotetext{
${ }^{1}$ These example test numbers were for illustration only and are not true test numbers.
} 
Table 5.1. Phase II Test Matrix: Planned and Actual Tests

\begin{tabular}{|c|c|c|c|c|c|}
\hline Simulant & $\begin{array}{l}\text { Pressures } \\
\text { (psig) }\end{array}$ & $\begin{array}{c}\text { Nominal } \\
\text { Orifice Sizes }\end{array}$ & $\begin{array}{l}\text { Planned No. } \\
\text { of Tests }^{(a)}\end{array}$ & $\begin{array}{l}\text { Actual No. } \\
\text { of Tests }^{(b, c)}\end{array}$ & $\begin{array}{l}\text { Range of Test } \\
\text { Numbers }{ }^{(\mathrm{d})}\end{array}$ \\
\hline Water (W) & $100,200,380$ & $\begin{array}{l}0.5,1 \mathrm{~mm} \\
0.5 \times 5 \mathrm{~mm}\end{array}$ & $\underset{4}{\text { Minimum of }}$ & 73 & $\begin{array}{l}\text { SS-091 - SS-151 and } \\
\text { SS-375 - SS-386 }\end{array}$ \\
\hline Clay Slurry (KBC-30) & $100,200,380$ & $\begin{array}{c}1 \mathrm{~mm} \\
0.5 \times 5 \mathrm{~mm}\end{array}$ & 24 & 68 & SS-152 - SS-219 \\
\hline Clay Slurry (KBC-6) & $100,200,380$ & $\begin{array}{c}1 \mathrm{~mm} \\
0.5 \times 5 \mathrm{~mm}\end{array}$ & 24 & 30 & SS-220 - SS-249 \\
\hline $\begin{array}{l}\text { Chemical Simulant } \\
\text { (FEG-30) }\end{array}$ & $100,200,380$ & $\begin{array}{c}1 \mathrm{~mm} \\
0.5 \times 5 \mathrm{~mm}\end{array}$ & 24 & 37 & SS-250 - SS-286 \\
\hline $\begin{array}{l}\text { Chemical Simulant } \\
\text { (FEG-6) }\end{array}$ & $100,200,380$ & $\begin{array}{c}1 \mathrm{~mm} \\
0.5 \times 5 \mathrm{~mm}\end{array}$ & 24 & 41 & SS-287 - SS-327 \\
\hline $\begin{array}{c}\text { Small-Treated, } 27 \\
\mathrm{wt} \% \text { (STR27) }\end{array}$ & $100,200,380$ & $\begin{array}{c}1 \mathrm{~mm} \\
0.5 \times 5 \mathrm{~mm}\end{array}$ & 24 & 26 & SS-328 - SS-353 \\
\hline $\begin{array}{l}\text { Dense Particle in } \\
\text { Water (DPW) }\end{array}$ & 380 & $\begin{array}{c}1 \mathrm{~mm} \\
0.5 \times 5 \mathrm{~mm}\end{array}$ & 8 & 11 & SS-354 - SS-364 \\
\hline \multirow[t]{2}{*}{$\begin{array}{l}\text { Dense Small Treated } \\
\text { (DST) }\end{array}$} & 380 & $\begin{array}{c}1 \mathrm{~mm} \\
0.5 \times 5 \mathrm{~mm}\end{array}$ & 8 & 10 & SS-365 - SS-374 \\
\hline & & TOTAL & 140 & 296 & SS-091 - SS-386 \\
\hline
\end{tabular}

(a) Based on the expectation that at each pressure and orifice combination, three in-chamber tests and one in-spray test would be conducted. For example, the KBC-30 tests would be expected to have three replicates $\times$ three pressures $\times$ two orifices $=18$ in-chamber tests and three pressures $\times$ two orifices $=6$ in-spray tests, for a total of 24.

(b) Note that the actual number of tests includes all tests performed in each simulant category that were assigned a test number. Based on several criteria, release fractions are not reported for every test.

(c) In the actual tests, in-spray measurements could not be reliably performed using the $1 \mathrm{~mm}$ hole or the $0.5 \times 5 \mathrm{~mm}$ slot. All the in-spray measurements that are reported were performed with the $0.5 \mathrm{~mm}$ hole instead.

(d) The range of test numbers is inclusive for each simulant tested.

With the exception of the water tests, the planned number of tests was derived assuming that each orifice would be tested at each pressure in triplicate for in-chamber measurements and once for an in-spray measurement. The number of water tests had a minimum expectation because the only planned testing was to perform a comparison of Phase I water data collected using the $100 \mathrm{~mm}$ lens with Phase II data collected using the $500 \mathrm{~mm}$ lens. However, due to the system changes in Phase II, an unknown number of additional shakedown tests were anticipated.

The actual number of tests reflects all tests that were conducted and assigned a number. This does not represent the number of tests for which release fractions are reported. Some of the tests included in this total were rejected for analysis due to an off-normal process condition or the data quality criteria listed in Section 6.3.2. Additional reasons for the larger number of actual tests compared to planned tests for some simulants are discussed in Section 5.4.2. Another important difference between the planned and actual columns is that in-spray measurements were planned to be performed with both orifices listed for all simulants; however, as discussed in Section 5.4.2, only one orifice (i.e., OTP-03, $0.5 \mathrm{~mm}$ hole) was used for all simulants. 


\subsection{Standard Test Operations}

Phase II small-scale aerosol tests were performed in a manner similar to Phase I tests. Each test was conducted in accordance with an approved test instruction (TI) and operating procedure to obtain observations under well-controlled and characterized conditions. ${ }^{1}$ The general test approach is described below and is composed of three parts:

1. daily startup checks/preparation

2. spray release tests

3. daily closeout checks.

The check/test procedures are summarized in the following sections. Other performance checks, in particular of the Malvern Insitec-S instrument, occurred less frequently and were performed as required by the governing procedure, OP-WTPSP-047. ${ }^{2}$ These performance checks involved removing the Malvern Insitec-S from the small-scale chamber and using a calibrated reticle to determine if the Malvern Insitec-S measurements were within acceptable ranges.

\subsubsection{Daily Startup Checks and Preparation}

These activities varied somewhat with the condition of the system as-left from the previous test day. For instance, preparation activities could include tasks such as loading in the simulant to be used (if the feed tank was empty) for the testing or modifying the simulant already in the tank. The latter would be required if a significant amount of mass had been lost due to evaporation or if a physical property (e.g., rheology) needed to be adjusted based on sample analysis results. When controlling for rheology, the mass lost from the previous day was always assumed to be solely due to evaporation of water and thus the simulant was adjusted by adding tap water (for the KBC simulants) or bottled (distilled) water (for the FEG simulants). Before testing began, process instruments (e.g., pressure transmitters and the Coriolis flow meter) were checked for the proper functionality; for example, the Coriolis flow meter was checked daily to verify it had a $0 \pm 0.1 \mathrm{gpm}$ reading when the system was not operating. The configuration of the Malvern Insitec-S instrument and other chamber equipment were also verified.

\subsubsection{Spray Release Tests}

\subsubsection{Pre-Test System Preparation}

The TI specified the tests (i.e., pressures, simulants, orifices, and configurations) to be conducted on a particular day. An initial tank weight and level was recorded and then the simulant in the feed tank was agitated at the highest speed possible without vortexing or splashing material out of the tank. Typically, even if the process lines were full of fluid, the hood vacuum supply was used to prime the test system pump and lines by drawing simulant from the feed tank into the pump inlet. The simulant then was circulated through the system lines until visual observation confirmed that air was purged from the system

\footnotetext{
${ }^{1}$ Kimura ML. 2012. Test Instruction and Procedure for Phase II Small Scale Aerosol Tests, TI-WTPSP-090 (unpublished), Pacific Northwest National Laboratory, Richland, Washington.

${ }^{2}$ Burns CA. 2012. Malvern Insitec-S Operating Procedure. OP-WTPSP-047, Rev 2.0 (unpublished), Pacific Northwest National Laboratory, Richland, Washington.
} 
(i.e., no bubbles were present in the simulant). This was done for all major pathways that simulant could take during a test (bypass line and both headers in the chamber).

Next, the data acquisition tools (e.g., data logger, scale-Excel interface, and Malvern software) were set up for testing. Meanwhile, the spray header (with the required OTP in place) was securely installed, and a pre-test checklist of system configuration checks was completed. Dip sample(s), if required, were taken from the feed tank while the agitator was shut off briefly. Samples were only taken for physical characterization of new simulants on their first day of testing or daily to measure and adjust rheology. Then, with the exception of water, the simulant was agitated and circulated at high shear (pump VFD set at $60 \mathrm{~Hz}$ ) for a minimum of $90 \mathrm{~s}$, while the simulant density (measured by the Coriolis meter in the flow loop) was checked against the known or estimated simulant density to verify that the system was fully suspending the material. If the density reading was fluctuating, the density check time was extended until the readings became stable. The simulant temperature in the test header was recorded at the beginning and end of the density check to document that it was taken within the testing operational tolerance of $75 \pm 10^{\circ} \mathrm{F}$.

\subsubsection{Aerosol Testing}

Once the simulant was verified to be properly suspended, the pump speed was set to $30 \mathrm{~Hz}$. The mixing fan was powered on and set to $6 \mathrm{~V}$. If the test to be performed was the first of the day, there was a change in configuration in the chamber, or $3 \mathrm{hr}$ had elapsed since the last test, an approximately 2-min pre-spray to wet the aerosol test enclosure was performed, followed by an evacuation of the enclosure (using the blower mounted on the chamber and a shared controller with the mixing fan) to remove residual aerosol. The pre-spray served three purposes:

1. Provide a consistent wall wetness to reduce variation in splash behavior when the spray struck the chamber walls.

2. Humidify the enclosure and minimize evaporation of droplets during the test spray.

3. Assist the operators in establishing valve configurations to achieve the proper pressure and flow rates targeted for the test.

Whether or not a pre-spray was conducted prior to a test spray, after the evacuation period the target test flow rate and pressure were set (or confirmed if previously set) by manipulating the needle valves on the bypass line and the return line. Valve configurations are discussed in more detail in Section 5.3.2.4. The system continued to circulate the simulant via the bypass header while the mixing blower was turned back on to $6 \mathrm{~V}$, the pre-test data were recorded, and an aerosol instrument background check performed.

When the background check was complete, data collection commenced for at least 1 min with the system ready to spray and the Malvern Insitec-S instrument collecting data in the absence of spray. Typically, the RH in the chamber just before the spray began, as measured by an FIO meter, was approximately $\geq 80$ percent (with some exceptions, particularly with the FEG simulants). The temperature in the system was confirmed to be $75 \pm 10^{\circ} \mathrm{F}$. After this period, the operators executed a coordinated valve protocol to initiate the spray through the orifice. Typically, the simulant was sprayed for 2 min. The spray was discontinued by executing the reverse of the valve protocol used to start the spray. At this point, post-test data was recorded on the TI and, if applicable, any special notes regarding the test were made in the Laboratory Record Book (LRB). Data collection continued for approximately 2 min after the 
spray, and then the chamber was evacuated in preparation for the next spray. This cycle was repeated for all tests performed on a given test day. Some test sprays were repeated without any data collection activities for the purpose of collecting images of the sprays. Imaged sprays were not assigned a unique test number as no data was collected during them. For some simulants, particularly the KBC and FEG, the material sprayed into the chamber was recycled to the feed tank periodically after selected spray tests.

\subsubsection{Aerosol Instrument Operation}

The Malvern Insitec-S was run by a dedicated operator according to procedure OP-WTPSP-047. ${ }^{1}$ During pre-test activities, the instrument settings (e.g., the purge flow, instrument configuration, and software settings) were verified to be correct for the upcoming spray. As mentioned above, a background measurement for the Malvern Insitec-S instrument was performed before each test while the lights inside the fume hood were turned off. The background measurement defined the baseline condition for the spray and captured the state of the chamber (with respect to the scattering pattern and laser obscuration) observed by the instrument before a spray. The test was not performed until a valid background measurement (indicated by RTSizer software and operator judgment) was obtained.

During the spray test, aerosol data were continuously monitored, especially the cumulative volume concentration $\left(\mathrm{C}_{\mathrm{v}}\right)$, the transmission, and the scattering pattern, to evaluate whether reasonable data were being collected. After the test, the data was exported and a real-time evaluation of the data was often performed. This evaluation plotted the size-dependent $\mathrm{C}_{\mathrm{v}}$ to ensure the initial concentration increased smoothly and was followed by a period of steady-state concentration. Real-time evaluations of this type helped identify tests where the data was not collected under ideal measurement conditions (e.g., the instrument was creeping out of alignment, droplets were depositing or condensing on the Malvern Insitec-S windows, or sharp changes in concentration were occurring). In those cases, test conditions could be repeated to produce additional sprays as needed.

\subsubsection{Valve Configurations}

The valve sequence was standardized in Phase II testing to minimize the pressure transients observed in the system in Phase I testing. In Phase I testing, even though the aerosol net generation rate was determined from the initial concentration rise, during which the spray was being generated by a different pressure than the target pressure, the measured release fraction was not significantly different from tests where the initial pressure was essentially the target pressure (Mahoney et al. 2013). Improved pressure control was desirable to reduce noise in both the aerosol data and the estimated WTP model predictions based on the measured pressure.

Even with a standardized valve protocol, the small-scale system, as configured in Phase II, still experienced small pressure transients, particularly in the first few seconds after a spray was initiated. This is a limitation of the system and the consequence of several factors, including the following:

1. Valve protocol requires that three valves must be manipulated simultaneously to start a spray.

2. The simulant or fluid in the spray header is not already in motion when the valves are opened.

\footnotetext{
${ }^{1}$ Burns CA. 2012. Malvern Insitec-S Operating Procedure. OP-WTPSP-047, Rev 2.0 (unpublished), Pacific Northwest National Laboratory, Richland, Washington.
} 
3. The quiescent simulant or fluid in the spray header may contain some air.

4. As set pressure increases, it is controlled by increasingly smaller gaps in needle valves.

Another complication to pressure control was the use of simulants with non-Newtonian rheology, which is discussed later in Section 5.4.2.

Valve protocol is presented in Figure 5.1 using a series of simplified small-scale system diagrams to illustrate the required valve actions. The figures show the three discrete stages of performing a spray test. The important ball valves (refer to Figure 4.3 for a more detailed schematic) are shown as either open or filled circles, denoting an open or closed valve. The state of the control valves are described in the text below. In the first stage, which is shown in Figure 5.1(a), the flow was circulating through the bypass header in the chamber and back to the tank. The pump was operating at a fixed speed (typically $30 \mathrm{~Hz}$ ). During this stage, the pressure control valve PCV-49 on the purge line was full open and the ball valve (BV-52) was closed. The needle valves on the bypass line (flow control valves) and the return line (pressure control valves) were manually adjusted to bring the pressure and flow rate to the target values. This required some iteration and the valve positions varied depending on the simulant and target pressure.

When the target values for pressure and flow rate were reached, the valves were configured to the second stage, which is shown in Figure 5.1(b). The flow was still circulating through the bypass header with the needle valves all in a fixed position. The purge line ball valve (BV-52) was opened to permit flow. Recall that PCV-49 was full open, so the pressure and flow rate in the bypass header must necessarily decrease. PCV-49 was manually adjusted to bring the pressure and flow rate to target values. For some simulants, particularly at low pressures, very little adjustment was required.

In the third stage, which is shown in Figure 5.1(c), all the control valves were in a fixed position as determined during the first two stages of the valve configuration. The figure shows the system in the valve configuration that initiated and maintained a spray. A spray was started by simultaneously manipulating three valves: BV-23 (closed to open), BV-52 (open to closed), and BV-24 (open to closed). In practice, BV-23 and BV-52 were manipulated slightly before BV-24 to avoid over pressurizing the system or sending an undesirable amount of fluid through the purge line at high velocity. Once the requisite spray time had passed, the spray was shut off by performing the same valve manipulations in reverse: BV-24 (closed to open), then BV-52 (closed to open), and BV-23 (open to closed). With the ball valves returned to the state that is shown in Figure 5.1(a), a spray could be repeated at the same target conditions rapidly. Because there was some elapsed time between repeated tests, PCV-49 was re-opened until it was time to conduct another spray.

The valve protocol described above and presented in Figure 5.1 was used consistently throughout Phase II testing. Some minor adjustments were required to solve difficulties, in particular with the chemical simulant, but overall the standardized procedure resulted in good pressure control with only small transients during the initial few seconds of the spray.

\subsubsection{Post-Test Activities}

When residual simulant remained upon completion of testing for a day, the chamber was cleaned. Simulant collected in the aerosol test chamber was reused (i.e., returned to the feed tank) or removed for disposal. In addition, the mixing blower and pump were turned off. The test piece was cleaned in place (when additional testing required the same test piece) or removed and cleaned as necessary. Pertinent test 
information was recorded in the LRB. An end-of-day sample was collected for the rheological simulants (KBC and FEG) to assess whether adjustments would be required before the next series of tests.
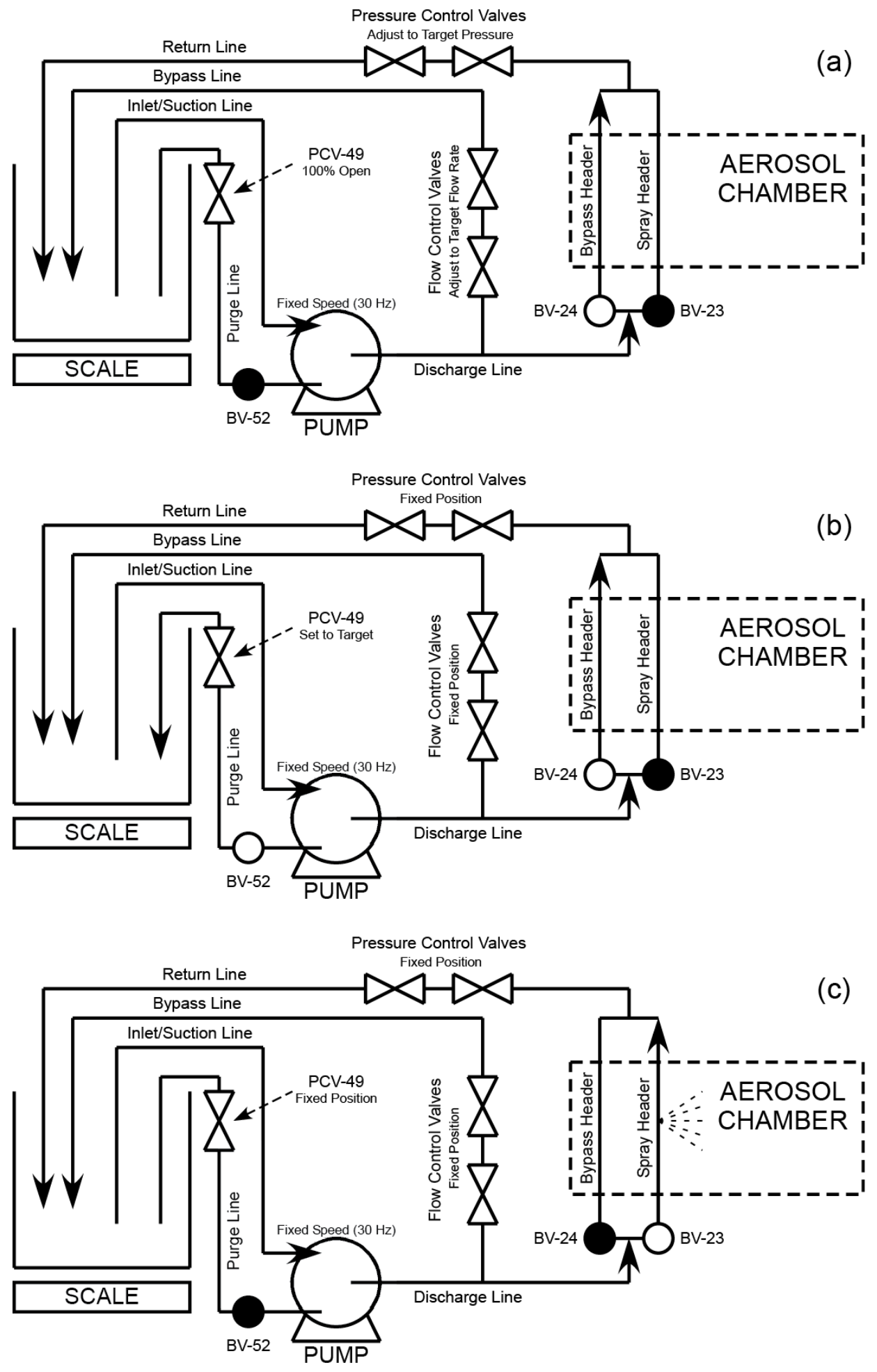

Figure 5.1. Simplified Diagrams of Small-Scale Valve Protocol: (a) Setting Target Conditions, (b) Equalizing the Purge Line, and (c) Spraying into the Chamber. Closed ball valves are denoted as filled circles and open ball valves are open circles. 
If a different type of simulant was planned for the next test, the simulant was removed into a drum using a diaphragm pump. Tap water was circulated at pump speeds above $30 \mathrm{~Hz}$ to rinse and flush the system until the water was no longer turbid. In some instances, more involved cleaning protocols were required (see Section 5.4.3).

\subsubsection{Daily Closeout Checks}

At the completion of testing each day, closeout checks were completed to ensure the system was left in the correct configuration and equipment was powered down. An end-of-day reading for the feed tank was taken, including level and mass, to assist in determining how much, if any, simulant loss occurred before the next tests. The tank was usually covered loosely with Saran ${ }^{\mathrm{TM}}$ wrap to help reduce evaporation of water. Data files were checked for proper naming convention and backed up on an external hard drive.

\subsection{Modifications to Test Operations}

The previous section outlined standard test operations for Phase II testing. Though the system was operated similarly to Phase I, there were a few differences between standard Phase I and Phase II operations. Further, some modifications to test operations and cleaning approaches were required to handle some simulants used in Phase II. The following subsections discuss differences from Phase I testing and non-standard operations due to challenging simulants.

\subsubsection{Differences from Phase I Testing}

The significant differences between standard Phase II and Phase I test operations were as follows:

- In general, pre-sprays were performed less frequently in Phase II, particularly with the chemical simulant.

- The RH (FIO) was recorded for the majority of the tests in Phase II. In Phase I, RH was not a parameter that was recorded with regularity.

- The valve protocols to set target conditions and initiate/discontinue the spray were used consistently for all tests in Phase II. The protocols, described in Section 5.3.2.4, resulted in a more consistent and steady pressure response in Phase II than in Phase I.

- The density check was performed for a shorter duration (90 s) in Phase II than in Phase I (5 min). In general, the density measured by the Coriolis flow meter stabilized within a few seconds while the pump was running at $30 \mathrm{~Hz}$.

- The chiller set-point had to be monitored and adjusted occasionally to control the rate of cooling in the heat exchanger depending on temperature measurements. Phase I did not have heat exchange capability, so operation of the entire cooling subsystem was only performed in Phase II.

- Sampling was performed at a lower frequency in Phase II than in Phase I. However, rapid turnaround sample analysis was more frequent during testing with rheological simulants in Phase II to assess if the simulant had to be adjusted to keep the rheology within the desired range.

- In Phase II, data acquisition and file management were performed on a single performance check (PC) by a single operator. In Phase I, these duties required two PCs and two operators. 


\subsubsection{Challenging Simulants}

\subsubsection{Kaolin-Bentonite Clay}

Chronologically, KBC slurry was tested first in Phase II, first at nominally $30 \mathrm{~Pa} / 30 \mathrm{cP}$ and then diluted down to nominally $6 \mathrm{~Pa} / 6 \mathrm{cP}$. In general, the clay was tested using all the standard procedures with no modifications. As discussed in Section 3.5.2, the clay had reasonably stable rheology and was well-controlled. This is reflected in Figure 3.10 and Figure 3.11, which show how the rheology measurements (yield stress and consistency, respectively) changed over operating time during the testing. The major difficulty experienced during testing with the clay was the qualitative observation that sprays could have varying spray geometries (as characterized by the observed spread, angle, and intensity of the jet) as they left the orifice. Some sprays looked noticeably different as they left the orifice and occasionally a spray would be deflected from a "straight" path. These abnormal sprays did not appear to be due to any abnormal pressure response. The inconsistent spray behavior made in-spray measurements particularly challenging, and was the driver for performing all the in-spray measurements with the $0.5 \mathrm{~mm}$ hole only $9.2 \mathrm{in}$. from the Malvern Insitec-S measurement zone. ${ }^{1}$ One observation about the clay sprays was the presence of a mound of simulant just underneath the orifice (for an example, see Figure 5.2). Whether this mound of clay contributed to the occasionally erratic behavior is hard to ascertain.

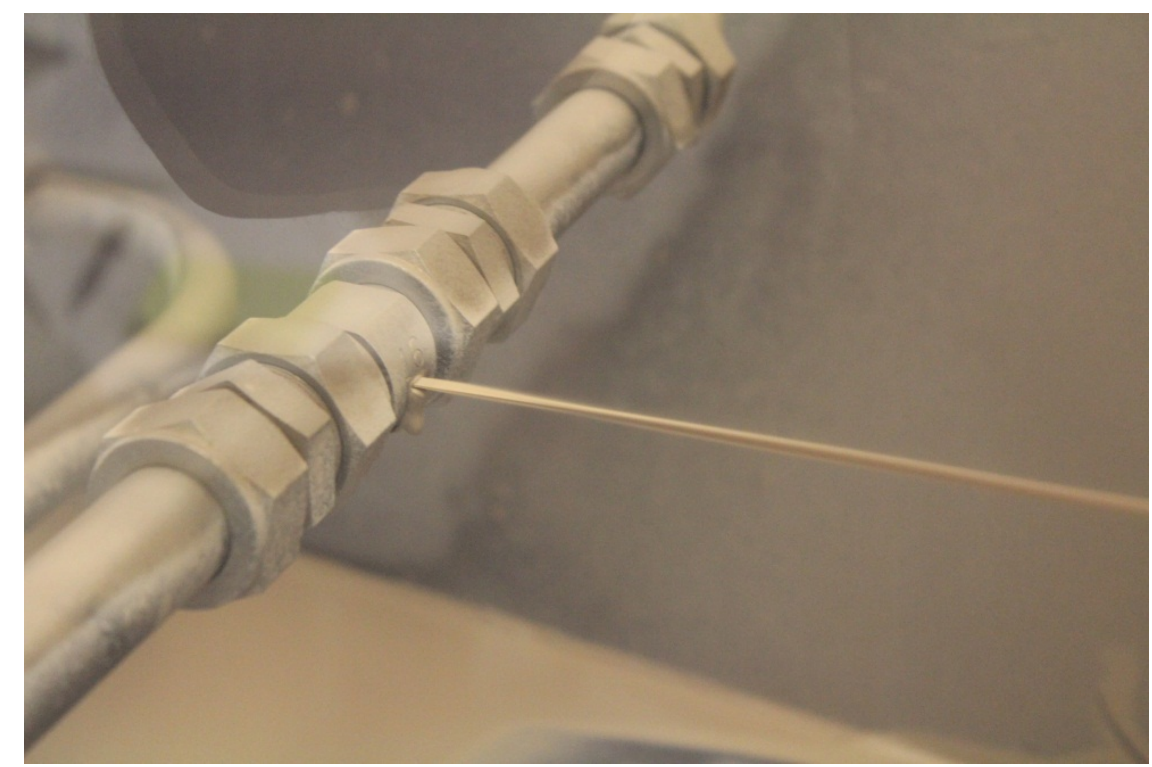

Figure 5.2. Image of the Spray from Test SS-222 (6/6 clay, OTP-16, 100 psig). The image shows how the clay simulant was bunched up underneath the orifice even as it sprayed.

\footnotetext{
${ }^{1}$ The spray header was extended closer to the Malvern instrument by adding 6-in. extensions on either side. Thus, only 4.4 in. remained from the OTP to the left edge of the Malvern faceplate. The faceplate is a 9.6-in. square, so the measurement zone is $4.8 \mathrm{in}$. from the left edge of the faceplate, and the OTP is $4.4+4.8=9.2 \mathrm{in}$. from the measurement zone.
} 


\subsubsection{Fe-Rich Simulant (FEG)}

The Fe-rich simulant was more challenging to test successfully, particularly when using standard test procedures. Both the $30 \mathrm{~Pa} / 30 \mathrm{cP}$ and $6 \mathrm{~Pa} / 6 \mathrm{cP}$ FEG had less stable rheology that was also time-dependent (suspected to be due to the effect of shear on the material). The contrast between the FEG and the clay is clear when the FEG rheology measurements are examined against operating time (see Figure 3.16 and Figure 3.17). Discrete sample analyses do not fully capture the magnitude of the problematic rheology, as the FEG simulant tended to thicken with time and exposure to shear. The thickening with shear rate was rapid, especially at higher target pressures, and reversible. For one example of this, see Figure 5.3. The left image was taken with only the agitator operating just before the pump was started. The surface is moving fluidly and the majority of the tank contents were in motion. There is a small region near the tank walls that is not mobilized or moving very slowly, which is similar to what was observed with clay slurry in the mixing tank. In the right image, the system has been operating for about $5 \mathrm{~min}$ with conditions set at approximately $200 \mathrm{psig}$ and $11.4 \mathrm{gpm}$. The surface is much less fluid and the FEG simulant has noticeably changed in thickness. The simulant from the surface, if removed, holds its shape and has a consistency like peanut butter.
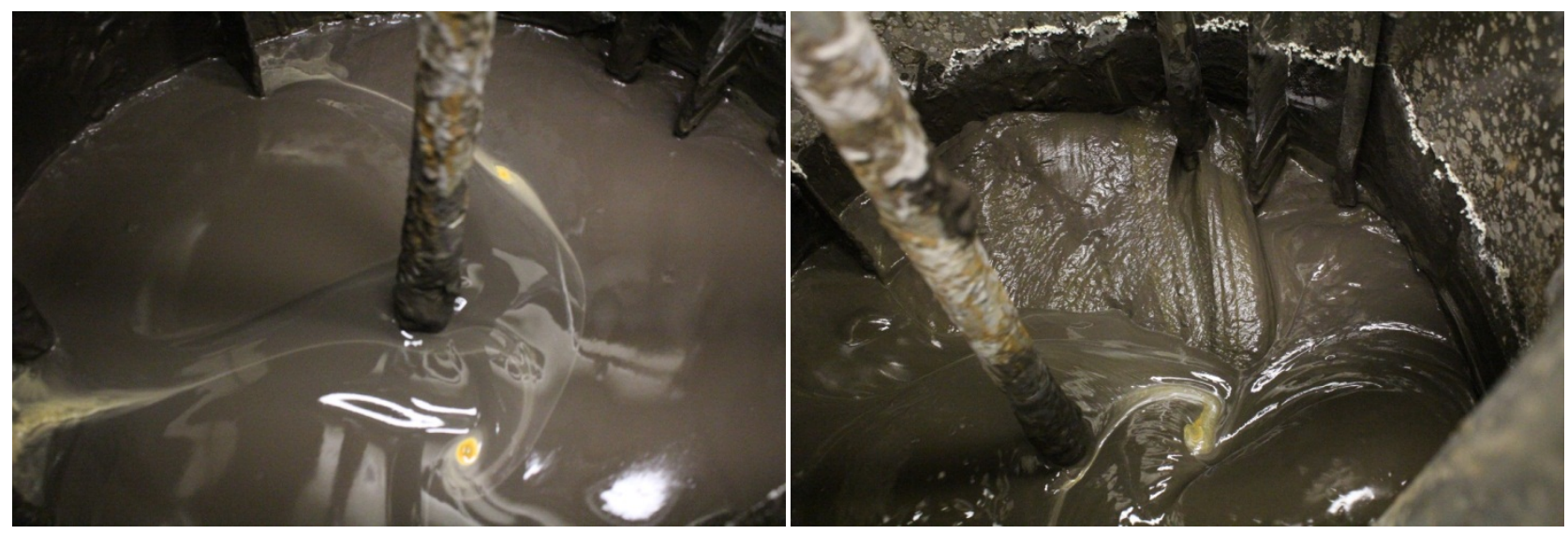

Figure 5.3. Comparison of Feed Tank Contents when Operating with FEG Simulant. The left image shows the tank just before circulation of simulant began and the right image after 5 min of operation at approximately $200 \mathrm{psig}$ and $11.4 \mathrm{gpm}$.

The situation shown in Figure 5.3 was not an isolated incident and occurred repeatedly during test operations. As the FEG thickened, the system struggled to maintain the flow rate at the target value and the pump would shudder and/or cavitate. Other difficulties encountered were:

- The target pressure and flow rate did not remain steady long enough to perform all activities required to complete a test.

- Upon initiating a spray at target conditions, there was little or no flow from the orifice. This was speculated to be either plugging or simply rheological resistance to flow. When observed, tests with this behavior were not analyzed and typically were repeated.

- Early on in the FEG testing, the heat exchanger became plugged with the simulant. It had to be removed from service for cleaning. This resulted in some increases in system temperature that could only be decreased by allowing the feed tank to cool down, thereby requiring time. 
- Time-dependent thickening with applied shear required time between sprays for the material to "relax."

- It was not clear how to obtain a representative sample of the tank contents for analysis.

- Gradually throughout the testing, the Coriolis flow meter became partially plugged and indicated densities that were greater than expected.

As testing proceeded, problems with the pump cavitating or laboring to meet target conditions persisted. The standard operating approach did not appear sufficient for testing with FEG simulant.

To permit completion of the FEG simulant testing, several modifications to the system were made. These included changes to the configuration of the equipment and the procedure used to perform the tests. The following changes were implemented:

- A diaphragm pump was added to assist the primary positive displacement pump. This pump was installed to remove simulant from the bottom of the feed tank $(\mathrm{BV}-36)$ and send it almost directly into the suction of the primary pump (via BV-30). The addition of a diaphragm pump allowed the system to maintain a target pressure long enough to test, with a noticeable decrease in cavitation. Later, upon post-test cleaning and examination, it was found that the inlet line had severe restrictions in diameter (see Figure 5.4) that certainly contributed to the difficulties. It appeared that the simulant solids deposited in a uniform ring around the inner diameter of the lines.

- The fixed speed of the pump was reduced from $30 \mathrm{~Hz}$ to a lower value (often $20 \mathrm{~Hz}$ ). The reduction in pump speed helped manage temperature increases and slowed the thickening of the material.

- The target flow rate requirement was relaxed. Even with the diaphragm pump to assist, the target flow rate of 11.4 gpm could not always be reached without straining the system or increasing the speed above $20 \mathrm{~Hz}$. The solution was to obtain the highest flow rate achievable at the pressure of interest.

- The heat exchanger was installed in a bypass configuration. Once cleaned, the heat exchanger was put back into service but not on the return line. It was placed on the bypass line and could be isolated with valves so it was used only when needed and did not run continuously.

- The time the simulant spent at target conditions was minimized. This often necessitated not performing a pre-spray (or only a few seconds of pre-spray) and reducing the amount of time before the spray was started. Some sprays were not conducted for the full $2 \mathrm{~min}$ if a steady-state concentration was observed on the Malvern Insitec-S instrument. Often the primary pump was reduced to $10 \mathrm{~Hz}$ immediately after the spray was completed.

The modifications listed above were successful in allowing the testing to be completed. However, because the rheology was erratic and the tests were conducted using a non-standard approach, caution should be exercised when assessing data from these tests. In particular, the sensitivity of the FEG simulant to shear may have greatly influenced the formation of the spray jet as it left the orifice, where the shear rate is very high. It is unlikely that the rheology of the FEG simulant is representative of any material the WTP will process. At the time of this writing, the authors are not aware of any tank waste samples that exhibited this type of rheological behavior. The higher solids loading in the FEG simulant needed to achieve the target rheological parameters is atypical of waste that will be processed in the WTP. 


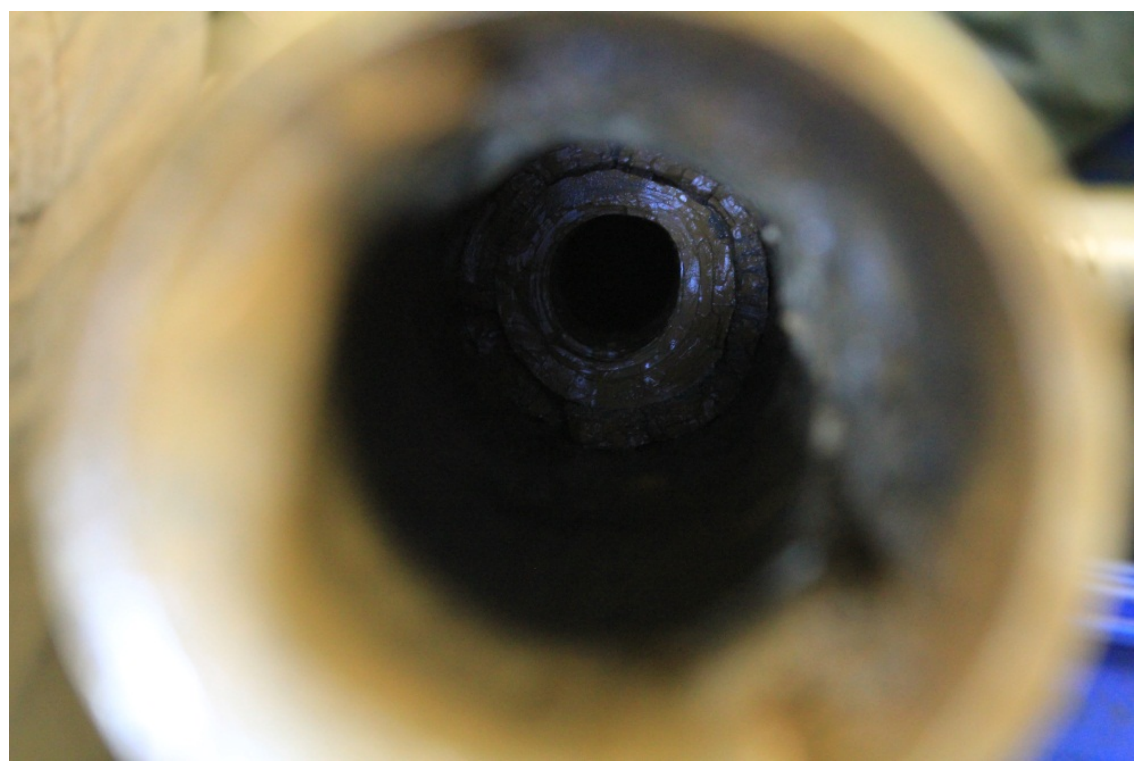

Figure 5.4. View Into the Inlet Line After Testing with the FEG Simulant. Note the significant reduction in diameter caused by the thick layer of caked FEG material.

\subsubsection{Dense Particle Simulants}

The other simulants that required some modification to the small-scale system were those containing the dense particles (Mo). Unlike the modifications for the FEG simulant, these changes were planned in anticipation of having a fast-settling solid particle in the system. The primary concern was that the Mo particles could settle in the inlet line, which had a 2.5-in.-diameter, and not reach the pump to get circulated throughout the system. This was mitigated by replacing the stainless steel piping on the inlet line with 1.5-in.-diameter flexible hose, which required a small expansion from 1.5 to 2.5 in. at the pump inlet. Secondary concerns were that the Mo particles would not be well-suspended in the feed tank and be under-represented in the fluid circulating through the system or that the Mo particles would not exit through the orifice due to their inertia.

The Mo particles were first tested in water (DPW). When the Mo particles (nominally $1 \mathrm{wt} \%$ in water) were first added to the feed tank, two dip samples were taken from the top of the tank: the first 1 min after particle addition and the second after shutting off the agitator, turning it back on, and re-suspending the particles for $5 \mathrm{~min}$. Both of those samples were analyzed for UDS and were measured to be 0.6 and $0.5 \mathrm{wt} \%$, respectively. ${ }^{1}$ These results indicated that some portion, but not all, of the Mo particles were suspended in the tank. It is likely that a fraction of the Mo particles, particularly the particles with the largest diameters, were at or near the tank bottom.

To test whether the Mo particles actually left the system through the orifice as part of the spray, a "spray catcher" was added to the system (see Figure 5.5). The system was sprayed at 100 and 380 psig using the $1 \mathrm{~mm}$ hole, and the tube deflected the spray into a collection vessel. The contents of the spray samples were weighed, and then dried in an oven. After drying, the residual solids left behind were used to estimate the original UDS in the spray, which was determined to be $0.36 \mathrm{wt} \%$ (100 psig test) and

\footnotetext{
${ }^{1}$ UDS was determined using a moisture analyzer but the result is FIO.
} 
$0.44 \mathrm{wt} \%$ (380 psig test). This confirmed that a portion of the Mo particles were leaving through the orifice, but not necessarily an amount representative of the parent slurry. All Mo tests were conducted at 380 psig and approximately 50 percent of the Mo particles were present in the spray. The DPW simulant was assumed to be a worst case and was more straightforward to measure, so the "spray catcher" tests were not repeated for the DST simulant. It was visually confirmed that Mo particles were found in the aerosol chamber when the DST simulant was tested.

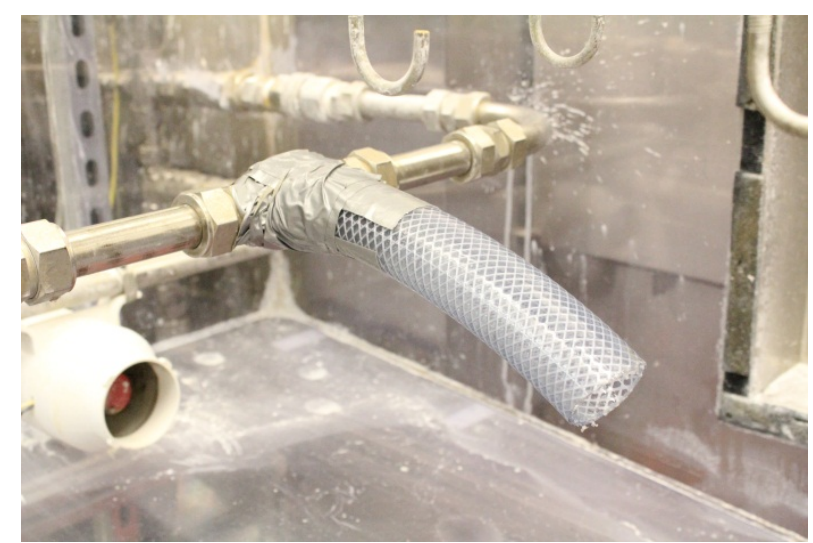

Figure 5.5. Picture of the "Spray Catcher" Used to Verify the Presence of Dense Mo Particles in the Spray Leaving an Orifice. The OTP is underneath the attachment point of the tube with the spray header.

\subsubsection{Cleaning}

Testing various simulants consecutively required some system cleaning. In general, this was performed by draining the simulant from the system, and then rinsing with small quantities of water (10-15 gallons) until the water was clear even after being circulated. The rinsing was often done by increasing the pump up to its highest speed $(60 \mathrm{~Hz})$ to mechanically remove any residual material.

The FEG simulant was the exception. Though the system was rinsed until the water was clear, it was suspected that not all FEG material was removed. Ultimately, the system was completely dismantled and manually scrubbed out. Hardened deposits and cake-like formations were found in many places (e.g., Figure 5.4 being one of the worst examples). Following system re-assembly, $0.5 \mathrm{M}$ oxalic acid was used to chemically dissolve any remaining simulant and the system was rinsed again before the $27 \mathrm{wt} \% \mathrm{STR}$ simulant was loaded. 


\subsection{Test and Analysis Methods}

The spray release tests were conducted to estimate the net amount of aerosol generated as a function of orifice geometry, system pressure, and fluid. Ultimately, the quantities of interest are the net generation rate and release fraction of the aerosol, the latter being the volumetric net generation rate of aerosol $(G)$ divided by the volumetric flow rate $\left(Q_{\text {spray }}\right)$ of the spray leak. The dependence of the release fraction or generation rate on test conditions can be compared directly to that predicted by the current WTP model.

In this section, the dependence of the measured release fractions with pressure will be compared to the pressure dependence predicted by the WTP model. In general, the release fraction results from the small-scale chamber will not be directly compared to the WTP model because of unquantified method bias that has not been accounted for, or bounded, in small-scale testing. In addition, the large-scale chamber is closer in size to the rooms in the WTP and thus thought to give more applicable results. For a discussion on the effect of chamber size on release fraction results, see Daniel et al. (2013). A comparison of small- and large-chamber release fractions is given in Schonewill et al. (2012). In addition, the small-scale release fraction results were collected with initial chamber humidities that were typically less that 100 percent RH, based on FIO humidity measurements, and the release fraction results are biased low as a result.

In the presentation of the large-scale results (see Section 10 and Appendix A in Daniel et al. 2013), an approach is implemented that provides a bounding estimate of the humidity and method bias. Though no bounding estimates have been made for the small-scale chamber results, the measurements are expected to have valid trends of release fraction with pressure, and these can be compared to the pressure trends predicted by the WTP model.

As in Phase I data analysis, the net generation rate of aerosol was determined by measuring the volume and size distribution of the aerosol (using a Malvern Insitec-S instrument) and performing a non-linear least squares fit to the data in the first $20 \mathrm{~s}$. In Phase II, the spray leak flow rate was calculated based on the slope of a line fitted to the process instrument data for feed tank weight versus time; for comparison, Phase I analysis used times and feed tank weights that were manually recorded in the TI at the start and end of spraying.

This section describes the techniques used to calculate the net generation rate, release fraction, and other auxiliary quantities (e.g., orifice coefficient). First, the analysis of process instrument data is discussed, including the approach used to estimate the spray leak flow rate. Next, the analysis of Malvern Insitec-S data is discussed, with a focus on how the net generation rate was calculated for the small-scale experiments. Next, elements of the test procedure and data analysis are discussed that had the potential to improve or detract from the meaningfulness of the results. Finally, the results of method validation tests carried out as part of Phase II are summarized and their significance for small-scale testing is discussed.

The analysis methods used here are similar (and identical in most respects) to the methods used for the large-scale aerosol data (Sections 3, 7, and 8 of Daniel et al. 2013). However the methods differed enough to warrant the following detailed description. 


\subsection{Analysis of Process Instrument Data}

Data from the process instruments installed on the flow loop and within the chamber were captured by a data logger that recorded measurements at a sampling rate of $2 \mathrm{~Hz}$ for pressure, header flow rate, temperature, and valve positions (see Section 4.2 for more detail). In addition, data for the weight of the feed tank were collected at a rate of $1 \mathrm{~Hz}$. The process instruments were used to meet the data objectives listed below:

1. Record the pressure upstream of the orifice for use in WTP model aerosol predictions and estimation of the orifice coefficient

2. Record the temperature in the test section to support estimates of water viscosity and density used in WTP model aerosol predictions

3. Record the feed tank, load-cell (scale) measurements of feed tank weight to calculate the mass of fluid lost from the system during a spray. This information was used to estimate the volumetric flow rate of the spray.

4. Record the header and bypass valve positions to provide spray start and end times for use in a variety of calculations.

To achieve data objective 1, the upstream header pressures recorded by the data logger during a test were averaged during the time period of interest, which was the initial 20-s period used for fitting the non-linear model of aerosol net generation rate. In Phase II, this fit-period average pressure was used to calculate the WTP model droplet size distribution, the measured orifice coefficient, and the WTP model total leak flow rate.

For comparison, in Phase I testing the time period of interest for the WTP model droplet size distribution was the fit period, but the entire spray duration was the time period of interest for calculations of orifice coefficient and WTP model total leak flow rate. This time period was appropriate because the measured leak flow rate was based on the difference in weights measured at the start and end of the test.

In all cases, the representative average pressure for spray leak calculations was found by squaring the average of the square root of the time series of measured pressures. This functionality was chosen because the flow rate through the orifice depends on the square root of pressure (see Equation (6.2)). The dependence of the spray size distribution on pressure is more complex (see Equations (1.1) through (1.6)). The square root dependence is approximate in this case. As discussed in Section 6.5.6 of Mahoney et al. (2013), the initial pressure variation during a small-scale test can have a significant effect on the average pressure during the time period selected for curve fitting.

Data objective 2 was met in Phase II by taking the average, over the entire spray duration, of the measured header temperatures. For comparison, in Phase I the average temperature was calculated as the average of two temperatures manually recorded as identified in the TI before and after the spray.

The average temperature was used to interpolate water viscosity and density from a lookup table of standard properties. Viscosity and density of simulants other than water were taken from laboratory measurements and were not adjusted to match the test temperature because there are no data for viscosity temperature dependence. The density-temperature dependence for non-water simulants was considered negligible, based on the changes seen in the water density. This approach is consistent with that used in 
Phase II large-scale testing and Phase I small-scale testing, but differs from that used in Phase I large-scale testing, where the temperature variation of water was used for other simulants.

Data objective 3 was met in Phase II by fitting a line to the time series of weight data taken during the initial 20-s period (i.e., 20 data points). The slope of the line provided a mass flow rate that was converted to a volume flow rate by dividing by the simulant density. In Phase I, the leak flow rate was calculated from two manually recorded measurements, the mass of fluid present in the tanks before and after an experiment. The Phase II approach was developed to improve on the Phase I approach by considering only the time period of the generation rate fit, making the basis of flow rate consistent with that of the generation rate and basing the leak flow rate on 20 data points rather than two. In addition, the Phase II approach avoided a problem observed in Phase I, which was inconsistency in the conditions at the times when start and end weights were measured. Differences in flow in and out of the tank cause differences in thrust against the bottom of the tank, which the feed tank load cell measured as weight. Using the slope of a time series of weight data avoided inaccuracies produced by inconsistent thrust conditions.

To meet data objective 4, changes in the open/closed status of the bypass (BV-24) and header (BV-23) valves were used to identify the times of spray start and spray end (refer to Figure 4.3 for a schematic showing the location of the valves). The signals recorded by the data logger indicated whether valves were closed or not-closed; the term "not-closed" is preferred to "open" because the signals did not distinguish between partly open and fully open. The use of valve signals and a clock that was common to all data measurements (data logger, feed tank scale, and Malvern Insitec-S instrument) made it possible to identify the spray start and end times with more precision than in Phase I. In Phase I, start and stop times of the spray were recorded in the TI during each test. Two separate unsynchronized clocks were used, one for the data logger and one for the Malvern Insitec-S instrument, and a set of start and stop times was recorded for each clock.

The determination of the orifice coefficient was not a formal objective of testing, but was included as being useful information. Using the following expression, which was derived by rearranging Equations (1.8) and (1.9) to solve for the orifice coefficient instead of assuming a value of 0.62, the spray leak flow rate was used to estimate the orifice coefficient, $C_{D}$, for each test

$$
C_{D}=\frac{Q_{\text {spray }} / A}{\sqrt{2\langle P\rangle / \rho(\langle T\rangle)}}
$$

where $A$ is the cross-sectional area of the orifice, $\langle P\rangle$ is the average pressure during the first $20 \mathrm{~s}$ of spray, and $Q_{\text {spray }}$ is the leak flow rate measured during the first $20 \mathrm{~s}$ of spray.

For most tests, a humidity instrument was used to measure the RH of the air in the chamber before and after the sprays. The measurements were recorded in the LRB. The instrument was not calibrated to NQA-1 standards, so collected data must be considered FIO. 


\subsection{Analysis of Malvern Insitec-S Data}

Data from the single Malvern Insitec-S instrument installed in the small-scale chamber were collected by a single computer at 4 or $1 \mathrm{~Hz}$ via commercial software (see Section 4.3 .1 for more detail). ${ }^{1}$ The data needed for calculations were the volume concentration of aerosol, $C_{v}$, measured in ppmv and the percent volume of aerosol as a function of predefined droplet size bins $(\phi)$, which is a differential or discrete measurement. In addition, arrays of date/time stamps and the Malvern Insitec-S laser transmission were required to perform the calculations outlined in this section, e.g., estimating the net generation rate. These calculations were very similar to those performed in the large-scale tests (see Section 7.2 of Schonewill et al. 2012), but were considerably simplified because there was no need to average the readings from three Malvern Insitec-S instruments. The calculation method was the same in Phase II as in Phase I, and the description given here is repeated from the Phase I report, except where Phase II modifications are noted.

In the first step of analysis, the laser transmission reading is examined to determine if any adjustments are required to get a more accurate measurement of $C_{v}$. As described in Section 4.3.1, the Malvern Insitec-S measures aerosol based on laser diffraction. Transmission is a measure of the received laser power, which is reduced by the presence of aerosol in its path between the source and the detector. Transmission is written as

$$
\tau=\frac{S(t)}{S_{0}}
$$

where $S(t)$ is the undeflected laser power measured at time $t$ and $S_{o}$ is the background undeflected laser power. The background is a fixed value that the operator sets by selecting the $S(t)$ reading at some time before $t=0$, when the spray starts. Ideally, the transmission should be 100 percent before the spray enters the chamber.

To check the need for correction, the laser power measured immediately before the start of the spray is compared to the fixed background laser power. If laser power drift has caused the initial undeflected laser power to change since the time when the background laser power was fixed, an adjustment is needed. In cases where the undeflected laser power drifts to less than the background value, the uncorrected transmission would be too small and the value of $C_{v}$ would be too large. Conversely, the initial undeflected laser power data could be greater than the background laser power. This would lead to an overestimation of transmission and under-estimation of $C_{v}$. If the drift discrepancy is great enough, an apparent transmission greater than 100 percent would be calculated using Equation (6.2). The Malvern software would take that as an error and report a PSD but not a concentration $C_{v}$. In this case, the true concentration could not be recovered by drift adjustment.

A more detailed discussion of the derivation of the adjustment factor is given in the Phase I large-scale report (Equations (7.7) through (7.11) of Schonewill et al. 2012). In summary, the correction for $C_{v}$ can be written as

$$
\frac{C_{v, a d j}}{C_{v, 0}}=\frac{\ln (\tau(t))}{\ln \left(\tau_{0}\right)} \approx \frac{(1-\tau(t))}{\left(1-\tau_{0}\right)} \approx \frac{1-S(t) / S(0)}{1-S(t) / S_{0}}
$$

\footnotetext{
${ }^{1}$ The Malvern collected data at $1 \mathrm{~Hz}$ only during shakedown testing. For all other testing (i.e., tests of the effect of pressure, solids, and non-Newtonian rheology), the Malvern collected data at $4 \mathrm{~Hz}$.
} 
where $C_{v, a d j}$ is the adjusted $C_{v}, C_{v, o}$ is the original measured $C_{v}$, and $S(0)$ is the initial undeflected laser power at the start of aerosol generation $(t=0)$. The corrected background $S(0)$ was chosen to be the average over the $5 \mathrm{~s}$ before the spray began. Note that if the transmission is 90 percent or greater, the final term on the right of Equation (6.3), which is the approximate linearized form of the equation derived with a Taylor series expansion, can be used. The approximate linearized form of Equation (6.3) was used for all the tests in which the Malvern Insitec-S probe was located outside the jet. The non-linearized logarithmic form of Equation (6.3) was used for calculations of in-spray concentrations.

The second step in the analysis of Malvern Insitec-S data is to transform the raw data into a form that can be fitted to obtain the volumetric net generation rate. The aerosol data, which are fractional concentrations in percent of total droplet volume, must be put on a basis of absolute volume concentration (i.e., volume of droplets per volume of chamber):

$$
\Phi_{i}=C_{v} \phi_{i}
$$

where $\phi_{i}$ is the fraction of volume between size bin $k$ and $k-1$ as reported by the Malvern Insitec-S, $\Phi_{i}$ is the differential ppmv in the bin, and $i$ is the size bin of interest ranging from 1 to $N$. The Malvern Insitec-S instrument has 60 size bins scaled logarithmically from 0.1 to $2000 \mu \mathrm{m}$, although in small-scale use, the effective range of the Malvern Insitec-S instrument was either 0.5 to 200 or 2.5 to $2500 \mu \mathrm{m}$. Equation (6.4) can be cumulated to give the cumulative ppmv below a certain droplet size

$$
\Phi_{c, k}=\sum_{i=1}^{k} \Phi_{i}
$$

where the subscript $c, k$ indicates the cumulative ppmv below the droplet diameter associated with size bin $k$. The cumulative ppmv also can be normalized to calculate a PSD (volume fraction) of the aerosol. Mathematically this is expressed as

$$
\phi_{c, k}=\frac{\Phi_{c, k}}{C_{v}}
$$

Both the differential (Equation (6.4)) and cumulative concentrations (Equation (6.5)) were used in the analysis of in-chamber (i.e., not in-spray) aerosol concentration data described in this section. The in-spray data were analyzed in terms of cumulative PSD (Equation (6.6)), because the assumption of uniform concentration in the chamber could not be applied to in-spray data, making it inappropriate to use concentration data to calculate aerosol net generation. However, these in-spray PSDs are of interest because they are a close approximation to the size distribution that would be present if the only aerosol generation mechanism present was jet breakup, not splash impact, and if there were no losses.

When a lens with a focal length of $100 \mathrm{~mm}$ is mounted on the Malvern Insitec-S instrument, the measurable range of droplet sizes is 0.5 to $200 \mu \mathrm{m}$. A $500 \mathrm{~mm}$ lens provides a measurable range from 2.5 to $2500 \mu \mathrm{m}$. All data collected in Phase I came from a Malvern Insitec-S mounted with a $100 \mathrm{~mm}$ lens; thus, $200 \mu \mathrm{m}$ represented the upper bound of the Phase I small-scale analysis. Most of the data in Phase II was gathered using a Malvern Insitec-S mounted with a $500 \mathrm{~mm}$ lens to permit measuring the larger droplets found in the jet.

The average cumulative or differential concentrations were obtained as functions of time. During the time the spray was active, data were observed to increase rapidly to a steady-state concentration. The 
term "steady-state" should not be interpreted to mean the concentration was consistently at a precise single value, but rather that the data fluctuated around some mean concentration. In some tests, the fluctuations were sizable and in others they were not. In general, the fluctuations became more significant as the orifice size increased (and consequently, the volumetric flow of the spray). Given the chaotic nature of turbulent jet flow and the data acquisition rate of the Malvern Insitec-S instrument, noise in the data was expected. Generation-rate calculations were performed on the range of valid data between 1.01 and $198.4 \mu \mathrm{m}$, although the region of the most interest in safety analysis was 10 - to $100-\mu \mathrm{m}$ droplets.

\subsection{Analysis of Aerosol Generation}

The first step in analysis of aerosol generation was to determine the volumetric net generation rate of aerosol, which was done by fitting a model to the Malvern Insitec-S concentration/time data. The functionality of the model was selected during Phase I after examination of some of the data and various theoretical considerations. The measured concentrations of most sizes of droplets tended to follow a curve strongly resembling an exponential approach to a steady-state value, suggesting an eventual balance between constant generation rate and losses with a roughly first-order dependence on aerosol

concentration. In some ranges of droplet sizes, generally less than $15 \mu \mathrm{m}$, the increase was approximately linear, implying negligible losses. As discussed in Section 3 of Schonewill et al. (2012), the observed quasi-exponential form of the concentration transients can be confirmed from theoretical arguments, which are briefly summarized in Section 6.3.1. Section 6.3.2 describes the application of the exponential model to the data and the quality checks performed to screen dubious runs out of the results database. Uncertainty is discussed in Section 6.3.3. The discussions in these sections match those in the Phase I report except where changes in approach are noted.

\subsubsection{Basis of the Exponential Model}

To determine the form of the rate equation that is fitted to the concentration transient data, the test enclosure (exclusive of the immediate vicinity of the jet) is treated as a continuously stirred tank reactor (CSTR). In the CSTR approximation, the control volume in which aerosol measurement is made is assumed to be homogeneous. Homogeneity results from instantaneous and complete mixing of the contents of the control volume. For the small-scale test enclosure, control volume homogeneity is an assumption that was expected to be reasonable given the turbulent mixing provided by the high-velocity jet and by the mixing fan.

The form and functionality of loss equations (and even the loss mechanisms considered) depend on the control volume assumed. If the entire box volume is considered, there is no convective transport of aerosol out of the control volume and only loss to the walls must be considered. As discussed in Section 6.5.2 of Mahoney et al. (2013), measurements made in three different locations (positions 1, 2, and 6) gave very similar release fraction results, indicating that it is acceptable to assume uniform concentration and behavior throughout the small-scale enclosure. A control volume including the entire enclosure was therefore used for small-scale analysis. The volume of the enclosure was $0.7025 \mathrm{~m}^{3}$ and was not changed during testing.

Aerosol is generated by primary and secondary jet breakup and by "splatter" droplets formed when the jet, or droplets formed by jet breakup, hit the splash wall at the downstream end of the enclosure. The in-flight and impact breakup events have not been distinguished in the current tests; however previous 
testing (see, for example, Mahoney et al. 2013) indicated that in-flight events may have a greater effect on aerosol generation than impact events. To avoid the complexity of droplet generation mechanics, the generation-rate model only considers the far-field (as expressed by the in-chamber measurement) concentration of aerosol. When considered far from the spray, net aerosol generation may be treated as a constant influx of spray droplets of given size to the control volume; the size of these droplets did not further decrease through additional breakup processes.

This size distribution can change with time as aerosols are preferentially retained or removed from the system. If no mechanism for droplet loss exists, the aerosol concentration will increase linearly with time, and the size distribution will not change. For systems that include losses, the aerosol concentration will increase until some equilibrium aerosol concentration is reached. Loss in the region outside the spray is assumed to occur through several means:

- Aerosol is deposited by convective transport on the surfaces of the test enclosure. The loss rates are proportional to the surface area, the droplet convective velocity, some form of a capture coefficient, and the droplet concentration.

- Aerosol settles out of the control volume at a rate proportional to the floor area, the droplet settling velocity, and the droplet concentration.

- Aerosol is entrained into the jet at a rate proportional to the entrainment velocity and the droplet concentration and then captured at the splash wall to an extent dependent on the local jet characteristics and on droplet size and properties.

- Aerosols coalesce or aggregate into larger aerosol structures. In general, coalescence is a two-particle interaction, so the rate of formation of larger droplets by coalescence is proportional to the product of the concentrations of two smaller droplet sizes. Coalescence yields a net decrease in the number of aerosol particles and a transfer of aerosol volume to larger size classifications. This means that the total loss/generation of aerosol volume resulting from coalescence is always zero. In typical small-scale tests, the in-chamber total aerosol concentrations are less than 5 ppmv and aerosol concentrations for specific size classifications are typically less than $0.5 \mathrm{ppmv}$ per classification. At these concentrations, the frequency of second-order reactions is expected to be low relative to first-order reactions. This combined with the fact that the overall impact of coalescence on the total volume concentration is zero, means that particle loss to aerosol coalescence can likely be neglected in far-field considerations of aerosol dynamics.

- Evaporative loss was also a possible mechanism but was not considered significant at the time the test procedure was planned because each test began with a wetted enclosure and the RH was expected to be high. Evaporation produces a loss of total concentration, but the effect on concentration in a given size range at a given time is complex: the concentration in a bin would increase because of evaporation from the next-larger size range but would also decrease because of evaporation that takes droplets down into the next-smaller size range. The effect of the initial humidity in the chamber was tested in Phase II, and the results of testing are discussed in Section 6.4.5.

The overall aerosol balance is derived by considering the sum of generation and loss terms. Because the evaporative mechanism and second-order mechanism of coalescence are not considered, and quantifying the amount lost via each first-order mechanism is not necessary for the data analysis, the loss terms may be joined into a single term for simplicity: 


$$
\frac{d \Phi_{i}}{d t}=\frac{g_{a, i}}{V_{e}}-\lambda_{i} \Phi_{i}
$$

where $\lambda_{i}$ is the loss rate coefficient (units of $1 / \mathrm{s}$ ), including all the loss mechanisms that are first-order in aerosol concentration for droplets of size $i$; $V_{e}$ is the enclosure volume; and $g_{a, i}$ is the net aerosol generation rate (volume/sec) of droplets of size $i$. The net aerosol generation rate equals the rate of production by the jet minus the rate of capture of droplets at the splash wall and minus the rate of other losses (e.g., evaporation, settling). Note that droplet capture at the splash wall is included in the net generation rate $g_{a, i}$, not the loss rate coefficient $\lambda_{i}$, because splash wall capture depends primarily on jet and droplet properties (sizes and velocities) and thus has zero-order dependence on the aerosol concentration.

The solution to this differential equation is

$$
\Phi_{i}=\frac{g_{a, i}}{V_{e} \lambda_{i}}\left(1-e^{-\lambda_{i} t}\right)
$$

An initial aerosol concentration of zero has been assumed. Using Equation (6.8), the equilibrium concentration becomes $\Phi_{i}^{e q}=g_{a, i} / V_{e} \lambda_{i}$.

Equation (6.8) can be used to analyze the dynamics of aerosol concentration at all times, including the initial period when the concentration is increasing and the period when concentration approaches

equilibrium. It is useful for sprays in which the aerosol concentration increases rapidly and the initial data have near-constant slope but are too few for good fitting. In such cases the leveling-off period, with nonconstant slope, must be included to provide enough points for a good determination.

Equation (6.8) expresses the aerosol material balance in terms of the concentration in each differential size bin of the size distribution. The cumulative aerosol concentration up to size $k$ is given by Equation (6.5). Substituting Equation (6.8) into this yields

$$
\Phi_{c, k}=\Phi_{c . k}^{e q}\left(1-\frac{1}{\Phi_{c, k}^{e q}} \sum_{i=1}^{k} \Phi_{i}^{e q} e^{-\lambda_{i} t}\right)
$$

Typically, the range of rate constants in the exponentials in the sum can be approximated by a single rate constant. When a single rate constant is assigned, Equation (6.9) becomes

$$
\Phi_{c, k}=\frac{G_{a, k}}{V_{e} \Lambda_{k}}\left(1-e^{-\Lambda_{k} t}\right)
$$

where Equation (6.10) has adopted the notation of Equation (6.8). Here $\Lambda_{k}$ is the cumulative loss rate constant (units $1 / \mathrm{s}$ ) for droplets up to size $k$ and $G_{a, k}$ is the net aerosol generation rate (volume/s) of droplets up to size $k$.

\subsubsection{Data Analysis}

The parameters $G_{a, k}$ and $\Lambda_{k}$ were found by fitting Equation (6.10) to cumulative concentrations $\Phi_{c, k}$. Similarly, the parameters $g_{a, i}$ and $\lambda_{i}$ were found by fitting Equation (6.8) to differential concentrations $\phi_{i}$. 
The differential-fit net generation rates were then cumulated to obtain the cumulative net generation rates $\dot{G}_{a, k}$ based on differential fits.

$$
\dot{G}_{a, k}=\sum_{i=1}^{k} g_{a, i}
$$

This cumulation process put the net generation rates obtained by differential fits on the same cumulative basis as the net generation rates from cumulative fits so that they could be compared.

Data fitting was carried out using a non-linear least squares algorithm ${ }^{1}$ for the first $20 \mathrm{~s}$ of the spray, subject to some constraints. (For comparison, the fitting time period was typically $60 \mathrm{~s}$ in large-scale testing.) The choice was made to constrain the values of the adjustable parameters for net generation rate and loss rate constant $\left(G_{a, k}\right.$ and $\Lambda_{k}$ or $g_{a, i}$ and $\lambda_{i}$ ) so that only meaningful values would be produced. The upper-limit and lower-limit constraints on the fitted net generation rate were based on the final concentration at the end of $20 \mathrm{~s}$. If the fitted net generation rate meant the 20 -s concentration was reached in $0.5 \mathrm{~s}$, the fitting procedure was terminated for producing an unreasonably high initial slope. A fitted slope this high was designated as inaccurate because it indicated a concentration increase that was too rapid to be captured accurately with the Malvern Insitec-S instrument. If the fitted net generation rate indicated that 1 percent of the 20 -s concentration was reached in $60 \mathrm{~s}$, the fitting procedure was terminated for producing an unreasonably low initial slope. The upper-limit and lower-limit constraints set on the loss rate constant were (arbitrarily) 100/s and 1E-05/s.

The bi-square weight method was used to make the fit more robust to outliers and/or spurious noise. In the bi-square weight method, the weight given each data point in the algorithm varies depending on proximity to the current best-fit curve. Data outside of that expected from random variation is given a weight of zero.

The algorithm assigned the net generation rate a 95 percent confidence interval, which included the uncertainty of predicting the curve based on the data and the random variation expected in a new observation. Goodness-of-fit was assessed in three ways: 1) comparison to lower-limit and upper-limit fitting constraints, 2) a convergence criterion, and 3) the coefficient of determination $\left(\overline{\mathrm{R}}^{2}\right)$ adjusted for degrees of freedom. Data for which the fit returned an upper-limit or lower-limit constraint value were rejected, as were data that did not converge. Fits that did not appear to adequately describe the data were detected by using the adjusted coefficient of determination to screen the results, with fits of $\overline{\mathrm{R}}^{2}<0.5$ rejected. The choice of 0.5 is arbitrary and does not prove that fits with $\overline{\mathrm{R}}^{2}<0.5$ were significantly poorer compared to those greater than 0.5 . However, it does suggest that less than half the variability in the data is described by the model fit.

A second step of data trimming was applied because the Malvern Insitec-S instrument measurements have high variability for the small cumulative volume fractions at low droplet size. Any release fraction calculated for a droplet size for which the cumulative volume fraction was less than 0.5 percent was not reported due to its higher uncertainty. This data trimming approach is similar with the large-scale data analysis methodology for Phase II. Neither the small-scale nor the large-scale tests applied this kind of trimming in Phase I.

\footnotetext{
${ }^{1}$ The algorithm was the fit function in MATLAB ${ }^{\circledR}$ version R2011b (The MathWorks, Inc.).
} 
Estimates of net generation rate were obtained primarily from the cumulative-fit method, which tended to produce good fits over a wider range of droplet sizes than did the differential fit. Based on the physical arguments made earlier, the net generation and loss terms in the model of Equation (6.8) should, in general, be a function of the droplet size. When the differential data is fit, the concentration is defined for narrow droplet size ranges. Thus, variations in net generation and loss behavior with size are isolated by treating the data separately in each size. However, the differential data have some statistical drawbacks: the data are smaller in absolute magnitude than the cumulative concentration data and much noisier, particularly for the smallest droplet sizes. The noisier data are more difficult to fit, and the results have a greater uncertainty. In addition, a bad fit for one differential concentration bin raises the question of how to cumulate it with other bins that have good fits. In this report, bad differential fits are excluded from plots as individual points but are included in the cumulation. This approach makes the cumulated differential-fit net generation rates $\hat{G}_{a, k}$ doubtful in some cases.

The cumulative-fit data are more attractive numerically, but treat the droplet net generation rates and losses in aggregate, which is physically less plausible. The larger the droplet size bin, the wider the range of sizes that are described by a single loss coefficient and net generation rate in the cumulative fit. See Appendix B for a collection of plots that show how cumulative and differential fits compared for the small-scale runs included in this report. In most cases, the two types of fits produce comparable results.

Once an estimate of the cumulative net generation rate was obtained using the model fit to the data, the cumulative release fraction was calculated as

$$
R F_{c, k}=G_{a . k} / Q_{s p r a y}
$$

An estimate of the fit-related uncertainty in the release fraction was determined using the confidence intervals from the model fit to the data

$$
\begin{aligned}
& R F_{c, k}^{+}=G_{a, k}^{+} / Q_{\text {spray }}-R F_{c, k} \\
& R F_{c, k}^{-}=R F_{c, k}-G_{a, k}^{-} / Q_{\text {spray }}
\end{aligned}
$$

where $G_{a, k}^{+}$and $G_{a, k}^{-}$are the upper and lower ends of the 95 percent confidence intervals on the net generation rate, respectively.

A screening process was applied to focus on the test results that were considered to be the highest quality for aerosol generation analysis. Some runs were excluded based on a review of the pressure, laser, and leak flow rate data. For any given test condition, runs preferred for analysis were those meeting the following criteria:

- no procedural errors or anomalous observations recorded in the LRB or test data document

- no evidence of lens wetting in the Malvern Insitec-S data

- negligible effects of laser drift during the first $20 \mathrm{~s}$, where negligible was defined as drift being less than 10 percent of the change produced by the presence of spray

- average flow loop pressure within 20 percent of the target

- a standard deviation of pressure data in the first $20 \mathrm{~s}$ of less than 10 percent of the target pressure 
- initial humidity (from the FIO instrument) greater than 80 percent RH

- leak flow rate variation of less than about 20 percent during the test

- orifice coefficients high enough in the first $20 \mathrm{~s}$ to indicate the absence of partial plugging

- orifice coefficients not exceeding unity.

For some test conditions, none of the tests conducted met all the criteria listed above (e.g., the initial RH was frequently less than 80 percent for tests in which FEG slurry was used). Test conditions for which one or more tests had to be used despite one or more of the criteria listed above not being met are noted in the discussion of results. For most test conditions, more than one run met the criteria equally well. In these cases, all runs were used to gain the advantage of replication. For comparison, in Phase I not all sets of replicates were included in the database, and for some test conditions only the first run (chronologically) was used, an arbitrary criterion that was set to avoid selection bias.

The release fraction predicted by the WTP model was also calculated for the selected experimental conditions. The WTP model is described in detail in Section 1.1 in Equations (1.1) through (1.9).

\subsubsection{Uncertainty}

There are several possible contributors to uncertainty (imprecision and bias) in the test and analysis methods. Bias could come from the following:

- physical processes that were not completely controlled by the test procedure (e.g., evaporation)

- misapplication of the exponential model (i.e., another model might have described the system better)

- Malvern Insitec-S measurements of aerosol concentration or droplet size (see Section 6.6)

- measurement of leak flow rate.

Imprecision, or scatter, could come from various causes. Examples include the following:

- variation in test conditions, compared to the target conditions

- variability produced by jet turbulence

- variability in instrument response.

Error bars representing uncertainty estimates are shown in most of the plots in this report. In Appendix B, the uncertainty of individual runs is displayed as error bars in plots of release fraction (as calculated by Equation (6.13)), based directly on the values of $G_{a, k}^{+}$and $G_{a, k}^{-}$. Only the fit uncertainty appears in these plots. The uncertainty of the leak flow rate is not included, nor is test-to-test variability.

For single runs plotted with error bars in Sections 6 or 7, error bars represent an approximate 95 percent confidence interval that combines the 95 percent confidence interval from the model fit to the run data (Equation 6.13) with an estimate of 95th percentile experimental variability. The latter estimate, which is 40 percent of the value of the release fraction or net generation rate, is based on a median value for two times the relative standard deviation of the release fractions in sets of replicate runs from Phase I. The value of two times the relative standard deviation was chosen because it is approximately equal to the 95 percent confidence interval for the data set. The error estimates for the model fit and experimental 
variability are combined by taking the square root of the sum of the squares of the two estimates. This results in greater uncertainties for individual data points whose fits had wider uncertainty.

This approach to finding the overall data uncertainty is a statistical approximation because the 95 percent confidence intervals on the fit are not exactly analogous to experimental standard deviations and because the standard deviations of the sets include a contribution from fit uncertainty, meaning that the fit uncertainty is double-counted. Therefore, the error bars do not represent rigorous uncertainties, but should give a reasonable idea of uncertainty in the data.

When the plotted data consists of the mean of the measurements of replicate data sets, the error bars are defined in a different way. The geometric mean of the replicates is calculated at each droplet size. The maximum and minimum values measured for the set of replicates are used as the top and bottom of the error bars. No mean or error bar is calculated for sizes where a value exists for only one of the runs. The geometric mean is

$$
\mu_{G}=\exp \left(\overline{\ln \left(R F_{l}\right)}\right)
$$

where $\overline{\ln \left(R F_{i}\right)}$ is the average of the logarithms of the release fractions.

\subsection{Test Method Considerations}

Certain elements of the test procedure or test conditions were investigated to identify the impact of various test conditions or to allow a comparison of Phase I and II results. These test conditions were investigated and included the focal length of the Malvern Insitec-S instrument lens (Section 6.4.1), the Malvern Insitec-S data collection rate (Section 6.4.2), the speed of the mixing fan (Section 6.4.3), the purge rate for the Malvern Insitec-S instrument used for in-spray measurements (Section 6.4.4), and initial humidity in the chamber (Section 6.4.5). Section 6.4.6 presents comparisons between Phase I and Phase II tests that were carried out at similar conditions.

One investigation that was not repeated was the effect of Malvern Insitec-S position, which was studied in Phase I (see Section 6.5.2 in Mahoney et al. 2013). The Phase I data demonstrated that there was not a significant effect of measurement position on the release fraction. Note that even though the measurement position in the small-scale chamber has no appreciable effect on release fraction, larger droplets (i.e., generally $\gtrsim 50 \mu \mathrm{m}$ ) are typically not detected in significant quantities in the small-scale chamber. This is primarily due to the proximity of the orifice to the splash wall, particularly when compared to the large-scale chamber.

\subsubsection{Malvern Insitec-S Lens Focal Length}

A $500 \mathrm{~mm}$ focal-length lens was used for most of the Phase II tests (and all of the post-shakedown tests), allowing for the measurement of larger size droplets than was possible with the $100 \mathrm{~mm}$ focal-length lens used in Phase I. As previously noted, the increase in the focal length of the lens changed the effective measurable size range from 0.5 to $200 \mu \mathrm{m}$ to 2.5 to $2500 \mu \mathrm{m}$. 
Figure 6.1, Figure 6.2, and Figure 6.3 show the cumulative release fractions from Phase II tests carried out with $100 \mathrm{~mm}$ and $500 \mathrm{~mm}$ lenses during shakedown testing. Shakedown testing used two different simulants (i.e., water and $\sim 30$ Pa clay slurry) and three different orifices (i.e., a round hole with a target diameter of $0.5 \mathrm{~mm}$, a round hole with a target diameter of $1 \mathrm{~mm}$, and a slot with target dimensions of $0.5 \times 5 \mathrm{~mm}$ ). In all three cases, the $500 \mathrm{~mm}$ lens gave higher release fractions in the size range between 10 and $100 \mu \mathrm{m}$, with release fractions obtained with the $500 \mathrm{~mm}$ lens being as much as two to three times the values obtained with the $100 \mathrm{~mm}$ lens. In general, the divergence between the 500 and $100 \mathrm{~mm}$ lens was greatest at droplet sizes near $10 \mu \mathrm{m}$ and the release fractions approached each other as droplet size increased. In the 10 to $100 \mu \mathrm{m}$ size range, the dependence of cumulative release fraction on size was similar for measurements made by the $500 \mathrm{~mm}$ and $100 \mathrm{~mm}$ lenses.

The release fractions given by the $500 \mathrm{~mm}$ lens decreased sharply for droplet sizes below about $4 \mu \mathrm{m}$, probably because the lower end of the measurable range at $2.5 \mu \mathrm{m}$ was being approached. In comparisons (not shown) made for the $\sim 30$ Pa clay slurry at 200 and $100 \mathrm{psig}$, using the $1 \mathrm{~mm}$ and $0.5 \times 5 \mathrm{~mm}$ orifices, the $500 \mathrm{~mm}$ lens gave cumulative release fractions greater than or equal to those given by the $100 \mathrm{~mm}$ lens.

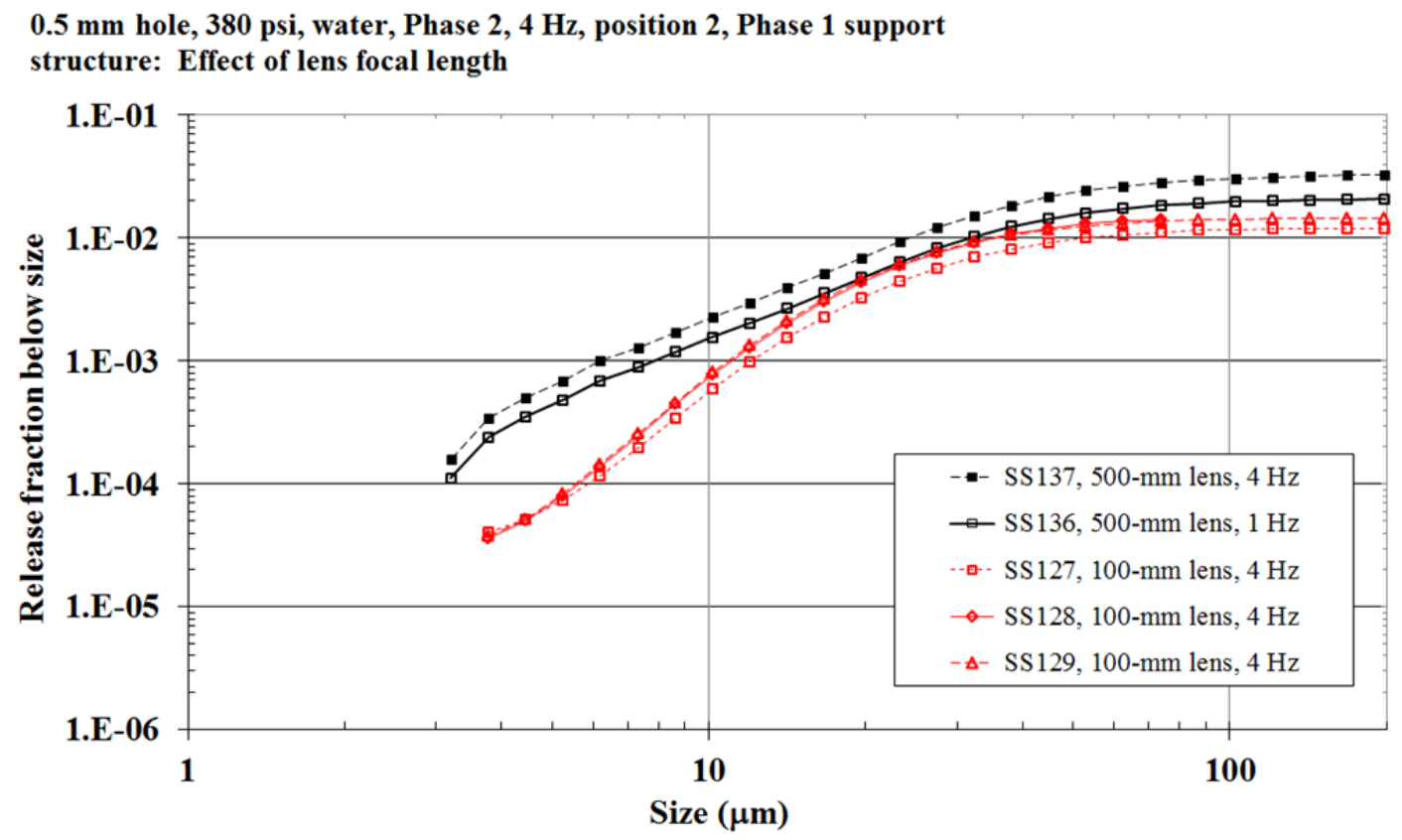

Figure 6.1. Effect of Lens Focal Length on Release Fractions for a $0.5 \mathrm{~mm}$ Hole, Water at 380 psig 
1 mm hole, 380 psi, clay@ 32 mPa s and 33 Pa, Phase 1 support structure, 500-mm vs 100-mm lens

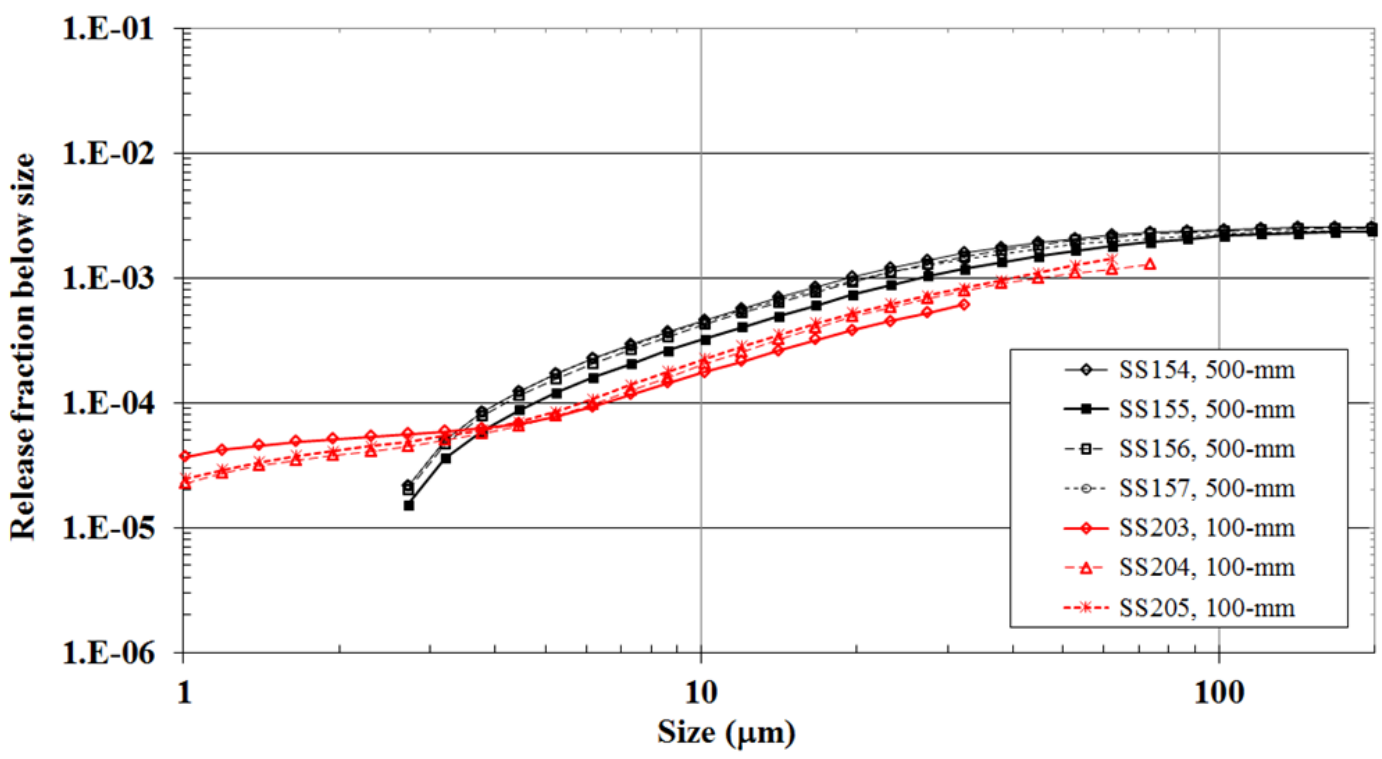

Figure 6.2. Effect of Lens Focal Length on Release Fractions for a $1 \mathrm{~mm}$ Hole, $\sim 30 \mathrm{~Pa}$ Clay Slurry at 380 psig

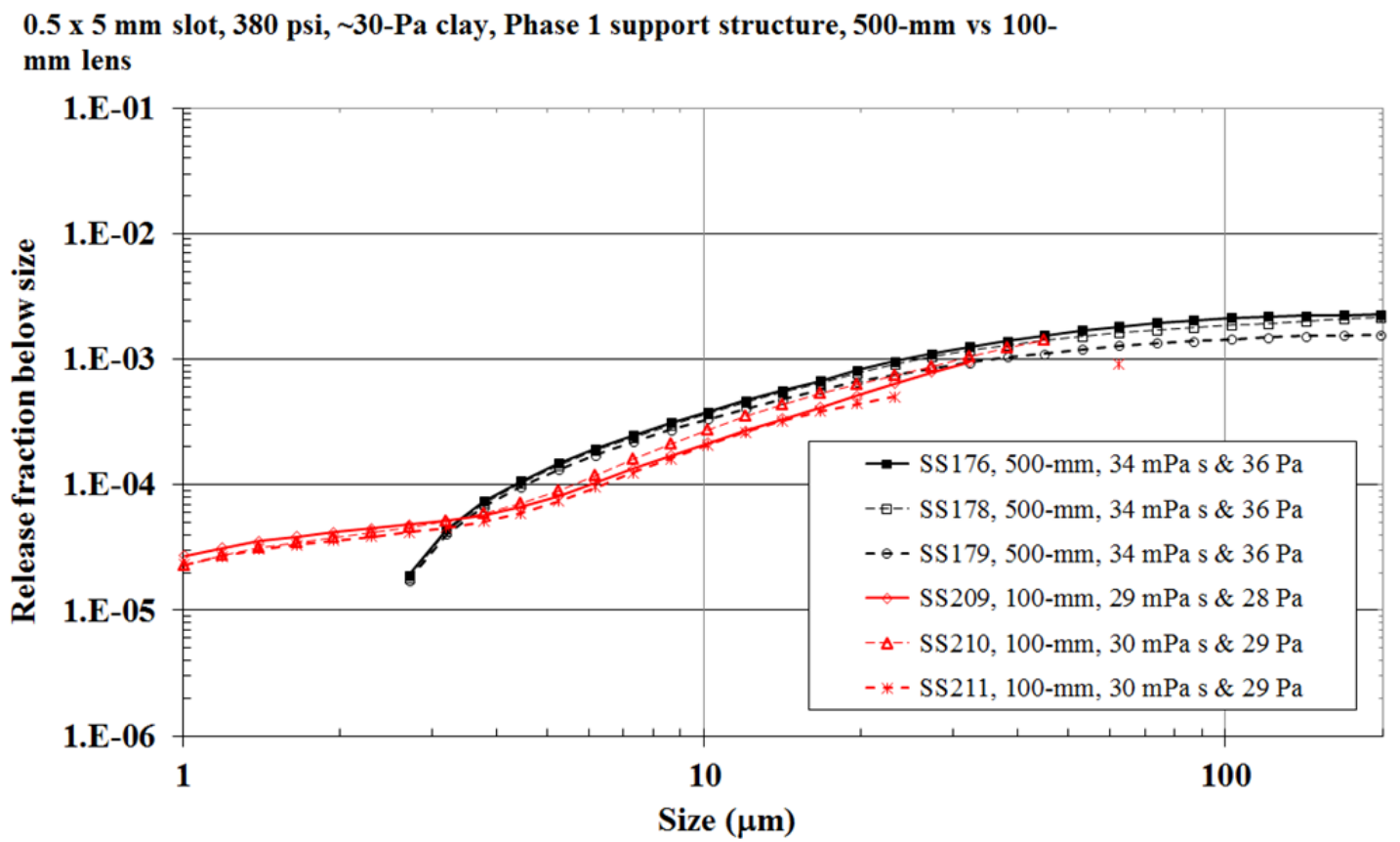

Figure 6.3. Effect of Lens Focal Length on Release Fractions for a $0.5 \times 5 \mathrm{~mm}$ Slot, $\sim 30$ Pa Clay Slurry at 380 psig 
The shakedown tests indicated that using the $500 \mathrm{~mm}$ lens in Phase II would not produce release fractions less conservative than those found in Phase I using the $100 \mathrm{~mm}$ lens. However, an important point to acknowledge is that, even at identical conditions, the aerosol data captured using the different lenses are not identical. The differences are primarily a function of the measurable size range. The $500 \mathrm{~mm}$ lens, which has a lower measurement limit of $2.5 \mu \mathrm{m}$, does not include droplets $<2.5 \mu \mathrm{m}$ in the PSD because the detector array is not configured to "see" droplets of this size. However, the laser is still obscured by these droplets, resulting in a reduction in transmission. This reduction in transmission is interpreted by the instrument as a higher concentration, which is further amplified by an estimate of the SMD too large relative to the "true" PSD of the material. As a result, the release fraction curves measured with a $500 \mathrm{~mm}$ lens have been shifted up across all droplet sizes compared to those measured with a $100 \mathrm{~mm}$ lens.

Although this was a known complication, the release fractions measured using the $500 \mathrm{~mm}$ lens data are consistently more conservative than those measured using the $100 \mathrm{~mm}$ lens. Because the aerosol data collected in this report supports safety and accident analyses (i.e., where conservatism is appropriate) and the $500 \mathrm{~mm}$ lens was necessary for the in-spray measurements, the $500 \mathrm{~mm}$ lens was used for all post-shakedown tests in Phase II to minimize instrument configuration changes.

\subsubsection{Malvern Insitec-S Data Collection Rate}

Because the $1 \mathrm{~Hz}$ data collection rate used in Phase I did not always capture the rapid initial rise in concentration for some orifices, most Phase II tests (and all post-shakedown tests) used a $4 \mathrm{~Hz}$ data collection rate. In particular, the refinement in data collection rate was expected to improve the determination of net generation rates for large droplets, where the rate often rises rapidly.

Figure 6.4 and Figure 6.5 show the release fractions determined by Phase II shakedown tests performed with 1- and $4 \mathrm{~Hz}$ data collection rates. In both cases, the simulant was water at $380 \mathrm{psig}$. Two different orifices were used, a $0.5 \mathrm{~mm}$ round hole and a $1 \mathrm{~mm}$ round hole. The release fractions determined using the different data collection rates are not distinguishable within the repeatability of the tests. 
$0.5 \mathrm{~mm}$ hole, $380 \mathrm{psi}$, water, Phase 2, 500-mm lens, position 2, Phase 1

support structure with no bubble cover: Effect of data collection rate

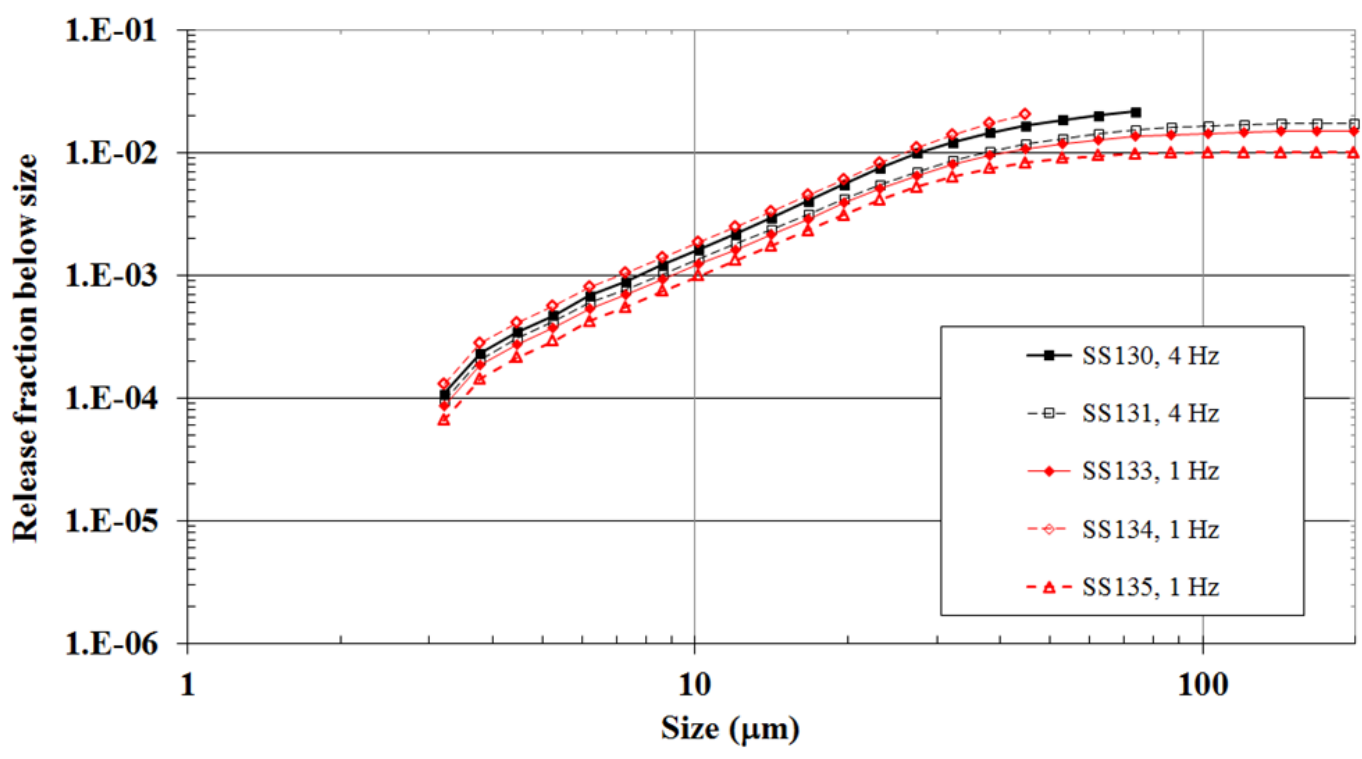

Figure 6.4. Effect of Malvern Insitec-S Data Collection Rate on Release Fractions for a $0.5 \mathrm{~mm}$ Hole, Water at $380 \mathrm{psig}$. The $500 \mathrm{~mm}$ lens was used.

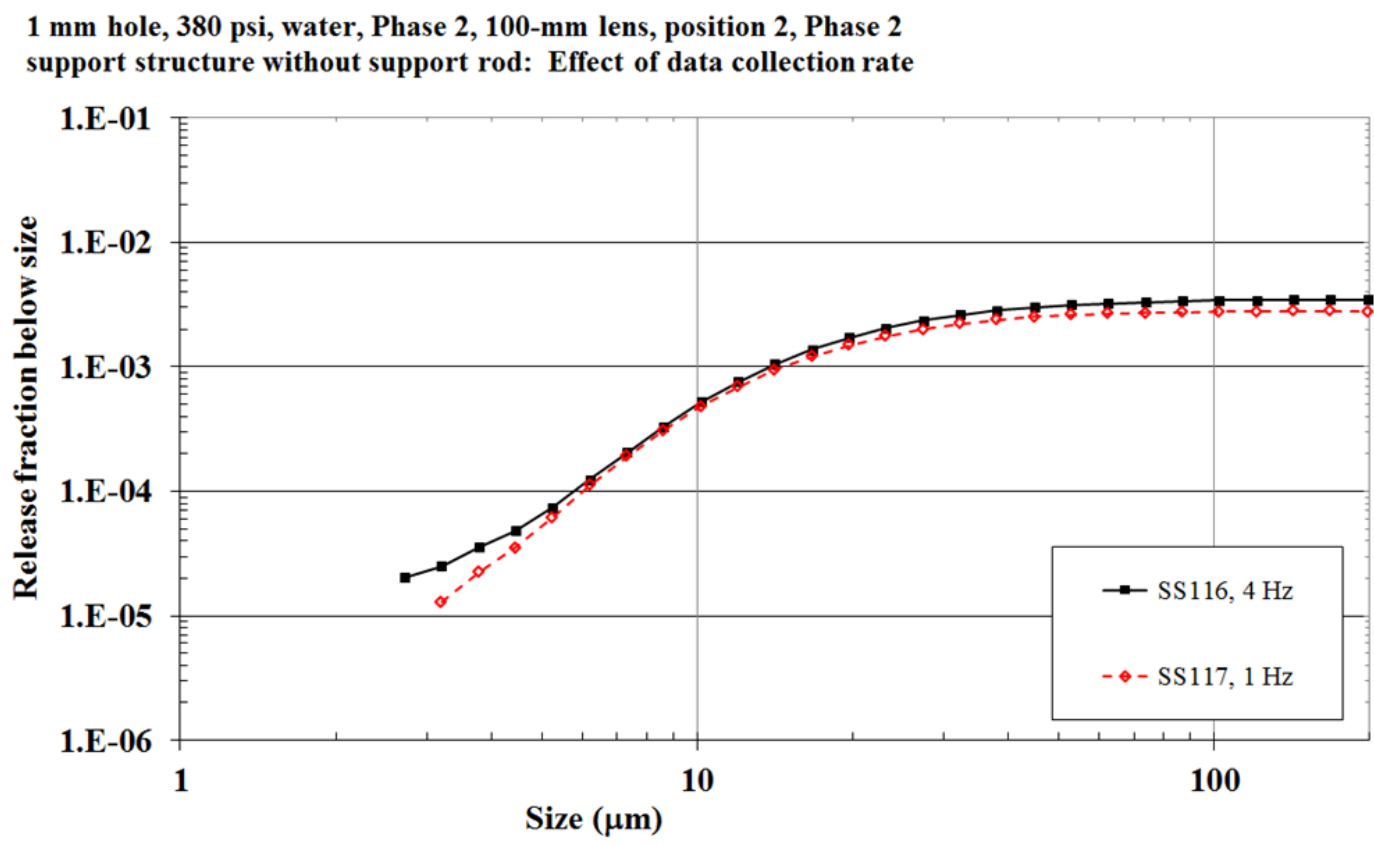

Figure 6.5. Effect of Malvern Insitec-S Data Collection Rate on Release Fractions for a $1 \mathrm{~mm}$ Hole, Water at $380 \mathrm{psig}$. The $100 \mathrm{~mm}$ lens was used.

\subsubsection{Mixing Fan}

Because it was important for the concentration in the enclosure to be reasonably uniform, at least outside the small region occupied by the jet itself, a mixing fan was installed in the enclosure and tested 
at different fan speeds (identified by the voltage that was set at the fan control). ${ }^{1}$ Tests of the effect of the mixing fan on release fractions were conducted in Phase I shakedown tests and repeated in Phase II shakedown tests.

Figure 6.6 shows the effect of different fan speeds on Phase II release fractions, using a 380 psig water spray from a $0.5 \mu \mathrm{m}$ hole (target size). The lowest fan speed (i.e., fan off) gave distinguishably lower release fractions over most of the droplet size range. The next lowest fan speed, $3 \mathrm{~V}$, gave release fractions that were not distinguishable from those at $6 \mathrm{~V}$ (within the repeatability of the 6-V tests). The highest fan speed, $11 \mathrm{~V}$, produced release fractions that were distinguishably higher than those from the 6-V speed. At droplet sizes between 30 and $60 \mu \mathrm{m}$, the release fractions measured with the 11-V fan speed were probably significantly higher than those at $6 \mathrm{~V}$. However, owing to data noise, no good fits were obtained for droplet sizes above $65 \mu \mathrm{m}$.

As observed in the Phase I tests (Section 6.5.3 of Mahoney et al. (2013)), the 11-V speed allowed more conservative release fractions (and probably better mixing); however, it also produced noisier data, for which the adjusted $\mathrm{R}^{2}$ of the fits were less than 0.5 . This caused the loss of some release fraction measurements at larger droplet sizes. Thus, the remaining Phase II testing used a default fan speed of $6 \mathrm{~V}$ for the sake of consistency with Phase I.

$0.5 \mathrm{~mm}$ hole, $380 \mathrm{psi}$, water, Phase 2, 100-mm lens, $4 \mathrm{~Hz}$, position 2, complete

Phase 2 support structure: Effect of mixing fan speed

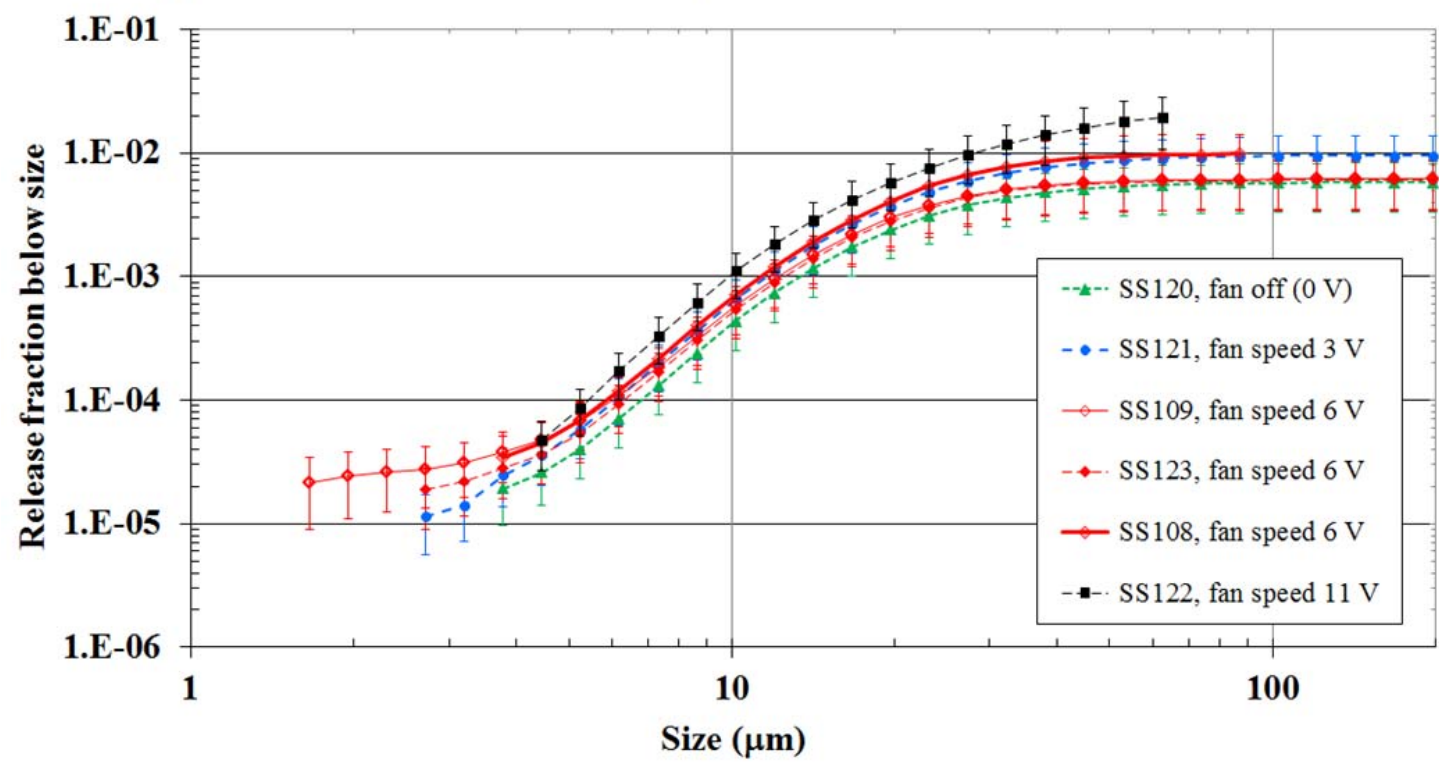

Figure 6.6. Effect of Fan Speed on Release Fractions for a $0.5 \mathrm{~mm}$ Hole, Water at $380 \mathrm{psig}$. The $100 \mathrm{~mm}$ lens was used. Error bars show an approximate 95 percent confidence interval for each test including model fit and experimental variability.

\footnotetext{
${ }^{1}$ The volumetric flow rate of the fan was not measured. The fan was operated at set voltages of $0,3,6$ and $11 \mathrm{~V}$.
} 


\subsubsection{Malvern Insitec-S Instrument Purge Rate}

As noted in Section 4.1.4, the Malvern Insitec-S instrument directs a low-flow stream of purge air to each of the windows to keep them clear of condensation and other contamination. Droplets that deposit on either the laser or detector window bias the measurement and require manual cleaning to mitigate. However, because the purge air sweeps across the surface of the windows, it is conceivable that an excessive purge air flow rate could dilute the aerosol concentration near the window and disrupt the measurement.

The purge rate for the Malvern Insitec-S instrument was not specifically determined in Phase II for the in-chamber configuration (Position 2). No changes to the Malvern Insitec-S instrument configuration warranted a change to the purge air flow rate; thus, the Phase II used same flow rate as Phase I (i.e., 1.2 SCFH per window). This flow rate has been demonstrated to strike a balance between keeping the windows clean while not adversely affecting the aerosol results (see Section 6.5.4 in Mahoney et al. 2013).

Conversely, the purge rate for the in-spray configuration was not assessed in Phase I testing (which included only a limited number of in-spray tests). In-spray measurement was expected to require higher purge air flow rates because the local concentration of aerosol is much greater and the Malvern Insitec-S measurement zone is in proximity to a chaotic, turbulent jet of fluid. To this end, higher capacity rotameters were installed for in-spray tests. During the shakedown testing performed with water, in-spray tests were conducted with the $0.5 \mathrm{~mm}$ hole at $380 \mathrm{psig}$. The purge air flow rate was set at 20,40 , and $60 \mathrm{SCFH}$ per window in consecutive tests. The aerosol data was observed to be unaffected (via real-time comparisons) by changes in the purge air flow rate. Ultimately, after a compressor failure and repair, the maximum achievable flow rate was approximately 35-40 SCFH per window. Because the purge air flow rate did not have any appreciable effect on the data in this range, the maximum achievable purge air flow rate was used for all in-spray (Position 4; see Figure 4.7) tests.

\subsubsection{Humidity}

In the standard test procedure used for Phase I and Phase II tests, a pre-spray was carried out before the first test of a day or before any test that was more than $3 \mathrm{hr}$ after the last preceding test. The pre-spray was followed by 2 min of evacuation to remove residual droplets. The pre-spray had two purposes: to provide consistent wall-wetting conditions, because droplet capture is affected by wall wetness, and to increase the humidity in the chamber and, correspondingly, reduce the effect of evaporation during the initial portion of the spray.

No pre-spray was needed or performed for many of the tests, because on any given test day a series of tests were conducted and the gaps between them were often less than $0.5 \mathrm{hr}$. The pre-spray could be omitted, subject to the judgment of the lead test engineer. During Phase II, it was omitted before some tests with non-Newtonian simulant to decrease the chance of plugging.

Because the test procedure did not explicitly control initial humidity (though steps were taken to keep the chamber air as saturated as possible) and because the effect of evaporation was not known, there was concern about a possible lack of conservatism in Phase II test results (particularly for droplets at or near the $10 \mu \mathrm{m}$ size). To address this concern, several tests were carried out with different initial humidities. 
A low spray rate, from a $0.5 \mathrm{~mm}$ orifice at $380 \mathrm{psig}$, was used to make any effect of evaporation more visible.

Figure 6.7 and Figure 6.8 show the release fractions determined at 380 and 100 psig, respectively, over a range of initial humidities from about 50 percent RH to near 100 percent RH. It must be noted at this point that the humidity instrument used in all Phase II small-scale tests was not calibrated to NQA-1 standards (although the sensor had been procured with a factory calibration), and its readings should be considered FIO. In addition, the constraint on the minimum fit $\mathrm{R}^{2}$ was taken off for the 100 psig plot (Figure 6.8) to allow the trend in the release fraction data to be shown despite the poor model fits caused by noisy data. Given these caveats, the two figures show the release fractions consistently increasing as initial humidity increases.

Both figures show some tendency for the release fractions of small droplets to decrease with humidity by a larger factor than the release fractions of large droplets. However, in general, the effect of humidity is to change the release fractions by nearly the same factor at all droplet sizes. Considered from a single-droplet perspective, small droplets might have been expected to be more affected because of their larger area-to-volume ratio. However, shrinkage of larger droplets into smaller droplets also occurs, complicating the trend of release fraction change with droplet size.

Figure 6.9 shows an example of the way in which initial humidity affects the initial change in concentration for the 380 psig tests. The figure includes both model fit curves (black lines) and data (colored symbols) for the concentration of $10-\mu \mathrm{m}$ droplets versus time. The model fit curves are shown only between 0 and $20 \mathrm{~s}$, the model-fitting period.

At the high end of the range of initial humidity, RH of 96 percent, the fit curve and the data both start at $0 \mathrm{~s}$. At the low end of the range, $\mathrm{RH}$ of 54 percent, the fit curve starts at $0 \mathrm{~s}$ but shows no non-zero concentration data until about $4 \mathrm{~s}$. Intermediate humidities show intermediate effects in the first few seconds: non-zero concentrations appear near $0 \mathrm{~s}$, but remain low and nearly constant for about $2 \mathrm{~s}$ rather than showing an ongoing rise. The delays and flattenings are evidence of a physical mechanism that is not closely fitted by the exponential model. Figure 6.10, the same type of plot for 102- $\mu \mathrm{m}$ droplets from the 380 psig spray, shows less initial flattening of the concentration rise.

Both Figure 6.9 and Figure 6.10 show that the effect of low initial humidity is not confined to the first few seconds, but continues throughout the 20 -s fitting period. This can be seen in the differences in the $10-\mu \mathrm{m}$ droplet concentrations at $50 \mathrm{~s}$, which is the end of the data plot. Equilibrium has not been reached at the two lowest humidities.

The data indicate that release fractions measured under conditions of subsaturated humidity are biased low. Figure 6.11 and Figure 6.12 show release fraction data plotted versus initial RH for the 380 and 100 psig sets of tests, respectively. The lines in the plots represent the Excel fits of exponential functions to the data at each droplet size. If the humidity data were NQA-1, the lines would provide a means to extrapolate release fractions measured at low known $\mathrm{RH}$ to the value that would have been measured at 100 percent RH. 
$0.5 \mathrm{~mm}$ hole, $380 \mathrm{psi}$, water, Phase 2, 500-mm lens, $4 \mathrm{~Hz}$, position 2, Phase 1 support structure: Effect of initial humidity

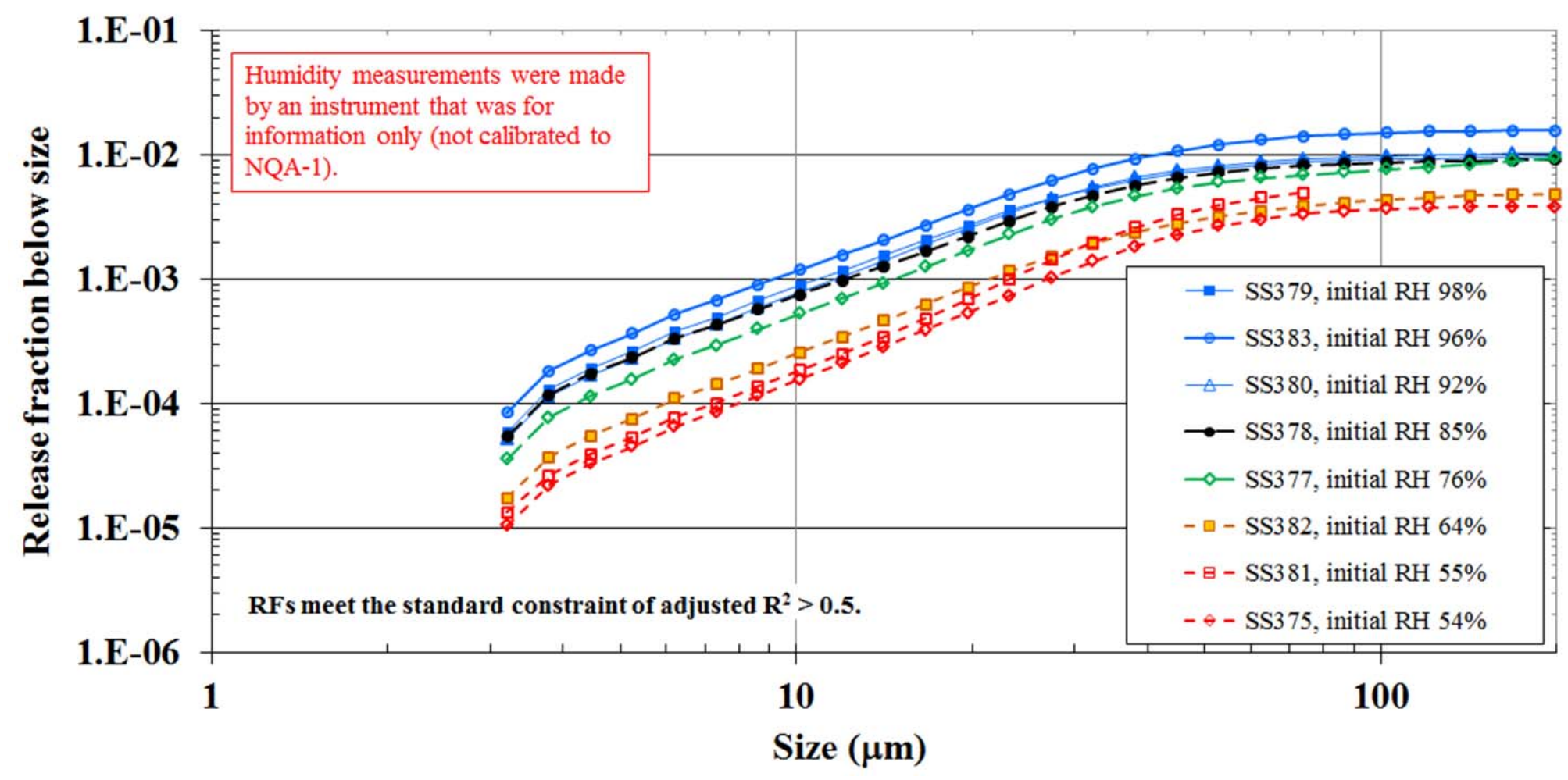

Figure 6.7. Effect of Initial RH on Release Fractions for a $0.5 \mathrm{~mm}$ Hole, Water at $380 \mathrm{psig}$ 
$0.5 \mathrm{~mm}$ hole, $100 \mathrm{psi}$, water, Phase 2, 500-mm lens, position 2, Phase 1 support structure: Effect of initial humidity

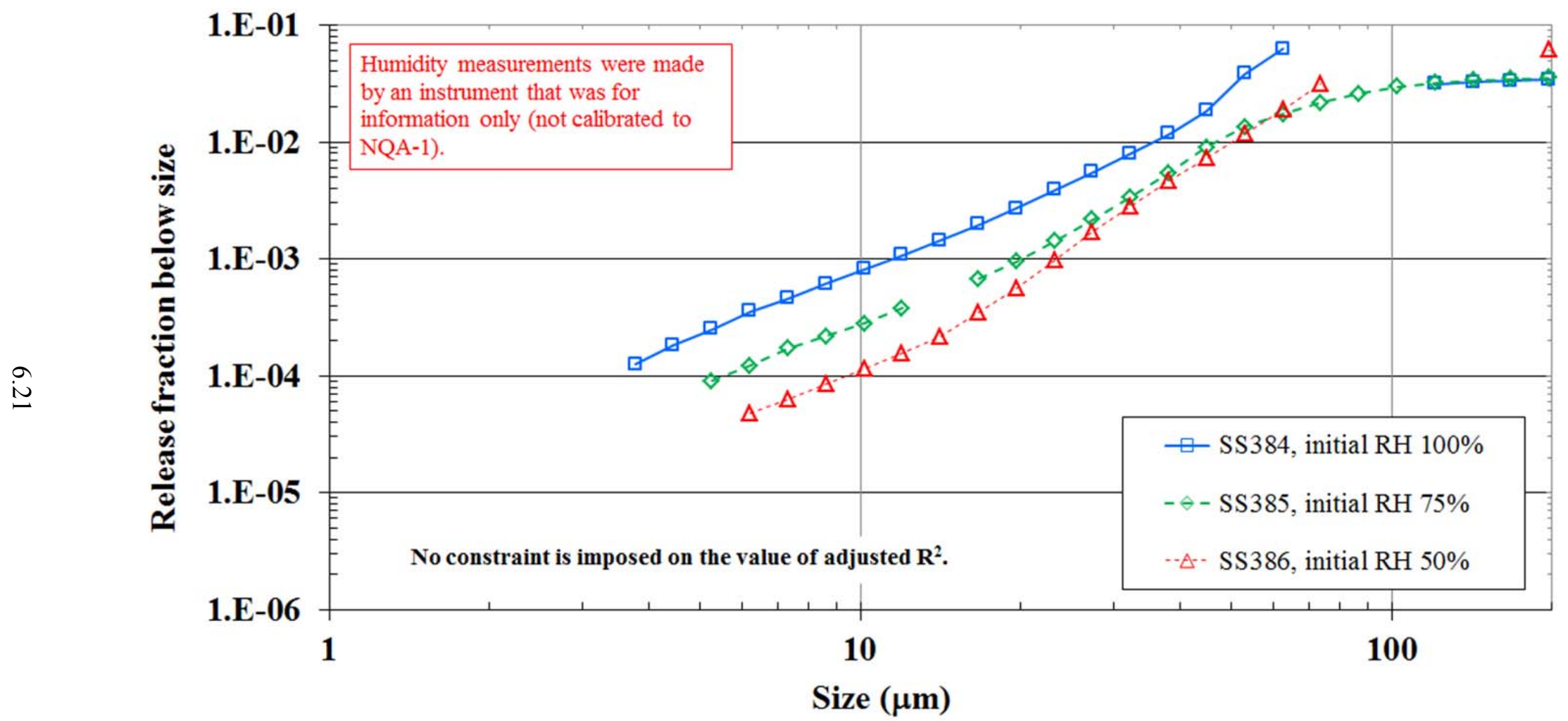

Figure 6.8. Effect of Initial RH on Release Fractions for a $0.5 \mathrm{~mm}$ Hole, Water at $100 \mathrm{psig}$ 


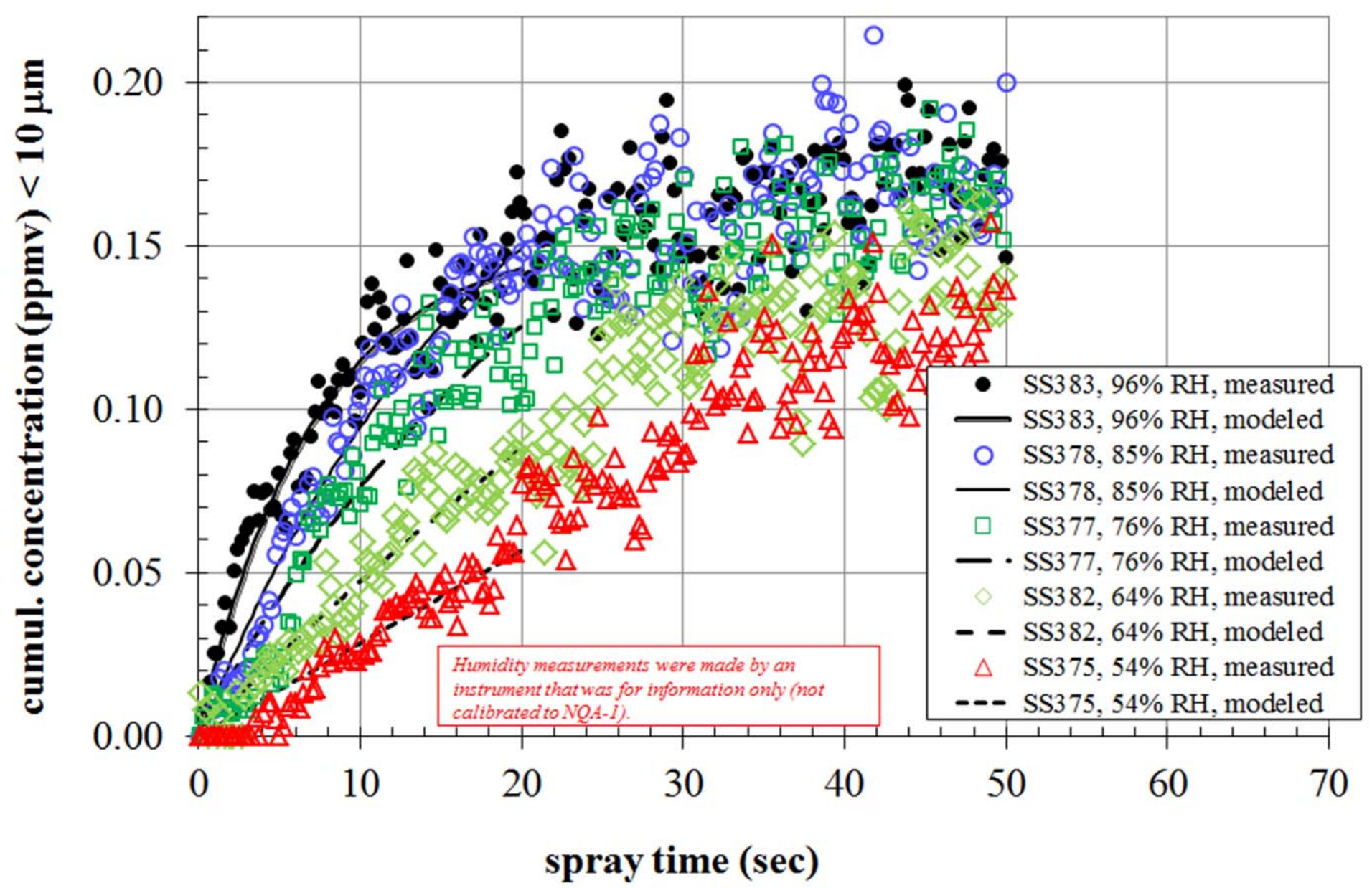

Figure 6.9. Effect of Initial RH on Concentrations of $10-\mu \mathrm{m}$ Droplets for a $0.5 \mathrm{~mm}$ Hole, Water at $380 \mathrm{psig}$ 


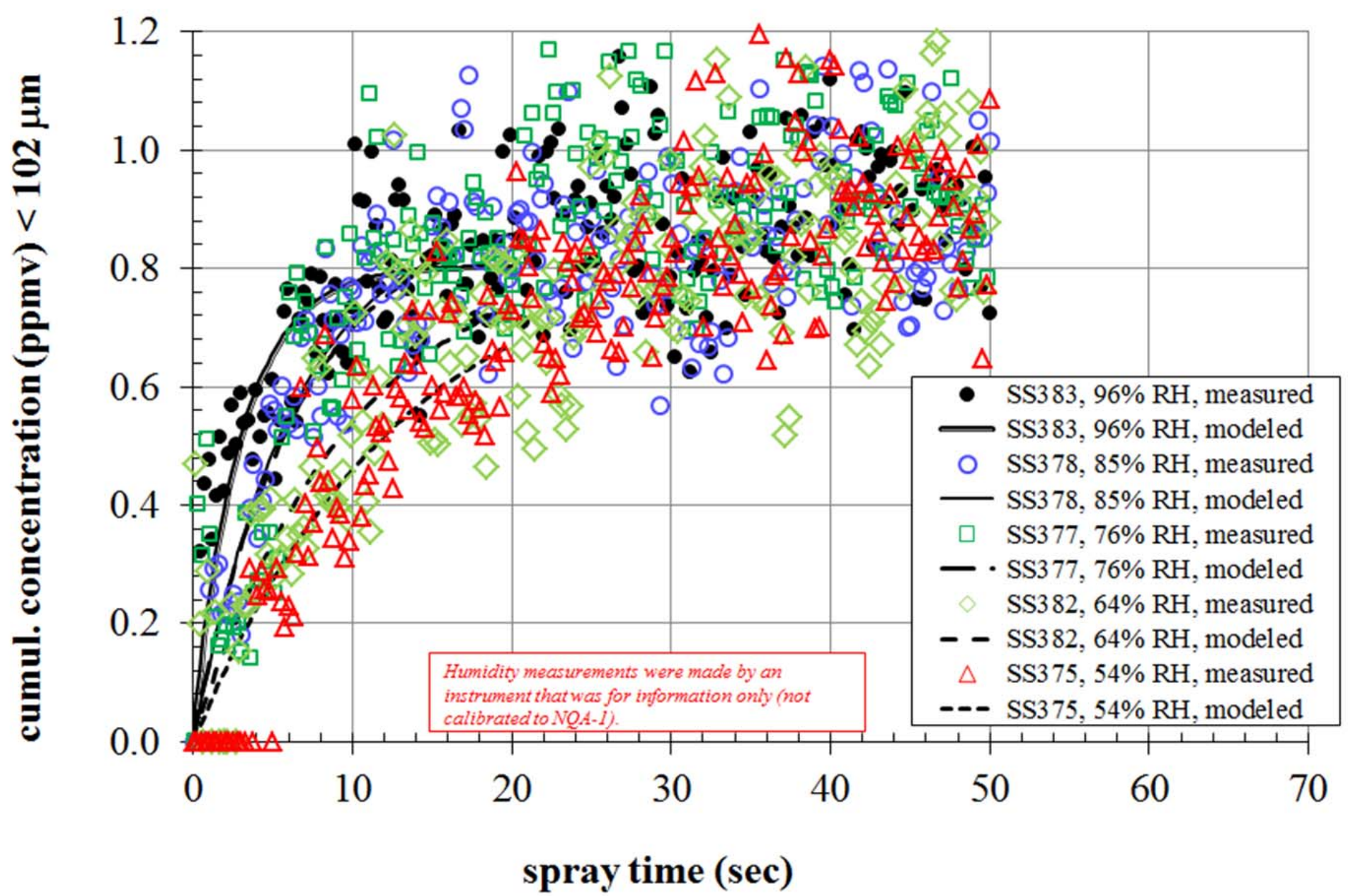

Figure 6.10. Effect of Initial RH on Concentrations of $102-\mu \mathrm{m}$ Droplets for a $0.5 \mathrm{~mm}$ Hole, Water at $380 \mathrm{psig}$ 


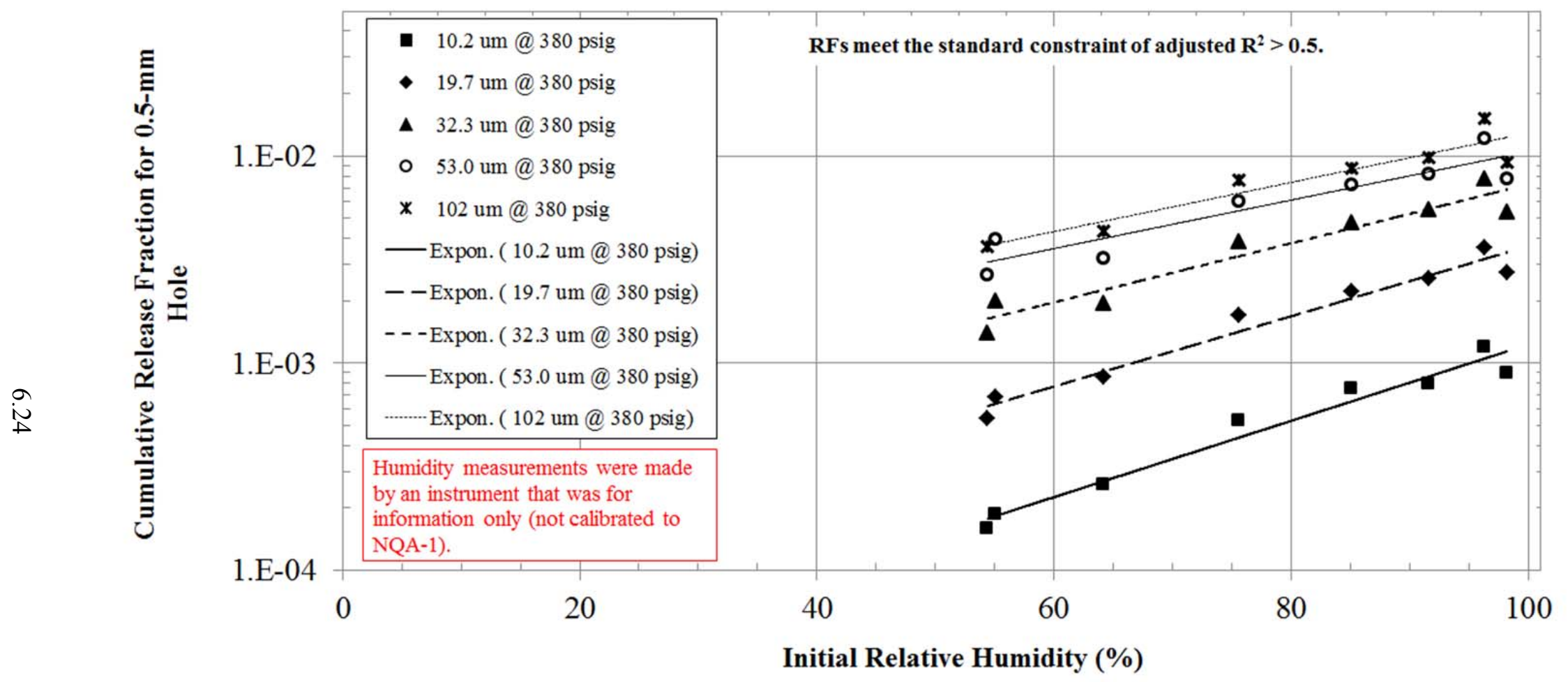

Figure 6.11. Trends of Release Fraction with Initial Humidity for a $0.5 \mathrm{~mm}$ Hole, Water at $380 \mathrm{psig}$ 


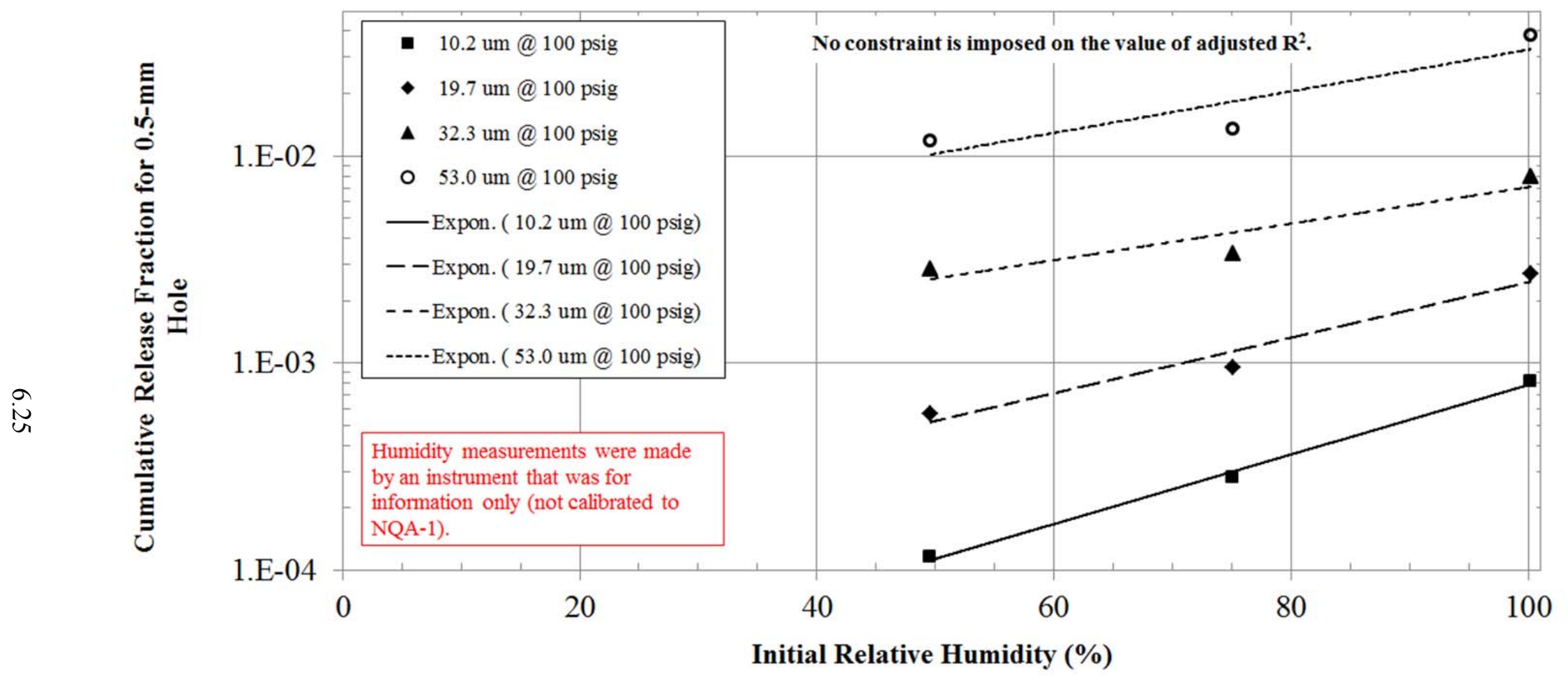

Figure 6.12. Trends of Release Fraction with Initial Humidity for a $0.5 \mathrm{~mm}$ Hole, Water at $100 \mathrm{psig}$ 
Based on the data in Figure 6.11 (380 psig), at $<10 \mu \mathrm{m}$ the release fraction at 98 percent initial $\mathrm{RH}$ was $9.0 \times 10^{-4}$, that at 85 percent initial RH was $7.5 \times 10^{-4}$, and that at 76 percent initial RH was $5.3 \times 10^{-4}$. Using these figures to interpolate, the decrease in release fraction from 100 percent RH to 80 percent RH (release fraction about $6.4 \times 10^{-4}$ ) was less than a factor of two, about the same magnitude as the test-to-test variability (and possibly being a cause of part of the variability). The low bias is more substantial when the initial RH is less than about 75 percent; there is almost an order-of-magnitude decrease in the release fraction of $10-\mu \mathrm{m}$ droplets at 54 percent RH, compared to 98 percent RH. Using the data to extrapolate to 100 percent initial $\mathrm{RH}$ and interpolate to 80 percent initial $\mathrm{RH}$, the decrease in release fraction is approximately a factor of two across the range of typical initial RHs.

The initial RH in Phase II testing depended on the plugging tendency of the simulant, because a propensity for plugging was often countered by omitting the standard pre-spray. Of the in-chamber tests with water, clay, and $27 \mathrm{wt} \%$ STR for which initial RH data were recorded, only one had initial humidity below 80 percent RH. On the other hand, of the $52 \mathrm{FEG}$ in-chamber tests for which initial RH data were recorded, 32 had initial RH less than 80 percent and nine had initial RH less than 70 percent. Initial humidities in the range of 68 to 75 percent $\mathrm{RH}$ were also present in three of the nine tests that used the STR/Mo simulant. Because the effect of initial RH on FEG release fractions was not measured as it was for water, it is difficult to estimate the impact. FEG (or other solids-containing simulants) will not be affected by evaporation in the same way as a liquid-only simulant because there is a fraction of solid particles whose size, when the liquid has evaporated away, represents a lower bound with respect to evaporation. For FEG in particular, the fraction of solid particles or agglomerates between 10-100 $\mu \mathrm{m}$ (the range of interest) is significant. Large-scale testing with 6 Pa clay indicated that the increase in release fraction when correcting the data to saturation (100 percent $\mathrm{RH})$ is smaller in magnitude compared to water (see Appendix A in Daniel et al. 2013). ${ }^{1}$ Extrapolating the behavior from these limited data sets has uncertainty, but suggests that the FEG release fractions, when corrected for initial RH, would be bounded by the magnitude of the water correction for larger droplets and the uncorrected FEG release fraction data for smaller droplets. Additional humidity testing is required to further resolve this uncertainty.

Because the humidity instrument data were FIO, and because the majority of the tests that had low initial humidity were performed with slurries rather than the water with which the humidity effect tests had been carried out, no attempt was made to correct the bias introduced by evaporation into small-scale release fraction data. In the remainder of this report, figure captions will be used to specify those tests where initial RH was less than 80 percent.

\subsubsection{Comparison of Phase II to Phase I}

As part of the Phase II system confirmation, release fraction results of Phase II tests were compared to those of Phase I tests at similar target conditions to check whether there were gross differences. This was done because of the following changes made in Phase II testing:

- an improved method for pressure control during the initial fit period

- a target header flow rate of $11.4 \mathrm{gpm}$ instead of $10 \mathrm{gpm}$

\footnotetext{
${ }^{1}$ For example, based on water test data, the maximum humidity bias correction factors are estimated to be 1.71, 1.51 , and 1.38 for 10,30 , and $100 \mu \mathrm{m}$ droplets, respectively. The $6 \mathrm{~Pa}$ clay was estimated to have maximum humidity bias correction factors of $0.92,1.24$, and 1.26 for the same droplet sizes.
} 
- a Malvern Insitec-S lens focal length of $500 \mathrm{~mm}$ instead of $100 \mathrm{~mm}$ (the effect was discussed in Section 6.4.1)

- modification of the chamber to make room for the longer lens assembly without adding to the net chamber volume

- determination of the start time from valve position

- determination of the leak flow rate from a linear fit to a weight-data time series instead of start and end weights

- faster data collection rates, $4 \mathrm{~Hz}$ instead of $1 \mathrm{~Hz}$ for the Malvern Insitec-S instrument and $2 \mathrm{~Hz}$ instead of $1 \mathrm{~Hz}$ for the process data logger (the lack of effect of the Insitec-S data collection rate was discussed in Section 6.4.2).

The effects of the system, procedure, and instrumentation changes that did not involve the Malvern Insitec-S instrument are shown in Figure 6.13, which presents the results of a Phase I/Phase II comparison for water at $380 \mathrm{psig}$ using a round orifice with target dimension of $0.5 \mathrm{~mm}$. In general, the release fractions from the two phases of testing are about equal.

$0.5 \mathrm{~mm}$ hole, $380 \mathrm{psi}$, water

Phase I, 100-mm lens, $1 \mathrm{~Hz}, 10 \mathrm{gpm}$ in header

Phase II, 100-mm lens, 4 Hz, 11.4 gpm in header, Phase I support structure

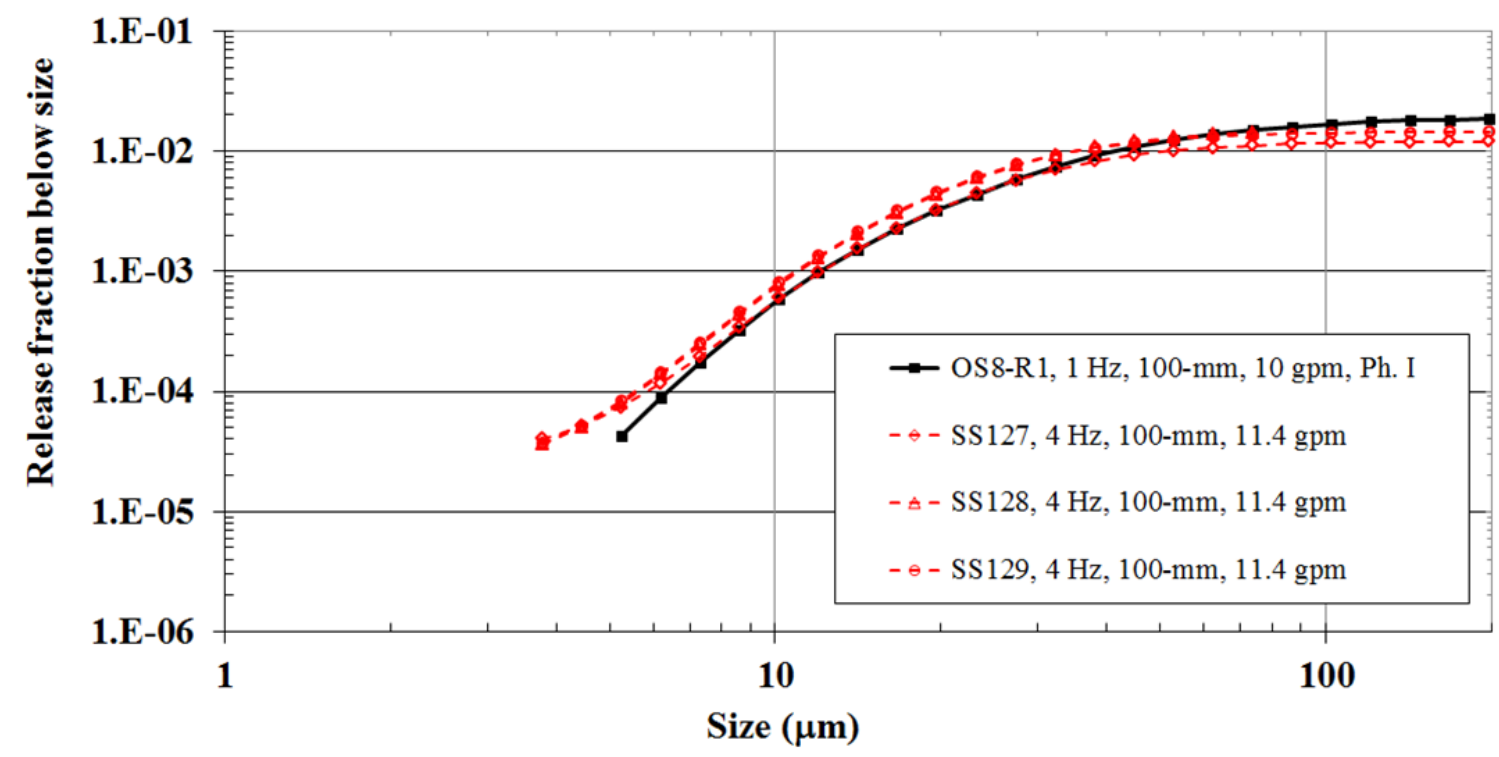

Figure 6.13. Comparison of Phase I and Phase II Results for a $0.5 \mathrm{~mm}$ Round Hole, Water at 380 psig

It was concluded that, if the changes in the Malvern Insitec-S lens focal length were excluded, the Phase II system, procedure, and instrumentation produced results similar to those from Phase I.

\subsection{Repeatability}

In Phase II, replicate tests were run and analyzed for a large number of test conditions. This section contains plots that are examples of the repeatability achieved at different test conditions. Not all test 
conditions are shown. For each simulant, tests that represent the best and worst repeatability are shown. The individual runs are plotted along with the geometric mean of the runs and error bars representing the maximum-minimum interval based on the data from individual runs. In cases where the initial RH (FIO measurement) was less than 80 percent, the lower values are noted in the captions. The captions and titles show the target rheological properties, while the legends show the measured properties, which are approximations for FEG owing to the history-dependence of the properties.

For water, as with most simulants, six test conditions provided replicate runs: i.e., three test pressures $(100,200$, and $380 \mathrm{psig}$ ) used with two orifices (target dimensions of $1 \mathrm{~mm}$ round and $0.5 \times 5 \mathrm{~mm}$ slot). Figure 6.14 shows the two sets of tests that bracket the repeatability for water tests. The $0.5 \times 5 \mathrm{~mm}$ slot at 100 psig gave the best repeatability; data from the three tests were within a factor of two or less. For the case with worst repeatability, the $1 \mathrm{~mm}$ round hole at $380 \mathrm{psig}$, the two tests were within a factor of two of each other. The only test condition for which there were more than two tests for the water simulant was the $0.5 \times 5 \mathrm{~mm}$ slot at $100 \mathrm{psig}$.

Figure 6.15 shows the best and worst repeatability for the tests using $\sim 6 \mathrm{~Pa}$ clay. All of the test conditions for this simulant had three tests each. The repeatability did not have any apparent dependence on orifice or pressure, however, as the figure shows, variability tended to increase for droplet sizes greater than $20 \mu \mathrm{m}$. Similar repeatability behavior was seen for the $\sim 30$ Pa clay tests, shown in Figure 6.16. For this simulant, there were three test conditions (not shown) where there were four tests apiece and three where there were three tests apiece. The maximum spread between tests remains about a factor of two.

The FEG simulant test repeatability is shown in Figure $6.17(\sim 6 \mathrm{~Pa})$ and Figure $6.18(\sim 30 \mathrm{~Pa})$. Because of the difficult behavior of the FEG simulant, obtaining more than two tests for a test condition was not always possible. Only one test was successful in each of the following cases: $0.5 \times 5 \mathrm{~mm} /$ 200 psig/6 Pa FEG and $1 \mathrm{~mm} / 380$ psig/30 Pa FEG. The spread between two tests could be a factor of three or four. For this pair of simulants, repeatability was generally better at 380 psig than at the lower pressures.

Figure 6.19 shows the best and worst repeatability for the tests of concentration in Newtonian slurry, $27 \mathrm{wt} \%$ STR. Most of the test conditions had three tests; the exception was the $1 \mathrm{~mm}$ hole at $380 \mathrm{psig}$, for which there were only two tests. The spread between any two tests was consistently less than a factor of two.

The dense particle tests used two simulants, $19 \mathrm{wt} \%$ STR plus $0.4 \mathrm{wt} \%$ Mo (i.e., the DST simulant) and $0.4 \mathrm{wt} \%$ Mo in water (i.e., the DPW simulant). Tests were run only at $380 \mathrm{psig}$, so only two tests were run per simulant. Both of the tests with $19 \mathrm{wt} \%$ STR $0.4 \mathrm{wt} \%$ Mo are shown in Figure 6.20. Most tests were within a factor of less than two of each other; however, one test for the slot orifice varied by more than a factor of three from the other three tests. Figure 6.21 shows the one test of $0.4 \mathrm{wt} \% \mathrm{Mo}$ in water $^{1}$ for which replicates were obtained. The repeatability was the same as for water (see Figure 6.14).

All simulants except FEG provided repeatability within a factor of two. In general, the FEG simulant, with its unusual thickening properties, showed higher variability from test to test.

\footnotetext{
${ }^{1}$ The target Mo concentration was $1 \mathrm{wt} \%$, but settling in the system decreased the in-spray concentration to $0.4 \mathrm{wt} \%$.
} 
$0.5 \times 5$ mm slot, 100 psi, Phase 2, 500-mm lens, position 2

Water

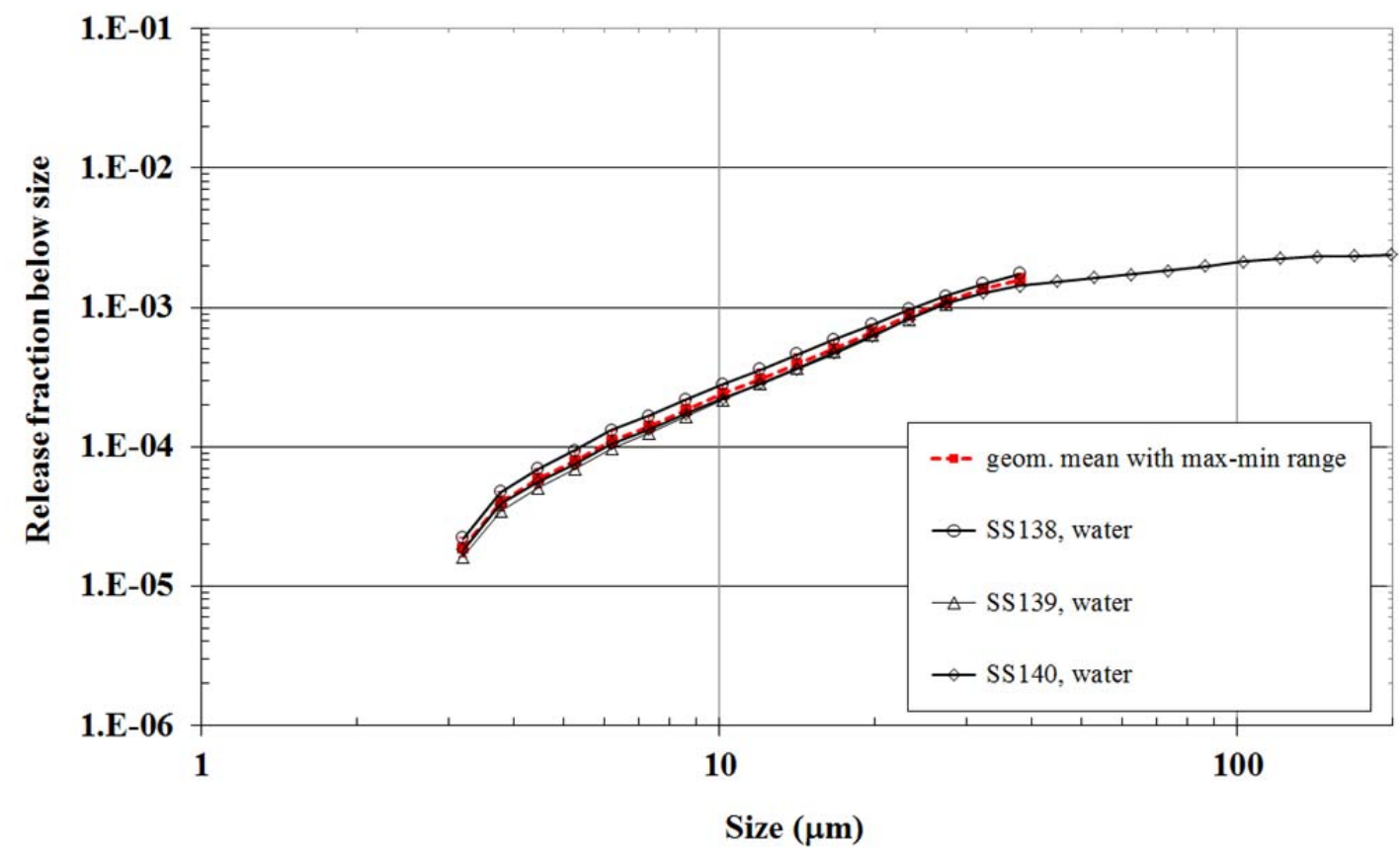

$1 \mathrm{~mm}$ hole, $380 \mathrm{psi}$, Phase 2, 500-mm lens, position 2

Water

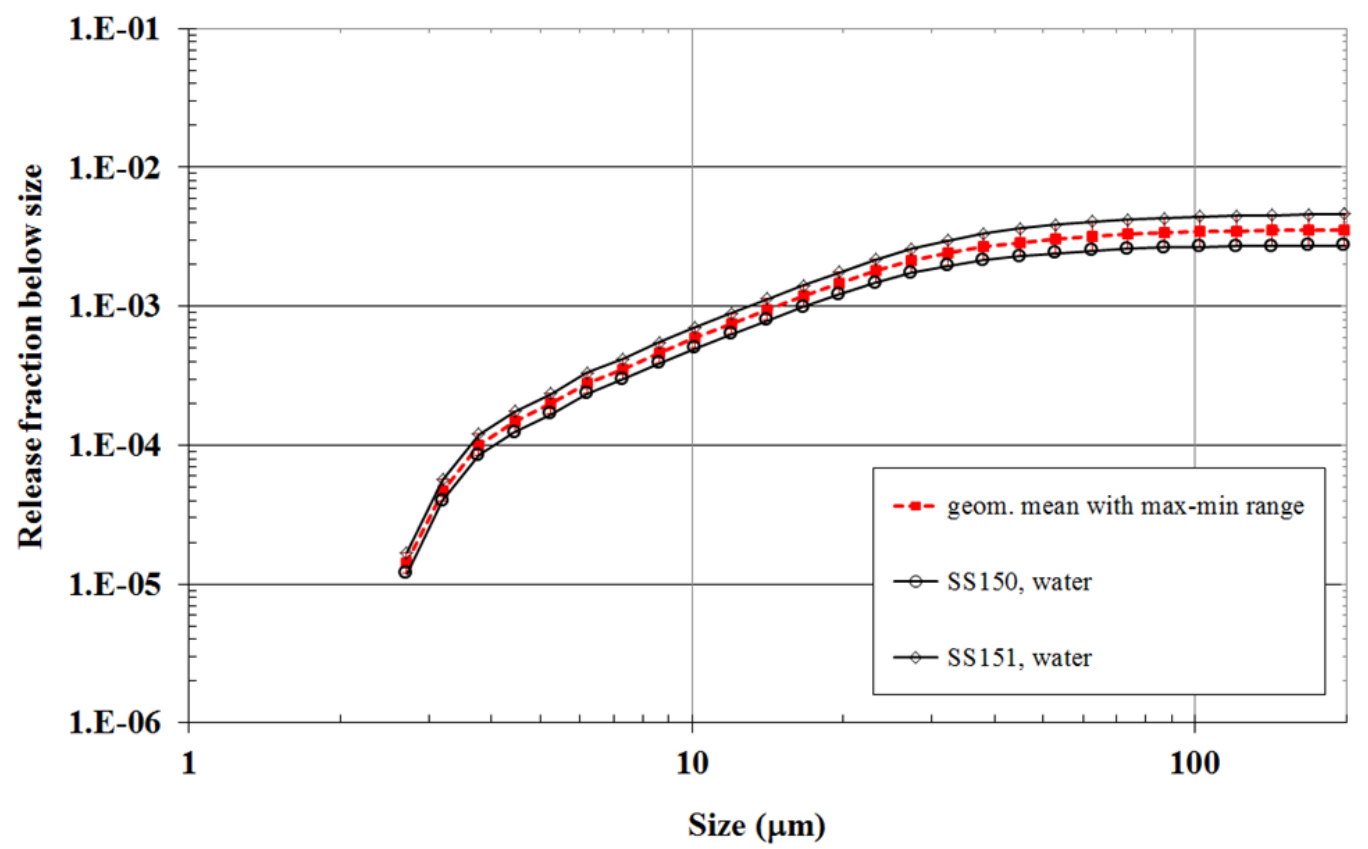

Figure 6.14. Best and Worst Repeatability Observed for Water Tests. Error bars represent the maximum and minimum measurements. 
$1 \mathrm{~mm}$ hole, $100 \mathrm{psi}$, Phase 2, 500-mm lens, position 2

$\sim$ 6-Pa/6-mPa s clay

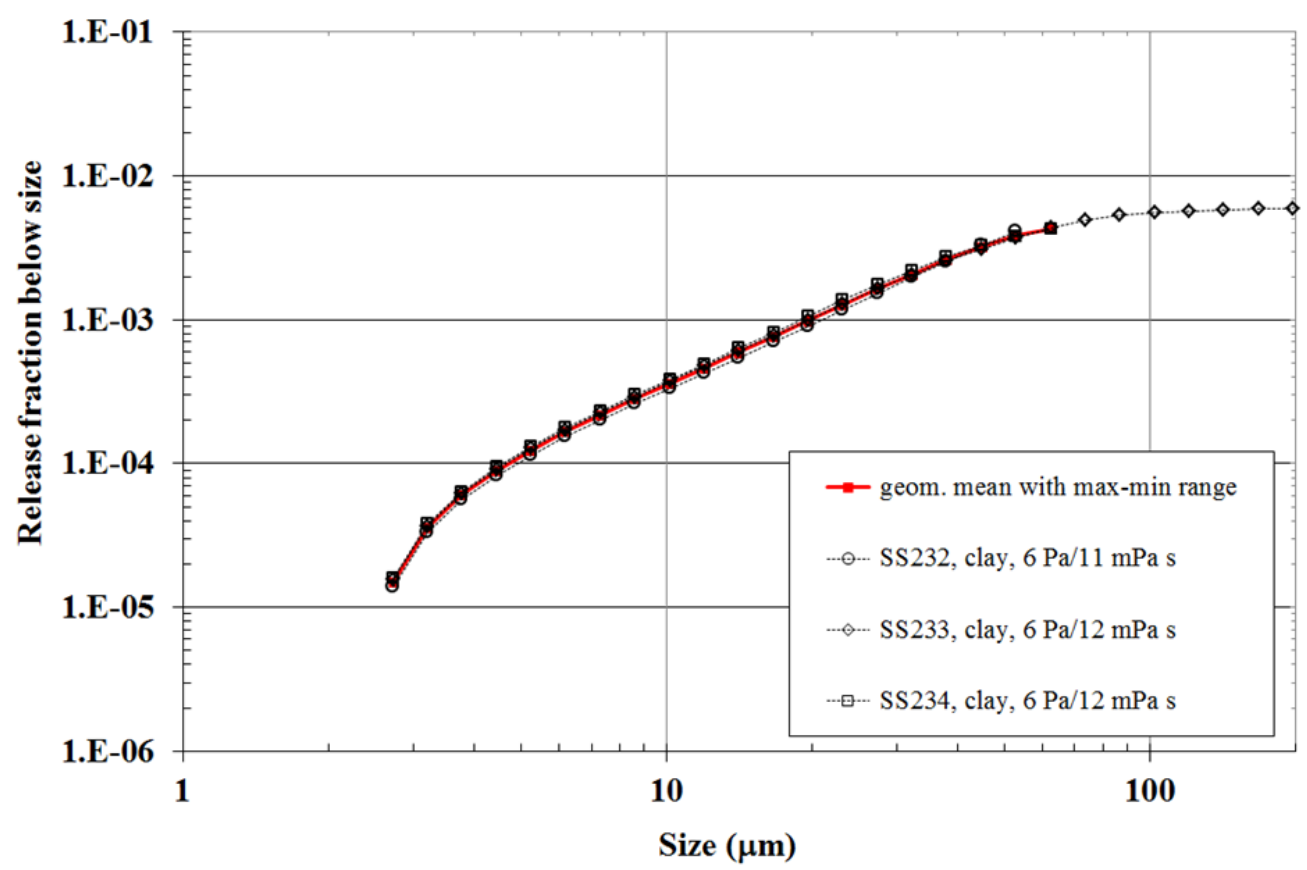

$0.5 \times 5$ mm slot, 100 psi, Phase 2, 500-mm lens, position 2 $\sim 6-\mathrm{Pa} / 6 \mathrm{mPa} s$ clay

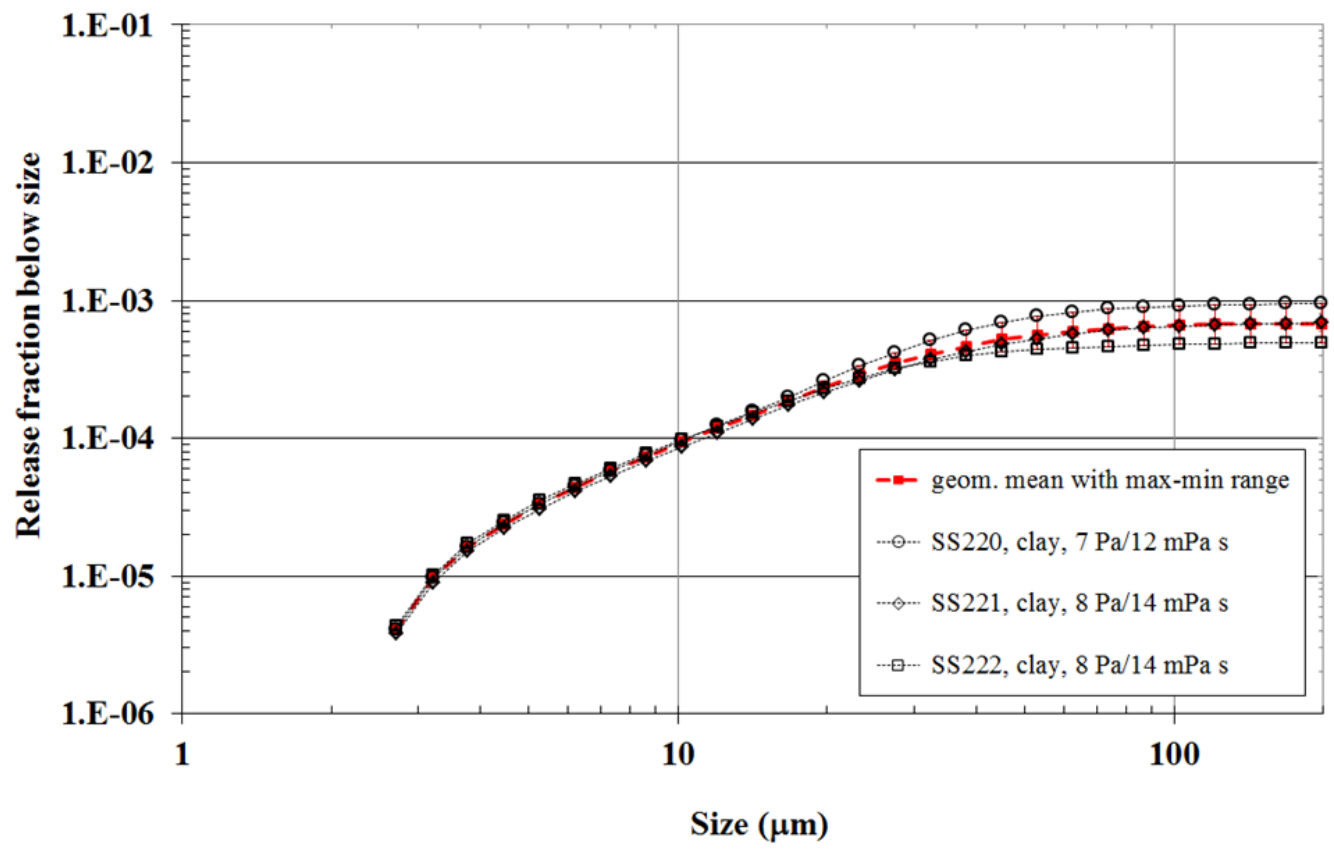

Figure 6.15. Best and Worst Repeatability Observed for $6 \mathrm{~Pa} / 6 \mathrm{mPa} \cdot \mathrm{s}$ Clay Tests. Error bars represent the maximum and minimum measurements. 
$1 \mathrm{~mm}$ hole, 200 psi, Phase 2, 500-mm lens, position 2

$\sim 30-P a / 30-m P a$ s clay

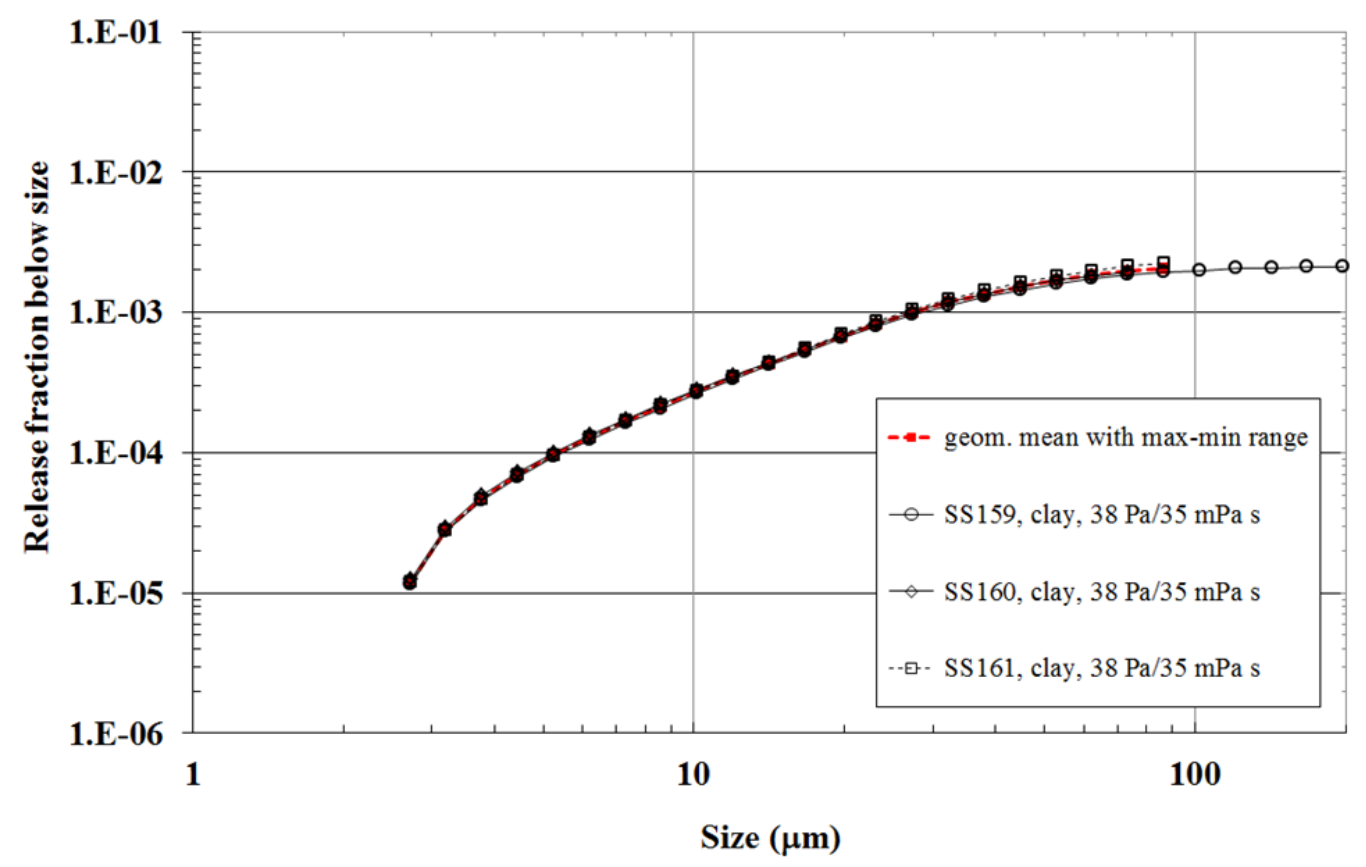

0.5 x 5 mm slot, 100 psi, Phase 2, 500-mm lens, position 2 $\sim 30-\mathrm{Pa} / 30 \mathrm{mPa} s$ clay

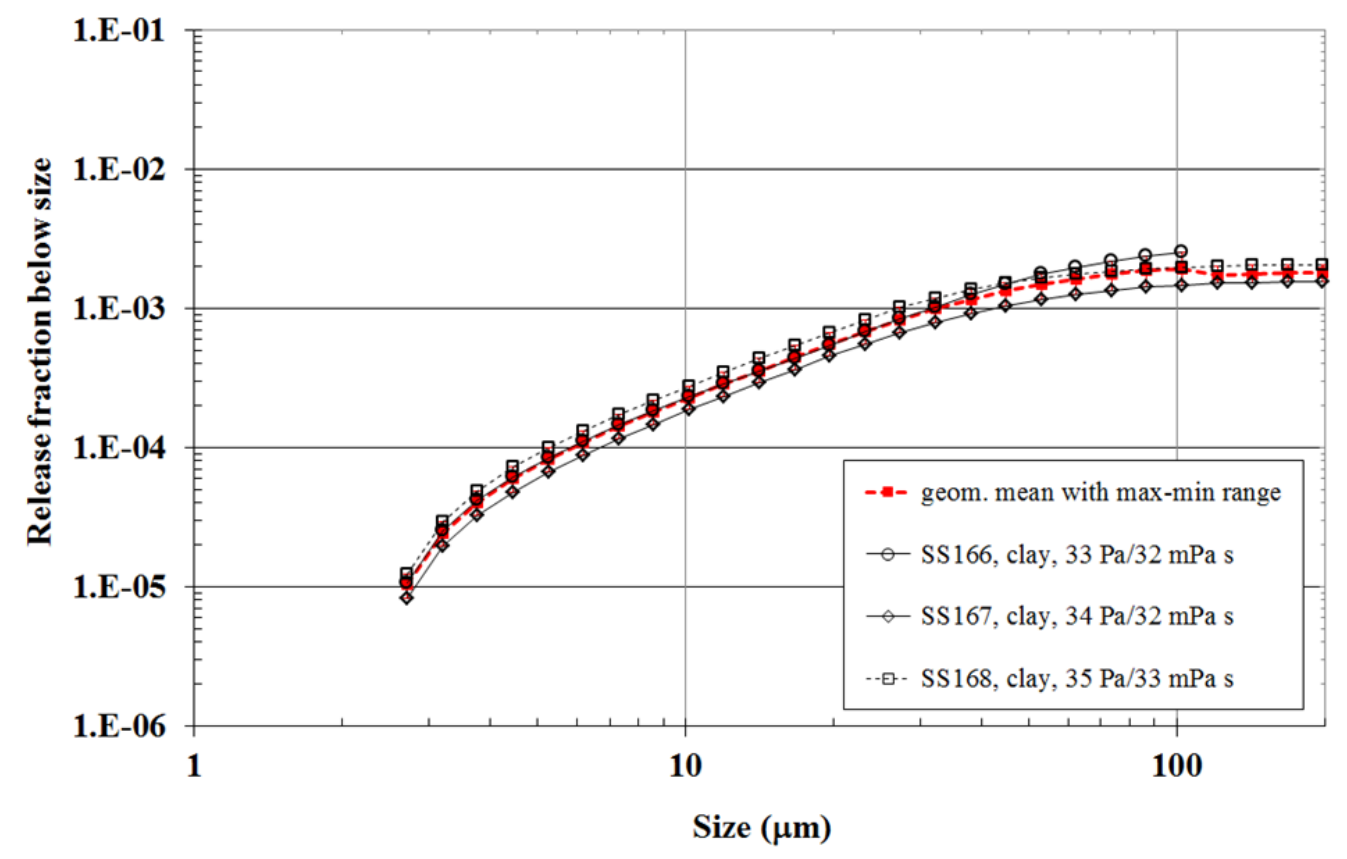

Figure 6.16. Best and Worst Repeatability Observed for $30 \mathrm{~Pa} / 30 \mathrm{mPa} \cdot \mathrm{s}$ Clay Tests. Error bars represent the maximum and minimum measurements. 
$1 \mathrm{~mm}$ hole, $380 \mathrm{psi}$, Phase 2, 500-mm lens, position 2

$\sim 6-P a / 6$ mPa s FEG

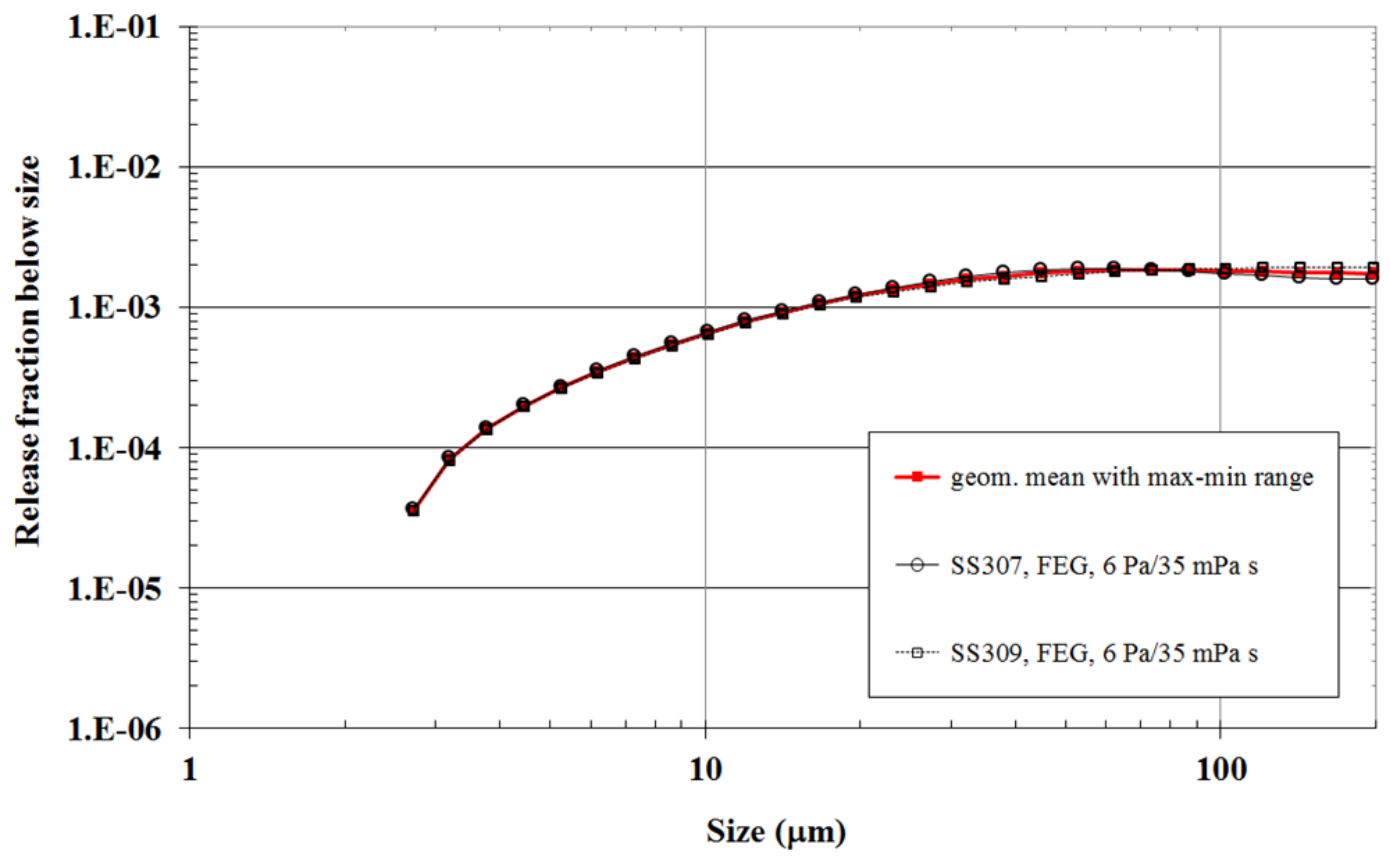

$1 \mathrm{~mm}$ hole, $200 \mathrm{psi}$, Phase 2, 500-mm lens, position 2 $\sim 6-\mathrm{Pa} / 6-\mathrm{mPa} s$ FEG

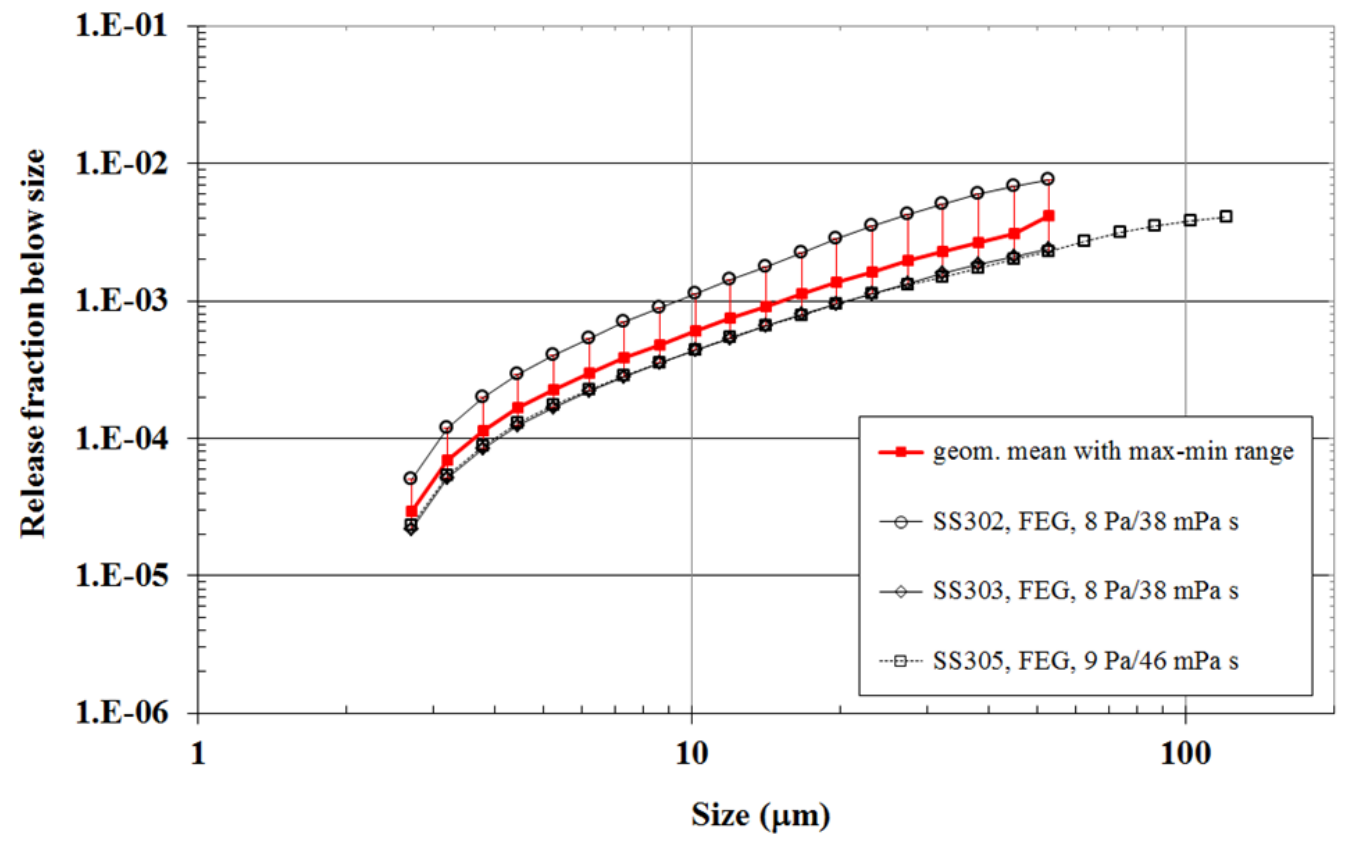

Figure 6.17. Best and Worst Repeatability Observed for $6 \mathrm{~Pa} / 6 \mathrm{mPa} \cdot \mathrm{s}$ FEG Tests. Initial RH (FIO) was 62 percent -75 percent in these tests. Error bars represent the maximum and minimum measurements. 
$0.5 \times 5$ mm slot, 380 psi, Phase 2, 500-mm lens, position 2 $\sim 30-P a / 30$ mPa $s$ FEG

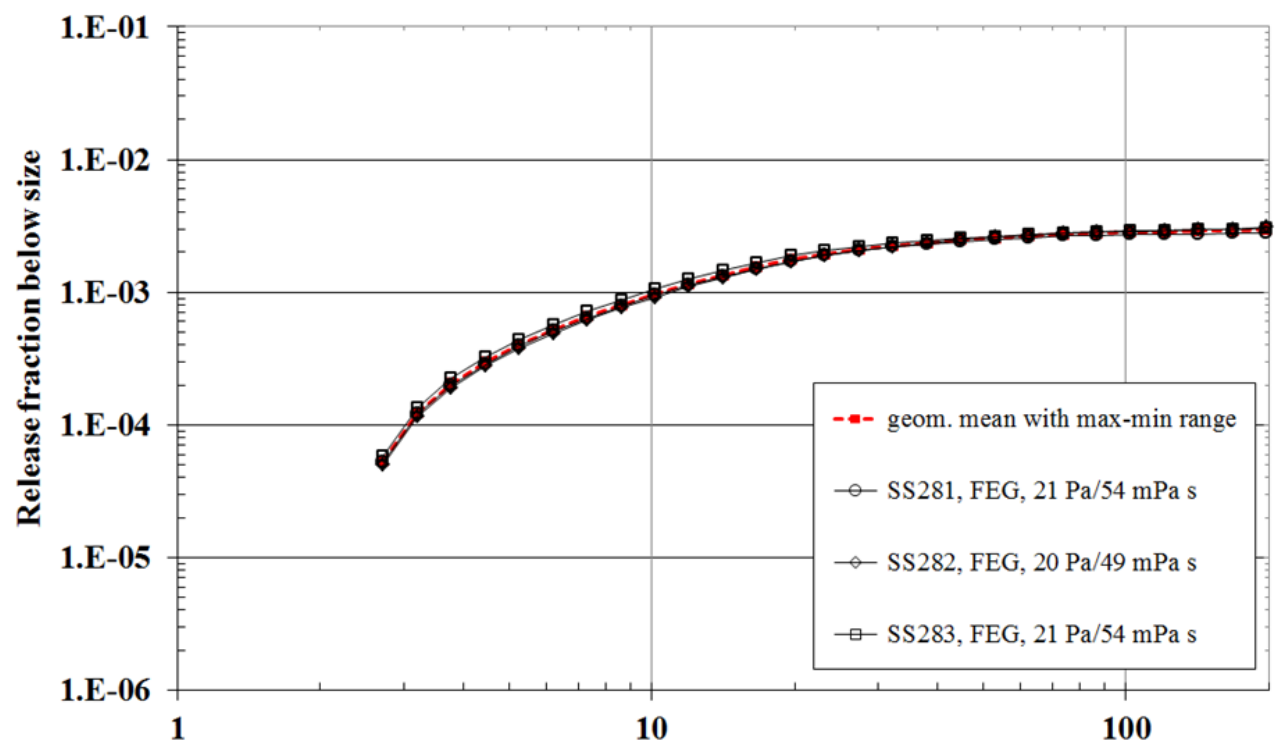

Size $(\mu \mathrm{m})$

$1 \mathrm{~mm}$ hole, 200 psi, Phase 2, 500-mm lens, position 2

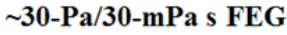

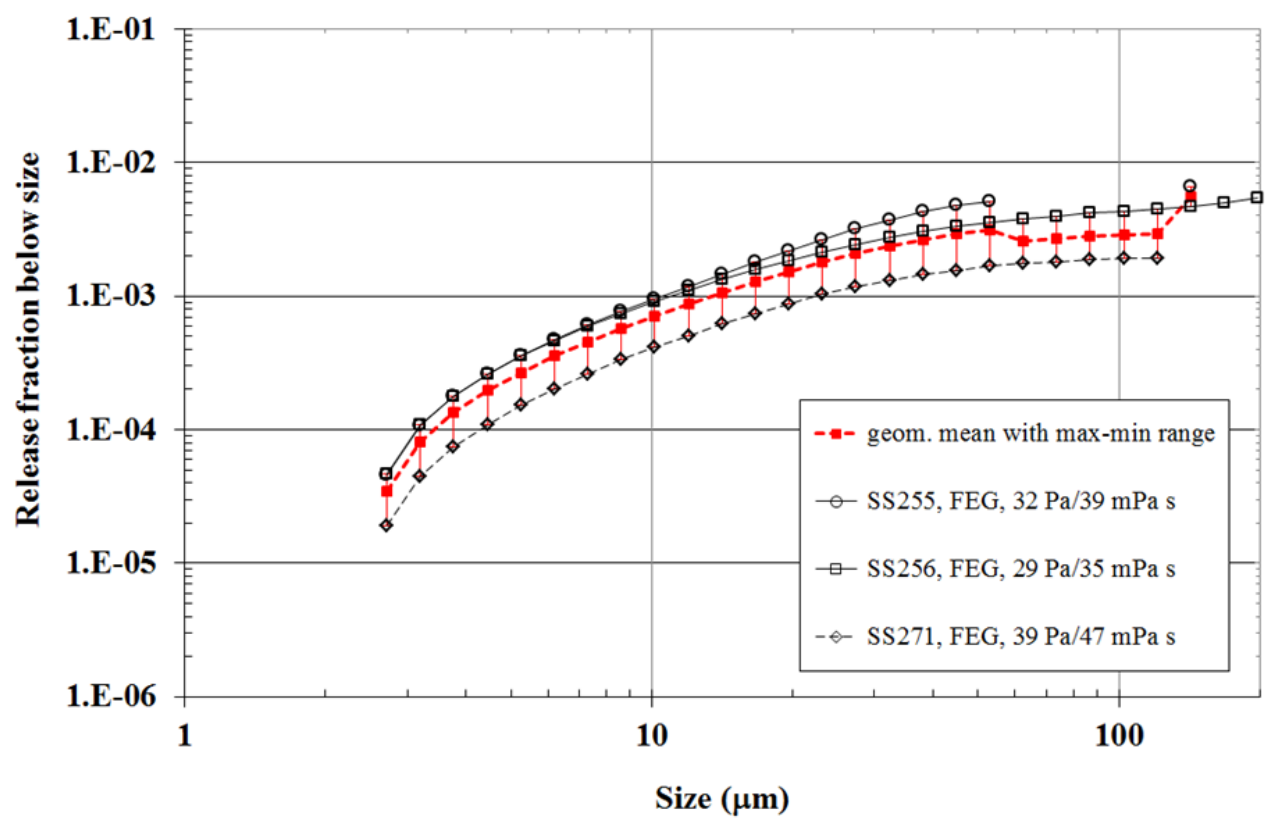

Figure 6.18. Best and Worst Repeatability Observed for $30 \mathrm{~Pa} / 30 \mathrm{mPa} \cdot \mathrm{s} F \mathrm{FE}$ Tests. Initial RH (FIO) was $>80$ percent in tests SS-281-SS-283 and 70 percent-82 percent for tests SS-255, SS256, and SS-271. Error bars represent the maximum and minimum measurements. 
$0.5 \times 5$ mm slot, 100 psi, Phase 2, 500-mm lens, position 2

$27 \mathrm{wt} \%$ STR

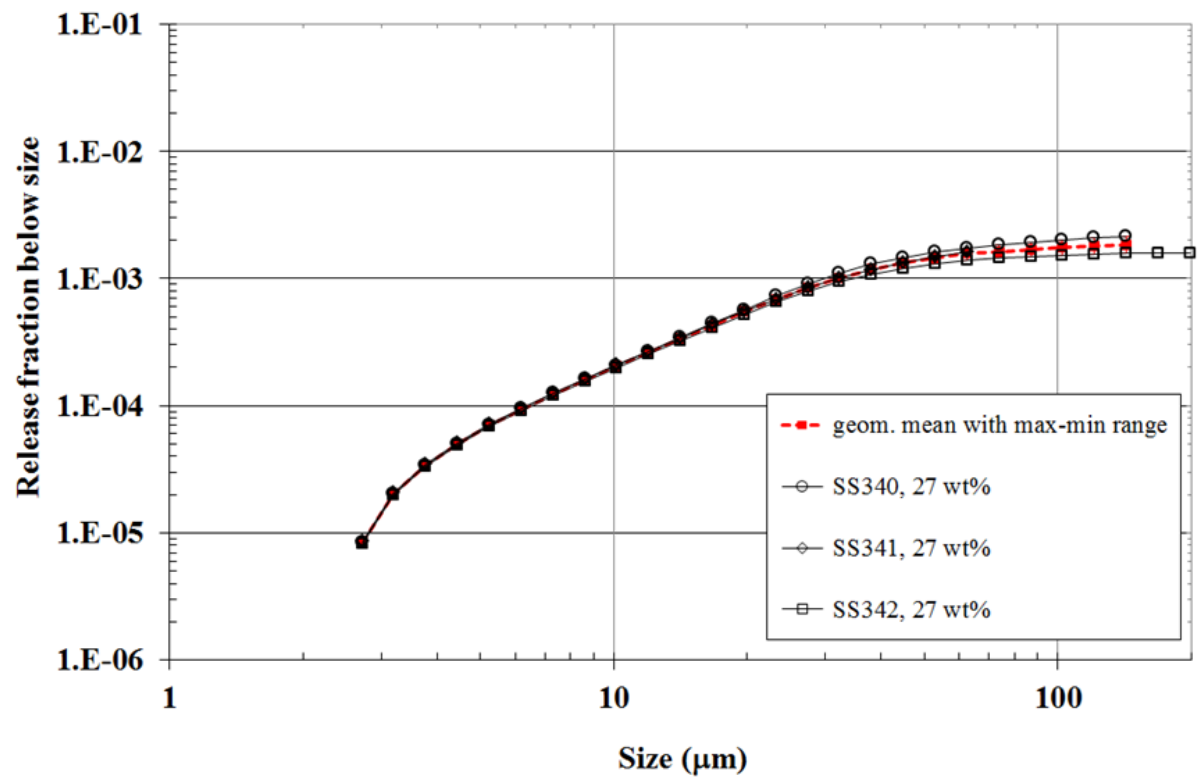

$1 \mathrm{~mm}$ hole, $380 \mathrm{psi}$, Phase 2, 500-mm lens, position 2 27 wt \% STR

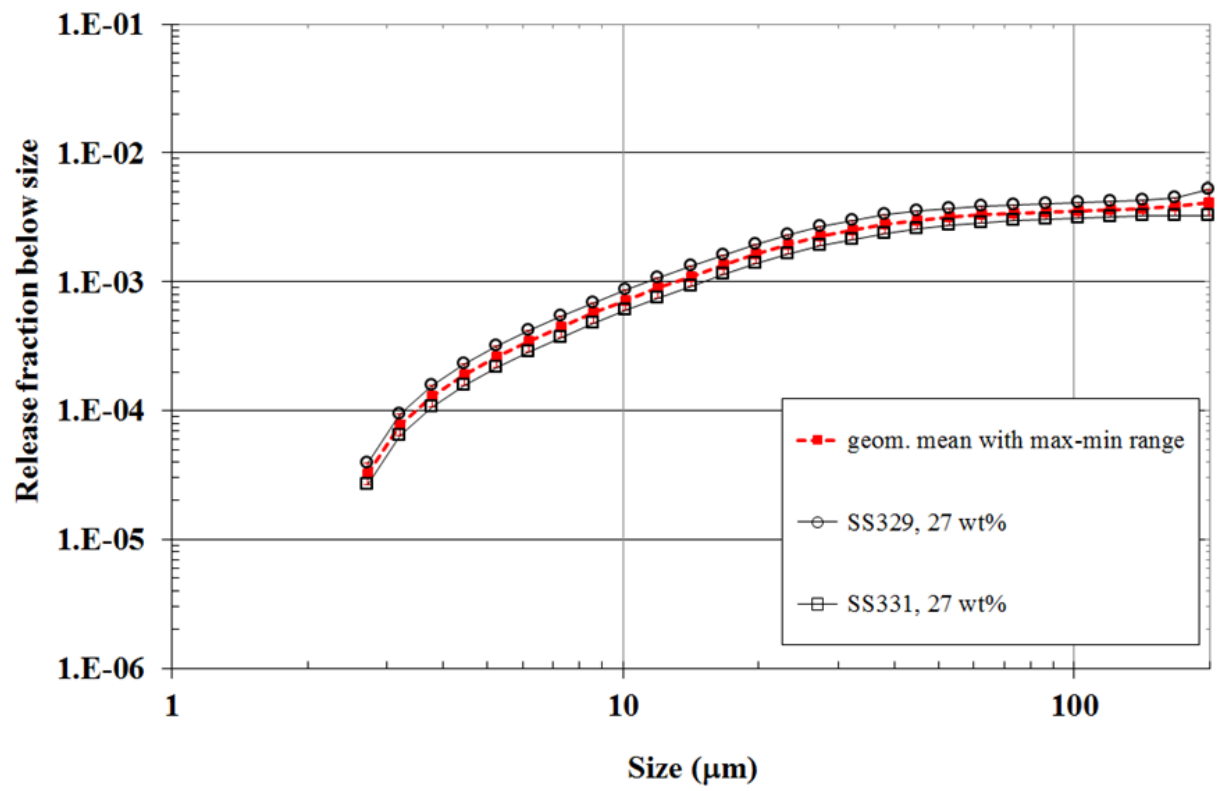

Figure 6.19. Best and Worst Repeatability Observed for $27 \mathrm{wt} \%$ STR Tests. Error bars represent the maximum and minimum measurements. 
$0.5 \times 5$ mm slot, 380 psi, Phase 2, 500-mm lens, position 2

$19 \mathrm{wt} \% \mathrm{STR} / 0.4 \mathrm{wt} \%$ Mo

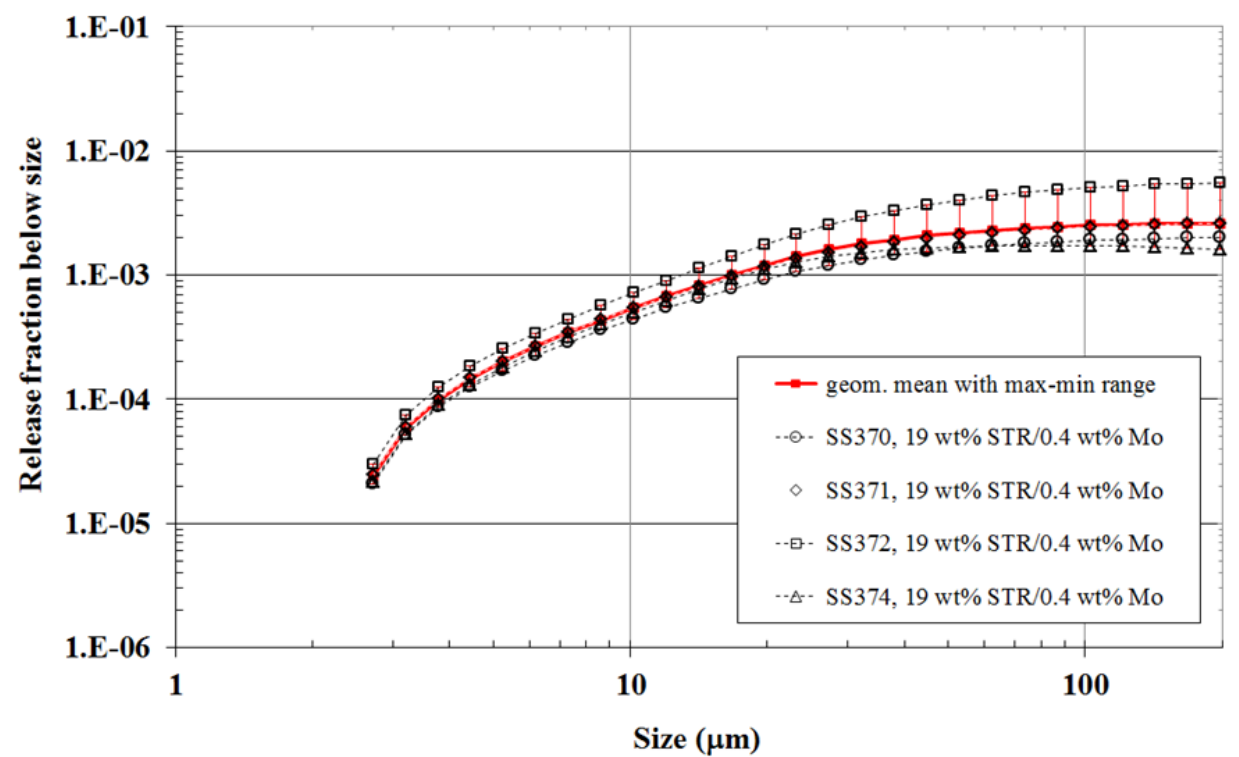

$1 \mathrm{~mm}$ hole, $380 \mathrm{psi}$, Phase 2, 500-mm lens, position 2

$19 \mathrm{wt} \% \mathrm{STR} / 0.4 \mathrm{wt} \%$ Mo

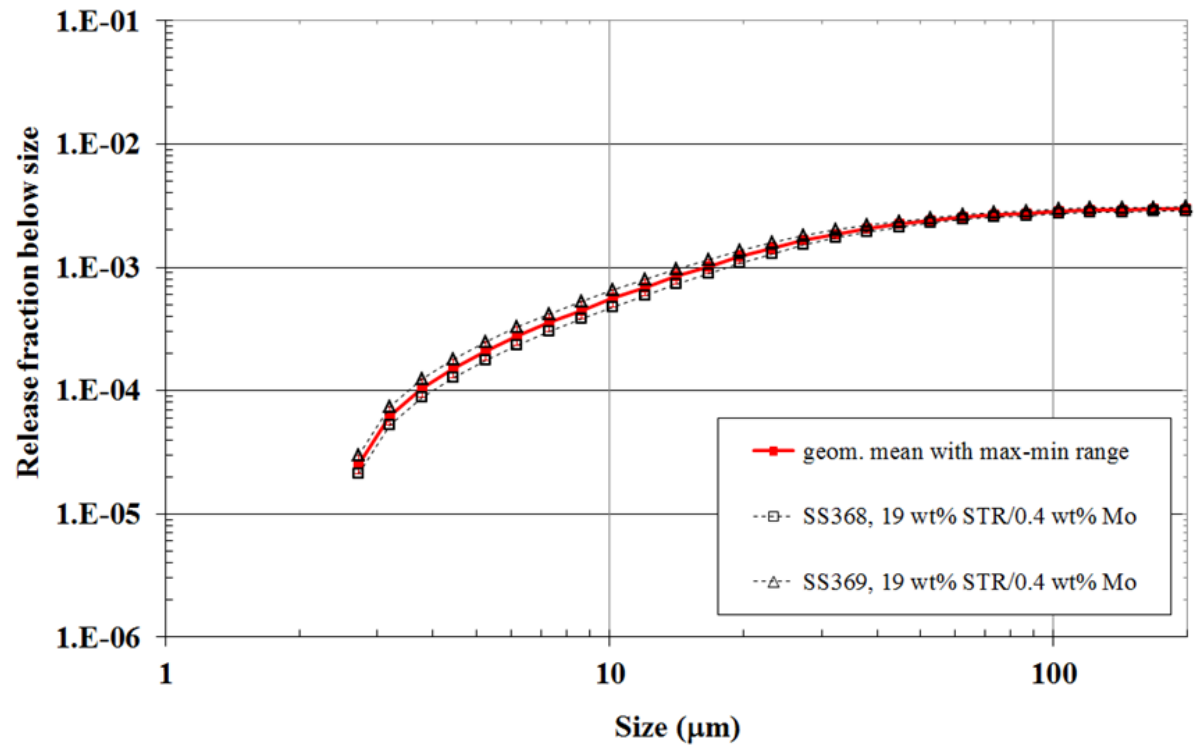

Figure 6.20. Repeatability for the Two $19 \mathrm{wt} \%$ STR/0.4 wt $\%$ Mo Tests. Error bars represent the maximum and minimum measurements. 
0.5 x 5 mm slot, 380 psi, Phase 2, 500-mm lens, position 2

0.4 wt $\%$ Mo

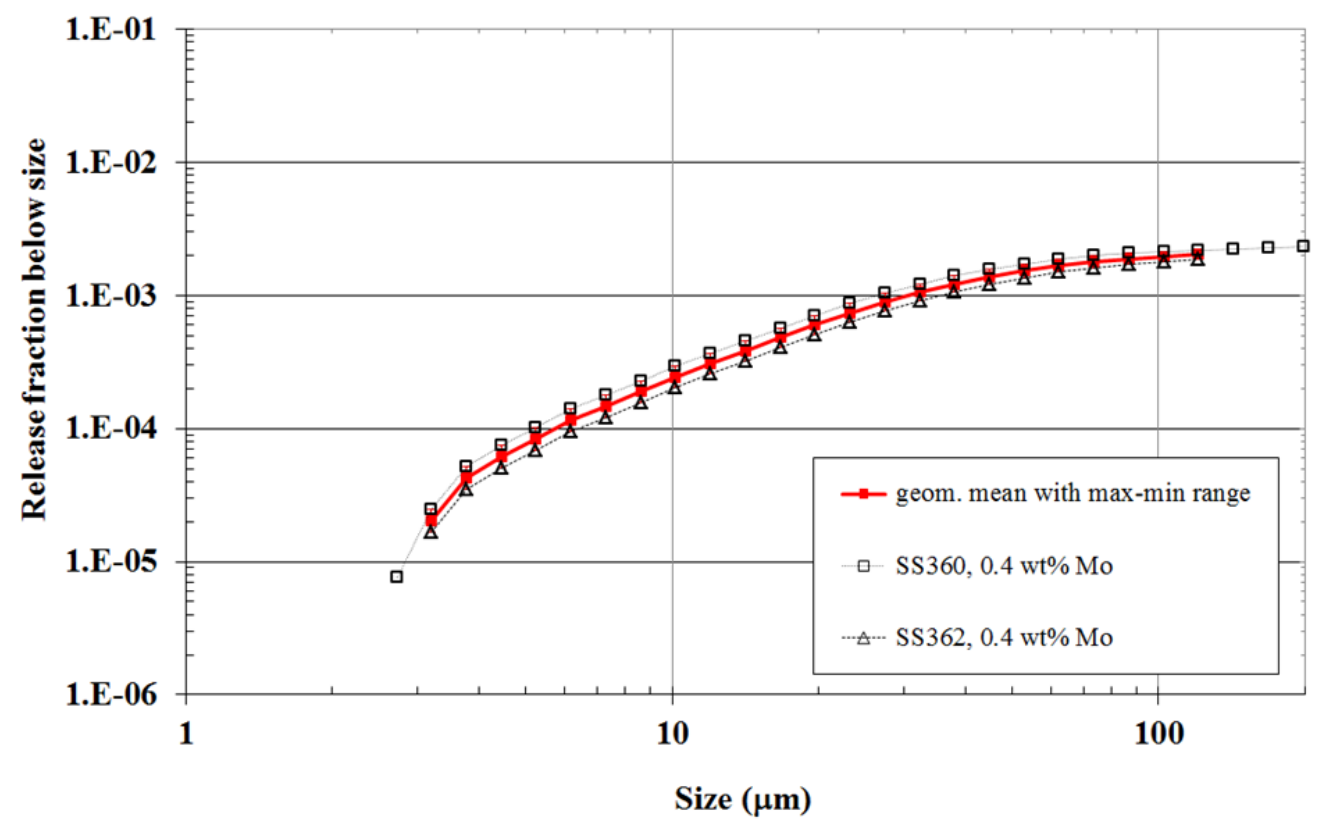

Figure 6.21. Repeatability for $0.4 \mathrm{wt} \%$ Mo in Water at $380 \mathrm{psig}, 0.5 \times 5 \mathrm{~mm}$ Slot. Error bars represent the maximum and minimum measurements.

\subsection{Malvern Insitec-S Validation Summary}

Phase II test activities included studies aimed at determining the accuracy of the Malvern Insitec-S size and concentration measurements under various test configurations. In this section, the test approach and results for these Malvern Insitec-S validation studies are summarized, with a particular focus on those results applicable to the small-scale Malvern Insitec-S configuration. For the complete discussion of the Malvern Insitec-S validation study, see Section 6 of Daniel et al. (2013).

In both Phase I and Phase II small-scale testing, a Malvern Insitec-S aerosol analyzer was used as the primary means of aerosol concentration and size distribution measurement. The Malvern Insitec-S employs open frame laser diffraction, whereby aerosol size and concentration are measured by time averaging laser power and scattered light intensities from diffraction events that occur in the measurement volume defined by the path of the $10 \mathrm{~mm}$-diameter laser beam across the $70 \mathrm{~mm}$ open path between laser source and detector. The measurement technique and configuration pose several difficulties when interpreting aerosol results:

- The PSD is not measured directly. Instead, the diffraction pattern produced by the aerosol or dispersion is measured and the size distribution is inferred by matching it to the diffraction pattern (often using a model).

- Aerosol concentration is determined using the Beer-Lambert law, which relates the loss of laser power due to the presence of aerosol in the Malvern Insitec-S measuring volume to the concentration 
of aerosol in the measuring volume. Use of the Beer-Lambert law depends on the determination of particle size, and errors in the measured size distribution may affect the accuracy of the concentration measurement.

- Diffraction events may occur at any point along the measurement (laser path) zone. The diffraction path length for the open frame (70 $\mathrm{mm}$ for small-scale) is long relative to typical laser diffraction techniques. Consequently, scattering events that occur far from the detector will show attenuation or complete loss of scattered light at high-angles relative to events that occur near the detector. The Malvern Insitec-S implements corrections for laser attenuation; however, these corrections are based on first principles and the accuracy of such corrections should be evaluated to determine their overall accuracy.

The difficulties presented above pose a challenge to those attempting to assess overall instrument accuracy with respect to size and concentration measurements. Because Malvern Insitec-S analyzers were the primary means of aerosol characterization for small-scale aerosol testing, it is important to understand their accuracy with respect to measurements of aerosol concentration and size distribution.

\subsubsection{Objectives of the Validation Testing}

The objectives of Malvern Insitec-S validation testing were 1) to assess baseline concentration and particle size accuracy of the analyzer and 2) to evaluate instrument performance in test configurations employed in Phase I and Phase II testing. Note that for small-scale testing the only difference between Phase I and Phase II is a change in lens focal length $(100 \mathrm{~mm}$ to $500 \mathrm{~mm})$. The path length remained the same $(70 \mathrm{~mm})$. Specifically, validation tests aimed to:

1. evaluate the accuracy of concentration, the linearity of concentration response, and the accuracy of size distribution measurements under instrument configurations where there is no need to correct the diffraction pattern for distance effects

2. evaluate the validity of diffraction pattern corrections that account for distance effects.

To meet these objectives, a series of validation tests were conducted (for both small- and large-scale configurations) that evaluated Malvern Insitec-S analyzer performance against known or certified materials and parametrically evaluated the impact of instrument settings on the result. These tests were used to determine if any corrections for bias should be made to the measured size and concentration data and if any optimal instrument settings exist to improve the overall aerosol measurements used for both Phase I and Phase II release fraction analysis. For details of the test approach (e.g., material, set-up) and many of the test results, refer to Sections 6.2 and 6.3, respectively, of Daniel et al. (2013).

\subsubsection{Outcomes of the Validation Testing}

This section summarizes the important results that relate specifically to the small-scale Malvern Insitec-S and its testing configurations. Tests were performed to evaluate the following aspects of the Malvern Insitec-S measurements:

- the baseline accuracy of size and concentration measurements for ideal (spherical) particulate systems in the size range of interest for aerosol testing (i.e., $10-100 \mu \mathrm{m}$ )

- the impact of irregular particles on measurement accuracy 
- differences in the size and concentration result when measuring with a $500 \mathrm{~mm}$ lens (used in Phase II testing) compared to measurements made by the $100 \mathrm{~mm}$ lens (used in Phase I testing)

- the impact of laser attenuation from long optical path lengths or increased distance from detector on "localized" and "distributed" aerosol size distribution and concentration measurements

- the appropriateness of corrections made to compensate for laser attenuation.

All of these aspects are applicable in both the small- and large-scale systems. The impact of laser attenuation and the corrections used to compensate for it is more nuanced than the first three aspects listed above, owing to the different path lengths of the small-scale $(70 \mathrm{~mm}$ ) and large-scale (up to $920 \mathrm{~mm}$ ) systems. Only outcomes relevant to the small-scale Malvern Insitec-S configuration will be discussed here. Based on the validation testing, the following general observations were made:

- The baseline accuracy of the Malvern Insitec-S aerosol analyzer, when measuring spherical particulate systems, was quite good. The concentration response was linear and projected to be within -13 percent/ +17 percent of the actual value at the 95 percent confidence level. With respect to the PSD, the Malvern Insitec-S measured the d(50) quite accurately (within 3 percent of the reference value), with poorer accuracy towards the tails of the distribution.

- Irregularity in particle shape led to inaccuracies in the concentration measurement (e.g., a factor of two too large in one case, a boehmite dispersion). Particle size measurement was not significantly affected. Some broadening of the distributions was observed relative to the reference measurement; however, the measurement of the $\mathrm{d}(50)$ was within 1 percent of the reference value.

- Use of a $500 \mathrm{~mm}$ lens (nominal range of 2.5 to $2500 \mu \mathrm{m}$ ) with a particle dispersion near the lower measuring range of the lens resulted in a concentration measurement around 25 percent lower and a broader size distribution compared to a measurement made with the $100 \mathrm{~mm}$ lens (nominal range of 0.5-200 $\mu \mathrm{m})$. The comparison between lenses improved when a dispersion well within the nominal ranges of both lenses was used.

- Concentration and PSD measurements were impacted when the distance of the scattering center from the detection unit was varied; this impact was found to be significant at distances greater than $150 \mathrm{~mm}$. For large-scale configurations, this issue required additional investigation. However, small-scale aerosol measurements for both Phase I and Phase II (which were conducted at path lengths shorter than $150 \mathrm{~mm}$ ) were not substantially impacted.

Based on these results, the recommendation is that the concentration and particle size measurements as performed by the Malvern Insitec-S aerosol instrument in the small-scale testing (both Phase I and Phase II) do not require any adjustments. The instrument accurately measures concentration and PSDs for spherical systems and applies appropriate corrections to account for attenuation. However, caution should be exercised for the following cases:

1. Aerosol particulate systems comprised of non-spherical particles or droplets. However, most particles in aerosol testing are expected to be spherical and irregular particles that may be present 
from slurry simulants will approach the primary particle size of the solids (all Phase II simulants had $\mathrm{d}(50)<5 \mu \mathrm{m}$ except for the DPW simulant, which had only a small fraction of solid $\mathrm{Mo}^{1}$ ).

2. Aerosol particulate systems with a large portion of droplets that approach the lower limit of the nominal size range of a particular lens. In small-scale testing, aerosol droplets exist in this range; however, in general, the majority of droplets are well within the working range of the lens.

3. Aerosol particulate systems that present a size distribution inconsistent with the assumptions used in the laser diffraction inversion technique performed by the Malvern software. In general, aerosol size distributions are broad and will be appropriately handled by the inversion technique; however, in some cases the inversion may slightly broaden the true size distribution of the measured aerosol.

The three situations listed above may increase bias or inaccuracy in Malvern Insitec-S measurements. However, as mentioned, the aerosol systems tested in the small-scale system were typically not expected to be affected.

\footnotetext{
${ }^{1}$ Note that the unsonicated FEG simulant PSD measurements indicate $\mathrm{d}(50) \geq 5 \mu \mathrm{m}$, but the sonicated PSD measurements (Figure 3.3) result in $\mathrm{d}(50)<5 \mu \mathrm{m}$. Aggregation is the suspected cause for this discrepancy, so the primary particle size is considered to be less than $5 \mu \mathrm{m}$ in the FEG simulants.
} 



\subsection{Parametric Results and Discussion}

This section discusses the results of the parametric analyses conducted following the bases defined in Sections 6.1 through 6.3 and the methods and repeatability detailed in Sections 6.4 and 6.5. This section focuses on examining the effects of various parameters on the measured release fractions and/or net generation rates.

Although the aerosol concentration data were analyzed by both cumulative and differential fits to obtain net aerosol generation rates (see Section 6.3), only results from the cumulative fits are shown. The cumulative method was chosen because the release fractions estimated by the two types of fits are typically indistinguishable within the 95 percent confidence intervals of the fits and the cumulative method had a wider range of droplet size bins whose concentration fits satisfied the $\bar{R}^{2}>0.5$ criterion. Appendix B contains a complete set of plots of release fractions, calculated by both the differential and cumulative method for each test.

In general, certain plotting conventions are followed in the subsequent discussions of parametric effects. Cumulative release fractions and net generation rates, determined using the cumulative method, are presented for three undersize bins, $10.17,32.28$, and $102.50 \mu \mathrm{m}$ (henceforth referred to as $<10,<32$, and $<102 \mu \mathrm{m}$ for simplicity). Measured data are shown with large symbols joined by thin lines; the corresponding WTP model predictions are shown with small symbols joined by heavy lines. The WTP model and the data are not on the same basis with respect to evaporation. The model (as used here) predicts aerosol concentration and size distribution before any evaporation, while aerosol measurements have been affected to some extent by evaporation. In addition, the in-chamber measurement method is suspected to have some settling-related bias due to the measurement zone being located above the spray, resulting in an under-prediction of release fractions for large droplets.

Because the WTP model predictions are based on average pressure during the fit period, not on the run target pressures, the lines may appear irregular (in cases where the average pressure departed noticeably from the target pressure). In cases where the fits were bad (per the criteria shown in Section 6.3), no measured-data symbols appear for the affected droplet sizes.

Plotted release fractions and net generation rates are either geometric means of a set of replicates or data from individual runs. The error bars on the geometric means represent the measured maximum and minimum of the set of runs. The error bars on individual runs represent an approximation to the 95 percent confidence level and include estimates of experimental variability and the uncertainty of the model fit that produced the release fractions. The error bars on individual runs are not exact uncertainties but provide a reasonable idea of the uncertainty of the data. More detail can be found in Section 6.3.1.

The parametric results are discussed in Sections 7.1 through 7.5. Each of these sections is organized around a single concept or variable. Section 7.1 discusses the effect of pressure based on tests with baseline orifice sizes $(1 \mathrm{~mm}$ round hole and $0.5 \times 5 \mathrm{~mm}$ slot $)$ using water and slurry data. Sections 7.2 and Section 7.3 investigate the effects of solids loading and dense particles in Newtonian slurries. Section 7.4 presents parametric studies of the effect of the non-Newtonian rheology of clay and Fe-rich (FEG) slurries. Section 7.5 describes droplet size distributions in in-jet sprays. Section 7.6 presents the orifice coefficients measured as a byproduct of aerosol testing. Finally, Section 7.7 summarizes the results of this study. 


\subsection{Effect of Pressure}

The small-scale aerosol testing of orifice effects was conducted for a limited set of pressures, using process water, two different concentrations of clay slurry, two different concentrations of FEG simulant slurry, and $27 \mathrm{wt} \%$ STR (boehmite) in water. In the WTP model, pressure affects the aerosol generation rate through the square-root dependence of leak flow rate on pressure and through the effect of jet velocity (dependent on the square root of pressure) upon the spray SMD. As discussed in Section 1.2.2, SMD is proportional to $\mathrm{U}^{-2 / 3}$ when the leading term in Equation (1.1) is much greater than the other terms. Thus, the dependence of the SMD on pressure is $\mathrm{SMD} \sim \Delta \mathrm{P}^{-1 / 3}$, and based on Equation (1.5), this is predicted to result in larger release fractions as pressure increases.

Three target pressures were employed in small-scale testing: 100, 200, and 380 psig. The effect of pressure on sprays can be observed by comparing the release fractions measured at these three pressures for a constant orifice size. The plots in this section make this comparison by showing the cumulative release fractions for individual runs, together with error bars that include the model fit uncertainty and an estimate of experimental variability. In this section, replicate runs are not averaged into a geometric mean because that would tend to obscure the fact that different runs were made of different pressures (i.e., they were not all made at the target pressure).

In this section, the pressure dependence of the measured release fractions are compared to the pressure dependence predicted by the WTP model. In general, however, the release fraction results from the small-scale chamber will not be directly compared to the WTP model, while the large-scale chamber is closer in size to the rooms in the WTP and thus is thought to give more applicable results. For a discussion on the effect of chamber size on release fraction results, see Daniel et al. (2013). A comparison of small- and large-chamber release fractions is given in Schonewill et al. (2012). In addition, the small-scale release fraction results were collected with initial chamber humidities that were typically less than 100 percent RH, based on FIO humidity measurements, and the release fraction results are biased low as a result. In tests where the initial RH was between 80 and 100 percent, the variation in release fraction between the low and high ends of the humidity range is expected to be less than a factor of two, which has to be considered in making comparisons among data in this humidity range (as for water and clay slurry data). In the FEG tests, where the initial RH was sometimes in the 60 to 80 percent range, and sometimes in the 80 to 100 percent range (Section 6.4.5), a wider variation of release fraction may have been caused by variability in humidity. In general, because all the data was affected by the initial humidity to various degrees, the relative differences are smaller. Thus, the small-scale chamber should give valid comparisons for this section - that is, for specific comparisons that focus on the trend of release fraction with pressure for a given simulant.

The first two examples, for the water simulant, are shown in Figure 7.1 (a round hole with a target diameter of $1 \mathrm{~mm}$ ) and Figure 7.2 (a rectangular slot with target dimensions of $0.5 \times 5 \mathrm{~mm}$ ). The plots include data from both Phase I and Phase II, denoted with unfilled and filled symbols, respectively. Each panel of the plot represents one cumulative size bin of droplets.

The general trend in the release fractions with increasing pressure differs from that of the WTP model. The release fractions at 100 psig are equal to or slightly greater than those at $200 \mathrm{psig}$, and then increase over the pressure step from 200 to 380 psig. 

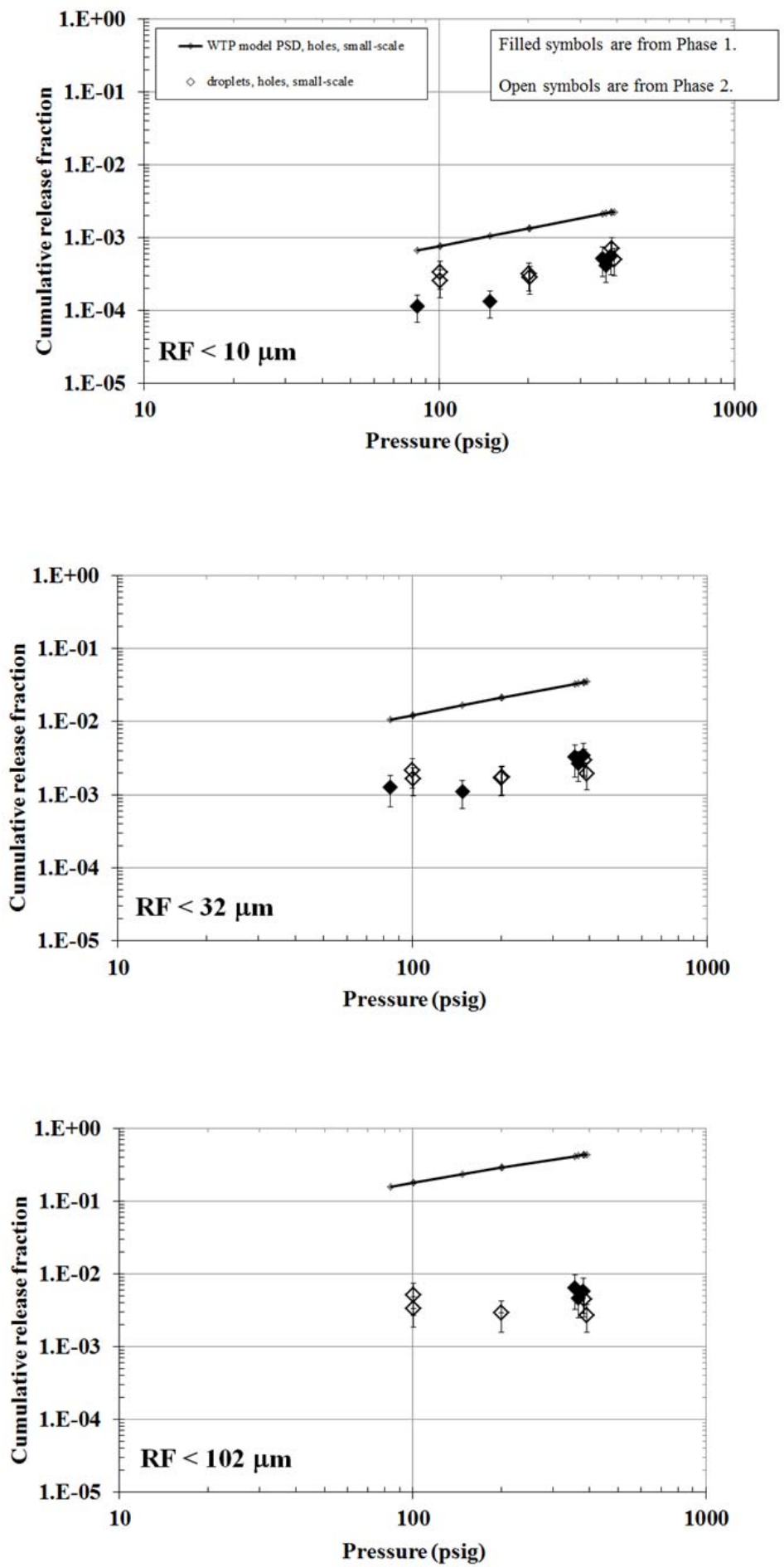

Figure 7.1. Effect of Pressure on Release Fractions for a Spray of Water from a Target $1 \mathrm{~mm}$ Hole. WTP model predictions are shown by the thick black lines. Error bars show an approximate 95 percent confidence interval for each test including model fit and experimental variability. Appendix D identifies the tests in the plot. 

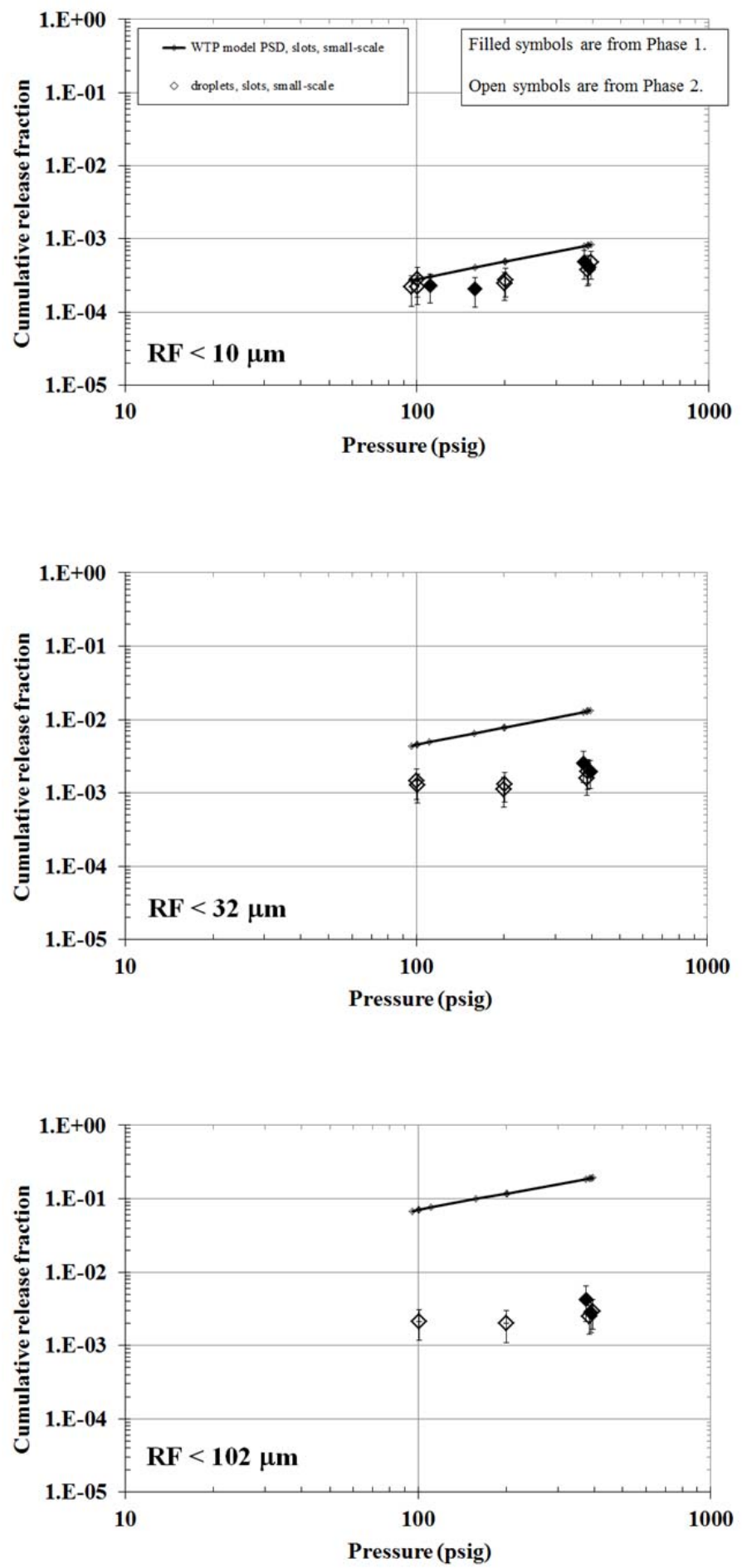

Figure 7.2. Effect of Pressure on Release Fractions for a Spray of Water from a Target $0.5 \times 5 \mathrm{~mm}$ Slot. WTP model predictions are shown by the thick black lines. Error bars show an approximate 95 percent confidence interval for each test including model fit and experimental variability. The 100 and 200 psig runs from Phase I were missing some initial data because of laser drift so the data may be suspect. Appendix D identifies the tests in the plot. 
It should be noted that the Phase I 100 and 200 psig runs for the slot were missing some initial data because of laser drift so the data may be suspect. In general, Phase II data are expected to be of better quality than Phase I data because Phase II pressure control was better and there was less effect of laser drift.

The trend in measured release fractions at low pressures runs counter to the trend predicted by the WTP model. If the trend is extrapolated to pressures $<100 \mathrm{psig}$, the WTP model may be exceeded in certain cases. However, the observed trend may be a result of chamber geometry (i.e., the physics of the aerosol generation). In the small-scale chamber, the jet travels only a few feet before it reaches the splash wall, where the majority of the spray is captured and does not aerosolize. At higher pressure, the higher spray velocity allows the jet less time in flight before it strikes the wall. Capture by the splash wall is balanced against stronger in-flight turbulent jet break-up (aerosolization) forces at higher pressure. Given these two opposing effects, it is reasonable to expect a range of pressures exist where they are approximately balanced (e.g., around 100 to 200 psig). At higher pressures, the jet spends less time in flight, but the break-up mechanisms are also much stronger (and result in finer droplet distributions that stay aloft longer). At lower pressures (at least approaching $100 \mathrm{psig}$ ), the flight time is longer and the break-up mechanism is still strong enough to create a significant amount of aerosol, leading to an approximately constant trend. It is likely that at even lower pressures (e.g., $<100 \mathrm{psig}$ ), even if the splash wall capture is negligible, the break-up forces will not be strong enough to result in significant aerosol. At the asymptotic limit of atmospheric pressure the orifice will not create sprays of significant velocity at all (e.g., a small stream or a series of drips) and negligible amounts of aerosol will be created.

Thus, it is not considered reasonable to extrapolate the trend to pressures lower than $100 \mathrm{psig}$. This conclusion is supported by results from similar tests in the large-scale chamber (see either Schonewill et al. 2012 or Daniel et al. 2013), which have a much longer flight time and do not exhibit the same trend. Furthermore, any conclusion drawn from the apparent trends is rendered ambiguous by the fact that the differences in release fraction from one pressure to another are of about the same size as the estimated uncertainty.

The results of the tests conducted using clay slurry whose target yield stress was $6 \mathrm{~Pa}$ are shown in Figure 7.3 and Figure 7.4. Repeatedly and consistently, the results for the $1 \mathrm{~mm}$ hole (Figure 7.3) show the same kind of decrease in release fraction from 100 to 200 psig as appeared for water. Equally consistently, the results for the $0.5 \times 5 \mathrm{~mm}$ slot show a continuous uptrend with pressure, with a slope equal to that of the WTP model or, for the $<10-\mu \mathrm{m}$ release fraction, greater than that of the model. Low initial humidity is not expected to be the cause because all the clay tests, like the water tests, started with a $\mathrm{RH}$ of $\geq 80$ percent.

Figure 7.5 and Figure 7.6 show the results of the tests conducted using clay slurry whose target yield stress was $30 \mathrm{~Pa}$. In this case, both orifices show the decrease in release fraction from 100 to $200 \mathrm{psig}$, although for the round hole the trend at $<102 \mu \mathrm{m}$ is partially masked by the fact that well-fit data were not available for all pressures.

The FEG simulant is the remaining non-Newtonian simulant that was tested. The results for the FEG simulant with a target yield stress of $6 \mathrm{~Pa}$ are shown in Figure 7.7 and Figure 7.8. The measured release fractions show a tendency to decrease with pressure for the $<32-\mu \mathrm{m}$ release fraction and possibly for the $<100-\mu \mathrm{m}$ release fraction, although the trend of the latter is unclear because of the difficulty of getting 
well-fit release fraction data for the larger droplets: at $<100 \mu \mathrm{m}$, many release fractions are missing and one has an unusually broad error band.

In addition, the tendency for the FEG release fraction to decrease (inconsistently) with pressure was noted in the Phase I observations (p. 7.15-16, Mahoney et al. 2013): “...the data show several cases of release fraction decreasing with increasing pressure. The trends are not perfectly clear, in that lines often could be drawn that would match the WTP model pressure dependence while remaining within the error bars. However, the repeated presence of decreasing best-fit release fractions, with increasing pressure, suggests the downward trend is real... To the extent that good fits for the release fractions were obtained for larger droplet sizes $(<30$ and $<100 \mu \mathrm{m})$, they are more likely to have release fractions that decrease with increasing pressure than are the $<10-\mu \mathrm{m}$ droplets."

It should be noted as a caveat that the 6 Pa FEG tests that used the $1 \mathrm{~mm}$ round hole had initial RHs (measured by an FIO instrument) that were all below 80 percent: humidity was 75 to 76 percent for the 100 psig tests, 68 to 75 percent for the 200 psig tests, and 62 to 68 percent for the 380 psig tests. The initial humidities for the tests that used the $0.5 \times 5 \mathrm{~mm}$ slot had initial humidities (FIO) between 70 and 80 percent, except that one test at 380 psig had an initial RH of 83 percent. If the sprays had been water, the low RH in these tests would be expected to cause a decrease in release fraction across all sizes (Section 6.4.5). The effect of evaporation on the FEG slurries is not known; the presence of particles and, potentially, of agglomerates could change the size dependence and magnitude of the effect of evaporation. Recall from Section 6.4.5 that large-scale testing with 6 Pa clay indicated that the increase in release fraction when correcting the data to saturation (100 percent $\mathrm{RH})$ is smaller in magnitude compared to water. Extrapolating the behavior from the large-scale measurements for $6 \mathrm{~Pa}$ clay to the FEG slurry is uncertain, but suggests that the FEG release fractions, when corrected for initial RH, would be bounded by the magnitude of the water correction for larger droplets and the uncorrected FEG release fraction data for smaller droplets. 

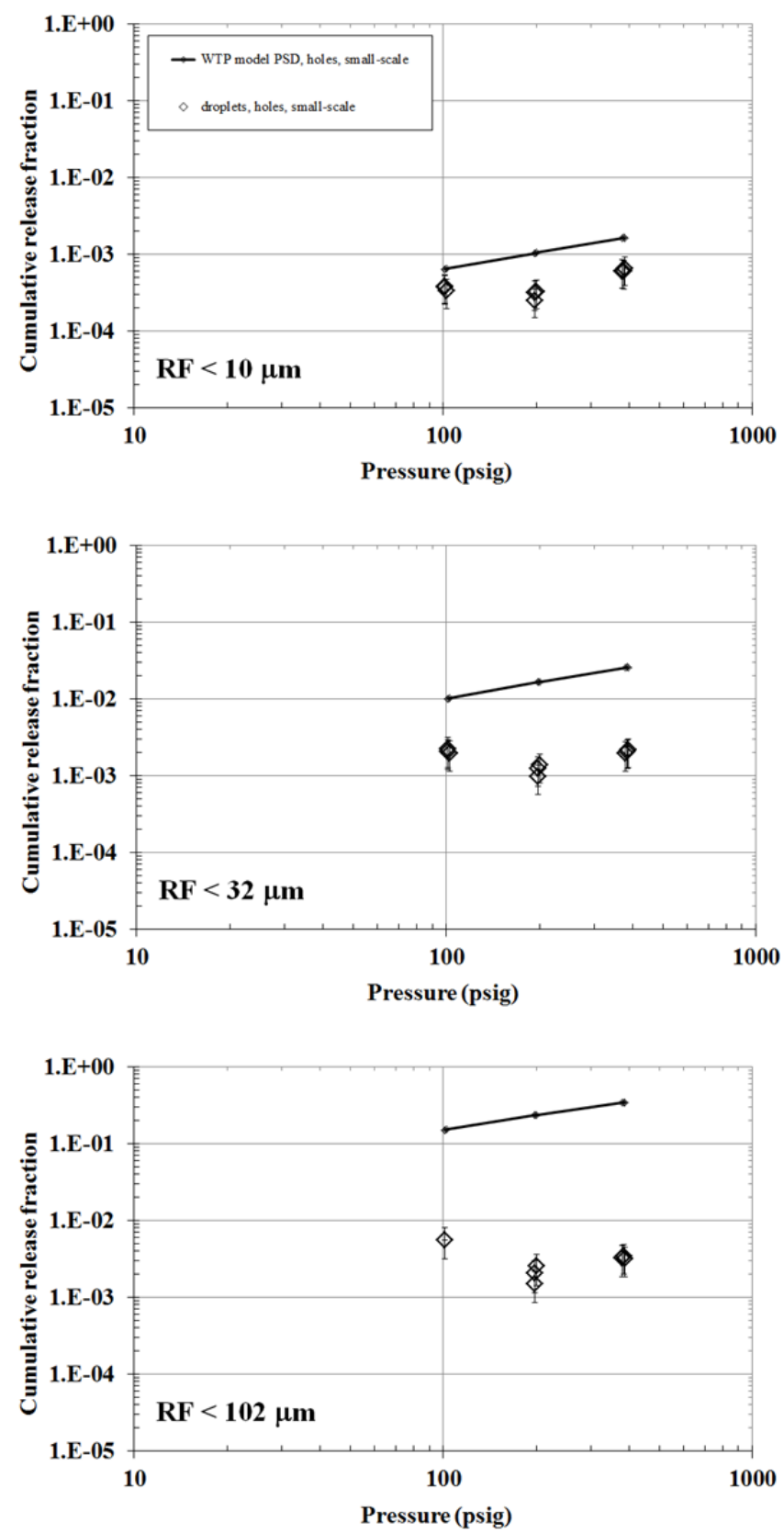

Figure 7.3. Effect of Pressure on Release Fractions for a Spray of 6 Pa Clay from a Target $1 \mathrm{~mm}$ Hole. WTP model predictions are shown by the thick black lines. Error bars show an approximate 95 percent confidence interval for each test including model fit and experimental variability. Appendix D identifies the tests in the plot. 

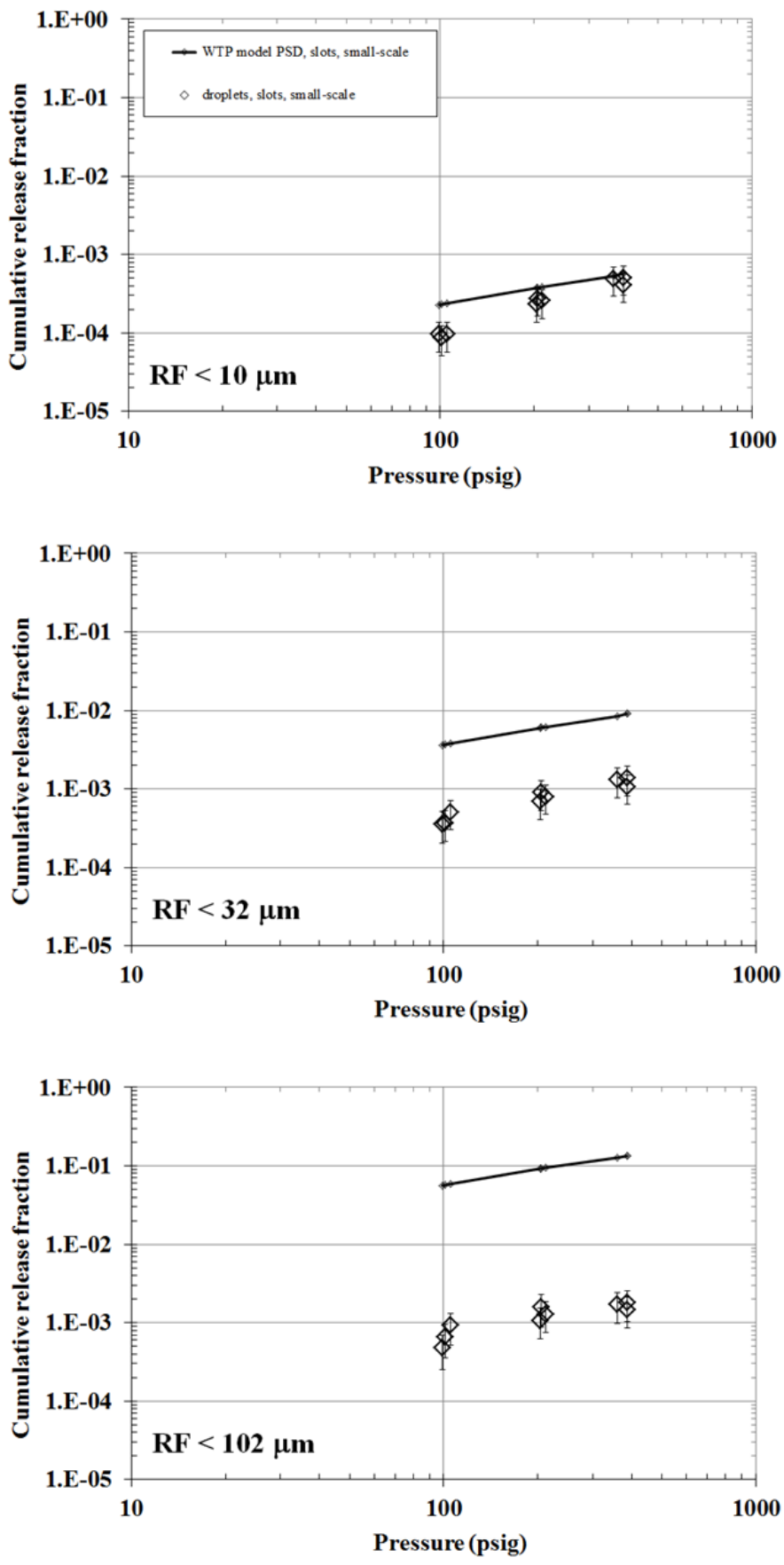

Figure 7.4. Effect of Pressure on Release Fractions for a Spray of 6 Pa Clay from a Target $0.5 \times 5 \mathrm{~mm}$ Slot. WTP model predictions are shown by the thick black lines. Error bars show an approximate 95 percent confidence interval for each test including model fit and experimental variability. Appendix D identifies the tests in the plot. 

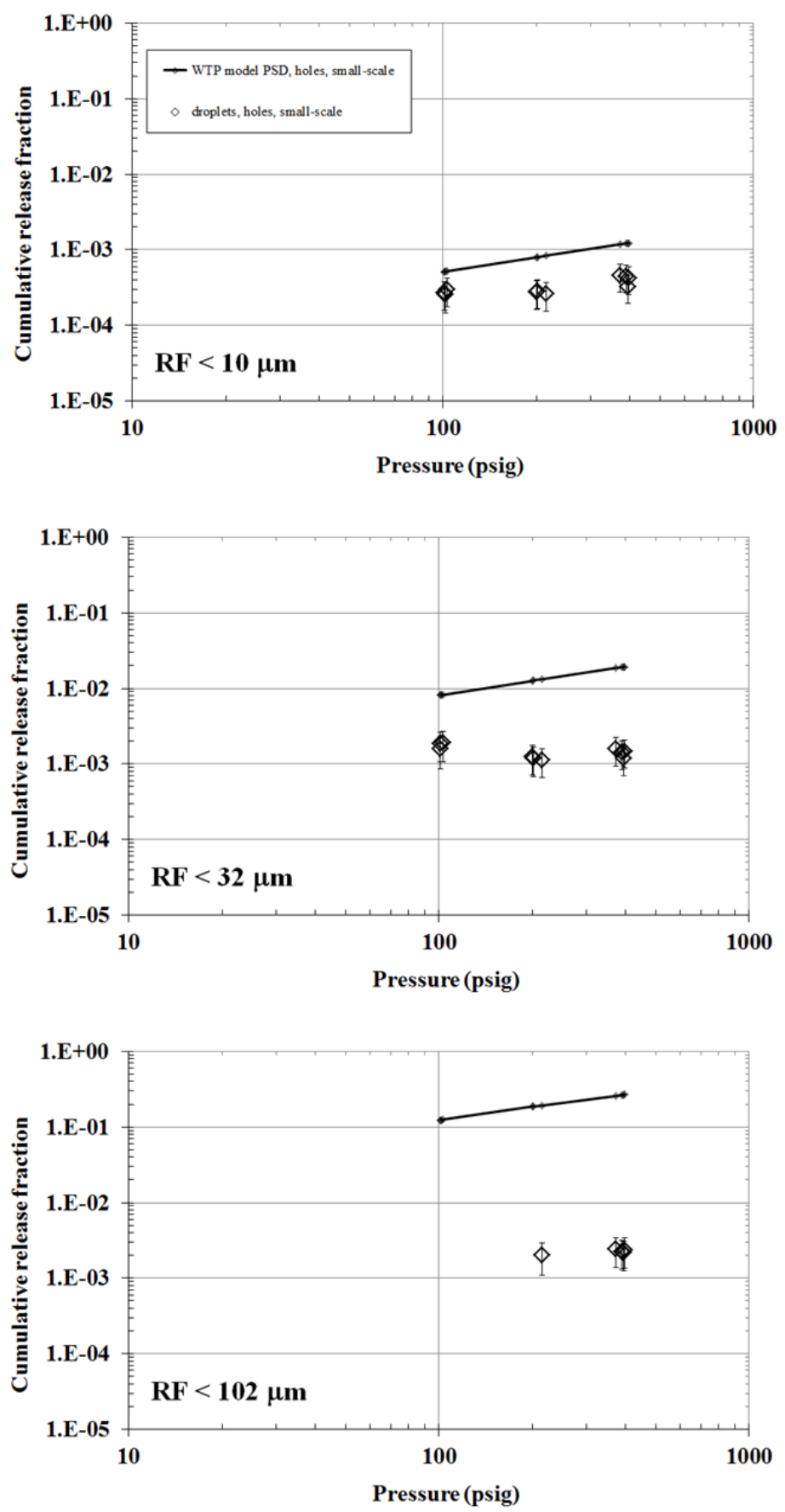

Figure 7.5. Effect of Pressure on Release Fractions for a Spray of $30 \mathrm{~Pa}$ Clay from a Target $1 \mathrm{~mm}$ Hole. WTP model predictions are shown by the thick black lines. Error bars show an approximate 95 percent confidence interval for each test including model fit and experimental variability. Appendix D identifies the tests in the plot. 

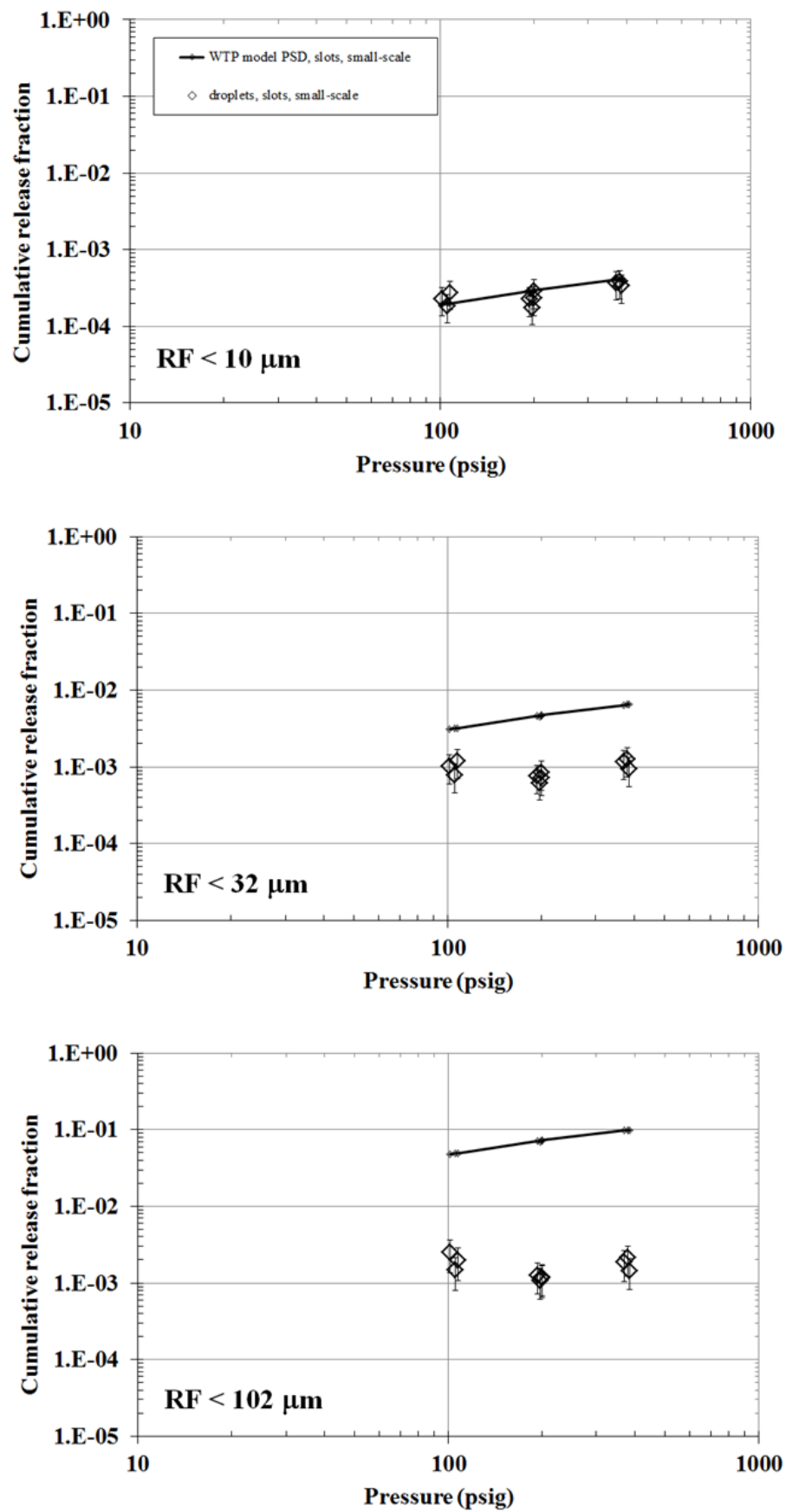

Figure 7.6. Effect of Pressure on Release Fractions for a Spray of $30 \mathrm{~Pa}$ Clay from a Target $0.5 \times 5 \mathrm{~mm}$ Slot. WTP model predictions are shown by the thick black lines. Error bars show an approximate 95 percent confidence interval for each test including model fit and experimental variability. Appendix D identifies the tests in the plot. 

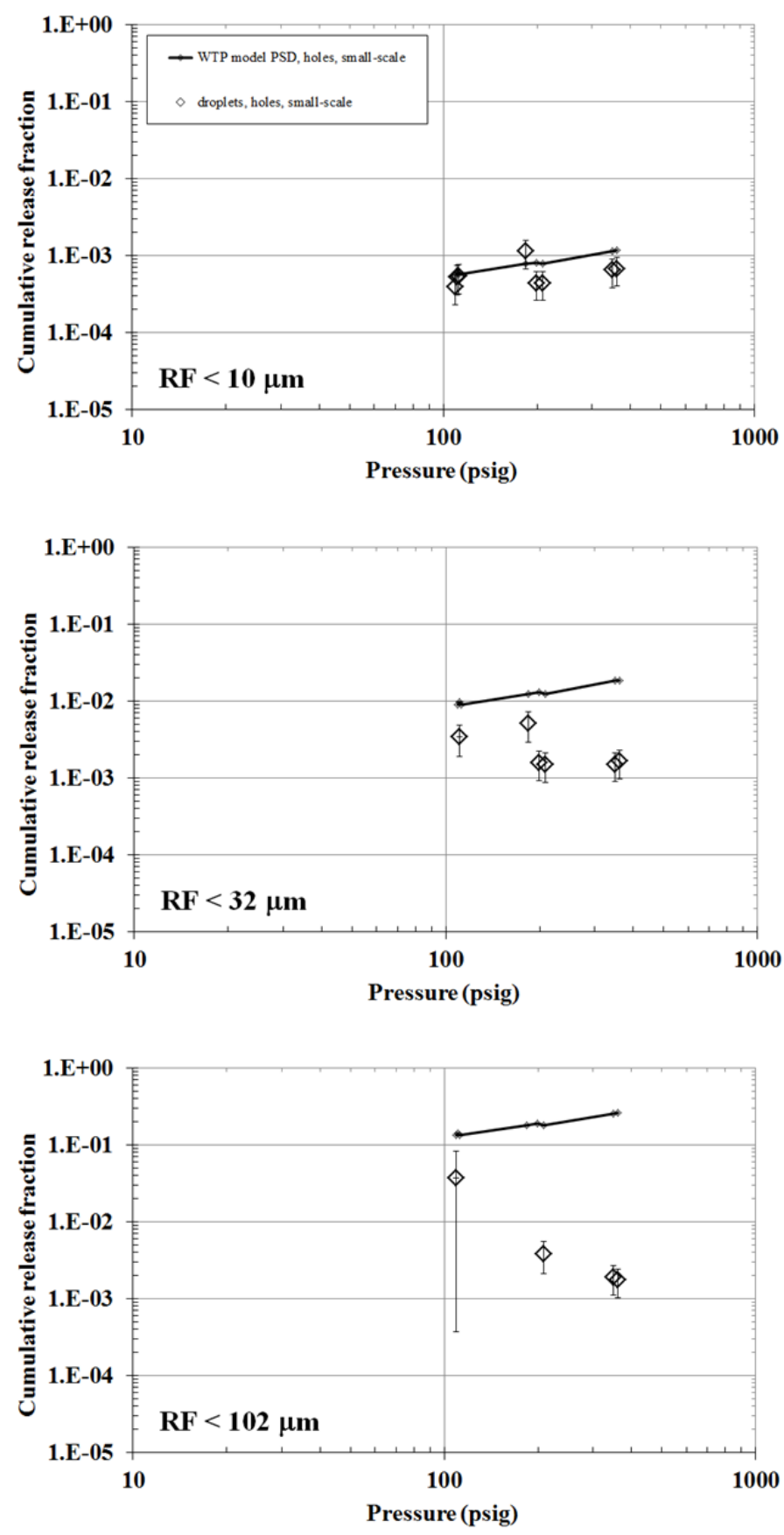

Figure 7.7. Effect of Pressure on Release Fractions for a Spray of 6 Pa FEG from a Target $1 \mathrm{~mm}$ Hole. WTP model predictions are shown by the thick black lines. Error bars show an approximate 95 percent confidence interval for each test including model fit and experimental variability. These tests had initial RHs (measured by an FIO instrument) between 62 and 76 percent. Appendix D identifies the tests in the plot. 

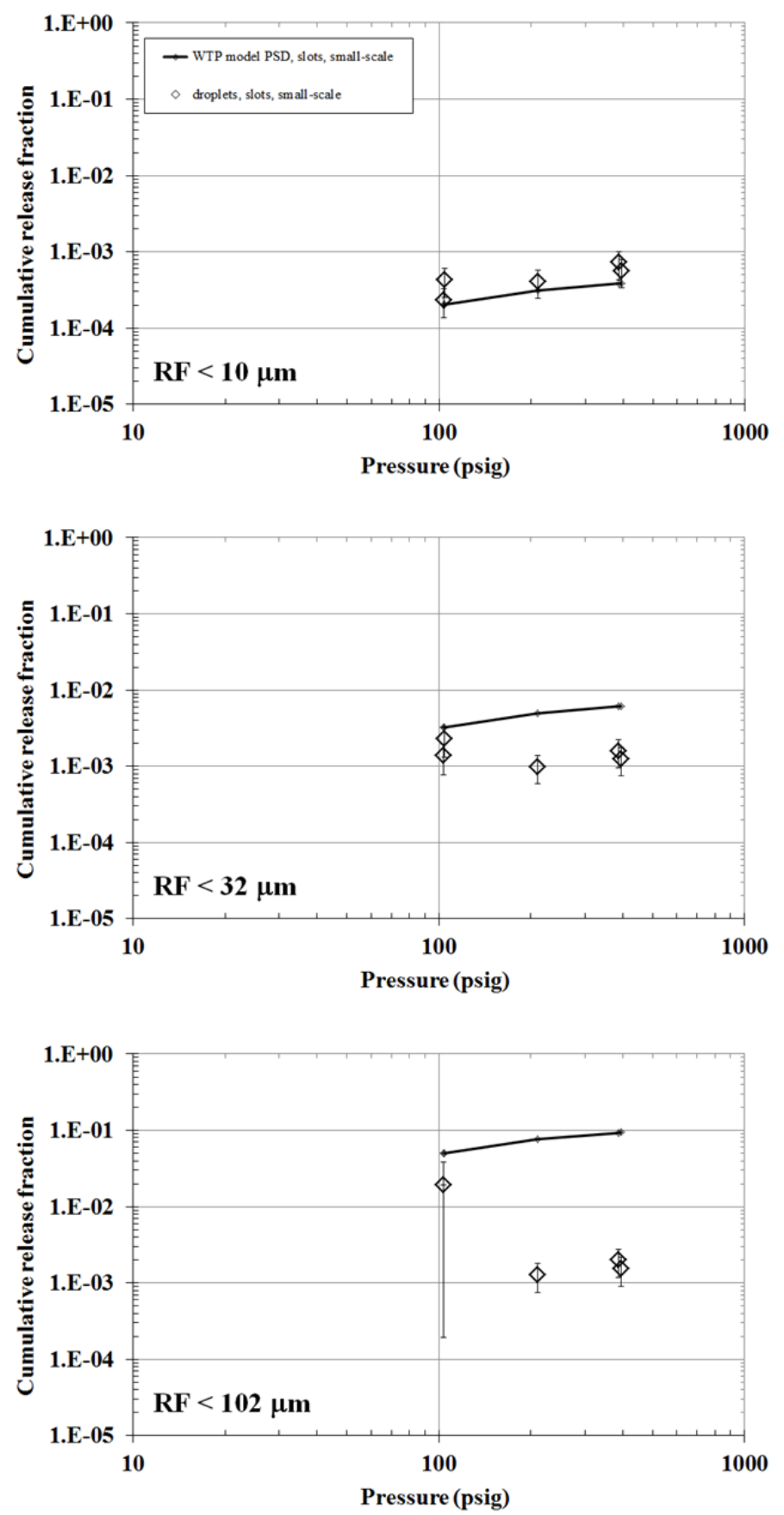

Figure 7.8. Effect of Pressure on Release Fractions for a Spray of 6 Pa FEG from a Target $0.5 \times 5 \mathrm{~mm}$ Slot. WTP model predictions are shown by the thick black lines. Error bars show an approximate 95 percent confidence interval for each test including model fit and experimental variability. In all of these tests except one (at $380 \mathrm{psig}$ ), the initial RH (an FIO measurement) was between 70 and 80 percent. Appendix D identifies the tests in the plot.

The results for the FEG simulant with a target yield stress of $30 \mathrm{~Pa}$ are shown in Figure 7.9 and Figure 7.10. In general, the same behavior is seen for $30 \mathrm{~Pa}$ FEG as for $6 \mathrm{~Pa}$ FEG. 

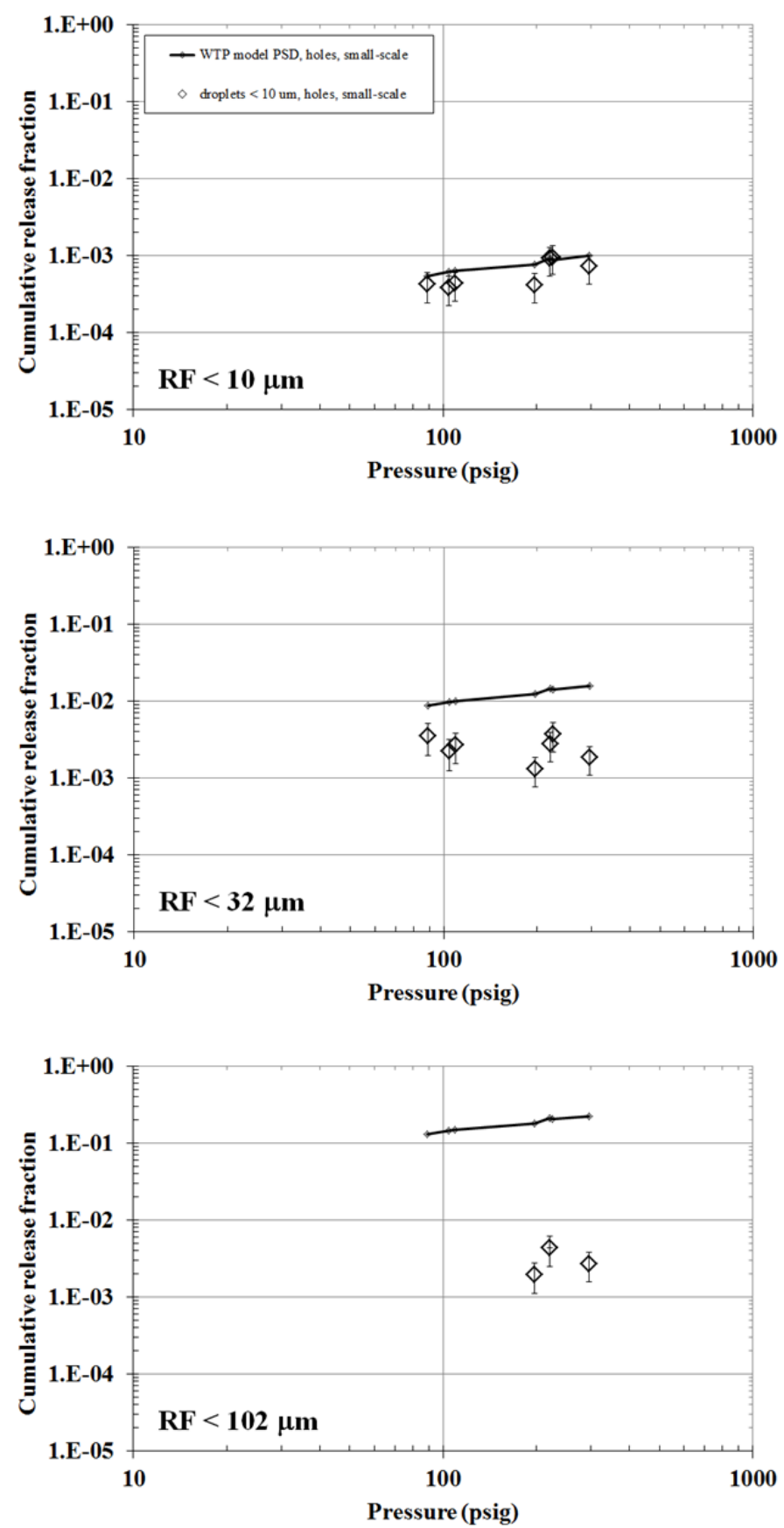

Figure 7.9. Effect of Pressure on Release Fractions for a Spray of 30 Pa FEG from a Target $1 \mathrm{~mm}$ Hole. WTP model predictions are shown by the thick black lines. Error bars show an approximate 95 percent confidence interval for each test including model fit and experimental variability. These tests had initial RHs (measured by an FIO instrument) between 67 and 87 percent. Appendix D identifies the tests in the plot. 

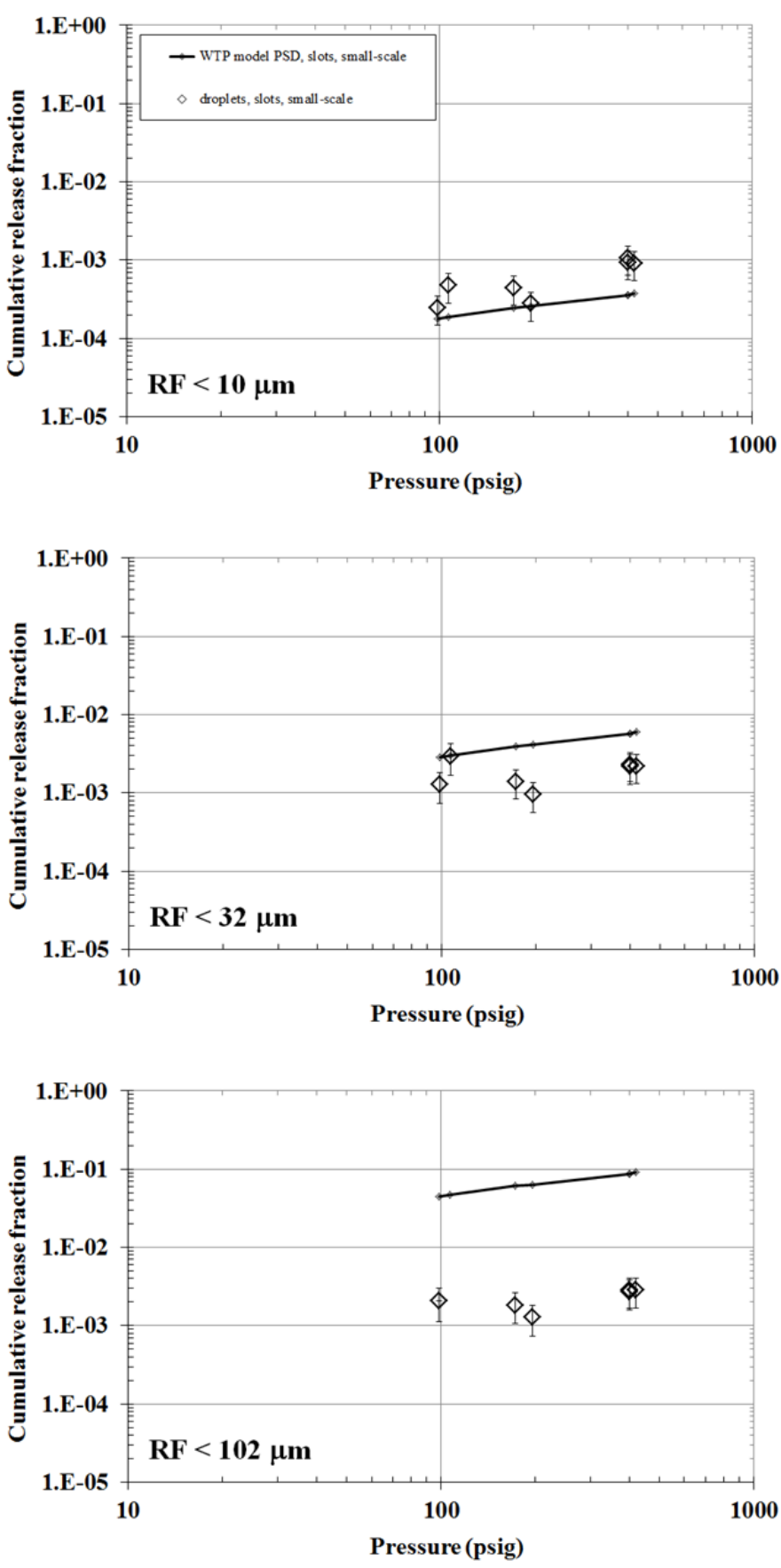

Figure 7.10. Effect of Pressure on Release Fractions for a Spray of 30 Pa FEG from a Target $0.5 \times 5 \mathrm{~mm}$ Slot. WTP model predictions are shown by the thick black lines. Error bars show an approximate 95 percent confidence interval for each test including model fit and experimental variability. Appendix D identifies the tests in the plot.

The $30 \mathrm{~Pa}$ FEG tests that used the $1 \mathrm{~mm}$ round hole had initial RHs (measured by an FIO instrument), for the most part, below 80 percent; humidity was 75 to 87 percent for the 100 psig tests, 70 to 82 percent for the 200 psig tests, and 67 percent for the 380 psig test. For the tests that used the $0.5 \times 5 \mathrm{~mm}$ slot, the 
initial humidities (FIO) ranged between 80 and 88 percent, a range in which evaporation would be expected to have produced little effect.

A high-solids Newtonian slurry simulant, $27 \mathrm{wt} \%$ STR in water, was also tested. The results for the STR-27 are shown in Figure 7.11 and Figure 7.12. For the $1 \mathrm{~mm}$ round hole, the release fractions were nearly constant with pressure for all three droplet size ranges. For the $0.5 \times 5 \mathrm{~mm}$ slot, the release fractions for $<10$ - $\mu \mathrm{m}$ droplets matched the pressure dependence of the WTP predictions, while the release fractions for $<32-\mu \mathrm{m}$ droplets and $<102-\mu \mathrm{m}$ droplets were approximately constant with pressure.

\subsection{Effect of Solids Loading in Newtonian Slurry}

Phase I testing of Newtonian slurries included slurries with solids concentrations of 8 and $20 \mathrm{wt} \%$. In Phase II, the concentration range for the STR boehmite slurry was extended upward to $27 \mathrm{wt} \%$ solids to address an increase in the maximum solids concentration in some process steps. Figure 7.13 and Figure 7.14 provide comparisons of release fractions at $380 \mathrm{psig}$ for water and three different STR concentrations, using the $1 \mathrm{~mm}$ round hole and the $0.5 \times 5 \mathrm{~mm}$ slot, respectively. The plots show the geometric means of each set of replicate runs, with error bars on the geometric means that represent the maximum and minimum measurement of the set of runs. When one run is shown for a test condition, as is usually the case for Phase I tests, the error band is an approximate 95 percent confidence interval including model fit and experimental variability.

As was noted in Phase I, the solids concentration does not seem to have had a monotonic effect on the release fraction. The $8 \mathrm{wt} \%$ slurry (Phase I) gave release fractions that were lower than those of water and of higher concentrations of STR. The tests with water (Phase II), $20 \mathrm{wt} \% \mathrm{STR}$ (Phase I), and $27 \mathrm{wt} \%$ STR (Phase II) gave release fractions that were within each other's error bars across the droplet size range of interest. The $27 \mathrm{wt} \%$ slurry produced slightly higher release fractions than water for the slot, at most about 50 percent higher in the size range between 10 and $100 \mu \mathrm{m}$. The data give the impression that, for this boehmite simulant, solids begin by depressing the release fraction (as for the $8 \mathrm{wt} \%$ simulant), then increase the release fraction as solids are increased to 20 and $27 \mathrm{wt} \%$. However, the small number of tests and the change in the Malvern Insitec-S instrument lens from Phase I to Phase II make the trend more ambiguous.

Recall that the Phase II data typically have higher release fractions than the Phase I data due to the use of the $500 \mathrm{~mm}$ lens (see Section 6.4.1), making it difficult to make a quantitative comparison between data from Phase I ( 8 and $20 \mathrm{wt} \%$ ) and data from Phase II (27 wt\% and water). Phase I measurements would likely have increased if they had been measured in Phase II, which might have given the $20 \mathrm{wt} \%$ STR the highest release fractions. Further, each of these Phase I tests were conducted only once, so it is difficult to assign significance to the differences between the 8 and $20 \mathrm{wt} \%$ release fractions and the other tests.

\subsection{Effect of Dense Particles}

Another concern addressed by Phase II testing was the effect of small concentrations of particles that had much higher density than any that were tested during Phase I. The dense particles chosen for testing were Mo metal with a density of $10.2 \mathrm{~g} / \mathrm{cc}$. Tests were carried out with $0.4 \mathrm{wt} \%$ Mo in water and $0.4 \mathrm{wt} \%$ Mo combined with STR to give a target total solids concentration of $20 \mathrm{wt} \%$. In both these simulants, the 
target concentration of Mo was $1 \mathrm{wt} \%$, but settling in the feed tank decreased the concentration in the spray header. The $0.4 \mathrm{wt} \%$ value was a concentration measured in sprayed material from the Mo/water slurry. The Mo concentration in the as-sprayed STR slurry was not measured, but was assumed to be the same as in water because the viscosity of the STR slurry was nearly the same as that of water.
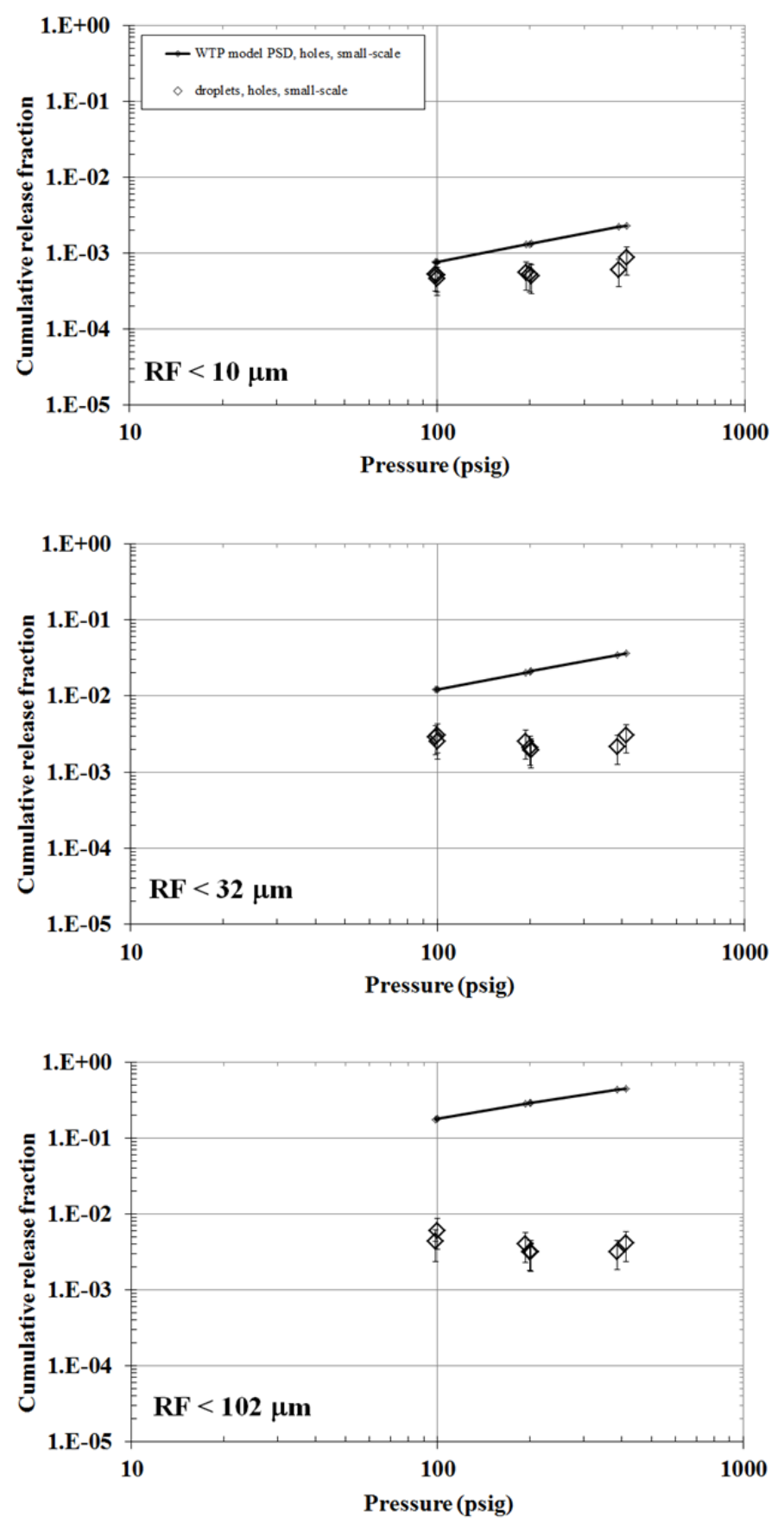

Figure 7.11. Effect of Pressure on Release Fractions for a Spray of $27 \mathrm{wt} \%$ STR from a Target $1 \mathrm{~mm}$ Hole. WTP model predictions are shown by the thick black lines. Error bars show an approximate 95 percent confidence interval for each test including model fit and experimental variability. Appendix D identifies the tests in the plot. 

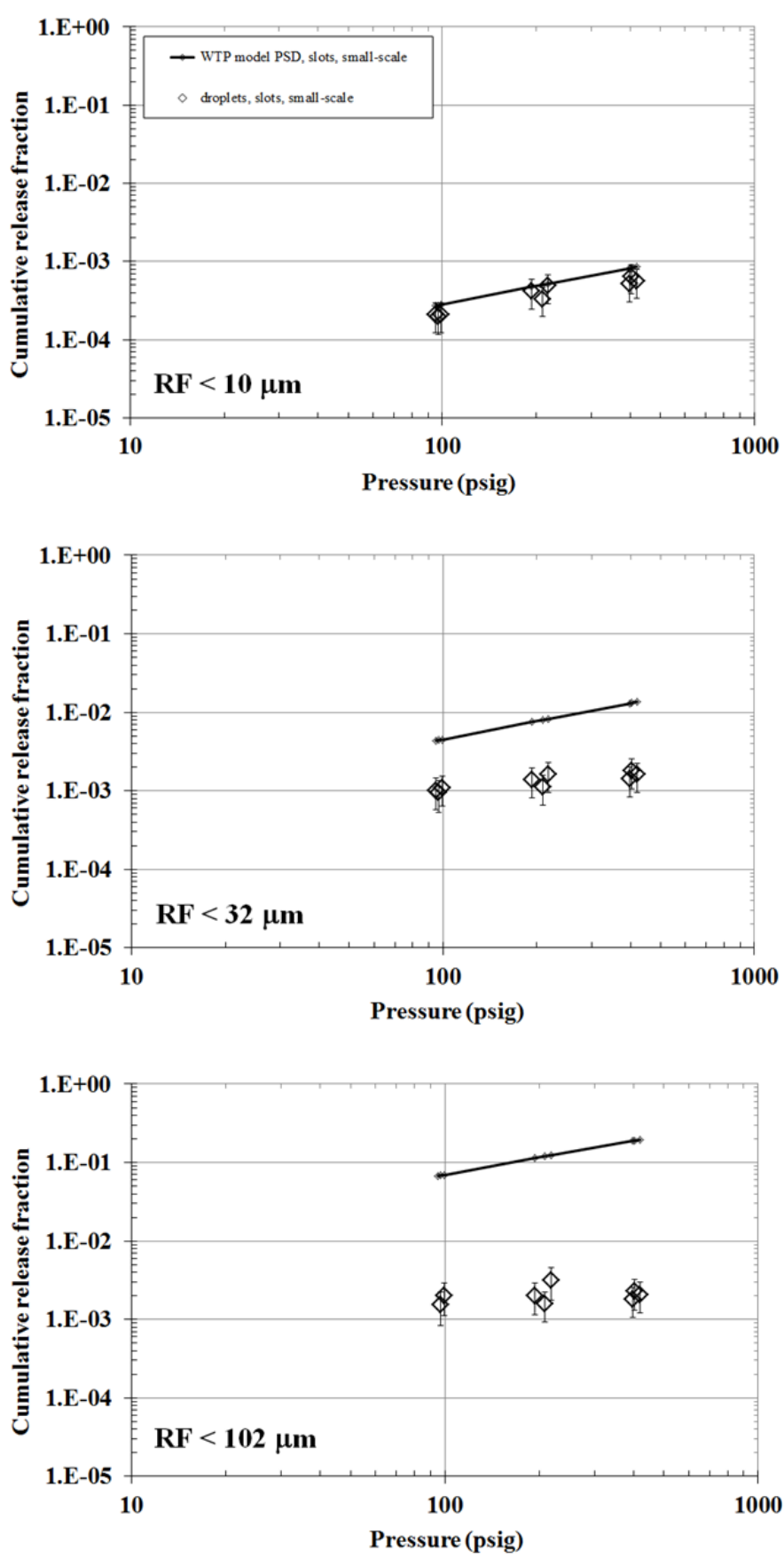

Figure 7.12. Effect of Pressure on Release Fractions for a Spray of $27 \mathrm{wt} \%$ STR from a Target $0.5 \times 5 \mathrm{~mm}$ Slot. WTP model predictions are shown by the thick black lines. Error bars show an approximate 95 percent confidence interval for each test including model fit and experimental variability. Appendix D identifies the tests in the plot. 
$1 \mathrm{~mm}$ hole, 380 psi, position 2, STR

Phase 2, 500-mm lens; Phase 1, 100-mm lens

27 wt\% (Phase 2) vs 20 wt\% (Phase 1) vs 8 wt\% (Phase 1) vs water (Phase 2)

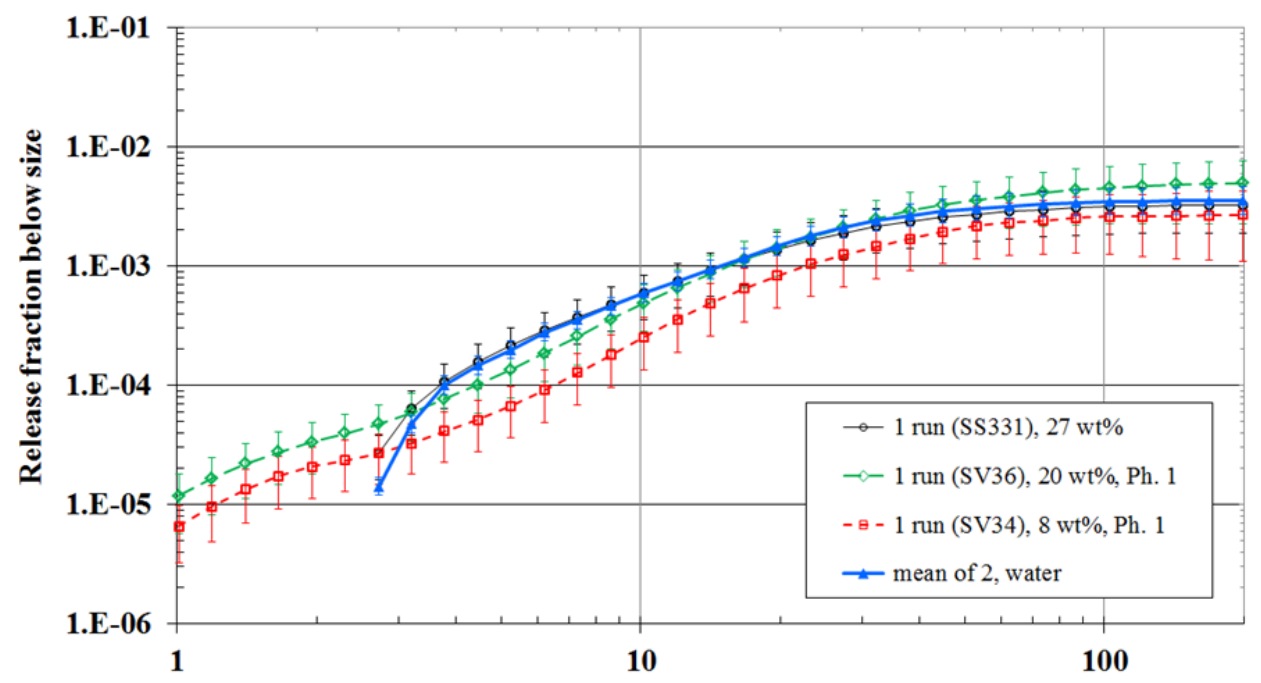

Size $(\mu \mathrm{m})$

Figure 7.13. Effect of STR Concentration for a $1 \mathrm{~mm}$ Round Hole at $380 \mathrm{psig}$. Error bars are maximum-minimum intervals for test conditions with replicate runs and approximate 95 percent confidence intervals for test conditions with only a single available run.

$0.5 \times 5 \mathrm{~mm}$ slot, $380 \mathrm{psi}$, position 2, STR

Phase 2, 500-mm lens; Phase 1, 100-mm lens

27 wt \% (Phase 2) vs 20 wt\% (Phase 1) vs 8 wt\% (Phase 1) vs water (Phase 2)

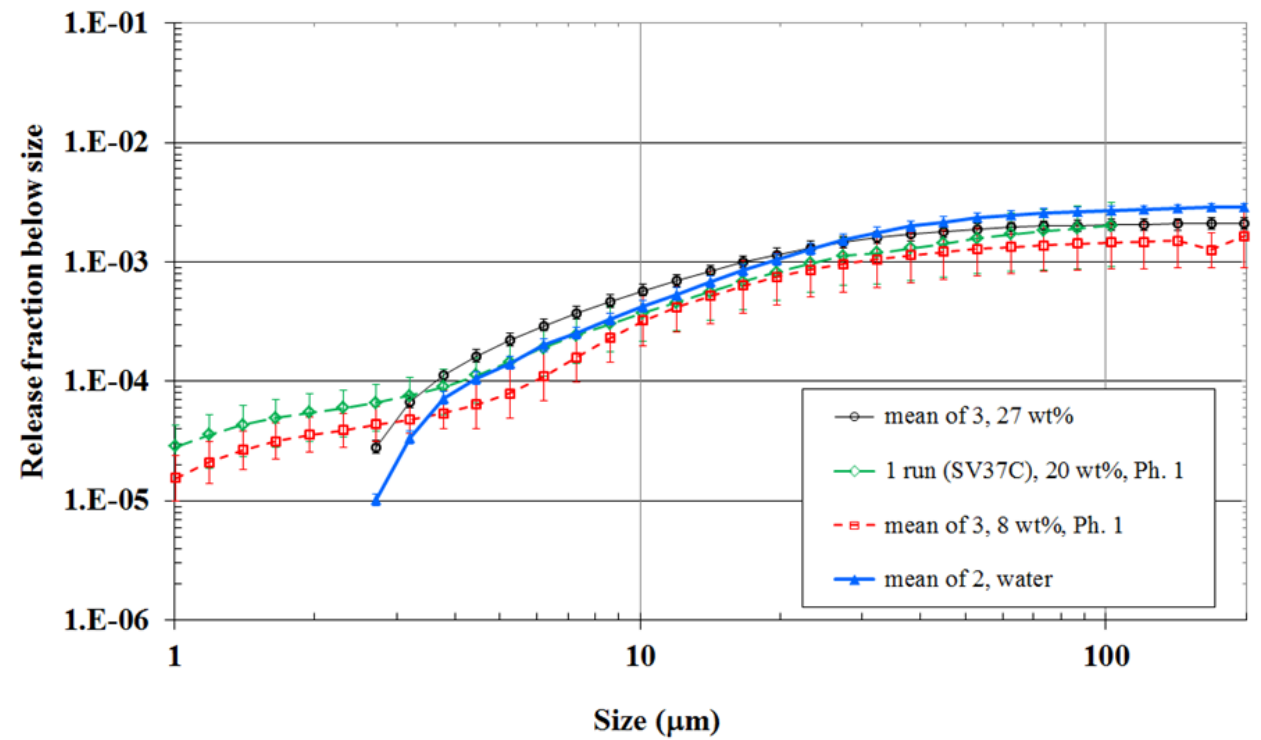

Figure 7.14. Effect of STR Concentration for a $0.5 \times 5 \mathrm{~mm}$ Slot at $380 \mathrm{psig}$. Error bars are maximum-minimum intervals for test conditions with replicate runs and approximate 95 percent confidence intervals for test conditions with only a single available run. 
Figure 7.15 and Figure 7.16 compare the release fractions of water with and without $0.4 \mathrm{wt} \% \mathrm{Mo}$, for jets issuing at 380 psig from a $1 \mathrm{~mm}$ round hole and from a $0.5 \times 5 \mathrm{~mm}$ slot. Figure 7.15 shows that, for the $1 \mathrm{~mm}$ hole, release fractions for droplet sizes above the $<30-\mu \mathrm{m}$ size range were higher for Mo/water than for water. In contrast in Figure 7.16, the mean of the two Mo/water runs that used the $0.5 \times 5 \mathrm{~mm}$ slot is lower at all droplet sizes than the mean of the water runs. Because there was only one successful test of Mo/water and only two tests with water, it is difficult to say whether the observed differences are statistically significant.

Figure 7.17 and Figure 7.18 compare the release fractions of $\sim 20 \mathrm{wt} \%$ STR slurry with and without $0.4 \mathrm{wt} \% \mathrm{Mo}$, for jets issuing at $380 \mathrm{psig}$ from the $1 \mathrm{~mm}$ hole and the $0.5 \times 5 \mathrm{~mm}$ slot. The dense particles appear to have no significant effect on aerosol generation although the limited amount of data prevents a definitive conclusion.

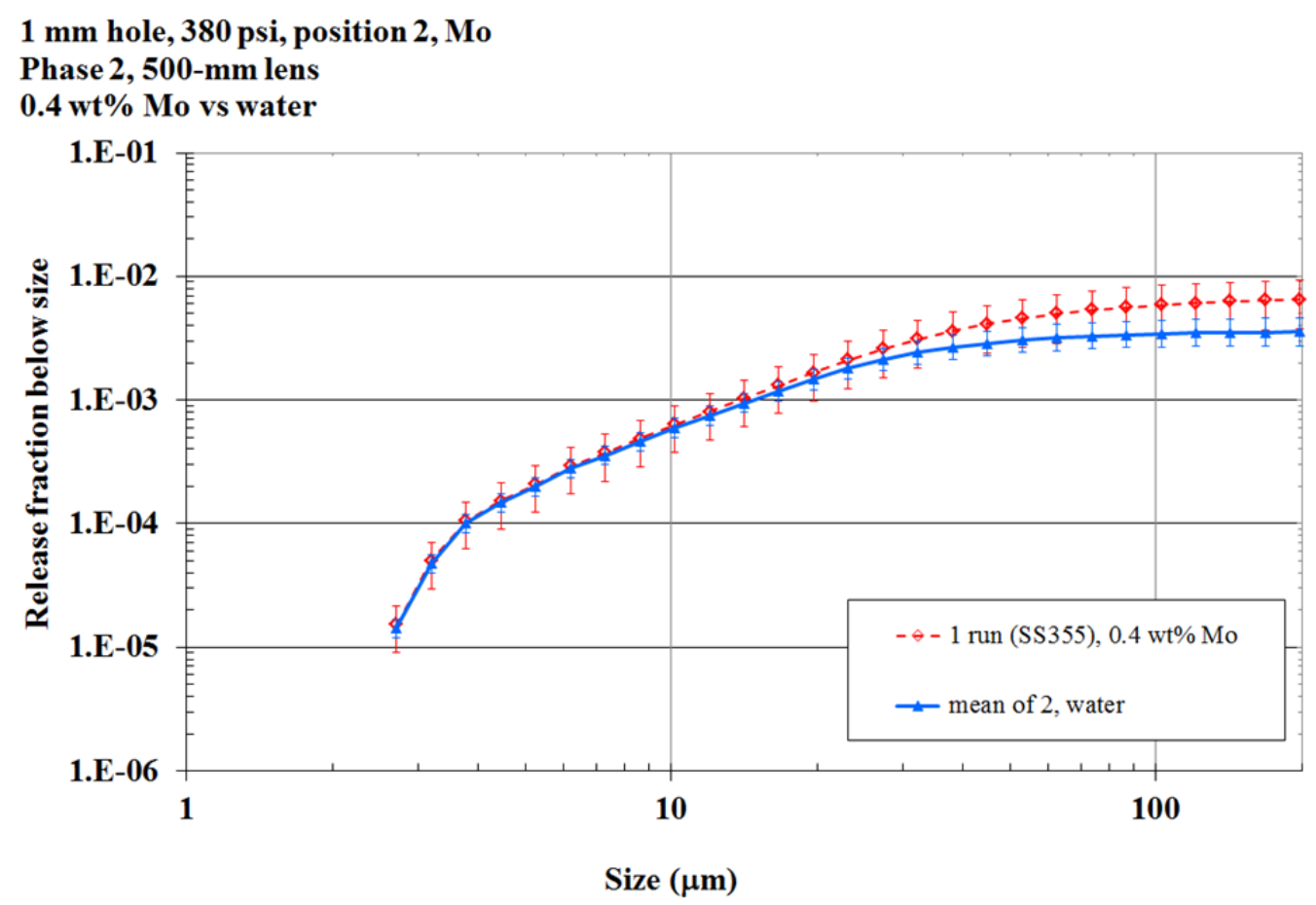

Figure 7.15. Effect of Dense Particle Concentration in Water for a $1 \mathrm{~mm}$ Round Hole at 380 psig. Error bars are maximum-minimum intervals for test conditions with replicate runs and approximate 95 percent confidence intervals for test conditions with only a single available run. 


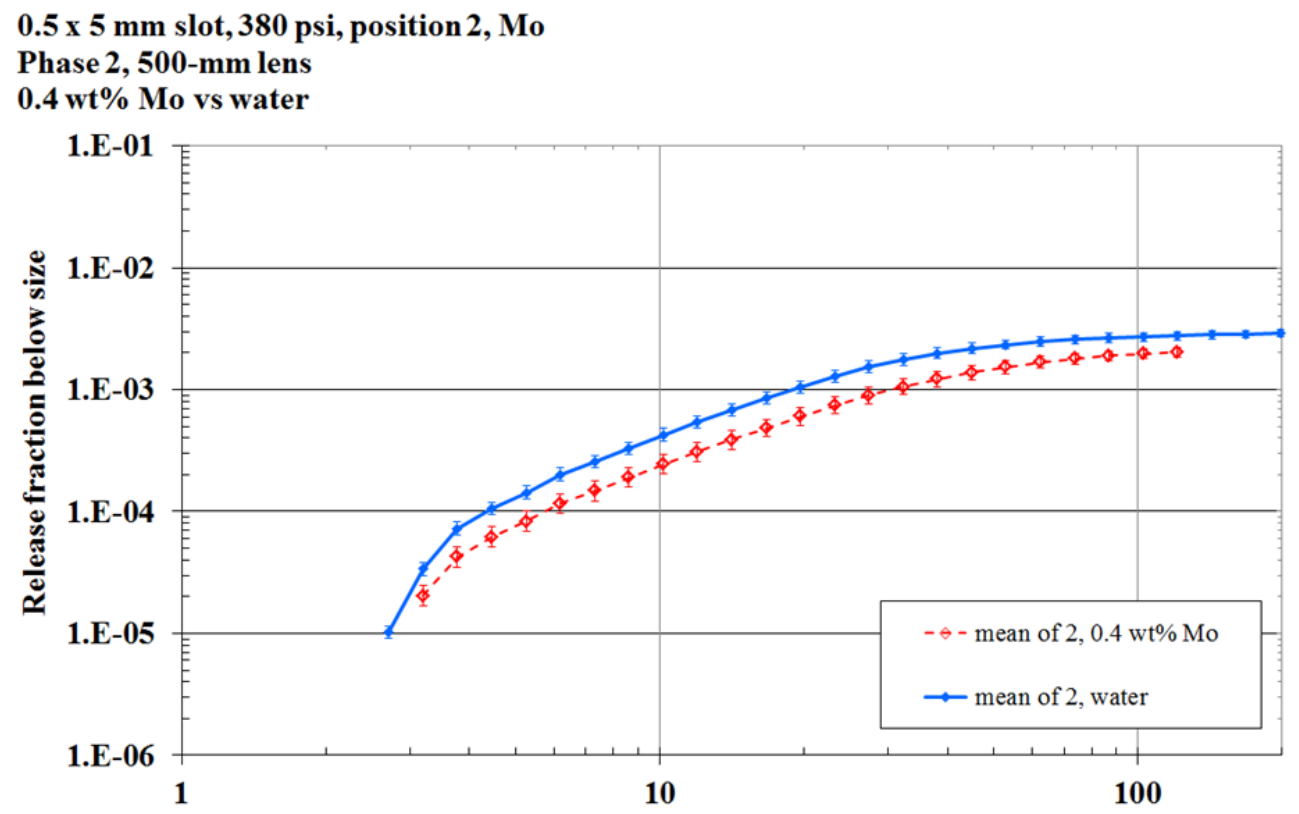

Size $(\mu \mathrm{m})$

Figure 7.16. Effect of Dense Particle Concentration in Water for a $0.5 \times 5 \mathrm{~mm}$ Slot at $380 \mathrm{psig}$. Error bars are maximum-minimum intervals.

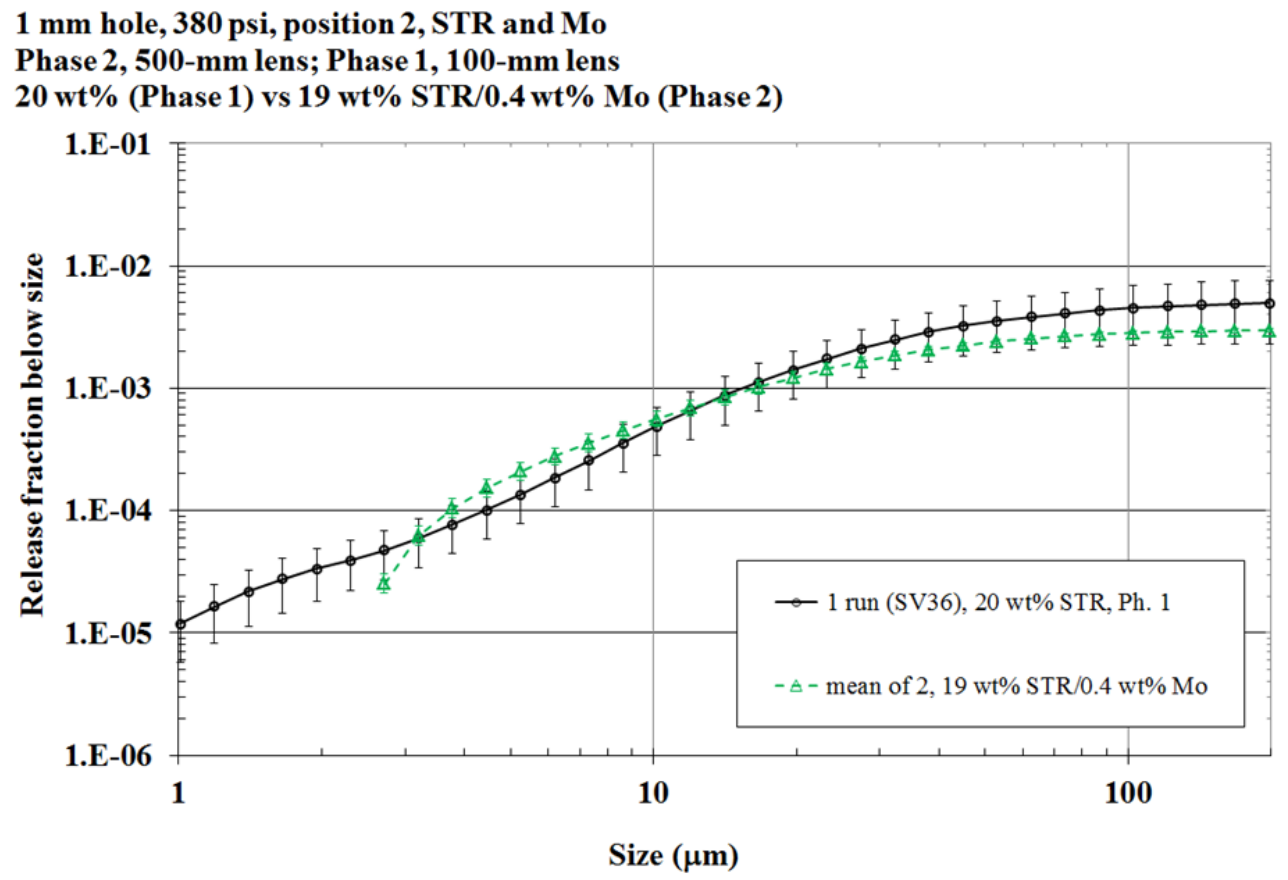

Figure 7.17. Effect of Dense Particle Concentration in $20 \mathrm{wt} \%$ STR Slurry for a $1 \mathrm{~mm}$ Round Hole at 380 psig. Error bars are maximum-minimum intervals for test conditions with replicate runs and approximate 95 percent confidence intervals for test conditions with only a single available run. 


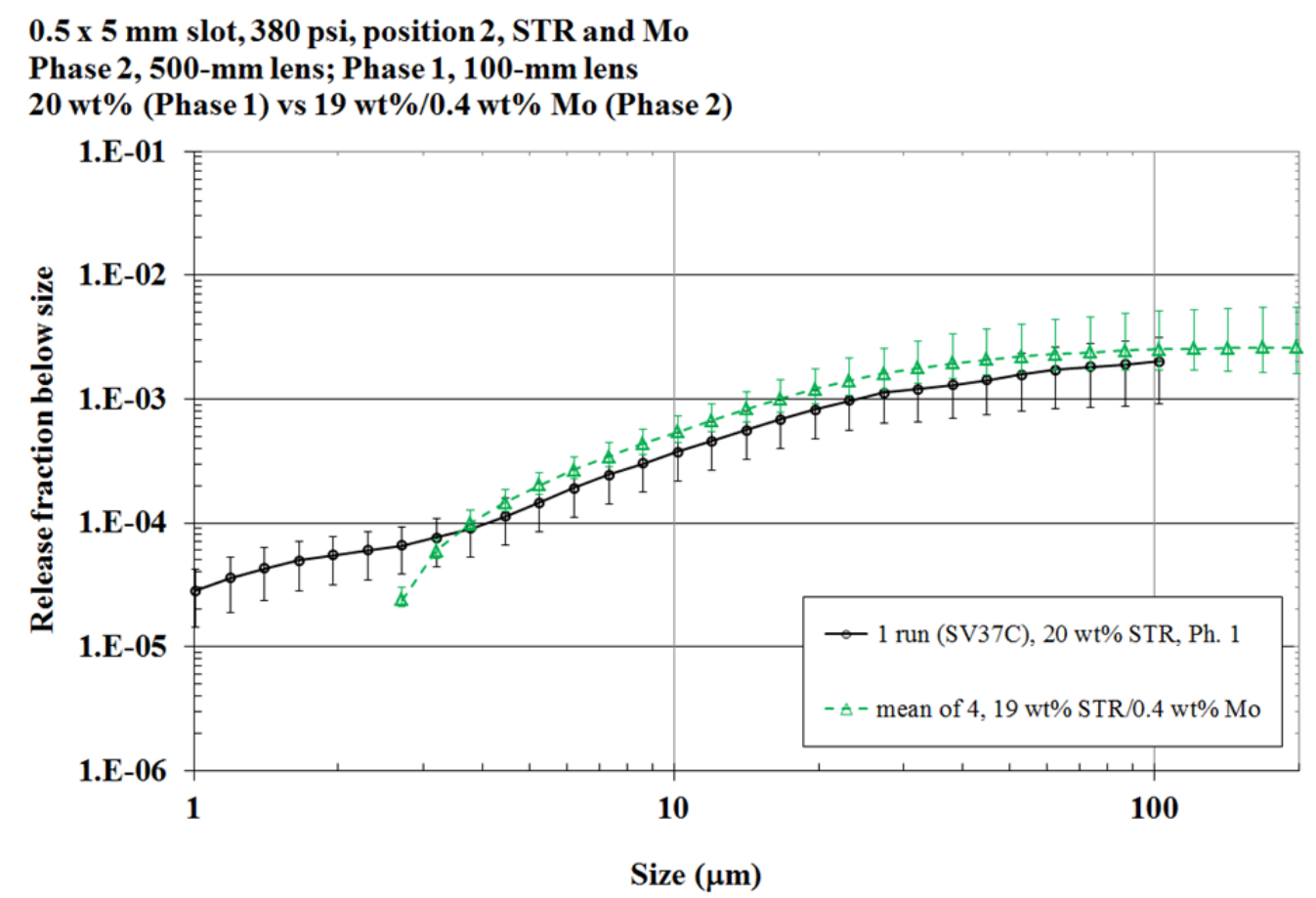

Figure 7.18. Effect of Dense Particle Concentration in $20 \mathrm{wt} \%$ STR Slurry for a $0.5 \times 5 \mathrm{~mm}$ Slot at 380 psig. Error bars are maximum-minimum intervals for test conditions with replicate runs and approximate 95 percent confidence intervals for test conditions with only a single available run.

\subsection{Effect of Non-Newtonian Rheology}

Phase I small-scale testing included one non-Newtonian FER simulant that was used in two formulations. The first, which combined Fe-rich metal oxides and hydroxides with boehmite, was used in one set of tests (identified as "FER-6b"). However, the behavior of FER-6b led to difficulties in accomplishing the testing goals and a different simulant was formulated that combined the same FER solids with gibbsite (identified as FER-6g or FER-30g). The remaining Phase I FER tests were conducted using this simulant at target yield stress of 6 or $30 \mathrm{~Pa}$, with and without AFA.

Phase II testing used two different non-Newtonian simulants, the gibbsite formulation of the FER simulant (identified simply as FEG in this report) and clay. These non-Newtonian simulants were tested at two different rheologies: nominally $30 \mathrm{~Pa} / 30 \mathrm{cP}$ FEG or clay and $6 \mathrm{~Pa} / 6 \mathrm{cP}$ FEG or clay. These simulants are often referred to using just their yield stress for brevity (e.g., 6 Pa clay). Section 3 contains more detail about these simulants. The objectives of small-scale non-Newtonian spray testing were to determine the effects of bounding Bingham rheology parameters and to define the relationship of the aerosol generation behavior between the non-Newtonian simulants. The relationship between FEG and clay release fractions was to be used as part of the analysis of large-scale data, because the only non-Newtonian simulant used at the large scale was clay.

Section 7.4.1 discusses the effect of rheological properties for each of the two non-Newtonian simulants. Section 7.4.2 shows the relationship of FEG and clay release fractions for different orifices 
and pressures. The question of whether the FEG, which exhibited unusual history-dependent thickening properties, provided results applicable to tank waste is discussed in Section 7.4.3.

\subsubsection{Rheology Effects}

This section considers the effect on the release fraction of changing the Bingham rheological parameters of non-Newtonian slurries. Given that the Bingham parameters did not vary independently of each other, observing the effect of only one rheological parameter (e.g., yield stress or consistency) is not possible. Tests to determine how release fraction varies with a rheological parameter independent of the other parameter would require alternative simulant formulations.

Even though the data are not compared directly to the model, the WTP model is a useful starting point for discussing the expected effect of rheology. The WTP spray release model is based on Newtonian rheological properties and does not account for non-Newtonian behavior. Thus, the Bingham consistency (or similar) of a non-Newtonian fluid is input into the WTP model, whereas the yield stress is not a model parameter. The model includes a small effect of liquid (or slurry) viscosity on the release fraction, such that the SMD has a weak positive dependence on viscosity. This results in a small decrease in the release fraction of smaller droplets with increasing viscosity if all other parameters remain the same.

Figure 7.19 through Figure 7.24 compare the release fractions obtained from water (0 Pa yield stress), $6 \mathrm{~Pa}$ clay slurry, and $30 \mathrm{~Pa}$ clay slurry, using two different orifices and three different pressures. The plots show the geometric means of each set of replicate runs, with error bars on the geometric means that represent the maximum and minimum measurement of the set of runs. All the plotted data are from Phase II tests.

In general, as was noted in Section 6.5, the clay tests showed high repeatability with three or four replicates. For this reason, the comparisons between the two strengths of clay are less ambiguous than the comparisons between either clay simulant or water (because for the Phase II water tests there were usually only two replicates).

For five out of the six test conditions, the release fractions for the 6 Pa clay equaled or slightly exceeded those for the $30 \mathrm{~Pa}$ clay. The exception was the $100 \mathrm{psig}$ spray from the $0.5 \times 5 \mathrm{~mm}$ slot, where the $30 \mathrm{~Pa}$ release fractions were about triple the $6 \mathrm{~Pa}$ release fractions. Each of the clays showed repeatability within a factor of two or less (Figure 6.15 and Figure 6.16), so spray variability is not the cause. In the droplet size range of interest $(<10$ to $<100 \mu \mathrm{m})$, the clay release fractions were always less than or equal to those of water, within the apparent variability.

Figure 7.25 through Figure 7.30 compare the release fractions obtained from water (0 Pa yield stress), 6 Pa FEG slurry, and 30 Pa FEG slurry, using two different orifices and three different pressures. The plots show the geometric means of each set of replicate runs, with error bars on the geometric means that represent the maximum and minimum of the set of runs. When there is only one run for a test condition, no error band is shown. All the plotted data are from Phase II tests. 
$1 \mathrm{~mm}$ hole, $100 \mathrm{psi}$, Phase 2, 500-mm lens, position 2

$\sim 30-\mathrm{Pa} / 30-\mathrm{mPa}$ s clay vs $\sim 6-\mathrm{Pa} / 6-\mathrm{mPa}$ s clay vs water

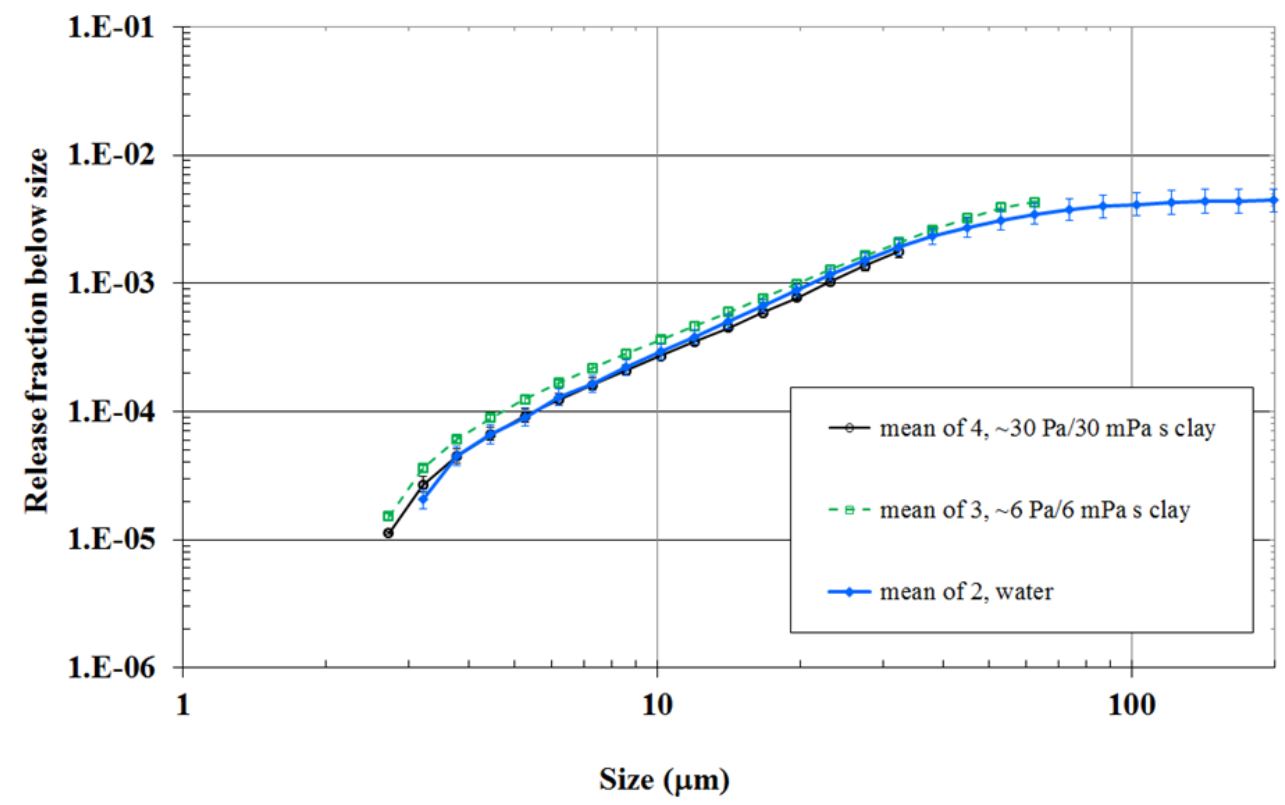

Figure 7.19. Effect of Rheological Parameters in Clay Slurry for a $1 \mathrm{~mm}$ Round Hole at 100 psig. Error bars are maximum-minimum intervals.

$1 \mathrm{~mm}$ hole, $200 \mathrm{psi}$, Phase 2, 500-mm lens, position 2 $\sim 30-\mathrm{Pa} / 30 \mathrm{mPa} s$ clay $\mathrm{vs} \sim 6-\mathrm{Pa} / 6-\mathrm{mPa}$ s clay vs water

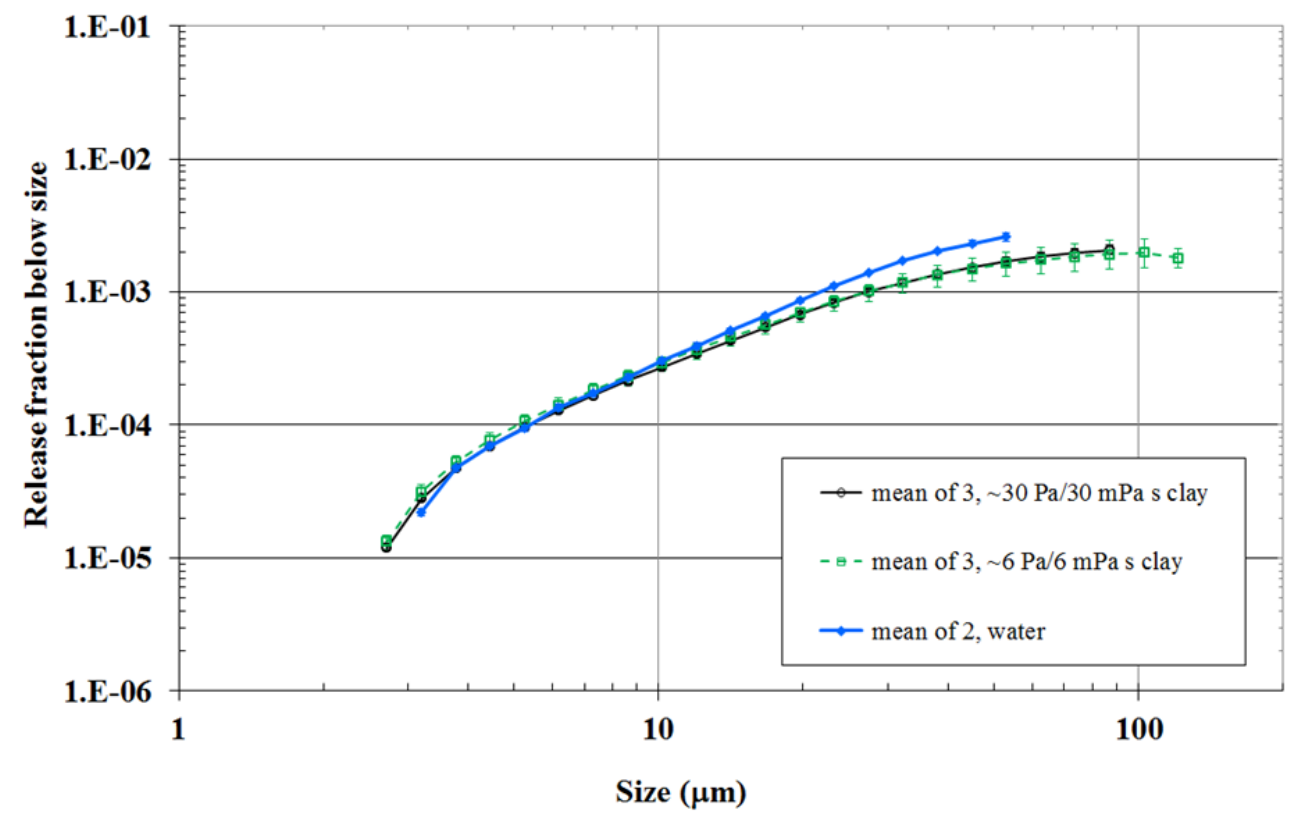

Figure 7.20. Effect of Rheological Parameters in Clay Slurry for a $1 \mathrm{~mm}$ Round Hole at 200 psig. Error bars are maximum-minimum intervals. 
$1 \mathrm{~mm}$ hole, 380 psi, Phase 2, 500-mm lens, position 2

$\sim 30-\mathrm{Pa} / 30-\mathrm{mPa}$ s clay vs $\sim 6-\mathrm{Pa} / 6-\mathrm{mPa}$ s clay vs water

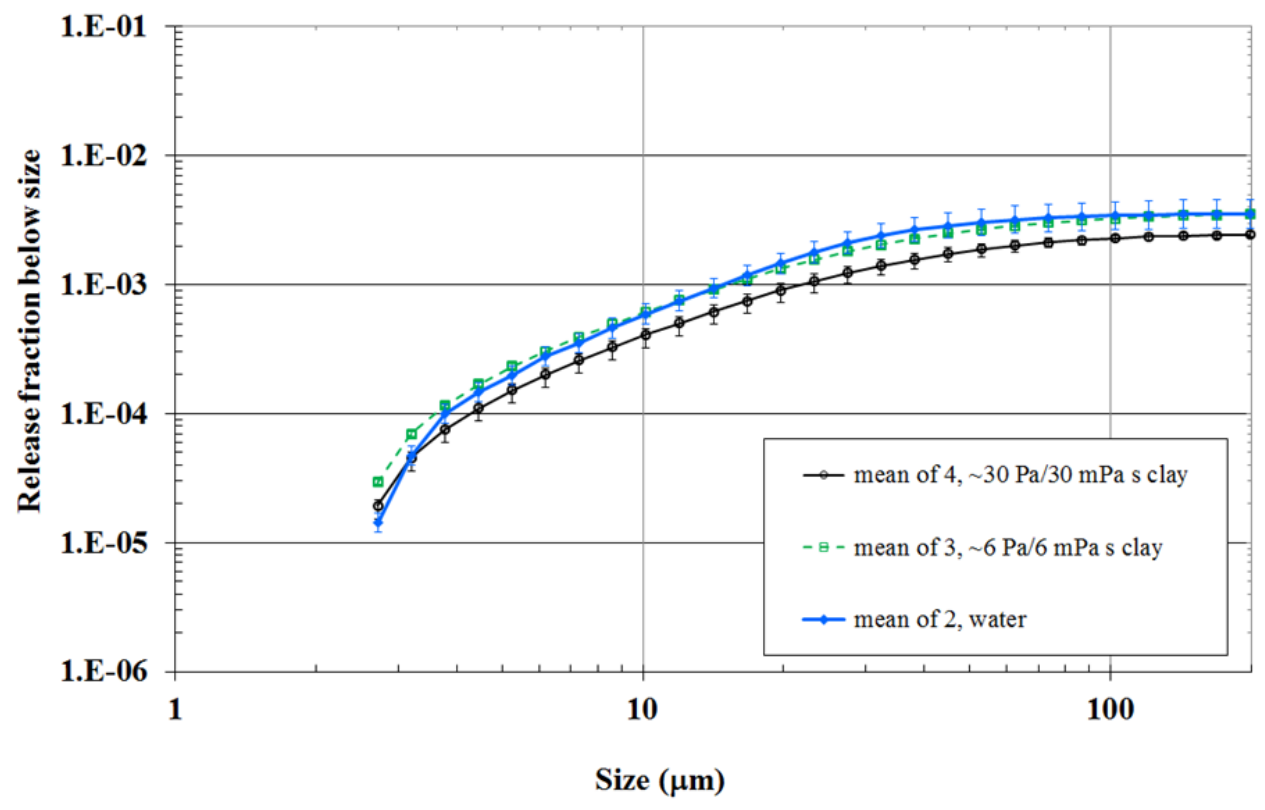

Figure 7.21. Effect of Rheological Parameters in Clay Slurry for a $1 \mathrm{~mm}$ Round Hole at 380 psig. Error bars are maximum-minimum intervals.

$0.5 \times 5 \mathrm{~mm}$ slot, $100 \mathrm{psi}$, Phase 2, 500-mm lens, position 2 $\sim 30-\mathrm{Pa} / 30$-mPa s clay vs $\sim 6-\mathrm{Pa} / 6-\mathrm{mPa}$ s clay vs water

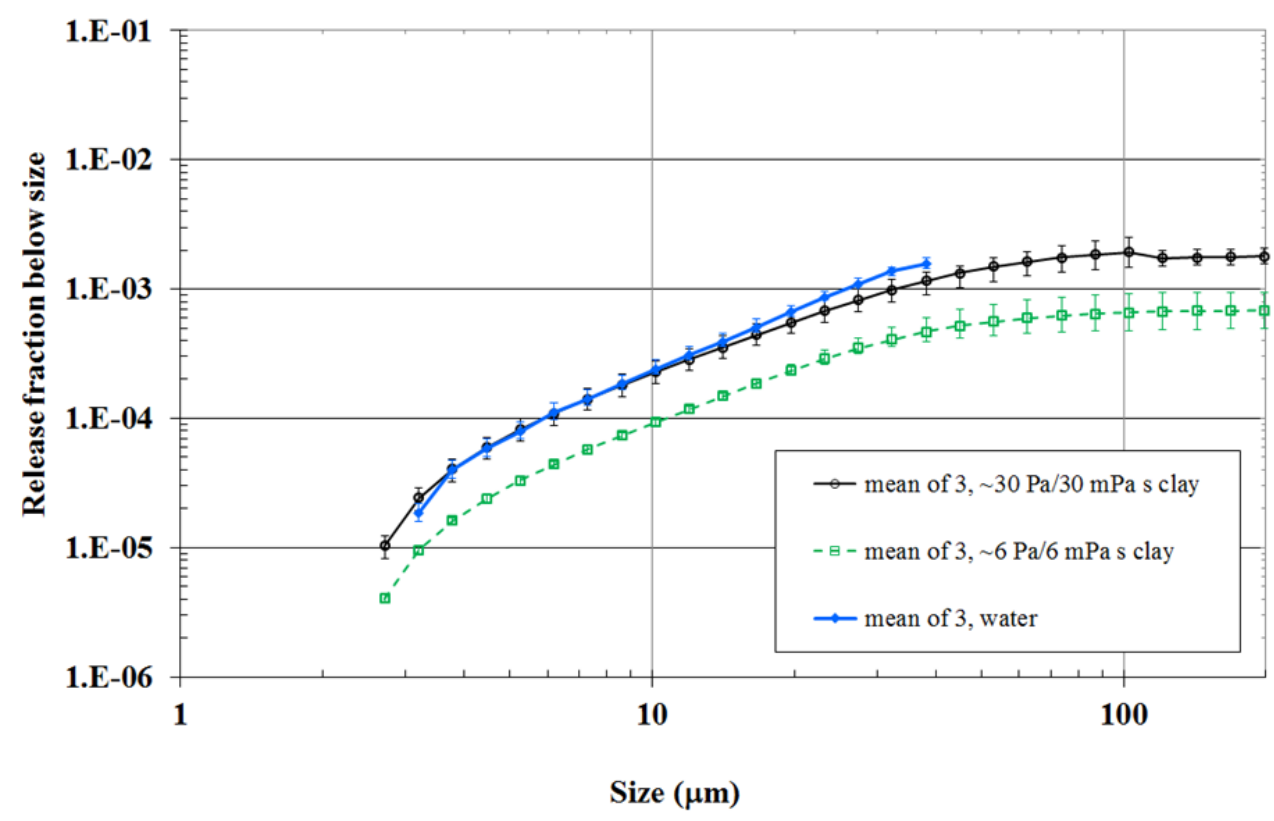

Figure 7.22. Effect of Rheological Parameters in Clay Slurry for a $0.5 \times 5 \mathrm{~mm}$ Slot at $100 \mathrm{psig}$. Error bars are maximum-minimum intervals. 
$0.5 \times 5$ mm slot, 200 psi, Phase 2, 500-mm lens, position 2

$\sim 30-\mathrm{Pa} / 30-\mathrm{mPa}$ s clay vs $\sim 6-\mathrm{Pa} / 6-\mathrm{mPa}$ s clay vs water

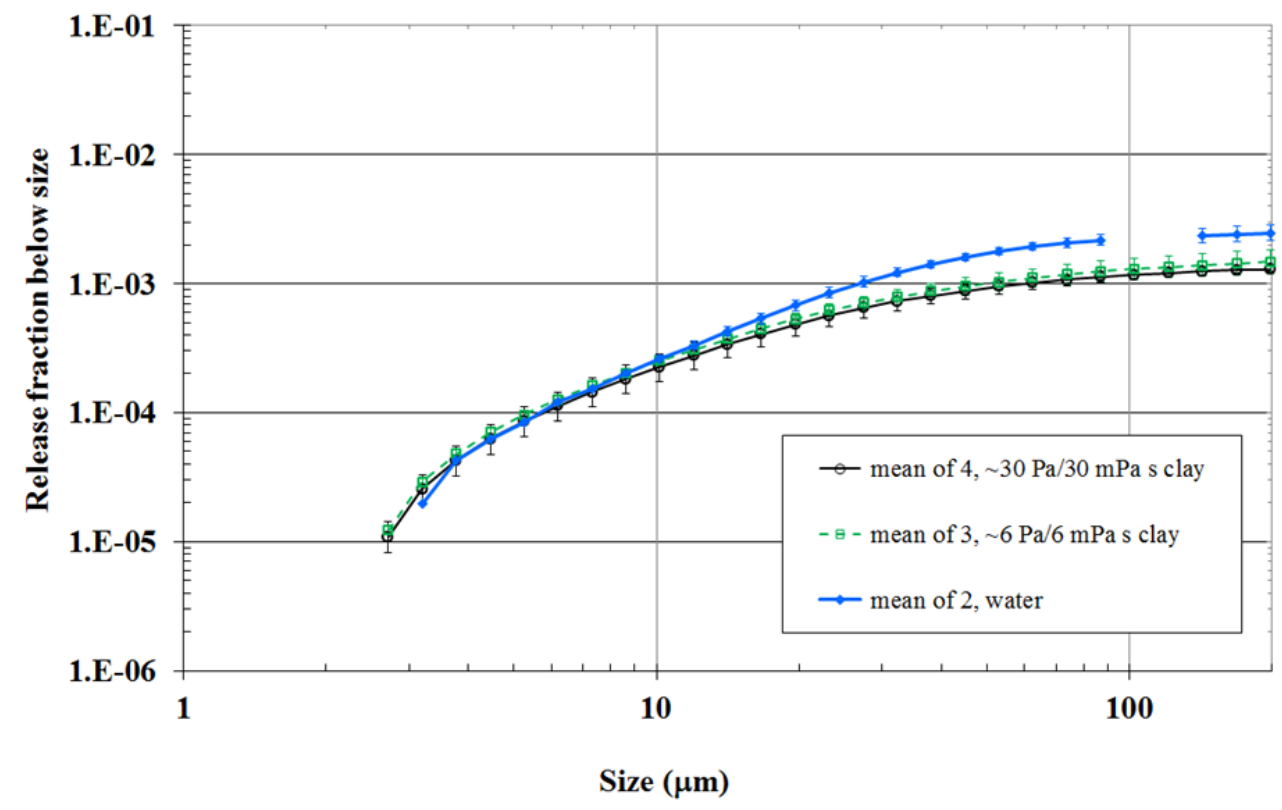

Figure 7.23. Effect of Rheological Parameters in Clay Slurry for a $0.5 \times 5 \mathrm{~mm}$ Slot at 200 psig. Error bars are maximum-minimum intervals.

0.5 x 5 mm slot, 380 psi, Phase 2, 500-mm lens, position 2 $\sim 30-\mathrm{Pa} / 30$-mPa s clay vs $\sim 6-\mathrm{Pa} / 6-\mathrm{mPa}$ s clay vs water

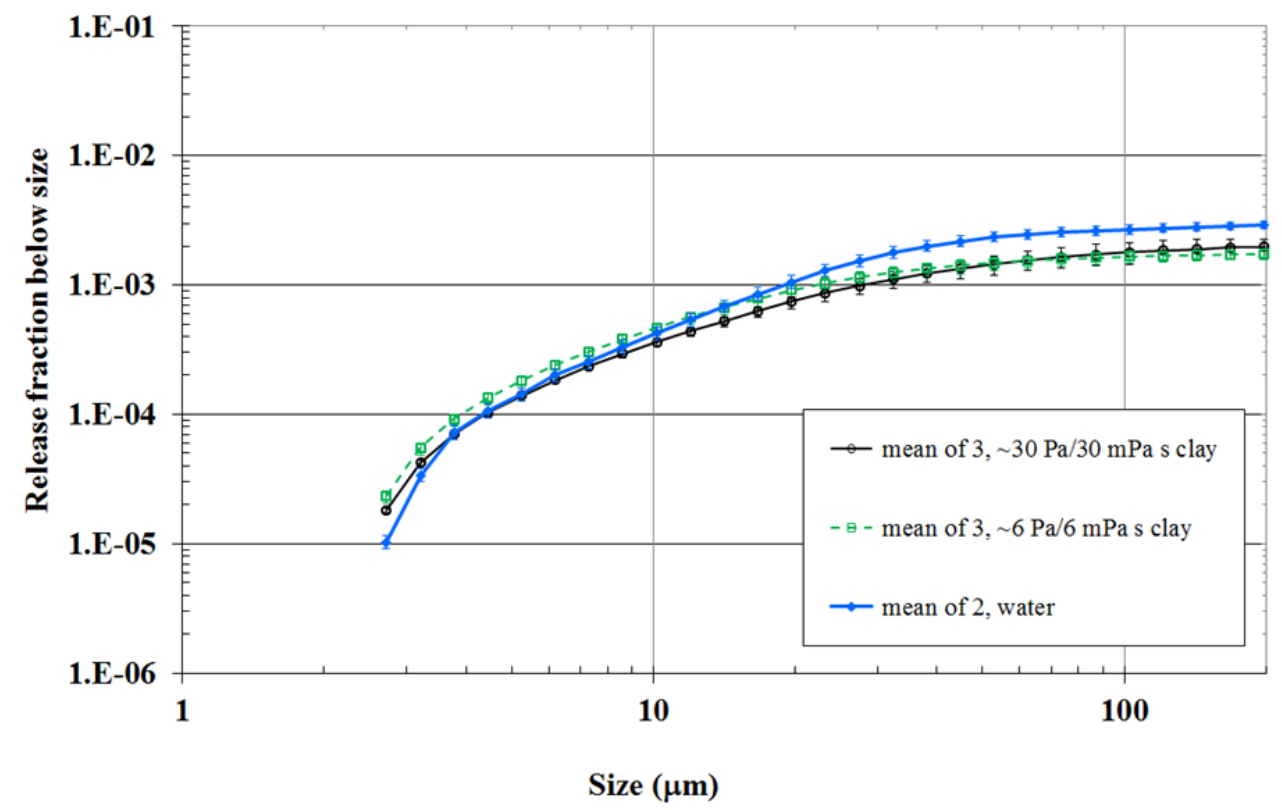

Figure 7.24. Effect of Rheological Parameters in Clay Slurry for a $0.5 \times 5 \mathrm{~mm}$ Slot at $380 \mathrm{psig}$. Error bars are maximum-minimum intervals. 
$1 \mathrm{~mm}$ hole, $100 \mathrm{psi}$, Phase 2, 500-mm lens, position 2

$\sim 30-\mathrm{Pa} / 30-\mathrm{mPa} s \mathrm{FEG}$ vs $\sim 6-\mathrm{Pa} / 6-\mathrm{mPa}$ s FEG vs water

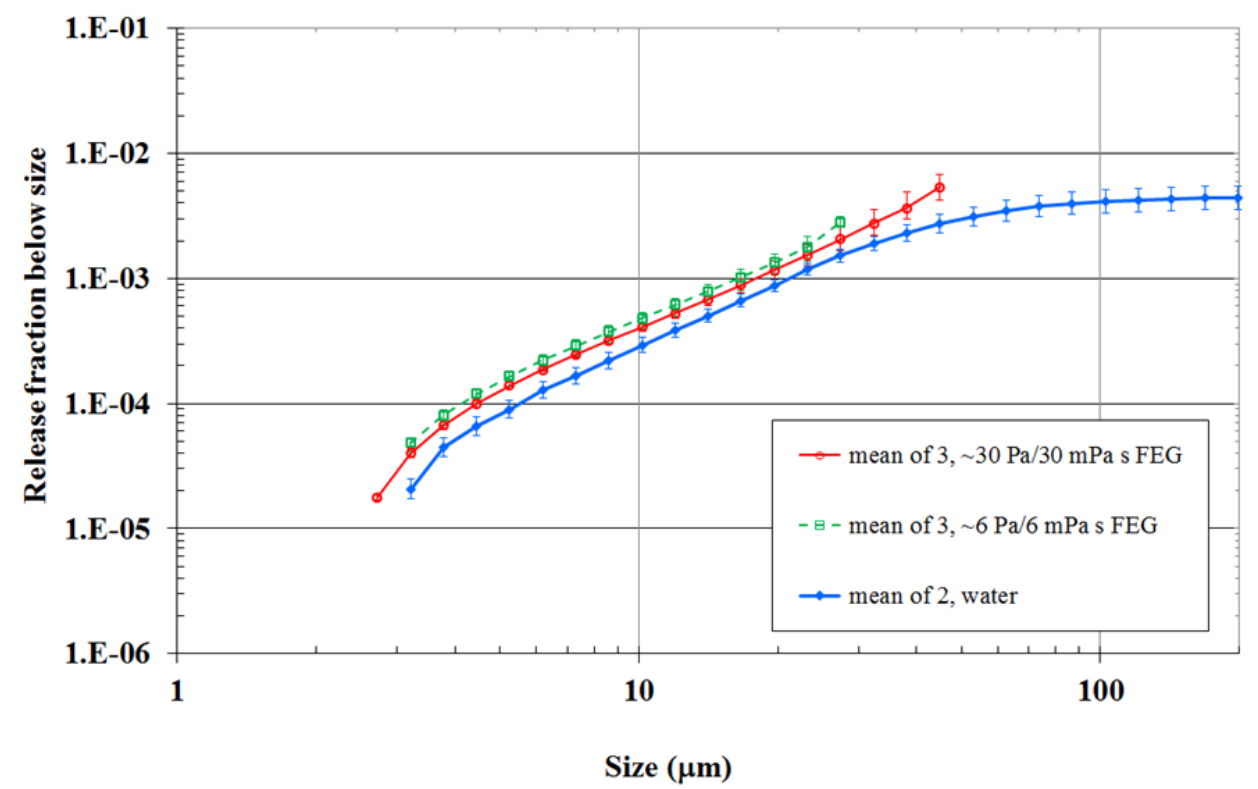

Figure 7.25. Effect of Rheological Parameters in FEG Slurry for a $1 \mathrm{~mm}$ Round Hole at $100 \mathrm{psig}$. Error bars are maximum-minimum intervals. The initial RH (FIO measurement) was 75 percent- 87 percent for the $30 \mathrm{~Pa}$ runs and about 76 percent for the $6 \mathrm{~Pa}$ runs.

$1 \mathrm{~mm}$ hole, $200 \mathrm{psi}$, Phase 2, 500-mm lens, position 2

$\sim 30-\mathrm{Pa} / 30 \mathrm{mPa} s$ FEG vs $\sim 6-\mathrm{Pa} / \mathbf{6} \mathrm{mPa}$ s FEG vs water

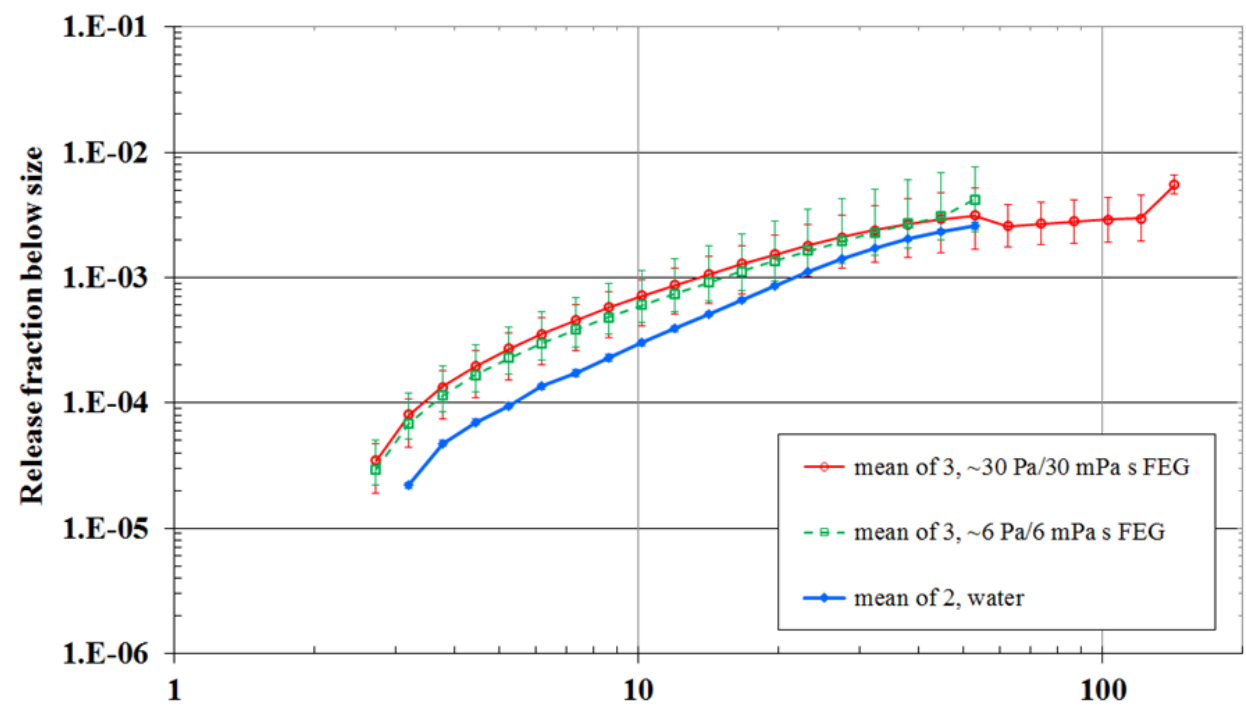

Size $(\mu \mathrm{m})$

Figure 7.26. Effect of Rheological Parameters in FEG Slurry for a $1 \mathrm{~mm}$ Round Hole at $200 \mathrm{psig}$. Error bars are maximum-minimum intervals. The initial RH (FIO measurement) was 70 percent -83 percent for the $30 \mathrm{~Pa}$ runs and 68 percent -75 percent for the $6 \mathrm{~Pa}$ runs. One of the runs (SS-271) used in the $30 \mathrm{~Pa}$ mean may have been partially plugged. 
$1 \mathrm{~mm}$ hole, $380 \mathrm{psi}$, Phase 2, 500-mm lens, position 2

$\sim 30-\mathrm{Pa} / 30-\mathrm{mPa}$ s FEG vs $\sim$-Pa/6-mPa s FEG vs water

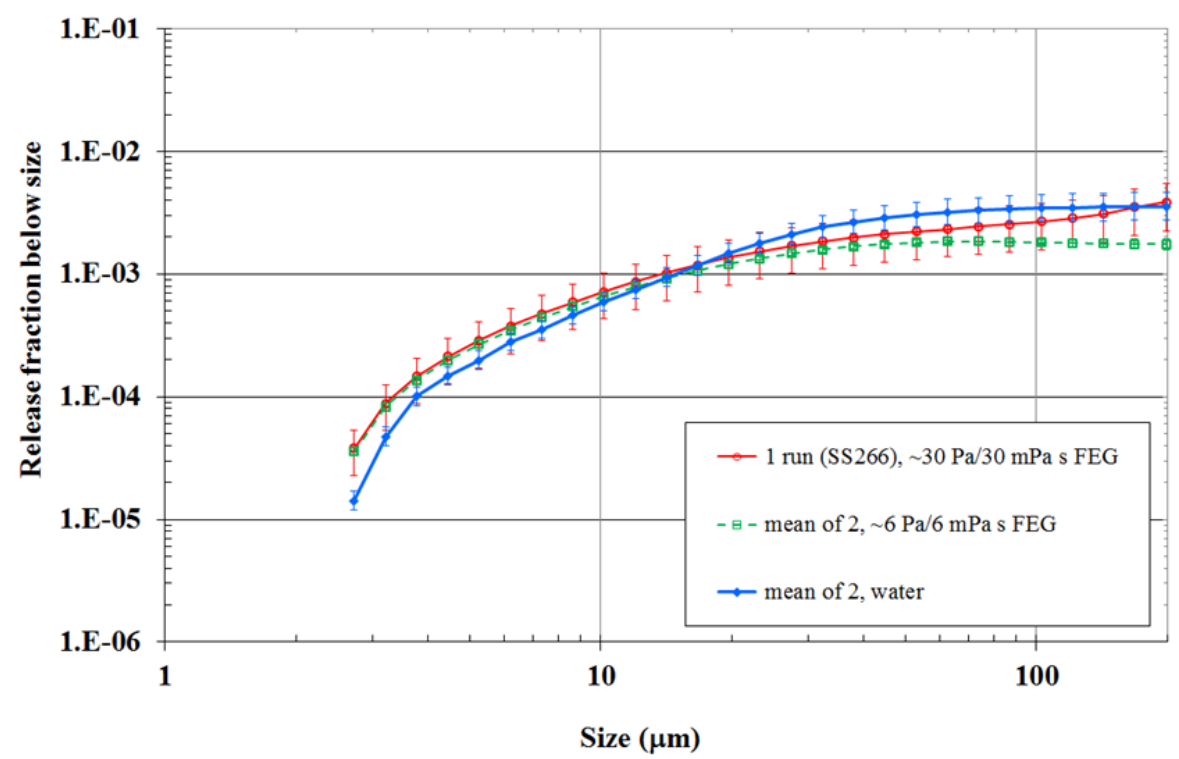

Figure 7.27. Effect of Rheological Parameters in FEG Slurry for a $1 \mathrm{~mm}$ Round Hole at 380 psig. Error bars are maximum-minimum intervals for test conditions where there are replicates or are approximate 95 percent confidence intervals for test conditions where only a single run is available. The initial RH (FIO measurement) was 67 percent for the single $30 \mathrm{~Pa}$ run and 62 percent -68 percent for the 6 Pa runs. The $30 \mathrm{~Pa}$ run (SS-266) had a low average pressure, $297 \mathrm{psig}$, during the first $20 \mathrm{~s}$.

$0.5 \times 5 \mathrm{~mm}$ slot, $100 \mathrm{psi}$, Phase 2, 500-mm lens, position 2 $\sim 30-\mathrm{Pa} / 30$-mPa $s$ FEG vs $\sim 6-\mathrm{Pa} / 6 \mathrm{mPa}$ s FEG vs water

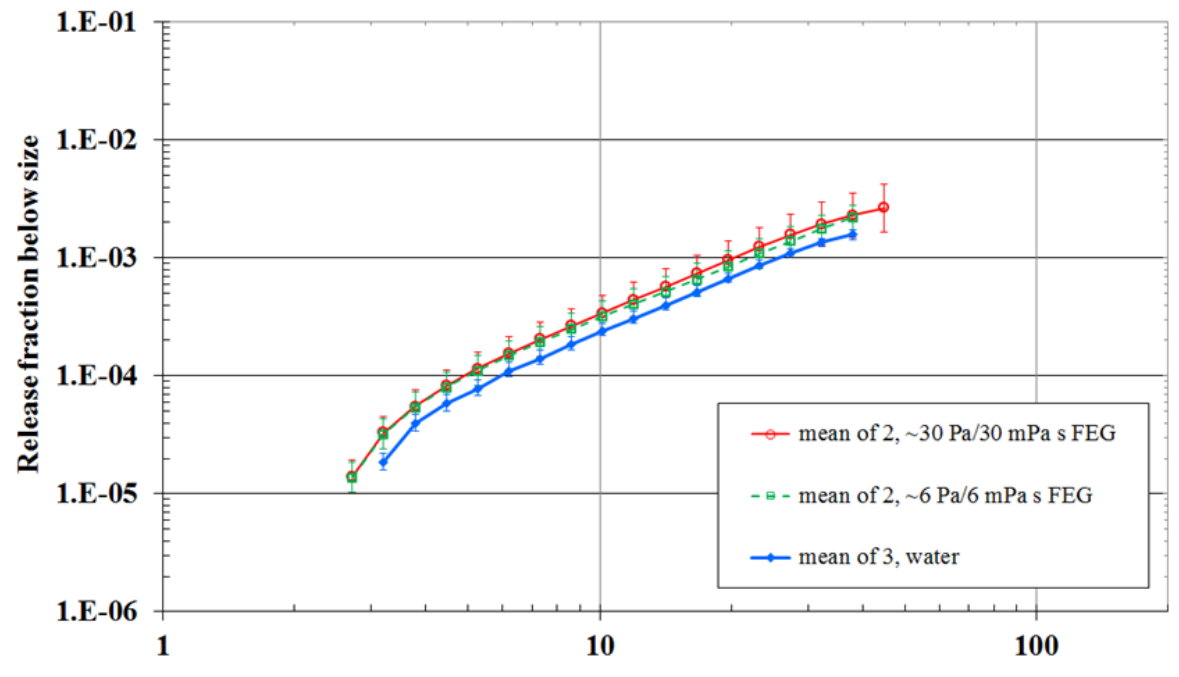

Size $(\mu \mathrm{m})$

Figure 7.28. Effect of Rheological Parameters in FEG Slurry for a $0.5 \times 5 \mathrm{~mm}$ Slot at $100 \mathrm{psig}$. Error bars are maximum-minimum intervals. The initial RH (FIO measurement) was $>80$ percent for the $30 \mathrm{~Pa}$ runs and 74 percent -78 percent for the $6 \mathrm{~Pa}$ runs. 
$0.5 \times 5 \mathrm{~mm}$ slot, $200 \mathrm{psi}$, Phase 2, 500-mm lens, position 2

$\sim 30-\mathrm{Pa} / 30$-mPa s FEG vs $\sim 6-\mathrm{Pa} / 6$-mPa $s$ FEG vs water

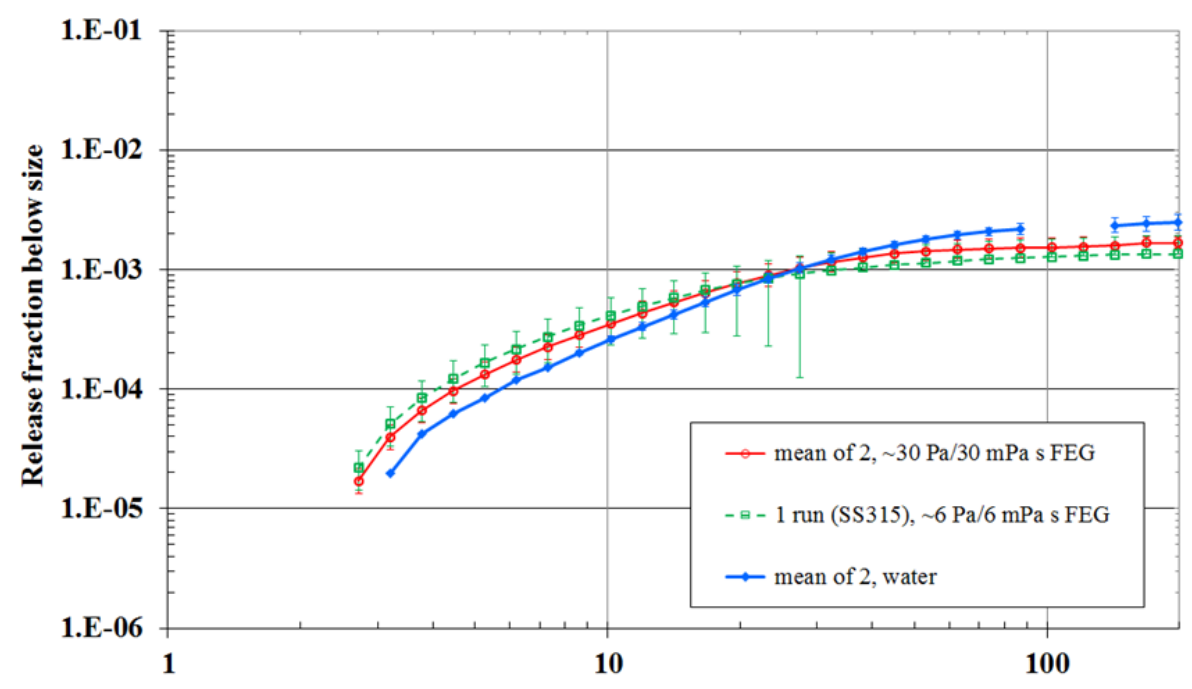

Size $(\mu \mathrm{m})$

Figure 7.29. Effect of Rheological Parameters in FEG Slurry for a $0.5 \times 5 \mathrm{~mm}$ Slot at $200 \mathrm{psig}$. Error bars are maximum-minimum intervals for test conditions where there were replicates or are approximate 95 percent confidence intervals for test conditions where only a single run is available. The initial RH (FIO measurement) was $>80$ percent for the $30 \mathrm{~Pa}$ runs and 73 percent for the single $6 \mathrm{~Pa}$ run.

$0.5 \times 5 \mathrm{~mm}$ slot, $380 \mathrm{psi}$, Phase 2, 500-mm lens, position 2

$\sim 30-\mathrm{Pa} / 30 \mathrm{mPa} s$ FEG vs $\sim 6-\mathrm{Pa} / 6 \mathrm{mPa}$ s FEG vs water

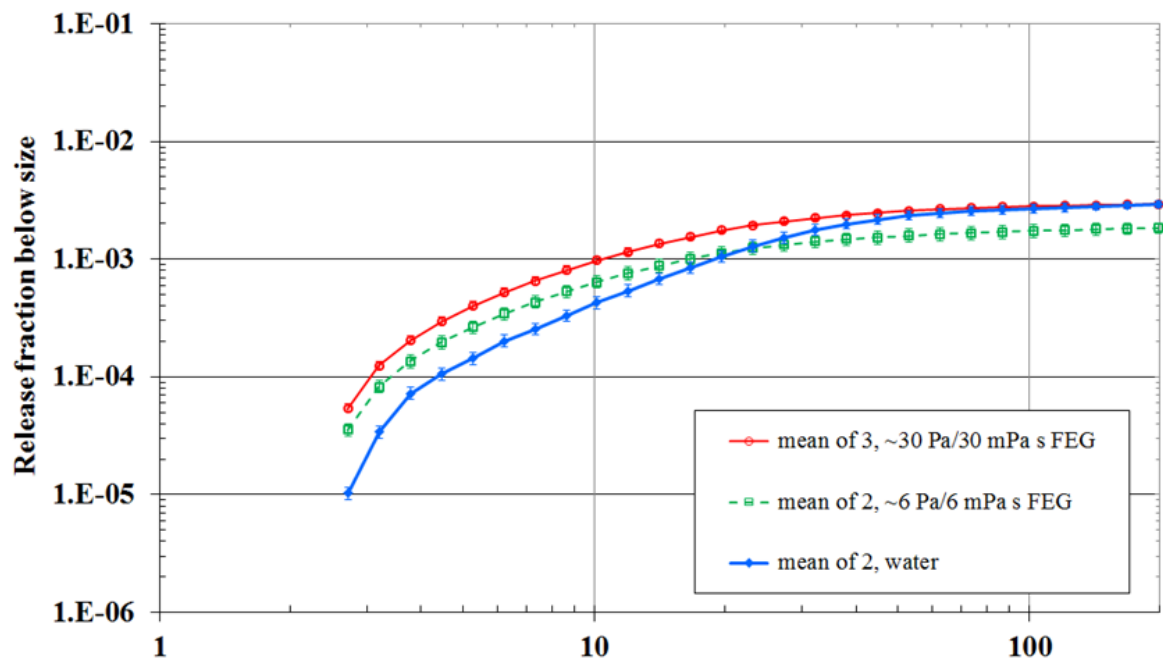

Size $(\mu \mathrm{m})$

Figure 7.30. Effect of Rheological Parameters in FEG Slurry for a $0.5 \times 5 \mathrm{~mm}$ Slot at $380 \mathrm{psig}$. Error bars are maximum-minimum intervals. The initial RH (FIO measurement) was $>80$ percent for the $30 \mathrm{~Pa}$ runs and 70 percent -83 percent for the $6 \mathrm{~Pa}$ runs. 
In general, the FEG tests showed less repeatability (more test-to-test variability) than the clay and water tests, as was discussed in Section 6.5. The low initial humidity $(<80 \% \mathrm{RH})$ in some of the $30 \mathrm{~Pa}$ tests and almost all of the 6 Pa tests suggests that the FEG data were biased low by an undetermined amount. Further, the difficulty pumping the FEG slurry through the system made it impossible to meet the header flow rate criterion of $11.4 \mathrm{gpm}$; the flow rate was between 5 and $10 \mathrm{gpm}$ in many tests, and was as low as 1 or 2 gpm in a few tests. Finally, the variable rheological behavior of the FEG slurry adds to the uncertainty of interpreting the differences in release fractions in terms of the target rheological properties.

For five out of the six test conditions, the release fractions for the $30 \mathrm{~Pa} F E G$ equaled those for the 6 Pa FEG, within variability. In the case of the $0.5 \times 5 \mathrm{~mm}$ slot at $380 \mathrm{psig}$, the $30 \mathrm{~Pa}$ FEG release fractions exceeded the $6 \mathrm{~Pa}$ FEG release fractions by approximately 50 to 70 percent. The $30 \mathrm{~Pa}$ and $6 \mathrm{~Pa}$ runs were repeatable within 50 percent or less, so the difference between the two FEGs may be statistically significant.

The release fractions for one or both of the two strengths of FEG are always greater than or equal to those of water over at least part of the droplet size range of interest $(<10$ to $<100 \mu \mathrm{m})$. The tendency for FEG release fractions to exceed those of water may be dampened, most prominently in smaller droplet sizes, if evaporation from water and FEG simulant are accounted for. Recall from Section 6.4.5 that water humidity corrections are expected to be larger than humidity corrections for slurries, particularly at droplet sizes near $10 \mu \mathrm{m}$ where slurry data requires little or no correction. The largest RF excess over water can be seen in Figure 7.26 (200 psig and the $1 \mathrm{~mm}$ hole) and Figure 7.30 (380 psig and the slot), in which the FEG release fractions are two to three times the water release fraction.

As will be discussed in Section 7.6, the orifice coefficients and volumetric flow rates for slurries were observed, in Phase II results, to be higher than those of water by 20 to 25 percent for the $1 \mathrm{~mm}$ hole. The implication is that at a given droplet size, where the release fractions for water and a slurry are equal, a higher volumetric flow rate for a slurry would result in a higher generation rate (as compared to water).

\subsubsection{Comparison of Clay and FEG Non-Newtonian Slurries}

The observations discussed in the preceding section imply that the FEG slurries produced higher release fractions than the water. This section provides a detailed comparison of FEG and clay. Figure 7.31 through Figure 7.42 are comparison plots for FEG versus clay, for two different simulant yield stresses, two different orifices ( $1 \mathrm{~mm}$ round and $0.5 \times 5 \mathrm{~mm}$ slot), and three different pressures ( 100 , 200 , and 380 psig). The plots show the geometric means of each set of replicate runs, with error bars on the geometric means that represent the maximum and minimum of the set of runs. When there is only one run for a test condition, no error band is shown. All the plotted data are from Phase II tests.

The release fractions for FEG slurries consistently exceeded or equaled those for clay slurries over part or all of the droplet size range of interest $(<10$ to $<100 \mu \mathrm{m})$. The difference between FEG and clay was often within the variability of the FEG release fractions. However, the consistency with which FEG produced higher release fractions over a range of different test conditions suggests that the higher releases from FEG are significant. 
$1 \mathrm{~mm}$ hole, $100 \mathrm{psi}$, Phase 2, 500-mm lens, position 2

$\sim 30-\mathrm{Pa} / 30-\mathrm{mPa}$ s clay vs $\sim 30-\mathrm{Pa} / 30-\mathrm{mPa}$ FEG

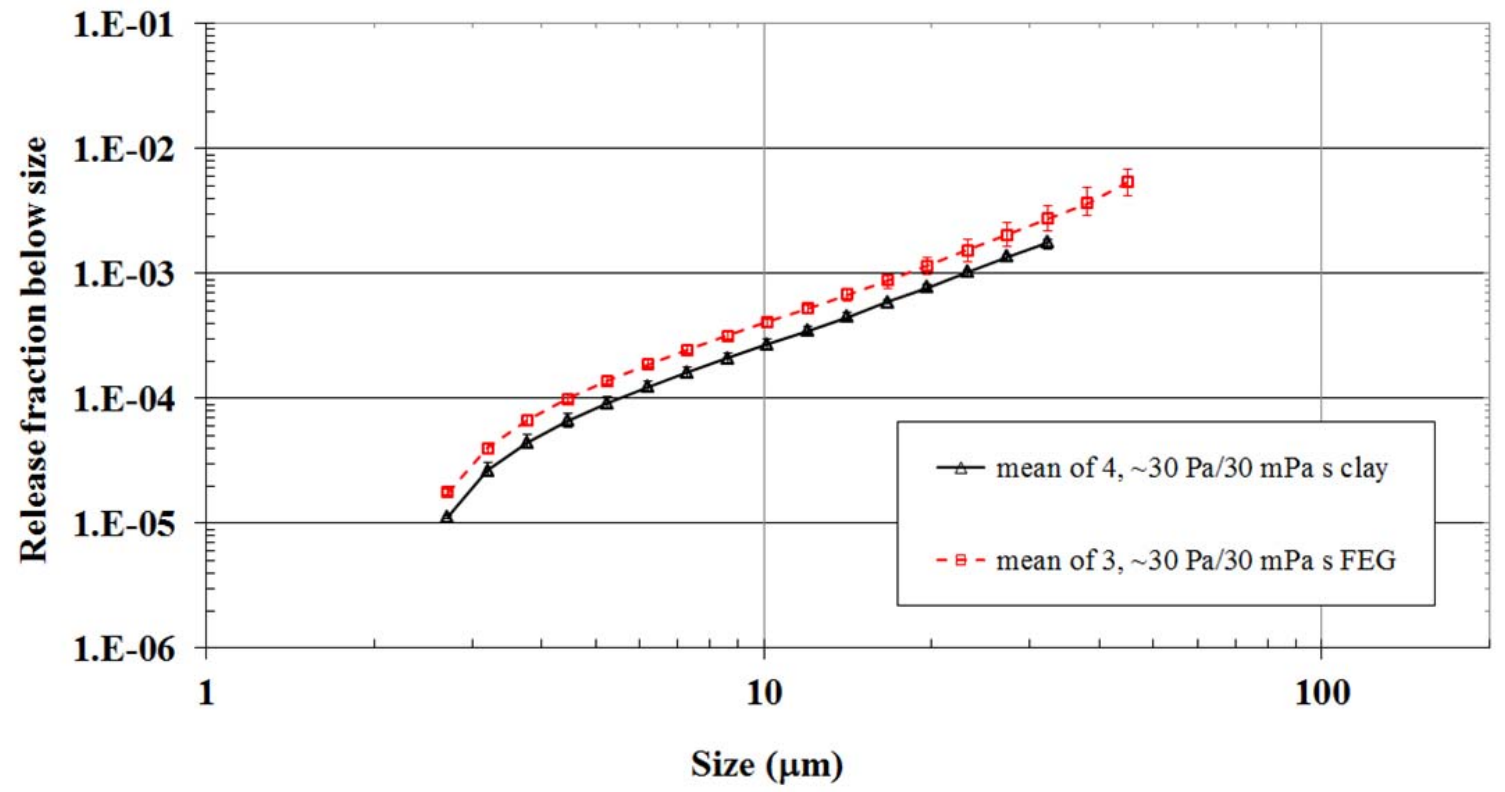

Figure 7.31. Clay and FEG Slurries at 30 Pa Target Yield Stress, Using a $1 \mathrm{~mm}$ Round Hole at 100 psig. Error bars are maximum-minimum intervals. The initial RH (FIO measurement) was 75 percent -87 percent for the FEG runs and $>80$ percent for the clay runs.

$1 \mathrm{~mm}$ hole, $200 \mathrm{psi}$, Phase 2, 500-mm lens, position 2 $\sim 30-\mathrm{Pa} / \mathbf{3 0}$-mPa s clay vs $\sim 30-\mathrm{Pa} / 30-\mathrm{mPa}$ s FEG

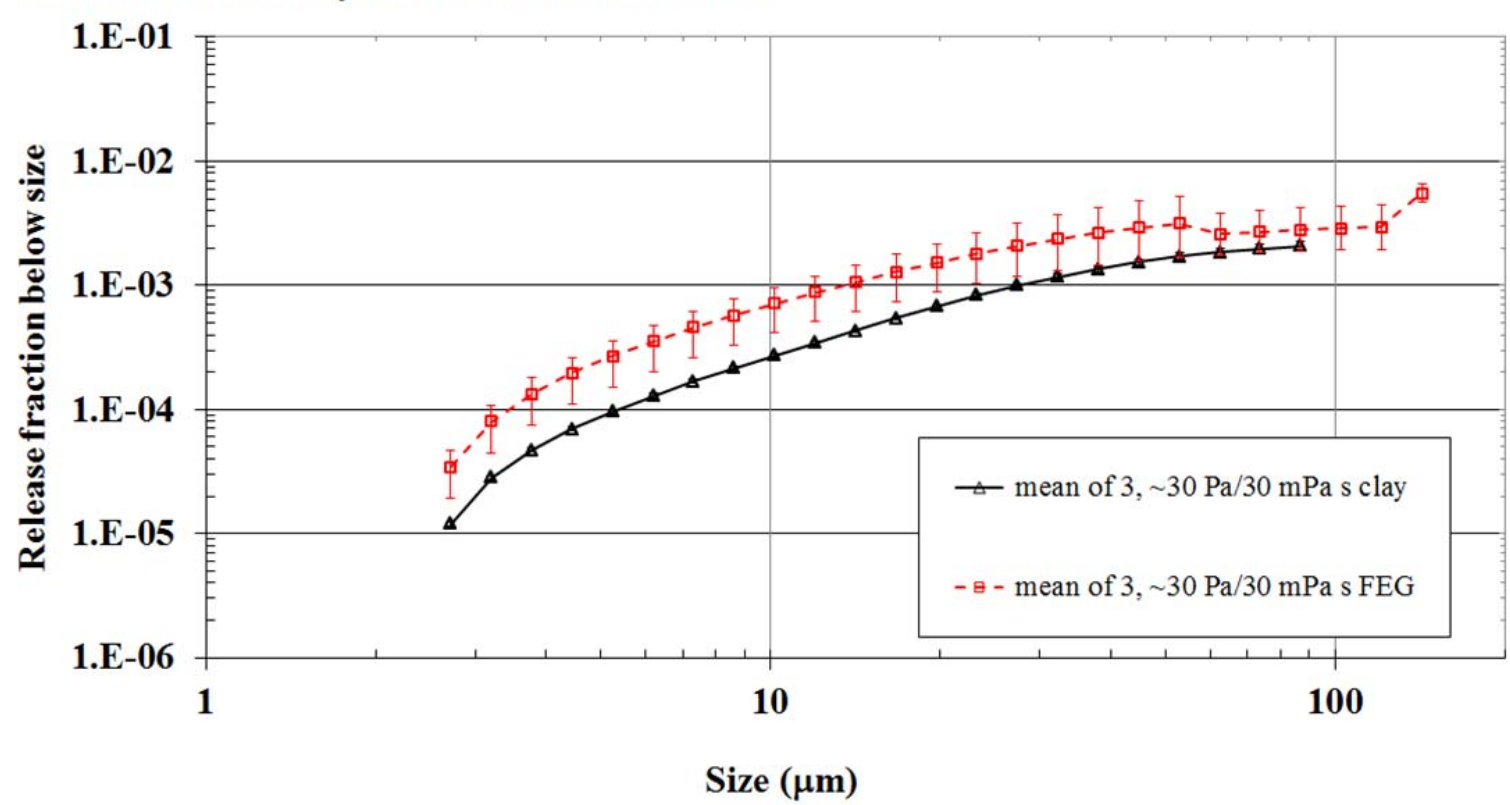

Figure 7.32. Clay and FEG Slurries at 30 Pa Target Yield Stress, Using a $1 \mathrm{~mm}$ Round Hole at 200 psig. Error bars are maximum-minimum intervals. The initial RH (FIO measurement) was 70 percent -83 percent for the FEG runs and $>80$ percent for the clay runs. One of the runs (SS-271) used in the FEG mean may have been partially plugged. 
$1 \mathrm{~mm}$ hole, $380 \mathrm{psi}$, Phase 2, 500-mm lens, position 2

$\sim 30-\mathrm{Pa} / \mathbf{3 0}$-mPa s clay vs $\sim 30-\mathrm{Pa} / \mathbf{3 0}$-mPa $\mathrm{FEG}$

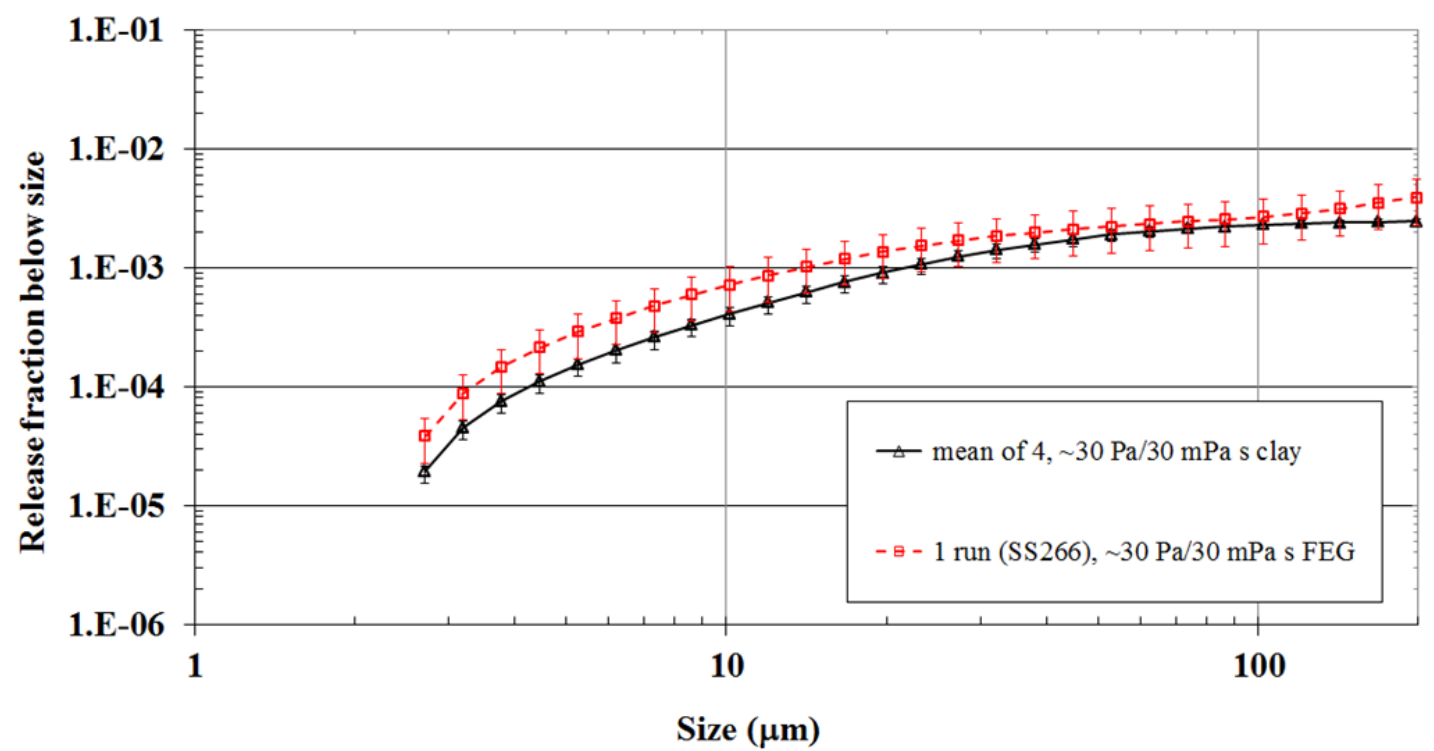

Figure 7.33. Clay and FEG Slurries at $30 \mathrm{~Pa}$ Target Yield Stress, Using a $1 \mathrm{~mm}$ Round Hole at 380 psig. Error bars are maximum-minimum intervals for test conditions where there were replicates or are approximate 95 percent confidence intervals for test conditions where only a single run is available. The initial RH (FIO measurement) was 67 percent for the single FEG run and $>80$ percent for the 6 Pa runs. The FEG run (SS-266) had a low average pressure, $297 \mathrm{psig}$, during the first $20 \mathrm{~s}$.

\section{5 x 5 mm slot, $100 \mathrm{psi}$, Phase 2, 500-mm lens, position 2} $\sim 30-\mathrm{Pa} / 30$-mPa s clay vs $\sim 30-\mathrm{Pa} / 30$-mPa s FEG

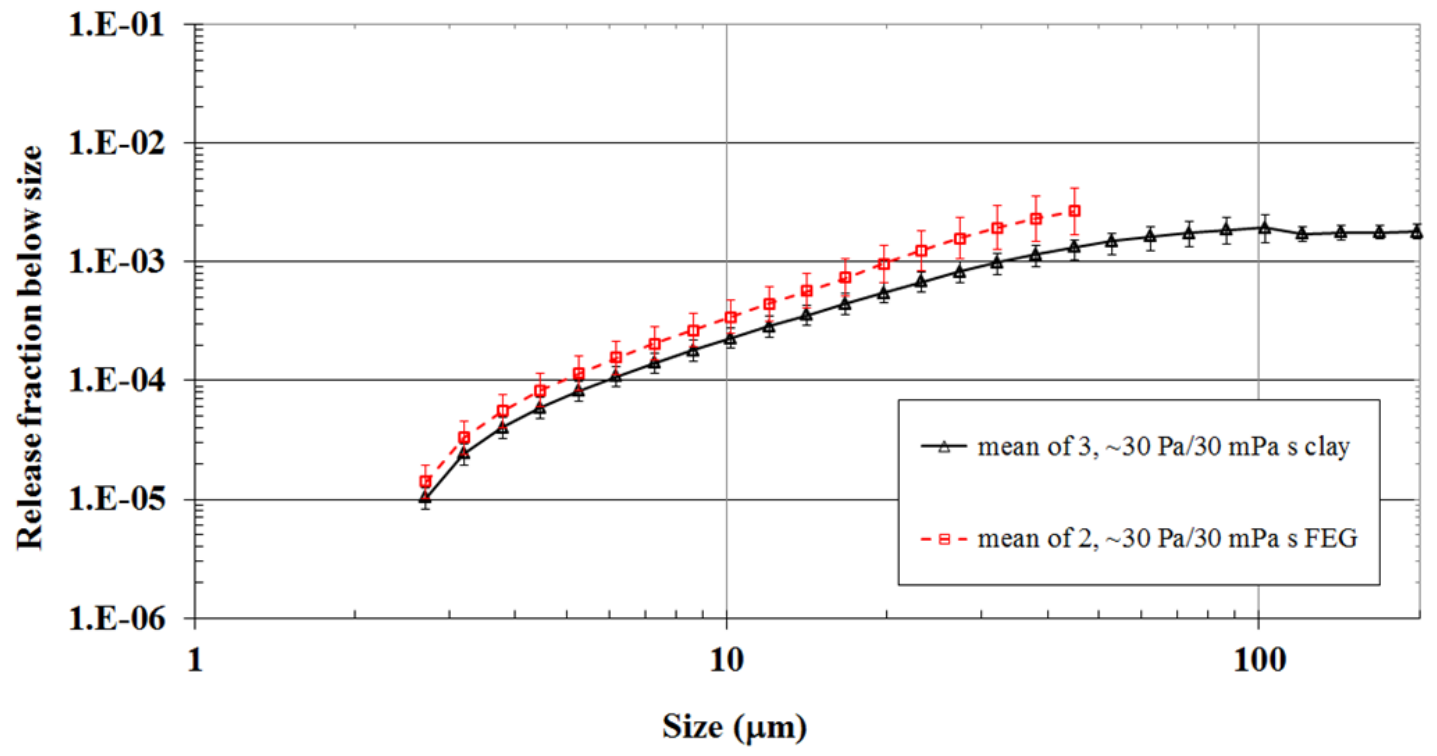

Figure 7.34. Clay and FEG Slurries at $30 \mathrm{~Pa}$ Target Yield Stress, Using a $0.5 \times 5 \mathrm{~mm}$ Slot at $100 \mathrm{psig}$. Error bars are maximum-minimum intervals. The initial RH (FIO measurement) was $>80$ percent for all runs. 
$0.5 \times 5$ mm slot, 200 psi, Phase 2, 500-mm lens, position 2 $\sim 30-\mathrm{Pa} / 30$-mPa s clay vs $\sim 30-\mathrm{Pa} / 30$-mPa s FEG

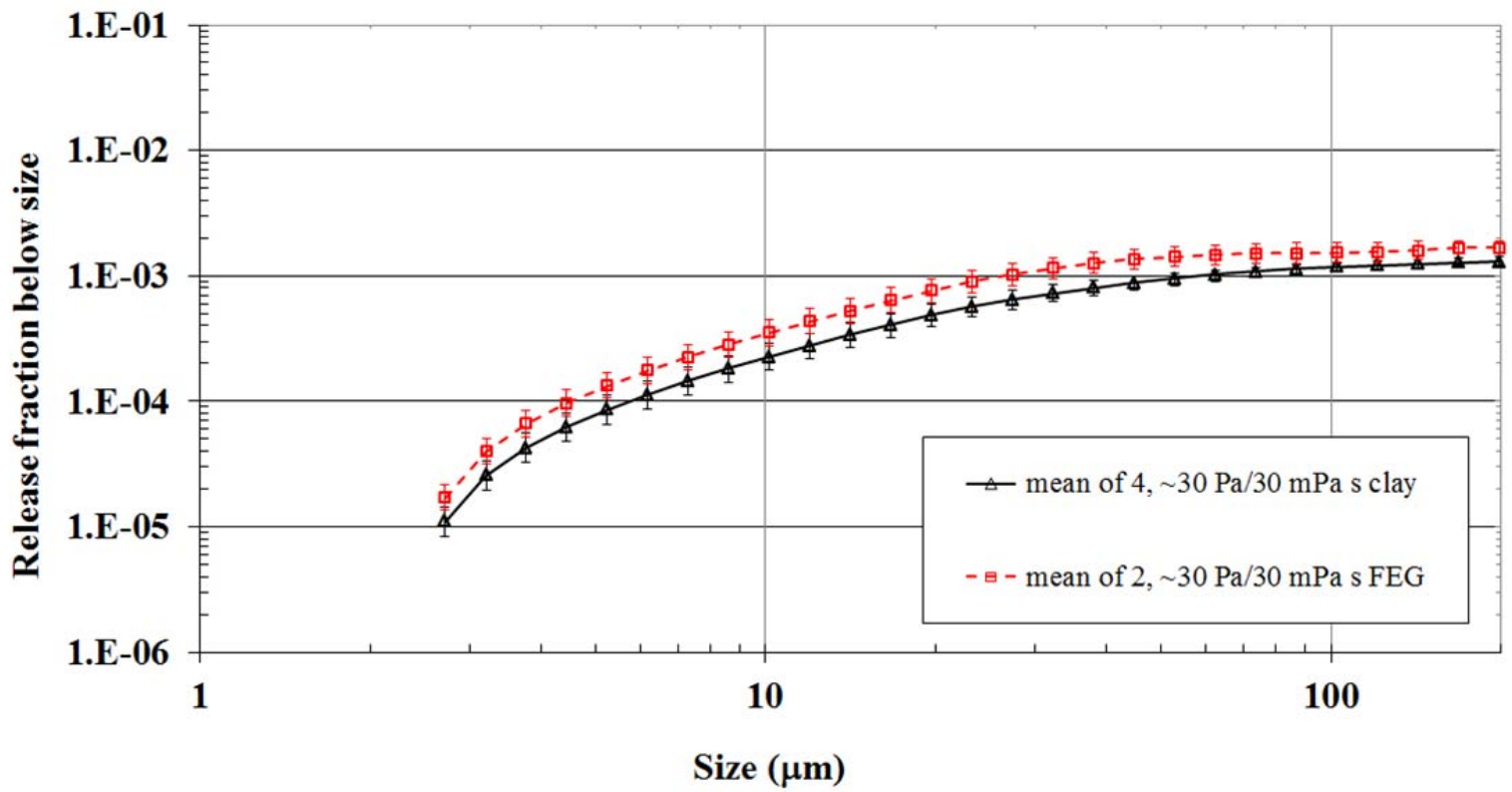

Figure 7.35. Clay and FEG Slurries at $30 \mathrm{~Pa}$ Target Yield Stress, Using a $0.5 \times 5 \mathrm{~mm}$ Slot at $200 \mathrm{psig}$. Error bars are maximum-minimum intervals. The initial RH (FIO measurement) was $>80$ percent for all runs.

$0.5 \times 5 \mathrm{~mm}$ slot, 380 psi, Phase 2, 500-mm lens, position 2 $\sim 30-\mathrm{Pa} / 30$-mPa s clay vs $\sim 30-\mathrm{Pa} / 30$-mPa $\mathrm{FEG}$

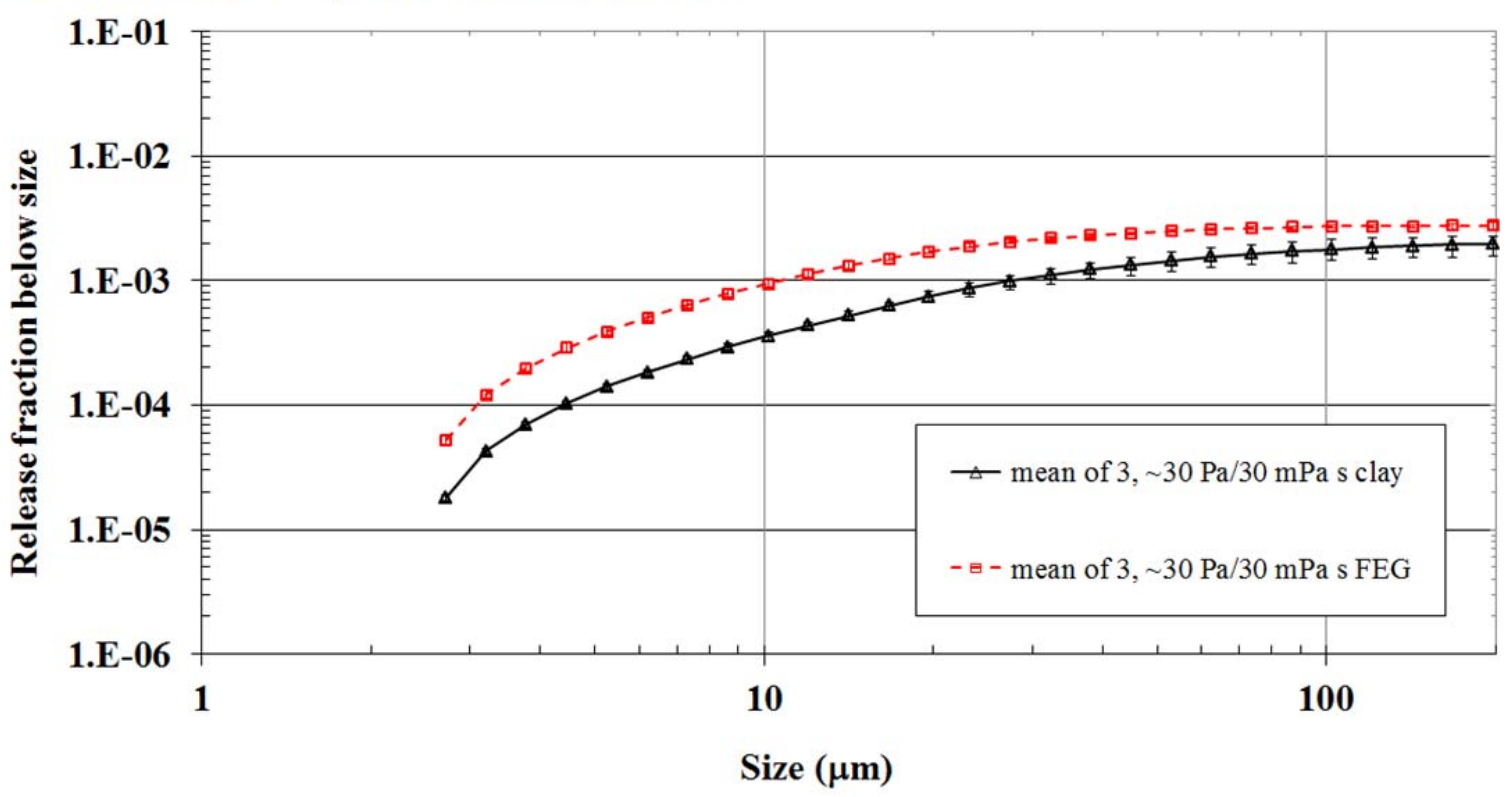

Figure 7.36. Clay and FEG Slurries at $30 \mathrm{~Pa}$ Target Yield Stress, Using a $0.5 \times 5 \mathrm{~mm}$ Slot at 380 psig. Error bars are maximum-minimum intervals. The initial RH (FIO measurement) was $>80$ percent for all runs. 
$1 \mathrm{~mm}$ hole, 100 psi, Phase 2, 500-mm lens, position 2

$\sim 6-\mathrm{Pa} / 6-\mathrm{mPa}$ s clay vs $\sim 6-\mathrm{Pa} / 6-\mathrm{mPa}$ s FEG

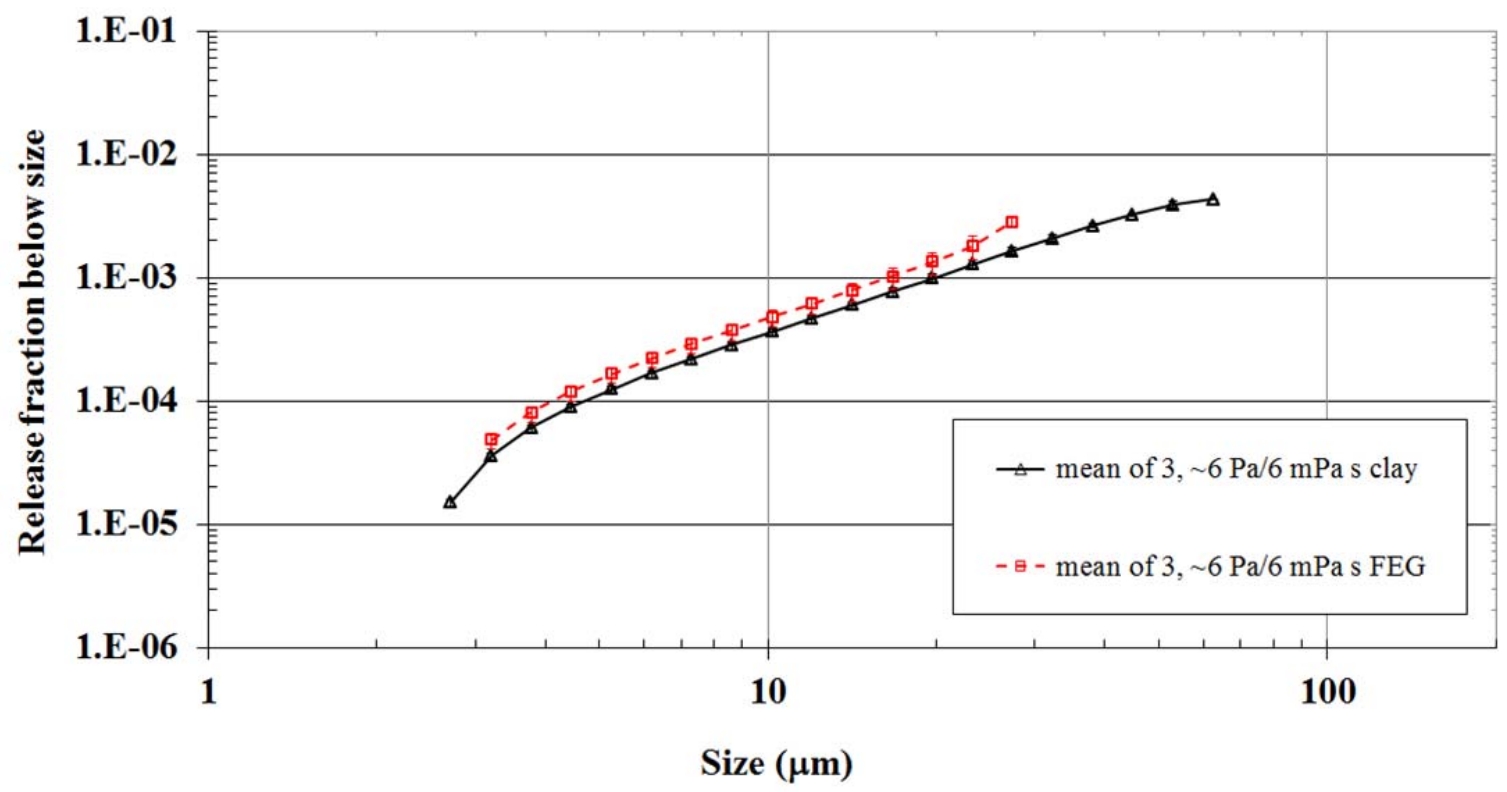

Figure 7.37. Clay and FEG Slurries at $6 \mathrm{~Pa}$ Target Yield Stress, Using a $1 \mathrm{~mm}$ Round Hole at 100 psig. Error bars are maximum-minimum intervals. The initial RH (FIO measurement) was $>80$ percent for the clay runs and about 76 percent for the FEG runs.

$1 \mathrm{~mm}$ hole, $200 \mathrm{psi}$, Phase 2, 500-mm lens, position 2

$\sim 6-\mathrm{Pa} / 6-\mathrm{mPa} s$ clay vs $\sim 6-\mathrm{Pa} / 6-\mathrm{mPa}$ s FEG

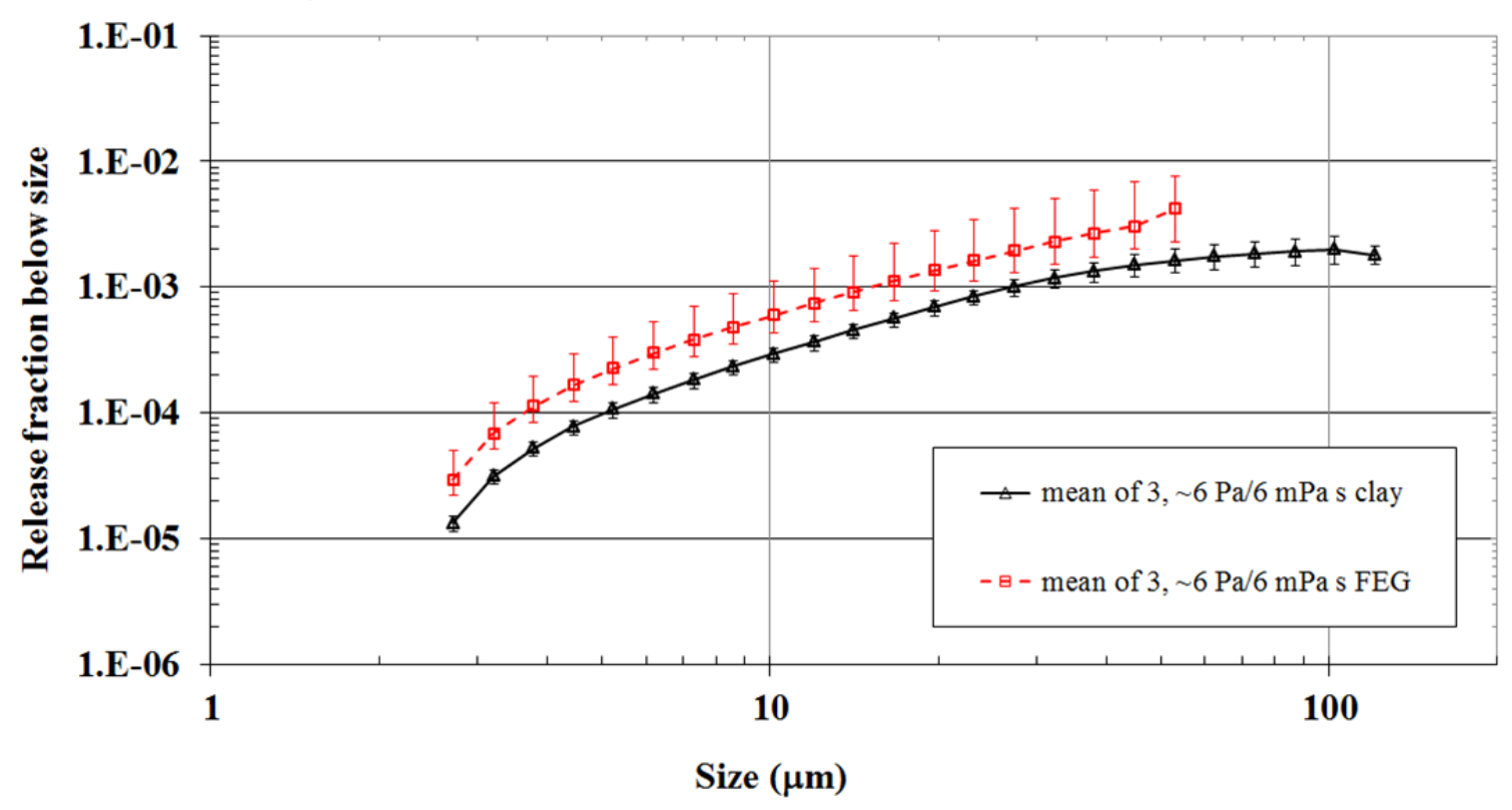

Figure 7.38. Clay and FEG Slurries at 6 Pa Target Yield Stress, Using a $1 \mathrm{~mm}$ Round Hole at 200 psig. Error bars are maximum-minimum intervals. The initial RH (FIO measurement) was $>80$ percent for the clay runs and 68 percent-75 percent for the FEG runs. 
$1 \mathrm{~mm}$ hole, $380 \mathrm{psi}$, Phase 2, 500-mm lens, position 2

$\sim 6-\mathrm{Pa} / 6-\mathrm{mPa} s$ clay vs $\sim 6-\mathrm{Pa} / 6-\mathrm{mPa}$ FEG

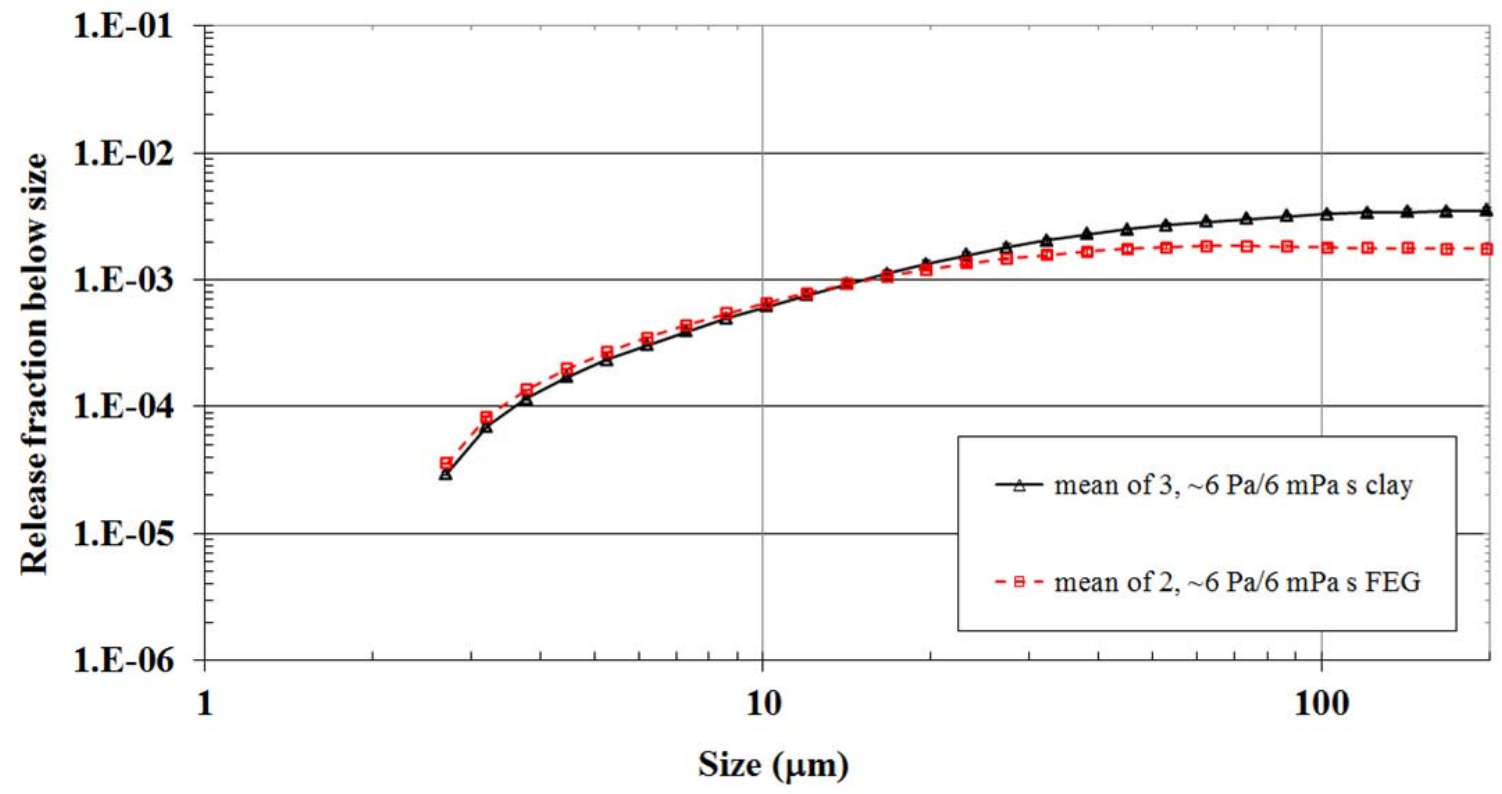

Figure 7.39. Clay and FEG Slurries at $6 \mathrm{~Pa}$ Target Yield Stress, Using a $1 \mathrm{~mm}$ Round Hole at 380 psig. Error bars are maximum-minimum intervals. The initial RH (FIO measurement) was $>80$ percent for the clay runs and 62 percent-68 percent for the FEG runs.

0.5 x 5 mm slot, 100 psi, Phase 2, 500-mm lens, position 2 $\sim$ 6-Pa/6-mPa s clay vs $\sim 6-\mathrm{Pa} / 6-\mathrm{mPa}$ s FEG

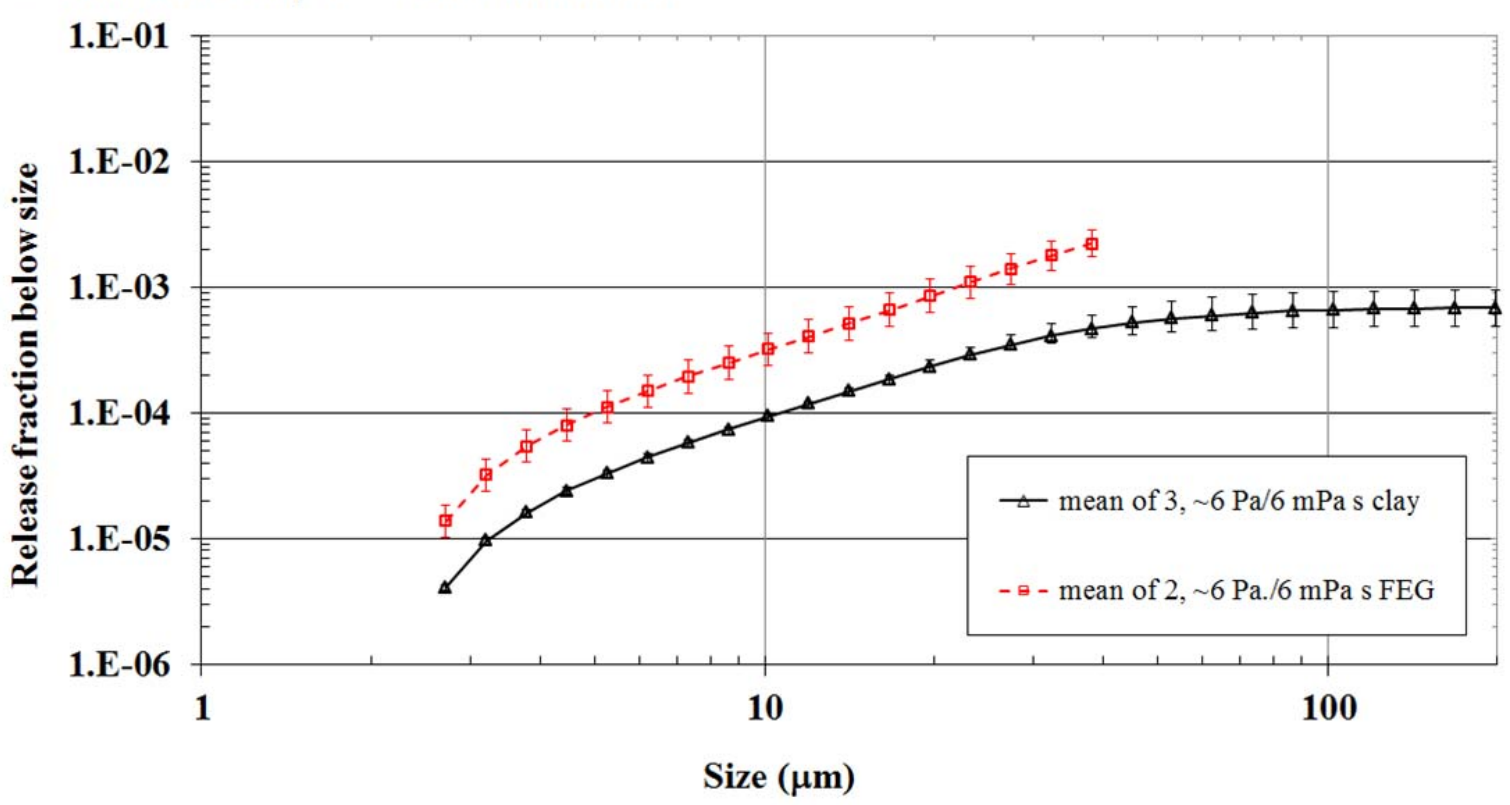

Figure 7.40. Clay and FEG Slurries at 6 Pa Target Yield Stress, Using a $0.5 \times 5 \mathrm{~mm}$ Slot at $100 \mathrm{psig}$. Error bars are maximum-minimum intervals. The initial RH (FIO measurement) was $>80$ percent for the clay runs and 74 percent-78 percent for the FEG runs. 
$0.5 \times 5 \mathrm{~mm}$ slot, $200 \mathrm{psi}$, Phase 2, 500 -mm lens, position 2

$\sim 6-\mathrm{Pa} / 6-\mathrm{mPa}$ s clay vs $\sim 6-\mathrm{Pa} / 6-\mathrm{mPa}$ S FEG

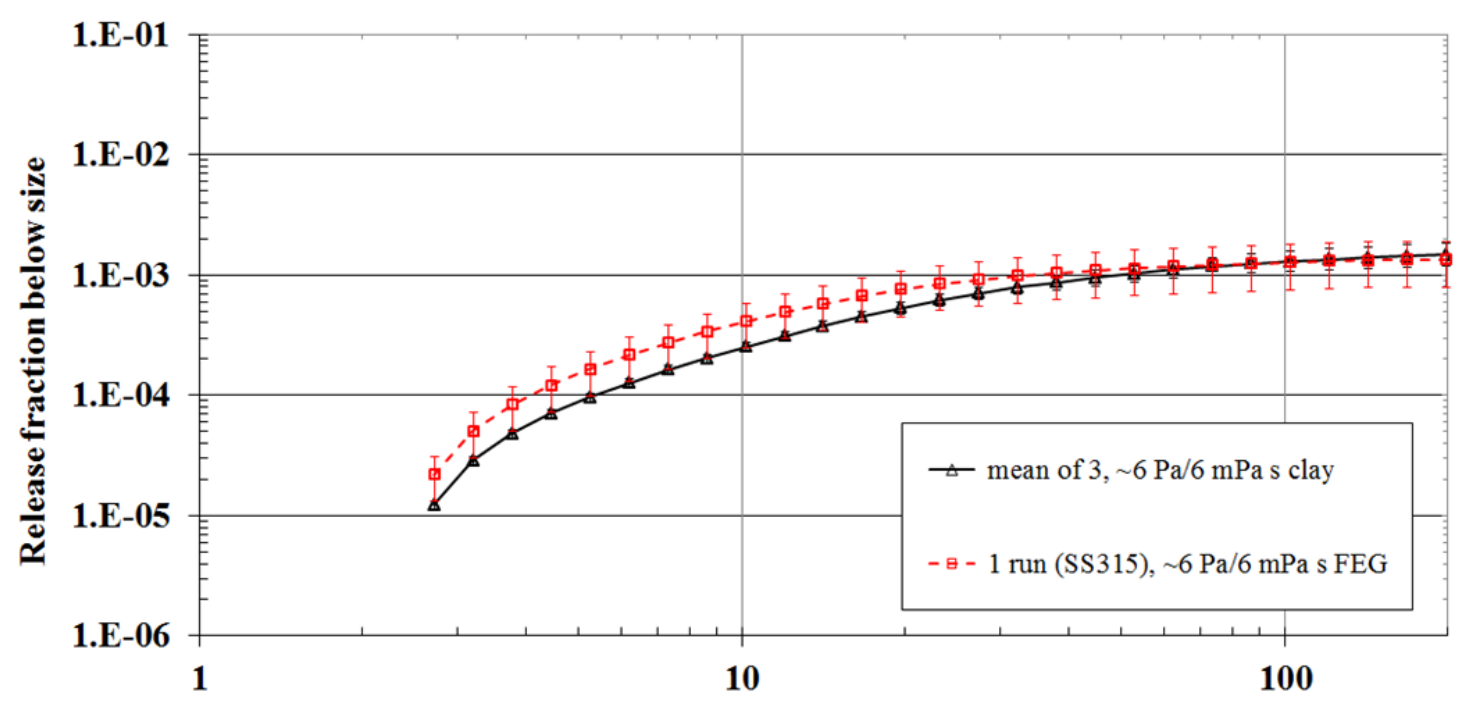

Size $(\mu \mathrm{m})$

Figure 7.41. Clay and FEG Slurries at $6 \mathrm{~Pa}$ Target Yield Stress, Using a $0.5 \times 5 \mathrm{~mm}$ Slot at 200 psig. Error bars are maximum-minimum intervals for test conditions where there were replicates or are approximate 95 percent confidence intervals for test conditions where only a single run is available. The initial RH (FIO measurement) was $>80$ percent for the clay runs and 73 percent for the single FEG run.

\section{$0.5 \times 5 \mathrm{~mm}$ slot, $380 \mathrm{psi}$, Phase 2, 500-mm lens, position 2 \\ $\sim$ 6-Pa/6-mPa s clay vs $\sim 6-\mathrm{Pa} / 6-\mathrm{mPa}$ S FEG}

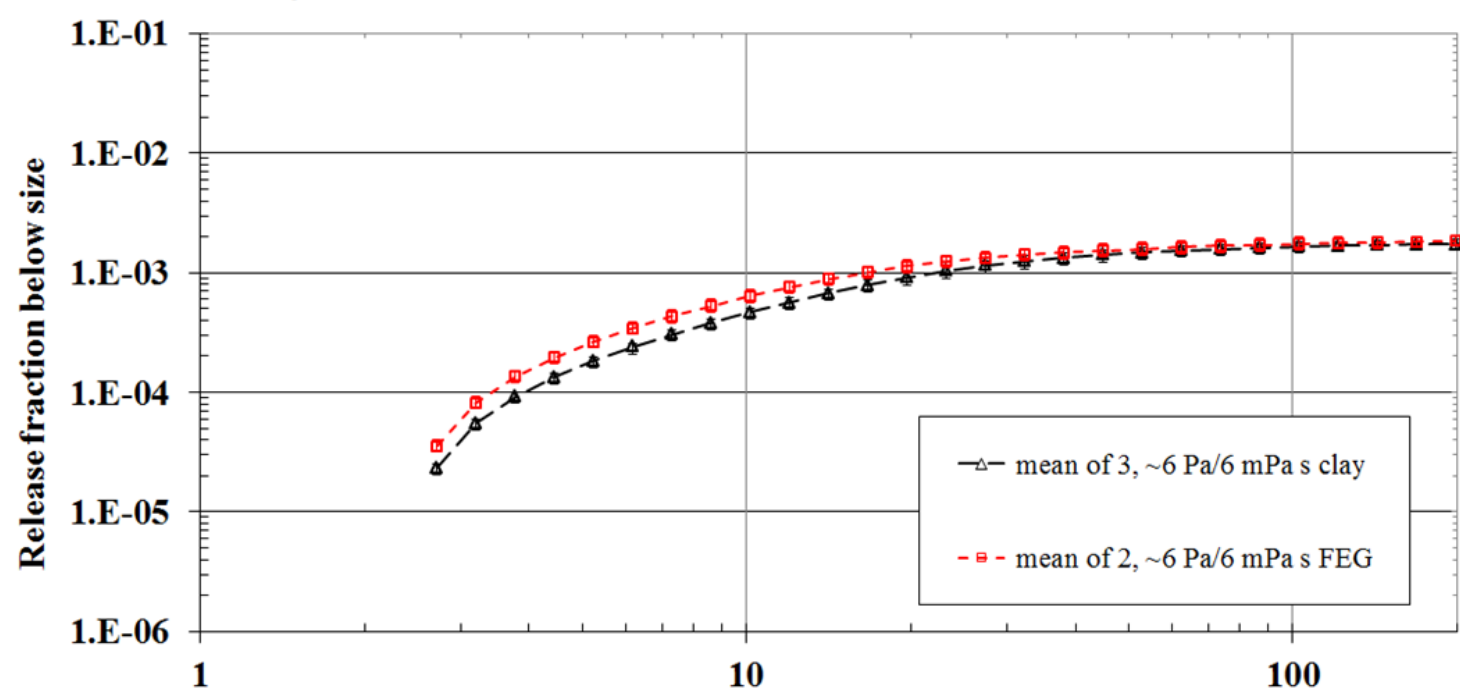

Size $(\mu \mathrm{m})$

Figure 7.42. Clay and FEG Slurries at 6 Pa Target Yield Stress, Using a $0.5 \times 5 \mathrm{~mm}$ Slot at 380 psig. Error bars are maximum-minimum intervals. The initial RH (FIO measurement) was $>80$ percent for the clay runs and 70 percent -83 percent for the FEG runs. 


\subsubsection{Discussion of Applicability of FEG Results}

The results for the FEG simulant should be regarded with some caution because of the atypical rheological behavior that was time-dependent, reversible, and thickened with the application of shear. The variability of the rheological parameters with time and applied shear made it difficult to ascertain whether the rheological properties of the FEG simulant matched the target conditions. As mentioned previously, other implications of the rheological behavior were that the rheology of the simulant was likely not constant during a test and other target experimental conditions (e.g., header flow rate, conducting pre-sprays) were not always met (see Section 5.4.2).

The time-dependent thickening seemed to be caused by the high shear conditions occurring during the sprays. The high shear conditions in the system were due primarily to simulant flow through partly closed needle valves and to operating the positive displacement pump at 20 to $30 \mathrm{~Hz}$. Periods of high shear occurred repeatedly for several minutes at a time over a few weeks, with lower shear conditions (open needle valves and less than $15 \mathrm{~Hz}$ pump speed) often occurring for hours a day between sprays on days when tests were carried out or unsuccessfully attempted.

When the FEG simulant was first put into the system, rheograms of samples showed no hysteresis between up-ramp and down-ramp Bingham properties, but a thickening of the slurry could be seen in the feed tank in $0.5 \mathrm{hr}$ or less. After several days of testing, samples of the simulant manifested considerable hysteresis (as shown in Figure 3.16). At this point, the slurry thickened visibly within minutes after it began to be pumped through the system and thinned after pumping was stopped.

The variable behavior of the FEG simulant probably contributed to variation between runs at the same test conditions that were nominally carried out with the same simulant properties. In addition, the rheological properties at the orifice are not necessarily well characterized by sample measurements, given the dependence on shear rate and shear history.

It is unknown whether the behavior of the FEG simulant, particularly its variable rheology, represents the behavior of any actual Hanford tank waste. A search of Hanford reports and consultation with topic experts has not located any samples of tank waste that exhibited the substantial thickening behavior shown in Figure 3.16. However, topic experts were not aware of any past tests that subjected waste to the repeated high shear conditions that were present in spray tests, so the absence of observations does not exclude the possibility that tank waste could behave in the manner observed for the FEG simulant.

\subsection{In-Spray Measurements}

In-spray (or in-jet) conditions represent jet aerosol generation at the limiting condition of zero effect of wall capture, splash droplet formation, and losses. In Phase II, in-spray measurements of PSD were made for every combination of simulant and pressure using either a $0.5 \mathrm{~mm}$ or $1 \mathrm{~mm}$ round orifice, depending on whether plugging was a problem. A $500 \mathrm{~mm}$ focal-length lens was used to see larger droplets. In Phase I, the upper measurable limit for the $100 \mathrm{~mm}$ focal-length lens in the small-scale configuration was $200 \mu \mathrm{m}$, whereas the upper limit for the $500 \mathrm{~mm}$ lens used in Phase II was $2500 \mu \mathrm{m}$. The effect of the upper measurement limit in Phase I is clear in Figure 7.32 of Mahoney et al. (2013). 
Figure 7.43 and Figure 7.44 show the cumulative and differential PSDs for test SS-100, a 380 psig spray of water from a $0.5 \mathrm{~mm}$ (target dimension) round hole. The figures show PSDs for several different times after the spray start; each curve is the average of measurements from $2 \mathrm{~s}$ before the stated time to $2 \mathrm{~s}$ after it. The main peak in the differential PSD (Figure 7.44) is at about $300 \mu \mathrm{m}$, but a small secondary peak is visible between 800 and $1000 \mu \mathrm{m}$. The secondary peak results in having about 10 percent of the volume above $2000 \mu \mathrm{m}$, and therefore unmeasured. This test was conducted with the Malvern Insitec-S instrument at the same distance from the orifice as in Phase I, 15 in. $(37 \mathrm{~cm})$ downstream.

Figure 7.45 shows the differential PSD for test SS-363, a 380 psig spray of water containing $0.4 \mathrm{wt} \%$ Mo particles $\left(\mathrm{d}_{50}=25 \mu \mathrm{m}\right)$ from a $0.5 \mathrm{~mm}$ (target dimension) round hole. This test was conducted with a 6-in. extension on the orifice, putting the Malvern Insitec-S instrument at about 9 in. $(23 \mathrm{~cm})$ from the orifice. Although the properties of the simulant were almost identical to those of test SS-100, the size distribution of this spray was much more coarse, with the peak apparently out beyond $2000 \mu \mathrm{m}$.

The change in downstream location of the Malvern Insitec-S instrument seems to have strongly affected the measured in-spray size distributions. In the Phase II tests, the in-jet water sprays (examples in Figure 7.43 and Figure 7.44) were measured at a Malvern Insitec-S instrument location that was the same as for Phase I, with the laser situated 15 in. $(37 \mathrm{~cm})$ downstream from the orifice. However, because the slurry sprays were often broad enough to wet the Malvern Insitec-S lens at this distance, the orifice was moved forward by 6 in., putting the Malvern Insitec-S laser about 9 in. $(23 \mathrm{~cm})$ from the orifice. All slurry runs considered for use in this report had measurements made at this shorter distance. Two inferences can be drawn from Figure 7.43 through Figure 7.45:

1. the laser (measurement point) was slightly upstream of the end of the liquid core for the water spray

2. the laser was further upstream, with less jet breakup, at the shorter along-jet distance used for measurement of all the slurry sprays.

Figure 7.46 and Figure 7.47 show the same kind of plots for test SS-200, a 380 psig spray of $30 \mathrm{~Pa}$ clay slurry from a $0.5 \mathrm{~mm}$ round hole ( 9 in. from the Malvern Insitec-S instrument). A single peak is visible between 1000 and $2000 \mu \mathrm{m}$, although the apparent peaking of the size distribution may have been caused by a measurement cutoff near the upper measurement limit of $2500 \mu \mathrm{m}$. Again, droplets of this size would not be expected from a $0.5 \mathrm{~mm}$ orifice. About half, or somewhat less, of the spray volume was unmeasured, being present in drops larger than $2000 \mu \mathrm{m}$. This type of size distribution was usual for the slurry sprays.

It is surmised that the jet was incompletely broken up at the location of measurement and that the instrument was either mistaking part of the liquid core for droplets or was measuring droplets that had been produced by primary breakup and had not yet undergone secondary breakup. In previous work, Epstein and Plys (2006) made PSD observations of in-spray measurements that indicated the partial or complete presence of liquid core. In that case, the questionable PSDs (Figure 5-3 of Epstein and Phys 2006) showed a small peak in the differential size distribution at sizes below $100 \mu \mathrm{m}$, a dip in the concentration between 100 and $200 \mu \mathrm{m}$, and a much larger peak at $300 \mu \mathrm{m}$, with no data for sizes larger than $350 \mu \mathrm{m}$. They found that measuring the "PSD" of a glass rod gave a similar PSD (Figure 5-4 of Epstein and Phys 2006). 
SS100: Malvern pos. 4, water @ 380 psig, axial 0.534 mm round hole, fans @ O V; P2struc;500mm,4 Hz;no6"extens;purge40scfh

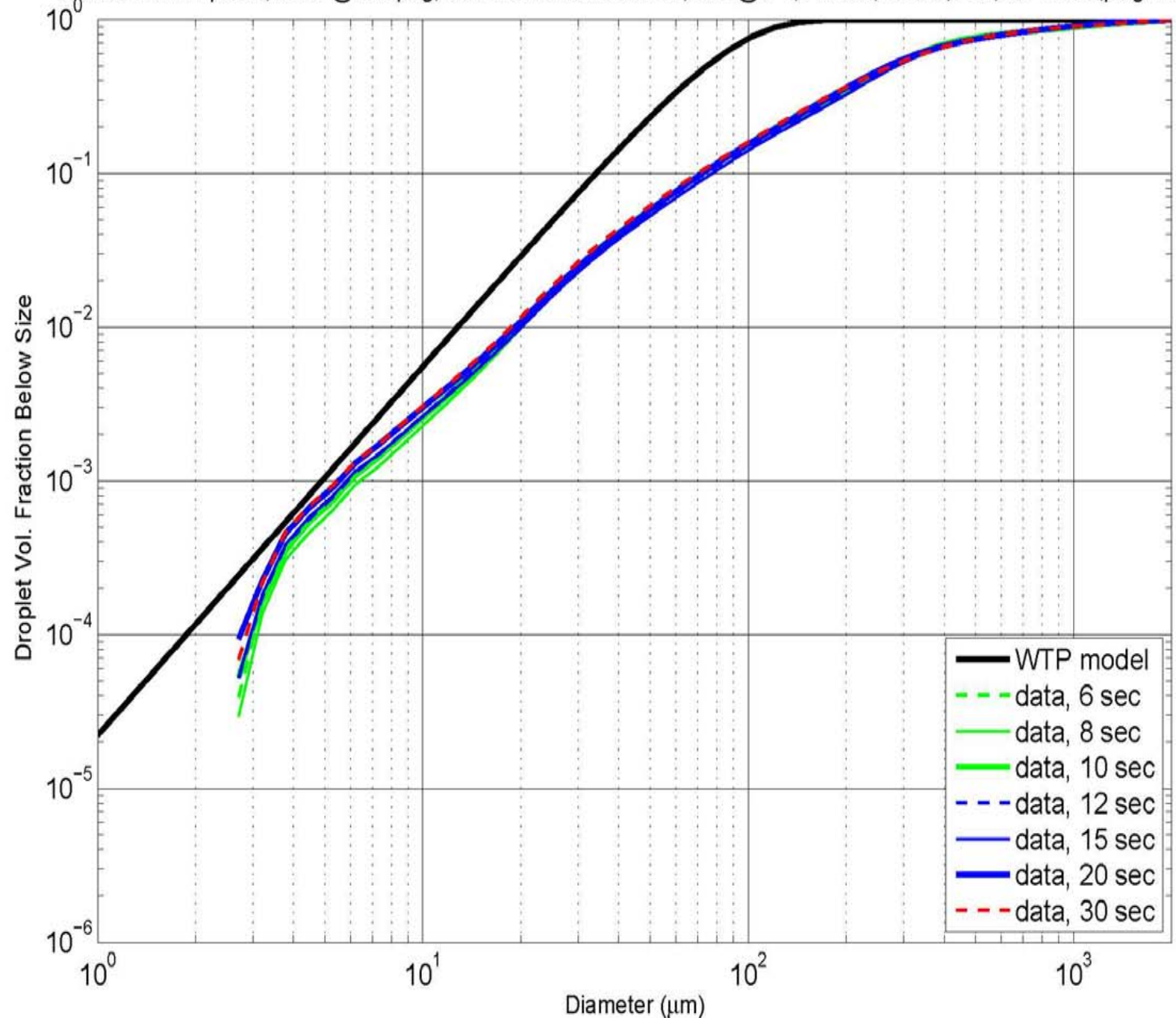

Figure 7.43. Cumulative In-Spray Size Distribution for a 380 psig Jet of Water from a $0.5 \mathrm{~mm}$ Round Hole 
SS100: Malvern pos. 4, water @ 380 psig, axial 0.534 mm round hole, fans @ 0 V; P2struc;500mm,4 Hz;no6"extens;purge40scfh

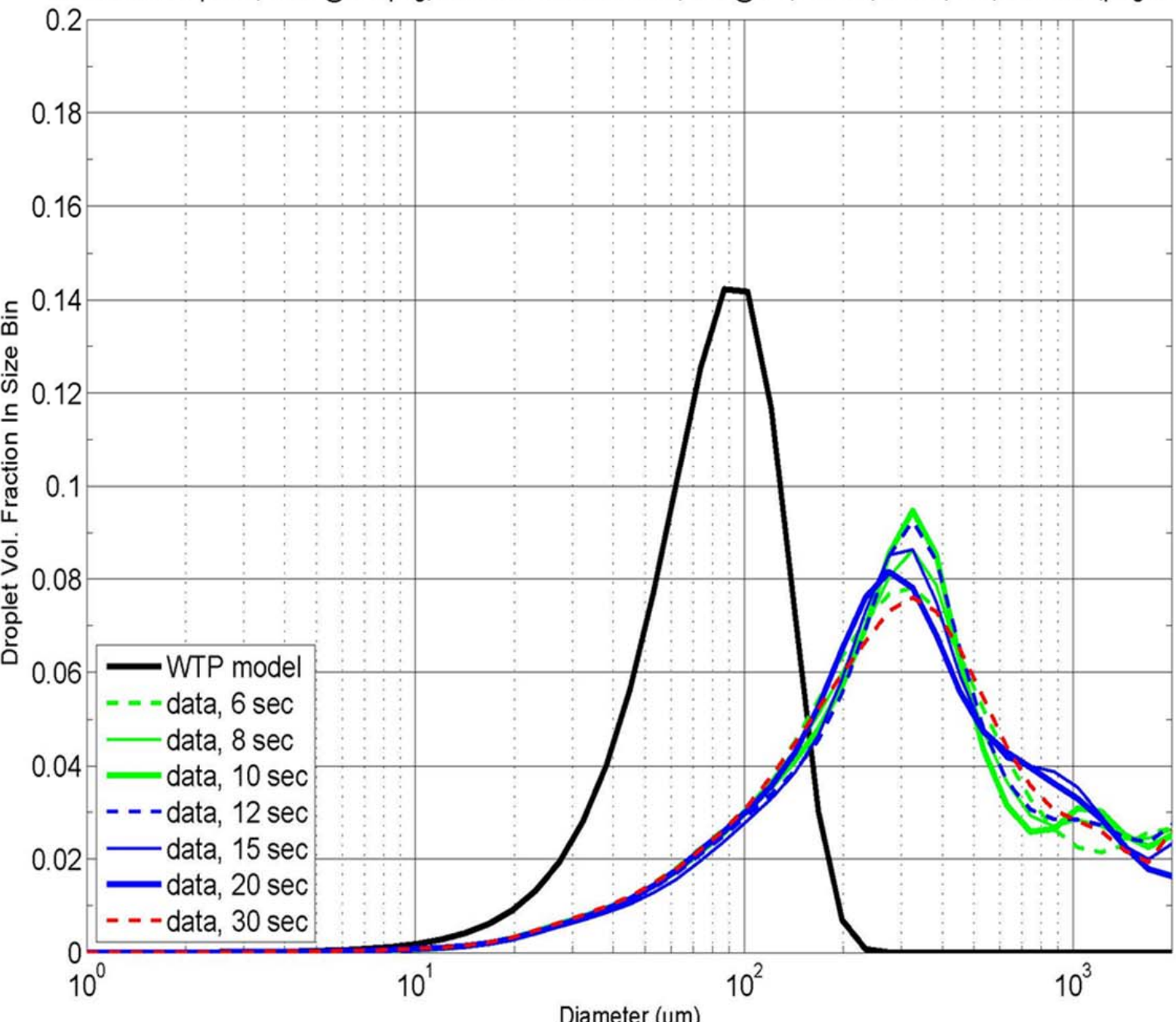

Diameter $(\mu \mathrm{m})$

Figure 7.44. Differential In-Spray Size Distribution for a 380 psig Jet of Water from a $0.5 \mathrm{~mm}$ Round Hole 


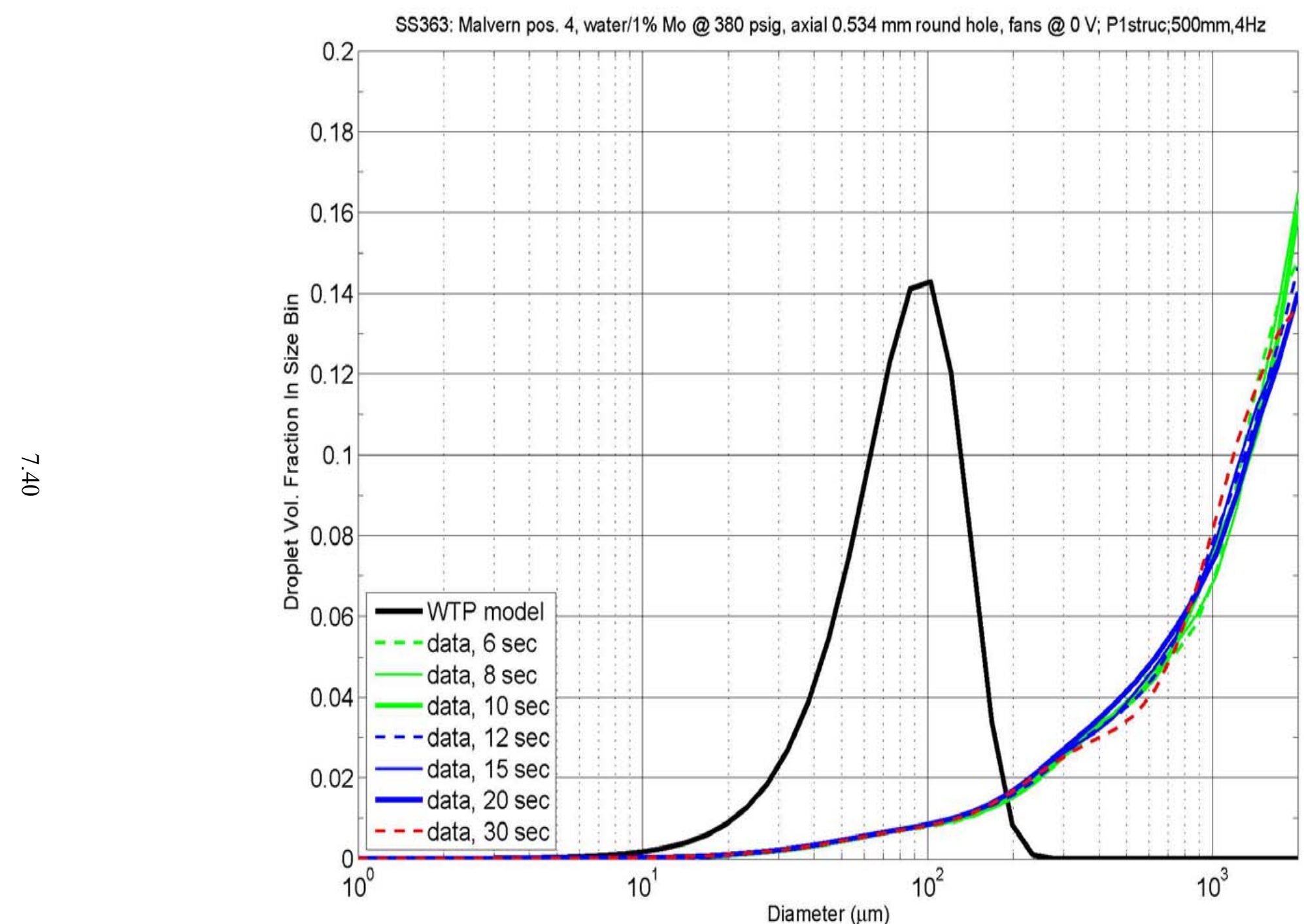

Figure 7.45. Differential In-Spray Size Distribution for a 380 psig Jet of Water/Mo from a $0.5 \mathrm{~mm}$ Round Hole 


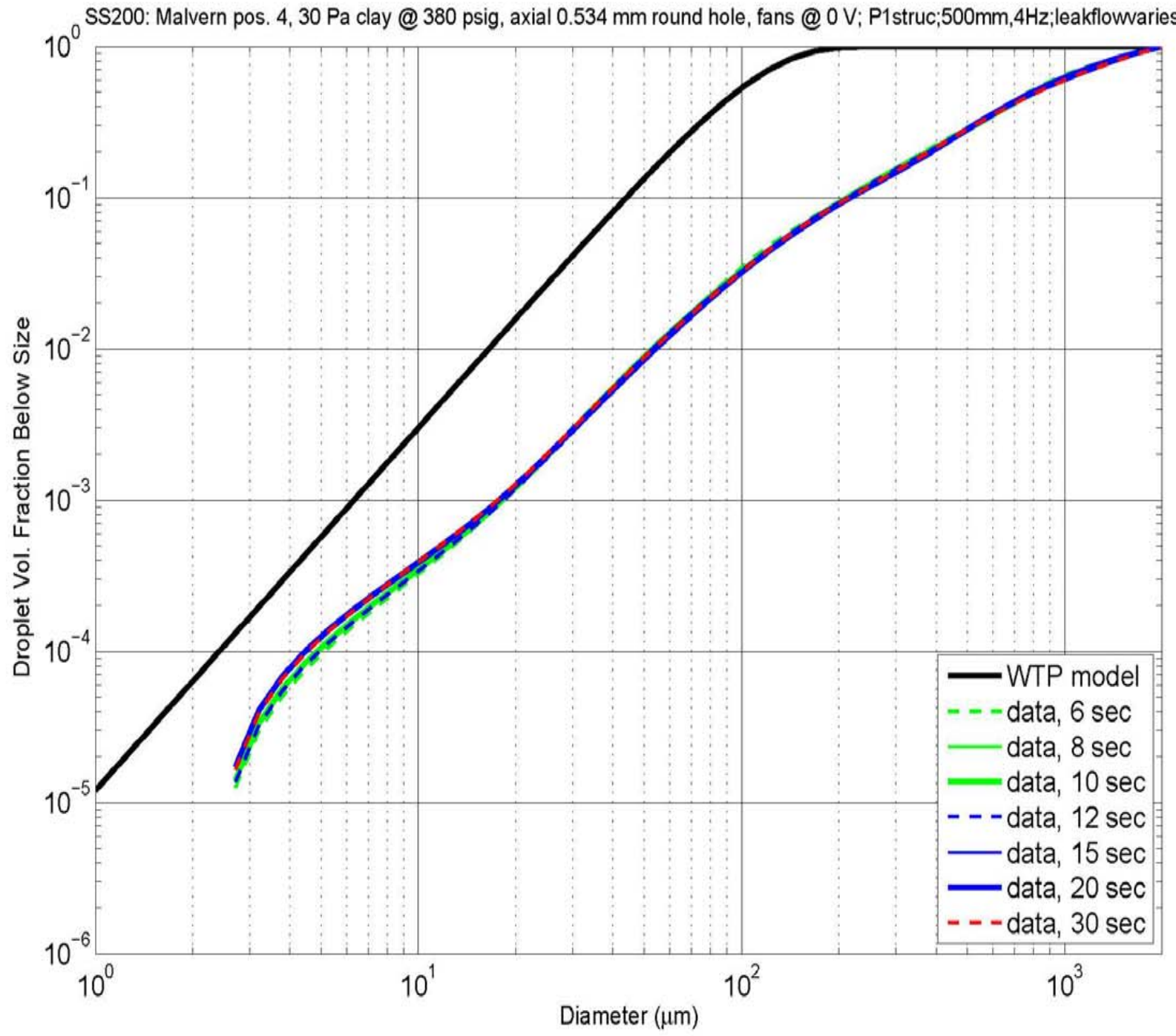

Figure 7.46. Cumulative In-Spray Size Distribution for a 380 psig Jet of 30 Pa Clay from a $0.5 \mathrm{~mm}$ Round Hole 
SS200: Malvern pos. 4,30 Pa clay @ 380 psig, axial 0.534 mm round hole, fans @ O V; P1struc;500mm,4Hz:leakflowvaries

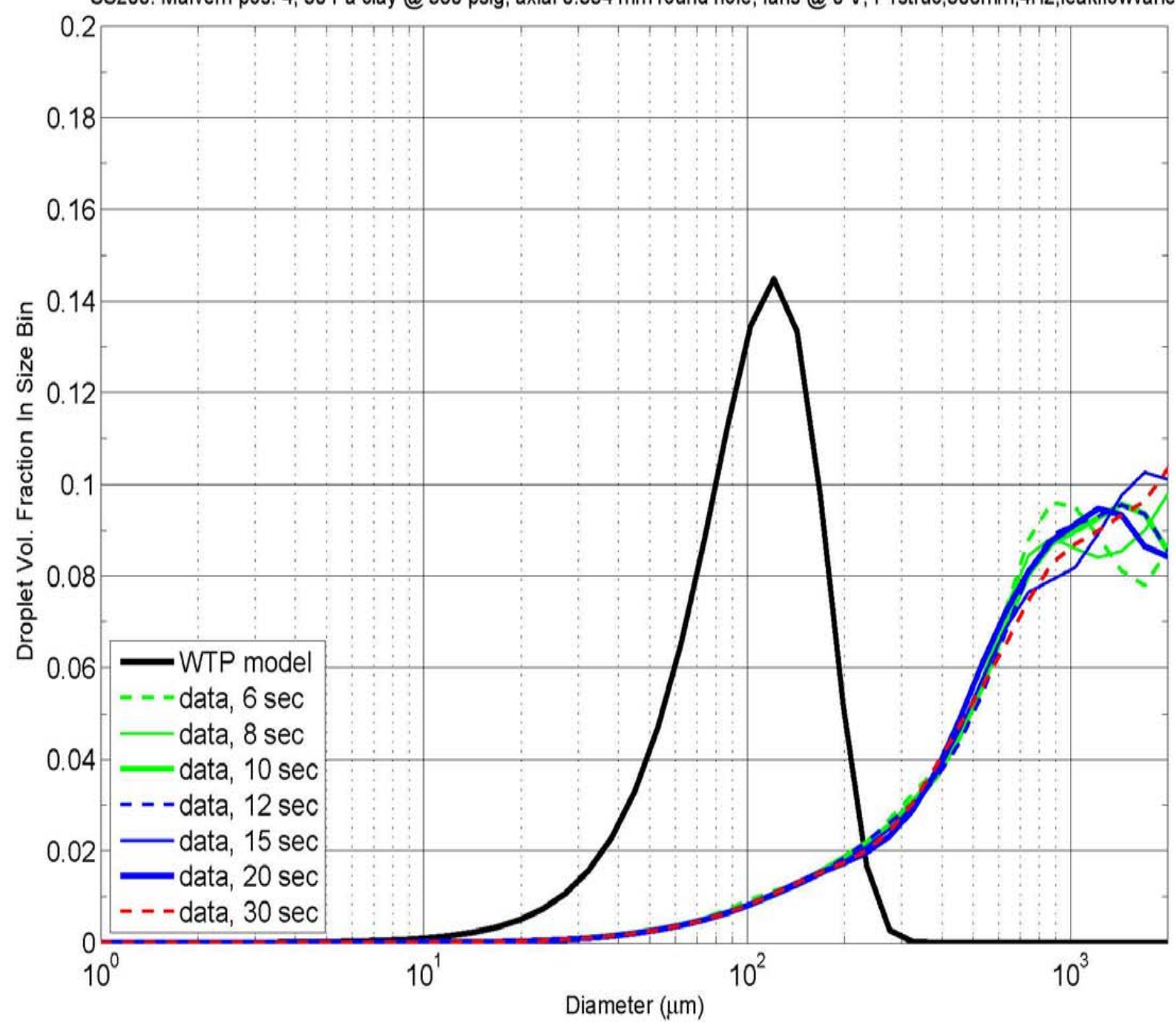

Figure 7.47. Differential In-Spray Size Distribution for a 380 psig Jet of 30 Pa Clay from a 0.5 mm Round Hole 
The meaningfulness of the small-scale in-spray data is in question, based on the peculiarly high droplet sizes for the PSD peaks. Supposing these droplet sizes to have been correct, much of the droplet volume was found in sizes too large to be measured. Therefore, these data have not been used in analyses and are not presented in their entirety in this report.

\subsection{Orifice Coefficients}

As an ancillary outcome of Phase I testing, orifice discharge coefficients were determined for many of the tests (Section 7.8, Mahoney et al. 2013). It was concluded that orifice coefficients were higher for orifices with area $<2 \mathrm{~mm}^{2}$ than for larger orifices and that there was data to show that the orifice coefficient was often higher than the value of 0.62 that has been used in WTP spray release modeling. Orifice coefficients have been calculated from Phase II data to check the results of Phase I. Table 7.1 and Table 7.2 provide a statistical comparison of Phase I and Phase II results. The coefficients are arranged by orifice and simulant; tests at all pressures are included but not specified in the tables.

The pattern of higher orifice coefficients for smaller holes was repeated in Phase II in the comparison between the $1 \mathrm{~mm}$ round hole and the $0.5 \times 5 \mathrm{~mm}$ slot. However, the coefficient was significantly smaller in Phase II for the $0.5 \mathrm{~mm}$ hole than for the $1 \mathrm{~mm}$ hole, whereas in Phase I, the two holes had nearly equal coefficients. The Phase II orifice data are expected to be more accurate than the Phase I data because the method of measuring the weight loss was improved (especially for the smallest flow rates, through small orifices, or at low pressure). In addition, the Phase II orifice data are expected to be more precise due to the higher number of runs for each simulant and orifice.

Table 7.1. Orifice Coefficients Measured in Round Holes in Phase I and Phase II

\begin{tabular}{lcccc}
\hline & \multicolumn{2}{c}{ Phase I Orifice Coefficients } & \multicolumn{2}{c}{ Phase II Orifice Coefficients } \\
\cline { 2 - 5 } & $\begin{array}{c}\text { No. of } \\
\text { Tests }\end{array}$ & $\begin{array}{c}\text { Mean and Standard } \\
\text { Deviation }\end{array}$ & $\begin{array}{c}\text { No. of } \\
\text { Tests }\end{array}$ & $\begin{array}{c}\text { Mean And Standard } \\
\text { Deviation }\end{array}$ \\
\hline Round hole, target dimension 0.5 mm diameter & & & \\
\hline Water & 2 & $0.70 \pm 0.006$ & 24 & $0.62 \pm 0.04$ \\
\hline Round hole, target dimension 1 mm diameter & & 10 & $0.74 \pm 0.02$ \\
\hline Water & 5 & $0.75 \pm 0.03$ & $\mathrm{n} / \mathrm{a}$ \\
1.8 mPa·s solution & 1 & 0.79 & $\mathrm{n} / \mathrm{a}$ & $\mathrm{n} / \mathrm{a}$ \\
2.6 mPa·s solution & 3 & $0.71 \pm 0.05$ & 10 & $0.92 \pm 0.04$ \\
STR (with and without AFA in Phase I, & 3 & $0.78 \pm 0.03$ & $\mathrm{n} / \mathrm{a}$ \\
with or without Mo in Phase II) & & & $\mathrm{n} / \mathrm{a}$ & $\mathrm{n} / \mathrm{a}$ \\
Small as-received (SAR) and TAR & 3 & $0.75 \pm 0.01$ & $\mathrm{n} / \mathrm{a}$ & $\mathrm{n} / \mathrm{a}$ \\
Phase I FER-6b (no AFA) & 2 & $0.80 \pm 0.14$ & $\mathrm{n} / \mathrm{a}$ & $0.90 \pm 0.04$ \\
Phase I FER-6g (AFA) & 2 & $0.73 \pm 0.008$ & 8 & $\mathrm{n} / \mathrm{a}$ \\
Phase II FEG-6 & $\mathrm{n} / \mathrm{a}$ & $\mathrm{n} / \mathrm{a}$ & $\mathrm{n} / \mathrm{a}$ \\
Phase I FER-30g (no AFA) & 2 & $0.85 \pm 0.06$ & $\mathrm{n} / \mathrm{a}$ & $0.86 \pm 0.12$ \\
Phase I FER-30g (AFA) & 2 & $0.70 \pm 0.03$ & $\mathrm{n} / \mathrm{a}$ & $0.91 \pm 0.05$ \\
Phase II FEG-30 & $\mathrm{n} / \mathrm{a}$ & $\mathrm{n} / \mathrm{a}$ & 7 & $0.94 \pm 0.04$ \\
Clay-6 & $\mathrm{n} / \mathrm{a}$ & $\mathrm{n} / \mathrm{a}$ & 9 & 16 \\
Clay-30 & $\mathrm{n} / \mathrm{a}$ & $\mathrm{n} / \mathrm{a}$ & &
\end{tabular}


Table 7.2. Orifice Coefficients Measured in the $0.5 \times 5 \mathrm{~mm}$ Slot (target dimensions) in Phase I and Phase II

\begin{tabular}{lcccc}
\hline & \multicolumn{2}{c}{ Phase I Orifice Coefficients } & \multicolumn{2}{c}{ Phase II Orifice Coefficients } \\
\cline { 2 - 5 } & $\begin{array}{c}\text { No. of } \\
\text { Tests }\end{array}$ & $\begin{array}{c}\text { Mean and Standard } \\
\text { Deviation }\end{array}$ & $\begin{array}{c}\text { No. of } \\
\text { Tests }\end{array}$ & $\begin{array}{c}\text { Mean and Standard } \\
\text { Deviation }\end{array}$ \\
\hline Water & 4 & $0.58 \pm 0.01$ & 7 & $0.63 \pm 0.003$ \\
STR (with and without AFA in Phase I, & 5 & $0.60 \pm 0.01$ & 13 & $0.74 \pm 0.03$ \\
with or without Mo in Phase II) & & & & \\
SAR and TAR & 3 & $0.65 \pm 0.03$ & $\mathrm{n} / \mathrm{a}$ & $\mathrm{n} / \mathrm{a}$ \\
Phase I FER-6b (no AFA) & 2 & $0.61 \pm 0.02$ & $\mathrm{n} / \mathrm{a}$ & $\mathrm{n} / \mathrm{a}$ \\
Phase I FER-6g (AFA) & 4 & $0.62 \pm 0.03$ & $\mathrm{n} / \mathrm{a}$ & $\mathrm{n} / \mathrm{a}$ \\
Phase II FEG-6 & $\mathrm{n} / \mathrm{a}$ & $\mathrm{n} / \mathrm{a}$ & 5 & $0.78 \pm 0.02$ \\
Phase I FER-30g (no AFA) & 2 & $0.61 \pm 0.08$ & $\mathrm{n} / \mathrm{a}$ & $\mathrm{n} / \mathrm{a}$ \\
Phase I FER-30g (AFA) & 3 & $0.57 \pm 0.009$ & $\mathrm{n} / \mathrm{a}$ & $\mathrm{n} / \mathrm{a}$ \\
Phase II FEG-30 & $\mathrm{n} / \mathrm{a}$ & $\mathrm{n} / \mathrm{a}$ & 7 & $0.78 \pm 0.04$ \\
Clay-6 & $\mathrm{n} / \mathrm{a}$ & $\mathrm{n} / \mathrm{a}$ & 9 & $0.78 \pm 0.02$ \\
Clay-30 & $\mathrm{n} / \mathrm{a}$ & $\mathrm{n} / \mathrm{a}$ & 19 & $0.75 \pm 0.05$ \\
\hline
\end{tabular}

The Phase II orifice coefficients for slurry simulants are higher than the Phase I coefficients, and in Phase II it is clear that the slurries (whether Newtonian or non-Newtonian) have higher orifice coefficients than water. Mean orifice coefficients for slurries flowing through the $1 \mathrm{~mm}$ hole and the $0.5 \times 5 \mathrm{~mm}$ slot were $\sim 0.9$ and $\sim 0.8$, respectively. These values are significantly greater than the coefficient of 0.62 used in WTP modeling. The increased orifice coefficients indicate flow rates which are 20 to 25 percent higher for slurries than for water through the same orifice.

\subsection{Summary of Results}

The results presented in this section focus on the effect of pressure and simulant properties on the cumulative release fraction as a function of droplet diameter. The trend in the release fractions as a function of pressure is compared to the trend predicted by the WTP model. The Phase II data yielded the following conclusions:

1. As pressure increased from 100 to $200 \mathrm{psig}$, the cumulative release fractions at $<10,<32$, and $<102 \mu \mathrm{m}$ usually decreased or remained constant. The relatively constant cumulative release fractions between 100 and 200 psig may be a function of the geometry of the small-scale chamber, as the large-scale chamber did not observe the same trend in this pressure range. As pressure increased from 200 to $380 \mathrm{psig}$, the cumulative release fraction usually increased at approximately the same rate as predicted by the WTP model. Overall, the trend with pressure is often not completely consistent with the predictions of the WTP model. It should be noted that the uncertainty of the results for any given combination of simulant and orifice is large enough to make the statistical significance of the trend unclear, but that the pattern is repeated over most of the combinations and so is worth considering (Section 7.1). 
2. A $27 \mathrm{wt} \%$ slurry of STR boehmite gave cumulative release fractions that were close to or identical with those of water and $20 \mathrm{wt} \%$ STR. At most, the release fractions for $27 \mathrm{wt} \%$ STR were 50 percent greater than those for water (Section 7.2).

3. The presence of a small fraction of dense particles (nominally $1 \mathrm{wt} \%$ Mo in water or in $20 \mathrm{wt} \% \mathrm{STR}$ ) did not produce any significant effect on the release fractions (Section 7.3).

4. In the droplet size range of interest $(<10$ to $<100 \mu \mathrm{m})$, the clay release fractions were always less than or equal to those of water within the variability of the measurements (Section 7.4.1).

5. For five out of the six test conditions (three pressures and two orifices), the release fractions for the 6 Pa clay equaled or slightly exceeded those for the $30 \mathrm{~Pa}$ clay. The exception was the 100 psig spray from the $0.5 \times 5 \mathrm{~mm}$ slot, where the $30 \mathrm{~Pa}$ release fractions were about triple the $6 \mathrm{~Pa}$ release fractions (Section 7.4.1).

6. The release fractions for one or both of the two strengths of FEG were always greater than or equal to those of water over at least part of the droplet size range of interest $(<10$ to $<100 \mu \mathrm{m})$. The tendency for FEG release fractions to exceed those of water would probably be increased if evaporation were accounted for, because the initial RH (as measured by an FIO instrument) was often 60 to 75 percent for the FEG tests and over 80 percent for the water tests. In the largest measured excess of FEG over water, the FEG release fractions were two to three times the water release fraction (Section 7.4.1).

7. For five out of the six test conditions, the release fractions for the $30 \mathrm{~Pa} \mathrm{FEG}$ equaled those for the $6 \mathrm{~Pa}$ FEG. In the case of the $0.5 \times 5 \mathrm{~mm}$ slot at $380 \mathrm{psig}$, the $30 \mathrm{~Pa}$ FEG release fractions exceeded the $6 \mathrm{~Pa}$ FEG release fractions by approximately 50 to 70 percent (Section 7.4.1).

8. The release fractions for FEG slurries consistently exceeded or equaled those for clay slurries over part or all of the droplet size range of interest $(<10$ to $<100 \mu \mathrm{m})$. The tendency for FEG release fractions to exceed those of clay would probably be increased if evaporation were accounted for, because the initial RH (as measured by an FIO instrument) was often 60 to 75 percent for the FEG tests and over 80 percent for the clay tests (Section 7.4.2).

9. A search of Hanford reports and consultation with topic experts has not located any samples of tank waste that exhibited the substantial thickening behavior of the FEG simulant. However, topic experts were not aware of any past tests that subjected waste samples to the repeated high shear conditions present in spray tests, so the absence of observations does not exclude the possibility that tank waste could behave in the manner observed for the FEG simulant if subjected to similar conditions (Section 7.4.3).

10. High concentrations of droplets with diameters twice the orifice size or larger were observed in the in-spray PSDs. The observations suggest that the liquid core of the jet had not completely broken up at the Malvern Insitec-S measurement location; thus, the small-scale in-spray data were not used in analyses (Section 7.5).

11. The Phase II orifice coefficients for slurry simulants are higher than the Phase I coefficients, and are considered to be better measurements than the Phase I coefficients. The slurries (whether Newtonian or non-Newtonian) have higher orifice coefficients than water. Mean orifice coefficients for slurries flowing through the $1 \mathrm{~mm}$ hole and the $0.5 \times 5 \mathrm{~mm}$ slot were $\sim 0.9$ and $\sim 0.8$, respectively. These values are significantly greater than the coefficient of 0.62 used in WTP modeling. The Phase II orifice coefficients and volumetric flow rates were 20 to 25 percent higher than those of water at similar test conditions (Section 7.6). 



\subsection{Conclusions and Recommendations}

Phase II aerosol spray tests were performed in the small-scale system to supplement Phase I testing described in Mahoney et al. (2013). As before, the release fractions and net generation rates were measured for orifice sizes, simulants, and spray pressures that represented expected WTP process stream properties and potential spray release scenarios. The trend in the release fractions as a function of pressure were compared to the trend predicted by the WTP model. For each set of conditions (i.e., particular orifice, simulant, and pressure), both in-chamber and in-spray measurements were made.

The test results are summarized as follows:

- The orifice coefficients were determined using a method expected to be more accurate than the method used in Phase I. Many of the Phase II orifice coefficients, especially for slurry simulants, are significantly greater than the coefficient of 0.62 used by the WTP model.

- The initial relative humidity in the chamber (based on an FIO measurement) affects the measured release fraction, in particular for RHs $<80$ percent. Using the data to extrapolate to 100 percent initial $\mathrm{RH}$ and interpolate to 80 percent initial $\mathrm{RH}$, the decrease in release fraction is approximately a factor of two across the range of typical initial RHs. The decrease in release fraction occurs across all droplet sizes. Consequently, release fractions measured at initial $\mathrm{RH}<80$ percent will be biased low by greater than a factor of two.

- In general, the Malvern Insitec-S focal length $(500 \mathrm{~mm})$ used in the Phase II testing gave higher release fractions compared to those found by the $100 \mathrm{~mm}$ focal length used in Phase I for tests at the same conditions. The data given by the $500 \mathrm{~mm}$ lens are higher because of the different measurable size ranges of the lens.

- Phase II release fraction measurements made using a $100 \mathrm{~mm}$ focal-length lens matched Phase I measurements for the same conditions within less than a factor of two.

- The cumulative release fractions did not have a monotonic relationship with pressure for the simulants tested in Phase II. Notably, there was no change or a small decrease in cumulative release fractions at $<10,<32$, and $<102 \mu \mathrm{m}$ as pressure increased from 100 to $200 \mathrm{psig}$; conversely, an increase in cumulative release fractions similar to the rate of the WTP model was observed as pressure increased from 200 to $380 \mathrm{psig}$. This suggests that release fractions at pressures $<100 \mathrm{psig}$ might be more likely to exceed the WTP model than they would be at pressures of $\geq 200$ psig; however, all of the apparent pressure trends are rendered ambiguous by the fact that the differences in release fraction from one pressure to another are of about the same size as the estimated uncertainty. Further, it is possible that this trend is dependent on chamber size (a similar trend was not observed in the large-scale chamber). In general, the cumulative release fraction at $<10 \mu \mathrm{m}$ was closer to the WTP model prediction than the cumulative release fraction at larger droplet sizes, particularly for the Fe-rich simulant.

- The $27 \mathrm{wt} \%$ STR slurry had cumulative release fractions that were very similar to, or at least indistinguishable from, both water and $20 \mathrm{wt} \%$ STR slurry (a Phase I measurement).

- The addition of a small fraction (nominally $1 \mathrm{wt} \%$ in the slurry) of dense particles (Mo with a density of $10.2 \mathrm{~g} / \mathrm{cm}^{3}$ ) to water and $19 \mathrm{wt} \%$ STR simulant did not result in a significant effect on the 
measured release fractions when compared to test data collected when the simulants were devoid of dense particles.

- Comparison of clay slurry and water cumulative release fractions in the droplet size range of interest, i.e., 10 to $100 \mu \mathrm{m}$, showed that the clay release fractions are less than or equal to those of water at both $6 \mathrm{~Pa}$ and $30 \mathrm{~Pa}$ yield stress.

- The cumulative release fractions of the chemical simulant (FEG) were consistently greater than those of water or the clay slurries. In general, the release fractions of the $30 \mathrm{~Pa}$ chemical simulant were similar to those of the $6 \mathrm{~Pa}$ chemical simulant. The release fractions of the chemical simulant were greater than those of water by as much as a factor of two to three.

- The PSD data collected for all simulants from in-spray measurements indicated that at least part of the liquid core of the spray was still intact at the point of measurement. Thus, the in-spray data could not be used as an estimate of an upper bound release fraction as it has been in the large-scale testing (see Daniel et al. 2013).

- Studies conducted to evaluate uncertainties surrounding the accuracy and performance of the Malvern Insitec-S aerosol instrument demonstrated that the concentration and particle size measurements of the instrument are not subject to any significant errors or biases and thus, do not require any adjustments. The Malvern Insitec-S accurately measures concentration and PSDs for spherical systems of droplets and applies appropriate corrections to account for attenuation.

The comparison between the chemical simulant and the clay slurry, and by extension, water, is complicated by several factors that increase the uncertainty of the results:

1. The rheology of the clay slurries (at both yield stresses) was more stable and well-controlled than the rheology of the chemical simulant slurries over the course of the testing. This influenced the achievable target flow rate in the system and how the tests were conducted.

2. In addition, the chemical simulant slurry exhibited time-dependent, reversible thickening with applied shear. Thus, it was difficult to assess the "true" rheology of the chemical simulant at the time a test was conducted and at the orifice as the simulant left the system (i.e., where shear rate is greatest).

3. Many chemical simulant tests were conducted at lower initial humidities (determined by an FIO measurement) than the corresponding tests conducted with the clay. The specific effect of humidity was measured on water, not on the chemical simulant; however, if the effect of humidity on the chemical simulant is analogous to the effect on water, the chemical simulant slurry release fractions would increase more than the release fractions of the clay or of water.

4. The spray for both rheological simulants was visually observed to be inconsistent in appearance and structure as it left the orifice. However, this tended to occur more frequently and to a greater degree with the chemical simulant. The effect this may have on the measurement of aerosol is unknown.

5. Perhaps because of the inconsistent spray structure, the chemical simulant release fraction data shows more test-to-test variability than the clay release fraction data, making trends more difficult to assess.

6. It is not clear how the chemical simulant, as formulated in both the Phase I and Phase II testing, compares to real waste that would be processed in the WTP. In particular, the processing dependent rheology has not, according to the available literature and discussion with topic experts, been encountered in any real waste studied to date. However, the tests that have been conducted on real 
waste may not have subjected the samples to enough shear to deduce whether the effects seen in the chemical simulant also appear in waste.

Recommendations based on the consideration of the Phase II small-scale test results, accounting for results of the Phase I testing already reported (Mahoney et al. 2013) are as follows:

- The cumulative release fractions measured for a variety of simulants in the in-chamber configuration support the conclusion that, on average, the release fraction of water is an upper bound for other simulant release fractions measured at the same pressure and using the same orifice. This does not hold universally; for instance, some of the Phase II chemical simulant release fractions exceed the water release fractions. However, if needed, the water release fractions could be adjusted using a simple multiplicative factor to encompass the entire release fraction data set measured in the small-scale system to date. It is recommended that the chemical simulant release fraction data are excluded in such an approach because the rheology was unusual, making the material difficult to test, and thus the simulant may not be representative of actual waste behavior. Furthermore, while such an approach has not been applied to the small-scale data discussed in this report, the Phase II large-scale data was used to develop a conservative correlation (see Section 10 in Daniel et al. 2013) and was shown to be a reasonably conservative estimate of aerosol generation rate.

- The chemical simulant as formulated in both Phase I and Phase II is challenging to use in small-scale aerosol testing. Further, the chemical simulant had unstable rheology and questions remain regarding how representative the chemical simulant is of expected WTP waste. If a simulant with chemical species similar to expected waste and a stable, bounding rheology is desired to be tested, it is recommended that the cause of the unusual rheology be identified and perhaps a new chemical simulant formulation be developed.

- In-spray testing was not feasible in the small-scale system as currently configured. In future work, in-spray tests should be restricted to the large-scale system. If additional in-spray testing is to be performed in the small-scale system, the chamber geometry will need to be modified to accommodate the acquisition of a more accurate measurement (i.e., further downstream from the orifice).

- The small-scale system has accumulated a large data set of orifice coefficients over the course of aerosol testing. The data set suggests, particularly for slurries, that coefficients significantly greater than 0.62 (the WTP model input) occur in orifices regardless of type, i.e., in both round and slot orientations. Caution is recommended when using a value of 0.62 for all expected fluids in the WTP, as it may under-estimate the release generated from slurries. 



\subsection{References}

10 CFR 830, Subpart A. 2010. "Nuclear Safety Management.” Subpart A, "Quality Assurance Requirements.” Code of Federal Regulations, U.S. Department of Energy, Washington, D.C.

ASME - American Society of Mechanical Engineers. 2001. Quality Assurance Requirements for Nuclear Facility Applications, Part 1, "Requirements for Quality Assurance Programs for Nuclear Facilities." New York, New York.

ASME - American Society of Mechanical Engineers. 2001. Quality Assurance Requirements for Nuclear Facility Applications, Part II, Subpart 2.7, "Quality Assurance Requirements for Computer Software for Nuclear Facility Applications.” New York, New York.

ASME - American Society of Mechanical Engineers. 2001. Quality Assurance Requirements for Nuclear Facility Applications, Part IV, Subpart 4.2, "Graded Approach Application of Quality Assurance Requirements for Research and Development." New York, New York.

Bamberger JA, PA Meyer, JR Bontha, CW Enderlin, AP Poloski, JA Fort, ST Yokuda, HD Smith, F Nigl, M Friedrich, DE Kurath, GL Smith, JM Bates, MA Gerber, and DA Wilson. 2005. Technical Basis for Testing Scaled Pulse Jet Mixing Systems for Non-Newtonian Slurries. PNWD-3551, WTP-RPT-113, Rev. 0, Battelle-Pacific Northwest Division, Richland, WA.

Bohren CF and DR Huffman. 1983. Absorption and Scattering of Light by Small Particles. Wiley, New York, p. 149.

Boyaval S and C Dumouchel. 2001. "Deconvolution Technique to Determine Local Spray Drop Size Distributions - Application to High-Pressure Swirl Atomizers." In Proceedings of the $17^{\text {th }}$ Annual Conference on Liquid Atomization and Spray Systems, September 2-6, 2001, Zurich, Switzerland.

Bruggeman DAG. 1935. "Berechnung verschiedener physikalischer Konstanten von heterogenen Substanzen." Annuals of Physics (Leipzig) 24:636-679.

Campbell T, M Parker, A Moon, B Fant, K Clossey, and J Cook. 2010. EFRT Issue M3 PJM Vessel Mixing Assessment, Volume 8-HLP-22. 24590-WTP-RPT-ENG-08-021-08, Rev. 1, River Protection Project, Waste Treatment Plant, Richland, Washington.

Crowe RD. 2010. Sludge Treatment Project (STP) Methodology for Spray Leak Scenarios. PRC-STP-00292, Rev. 0, CH2M HILL Plateau Remediation Company, Richland, Washington.

Daniel RC, JM Billing, JR Bontha, CA Burns, MS Fountain, PA Gauglitz, JJ Jenks, DE Kurath, PJ MacFarlan, RW Shimskey, and LA Mahoney. 2013. Large-Scale Spray Releases: Additional Aerosol Test Results. PNNL-22415, WTP-RPT-221, Rev. 0, Pacific Northwest National Laboratory, Richland, Washington.

Denn MM. 1980. Process Fluid Mechanics. Prentice-Hall, Inc., Englewood Cliffs, New Jersey.

DOE Order 414.1D. 2011. “Quality Assurance.” U.S. Department of Energy, Washington, D.C. 
Dombrowski N and WR Johns. 1963. "The Aerodynamic Instability and Disintegration of Viscous Liquid Sheets.” Chemical Engineering Science 18:203-214.

Epstein M and MG Plys. 2006. Measured Drop Size Distributions with Cold Sprays Emanating from Small Leak Openings. FAI/06-55, Fauske \& Associates, LLC., Burr Ridge, Illinois.

Garnett JCM. 1904. "Colours in metal glasses and in metallic films." Philosophical Transactions of the Royal Society London 203:385-420.

Gimpel R. 2010. Slurry Property Ranges in Non-Newtonian Pretreatment Vessels at WTP. 24590-WTP-RPT-PET-10-014, Rev. 2, River Protection Project, Waste Treatment Plant, Richland, Washington.

Kurath DE, BD Hanson, MJ Minette, DL Baldwin, BM Rapko, LA Mahoney, PP Schonewill, RC Daniel, PW Eslinger, JL Huckaby, JM Billing, PS Sundar, GB Josephson, JJ Toth, ST Yokuda, EBK Baer, SM Barnes, EC Golovich, SD Rassat, CF Brown, JGH Geeting, GJ Sevigny, AJ Casella, JR Bontha, RL Aaberg, PM Aker, CE Guzman-Leong, ML Kimura, SK Sundaram, RP Pires, BE Wells, and OP Bredt. 2009. Pretreatment Engineering Platform Phase-1 Final Test Report. PNNL-18894, WTP-RPT-197, Pacific Northwest National Laboratory, Richland, Washington.

Larson AR and BT Allen. 2010. WTP Methodology for Spray Leak Scenarios. 24590-WTP-RPT-ENS-10-001, Rev. 1, River Protection Project, Waste Treatment Plant, Richland, Washington.

Levy N, S Amara, J-C Champoussin, and N Guerrassi. 1997. "Non-Reactive Diesel Spray Computations Supported by PDA Measurements." SAE Paper 970049.

Looyenga H. 1965. "Dielectric Constants of Heterogeneous Mixtures.” Physica 31(3):401-406.

Mahoney LA, PA Gauglitz, J Blanchard, ML Kimura, and DE Kurath. 2012. Small-scale Spray Releases: Orifice Plugging Test Results. PNNL-21361, WTP-RPT-219, Rev. 0, Pacific Northwest National Laboratory, Richland, Washington.

Mahoney LA, PA Gauglitz, ML Kimura, GN Brown, DE Kurath, J Blanchard, C Song, RC Daniel, BE Wells, D Tran, WC Buchmiller, CA Burns and DM Smith. 2013. Small-scale Spray Releases: Initial Aerosol Test Results. PNNL-21367, WTP-RPT-216 Rev. 1, Pacific Northwest National Laboratory, Richland, Washington.

Malvern Instruments Ltd. 2010. RTSizer and Insitec Analyser User Manual. MAN0467 Issue 1.0, Malvern Instruments Ltd., Malvern, Worcestershire, United Kingdom.

McAllister J. 2010. Severity Level Calculations for the Pretreatment Facility Based on Updated MAR. Calculation No. 24590-PTF-Z0C-W14T-00036, Rev. B, River Protection Project, Waste Treatment Plant, Richland, Washington.

Monecke J. 1994. "Bergman Spectral Representation of a Simple Expression for the Dielectric Response of a Symmetric Two-Component Composite." Journal of Physics: Condensed Matter 6:907-912. 
Nasr GG, AJ Yule, and L Bendig. 2002. Industrial Sprays and Atomization: Design, Analysis and Applications. Springer, New York.

Poloski AP, PA Meyer, LK Jagoda, and PR Hrma. 2004. Non-Newtonian Slurry Simulant Development and Selection for Pulse Jet Mixer Testing. PNWD-3495, WTP-RPT-111, Rev. 0, Battelle--Pacific Northwest Division, Richland, Washington.

Rosin P and E Rammler. 1933. "The Laws Governing the Fineness of Powdered Coal." Journal of the Institute of Fuel 7:29-36.

Russell RL, SD Rassat, ST Arm, MS Fountain, BK Hatchell, CW Stewart, CD Johnson, PA Meyer, and CE Guzman-Leong. 2005. Final Report: Gas Retention and Release in Hybrid Pulse Jet Mixed Tanks Containing Non-Newtonian Waste Simulants. PNWD-3552, WTP-RPT-114, Rev. 1, Battelle-Pacific Northwest Division, Richland, Washington.

Schonewill PP, PA Gauglitz, JR Bontha, RD Daniel, JJ Jenks, DE Kurath, JM Billing, HE Adkins Jr, CW Enderlin, CA Burns, C Fischer, CD Lukins, JL Shutthanandam, and DM Smith. 2012. Large-Scale Spray Releases: Initial Aerosol Test Results. PNNL-21333, WTP-RPT-217, Rev. 0, Pacific Northwest National Laboratory, Richland, Washington.

Williams JC. 2000. Analysis of Waste Leak and Toxic Chemical Release Accidents from Waste Feed Delivery (WFD) Dilute System. RPP-5098, Rev. 1, Fluor Hanford, Richland, Washington.

Zhao H, HF Liu, JL Xu, and WF Li. 2011. "Secondary breakup of coal water slurry drops." Physics of Fluids 23, 113101.

Zimmerman BD. 2003. Waste Transfer Leaks Technical Basis Document. RPP-13750, Rev. 0, CH2M HILL Hanford Group, Inc., Richland, Washington. 

Appendix A

Run Log and Test Conditions 



\section{Appendix A}

\section{Run Log and Test Conditions}

This appendix contains the complete list of Phase II tests conducted using the small-scale system. Table A.1 lists relevant metadata for each test. Each gray-shaded entry denotes a test that was not analyzed for release fraction because the test was aborted or an off-normal condition was observed during testing. Gray-shaded entries do not include every test that was not analyzed, only those deemed unsuitable during test operations. Additional criteria were applied to the unshaded entries in Table A.1 according to the data-screening process described in Section 6.3.2.

Each entry in Table A.1 shows the nominal or target test conditions, including the following:

- Test ID: the unique identifier for each test.

- Simulant: the simulant used. Table 5.1 provides the definitions of, and planned testing for, each simulant. Section 3 discusses the physical properties of each simulant.

- Mal. Pos.: the Malvern Insitec-S instrument position (see Figure 4.7). Position 2 is also referred to as an in-chamber measurement or test and position 4 as an in-spray measurement or test.

- Ext. Len.: the extension length added to the standard spray header to facilitate the collection of in-spray measurements. An entry of "N/A" indicates no extensions were installed.

- Mixing Fan: the voltage applied to the mixing fan.

- Nom. P: the target pressure, in psig. Actual measured pressure varies approximately \pm 10 percent from the target pressure (or in some cases, was outside of this range and the test was repeated).

- OTP: the orifice test piece used. See Table 4.4 for nominal and actual dimensions of the OTPs.

- Date: the calendar day the test was conducted.

- Spray Start: the manually recorded time that spray was started. This was, in most cases, the start time used in analysis. However, the actual start time was determined using the valve position indication as described in Section 6.1.

- Spray Stop: the manually recorded time that spray was stopped.

In addition to the test metadata, some actual test data are presented in the run log. These data were manually recorded during the test and were not the values used in the data analysis; rather, they give a "snapshot" of the test conditions. These include the following:

- Header Flow: the flow rate during the spray as recorded from the Coriolis flow meter display, in gpm.

- Loop Temp: the temperature in the flow loop just upstream of the spray header, in degrees Fahrenheit.

- Pretest Tank Mass: the mass of simulant, either gross (including the feed tank) or net (simulant only), recorded before the spray was started, in pounds. The convention used to measure the pretest tank mass (i.e., gross or net) was also used to measure final tank mass. 
- Final Tank Mass: the mass of simulant recorded after the spray was stopped, in pounds.

- Notes: any observations on the test, typically recording significant abnormal conditions or changes to conditions outside standard operational configuration or procedure.

- TDP: the test data package that contains the documentation for the test.

For the release fractions corresponding to tests shown in Table A.1, refer to Appendix B.

Additional data from each test are given in Table A.2. Data are cross-referenced using the Test ID number. In contrast to the information given in Table A.1, many of the values in Table A.2 are calculated from data collected by the data acquisition system. The data in Table A.2 include:

- Test ID: the unique identifier for each test.

- Target P: the pressure targeted for the test, in psig.

- Simulant: the simulant used in the test, where references to physical properties in the simulant name refer to target values.

- Orifice Dimensions: either diameter (the value is repeated) or width and length, in $\mathrm{mm}$.

- Average Header Flow: average flow rate in the spray header during the entire test, in gpm.

- Average P: the square of the average of the square root of the pressure for the period where the aerosol data was fit, in psig.

- Average T: the average temperature during the entire test, in degrees Celsius.

- Average Leakage Flow Rate: the average leak flow rate emitted from the orifice during the period over which the aerosol data was fit, in $\mathrm{g} / \mathrm{s}$.

- Orifice Coefficient: orifice coefficient calculated from the average leak flow rate from the preceding column.

- Initial RH: the initial relative humidity (if available) recorded before the spray was started, in percent. 
Table A.1. Phase II Small-Scale Run Log

\begin{tabular}{|c|c|c|c|c|c|c|c|c|c|c|c|c|c|c|c|c|c|}
\hline Test ID & Simulant & $\begin{array}{l}\text { Mal. } \\
\text { Pos. }\end{array}$ & $\begin{array}{l}\text { Ext. } \\
\text { Len. } \\
\text { (in.) }\end{array}$ & $\begin{array}{l}\text { Malvern } \\
\text { Lens } \\
(\mathrm{mm})\end{array}$ & $\begin{array}{l}\text { Mixing } \\
\text { Fan (V) }\end{array}$ & $\begin{array}{l}\text { Actual } \\
\text { Purge } \\
\text { Flow per } \\
\text { Window } \\
\text { (SCFH) } \\
\end{array}$ & $\begin{array}{c}\text { Nom. P } \\
\text { (psig) }\end{array}$ & ОТР & $\begin{array}{c}\text { Date } \\
(\mathrm{mm} / \mathrm{dd} / \mathrm{yyyy})\end{array}$ & \begin{tabular}{|} 
Spray Start \\
$($ hh:mm:ss)
\end{tabular} & $\begin{array}{l}\text { Spray Stop } \\
\text { (hh:mm:ss) }\end{array}$ & $\begin{array}{l}\text { Header } \\
\text { Flow } \\
(\mathrm{gpm})\end{array}$ & \begin{tabular}{|l|} 
Loop \\
Temp \\
During \\
Spray \\
$\left({ }^{\circ} \mathrm{F}\right)$ \\
\end{tabular} & $\begin{array}{c}\text { Pretest } \\
\text { Tank } \\
\text { Mass } \\
\text { (lb) }\end{array}$ & $\begin{array}{c}\text { Final } \\
\text { Tank } \\
\text { Mass (lb) } \\
\end{array}$ & Notes & TDP \\
\hline SS-091 & Water & 2 & N/A & 500 & 6 & 1.2 & 380 & OTP-03 & $07 / 23 / 2012$ & 11:02:08 & $11: 04: 22$ & 11.5 & 68.0 & 325.00 & 323.00 & OTP was upside down. & \multirow{6}{*}{$\begin{array}{l}\text { TDP- } \\
862\end{array}$} \\
\hline SS-092 & Water & 2 & N/A & 500 & 6 & 1.2 & 380 & OTP-03 & $07 / 23 / 2012$ & $11: 32: 32$ & $11: 34: 43$ & 11.4 & 68.7 & 322.00 & 320.00 & OTP was upside down. & \\
\hline SS-093 & Water & 2 & N/A & 500 & 6 & 1.2 & 380 & OTP-03 & $07 / 23 / 2012$ & 11:46:14 & 11:48:35 & 11.4 & 68.9 & 319.00 & 317.06 & OTP was upside down. & \\
\hline SS-094 & Water & 2 & N/A & 500 & 6 & 1.2 & 380 & OTP-03 & $07 / 23 / 2012$ & 14:07:54 & 14:10:00 & 11.4 & 70.7 & 313.04 & 310.82 & OTP was upside down. & \\
\hline SS-095 & Water & 2 & N/A & 500 & 6 & 1.2 & 380 & OTP-03 & $07 / 23 / 2012$ & $14: 20: 51$ & 14:23:13 & 10 & 70.4 & 310.04 & 307.80 & $\begin{array}{l}\text { Malvern Update set to } 1 \mathrm{~Hz} \text {. } \\
\text { OTP was upside down. }\end{array}$ & \\
\hline SS-096 & Water & 4 & $\mathrm{~N} / \mathrm{A}$ & 500 & 6 & 1.2 & 380 & OTP-03 & $07 / 23 / 2012$ & 15:48:37 & $15: 50: 49$ & 11.4 & 69.0 & 302.72 & 300.58 & $\begin{array}{l}\text { Malvern set to } 4 \mathrm{~Hz} \text {. Data } \\
\text { was bad-lens got wet. } \\
\text { Mixing fan was on. } \\
\text { Pre-spray was performed. }\end{array}$ & \\
\hline SS-097 & Water & 4 & N/A & 500 & off & 1.2 & 380 & OTP-03 & $07 / 24 / 2012$ & 11:09:25 & 11:10:05 & 11.6 & 66.7 & 389.92 & 389.34 & $\begin{array}{l}\text { No pre-spray, mixing fan off. } \\
30 \text { second spray. }\end{array}$ & \multirow[t]{9}{*}{$\begin{array}{c}\text { TDP- } \\
863\end{array}$} \\
\hline SS-098 & -- & 4 & N/A & 500 & off & $\mathrm{n} / \mathrm{a}$ & 380 & OTP-03 & $07 / 24 / 2012$ & N/A & N/A & $\mathrm{N} / \mathrm{A}$ & N/A & N/A & N/A & $\begin{array}{l}\text { Non-QA - scoping tests for } \\
\text { air purge. Large rotameter } \\
\text { was used with a range of } \\
\text { purge flows. }\end{array}$ & \\
\hline SS-099 & Water & 4 & N/A & 500 & off & 60 & 380 & OTP-03 & $07 / 24 / 2012$ & $13: 37: 20$ & 13:38:07 & 11.5 & 68.7 & 382.68 & 381.60 & $\begin{array}{l}\text { No pre-spray, mixing fan off. } \\
30 \text { second spray. }\end{array}$ & \\
\hline SS-100 & Water & 4 & N/A & 500 & off & 40 & 380 & OTP-03 & $07 / 24 / 2012$ & $13: 50: 02$ & 13:50:47 & 11.6 & 68.7 & 381.58 & 380.56 & $\begin{array}{l}\text { No pre-spray, mixing fan off. } \\
30 \text { second spray. }\end{array}$ & \\
\hline SS-101 & Water & 4 & N/A & 500 & off & 20 & 380 & OTP-03 & $07 / 24 / 2012$ & 14:01:50 & 14:02:43 & 11.6 & 68.9 & 380.52 & 379.34 & Failed test. & \\
\hline SS-102 & Water & 4 & N/A & 500 & off & 20 & 380 & OTP-03 & $07 / 24 / 2012$ & $14: 10: 25$ & 14:11:46 & 11.6 & 69.2 & 379.36 & 377.50 & $\begin{array}{l}\text { No pre-spray, mixing fan off. } \\
30 \text { second spray. }\end{array}$ & \\
\hline SS-103 & Water & 2 & $\mathrm{~N} / \mathrm{A}$ & 500 & 6 & 1.2 & 380 & OTP-03 & $07 / 24 / 2012$ & 15:39:10 & 15:41:18 & 11.5 & 69.0 & 373.32 & 370.40 & OTP in upright position. & \\
\hline SS-104 & Water & 2 & $\mathrm{~N} / \mathrm{A}$ & 500 & 6 & 1.2 & 380 & OTP-03 & $07 / 24 / 2012$ & $16: 01: 52$ & 16:04:03 & 11.5 & 69.0 & 370.38 & 367.32 & $\begin{array}{l}\text { Test to control humidity. The } \\
\text { test was run after evacuating } \\
\text { to RH }<60 \% \text {. } \\
\end{array}$ & \\
\hline SS-105 & Water & 2 & $\mathrm{~N} / \mathrm{A}$ & 500 & 6 & 1.2 & 380 & OTP-03 & $07 / 24 / 2012$ & $16: 24: 50$ & $16: 27: 08$ & 11.5 & 69.3 & 361.60 & 358.42 & $\begin{array}{l}\text { Highest humidity. Chamber } \\
\text { was not evacuated. The } \\
\text { aerosol was allowed to drop } \\
\text { to below detection limits and } \\
\text { then waited } \sim 5 \text { min prior to } \\
\text { initiating the test. }\end{array}$ & \\
\hline SS-106 & Water & 2 & N/A & 500 & 6 & 1.2 & 380 & OTP-03 & $07 / 25 / 2012$ & 10:17:25 & 10:19:49 & 11.4 & 83.5 & 353.68 & 351.22 & $\begin{array}{l}\text { Warm water test. Target } \\
\text { initial temp } 80^{\circ} \text {. Pre-spray } \\
\text { evacuation was conducted } \\
\text { before initiating the test. }\end{array}$ & $\begin{array}{l}\text { TDP- } \\
864\end{array}$ \\
\hline
\end{tabular}


Table A.1. (contd)

\begin{tabular}{|c|c|c|c|c|c|c|c|c|c|c|c|c|c|c|c|c|c|}
\hline Test ID & Simulant & $\begin{array}{l}\text { Mal. } \\
\text { Pos. }\end{array}$ & $\begin{array}{l}\text { Ext. } \\
\text { Len. } \\
\text { (in.) }\end{array}$ & $\begin{array}{l}\text { Malvern } \\
\text { Lens } \\
(\mathrm{mm})\end{array}$ & $\begin{array}{l}\text { Mixing } \\
\text { Fan (V) }\end{array}$ & $\begin{array}{l}\text { Actual } \\
\text { Purge } \\
\text { Flow per } \\
\text { Window } \\
\text { (SCFH) }\end{array}$ & $\begin{array}{l}\text { Nom. P } \\
\text { (psig) }\end{array}$ & OTP & $\begin{array}{c}\text { Date } \\
\text { (mm/dd/yyy) }\end{array}$ & $\begin{array}{l}\text { Spray Start } \\
\text { (hh:mm:ss) }\end{array}$ & $\begin{array}{l}\text { Spray Stop } \\
\text { (hh:mm:ss) }\end{array}$ & $\begin{array}{l}\text { Header } \\
\text { Flow } \\
(\mathrm{gpm})\end{array}$ & $\begin{array}{l}\text { Loop } \\
\text { Temp } \\
\text { During } \\
\text { Spray } \\
\left({ }^{\circ} \mathrm{F}\right)\end{array}$ & $\begin{array}{l}\text { Pretest } \\
\text { Tank } \\
\text { Mass } \\
\text { (lb) }\end{array}$ & $\begin{array}{c}\text { Final } \\
\text { Tank } \\
\text { Mass (lb) }\end{array}$ & Notes & TDP \\
\hline SS-107 & Water & 2 & N/A & 500 & 6 & 1.2 & 380 & OTP-03 & $07 / 25 / 2012$ & $11: 21: 23$ & 11:23:42 & 11.5 & 69.9 & 350.06 & 347.86 & Splash guard. & \\
\hline SS-108 & Water & 2 & N/A & 100 & 6 & 1.2 & 380 & OTP-03 & $07 / 26 / 2012$ & $16: 02: 45$ & 16:04:49 & 11.5 & 67.8 & 339.90 & 337.94 & $\begin{array}{l}\text { This test used the same } \\
\text { conditions as SS- } 103 \text { except } \\
\text { that the } 100 \text { - lens was in the } \\
\text { Malvern. Humidity }=94.2 \\
\text { during test. }\end{array}$ & \multirow[t]{5}{*}{$\begin{array}{l}\text { TDP- } \\
865\end{array}$} \\
\hline SS-109 & Water & 2 & N/A & 100 & 6 & 1.2 & 380 & OTP-03 & $07 / 26 / 2012$ & $16: 23: 38$ & $16: 25: 45$ & 11.4 & 68.4 & 337.04 & 334.08 & $\begin{array}{l}\text { Duplicate test to SS-108. } \\
\text { R humidity }=95.3 .\end{array}$ & \\
\hline SS-110 & Water & 2 & N/A & 100 & 6 & 1.2 & 380 & OTP-03 & $07 / 26 / 2012$ & $16: 40: 23$ & $16: 42: 51$ & 11.5 & 68.5 & 334.08 & 330.72 & $\begin{array}{l}\text { Same conditions as test } \\
\text { SS-108 except that the } \\
\text { support rod was removed to } \\
\text { better simulate Phase I } \\
\text { conditions. The Malvern was } \\
\text { supported by the lifting } \\
\text { mechanism only. } 100 \mathrm{~mm} \\
\text { lens, } 11.4 \text { gpm and } 4 \mathrm{~Hz} \text {. } \\
\text { Humidity }=93.6 \text {. }\end{array}$ & \\
\hline SS-111 & Water & 2 & $\mathrm{~N} / \mathrm{A}$ & 100 & 6 & 1.2 & 380 & OTP-03 & $07 / 26 / 2012$ & $16: 52: 49$ & $16: 55: 01$ & 10.1 & 69.3 & 330.64 & 327.60 & $\begin{array}{l}\text { Malvern was supported by } \\
\text { lifting mechanism only. } \\
\text { Malvern freq. }=1 \mathrm{~Hz} \\
\text { humidity }=94.6 . \text { Flow at } \\
10 \mathrm{gpm} .\end{array}$ & \\
\hline SS-112 & Water & 2 & N/A & 100 & 6 & 1.2 & 380 & OTP-03 & $07 / 26 / 2012$ & 17:04:09 & $17: 06: 25$ & 10.0 & 69.7 & 327.62 & 324.42 & $\begin{array}{l}\text { Same test as SS- } 111 \text { only the } \\
\text { Malvern freq. }=4 \mathrm{~Hz} \\
\text { humidity }=95.2 . \text { Flow at } \\
10 \mathrm{gpm} .\end{array}$ & \\
\hline SS-113 & Water & 2 & N/A & 100 & 6 & 1.2 & 380 & OTP-05 & $07 / 27 / 2012$ & $11: 34: 20$ & $11: 36: 26$ & 10.2 & 66.6 & 318.70 & 307.60 & $\begin{array}{l}\text { Malvern was supported by } \\
\text { lifting mechanism only. } \\
1 \mathrm{~mm} \text { orifice. Malvern rate }= \\
1 \mathrm{~Hz} . \mathrm{RH}=96.2 \text {. }\end{array}$ & \multirow[t]{3}{*}{$\begin{array}{l}\text { TDP- } \\
866\end{array}$} \\
\hline SS-114 & Water & 2 & N/A & 100 & 6 & 1.2 & 380 & OTP-05 & $07 / 27 / 2012$ & 11:46:58 & 11:49:11 & 10.0 & 67.6 & 306.46 & 295.90 & $\begin{array}{l}\text { Malvern was supported by } \\
\text { lifting mechanism only. } \\
1 \mathrm{~mm} \text { orifice. Malvern rate }= \\
4 \mathrm{~Hz} \text {. RH }=96.9 \text {. It was } \\
\text { noted in the LRB that B- } 49 \\
\text { bonnet was loose and the ale } \\
\text { changed position during } \\
\text { testing due to system } \\
\text { vibrations. The ale was } \\
\text { tightened after test SS-114. }\end{array}$ & \\
\hline SS-115 & Water & 2 & N/A & 100 & 6 & 1.2 & 380 & OTP-05 & $07 / 27 / 2012$ & $13: 25: 10$ & N/A & N/A & N/A & N/A & N/A & Failed test. & \\
\hline
\end{tabular}


Table A.1. (contd)

\begin{tabular}{|c|c|c|c|c|c|c|c|c|c|c|c|c|c|c|c|c|c|}
\hline Test ID & Simulant & $\begin{array}{l}\text { Mal. } \\
\text { Pos. }\end{array}$ & $\begin{array}{l}\text { Ext. } \\
\text { Len. } \\
\text { (in.) }\end{array}$ & $\begin{array}{l}\text { Malvern } \\
\text { Lens } \\
(\mathrm{mm})\end{array}$ & $\begin{array}{l}\text { Mixing } \\
\text { Fan (V) }\end{array}$ & $\begin{array}{l}\text { Actual } \\
\text { Purge } \\
\text { Flow per } \\
\text { Window } \\
\text { (SCFH) }\end{array}$ & $\begin{array}{c}\text { Nom. P } \\
\text { (psig) }\end{array}$ & OTP & $\begin{array}{c}\text { Date } \\
\text { (mm/dd/yyyy) }\end{array}$ & $\begin{array}{l}\text { Spray Start } \\
\text { (hh:mm:ss) }\end{array}$ & $\begin{array}{l}\text { Spray Stop } \\
\text { (hh:mm:ss) }\end{array}$ & $\begin{array}{l}\text { Header } \\
\text { Flow } \\
(\mathrm{gpm})\end{array}$ & $\begin{array}{l}\text { Loop } \\
\text { Temp } \\
\text { During } \\
\text { Spray } \\
\left({ }^{\circ} \mathrm{F}\right)\end{array}$ & $\begin{array}{l}\text { Pretest } \\
\text { Tank } \\
\text { Mass } \\
\text { (lb) }\end{array}$ & $\begin{array}{c}\text { Final } \\
\text { Tank } \\
\text { Mass (lb) }\end{array}$ & Notes & TDP \\
\hline SS-116 & Water & 2 & N/A & 100 & 6 & 1.2 & 380 & OTP-05 & $07 / 27 / 2012$ & $13: 41: 44$ & $13: 43: 49$ & 9.9 & 69.1 & 274.26 & 264.38 & $\begin{array}{l}\text { Malvern was supported by } \\
\text { lifting mechanism only. } \\
1 \mathrm{~mm} \text { orifice. Malvern rate }= \\
4 \mathrm{~Hz} . \mathrm{RH}=96.3 .\end{array}$ & \\
\hline SS-117 & Water & 2 & N/A & 100 & 6 & 1.2 & 380 & OTP-05 & $07 / 27 / 2012$ & $13: 52: 27$ & $13: 54: 45$ & 9.9 & 68.8 & 263.30 & 252.32 & $\begin{array}{l}\text { Malvern was supported by } \\
\text { lifting mechanism only. } \\
1 \mathrm{~mm} \text { orifice. Malvern rate }= \\
1 \mathrm{~Hz} . \mathrm{RH}=97.0 .\end{array}$ & \\
\hline SS-118 & Water & 2 & N/A & 100 & 6 & 1.2 & 380 & OTP-05 & $07 / 27 / 2012$ & 14:05:49 & 14:08:00 & 10.1 & 68.8 & 251.29 & 239.26 & $\begin{array}{l}\text { Stabilizer was installed. } \\
\text { Malvern was supported by } \\
\text { lifting mechanism and } \\
\text { support rod. The } 1 \mathrm{~mm} \\
\text { orifice. Malvern rate }=1 \mathrm{~Hz} . \\
\mathrm{RH}=97.6 .\end{array}$ & \\
\hline SS-119 & Water & 2 & N/A & 100 & 6 & 1.2 & 380 & OTP-05 & $07 / 27 / 2012$ & 14:17:07 & $14: 19: 25$ & 10.0 & 69.4 & 239.96 & 228.90 & $\begin{array}{l}\text { Malvern was supported by } \\
\text { lifting mechanism and } \\
\text { support rod. } 1 \mathrm{~mm} \text { orifice. } \\
\text { Malvern rate }=4 \mathrm{~Hz} . \mathrm{RH}= \\
96.6 .\end{array}$ & \\
\hline SS-120 & Water & 2 & N/A & 100 & 0 & 1.2 & 380 & OTP-03 & $07 / 31 / 2012$ & 10:11:49 & 10:14:25 & 11.5 & 67.2 & 316.26 & 313.56 & $\begin{array}{l}\text { Fan speed: } 0 \text { V. Use the } \\
\text { Malvern support rod. }\end{array}$ & \multirow[t]{9}{*}{$\begin{array}{l}\text { TDP- } \\
867\end{array}$} \\
\hline SS-121 & Water & 2 & N/A & 100 & 3 & 1.2 & 380 & OTP-03 & $07 / 31 / 2012$ & 10:26:17 & 10:28:27 & 11.5 & 67.4 & 312.62 & 310.54 & $\begin{array}{l}\text { Fan speed: } 3 \mathrm{~V} \text {. Use the } \\
\text { Malvern support rod. }\end{array}$ & \\
\hline SS-122 & Water & 2 & N/A & 100 & 11 & 1.2 & 380 & OTP-03 & $07 / 31 / 2012$ & 10:54:59 & 10:57:07 & 11.5 & 68.0 & 309.58 & 306.66 & $\begin{array}{l}\text { Fan speed: } 11 \mathrm{~V} \text {. Use the } \\
\text { Malvern support rod. }\end{array}$ & \\
\hline SS-123 & Water & 2 & $\mathrm{~N} / \mathrm{A}$ & 100 & 6 & 1.2 & 380 & OTP-03 & $07 / 31 / 2012$ & 11:54:13 & $11: 56: 26$ & 11.5 & 68.9 & 303.10 & 300.94 & $\begin{array}{l}\text { Fan speed: } 6 \mathrm{~V} \text {. Use the } \\
\text { Malvern support rod. }\end{array}$ & \\
\hline SS-124 & Water & 2 & N/A & 100 & 6 & 1.2 & 380 & OTP-03 & $07 / 31 / 2012$ & $15: 13: 52$ & $15: 16: 01$ & 11.4 & 67.4 & 297.12 & 294.14 & $\begin{array}{l}\text { The Phase II attachment } \\
\text { scissor lift and internal } \\
\text { unistrut was removed, and the } \\
\text { Malvern was installed using } \\
\text { the Phase I all-thread hooks. } \\
100 \text { mm lens. Malvern } \\
\text { position } 2 \text {. Fan speed } 6 \text { V. } \\
\text { Malvern cap was off. }\end{array}$ & \\
\hline SS-125 & Water & 2 & N/A & 100 & 6 & 1.2 & 380 & OTP-03 & $07 / 31 / 2012$ & $15: 24: 50$ & 15:27:01 & 11.5 & 68.0 & 294.14 & 291.16 & $\begin{array}{l}\text { Duplicate test to SS-124. } \\
\text { Cap on Malvern was left off. }\end{array}$ & \\
\hline SS-126 & Water & 2 & N/A & 100 & 6 & 1.2 & 380 & OTP-03 & $07 / 31 / 2012$ & $15: 38: 21$ & N/A & 11.5 & 68.2 & 291.22 & N/A & Failed test. & \\
\hline SS-127 & Water & 2 & N/A & 100 & 6 & 1.2 & 380 & OTP-03 & $07 / 31 / 2012$ & $15: 46: 56$ & 15:49:06 & 11.5 & 68.5 & 288.40 & 285.42 & $\begin{array}{l}\text { Duplicate test to SS-124 } \\
\text { except Malvern cap was on. }\end{array}$ & \\
\hline SS-128 & Water & 2 & N/A & 100 & 6 & 1.2 & 380 & OTP-03 & $07 / 31 / 2012$ & $15: 57: 52$ & $16: 00: 05$ & 11.4 & 68.8 & 285.40 & 282.42 & Duplicate test to SS-127. & \\
\hline
\end{tabular}


Table A.1. (contd)

\begin{tabular}{|c|c|c|c|c|c|c|c|c|c|c|c|c|c|c|c|c|c|}
\hline Test ID & Simulant & $\begin{array}{l}\text { Mal. } \\
\text { Pos. }\end{array}$ & $\begin{array}{l}\text { Ext. } \\
\text { Len. } \\
\text { (in.) }\end{array}$ & $\begin{array}{l}\text { Malvern } \\
\text { Lens } \\
(\mathrm{mm})\end{array}$ & $\begin{array}{l}\text { Mixing } \\
\text { Fan (V) }\end{array}$ & $\begin{array}{l}\text { Actual } \\
\text { Purge } \\
\text { Flow per } \\
\text { Window } \\
\text { (SCFH) }\end{array}$ & $\begin{array}{c}\text { Nom. P } \\
\text { (psig) }\end{array}$ & ОТР & $\begin{array}{c}\text { Date } \\
(\mathrm{mm} / \mathrm{dd} / \mathrm{yyyy})\end{array}$ & $\begin{array}{l}\text { Spray Start } \\
\text { (hh:mm:ss) }\end{array}$ & $\begin{array}{l}\text { Spray Stop } \\
\text { (hh:mm:ss) }\end{array}$ & $\begin{array}{l}\text { Header } \\
\text { Flow } \\
\text { (gpm) }\end{array}$ & \begin{tabular}{|c|} 
Loop \\
Temp \\
During \\
Spray \\
$\left({ }^{\circ} \mathrm{F}\right)$
\end{tabular} & $\begin{array}{c}\text { Pretest } \\
\text { Tank } \\
\text { Mass } \\
\text { (lb) }\end{array}$ & $\begin{array}{c}\text { Final } \\
\text { Tank } \\
\text { Mass (lb) }\end{array}$ & Notes & TDP \\
\hline SS-129 & Water & 2 & $\mathrm{~N} / \mathrm{A}$ & 100 & 6 & 1.2 & 380 & OTP-03 & $07 / 31 / 2012$ & $16: 11: 57$ & 16:14:04 & 11.5 & 69.5 & 282.40 & 297.50 & Duplicate test to SS-127. & \\
\hline SS-130 & Water & 2 & N/A & 500 & 6 & 1.2 & 380 & \begin{tabular}{|l|} 
OTP-03 \\
\end{tabular} & $08 / 03 / 2012$ & $13: 43: 49$ & $13: 46: 13$ & 11.4 & 65.7 & 318.16 & 314.86 & $\begin{array}{l}4 \mathrm{~Hz} \text {, lens cut-out cover was } \\
\text { not on. }\end{array}$ & \multirow[t]{8}{*}{$\begin{array}{c}\text { TDP- } \\
868\end{array}$} \\
\hline SS-131 & Water & 2 & $\mathrm{~N} / \mathrm{A}$ & 500 & 6 & 1.2 & 380 & \begin{tabular}{|l|} 
OTP-03 \\
\end{tabular} & $08 / 03 / 2012$ & $13: 56: 25$ & $13: 58: 53$ & 11.4 & 66.4 & 314.82 & 311.44 & $\begin{array}{l}\text { Duplicate test to SS- } 130 \text {. } \\
\text { Lens cut-out cover was not } \\
\text { on. }\end{array}$ & \\
\hline SS-132 & Water & 2 & N/A & 500 & 6 & 1.2 & 380 & OTP-03 & $08 / 03 / 2012$ & $14: 07: 54$ & 14:10:34 & 11.3 & 67.4 & 311.44 & 307.80 & $\begin{array}{l}\text { Duplicate test to SS- } 130 . \\
\text { Lens cut-out cover was not } \\
\text { on. }\end{array}$ & \\
\hline SS-133 & Water & 2 & N/A & 500 & 6 & 1.2 & 380 & \begin{tabular}{|l|} 
OTP-03 \\
\end{tabular} & $08 / 03 / 2012$ & $14: 19: 12$ & $14: 21: 25$ & 11.4 & 67.7 & 307.80 & 304.80 & $\begin{array}{l}1 \mathrm{~Hz} \text {, Lens cut-out cover was } \\
\text { not on. }\end{array}$ & \\
\hline SS-134 & Water & 2 & N/A & 500 & 6 & 1.2 & 380 & \begin{tabular}{|l|} 
OTP-03 \\
\end{tabular} & $08 / 03 / 2012$ & $14: 29: 58$ & $14: 32: 03$ & 11.4 & 67.9 & 304.80 & 301.94 & $\begin{array}{l}\text { Duplicate test to SS- } 133 \text {. } \\
\text { Lens cut-out cover was not } \\
\text { on. }\end{array}$ & \\
\hline SS-135 & Water & 2 & $\mathrm{~N} / \mathrm{A}$ & 500 & 6 & 1.2 & 380 & \begin{tabular}{|l|} 
OTP-03 \\
\end{tabular} & $08 / 03 / 2012$ & $14: 39: 51$ & $14: 41: 50$ & 11.2 & 68.3 & 301.96 & 299.26 & $\begin{array}{l}\text { Duplicate test to SS-133. } \\
\text { Lens cut-out cover was not } \\
\text { on. }\end{array}$ & \\
\hline SS-136 & Water & 2 & N/A & 500 & 6 & 1.2 & 380 & OTP-03 & $08 / 03 / 2012$ & $14: 59: 42$ & 15:02:05 & 11.39 & 68.7 & 299.22 & 295.96 & $1 \mathrm{~Hz}$. & \\
\hline SS-137 & Water & 2 & $\mathrm{~N} / \mathrm{A}$ & 500 & 6 & 1.2 & 380 & \begin{tabular}{|l|} 
OTP-03 \\
\end{tabular} & $08 / 03 / 2012$ & $15: 09: 59$ & $15: 12: 21$ & 11.5 & 68.8 & 295.92 & 292.62 & $4 \mathrm{~Hz}$. & \\
\hline SS-138 & Water & 2 & N/A & 500 & 6 & 1.2 & 100 & OTP-16 & $08 / 09 / 2012$ & $10: 22: 08$ & 10:24:18 & 11.4 & 68.3 & $\mathrm{n} / \mathrm{a}$ & 322.58 & $\begin{array}{l}\text { NOTE: B-49 was partially } \\
\text { closed when pretest weight } \\
\text { information was recorded. }\end{array}$ & \multirow[t]{14}{*}{\begin{tabular}{|c|} 
TDP- \\
869
\end{tabular}} \\
\hline SS-139 & Water & 2 & $\mathrm{~N} / \mathrm{A}$ & 500 & 6 & 1.2 & 100 & OTP-16 & 08/09/2012 & 10:40:00 & $10: 42: 08$ & 11.7 & 66.9 & 322.54 & 305.50 & $\begin{array}{l}\text { Pressure fluctuations } \\
\text { observed - test was repeated. }\end{array}$ & \\
\hline SS-140 & Water & 2 & N/A & 500 & 6 & 1.2 & 100 & \begin{tabular}{|l|} 
OTP-16 \\
\end{tabular} & $08 / 09 / 2012$ & $10: 58: 05$ & $11: 00: 17$ & 11.7 & 66.1 & 305.50 & 287.76 & & \\
\hline SS-141 & Water & 2 & N/A & 500 & 6 & 1.2 & 200 & OTP-16 & $08 / 09 / 2012$ & 11:11:04 & 11:13:10 & 11.4 & 66.7 & 342.40 & 318.56 & & \\
\hline SS-142 & Water & 2 & N/A & 500 & 6 & 1.2 & 200 & OTP-16 & $08 / 09 / 2012$ & $11: 21: 50$ & 11:24:00 & 11.4 & 66.9 & 318.54 & 294.02 & & \\
\hline SS-143 & Water & 2 & $\mathrm{~N} / \mathrm{A}$ & 500 & 6 & 1.2 & 380 & OTP-16 & $08 / 09 / 2012$ & $13: 53: 45$ & 13:56:09 & 11.7 & 66.9 & 315.12 & 277.12 & & \\
\hline SS-144 & Water & 2 & N/A & 500 & 6 & 1.2 & 380 & OTP-16 & $08 / 09 / 2012$ & 14:06:30 & $14: 08: 41$ & 11.8 & 66.9 & 342.70 & 308.56 & & \\
\hline SS-145 & Water & 2 & N/A & 500 & 6 & 1.2 & 100 & OTP-05 & $08 / 09 / 2012$ & $14: 34: 59$ & 14:37:11 & 11.5 & 66.3 & 305.68 & 299.62 & & \\
\hline SS-146 & Water & 2 & N/A & 500 & 6 & 1.2 & 100 & OTP-05 & $08 / 09 / 2012$ & $14: 46: 58$ & 14:49:08 & 11.4 & 66.2 & 299.60 & 293.70 & & \\
\hline SS-147 & Water & 2 & N/A & 500 & 6 & 1.2 & 200 & OTP-05 & $08 / 09 / 2012$ & $15: 28: 16$ & $15: 30: 24$ & 11.4 & 65.4 & 290.74 & 282.50 & & \\
\hline SS-148 & Water & 2 & N/A & 500 & 6 & 1.2 & 200 & OTP-05 & $08 / 09 / 2012$ & $15: 38: 41$ & $15: 40: 49$ & 11.5 & 60.0 & 282.48 & 274.24 & & \\
\hline SS-149 & Water & 2 & N/A & 500 & 6 & 1.2 & 380 & OTP-05 & $08 / 09 / 2012$ & $15: 48: 54$ & 15:51:07 & 11.4 & 66.6 & 270.62 & 258.88 & $\begin{array}{l}\text { Ventilation valve was open, } \\
\text { test was repeated. }\end{array}$ & \\
\hline SS-150 & Water & 2 & $\mathrm{~N} / \mathrm{A}$ & 500 & 6 & 1.2 & 380 & \begin{tabular}{|l|} 
OTP-05 \\
\end{tabular} & $08 / 09 / 2012$ & $15: 58: 32$ & 16:00:40 & 11.5 & 67.5 & 258.88 & 247.40 & & \\
\hline SS-151 & Water & 2 & $\mathrm{~N} / \mathrm{A}$ & 500 & 6 & 1.2 & 380 & OTP-05 & $08 / 09 / 2012$ & $16: 09: 10$ & 16:11:19 & 11.6 & 67.8 & 243.56 & 232.00 & & \\
\hline
\end{tabular}


Table A.1. (contd)

\begin{tabular}{|c|c|c|c|c|c|c|c|c|c|c|c|c|c|c|c|c|c|}
\hline Test ID & Simulant & $\begin{array}{l}\text { Mal. } \\
\text { Pos. }\end{array}$ & $\begin{array}{l}\text { Ext. } \\
\text { Len. } \\
\text { (in.) }\end{array}$ & $\begin{array}{l}\text { Malvern } \\
\text { Lens } \\
(\mathrm{mm})\end{array}$ & $\begin{array}{l}\text { Mixing } \\
\text { Fan (V) }\end{array}$ & $\begin{array}{l}\text { Actual } \\
\text { Purge } \\
\text { Flow per } \\
\text { Window } \\
\text { (SCFH) }\end{array}$ & $\begin{array}{c}\text { Nom. P } \\
\text { (psig) }\end{array}$ & OTP & $\begin{array}{c}\text { Date } \\
(\mathrm{mm} / \mathrm{dd} / \text { yyyy })\end{array}$ & $\begin{array}{l}\text { Spray Start } \\
\text { (hh:mm:ss) }\end{array}$ & $\begin{array}{l}\text { Spray Stop } \\
\text { (hh:mm:ss) }\end{array}$ & $\begin{array}{l}\text { Header } \\
\text { Flow } \\
(\mathrm{gpm})\end{array}$ & $\begin{array}{c}\text { Loop } \\
\text { Temp } \\
\text { During } \\
\text { Spray } \\
\left({ }^{\circ} \mathrm{F}\right)\end{array}$ & $\begin{array}{c}\text { Pretest } \\
\text { Tank } \\
\text { Mass } \\
\text { (lb) }\end{array}$ & $\begin{array}{c}\text { Final } \\
\text { Tank } \\
\text { Mass (lb) }\end{array}$ & Notes & TDP \\
\hline SS-152 & KBC-30 & 2 & $\mathrm{~N} / \mathrm{A}$ & 500 & 6 & 1.2 & 380 & OTP-05 & $08 / 15 / 2012$ & 09:21:01 & $09: 23: 43$ & $>15$ & 67.9 & 321.88 & 302.88 & $\begin{array}{l}\text { Large pressure spike to } \\
\sim 540 \text { psi upon spray. }\end{array}$ & \multirow[t]{14}{*}{$\begin{array}{l}\text { TDP- } \\
870\end{array}$} \\
\hline SS-153 & KBC-30 & 2 & $\mathrm{~N} / \mathrm{A}$ & 500 & 6 & 1.2 & 380 & OTP-05 & $08 / 15 / 2012$ & 09:33:54 & 09:36:04 & 15.5 & 68.5 & 302.88 & 288.42 & $\begin{array}{l}\text { Flow rate out of spec: } \\
>15 \text { gpm. }\end{array}$ & \\
\hline SS-154 & KBC-30 & 2 & $\mathrm{~N} / \mathrm{A}$ & 500 & 6 & 1.2 & 380 & OTP-05 & $08 / 15 / 2012$ & 09:45:48 & $09: 47: 57$ & 11.4 & 69 & 288.32 & 273.90 & $\begin{array}{l}\text { Some initial pressure } \\
\text { variation - still may be } \\
\text { useable. }\end{array}$ & \\
\hline SS-155 & KBC-30 & 2 & N/A & 500 & 6 & 1.2 & 380 & OTP-05 & $08 / 15 / 2012$ & 10:11:00 & 10:13:24 & 11.4 & 69.1 & 310.92 & 294.26 & & \\
\hline SS-156 & KBC-30 & 2 & N/A & 500 & 6 & 1.2 & 380 & OTP-05 & $08 / 15 / 2012$ & $10: 24: 43$ & 10:26:59 & 11.5 & 69.5 & 294.36 & 278.68 & & \\
\hline SS-157 & KBC-30 & 2 & $\mathrm{~N} / \mathrm{A}$ & 500 & 6 & 1.2 & 380 & OTP-05 & $08 / 15 / 2012$ & $10: 37: 21$ & 10:39:48 & 11.5 & 70 & 278.76 & 261.88 & & \\
\hline SS-158 & KBC-30 & 2 & N/A & 500 & 6 & 1.2 & 200 & OTP-05 & $08 / 15 / 2012$ & $11: 20: 05$ & $11: 22: 11$ & 11.5 & 68.3 & 316.60 & 305.74 & $\begin{array}{l}\text { Ventilation valve open and } \\
\text { pressure a bit high. }\end{array}$ & \\
\hline SS-159 & KBC-30 & 2 & $\mathrm{~N} / \mathrm{A}$ & 500 & 6 & 1.2 & 200 & OTP-05 & $08 / 15 / 2012$ & 11:29:41 & 11:31:46 & 11.5 & 68.1 & 305.86 & 295.28 & & \\
\hline SS-160 & KBC-30 & 2 & N/A & 500 & 6 & 1.2 & 200 & OTP-05 & $08 / 15 / 2012$ & 11:40:11 & 11:42:14 & 11.5 & 68 & 295.34 & 284.96 & & \\
\hline SS-161 & KBC-30 & 2 & N/A & 500 & 6 & 1.2 & 200 & OTP-05 & $08 / 15 / 2012$ & 11:49:38 & 11:51:52 & 11.5 & 67.6 & 284.98 & 273.74 & & \\
\hline SS-162 & KBC-30 & 2 & N/A & 500 & 6 & 1.2 & 100 & OTP-05 & $08 / 15 / 2012$ & $13: 35: 16$ & 13:37:30 & 11.4 & 66.4 & 313.10 & 304.80 & & \\
\hline SS-163 & KBC-30 & 2 & N/A & 500 & 6 & 1.2 & 100 & OTP-05 & $08 / 15 / 2012$ & 13:46:24 & 13:48:31 & 11.4 & 66 & 304.90 & 297.10 & & \\
\hline SS-164 & KBC-30 & 2 & $\mathrm{~N} / \mathrm{A}$ & 500 & 6 & 1.2 & 100 & OTP-05 & $08 / 15 / 2012$ & 13:56:46 & 13:58:44 & 11.5 & 65.6 & 297.10 & 289.74 & $\begin{array}{l}\text { Malvern data of this test } \\
\text { looked strange. }\end{array}$ & \\
\hline SS-165 & KBC-30 & 2 & N/A & 500 & 6 & 1.2 & 100 & OTP-05 & $08 / 15 / 2012$ & 14:07:24 & 14:09:23 & 11.5 & 65.6 & 289.96 & 282.68 & & \\
\hline SS-166 & KBC-30 & 2 & N/A & 500 & 6 & 1.2 & 100 & OTP-16 & $08 / 16 / 2012$ & 13:08:16 & 13:10:28 & 11.5 & 67.9 & 302.06 & 281.38 & & \multirow{6}{*}{\begin{tabular}{|c} 
TDP- \\
871
\end{tabular}} \\
\hline SS-167 & KBC-30 & 2 & N/A & 500 & 6 & 1.2 & 100 & OTP-16 & $08 / 16 / 2012$ & $13: 20: 53$ & 13:23:06 & 11.8 & 66.7 & 281.48 & 260.44 & & \\
\hline SS-168 & KBC-30 & 2 & $\mathrm{~N} / \mathrm{A}$ & 500 & 6 & 1.2 & 100 & OTP-16 & $08 / 16 / 2012$ & 13:47:21 & 13:49:29 & 12.0 & 65.8 & 320.40 & 299.12 & & \\
\hline SS-169 & KBC-30 & 2 & N/A & 500 & 6 & 1.2 & 200 & OTP-16 & $08 / 16 / 2012$ & 14:44:18 & 14:46:31 & 11.4 & 67.7 & 314.54 & 283.58 & & \\
\hline SS-170 & KBC-30 & 2 & N/A & 500 & 6 & 1.2 & 200 & OTP-16 & $08 / 16 / 2012$ & 15:52:45 & 15:55:02 & 11.4 & 67 & 303.68 & 271.80 & & \\
\hline SS-171 & KBC-30 & 2 & N/A & 500 & 6 & 1.2 & 200 & OTP-16 & $08 / 16 / 2012$ & 16:06:39 & 16:08:47 & 11.3 & 67.9 & 271.80 & 241.92 & & \\
\hline SS-172 & KBC-30 & 2 & N/A & 500 & 6 & 1.2 & 200 & OTP-16 & $08 / 17 / 2012$ & $11: 38: 32$ & N/A & $\begin{array}{l}\text { Too } \\
\text { high }\end{array}$ & N/A & 299.48 & N/A & $\begin{array}{l}\text { Flow too high - test should } \\
\text { not be used. }\end{array}$ & \multirow[t]{8}{*}{$\begin{array}{c}\text { TDP- } \\
872\end{array}$} \\
\hline SS-173 & KBC-30 & 2 & N/A & 500 & 6 & 1.2 & 200 & OTP-16 & $08 / 17 / 2012$ & 11:42:34 & 11:44:38 & 11.9 & 68.4 & 281.54 & 252.10 & & \\
\hline SS-174 & KBC-30 & 2 & $\mathrm{~N} / \mathrm{A}$ & 500 & 6 & 1.2 & 200 & OTP-16 & $08 / 17 / 2012$ & 11:58:04 & 12:00:27 & 12 & 68.6 & 251.86 & 217.88 & & \\
\hline SS-175 & KBC-30 & 2 & & 500 & 6 & 1.2 & 380 & OTP-16 & $08 / 17 / 2012$ & 13:26:42 & N/A & 11.9 & 67.6 & 308.70 & N/A & Poor Malvern data. & \\
\hline SS-176 & KBC-30 & 2 & $\mathrm{~N} / \mathrm{A}$ & 500 & 6 & 1.2 & 380 & OTP-16 & $08 / 17 / 2012$ & 13:35:56 & 13:37:17 & 11.5 & 69.2 & 281.92 & $\mathrm{~N} / \mathrm{A}$ & & \\
\hline SS-177 & KBC-30 & 2 & N/A & 500 & 6 & 1.2 & 380 & OTP-16 & $08 / 17 / 2012$ & $13: 57: 34$ & $\mathrm{~N} / \mathrm{A}$ & $\begin{array}{l}\text { Too } \\
\text { high }\end{array}$ & N/A & 318.54 & $\mathrm{~N} / \mathrm{A}$ & $\begin{array}{l}\text { Pressure and flow too high - } \\
\text { test should not be used. }\end{array}$ & \\
\hline SS-178 & KBC-30 & 2 & $\mathrm{~N} / \mathrm{A}$ & 500 & 6 & 1.2 & 380 & OTP-16 & $08 / 17 / 2012$ & 14:03:49 & 14:06:06 & 11.5 & 70.3 & 306.76 & 260.32 & & \\
\hline SS-179 & KBC-30 & 2 & N/A & 500 & 6 & 1.2 & 380 & OTP-16 & $08 / 17 / 2012$ & 14:19:14 & 14:21:16 & 11.7 & 71.8 & 260.60 & 216.48 & & \\
\hline
\end{tabular}


Table A.1. (contd)

\begin{tabular}{|c|c|c|c|c|c|c|c|c|c|c|c|c|c|c|c|c|c|}
\hline Test ID & Simulant & $\begin{array}{l}\text { Mal. } \\
\text { Pos. }\end{array}$ & $\begin{array}{l}\text { Ext. } \\
\text { Len. } \\
\text { (in.) }\end{array}$ & $\begin{array}{l}\text { Malvern } \\
\text { Lens } \\
(\mathrm{mm})\end{array}$ & $\begin{array}{l}\text { Mixing } \\
\text { Fan (V) }\end{array}$ & $\begin{array}{l}\text { Actual } \\
\text { Purge } \\
\text { Flow per } \\
\text { Window } \\
\text { (SCFH) }\end{array}$ & $\begin{array}{l}\text { Nom. P } \\
\text { (psig) }\end{array}$ & OTP & $\begin{array}{c}\text { Date } \\
(\mathrm{mm} / \mathrm{dd} / \mathrm{yyyy})\end{array}$ & $\begin{array}{l}\text { Spray Start } \\
\text { (hh:mm:ss) }\end{array}$ & $\begin{array}{l}\text { Spray Stop } \\
\text { (hh:mm:ss) }\end{array}$ & $\begin{array}{l}\text { Header } \\
\text { Flow } \\
(\mathrm{gpm})\end{array}$ & $\begin{array}{l}\text { Loop } \\
\text { Temp } \\
\text { During } \\
\text { Spray } \\
\left({ }^{\circ} \mathrm{F}\right)\end{array}$ & $\begin{array}{l}\text { Pretest } \\
\text { Tank } \\
\text { Mass } \\
\text { (lb) }\end{array}$ & $\begin{array}{c}\text { Final } \\
\text { Tank } \\
\text { Mass (lb) }\end{array}$ & Notes & TDP \\
\hline SS-180 & KBC-30 & 4 & N/A & 500 & off & 35 & 100 & OTP-16 & $08 / 20 / 2012$ & 10:51:56 & $10: 52: 40$ & 11.6 & 66.5 & 312.68 & 304.40 & $\begin{array}{l}\text { Malvern lenses were coated } \\
\text { with clay. }\end{array}$ & \multirow[t]{2}{*}{$\begin{array}{c}\text { TDP- } \\
873\end{array}$} \\
\hline SS-181 & KBC-30 & 4 & N/A & 500 & off & 35 & 200 & OTP-16 & $08 / 20 / 2012$ & $14: 33: 51$ & $14: 34: 36$ & 11.7 & 66.5 & 270.50 & 256.60 & Lenses were coated with clay. & \\
\hline SS-182 & KBC-30 & 4 & N/A & 500 & off & 35 & 100 & OTP-05 & $08 / 21 / 2012$ & 10:49:01 & 10:49:52 & 11.4 & 66.6 & 313.72 & 311.40 & & \multirow{3}{*}{\begin{tabular}{|c} 
TDP- \\
874
\end{tabular}} \\
\hline SS-183 & KBC-30 & 4 & 6 & 500 & off & 35 & 100 & OTP-05 & $08 / 21 / 2012$ & $14: 45: 56$ & 14:46:58 & 11.5 & 67.4 & $\begin{array}{c}\text { not } \\
\text { recorded }\end{array}$ & 297.64 & First use of 6-in. extensions. & \\
\hline SS-184 & KBC-30 & 4 & 6 & 500 & off & 35 & 200 & OTP-05 & $08 / 21 / 2012$ & 15:07:45 & 15:08:53 & 11.6 & 67.5 & 293.66 & 287.68 & Lenses were coated with clay. & \\
\hline SS-185 & KBC-30 & 4 & 6 & 500 & off & 40 & 380 & OTP-05 & $08 / 22 / 2012$ & 10:13:59 & $10: 15: 16$ & 11.4 & 70.8 & 307.04 & 298.06 & $\begin{array}{l}\text { Pressure too high, lens coated } \\
\text { with clay. Malvern removed } \\
\text { for cleaning after test. } \\
\end{array}$ & \multirow[t]{9}{*}{$\begin{array}{l}\text { TDP- } \\
875\end{array}$} \\
\hline SS-186 & KBC-30 & 4 & 6 & 500 & off & 40 & 380 & OTP-05 & $08 / 22 / 2012$ & $12: 54: 58$ & $12: 55: 16$ & 11.8 & N/A & 299.20 & N/A & \begin{tabular}{|l|} 
Malvern remounted in \\
chamber. Lens cleaned. \\
Determined after SS-192 that \\
the laser was not centered on \\
the spray. Pressure too high.
\end{tabular} & \\
\hline SS-187 & KBC-30 & 4 & 6 & 500 & off & 40 & 380 & OTP-03 & $08 / 22 / 2012$ & $14: 32: 12$ & 14:33:19 & 11.5 & 69.2 & 304.72 & 302.70 & $\begin{array}{l}\text { Malvern not centered on } \\
\text { spray. }\end{array}$ & \\
\hline SS-188 & KBC-30 & 4 & 6 & 500 & off & 40 & 380 & OTP-03 & $08 / 22 / 2012$ & $14: 50: 57$ & 14:51:14 & 11.3 & 71 & 304.52 & 302.10 & $\begin{array}{l}\text { Malvern not centered on } \\
\text { spray. }\end{array}$ & \\
\hline SS-189 & KBC-30 & 4 & 6 & 500 & off & off & 200 & OTP-03 & $08 / 22 / 2012$ & 15:04:42 & 15:06:08 & 11.7 & 71.4 & 301.88 & 300.02 & Purge air was mistakenly off. & \\
\hline SS-190 & KBC-30 & 4 & 6 & 500 & off & 40 & 200 & OTP-03 & $08 / 22 / 2012$ & 15:19:56 & $15: 21: 37$ & 11.4 & 70.6 & 300.06 & 297.90 & $\begin{array}{l}\text { Malvern not centered on } \\
\text { spray. }\end{array}$ & \\
\hline SS-191 & KBC-30 & 4 & 6 & 500 & off & 40 & 200 & OTP-03 & $08 / 22 / 2012$ & $15: 34: 52$ & $15: 37: 37$ & 11.6 & 70.3 & 297.76 & 294.20 & $\begin{array}{l}\text { Malvern not centered on } \\
\text { spray. }\end{array}$ & \\
\hline SS-192 & KBC-30 & 4 & 6 & 500 & off & 40 & 100 & OTP-03 & $08 / 22 / 2012$ & $15: 55: 05$ & $15: 57: 32$ & 11.2 & 69.5 & 293.44 & 291.38 & $\begin{array}{l}\text { Malvern not centered on } \\
\text { spray. }\end{array}$ & \\
\hline SS-193 & KBC-30 & 4 & 6 & 500 & off & 40 & 100 & OTP-03 & $08 / 22 / 2012$ & 16:16:41 & $16: 18: 47$ & 11.5 & 68.5 & 288.72 & 287.04 & $\begin{array}{l}\text { Malvern adjusted to be } \\
\text { centered with spray jet. }\end{array}$ & \\
\hline SS-194 & KBC-30 & 4 & 6 & 500 & off & 40 & 100 & OTP-03 & $08 / 23 / 2012$ & 09:42:11 & N/A & 11.8 & 68.8 & 307.20 & N/A & $\begin{array}{l}\text { Repeat of SS-193 to confirm } \\
\text { data - orifice plugged after } \\
\text { around } 20 \text { seconds, some data } \\
\text { may be useable. }\end{array}$ & \multirow[t]{4}{*}{$\begin{array}{l}\text { TDP- } \\
876\end{array}$} \\
\hline SS-195 & KBC-30 & 4 & 6 & 500 & off & 40 & 100 & OTP-03 & $08 / 23 / 2012$ & 09:49:36 & N/A & 11.9 & $\mathrm{n} / \mathrm{a}$ & 306.92 & N/A & $\begin{array}{l}\text { Repeat of SS-193 to confirm } \\
\text { data - orifice plugged after a } \\
\text { few seconds. }\end{array}$ & \\
\hline SS-196 & KBC-30 & 4 & 6 & 500 & off & 40 & 100 & OTP-03 & $08 / 23 / 2012$ & 10:29:55 & N/A & 11.3 & 68.2 & 306.34 & N/A & $\begin{array}{l}\text { Repeat of SS-193 to confirm } \\
\text { data - pressure too high. }\end{array}$ & \\
\hline SS-197 & KBC-30 & 4 & 6 & 500 & off & 40 & 100 & OTP-03 & $08 / 23 / 2012$ & $10: 37: 15$ & 10:39:28 & 11.7 & 68.4 & 305.66 & 303.72 & $\begin{array}{l}\text { Repeat of SS-193 to confirm } \\
\text { data. }\end{array}$ & \\
\hline
\end{tabular}


Table A.1. (contd)

\begin{tabular}{|c|c|c|c|c|c|c|c|c|c|c|c|c|c|c|c|c|c|}
\hline Test ID & Simulant & $\begin{array}{l}\text { Mal. } \\
\text { Pos. }\end{array}$ & $\begin{array}{l}\text { Ext. } \\
\text { Len. } \\
\text { (in.) }\end{array}$ & $\begin{array}{l}\text { Malvern } \\
\text { Lens } \\
(\mathrm{mm})\end{array}$ & $\begin{array}{l}\text { Mixing } \\
\text { Fan (V) }\end{array}$ & $\begin{array}{l}\text { Actual } \\
\text { Purge } \\
\text { Flow per } \\
\text { Window } \\
\text { (SCFH) }\end{array}$ & $\begin{array}{c}\text { Nom. P } \\
\text { (psig) }\end{array}$ & OTP & $\begin{array}{c}\text { Date } \\
\text { (mm/dd/yyy) }\end{array}$ & $\begin{array}{l}\text { Spray Start } \\
\text { (hh:mm:ss) }\end{array}$ & $\begin{array}{l}\text { Spray Stop } \\
\text { (hh:mm:ss) }\end{array}$ & $\begin{array}{l}\text { Header } \\
\text { Flow } \\
(\mathrm{gpm})\end{array}$ & $\begin{array}{c}\text { Loop } \\
\text { Temp } \\
\text { During } \\
\text { Spray } \\
\left({ }^{\circ} \mathrm{F}\right)\end{array}$ & $\begin{array}{c}\text { Pretest } \\
\text { Tank } \\
\text { Mass } \\
\text { (lb) }\end{array}$ & $\begin{array}{c}\text { Final } \\
\text { Tank } \\
\text { Mass (lb) }\end{array}$ & Notes & TDP \\
\hline SS-198 & KBC-30 & 4 & 6 & 500 & off & 40 & 200 & OTP-03 & 08/23/2012 & 10:53:58 & 10:55:53 & 11.8 & 68.2 & 303.74 & 300.53 & & \\
\hline SS-199 & KBC-30 & 4 & 6 & 500 & off & 40 & 200 & OTP-03 & $08 / 23 / 2012$ & 11:08:47 & 11:10:44 & 11.3 & 69.2 & 300.12 & 298.60 & $\begin{array}{l}\text { Lost spray after } \sim 45 \text { seconds } \\
\text { due to plugging; data before } \\
\text { this is useable. }\end{array}$ & \\
\hline SS-200 & KBC-30 & 4 & 6 & 500 & off & 40 & 380 & OTP-03 & $08 / 23 / 2012$ & 11:32:17 & 11:34:30 & 11.5 & 70.2 & 298.00 & 294.36 & $\begin{array}{l}\text { Partial plug after } \sim 1 \mathrm{~min} \text { of } \\
\text { spraying. }\end{array}$ & \\
\hline SS-201 & KBC-30 & 4 & 6 & 500 & off & 40 & 380 & OTP-03 & $08 / 23 / 2012$ & 11:46:09 & 11:47:38 & 11.7 & 72.6 & 294.52 & 293.02 & $\begin{array}{l}\text { Flow looked low-partially } \\
\text { plugged? }\end{array}$ & \\
\hline SS-202 & KBC-30 & 2 & $\mathrm{~N} / \mathrm{A}$ & 100 & 6 & 1.2 & 380 & OTP-05 & $08 / 24 / 2012$ & 09:38:35 & $\mathrm{N} / \mathrm{A}$ & $\mathrm{N} / \mathrm{A}$ & N/A & 286.54 & $\mathrm{~N} / \mathrm{A}$ & $\begin{array}{l}\text { Test stopped because of high } \\
\text { pressure }>430 \text { psi. }\end{array}$ & \multirow[t]{7}{*}{$\begin{array}{l}\text { TDP- } \\
877\end{array}$} \\
\hline SS-203 & KBC-30 & 2 & N/A & 100 & 6 & 1.2 & 380 & OTP-05 & $08 / 24 / 2012$ & 09:48:53 & 09:51:38 & 11.8 & 73.2 & 279.82 & 259.62 & & \\
\hline SS-204 & KBC-30 & 2 & N/A & 100 & 6 & 1.2 & 380 & OTP-05 & $08 / 24 / 2012$ & 10:03:38 & 10:06:08 & 11.2 & 73.5 & 259.40 & 241.82 & & \\
\hline SS-205 & KBC-30 & 2 & N/A & 100 & 6 & 1.2 & 380 & OTP-05 & $08 / 24 / 2012$ & 10:16:42 & 10:18:50 & 11.4 & 74.0 & 241.80 & 226.54 & & \\
\hline SS-206 & KBC-30 & 2 & & 100 & 6 & 1.2 & 200 & OTP-05 & $08 / 24 / 2012$ & N/A & N/A & $\mathrm{N} / \mathrm{A}$ & N/A & 295.10 & N/A & $\begin{array}{l}\text { Test stopped } b / c \text { of high } \\
\text { pressure. }\end{array}$ & \\
\hline SS-207 & KBC-30 & 2 & N/A & 100 & 6 & 1.2 & 200 & OTP-05 & $08 / 24 / 2012$ & 13:17:55 & 13:20:03 & 11.4 & 67.7 & 290.96 & $\mathrm{n} / \mathrm{a}$ & & \\
\hline SS-208 & KBC-30 & 2 & N/A & 100 & 6 & 1.2 & 200 & OTP-05 & $08 / 24 / 2012$ & 13:30:06 & 13:32:11 & 11.4 & 68.1 & 279.48 & 268.20 & & \\
\hline SS-209 & KBC-30 & 2 & N/A & 100 & 6 & 1.2 & 380 & OTP-16 & $08 / 28 / 2012$ & 13:52:51 & 13:54:50 & 12.1 & 69.0 & 249.88 & 207.78 & & \multirow{8}{*}{$\begin{array}{c}\text { TDP- } \\
878\end{array}$} \\
\hline SS-210 & KBC-30 & 2 & N/A & 100 & 6 & 1.2 & 380 & OTP-16 & $08 / 28 / 2012$ & 14:17:03 & 14:19:14 & 12 & 69.8 & 297.14 & 251.62 & & \\
\hline SS-211 & KBC-30 & 2 & N/A & 100 & 6 & 1.2 & 380 & OTP-16 & $08 / 28 / 2012$ & 14:29:31 & 14:31:39 & 11.8 & 71.3 & 251.64 & 208.44 & & \\
\hline SS-212 & KBC-30 & 2 & N/A & 100 & 6 & 1.2 & 200 & OTP-16 & $08 / 28 / 2012$ & $15: 20: 54$ & N/A & 13.4 & N/A & 290.88 & N/A & $\begin{array}{l}\text { Flow rate was on high side so } \\
\text { test repeated. }\end{array}$ & \\
\hline SS-213 & KBC-30 & 2 & N/A & 100 & 6 & 1.2 & 200 & OTP-16 & $08 / 28 / 2012$ & $15: 27: 15$ & $15: 29: 25$ & 11.7 & 68.3 & 277.30 & 243.26 & & \\
\hline SS-214 & KBC-30 & 2 & N/A & 100 & 6 & 1.2 & 200 & OTP-16 & $08 / 28 / 2012$ & $15: 39: 43$ & $15: 41: 59$ & 11.3 & 69.0 & 243.22 & 208.88 & $\begin{array}{l}\text { NOTE: The plastic cover of } \\
\text { the cut-out area fell during } \\
\text { spray; data may be affected. }\end{array}$ & \\
\hline SS-215 & KBC-30 & 2 & N/A & 100 & 6 & 1.2 & 200 & OTP-16 & $08 / 28 / 2012$ & $16: 05: 31$ & $16: 07: 38$ & 11.3 & 67.7 & 302.00 & 269.14 & & \\
\hline SS-216 & KBC-30 & 2 & N/A & 100 & 6 & 1.2 & 200 & OTP-16 & $08 / 28 / 2012$ & 16:17:32 & 16:19:38 & 11.5 & 68.7 & 269.26 & 236.82 & & \\
\hline SS-217 & KBC-30 & 2 & N/A & 100 & 6 & 1.2 & 100 & OTP-16 & $08 / 29 / 2012$ & 09:29:17 & 09:31:20 & 11.5 & 66.7 & 275.92 & 252.34 & & \multirow{3}{*}{$\begin{array}{l}\text { TDP- } \\
879\end{array}$} \\
\hline SS-218 & KBC-30 & 2 & N/A & 100 & 6 & 1.2 & 100 & OTP-16 & 08/29/2012 & 09:47:50 & 09:49:56 & 11.5 & 67.0 & 252.26 & 228.34 & & \\
\hline SS-219 & KBC-30 & 2 & N/A & 100 & 6 & 1.2 & 100 & OTP-16 & $08 / 29 / 2012$ & 10:42:46 & 10:44:54 & 11.6 & 65.8 & 298.50 & 273.24 & & \\
\hline SS-220 & KBC-6 & 2 & N/A & 500 & 6 & 1.2 & 100 & OTP-16 & $08 / 30 / 2012$ & 10:29:34 & 10:32:00 & 11.8 & 67.3 & 334.78 & 307.14 & & \multirow{5}{*}{$\begin{array}{c}\text { TDP- } \\
880\end{array}$} \\
\hline SS-221 & KBC-6 & 2 & N/A & 500 & 6 & 1.2 & 100 & OTP-16 & 08/30/2012 & 10:43:04 & 10:45:14 & 11.6 & 67 & 307.38 & 282.86 & & \\
\hline SS-222 & KBC-6 & 2 & N/A & 500 & 6 & 1.2 & 100 & OTP-16 & $08 / 30 / 2012$ & $10: 57: 12$ & 10:59:18 & 11.4 & 66.8 & 282.96 & 260.96 & & \\
\hline SS-223 & KBC-6 & 2 & N/A & 500 & 6 & 1.2 & 200 & OTP-16 & $08 / 30 / 2012$ & 12:39:04 & 12:41:15 & 11.8 & 67.8 & 345.10 & 310.64 & & \\
\hline SS-224 & KBC-6 & 2 & N/A & 500 & 6 & 1.2 & 200 & OTP-16 & $08 / 30 / 2012$ & $12: 51: 13$ & 12:53:54 & 11.6 & 68 & 310.68 & 268.78 & & \\
\hline
\end{tabular}


Table A.1. (contd)

\begin{tabular}{|c|c|c|c|c|c|c|c|c|c|c|c|c|c|c|c|c|c|}
\hline Test ID & Simulant & $\begin{array}{l}\text { Mal. } \\
\text { Pos. }\end{array}$ & $\begin{array}{l}\text { Ext. } \\
\text { Len. } \\
\text { (in.) }\end{array}$ & $\begin{array}{l}\text { Malvern } \\
\text { Lens } \\
(\mathrm{mm})\end{array}$ & $\begin{array}{l}\text { Mixing } \\
\text { Fan (V) }\end{array}$ & $\begin{array}{l}\text { Actual } \\
\text { Purge } \\
\text { Flow per } \\
\text { Window } \\
\text { (SCFH) }\end{array}$ & $\begin{array}{c}\text { Nom. P } \\
\text { (psig) }\end{array}$ & OTP & $\begin{array}{c}\text { Date } \\
(\mathrm{mm} / \mathrm{dd} / \mathrm{yyyy})\end{array}$ & $\begin{array}{l}\text { Spray Start } \\
\text { (hh:mm:ss) }\end{array}$ & $\begin{array}{l}\text { Spray Stop } \\
\text { (hh:mm:ss) }\end{array}$ & $\begin{array}{l}\text { Header } \\
\text { Flow } \\
(\text { gpm })\end{array}$ & $\begin{array}{c}\text { Loop } \\
\text { Temp } \\
\text { During } \\
\text { Spray } \\
\left({ }^{\circ} \mathrm{F}\right)\end{array}$ & $\begin{array}{c}\text { Pretest } \\
\text { Tank } \\
\text { Mass } \\
\text { (lb) }\end{array}$ & $\begin{array}{c}\text { Final } \\
\text { Tank } \\
\text { Mass (lb) }\end{array}$ & Notes & TDP \\
\hline SS-225 & KBC-6 & 2 & N/A & 500 & 6 & 1.2 & 200 & OTP-16 & 08/30/2012 & 13:03:21 & 13:05:28 & 11.6 & 68.4 & 268.96 & 236.70 & & \\
\hline SS-226 & KBC-6 & 2 & N/A & 500 & 6 & 1.2 & 380 & OTP-16 & $08 / 30 / 2012$ & 13:46:14 & $\mathrm{N} / \mathrm{A}$ & 11.8 & 68.6 & N/A & N/A & $\begin{array}{l}\text { Exhaust ale found to be open; } \\
\text { test was aborted. }\end{array}$ & \\
\hline SS-227 & KBC-6 & 2 & N/A & 500 & 6 & 1.2 & 380 & OTP-16 & $08 / 30 / 2012$ & $13: 53: 26$ & $13: 55: 34$ & 11.7 & 69.7 & 275.94 & 230.36 & $\begin{array}{l}\text { NOTE: After spray was } \\
\text { stopped, ale B- } 23 \text { switch was } \\
\text { tested. This led to some } \\
\text { spray after the decay phase. } \\
\text { Spray data should still be } \\
\text { good. }\end{array}$ & \\
\hline SS-228 & KBC-6 & 2 & N/A & 500 & 6 & 1.2 & 380 & OTP-16 & $08 / 30 / 2012$ & $14: 21: 25$ & N/A & N/A & $\mathrm{N} / \mathrm{A}$ & 349.52 & N/A & $\begin{array}{l}\text { Valve problem, test to be } \\
\text { repeated. }\end{array}$ & \\
\hline SS-229 & KBC-6 & 2 & N/A & 500 & 6 & 1.2 & 380 & OTP-16 & $08 / 30 / 2012$ & 14:31:45 & 14:34:03 & 11.6 & 70.6 & 316.70 & 267.46 & $\begin{array}{l}\text { Flow changed a bit during } \\
\text { test. }\end{array}$ & \\
\hline SS-230 & KBC-6 & 2 & N/A & 500 & 6 & 1.2 & 380 & OTP-16 & $08 / 30 / 2012$ & 14:39:30 & 14:41:39 & 11.6 & 71.3 & 267.46 & 225.52 & & \\
\hline SS-231 & KBC-6 & 2 & N/A & 500 & 6 & 1.2 & 380 & OTP-16 & 08/30/2012 & $14: 57: 43$ & $14: 59: 52$ & 11.1 & 70.5 & 313.02 & 269.20 & & \\
\hline SS-232 & KBC-6 & 2 & N/A & 500 & 6 & 1.2 & 100 & OTP-05 & $08 / 31 / 2012$ & 9:53:05 & 9:55:14 & 11.5 & 66.9 & 366.60 & 358.48 & & \multirow{12}{*}{$\begin{array}{c}\text { TDP- } \\
881\end{array}$} \\
\hline SS-233 & KBC-6 & 2 & N/A & 500 & 6 & 1.2 & 100 & OTP-05 & $08 / 31 / 2012$ & 10:07:00 & 10:09:05 & 11.5 & 67.8 & 358.40 & 350.56 & & \\
\hline SS-234 & KBC-6 & 2 & N/A & 500 & 6 & 1.2 & 100 & OTP-05 & $08 / 31 / 2012$ & 10:20:31 & 10:22:35 & 11.5 & 69.7 & 350.58 & 342.58 & & \\
\hline SS-235 & KBC-6 & 2 & N/A & 500 & 6 & 1.2 & 200 & OTP-05 & 08/31/2012 & 10:42:10 & 10:44:05 & 11.4 & 69.5 & 337.00 & 326.82 & & \\
\hline SS-236 & KBC-6 & 2 & N/A & 500 & 6 & 1.2 & 200 & OTP-05 & 08/31/2012 & $10: 54: 30$ & 10:56:34 & 11.2 & 69.6 & 326.90 & 316.02 & & \\
\hline SS-237 & KBC-6 & 2 & N/A & 500 & 6 & 1.2 & 200 & OTP-05 & 08/31/2012 & 11:08:30 & 11:10:31 & 11.4 & 69.5 & 316.10 & 305.42 & & \\
\hline SS-238 & KBC-6 & 2 & N/A & 500 & 6 & 1.2 & 380 & OTP-05 & $08 / 31 / 2012$ & 11:39:30 & $\mathrm{N} / \mathrm{A}$ & N/A & N/A & 358.10 & N/A & $\begin{array}{l}\text { Pressure incorrect, test } \\
\text { aborted. }\end{array}$ & \\
\hline SS-239 & KBC-6 & 2 & N/A & 500 & 6 & 1.2 & 380 & OTP-05 & $08 / 31 / 2012$ & 11:46:52 & $\mathrm{N} / \mathrm{A}$ & N/A & $\mathrm{N} / \mathrm{A}$ & 350.78 & $\mathrm{~N} / \mathrm{A}$ & $\begin{array}{l}\text { Pressure incorrect, test } \\
\text { aborted. }\end{array}$ & \\
\hline SS-240 & KBC-6 & 2 & $\mathrm{~N} / \mathrm{A}$ & 500 & 6 & 1.2 & 380 & OTP-05 & $08 / 31 / 2012$ & N/A & N/A & N/A & N/A & 325.16 & $\mathrm{~N} / \mathrm{A}$ & Test aborted. & \\
\hline SS-241 & KBC-6 & 2 & N/A & 500 & 6 & 1.2 & 380 & OTP-05 & $08 / 31 / 2012$ & $13: 22: 21$ & $13: 24: 22$ & 11.5 & 76.1 & 321.50 & 306.86 & & \\
\hline SS-242 & KBC-6 & 2 & N/A & 500 & 6 & 1.2 & 380 & OTP-05 & $08 / 31 / 2012$ & 13:36:23 & 13:38:24 & 11.3 & 74.1 & 362.92 & 348.58 & & \\
\hline SS-243 & KBC-6 & 2 & N/A & 500 & 6 & 1.2 & 380 & OTP-05 & $08 / 31 / 2012$ & 13:50:01 & 13:52:07 & 11.3 & 73.2 & 348.48 & 333.52 & & \\
\hline SS-244 & KBC-6 & 4 & 6 & 500 & off & 35 & 380 & OTP-03 & 09/04/2012 & 10:09:24 & 10:11:25 & 12 & 68.8 & 363.08 & 360.60 & & \multirow{6}{*}{$\begin{array}{l}\text { TDP- } \\
882\end{array}$} \\
\hline SS-245 & KBC-6 & 4 & 6 & 500 & off & 35 & 380 & OTP-03 & $09 / 04 / 2012$ & 10:28:30 & 10:30:46 & 11.7 & 70.7 & 359.30 & 354.86 & & \\
\hline SS-246 & KBC-6 & 4 & 6 & 500 & off & 35 & 200 & OTP-03 & $09 / 04 / 2012$ & 10:45:30 & 10:47:39 & 11.4 & 70.4 & 352.52 & 350.02 & & \\
\hline SS-247 & KBC-6 & 4 & 6 & 500 & off & 35 & 200 & OTP-03 & $09 / 04 / 2012$ & 10:56:57 & 10:59:08 & 11.6 & 70.2 & 349.68 & 347.08 & & \\
\hline SS-248 & KBC-6 & 4 & 6 & 500 & off & 35 & 100 & OTP-03 & 09/04/2012 & 11:13:27 & 11:15:31 & 11.6 & 68.6 & 346.26 & 344.38 & & \\
\hline SS-249 & KBC-6 & 4 & 6 & 500 & off & 35 & 100 & OTP-03 & 09/04/2012 & $11: 27: 17$ & $11: 29: 21$ & 11.7 & 67.9 & 344.44 & 342.58 & & \\
\hline
\end{tabular}


Table A.1. (contd)

\begin{tabular}{|c|c|c|c|c|c|c|c|c|c|c|c|c|c|c|c|c|c|}
\hline Test ID & Simulant & $\begin{array}{l}\text { Mal. } \\
\text { Pos. }\end{array}$ & $\begin{array}{l}\text { Ext. } \\
\text { Len. } \\
\text { (in.) }\end{array}$ & $\begin{array}{l}\text { Malvern } \\
\text { Lens } \\
(\mathrm{mm}) \\
\end{array}$ & \begin{tabular}{|l|} 
Mixing \\
Fan (V) \\
\end{tabular} & $\begin{array}{l}\text { Actual } \\
\text { Purge } \\
\text { Flow per } \\
\text { Window } \\
\text { (SCFH) } \\
\end{array}$ & $\begin{array}{c}\text { Nom. P } \\
\text { (psig) }\end{array}$ & ОТР & $\begin{array}{c}\text { Date } \\
(\mathrm{mm} / \mathrm{dd} / \mathrm{yyyy})\end{array}$ & $\begin{array}{l}\text { Spray Start } \\
(\mathrm{hh}: \mathrm{mm}: \mathrm{ss}) \\
\end{array}$ & $\begin{array}{l}\text { Spray Stop } \\
\text { (hh:mm:ss) }\end{array}$ & $\begin{array}{l}\text { Header } \\
\text { Flow } \\
(\mathrm{gpm})\end{array}$ & $\begin{array}{l}\text { Loop } \\
\text { Temp } \\
\text { During } \\
\text { Spray } \\
\left({ }^{\circ} \mathrm{F}\right) \\
\end{array}$ & $\begin{array}{l}\text { Pretest } \\
\text { Tank } \\
\text { Mass } \\
\text { (lb) } \\
\end{array}$ & $\begin{array}{c}\text { Final } \\
\text { Tank } \\
\text { Mass (lb) } \\
\end{array}$ & Notes & TDP \\
\hline SS-250 & FEG-30 & 2 & N/A & 500 & 6 & 1.2 & 100 & OTP-05 & $09 / 13 / 2012$ & 14:18:11 & $14: 19: 05$ & 10.3 & 68.4 & -2.12 & $\mathrm{n} / \mathrm{a}$ & $\begin{array}{l}\text { Note: Orifice plugged around } \\
1 \text { min into spray. } \\
\end{array}$ & \multirow{5}{*}{$\begin{array}{l}\text { TDP- } \\
883^{(a)}\end{array}$} \\
\hline SS-251 & FEG-30 & 2 & N/A & 500 & 6 & 1.2 & 100 & OTP-05 & 09/13/2012 & 14:33:44 & 14:35:44 & 9.6 & 68.4 & -5.26 & -14.06 & $\begin{array}{l}\text { Flow too low but no } \\
\text { adjustments could be made to } \\
\text { rectify. }\end{array}$ & \\
\hline SS-252 & FEG-30 & 2 & N/A & 500 & 6 & 1.2 & 100 & OTP-05 & 09/13/2012 & $14: 45: 00$ & $14: 47: 37$ & 9.0 & 68.8 & -14.32 & -25.78 & $\begin{array}{l}\text { Flow too low but no } \\
\text { adjustments could be made to } \\
\text { rectify. }\end{array}$ & \\
\hline SS-253 & FEG-30 & 2 & N/A & 500 & 6 & 1.2 & 100 & OTP-05 & $09 / 13 / 2012$ & $14: 57: 36$ & $14: 59: 42$ & 10.8 & 69.2 & -25.84 & -35.00 & $\begin{array}{l}\text { Flow too low but no } \\
\text { adjustments could be made to } \\
\text { rectify. }\end{array}$ & \\
\hline SS-254 & FEG-30 & 2 & N/A & 500 & 6 & 1.2 & 200 & OTP-05 & $09 / 13 / 2012$ & $15: 20: 56$ & $15: 23: 00$ & 6.9 & 72.1 & -45.54 & -51.14 & $\begin{array}{l}\text { Flow too low but no } \\
\text { adjustments could be made to } \\
\text { rectify. }\end{array}$ & \\
\hline SS-255 & FEG-30 & 2 & N/A & 500 & 6 & 1.2 & 200 & OTP-05 & 09/19/2012 & 10:07:17 & 10:09:27 & 12.5 & 78.6 & 542.64 & 532.98 & & \multirow{6}{*}{$\begin{array}{l}\text { TDP } \\
884^{(a)}\end{array}$} \\
\hline SS-256 & FEG-30 & 2 & N/A & 500 & 6 & 1.2 & 200 & OTP-05 & 09/19/2012 & 10:30:39 & 10:33:07 & 13.2 & 81.4 & 544.20 & 531.78 & $\begin{array}{l}\text { Check ale stuck open for a } \\
\text { few seconds after the spray } \\
\text { stop time. C scatters by order } \\
\text { of magnitude. }\end{array}$ & \\
\hline SS-257 & FEG-30 & 2 & N/A & 500 & 6 & 1.2 & 200 & OTP-05 & $09 / 19 / 2012$ & 10:52:30 & 10:54:36 & 12.1 & 83.8 & 532.10 & 525.86 & Same comment as above. & \\
\hline SS-258 & FEG-30 & 2 & N/A & 500 & 6 & 1.2 & 200 & OTP-05 & $09 / 19 / 2012$ & $11: 32: 12$ & N/A & 11.4 & 83.7 & 519.62 & 518.94 & $\begin{array}{l}\text { Malvern could not see spray. } \\
\text { Data not exported. }\end{array}$ & \\
\hline SS-259 & FEG-30 & 2 & N/A & 500 & 6 & 1.2 & 200 & OTP-05 & 09/19/2012 & 11:43:13 & N/A & N/A & N/A & 518.16 & N/A & & \\
\hline SS-260 & FEG-30 & 2 & N/A & 500 & 6 & 1.2 & 200 & OTP-05 & 09/19/2012 & $13: 55: 30$ & 13:58:05 & 11.5 & 80.6 & 518.56 & 509.32 & $\begin{array}{l}\text { Spray was on/off at the } \\
\text { beginning of the spray time. }\end{array}$ & \\
\hline SS-261 & FEG-30 & 2 & N/A & 500 & 6 & 1.2 & 100 & OTP-05 & 09/21/2012 & 11:43:54 & $11: 46: 04$ & 12.1 & 73.8 & 526.60 & 517.10 & & \multirow{4}{*}{\begin{tabular}{|l} 
TDP \\
$885^{\text {(a) }}$
\end{tabular}} \\
\hline SS-262 & FEG-30 & 2 & N/A & 500 & 6 & 1.2 & 100 & OTP-05 & 09/21/2012 & 14:15:09 & 14:17:11 & 12.8 & 70.8 & 525.44 & 518.72 & & \\
\hline SS-263 & FEG-30 & 2 & N/A & 500 & 6 & 1.2 & 100 & OTP-05 & $09 / 21 / 2012$ & $14: 28: 15$ & $14: 30: 29$ & 11.6 & 75.3 & 518.02 & 516.54 & $\begin{array}{l}\text { Malvern could not see spray. } \\
\text { Data not exported. }\end{array}$ & \\
\hline SS-264 & FEG-30 & 2 & N/A & 500 & 6 & 1.2 & 100 & OTP-05 & $09 / 21 / 2012$ & $14: 44: 22$ & $14: 46: 28$ & 11.2 & 77.8 & 515.82 & 507.44 & $\begin{array}{l}\text { Malvern could not see spray. } \\
\text { Data not exported. }\end{array}$ & \\
\hline SS-265 & FEG-30 & 2 & N/A & 500 & 6 & 1.2 & 380 & OTP-05 & $09 / 25 / 2012$ & 10:23:09 & 10:24:18 & 1.0 & 74.9 & 511.12 & 500.78 & & \multirow{7}{*}{$\begin{array}{l}\text { TDP- } \\
886^{(\text {a) }}\end{array}$} \\
\hline SS-266 & FEG-30 & 2 & N/A & 500 & 6 & 1.2 & 380 & OTP-05 & $09 / 25 / 2012$ & 10:38:57 & $10: 40: 39$ & 2.0 & 75.1 & 511.88 & 497.40 & & \\
\hline SS-267 & FEG-30 & 2 & N/A & 500 & 6 & 1.2 & 380 & OTP-05 & $09 / 25 / 2012$ & 10:56:08 & $10: 57: 26$ & 0.9 & 75.1 & 509.98 & 498.92 & & \\
\hline SS-268 & FEG-30 & 2 & N/A & 500 & 6 & 1.2 & 200 & OTP-05 & $09 / 25 / 2012$ & 13:03:16 & & & & & & $\begin{array}{l}\text { Malvern could not see spray. } \\
\text { Data not exported. }\end{array}$ & \\
\hline SS-269 & FEG-30 & 2 & N/A & 500 & 6 & 1.2 & 200 & OTP-05 & 09/25/2012 & 13:12:52 & 13:15:08 & 7.7 & 76.3 & 512.74 & 508.94 & & \\
\hline SS-270 & FEG-30 & 2 & N/A & 500 & 6 & 1.2 & 200 & OTP-05 & $09 / 25 / 2012$ & $13: 28: 30$ & 13:30:39 & 7.4 & 78.8 & 511.52 & 505.02 & & \\
\hline SS-271 & FEG-30 & 2 & N/A & 500 & 6 & 1.2 & 200 & OTP-05 & $09 / 25 / 2012$ & 13:41:33 & 13:43:53 & 7.1 & 79.9 & 505.08 & 495.08 & & \\
\hline
\end{tabular}


Table A.1. (contd)

\begin{tabular}{|c|c|c|c|c|c|c|c|c|c|c|c|c|c|c|c|c|c|}
\hline Test ID & Simulant & $\begin{array}{l}\text { Mal. } \\
\text { Pos. }\end{array}$ & $\begin{array}{l}\text { Ext. } \\
\text { Len. } \\
\text { (in.) }\end{array}$ & $\begin{array}{l}\text { Malvern } \\
\text { Lens } \\
(\mathrm{mm})\end{array}$ & \begin{tabular}{|l|} 
Mixing \\
Fan (V)
\end{tabular} & $\begin{array}{l}\text { Actual } \\
\text { Purge } \\
\text { Flow per } \\
\text { Window } \\
\text { (SCFH) }\end{array}$ & $\begin{array}{l}\text { Nom. P } \\
\text { (psig) }\end{array}$ & OTP & $\begin{array}{c}\text { Date } \\
(\mathrm{mm} / \mathrm{dd} / \mathrm{yyyy})\end{array}$ & $\begin{array}{l}\text { Spray Start } \\
\text { (hh:mm:ss) }\end{array}$ & $\begin{array}{l}\text { Spray Stop } \\
\text { (hh:mm:ss) }\end{array}$ & $\begin{array}{l}\text { Header } \\
\text { Flow } \\
\text { (gpm) }\end{array}$ & $\begin{array}{c}\text { Loop } \\
\text { Temp } \\
\text { During } \\
\text { Spray } \\
\left({ }^{\circ} \mathrm{F}\right)\end{array}$ & $\begin{array}{c}\text { Pretest } \\
\text { Tank } \\
\text { Mass } \\
\text { (lb) }\end{array}$ & $\begin{array}{c}\text { Final } \\
\text { Tank } \\
\text { Mass (lb) }\end{array}$ & Notes & TDP \\
\hline SS-272 & FEG-30 & 2 & N/A & 500 & 6 & 0 & 100 & OTP-16 & $09 / 26 / 2012$ & $13: 11: 25$ & $13: 12: 54$ & 8.0 & 72.8 & 506.88 & 487.04 & $\begin{array}{l}\text { Test not valid. Air not turned } \\
\text { on. }\end{array}$ & \multirow{7}{*}{$\begin{array}{l}\text { TDP- } \\
887^{(a)}\end{array}$} \\
\hline SS-273 & FEG-30 & 2 & $\mathrm{~N} / \mathrm{A}$ & 500 & 6 & 1.2 & 100 & OTP-16 & $09 / 26 / 2012$ & 13:39:17 & $13: 40: 44$ & 8.4 & 73.6 & 504.00 & N/A & $\begin{array}{l}\text { Final weight was not written } \\
\text { down. } \mathrm{LPi}=738, \mathrm{LPf}=741 \text {. } \\
\text { Laser is drifting. }\end{array}$ & \\
\hline SS-274 & FEG-30 & 2 & N/A & 500 & 6 & 1.2 & 100 & OTP-16 & $09 / 26 / 2012$ & 14:57:58 & $14: 59: 24$ & 10.1 & 74.4 & 499.26 & 482.88 & & \\
\hline SS-275 & FEG-30 & 2 & N/A & 500 & 6 & 1.2 & 100 & OTP-16 & 09/26/2012 & $15: 17: 14$ & $15: 18: 35$ & 7.6 & 77.1 & 497.96 & 481.99 & & \\
\hline SS-276 & FEG-30 & 2 & N/A & 500 & 6 & 1.2 & 200 & OTP-16 & $09 / 26 / 2012$ & $15: 36: 15$ & $15: 38: 17$ & 6.8 & 78.6 & 497.78 & 466.82 & $\begin{array}{l}\text { Evacuation ale left open } \\
\text { during test. Pressure adj @ } \\
\sim 15: 38 \text {. }\end{array}$ & \\
\hline SS-277 & FEG-30 & 2 & N/A & 500 & 6 & 1.2 & 200 & OTP-16 & 09/26/2012 & $15: 55: 25$ & $15: 56: 45$ & 5.2 & 78.2 & 492.56 & 471.76 & $\mathrm{LPi}=726, \mathrm{LPf}=742$ & \\
\hline SS-278 & FEG-30 & 2 & N/A & 500 & 6 & 1.2 & 200 & OTP-16 & 09/26/2012 & $16: 38: 13$ & $16: 39: 40$ & 5.8 & 80.1 & 483.20 & 455.18 & $\mathrm{LPi}=721, \mathrm{LPf}=740$ & \\
\hline SS-279 & FEG-30 & 2 & N/A & 500 & 6 & 1.2 & 200 & OTP-16 & 09/27/2012 & $9: 40: 36$ & 9:47:55 & 7.1 & 73.3 & 507.32 & 473.88 & $\begin{array}{l}\text { Stop time is when evacuation } \\
\text { was stopped. Pressure is } \\
279 \text { psi. }\end{array}$ & \multirow{8}{*}{$\begin{array}{l}\text { TDP- } \\
889^{(\mathrm{a})}\end{array}$} \\
\hline SS-280 & FEG-30 & 2 & N/A & 500 & 6 & 1.2 & 200 & OTP-16 & $09 / 27 / 2012$ & 10:04:18 & $10: 10: 15$ & 6.0 & 76.6 & 505.62 & 480.02 & $\begin{array}{l}\text { Stop time is when evacuation } \\
\text { was stopped. }\end{array}$ & \\
\hline SS-281 & FEG-30 & 2 & N/A & 500 & 6 & 1.2 & 380 & OTP-16 & $09 / 27 / 2012$ & 10:29:49 & $10: 31: 26$ & 4.3 & 78.8 & 504.48 & 467.50 & $\begin{array}{l}\text { Stop time was when spray } \\
\text { was stopped. }\end{array}$ & \\
\hline SS-282 & FEG-30 & 2 & N/A & 500 & 6 & 1.2 & 380 & OTP-16 & 09/28/2012 & $9: 21: 24$ & $9: 22: 44$ & 3.1 & 69 & 500.38 & 469.08 & & \\
\hline SS-283 & FEG-30 & 2 & N/A & 500 & 6 & 1.2 & 380 & OTP-16 & $09 / 28 / 2012$ & $9: 42: 32$ & $9: 43: 40$ & 4.9 & 70.1 & 504.08 & 477.66 & & \\
\hline SS-284 & FEG-30 & 4 & 6 & 500 & off & 40 & 100 & OTP-03 & $09 / 28 / 2012$ & $12: 54: 26$ & $12: 55: 42$ & 8.8 & 72.7 & 508.52 & 507.42 & & \\
\hline SS-285 & FEG-30 & 4 & 6 & 500 & off & 40 & 200 & OTP-03 & $09 / 28 / 2012$ & $13: 07: 23$ & 13:08:32 & 6.1 & 75 & 507.88 & 507.26 & & \\
\hline SS-286 & FEG-30 & 4 & 6 & 500 & off & 40 & 380 & OTP-03 & $09 / 28 / 2012$ & 13:28:05 & $13: 29: 29$ & 4.3 & 75.5 & 507.08 & 505.36 & & \\
\hline SS-287 & FEG-6 & 4 & 6 & 500 & off & 35 & 100 & OTP-03 & $10 / 02 / 2012$ & 11:15:15 & N/A & 8.3 & N/A & 546.48 & N/A & Abort, laser alignment. & \multirow{8}{*}{$\begin{array}{l}\text { TDP- } \\
890^{(\mathrm{a})}\end{array}$} \\
\hline SS-288 & FEG-6 & 4 & 6 & 500 & off & 35 & 100 & OTP-03 & $10 / 02 / 2012$ & 11:33:12 & N/A & N/A & N/A & 545.70 & N/A & Abort, plug orifice. & \\
\hline SS-289 & FEG-6 & 4 & 6 & 500 & off & 35 & 100 & OTP-03 & $10 / 02 / 2012$ & 11:44:32 & N/A & N/A & N/A & 545.34 & N/A & Abort, plug orifice. & \\
\hline SS-290 & FEG-6 & 4 & 6 & 500 & off & 35 & 100 & OTP-03 & $10 / 02 / 2012$ & 13:22:57 & $13: 24: 40$ & N/A & N/A & 544.00 & N/A & Abort, plug orifice. & \\
\hline SS-291 & FEG-6 & 4 & 6 & 500 & off & 35 & 380 & OTP-03 & $10 / 02 / 2012$ & 13:39:44 & N/A & N/A & N/A & 543.83 & N/A & Abort, plug orifice. & \\
\hline SS-292 & FEG-6 & 4 & 6 & 500 & off & 35 & 380 & OTP-03 & $10 / 02 / 2012$ & 14:02:06 & $14: 03: 45$ & 6.4 & 79.2 & 544.38 & 542.36 & $\begin{array}{l}\text { Run okay, pressure rather } \\
\text { high @ } \sim 460 \text { psi. }\end{array}$ & \\
\hline SS-293 & FEG-6 & 4 & 6 & 500 & off & 35 & 380 & OTP-03 & $10 / 02 / 2012$ & 14:20:05 & N/A & N/A & N/A & 542.24 & N/A & Abort, plug orifice. & \\
\hline SS-294 & FEG-6 & 4 & 6 & 500 & off & 35 & 380 & OTP-03 & $10 / 02 / 2012$ & $14: 37: 57$ & N/A & N/A & N/A & 540.28 & N/A & Abort, plug orifice. & \\
\hline
\end{tabular}


Table A.1. (contd)

\begin{tabular}{|c|c|c|c|c|c|c|c|c|c|c|c|c|c|c|c|c|c|}
\hline Test ID & Simulant & $\begin{array}{l}\text { Mal. } \\
\text { Pos. }\end{array}$ & $\begin{array}{l}\text { Ext. } \\
\text { Len. } \\
\text { (in.) }\end{array}$ & $\begin{array}{l}\text { Malvern } \\
\text { Lens } \\
(\mathrm{mm}) \\
\end{array}$ & $\begin{array}{l}\text { Mixing } \\
\text { Fan (V) }\end{array}$ & $\begin{array}{l}\text { Actual } \\
\text { Purge } \\
\text { Flow per } \\
\text { Window } \\
\text { (SCFH) }\end{array}$ & $\begin{array}{c}\text { Nom. P } \\
\text { (psig) }\end{array}$ & ОТР & $\begin{array}{c}\text { Date } \\
(\mathrm{mm} / \mathrm{dd} / \mathrm{yyyy})\end{array}$ & $\begin{array}{l}\text { Spray Start } \\
(\text { hh:mm:ss) }\end{array}$ & $\begin{array}{l}\text { Spray Stop } \\
\text { (hh:mm:ss) }\end{array}$ & $\begin{array}{l}\text { Header } \\
\text { Flow } \\
(\text { gpm) }\end{array}$ & \begin{tabular}{|c|} 
Loop \\
Temp \\
During \\
Spray \\
$\left({ }^{\circ} \mathrm{F}\right)$ \\
\end{tabular} & $\begin{array}{c}\text { Pretest } \\
\text { Tank } \\
\text { Mass } \\
(\mathrm{lb})\end{array}$ & $\begin{array}{c}\text { Final } \\
\text { Tank } \\
\text { Mass (lb) }\end{array}$ & Notes & TDP \\
\hline SS-295 & FEG-6 & 4 & 6 & 500 & off & 40 & 100 & OTP-03 & $10 / 04 / 2012$ & 10:21:50 & N/A & N/A & $\mathrm{N} / \mathrm{A}$ & 539.66 & N/A & Abort, plug orifice. & \multirow{4}{*}{$\begin{array}{l}\text { TDP } \\
891^{\mathrm{a}}\end{array}$} \\
\hline SS-296 & FEG-6 & 4 & 6 & 500 & off & 40 & 100 & OTP-03 & $10 / 04 / 2012$ & $10: 28: 35$ & N/A & N/A & N/A & 539.55 & N/A & Abort, plug orifice. & \\
\hline SS-297 & FEG-6 & 4 & 6 & 500 & off & 40 & 380 & OTP-03 & $10 / 04 / 2012$ & 10:42:05 & $\mathrm{N} / \mathrm{A}$ & $\mathrm{N} / \mathrm{A}$ & $\mathrm{N} / \mathrm{A}$ & 540.18 & $\mathrm{~N} / \mathrm{A}$ & Abort, plug orifice. & \\
\hline SS-298 & FEG-6 & 4 & 6 & 500 & off & 40 & 380 & OTP-03 & $10 / 04 / 2012$ & $11: 40: 37$ & N/A & N/A & N/A & 539.68 & N/A & Abort, plug orifice. & \\
\hline SS-299 & FEG-6 & 2 & $\mathrm{~N} / \mathrm{A}$ & 500 & 6 & 1.2 & 100 & OTP-05 & $10 / 05 / 2012$ & $13: 46: 30$ & $13: 48: 10$ & 9.6 & 72.6 & 527.92 & 521.24 & & \multirow{7}{*}{$\begin{array}{l}\text { TDP- } \\
892^{\text {(a) }}\end{array}$} \\
\hline SS-300 & FEG-6 & 2 & $\mathrm{~N} / \mathrm{A}$ & 500 & 6 & 1.2 & 100 & OTP-05 & $10 / 05 / 2012$ & 14:02:28 & 14:04:05 & 9.7 & 74.2 & 520.62 & 513.50 & & \\
\hline SS-301 & FEG-6 & 2 & N/A & 500 & 6 & 1.2 & 100 & OTP-05 & $10 / 05 / 2012$ & 14:14:50 & 14:16:27 & 9.5 & 75.7 & 513.44 & 506.84 & & \\
\hline SS-302 & FEG-6 & 2 & $\mathrm{~N} / \mathrm{A}$ & 500 & 6 & 1.2 & 200 & OTP-05 & $10 / 05 / 2012$ & $15: 02: 34$ & $15: 04: 05$ & 9.1 & 74.6 & 532.76 & 525.18 & & \\
\hline SS-303 & FEG-6 & 2 & $\mathrm{~N} / \mathrm{A}$ & 500 & 6 & 1.2 & 200 & OTP-05 & $10 / 05 / 2012$ & $15: 15: 25$ & $15: 17: 14$ & 8.6 & 75.4 & 524.86 & 515.10 & & \\
\hline SS-304 & FEG-6 & 2 & $\mathrm{~N} / \mathrm{A}$ & 500 & 6 & 1.2 & 200 & OTP-05 & $10 / 05 / 2012$ & $15: 29: 31$ & $15: 31: 44$ & 8.5 & 76.7 & 515.06 & 503.92 & $\begin{array}{l}\text { Evacuation ale left open } \\
\text { during test. }\end{array}$ & \\
\hline SS-305 & FEG-6 & 2 & N/A & 500 & 6 & 1.2 & 200 & OTP-05 & $10 / 05 / 2012$ & $15: 55: 41$ & $15: 57: 38$ & 8.1 & 75 & 531.16 & 519.98 & & \\
\hline SS-306 & FEG-6 & 2 & $\mathrm{~N} / \mathrm{A}$ & 500 & off & 1.2 & 380 & OTP-05 & $10 / 08 / 2012$ & 10:11:29 & $10: 13: 44$ & 8.5 & 66.1 & 523.60 & & Abort, Mixing fan not on. & \multirow{11}{*}{$\begin{array}{l}\text { TDP } \\
893^{(\mathrm{a}}\end{array}$} \\
\hline SS-307 & FEG-6 & 2 & N/A & 500 & 6 & 1.2 & 380 & OTP-05 & $10 / 08 / 2012$ & $10: 56: 48$ & $10: 57: 59$ & 8.9 & 66.7 & 529.10 & 520.58 & & \\
\hline SS-308 & FEG-6 & 2 & N/A & 500 & 6 & 1.2 & 380 & OTP-05 & $10 / 08 / 2012$ & 11:13:37 & $11: 14: 41$ & 7.7 & 68.3 & 519.34 & 512.48 & & \\
\hline SS-309 & FEG-6 & 2 & N/A & 500 & 6 & 1.2 & 380 & OTP-05 & $10 / 08 / 2012$ & 11:34:20 & $11: 35: 41$ & 7.8 & 69.7 & 527.98 & 518.22 & & \\
\hline SS-310 & FEG-6 & 2 & N/A & 500 & 6 & 1.2 & 100 & OTP-16 & $10 / 08 / 2012$ & $13: 22: 26$ & $13: 23: 55$ & 9.1 & 71.4 & 526.66 & 508.74 & & \\
\hline SS-311 & FEG-6 & 2 & N/A & 500 & 6 & 1.2 & 100 & OTP-16 & $10 / 08 / 2012$ & 13:39:44 & $13: 41: 15$ & 8.9 & 72.3 & 524.88 & 504.90 & & \\
\hline SS-312 & FEG-6 & 2 & $\mathrm{~N} / \mathrm{A}$ & 500 & 6 & 1.2 & 100 & OTP-16 & $10 / 08 / 2012$ & 13:52:47 & $13: 54: 39$ & 8.0 & 73.6 & 504.48 & 481.98 & & \\
\hline SS-313 & FEG-6 & 2 & N/A & 500 & 6 & 1.2 & 100 & OTP-16 & $10 / 08 / 2012$ & $14: 45: 49$ & 14:48:01 & 8.6 & 71.8 & 521.84 & 495.30 & & \\
\hline SS-314 & FEG-6 & 2 & N/A & 500 & 6 & 1.2 & 200 & OTP-16 & $10 / 08 / 2012$ & 15:09:38 & $15: 11: 04$ & 6.1 & 72.7 & 490.22 & 467.60 & & \\
\hline SS-315 & FEG-6 & 2 & N/A & 500 & 6 & 1.2 & 200 & OTP-16 & $10 / 08 / 2012$ & 15:41:43 & $15: 43: 15$ & 7.4 & 71.3 & 519.64 & 494.38 & & \\
\hline SS-316 & FEG-6 & 2 & N/A & 500 & 6 & 1.2 & 200 & OTP-16 & $10 / 08 / 2012$ & 16:02:21 & 16:04:07 & 6.2 & 72.6 & 518.72 & 489.44 & & \\
\hline SS-317 & FEG-6 & 2 & N/A & 500 & 6 & 1.2 & 380 & OTP-16 & $10 / 09 / 2012$ & 9:55:04 & $9: 56: 43$ & 7.6 & 65.3 & 518.68 & 478.58 & & \multirow{6}{*}{$\begin{array}{l}\text { TDP- } \\
894^{(\mathrm{a})}\end{array}$} \\
\hline SS-318 & FEG-6 & 2 & N/A & 500 & 6 & 1.2 & 380 & OTP-16 & $10 / 09 / 2012$ & 10:33:11 & N/A & N/A & N/A & 517.70 & N/A & Abort. & \\
\hline SS-319 & FEG-6 & 2 & N/A & 500 & 6 & 1.2 & 380 & OTP-16 & $10 / 09 / 2012$ & $12: 40: 13$ & $12: 42: 10$ & 8.6133 & 68.7 & 518.78 & 476.00 & & \\
\hline SS-320 & FEG-6 & 2 & $\mathrm{~N} / \mathrm{A}$ & 500 & 6 & 1.2 & 380 & OTP-16 & $10 / 09 / 2012$ & $13: 39: 47$ & $13: 41: 56$ & 9.2833 & 70.4 & 520.98 & 472.14 & & \\
\hline SS-321 & FEG-6 & 4 & 6 & 500 & off & 35 & 380 & OTP-03 & $10 / 09 / 2012$ & $15: 36: 02$ & $15: 37: 11$ & 9.4344 & 70.6 & 519.10 & N/A & & \\
\hline SS-322 & FEG-6 & 4 & 6 & 500 & off & 35 & 380 & OTP-03 & $10 / 09 / 2012$ & 16:08:18 & 16:09:16 & 9.4408 & 70.4 & 515.40 & 512.74 & & \\
\hline SS-323 & FEG-6 & 4 & 6 & 500 & off & 35 & 100 & OTP-03 & $10 / 10 / 2012$ & $16: 30: 20$ & $\mathrm{~N} / \mathrm{A}$ & 9.4 & $\mathrm{~N} / \mathrm{A}$ & 522.16 & N/A & Abort, plug orifice. & \multirow{2}{*}{$\begin{array}{l}\text { TDP- } \\
895^{(\text {a) }}\end{array}$} \\
\hline SS-324 & FEG-6 & 4 & 6 & 500 & off & 35 & 100 & OTP-03 & $10 / 10 / 2012$ & $16: 36: 17$ & $\mathrm{~N} / \mathrm{A}$ & 9.2 & $\mathrm{~N} / \mathrm{A}$ & 521.68 & N/A & Abort, plug orifice. & \\
\hline
\end{tabular}


Table A.1. (contd)

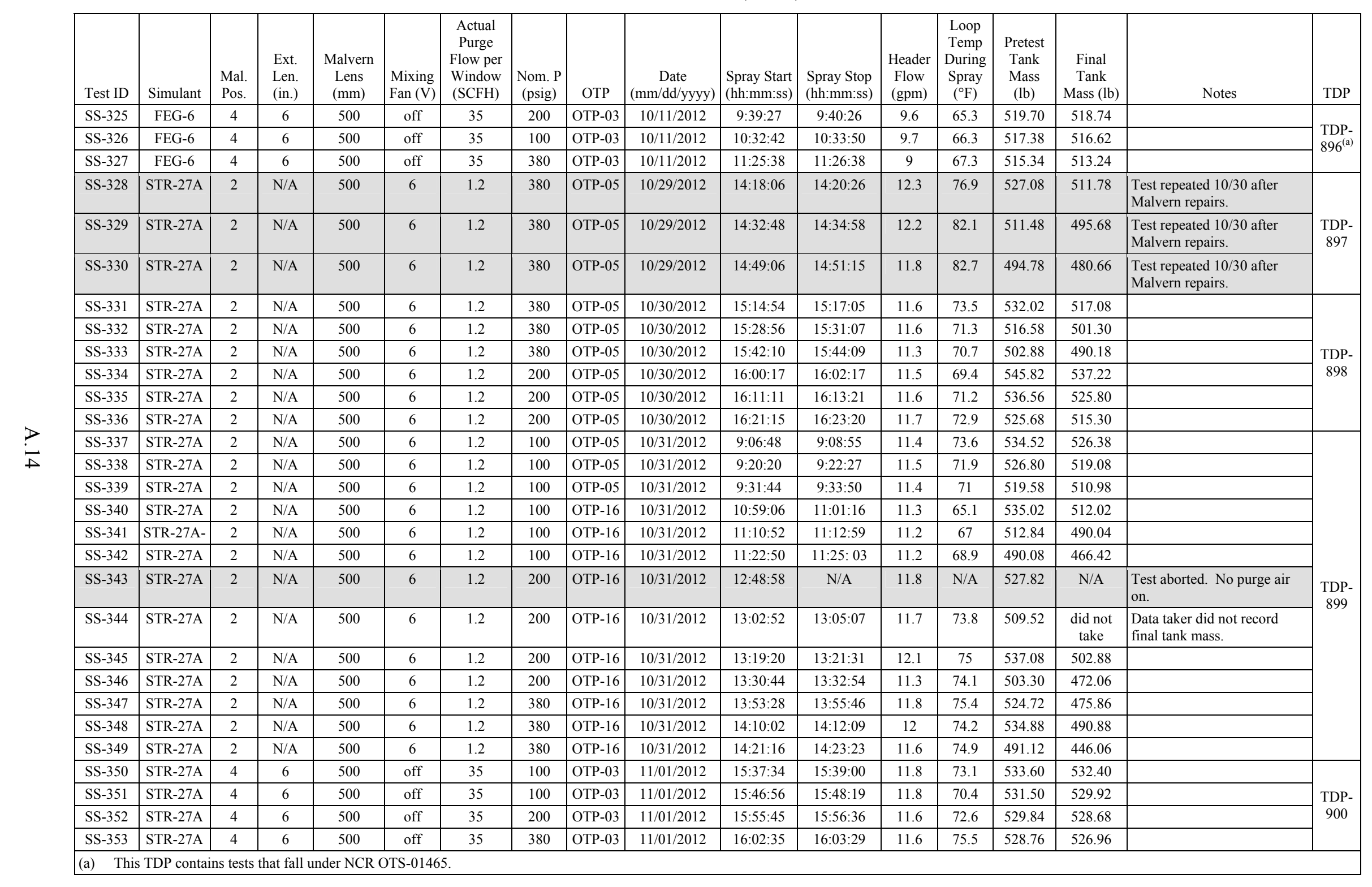


Table A.1. (contd)

\begin{tabular}{|c|c|c|c|c|c|c|c|c|c|c|c|c|c|c|c|c|c|}
\hline Test ID & Simulant & $\begin{array}{l}\text { Mal. } \\
\text { Pos. }\end{array}$ & $\begin{array}{l}\text { Ext. } \\
\text { Len. } \\
\text { (in.) }\end{array}$ & $\begin{array}{l}\text { Malvern } \\
\text { Lens } \\
(\mathrm{mm})\end{array}$ & $\begin{array}{l}\text { Mixing } \\
\text { Fan (V) }\end{array}$ & $\begin{array}{l}\text { Actual } \\
\text { Purge } \\
\text { Flow per } \\
\text { Window } \\
\text { (SCFH) } \\
\end{array}$ & $\begin{array}{c}\text { Nom. P } \\
\text { (psig) }\end{array}$ & OTP & $\begin{array}{c}\text { Date } \\
(\mathrm{mm} / \mathrm{dd} / \mathrm{yyyy})\end{array}$ & $\begin{array}{l}\text { Spray Start } \\
\text { (hh:mm:ss) }\end{array}$ & $\begin{array}{l}\text { Spray Stop } \\
\text { (hh:mm:ss) }\end{array}$ & $\begin{array}{c}\text { Header } \\
\text { Flow } \\
(\text { gpm })\end{array}$ & \begin{tabular}{|l|} 
Loop \\
Temp \\
During \\
Spray \\
$\left({ }^{\circ} \mathrm{F}\right)$ \\
\end{tabular} & $\begin{array}{l}\text { Pretest } \\
\text { Tank } \\
\text { Mass } \\
(\mathrm{lb}) \\
\end{array}$ & $\begin{array}{c}\text { Final } \\
\text { Tank } \\
\text { Mass (lb) } \\
\end{array}$ & Notes & TDP \\
\hline SS-354 & DPW & 2 & $\mathrm{~N} / \mathrm{A}$ & 500 & 6 & 1.2 & 380 & OTP-05 & $11 / 08 / 2012$ & $11: 28: 35$ & $11: 29: 45$ & 11.8 & 79.6 & 498.58 & 490.08 & & \multirow{9}{*}{$\begin{array}{r}\text { TDP } \\
901\end{array}$} \\
\hline SS-355 & DPW & 2 & $\mathrm{~N} / \mathrm{A}$ & 500 & 6 & 1.2 & 380 & OTP-05 & $11 / 08 / 2012$ & 11:38:21 & 11:39:31 & 12.1 & 82.2 & 491.59 & 482.40 & & \\
\hline SS-356 & DPW & 2 & $\mathrm{~N} / \mathrm{A}$ & 500 & 6 & 1.2 & 380 & OTP-05 & $11 / 08 / 2012$ & $11: 48: 55$ & $11: 49: 58$ & 11.9 & 84.8 & 482.98 & 475.88 & Background was after spray. & \\
\hline SS-357 & DPW & 2 & $\mathrm{~N} / \mathrm{A}$ & 500 & 6 & 1.2 & 380 & OTP-16 & $11 / 08 / 2012$ & $13: 36: 51$ & $13: 38: 03$ & 12.5 & 72.6 & 475.08 & 452.88 & $\begin{array}{l}\text { Background slowly drifting } \\
\text { down at beginning. After } \\
\text { spool change, the line was } \\
\text { not filled before spraying. }\end{array}$ & \\
\hline SS-358 & DPW & 2 & $\mathrm{~N} / \mathrm{A}$ & 500 & 6 & 1.2 & 380 & OTP-16 & $11 / 08 / 2012$ & $14: 21: 22$ & 14:22:24 & 12.1 & 76.4 & 452.30 & 426.86 & Background drifting down. & \\
\hline SS-359 & DPW & 2 & $\mathrm{~N} / \mathrm{A}$ & 500 & 6 & 1.2 & 380 & OTP-16 & $11 / 08 / 2012$ & $15: 20: 05$ & N/A & 12 & 78.7 & 423.32 & 417.28 & $\begin{array}{l}\text { Background drifting down. } \\
\text { Test aborted. }\end{array}$ & \\
\hline SS-360 & DPW & 2 & $\mathrm{~N} / \mathrm{A}$ & 500 & 6 & 5 & 380 & OTP-16 & $11 / 08 / 2012$ & 16:00:00 & 16:01:01 & 11.9 & 79.3 & 414.94 & 395.66 & Background drifting down. & \\
\hline SS-361 & DPW & 2 & $\mathrm{~N} / \mathrm{A}$ & 500 & 6 & 1.2 & 380 & OTP-16 & $11 / 08 / 2012$ & $16: 30: 54$ & N/A & 11.6 & 83.1 & 395.20 & 389.48 & $\begin{array}{l}\text { Background drifting down. } \\
\text { Test aborted. }\end{array}$ & \\
\hline SS-362 & DPW & 2 & $\mathrm{~N} / \mathrm{A}$ & 500 & 6 & 5 & 380 & OTP-16 & $11 / 08 / 2012$ & $16: 56: 38$ & $16: 57: 47$ & 12 & 78.8 & 385.88 & 364.96 & & \\
\hline SS-363 & DPW & 4 & 6 & 500 & off & 35 & 380 & OTP-03 & $11 / 09 / 2012$ & 10:45:09 & $10: 46: 10$ & 11.7 & 72.3 & 359.10 & 357.36 & & \multirow{2}{*}{$\begin{array}{r}\text { TDP- } \\
902\end{array}$} \\
\hline SS-364 & DPW & 4 & 6 & 500 & off & 35 & 380 & OTP-03 & $11 / 09 / 2012$ & 11:01:05 & 11:02:09 & 11.8 & 76 & 357.38 & 355.52 & & \\
\hline SS-365 & DST & 4 & 6 & 500 & off & 35 & 380 & OTP-03 & $11 / 13 / 2012$ & 11:08:45 & 11:09:50 & 14 & 73.3 & 542.48 & 540.22 & & \multirow{10}{*}{$\begin{array}{r}\text { TDP- } \\
903\end{array}$} \\
\hline SS-366 & DST & 2 & N/A & 500 & 6 & 1.2 & 380 & OTP-05 & $11 / 13 / 2012$ & $13: 22: 20$ & 13:23:39 & 11.9 & 77.9 & 538.60 & 527.98 & & \\
\hline SS-367 & DST & 2 & $\mathrm{~N} / \mathrm{A}$ & 500 & 6 & 1.2 & 380 & OTP-05 & $11 / 13 / 2012$ & $13: 40: 47$ & 13:41:53 & 12.5 & 80.5 & 527.72 & 519.78 & & \\
\hline SS-368 & DST & 2 & N/A & 500 & 6 & 1.2 & 380 & OTP-05 & $11 / 13 / 2012$ & 14:04:46 & 14:05:56 & 12.3 & 82.1 & 520.16 & 511.74 & & \\
\hline SS-369 & DST & 2 & $\mathrm{~N} / \mathrm{A}$ & 500 & 6 & 1.2 & 380 & OTP-05 & $11 / 13 / 2012$ & $14: 25: 20$ & 14:26:41 & 12.1 & 83.8 & 510.88 & 501.98 & & \\
\hline SS-370 & DST & 2 & N/A & 500 & 6 & 1.2 & 380 & OTP-16 & $11 / 13 / 2012$ & $15: 27: 40$ & $15: 28: 49$ & 12.6 & 66.9 & 498.68 & 474.68 & & \\
\hline SS-371 & DST & 2 & N/A & 500 & 6 & 1.2 & 380 & OTP-16 & $11 / 13 / 2012$ & $15: 52: 45$ & $15: 53: 50$ & 11.9 & 71 & 478.68 & 456.08 & & \\
\hline SS-372 & DST & 2 & N/A & 500 & 6 & 1.2 & 380 & OTP-16 & $11 / 13 / 2012$ & 16:02:48 & 16:03:59 & 11.4 & 73.6 & 456.72 & 433.94 & & \\
\hline SS-373 & DST & 2 & N/A & 500 & 6 & 1.2 & 380 & OTP-16 & $11 / 13 / 2012$ & $16: 17: 55$ & N/A & 11.9 & 76.4 & 433.88 & 411.80 & Test aborted. & \\
\hline SS-374 & DST & 2 & $\mathrm{~N} / \mathrm{A}$ & 500 & 6 & 1.2 & 380 & OTP-16 & $11 / 13 / 2012$ & $16: 31: 17$ & $16: 32: 41$ & 11.8 & 77.8 & 410.38 & 381.66 & & \\
\hline
\end{tabular}


Table A.1. (contd)

\begin{tabular}{|c|c|c|c|c|c|c|c|c|c|c|c|c|c|c|c|c|c|}
\hline Test ID & Simulant & $\begin{array}{l}\text { Mal. } \\
\text { Pos. }\end{array}$ & $\begin{array}{l}\text { Ext. } \\
\text { Len. } \\
\text { (in.) }\end{array}$ & $\begin{array}{l}\text { Malvern } \\
\text { Lens } \\
(\mathrm{mm})\end{array}$ & $\begin{array}{l}\text { Mixing } \\
\text { Fan (V) }\end{array}$ & $\begin{array}{l}\text { Actual } \\
\text { Purge } \\
\text { Flow per } \\
\text { Window } \\
\text { (SCFH) }\end{array}$ & $\begin{array}{c}\text { Nom. P } \\
\text { (psig) }\end{array}$ & OTP & $\begin{array}{c}\text { Date } \\
\text { (mm/dd/yyyy) }\end{array}$ & $\begin{array}{l}\text { Spray Start } \\
\text { (hh:mm:ss) }\end{array}$ & $\begin{array}{l}\text { Spray Stop } \\
\text { (hh:mm:ss) }\end{array}$ & $\begin{array}{l}\text { Header } \\
\text { Flow } \\
\text { (gpm) }\end{array}$ & \begin{tabular}{|c|} 
Loop \\
Temp \\
During \\
Spray \\
$\left({ }^{\circ} \mathrm{F}\right)$
\end{tabular} & $\begin{array}{c}\text { Pretest } \\
\text { Tank } \\
\text { Mass } \\
\text { (lb) }\end{array}$ & $\begin{array}{c}\text { Final } \\
\text { Tank } \\
\text { Mass (lb) }\end{array}$ & Notes & TDP \\
\hline SS-375 & Water & 2 & N/A & 500 & 6 & 1.2 & 380 & OTP-03 & $01 / 17 / 2013$ & $10: 12: 26$ & 10:14:31 & 11.41 & 72.4 & 530.80 & 527.36 & Initial RH-54.4\%. & \multirow{12}{*}{$\begin{array}{r}\text { TDP } \\
910\end{array}$} \\
\hline SS-376 & Water & 2 & N/A & 500 & 6 & 1.2 & 380 & OTP-03 & $01 / 17 / 2013$ & $10: 26: .07$ & $10: 28: 12$ & 11.44 & 75.5 & 527.32 & 524.54 & $\begin{array}{l}\text { Initial RH }-66.0 \% \text {; Mixing } \\
\text { fan not on at start of spray - } \\
\text { turned on } 15-20 \text { seconds into } \\
\text { the spray. }\end{array}$ & \\
\hline SS-377 & Water & 2 & N/A & 500 & 6 & 1.2 & 380 & OTP-03 & $01 / 17 / 2013$ & $10: 38: 52$ & $10: 40: 59$ & 11.43 & 77.5 & 524.76 & 520.92 & Initial RH $-75.6 \%$. & \\
\hline SS-378 & Water & 2 & N/A & 500 & 6 & 1.2 & 380 & OTP-03 & $01 / 17 / 2013$ & $10: 50: 58$ & $10: 53: 05$ & 11.42 & 79.9 & 520.88 & 517.40 & Initial RH-85.1\%. & \\
\hline SS-379 & Water & 2 & N/A & 500 & 6 & 1.2 & 380 & OTP-03 & $01 / 17 / 2013$ & 11:01:42 & $11: 03: 55$ & 11.45 & 82.0 & 517.52 & 513.48 & Initial RH-98.2\%. & \\
\hline SS-380 & Water & 2 & N/A & 500 & 6 & 1.2 & 380 & OTP-03 & $01 / 17 / 2013$ & 11:13:32 & $11: 15: 50$ & 11.38 & 83.8 & 513.68 & 509.80 & Initial RH - 91.6\%. & \\
\hline SS-381 & Water & 2 & N/A & 500 & 6 & 1.2 & 380 & OTP-03 & $01 / 17 / 2013$ & 14:14:09 & 14:16:14 & 11.42 & 72.5 & 534.48 & 531.10 & Initial RH-55.1\%. & \\
\hline SS-382 & Water & 2 & N/A & 500 & 6 & 1.2 & 380 & OTP-03 & $01 / 17 / 2013$ & $14: 26: 54$ & 14:29:05 & 11.43 & 75 & 532.28 & 527.98 & Initial RH $-64.2 \%$. & \\
\hline SS-383 & Water & 2 & N/A & 500 & 6 & 1.2 & 380 & OTP-03 & $01 / 17 / 2013$ & $14: 37: 45$ & $14: 39: 54$ & 11.39 & 77 & 528.34 & 524.92 & Initial RH-96.3\%. & \\
\hline SS-384 & Water & 2 & $\mathrm{~N} / \mathrm{A}$ & 500 & 6 & 1.2 & 100 & OTP-03 & $01 / 17 / 2013$ & 14:49:01 & 14:51:07 & 11.54 & 78.8 & 523.88 & 521.62 & Initial RH - 100.2\%. & \\
\hline SS-385 & Water & 2 & N/A & 500 & 6 & 1.2 & 100 & OTP-03 & $01 / 17 / 2013$ & 15:02:01 & 15:04:06 & 11.53 & 79.7 & 522.28 & 520.02 & Initial RH-75.1\%. & \\
\hline SS-386 & Water & 2 & N/A & 500 & 6 & 1.2 & 100 & OTP-03 & $01 / 17 / 2013$ & $15: 32: 18$ & $15: 34: 25$ & 11.56 & 81.6 & 520.08 & 518.08 & Initial RH-49.6\%. & \\
\hline
\end{tabular}


Table A.2. Additional Data for Phase II Spray Release Tests

\begin{tabular}{|c|c|c|c|c|c|c|c|c|c|c|}
\hline Test ID & $\begin{array}{c}\text { Target } \\
\mathrm{P} \text { (psig) }\end{array}$ & $\begin{array}{c}\text { Simulant (@) } \\
\text { Target } \\
\text { Properties) }\end{array}$ & $\begin{array}{c}\text { Outer Orifice } \\
\text { Diameter / } \\
\text { Width } \\
(\mathrm{mm})\end{array}$ & $\begin{array}{l}\text { Outer Orifice } \\
\text { Diameter / } \\
\text { Length } \\
(\mathrm{mm})\end{array}$ & $\begin{array}{c}\text { Average } \\
\text { Header Flow } \\
\text { During Whole } \\
\text { Spray (gpm) }\end{array}$ & $\begin{array}{c}\text { Square of } \\
\text { avg(sqrt(P)) } \\
\text { Over Fit } \\
\text { Period (psig) }\end{array}$ & $\begin{array}{c}\text { Average } \\
\text { Temperature } \\
\text { During Whole } \\
\text { Spray }\left({ }^{\circ} \mathrm{C}\right)\end{array}$ & $\begin{array}{c}\text { Average } \\
\text { Leakage Flow } \\
\text { Rate Over Fit } \\
\text { Period }(\mathrm{g} / \mathrm{s})\end{array}$ & $\begin{array}{c}\text { Orifice } \\
\text { Coefficient } \\
\text { During Fit } \\
\text { Period } \\
\end{array}$ & $\begin{array}{c}\text { Initial } \\
\text { Relative } \\
\text { Humidity } \\
(\%)\end{array}$ \\
\hline SS-103 & 380 & Water & 0.534 & 0.534 & 11.5 & 382.2 & 21.6 & 9.08 & 0.559 & $\mathrm{n} / \mathrm{m}$ \\
\hline SS-104 & 380 & Water & 0.534 & 0.534 & 11.5 & 372.5 & 21.8 & 9.68 & 0.604 & 59.1 \\
\hline SS-105 & 380 & Water & 0.534 & 0.534 & 11.6 & 383.6 & 21.9 & 10.49 & 0.645 & 95.0 \\
\hline SS-106 & 380 & Water & 0.534 & 0.534 & 11.5 & 378.9 & 29.9 & 10.21 & 0.632 & 83.2 \\
\hline SS-107 & 380 & Water & 0.534 & 0.534 & 11.6 & 378.8 & 22.3 & 10.11 & 0.625 & 85.9 \\
\hline SS-108 & 380 & Water & 0.534 & 0.534 & 11.5 & 371.7 & 21.1 & 9.50 & 0.593 & $\mathrm{n} / \mathrm{m}$ \\
\hline SS-109 & 380 & Water & 0.534 & 0.534 & 11.4 & 389.2 & 21.2 & 11.22 & 0.684 & $\mathrm{n} / \mathrm{m}$ \\
\hline SS-110 & 380 & Water & 0.534 & 0.534 & 11.5 & 375.8 & 21.5 & 10.74 & 0.667 & 91.8 \\
\hline SS-111 & 380 & Water & 0.534 & 0.534 & 10.1 & 372.4 & 21.9 & 10.33 & 0.644 & 91.7 \\
\hline SS-112 & 380 & Water & 0.534 & 0.534 & 10.1 & 378.8 & 22.1 & 10.98 & 0.679 & 93.5 \\
\hline SS-114 & 380 & Water & 0.975 & 0.975 & 10.0 & 379.2 & 20.8 & 40.20 & 0.745 & 94.7 \\
\hline SS-116 & 380 & Water & 0.975 & 0.975 & 10.0 & 378.7 & 21.7 & 38.55 & 0.715 & 91.9 \\
\hline SS-117 & 380 & Water & 0.975 & 0.975 & 10.0 & 378.5 & 21.6 & 41.78 & 0.775 & 92.7 \\
\hline SS-118 & 380 & Water & 0.975 & 0.975 & 10.1 & 389.7 & 21.6 & 41.32 & 0.756 & $\mathrm{n} / \mathrm{m}$ \\
\hline SS-119 & 380 & Water & 0.975 & 0.975 & 10.1 & 383.0 & 21.9 & 38.39 & 0.708 & 93.3 \\
\hline SS-120 & 380 & Water & 0.534 & 0.534 & 11.5 & 379.2 & 20.7 & 9.71 & 0.600 & $\mathrm{n} / \mathrm{m}$ \\
\hline SS-121 & 380 & Water & 0.534 & 0.534 & 11.5 & 379.4 & 20.7 & 9.91 & 0.612 & $\mathrm{n} / \mathrm{m}$ \\
\hline SS-122 & 380 & Water & 0.534 & 0.534 & 11.5 & 376.6 & 21.0 & 9.66 & 0.599 & $\mathrm{n} / \mathrm{m}$ \\
\hline SS-123 & 380 & Water & 0.534 & 0.534 & 11.5 & 376.4 & 21.5 & 9.83 & 0.610 & $\mathrm{n} / \mathrm{m}$ \\
\hline SS-127 & 380 & Water & 0.534 & 0.534 & 11.5 & 381.3 & 21.5 & 10.58 & 0.652 & $\mathrm{n} / \mathrm{m}$ \\
\hline SS-128 & 380 & Water & 0.534 & 0.534 & 11.5 & 388.3 & 21.6 & 10.19 & 0.622 & $\mathrm{n} / \mathrm{m}$ \\
\hline SS-129 & 380 & Water & 0.534 & 0.534 & 11.5 & 373.9 & 22.1 & 9.47 & 0.589 & $\mathrm{n} / \mathrm{m}$ \\
\hline SS-130 & 380 & Water & 0.534 & 0.534 & 11.4 & 382.7 & 19.9 & 9.77 & 0.601 & 87.7 \\
\hline SS-131 & 380 & Water & 0.534 & 0.534 & 11.4 & 379.3 & 20.4 & 10.32 & 0.638 & 89.2 \\
\hline SS-133 & 380 & Water & 0.534 & 0.534 & 11.4 & 386.2 & 21.1 & 11.25 & 0.689 & 89.6 \\
\hline SS-134 & 380 & Water & 0.534 & 0.534 & 11.4 & 382.4 & 21.3 & 9.29 & 0.572 & 89.2 \\
\hline SS-135 & 380 & Water & 0.534 & 0.534 & 11.3 & 369.6 & 21.5 & 9.96 & 0.623 & 89.7 \\
\hline SS-136 & 380 & Water & 0.534 & 0.534 & 11.4 & 381.1 & 21.6 & 10.54 & 0.650 & 86.0 \\
\hline SS-137 & 380 & Water & 0.534 & 0.534 & 11.5 & 394.9 & 21.7 & 8.98 & 0.544 & 88.1 \\
\hline SS-138 & 100 & Water & 0.534 & 4.886 & 11.5 & 100.2 & 21.3 & 61.17 & 0.631 & $\mathrm{n} / \mathrm{m}$ \\
\hline
\end{tabular}


Table A.2. (contd)

\begin{tabular}{|c|c|c|c|c|c|c|c|c|c|c|}
\hline Test ID & $\begin{array}{l}\text { Target } \\
\mathrm{P}(\mathrm{psig})\end{array}$ & $\begin{array}{c}\text { Simulant }(@ \\
\text { Target Properties) }\end{array}$ & $\begin{array}{c}\text { Outer } \\
\text { Orifice } \\
\text { Diameter / } \\
\text { Width } \\
(\mathrm{mm})\end{array}$ & $\begin{array}{l}\text { Outer Orifice } \\
\text { Diameter / } \\
\text { Length } \\
(\mathrm{mm})\end{array}$ & $\begin{array}{c}\text { Average } \\
\text { Header Flow } \\
\text { During } \\
\text { Whole Spray } \\
\text { (gpm) }\end{array}$ & $\begin{array}{c}\text { Square of } \\
\text { avg(sqrt(P)) } \\
\text { Over Fit } \\
\text { Period (psig) }\end{array}$ & $\begin{array}{c}\text { Average } \\
\text { Temperature } \\
\text { During Whole } \\
\text { Spray }\left({ }^{\circ} \mathrm{C}\right)\end{array}$ & $\begin{array}{c}\text { Average } \\
\text { Leakage Flow } \\
\text { Rate Over Fit } \\
\text { Period }(\mathrm{g} / \mathrm{s})\end{array}$ & $\begin{array}{c}\text { Orifice } \\
\text { Coefficient } \\
\text { During Fit } \\
\text { Period }\end{array}$ & $\begin{array}{c}\text { Initial } \\
\text { Relative } \\
\text { Humidity } \\
(\%)\end{array}$ \\
\hline SS-139 & 100 & Water & 0.534 & 4.886 & 11.7 & 95.9 & 20.5 & 59.71 & 0.630 & $\mathrm{n} / \mathrm{m}$ \\
\hline SS-140 & 100 & Water & 0.534 & 4.886 & 11.7 & 100.6 & 20.1 & 60.56 & 0.624 & 96.0 \\
\hline SS-141 & 200 & Water & 0.534 & 4.886 & 11.4 & 199.7 & 20.2 & 85.37 & 0.624 & $\mathrm{n} / \mathrm{m}$ \\
\hline SS-142 & 200 & Water & 0.534 & 4.886 & 11.5 & 201.2 & 20.4 & 85.60 & 0.623 & $\mathrm{n} / \mathrm{m}$ \\
\hline SS-143 & 380 & Water & 0.534 & 4.886 & 11.7 & 396.6 & 20.5 & 120.59 & 0.626 & $\mathrm{n} / \mathrm{m}$ \\
\hline SS-144 & 380 & Water & 0.534 & 4.886 & 11.8 & 384.6 & 20.6 & 118.56 & 0.625 & $\mathrm{n} / \mathrm{m}$ \\
\hline SS-145 & 100 & Water & 0.975 & 0.975 & 11.5 & 100.4 & 20.2 & 20.86 & 0.752 & $\mathrm{n} / \mathrm{m}$ \\
\hline SS-146 & 100 & Water & 0.975 & 0.975 & 11.5 & 100.3 & 19.9 & 20.65 & 0.745 & $\mathrm{n} / \mathrm{m}$ \\
\hline SS-147 & 200 & Water & 0.975 & 0.975 & 11.5 & 200.4 & 19.7 & 29.04 & 0.741 & $\mathrm{n} / \mathrm{m}$ \\
\hline SS-148 & 200 & Water & 0.975 & 0.975 & 11.5 & 201.9 & 20.0 & 29.22 & 0.742 & $\mathrm{n} / \mathrm{m}$ \\
\hline SS-150 & 380 & Water & 0.975 & 0.975 & 11.5 & 392.1 & 20.9 & 40.87 & 0.745 & $\mathrm{n} / \mathrm{m}$ \\
\hline SS-151 & 380 & Water & 0.975 & 0.975 & 11.6 & 384.1 & 21.2 & 40.63 & 0.748 & $\mathrm{n} / \mathrm{m}$ \\
\hline SS-154 & 380 & 30 Pa clay & 0.975 & 0.975 & 11.5 & 373.1 & 22.5 & 51.20 & 0.855 & $\mathrm{n} / \mathrm{m}$ \\
\hline SS-155 & 380 & 30 Pa clay & 0.975 & 0.975 & 11.4 & 394.6 & 22.6 & 53.25 & 0.865 & $\mathrm{n} / \mathrm{m}$ \\
\hline SS-156 & 380 & 30 Pa clay & 0.975 & 0.975 & 11.5 & 397.0 & 22.9 & 54.76 & 0.886 & $\mathrm{n} / \mathrm{m}$ \\
\hline SS-157 & 380 & $30 \mathrm{~Pa}$ clay & 0.975 & 0.975 & 11.5 & 389.0 & 22.8 & 54.58 & 0.893 & $\mathrm{n} / \mathrm{m}$ \\
\hline SS-159 & 200 & 30 Pa clay & 0.975 & 0.975 & 11.6 & 215.2 & 21.3 & 44.35 & 0.975 & $\mathrm{n} / \mathrm{m}$ \\
\hline SS-160 & 200 & 30 Pa clay & 0.975 & 0.975 & 11.5 & 202.0 & 21.4 & 40.85 & 0.927 & $\mathrm{n} / \mathrm{m}$ \\
\hline SS-161 & 200 & 30 Pa clay & 0.975 & 0.975 & 11.5 & 200.8 & 21.3 & 41.64 & 0.948 & $\mathrm{n} / \mathrm{m}$ \\
\hline SS-162 & 100 & $30 \mathrm{~Pa}$ clay & 0.975 & 0.975 & 11.4 & 101.4 & 20.4 & 29.92 & 0.958 & $\mathrm{n} / \mathrm{m}$ \\
\hline SS-163 & 100 & 30 Pa clay & 0.975 & 0.975 & 11.4 & 101.4 & 20.1 & 29.94 & 0.959 & $\mathrm{n} / \mathrm{m}$ \\
\hline SS-164 & 100 & 30 Pa clay & 0.975 & 0.975 & 11.5 & 102.0 & 20.0 & 29.75 & 0.950 & $\mathrm{n} / \mathrm{m}$ \\
\hline SS-165 & 100 & 30 Pa clay & 0.975 & 0.975 & 11.5 & 103.0 & 20.0 & 30.45 & 0.968 & $\mathrm{n} / \mathrm{m}$ \\
\hline SS-166 & 100 & $30 \mathrm{~Pa}$ clay & 0.534 & 4.886 & 11.6 & 101.6 & 21.0 & 69.30 & 0.635 & $\mathrm{n} / \mathrm{m}$ \\
\hline SS-167 & 100 & $30 \mathrm{~Pa}$ clay & 0.534 & 4.886 & 11.8 & 105.1 & 20.4 & 75.78 & 0.682 & $\mathrm{n} / \mathrm{m}$ \\
\hline SS-168 & 100 & 30 Pa clay & 0.534 & 4.886 & 12.0 & 107.1 & 19.9 & 77.95 & 0.695 & $\mathrm{n} / \mathrm{m}$ \\
\hline SS-170 & 200 & 30 Pa clay & 0.534 & 4.886 & 11.4 & 200.0 & 21.3 & 112.05 & 0.731 & $\mathrm{n} / \mathrm{m}$ \\
\hline SS-171 & 200 & $30 \mathrm{~Pa}$ clay & 0.534 & 4.886 & 11.4 & 198.0 & 21.7 & 108.74 & 0.713 & $\mathrm{n} / \mathrm{m}$ \\
\hline SS-173 & 200 & 30 Pa clay & 0.534 & 4.886 & 11.9 & 194.1 & 21.9 & 109.22 & 0.724 & $\mathrm{n} / \mathrm{m}$ \\
\hline
\end{tabular}


Table A.2. (contd)

\begin{tabular}{|c|c|c|c|c|c|c|c|c|c|c|}
\hline Test ID & $\begin{array}{l}\text { Target } \\
\mathrm{P} \text { (psig) }\end{array}$ & $\begin{array}{c}\text { Simulant }(@ \\
\text { Target Properties) }\end{array}$ & $\begin{array}{c}\text { Outer } \\
\text { Orifice } \\
\text { Diameter / } \\
\text { Width } \\
(\mathrm{mm})\end{array}$ & $\begin{array}{l}\text { Outer Orifice } \\
\text { Diameter / } \\
\text { Length } \\
(\mathrm{mm})\end{array}$ & $\begin{array}{c}\text { Average } \\
\text { Header Flow } \\
\text { During } \\
\text { Whole Spray } \\
\text { (gpm) }\end{array}$ & $\begin{array}{c}\text { Square of } \\
\text { avg(sqrt(P)) } \\
\text { Over Fit } \\
\text { Period (psig) }\end{array}$ & $\begin{array}{c}\text { Average } \\
\text { Temperature } \\
\text { During Whole } \\
\text { Spray }\left({ }^{\circ} \mathrm{C}\right)\end{array}$ & $\begin{array}{c}\text { Average } \\
\text { Leakage Flow } \\
\text { Rate Over Fit } \\
\text { Period }(\mathrm{g} / \mathrm{s})\end{array}$ & $\begin{array}{c}\text { Orifice } \\
\text { Coefficient } \\
\text { During Fit } \\
\text { Period }\end{array}$ & $\begin{array}{c}\text { Initial } \\
\text { Relative } \\
\text { Humidity } \\
(\%)\end{array}$ \\
\hline SS-174 & 200 & $30 \mathrm{~Pa}$ clay & 0.534 & 4.886 & 12.0 & 200.6 & 21.9 & 108.13 & 0.705 & $\mathrm{n} / \mathrm{m}$ \\
\hline SS-176 & 380 & $30 \mathrm{~Pa}$ clay & 0.534 & 4.886 & 11.6 & 379.7 & 23.1 & 153.83 & 0.729 & 94.7 \\
\hline SS-178 & 380 & $30 \mathrm{~Pa}$ clay & 0.534 & 4.886 & 11.5 & 370.0 & 23.5 & 153.31 & 0.736 & 87.6 \\
\hline SS-179 & 380 & 30 Pa clay & 0.534 & 4.886 & 11.6 & 384.5 & 24.4 & 154.03 & 0.725 & 94.1 \\
\hline SS-203 & 380 & $30 \mathrm{~Pa}$ clay & 0.975 & 0.975 & 11.8 & 396.5 & 24.7 & 57.91 & 0.938 & $\mathrm{n} / \mathrm{m}$ \\
\hline SS-204 & 380 & $30 \mathrm{~Pa}$ clay & 0.975 & 0.975 & 11.2 & 360.5 & 24.8 & 55.93 & 0.950 & 89.1 \\
\hline SS-205 & 380 & 30 Pa clay & 0.975 & 0.975 & 11.4 & 371.5 & 24.9 & 55.43 & 0.928 & 89.9 \\
\hline SS-207 & 200 & $30 \mathrm{~Pa}$ clay & 0.975 & 0.975 & 11.5 & 198.2 & 21.4 & 43.08 & 0.987 & 83.2 \\
\hline SS-208 & 200 & 30 Pa clay & 0.975 & 0.975 & 11.5 & 198.9 & 21.9 & 42.90 & 0.981 & 89.9 \\
\hline SS-209 & 380 & $30 \mathrm{~Pa}$ clay & 0.534 & 4.886 & 12.1 & 408.9 & 23.0 & 163.18 & 0.745 & 94.0 \\
\hline SS-210 & 380 & 30 Pa clay & 0.534 & 4.886 & 12.0 & 397.2 & 23.5 & 163.37 & 0.757 & 91.7 \\
\hline SS-211 & 380 & 30 Pa clay & 0.534 & 4.886 & 11.7 & 380.5 & 24.2 & 161.58 & 0.765 & 96.1 \\
\hline SS-213 & 200 & 30 Pa clay & 0.534 & 4.886 & 11.7 & 215.8 & 22.1 & 120.99 & 0.760 & 95.4 \\
\hline SS-215 & 200 & $30 \mathrm{~Pa}$ clay & 0.534 & 4.886 & 11.3 & 201.3 & 21.4 & 122.42 & 0.796 & 94.2 \\
\hline SS-216 & 200 & $30 \mathrm{~Pa}$ clay & 0.534 & 4.886 & 11.5 & 205.8 & 22.1 & 122.29 & 0.787 & $\mathrm{n} / \mathrm{m}$ \\
\hline SS-217 & 100 & $30 \mathrm{~Pa}$ clay & 0.534 & 4.886 & 11.5 & 101.5 & 20.5 & 92.03 & 0.843 & 80.7 \\
\hline SS-218 & 100 & $30 \mathrm{~Pa}$ clay & 0.534 & 4.886 & 11.6 & 101.9 & 20.6 & 86.15 & 0.788 & 86.8 \\
\hline SS-219 & 100 & $30 \mathrm{~Pa}$ clay & 0.534 & 4.886 & 11.6 & 102.7 & 20.0 & 92.61 & 0.844 & 85.1 \\
\hline SS-220 & 100 & 6 Pa clay & 0.534 & 4.886 & 11.7 & 105.0 & 20.5 & 87.84 & 0.809 & 84.6 \\
\hline SS-221 & 100 & 6 Pa clay & 0.534 & 4.886 & 11.5 & 101.1 & 20.5 & 85.92 & 0.806 & 88.8 \\
\hline SS-222 & 100 & 6 Pa clay & 0.534 & 4.886 & 11.4 & 99.2 & 20.4 & 83.18 & 0.788 & 89.4 \\
\hline SS-223 & 200 & 6 Pa clay & 0.534 & 4.886 & 11.8 & 211.9 & 21.1 & 121.01 & 0.784 & 86.4 \\
\hline SS-224 & 200 & 6 Pa clay & 0.534 & 4.886 & 11.6 & 205.4 & 21.3 & 117.16 & 0.771 & 93.1 \\
\hline SS-225 & 200 & 6 Pa clay & 0.534 & 4.886 & 11.6 & 203.6 & 21.5 & 117.18 & 0.775 & 95.4 \\
\hline SS-229 & 380 & 6 Pa clay & 0.534 & 4.886 & 11.6 & 386.8 & 23.4 & 161.17 & 0.773 & 95.5 \\
\hline SS-230 & 380 & 6 Pa clay & 0.534 & 4.886 & 11.5 & 386.6 & 23.8 & 157.36 & 0.755 & 97.3 \\
\hline SS-231 & 380 & 6 Pa clay & 0.534 & 4.886 & 11.1 & 359.6 & 23.2 & 155.08 & 0.772 & 97.4 \\
\hline SS-232 & 100 & 6 Pa clay & 0.975 & 0.975 & 11.5 & 102.6 & 20.7 & 30.09 & 0.980 & 88.0 \\
\hline SS-233 & 100 & 6 Pa clay & 0.975 & 0.975 & 11.4 & 101.2 & 21.0 & 27.80 & 0.912 & 87.1 \\
\hline
\end{tabular}


Table A.2. (contd)

\begin{tabular}{|c|c|c|c|c|c|c|c|c|c|c|}
\hline Test ID & $\begin{array}{l}\text { Target } \\
\mathrm{P} \text { (psig) }\end{array}$ & $\begin{array}{c}\text { Simulant }(@ \\
\text { Target Properties) }\end{array}$ & $\begin{array}{c}\text { Outer } \\
\text { Orifice } \\
\text { Diameter / } \\
\text { Width } \\
(\mathrm{mm})\end{array}$ & $\begin{array}{l}\text { Outer Orifice } \\
\text { Diameter / } \\
\text { Length } \\
(\mathrm{mm})\end{array}$ & $\begin{array}{c}\text { Average } \\
\text { Header Flow } \\
\text { During } \\
\text { Whole Spray } \\
\text { (gpm) }\end{array}$ & $\begin{array}{c}\text { Square of } \\
\text { avg(sqrt(P)) } \\
\text { Over Fit } \\
\text { Period (psig) }\end{array}$ & $\begin{array}{c}\text { Average } \\
\text { Temperature } \\
\text { During Whole } \\
\text { Spray }\left({ }^{\circ} \mathrm{C}\right)\end{array}$ & $\begin{array}{c}\text { Average } \\
\text { Leakage Flow } \\
\text { Rate Over Fit } \\
\text { Period }(\mathrm{g} / \mathrm{s})\end{array}$ & $\begin{array}{c}\text { Orifice } \\
\text { Coefficient } \\
\text { During Fit } \\
\text { Period }\end{array}$ & $\begin{array}{c}\text { Initial } \\
\text { Relative } \\
\text { Humidity } \\
(\%)\end{array}$ \\
\hline SS-234 & 100 & 6 Pa clay & 0.975 & 0.975 & 11.5 & 100.9 & 22.0 & 25.56 & 0.839 & 89.1 \\
\hline SS-235 & 200 & 6 Pa clay & 0.975 & 0.975 & 10.3 & 197.6 & 22.0 & 36.52 & 0.857 & 86.7 \\
\hline SS-236 & 200 & 6 Pa clay & 0.975 & 0.975 & 10.4 & 198.2 & 22.1 & 42.20 & 0.988 & 88.9 \\
\hline SS-237 & 200 & 6 Pa clay & 0.975 & 0.975 & 10.4 & 200.1 & 22.1 & 38.84 & 0.905 & 86.4 \\
\hline SS-241 & 380 & 6 Pa clay & 0.975 & 0.975 & 11.5 & 383.9 & 26.7 & 53.13 & 0.894 & 94.4 \\
\hline SS-242 & 380 & 6 Pa clay & 0.975 & 0.975 & 11.4 & 377.9 & 25.1 & 53.51 & 0.908 & 91.3 \\
\hline SS-243 & 380 & 6 Pa clay & 0.975 & 0.975 & 11.4 & 386.5 & 24.5 & 53.23 & 0.893 & 91.2 \\
\hline SS-251 & 100 & $30 \mathrm{~Pa}$ FER & 0.975 & 0.975 & 9.5 & 104.3 & 21.3 & 32.02 & 0.938 & 74.8 \\
\hline SS-253 & 100 & $30 \mathrm{~Pa}$ FER & 0.975 & 0.975 & 7.8 & 109.0 & 21.8 & 34.27 & 0.981 & 87.4 \\
\hline SS-255 & 200 & $30 \mathrm{~Pa}$ FER & 0.975 & 0.975 & 11.9 & 224.9 & 27.9 & 37.47 & 0.747 & 69.5 \\
\hline SS-256 & 200 & $30 \mathrm{~Pa}$ FER & 0.975 & 0.975 & 12.6 & 221.2 & 29.7 & 39.91 & 0.803 & 82.5 \\
\hline SS-261 & 100 & $30 \mathrm{~Pa}$ FER & 0.975 & 0.975 & 12.5 & 89.1 & 24.9 & 28.67 & 0.908 & 76.6 \\
\hline SS-266 & 380 & $30 \mathrm{~Pa}$ FER & 0.975 & 0.975 & 2.1 & 297.1 & 26.7 & 55.79 & 0.968 & 67.0 \\
\hline SS-271 & 200 & $30 \mathrm{~Pa}$ FER & 0.975 & 0.975 & 7.1 & 197.9 & 29.7 & 32.55 & 0.692 & 76.9 \\
\hline SS-274 & 100 & $30 \mathrm{~Pa}$ FER & 0.534 & 4.886 & 10.0 & 106.9 & 27.1 & 96.85 & 0.802 & 88.3 \\
\hline SS-275 & 100 & $30 \mathrm{~Pa}$ FER & 0.534 & 4.886 & 7.6 & 98.9 & 27.9 & 88.40 & 0.761 & 84.7 \\
\hline SS-277 & 200 & $30 \mathrm{~Pa}$ FER & 0.534 & 4.886 & 5.3 & 172.8 & 28.6 & 110.80 & 0.721 & 85.9 \\
\hline SS-280 & 200 & $30 \mathrm{~Pa}$ FER & 0.534 & 4.886 & 5.7 & 195.6 & 27.3 & 137.75 & 0.843 & 84.2 \\
\hline SS-281 & 380 & $30 \mathrm{~Pa}$ FER & 0.534 & 4.886 & 4.1 & 400.9 & 28.6 & 188.63 & 0.806 & 85.0 \\
\hline SS-282 & 380 & $30 \mathrm{~Pa}$ FER & 0.534 & 4.886 & 4.2 & 418.9 & 22.9 & 180.99 & 0.757 & 80.0 \\
\hline SS-283 & 380 & $30 \mathrm{~Pa}$ FER & 0.534 & 4.886 & 5.0 & 399.9 & 23.4 & 176.04 & 0.753 & 81.6 \\
\hline SS-299 & 100 & 6 Pa FER & 0.975 & 0.975 & 9.6 & 110.7 & 24.9 & 29.93 & 0.879 & 75.1 \\
\hline SS-300 & 100 & 6 Pa FER & 0.975 & 0.975 & 9.7 & 112.1 & 25.2 & 30.96 & 0.903 & 76.1 \\
\hline SS-301 & 100 & 6 Pa FER & 0.975 & 0.975 & 9.5 & 109.5 & 25.9 & 31.10 & 0.918 & 76.1 \\
\hline SS-302 & 200 & 6 Pa FER & 0.975 & 0.975 & 9.1 & 183.5 & 26.0 & 37.97 & 0.866 & 67.6 \\
\hline SS-303 & 200 & 6 Pa FER & 0.975 & 0.975 & 8.7 & 199.4 & 26.9 & 43.66 & 0.955 & 75.2 \\
\hline SS-305 & 200 & 6 Pa FER & 0.975 & 0.975 & 8.2 & 209.0 & 24.5 & 40.26 & 0.860 & 69.0 \\
\hline SS-307 & 380 & 6 Pa FER & 0.975 & 0.975 & 8.9 & 361.4 & 22.0 & 54.98 & 0.893 & 62.4 \\
\hline SS-309 & 380 & 6 Pa FER & 0.975 & 0.975 & 7.6 & 348.5 & 23.5 & 57.03 & 0.944 & 68.2 \\
\hline
\end{tabular}


Table A.2. (contd)

\begin{tabular}{|c|c|c|c|c|c|c|c|c|c|c|}
\hline Test ID & $\begin{array}{l}\text { Target } \\
\mathrm{P} \text { (psig) }\end{array}$ & $\begin{array}{c}\text { Simulant }(@ \\
\text { Target Properties) }\end{array}$ & $\begin{array}{c}\text { Outer } \\
\text { Orifice } \\
\text { Diameter / } \\
\text { Width } \\
(\mathrm{mm})\end{array}$ & $\begin{array}{l}\text { Outer Orifice } \\
\text { Diameter / } \\
\text { Length } \\
(\mathrm{mm})\end{array}$ & $\begin{array}{c}\text { Average } \\
\text { Header Flow } \\
\text { During } \\
\text { Whole Spray } \\
\text { (gpm) }\end{array}$ & $\begin{array}{c}\text { Square of } \\
\text { avg(sqrt(P)) } \\
\text { Over Fit } \\
\text { Period (psig) }\end{array}$ & $\begin{array}{c}\text { Average } \\
\text { Temperature } \\
\text { During Whole } \\
\text { Spray }\left({ }^{\circ} \mathrm{C}\right)\end{array}$ & $\begin{array}{c}\text { Average } \\
\text { Leakage Flow } \\
\text { Rate Over Fit } \\
\text { Period }(\mathrm{g} / \mathrm{s})\end{array}$ & $\begin{array}{c}\text { Orifice } \\
\text { Coefficient } \\
\text { During Fit } \\
\text { Period }\end{array}$ & $\begin{array}{c}\text { Initial } \\
\text { Relative } \\
\text { Humidity } \\
(\%)\end{array}$ \\
\hline SS-312 & 100 & $6 \mathrm{~Pa}$ FER & 0.534 & 4.886 & 8.2 & 103.6 & 25.0 & 93.46 & 0.811 & 78.1 \\
\hline SS-313 & 100 & 6 Pa FER & 0.534 & 4.886 & 8.6 & 104.1 & 24.5 & 87.66 & 0.759 & 73.7 \\
\hline SS-315 & 200 & 6 Pa FER & 0.534 & 4.886 & 7.1 & 210.7 & 24.2 & 124.97 & 0.761 & 73.4 \\
\hline SS-317 & 380 & 6 Pa FER & 0.534 & 4.886 & 7.5 & 394.6 & 21.3 & 176.49 & 0.785 & 70.4 \\
\hline SS-320 & 380 & 6 Pa FER & 0.534 & 4.886 & 9.2 & 385.8 & 23.2 & 169.71 & 0.764 & 82.9 \\
\hline SS-329 & 380 & STR-27 & 0.975 & 0.975 & 12.1 & 413.4 & 29.3 & 52.95 & 0.854 & 84.5 \\
\hline SS-331 & 380 & STR-27 & 0.975 & 0.975 & 11.5 & 387.4 & 24.6 & 58.49 & 0.975 & 88.2 \\
\hline SS-334 & 200 & STR-27 & 0.975 & 0.975 & 11.6 & 194.0 & 21.9 & 39.23 & 0.924 & 84.4 \\
\hline SS-335 & 200 & STR-27 & 0.975 & 0.975 & 11.6 & 201.7 & 23.2 & 40.81 & 0.942 & 90.4 \\
\hline SS-336 & 200 & STR-27 & 0.975 & 0.975 & 11.6 & 200.0 & 24.2 & 38.11 & 0.884 & 92.4 \\
\hline SS-337 & 100 & STR-27 & 0.975 & 0.975 & 11.5 & 99.7 & 24.4 & 27.29 & 0.896 & 85.8 \\
\hline SS-338 & 100 & STR-27 & 0.975 & 0.975 & 11.5 & 99.7 & 23.5 & 28.67 & 0.942 & 89.4 \\
\hline SS-339 & 100 & STR-27 & 0.975 & 0.975 & 11.4 & 98.6 & 22.9 & 26.77 & 0.884 & 88.6 \\
\hline SS-340 & 100 & STR-27 & 0.534 & 4.886 & 11.4 & 99.3 & 19.5 & 79.63 & 0.750 & 87.0 \\
\hline SS-341 & 100 & STR-27 & 0.534 & 4.886 & 11.2 & 95.1 & 20.6 & 74.76 & 0.720 & 88.4 \\
\hline SS-342 & 100 & STR-27 & 0.534 & 4.886 & 11.2 & 97.1 & 21.7 & 71.86 & 0.685 & 88.8 \\
\hline SS-344 & 200 & STR-27 & 0.534 & 4.886 & 11.7 & 208.9 & 24.7 & 111.27 & 0.723 & 86.2 \\
\hline SS-345 & 200 & STR-27 & 0.534 & 4.886 & 12.0 & 218.3 & 25.2 & 119.44 & 0.759 & 89.2 \\
\hline SS-346 & 200 & STR-27 & 0.534 & 4.886 & 11.3 & 193.6 & 24.7 & 109.74 & 0.740 & 96.2 \\
\hline SS-347 & 380 & STR-27 & 0.534 & 4.886 & 11.6 & 397.8 & 25.3 & 160.06 & 0.753 & 85.9 \\
\hline SS-348 & 380 & STR-27 & 0.534 & 4.886 & 11.8 & 419.2 & 24.5 & 167.41 & 0.767 & 95.6 \\
\hline SS-349 & 380 & STR-27 & 0.534 & 4.886 & 11.6 & 403.5 & 25.1 & 164.76 & 0.770 & 99.0 \\
\hline SS-355 & 380 & Water $/ 1 \mathrm{wt} \% \mathrm{Mo}$ & 0.975 & 0.975 & 12.1 & 399.1 & 29.0 & 47.74 & 0.861 & $\mathrm{n} / \mathrm{m}$ \\
\hline SS-360 & 380 & Water $/ 1 \mathrm{wt} \% \mathrm{Mo}$ & 0.534 & 4.886 & 12.0 & 384.5 & 27.8 & 136.02 & 0.715 & $\mathrm{n} / \mathrm{m}$ \\
\hline SS-362 & 380 & Water $/ 1 \mathrm{wt} \%$ Mo & 0.534 & 4.886 & 12.0 & 334.6 & 27.5 & 129.92 & 0.733 & $\mathrm{n} / \mathrm{m}$ \\
\hline SS-368 & 380 & STR $20 / 1 \mathrm{wt} \% \mathrm{Mo}$ & 0.975 & 0.975 & 12.5 & 389.8 & 29.2 & 58.01 & 0.988 & 71.4 \\
\hline SS-369 & 380 & STR 20/1 wt $\%$ Mo & 0.975 & 0.975 & 12.3 & 402.8 & 30.2 & 53.74 & 0.900 & 74.7 \\
\hline SS-370 & 380 & STR $20 / 1 \mathrm{wt} \%$ Mo & 0.534 & 4.886 & 12.7 & 379.5 & 20.8 & 157.13 & 0.776 & 87.7 \\
\hline SS-371 & 380 & STR 20/1 wt $\%$ Mo & 0.534 & 4.886 & 11.8 & 393.8 & 22.8 & 152.10 & 0.737 & 86.8 \\
\hline
\end{tabular}


Table A.2. (contd)

\begin{tabular}{|c|c|c|c|c|c|c|c|c|c|c|}
\hline Test ID & $\begin{array}{l}\text { Target } \\
\mathrm{P} \text { (psig) }\end{array}$ & $\begin{array}{c}\text { Simulant }(@ \\
\text { Target Properties) }\end{array}$ & $\begin{array}{c}\text { Outer } \\
\text { Orifice } \\
\text { Diameter / } \\
\text { Width } \\
(\mathrm{mm})\end{array}$ & $\begin{array}{l}\text { Outer Orifice } \\
\text { Diameter / } \\
\text { Length } \\
(\mathrm{mm})\end{array}$ & $\begin{array}{c}\text { Average } \\
\text { Header Flow } \\
\text { During } \\
\text { Whole Spray } \\
\text { (gpm) }\end{array}$ & $\begin{array}{c}\text { Square of } \\
\text { avg(sqrt(P)) } \\
\text { Over Fit } \\
\text { Period (psig) }\end{array}$ & $\begin{array}{c}\text { Average } \\
\text { Temperature } \\
\text { During Whole } \\
\text { Spray }\left({ }^{\circ} \mathrm{C}\right)\end{array}$ & $\begin{array}{c}\text { Average } \\
\text { Leakage Flow } \\
\text { Rate Over Fit } \\
\text { Period }(\mathrm{g} / \mathrm{s})\end{array}$ & $\begin{array}{c}\text { Orifice } \\
\text { Coefficient } \\
\text { During Fit } \\
\text { Period }\end{array}$ & $\begin{array}{c}\text { Initial } \\
\text { Relative } \\
\text { Humidity } \\
(\%)\end{array}$ \\
\hline SS-372 & 380 & STR 20/1 wt $\%$ Mo & 0.534 & 4.886 & 11.5 & 365.0 & 24.3 & 147.37 & 0.742 & 93.5 \\
\hline SS-374 & 380 & STR $20 / 1 \mathrm{wt} \% \mathrm{Mo}$ & 0.534 & 4.886 & 11.8 & 369.6 & 26.8 & 147.15 & 0.736 & 87.9 \\
\hline SS-375 & 380 & Water & 0.534 & 0.534 & 11.5 & 372.6 & 24.2 & 12.67 & 0.790 & 54.4 \\
\hline SS-376 & 380 & Water & 0.534 & 0.534 & 11.5 & 374.9 & 25.6 & 13.08 & 0.813 & 66.0 \\
\hline SS-377 & 380 & Water & 0.534 & 0.534 & 11.5 & 381.4 & 26.9 & 12.15 & 0.749 & 75.6 \\
\hline SS-378 & 380 & Water & 0.534 & 0.534 & 11.5 & 377.9 & 28.1 & 11.46 & 0.710 & 85.1 \\
\hline SS-379 & 380 & Water & 0.534 & 0.534 & 11.4 & 375.4 & 29.3 & 13.83 & 0.860 & 98.2 \\
\hline SS-380 & 380 & Water & 0.534 & 0.534 & 11.4 & 377.9 & 30.4 & 10.73 & 0.665 & 91.6 \\
\hline SS-381 & 380 & Water & 0.534 & 0.534 & 11.5 & 374.5 & 24.0 & 11.69 & 0.727 & 55.1 \\
\hline SS-382 & 380 & Water & 0.534 & 0.534 & 11.4 & 376.6 & 25.4 & 13.62 & 0.845 & 64.2 \\
\hline SS-383 & 380 & Water & 0.534 & 0.534 & 11.4 & 376.6 & 26.6 & 12.36 & 0.767 & 96.3 \\
\hline SS-384 & 100 & Water & 0.534 & 0.534 & 11.6 & 100.9 & 27.4 & 8.34 & 1.000 & 100.2 \\
\hline SS-385 & 100 & Water & 0.534 & 0.534 & 11.6 & 100.7 & 27.9 & 7.11 & 0.854 & 75.1 \\
\hline SS-386 & 100 & Water & 0.534 & 0.534 & 11.6 & 100.6 & 28.9 & 7.89 & 0.948 & 49.6 \\
\hline
\end{tabular}


Appendix B

Selected Release Fraction Plots 



\section{Appendix B}

\section{Selected Release Fraction Plots}

This appendix contains plots of cumulative release fraction versus droplet size for tests discussed in Chapters 6 and 7. These tests are a subset of those detailed in Appendix A.

Each figure shows a measured droplet PSD, the average over $18-22 \mathrm{~s}$. In addition, each figure shows the cumulative release fraction obtained in two ways: 1) by a fit to the cumulative concentration under each size (i.e., the cumulative fit) and 2) by cumulating fits to the differential concentrations in all the bins under each size (i.e., the cumulated differential fit).

As noted in Section 6.3, some of the cumulative and differential fits were considered to be bad fits. A fit was considered bad if 1 ) the output of the fit was equal to the lower-limit or upper-limit fitting constraints, 2) the fitting routine did not achieve convergence, 3 ) the adjusted coefficient of determination $\left(\overline{\mathrm{R}}^{2}\right)$ of the fit was less than 0.5 , or 4 ) the cumulative droplet concentration (based on the PSD) at a droplet size was less than 0.005 . In the first and second cases, the fitting routine did not return any numeric values for the upper and lower ends of the 95 percent confidence interval on the fitted value. In the third and fourth cases, and for the good fits, the 95 percent confidence interval was returned.

The cumulative fits for different size bins are independent of each other, so a bad fit at one size does not affect the fits for cumulative release fractions for larger sizes. The differential fits, however, are added (cumulated) to give cumulative release fractions. In addition, the ends of their confidence interval are cumulated. Thus, for this report, a decision had to be made regarding how to cumulate a bad fit for one differential concentration bin with good fits from other bins. In this case, bad differential fits are included in cumulations, but the individual points for bad differential fits are excluded from the plots. In some cases, using bad differential fits in cumulations cast doubt on the cumulated differential-fit net generation rates and release fractions. For this reason, and others, only cumulative fits appear in the analyses included in Chapters 6 and 7.

A bad fit at the smallest size affects the cumulated differential release fractions at larger sizes, and also affects the error bars for the cumulated differential fits. Because no numeric confidence limit was given for the size where the fit was bad, there are no error bars for that size. The non-numeric value of confidence interval was accumulated with the good values for larger sizes, causing all the error bars in the cumulated differential curve to be zeroed out.

A review of the plots in this appendix shows that, except in cases where a bad fit of type 1 or 2 causes inaccuracy in the cumulated differential fit, the two approaches to fitting (cumulative and cumulated differential) give very similar results in the size range from $<10$ to $<100 \mu \mathrm{m}$, except where there is a bad differential fit. The bad fit of type 1 or 2 can be recognized in a plot by the zero-length error bars. 

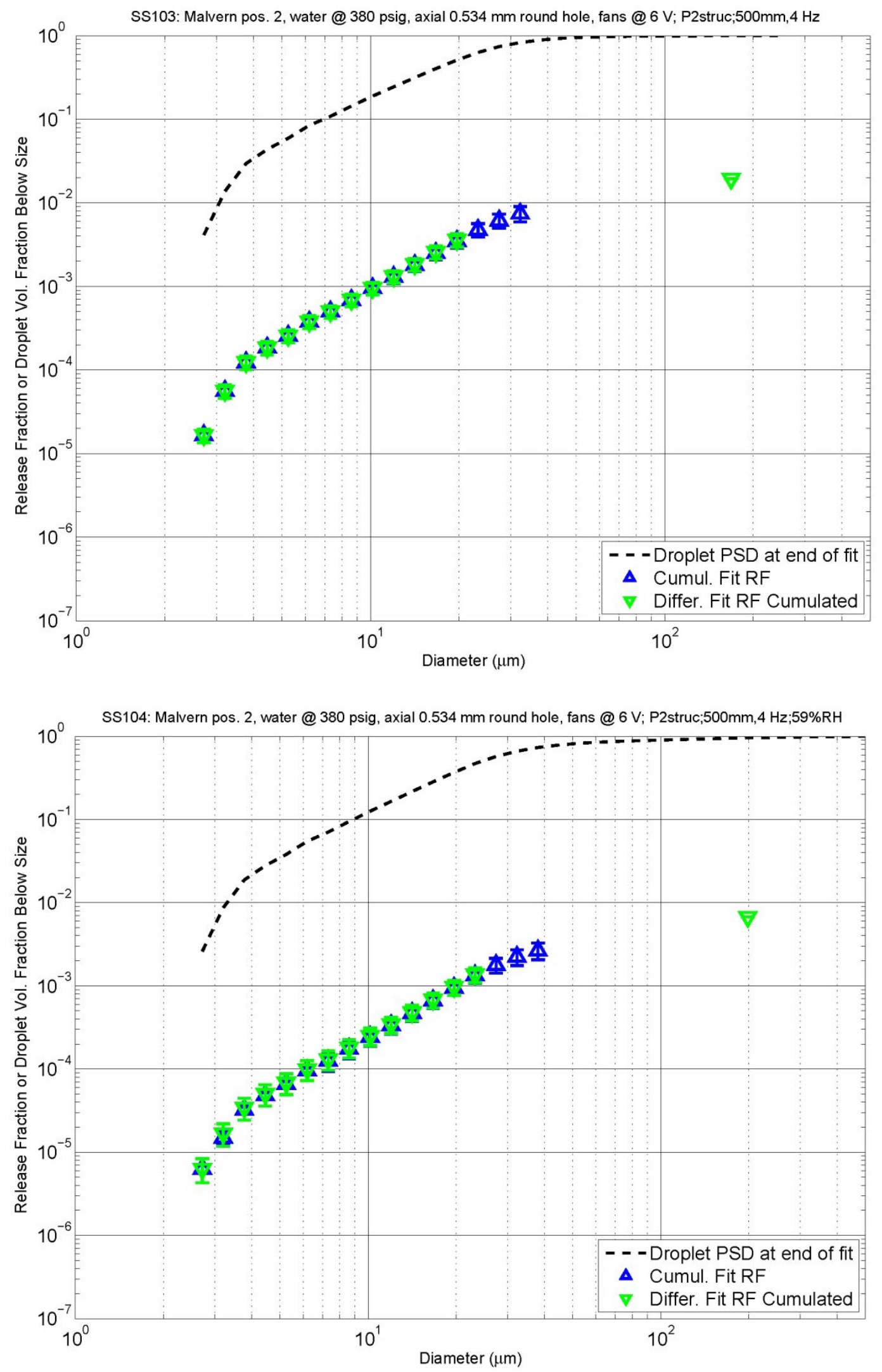

B. 2 

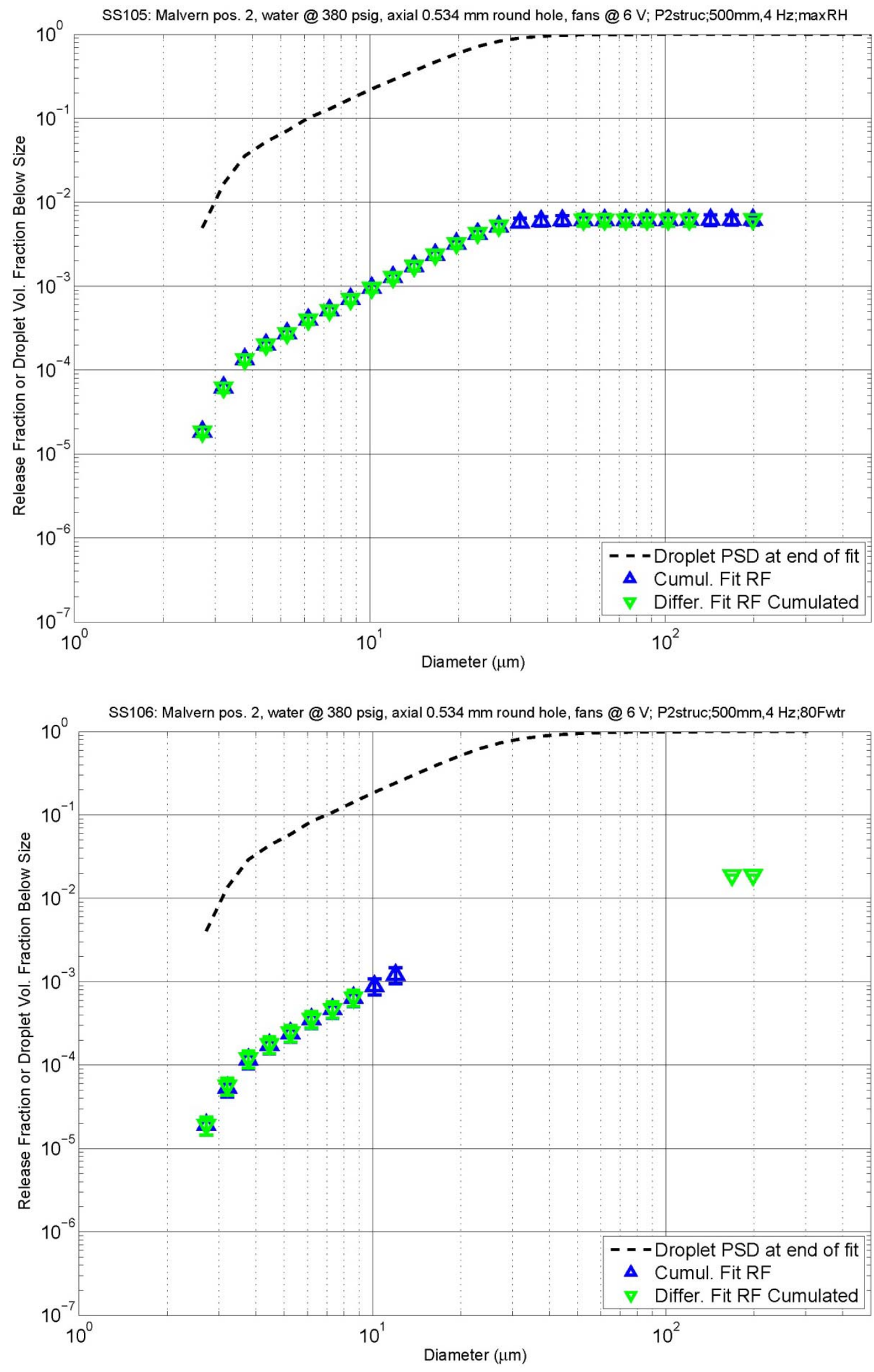

B. 3 

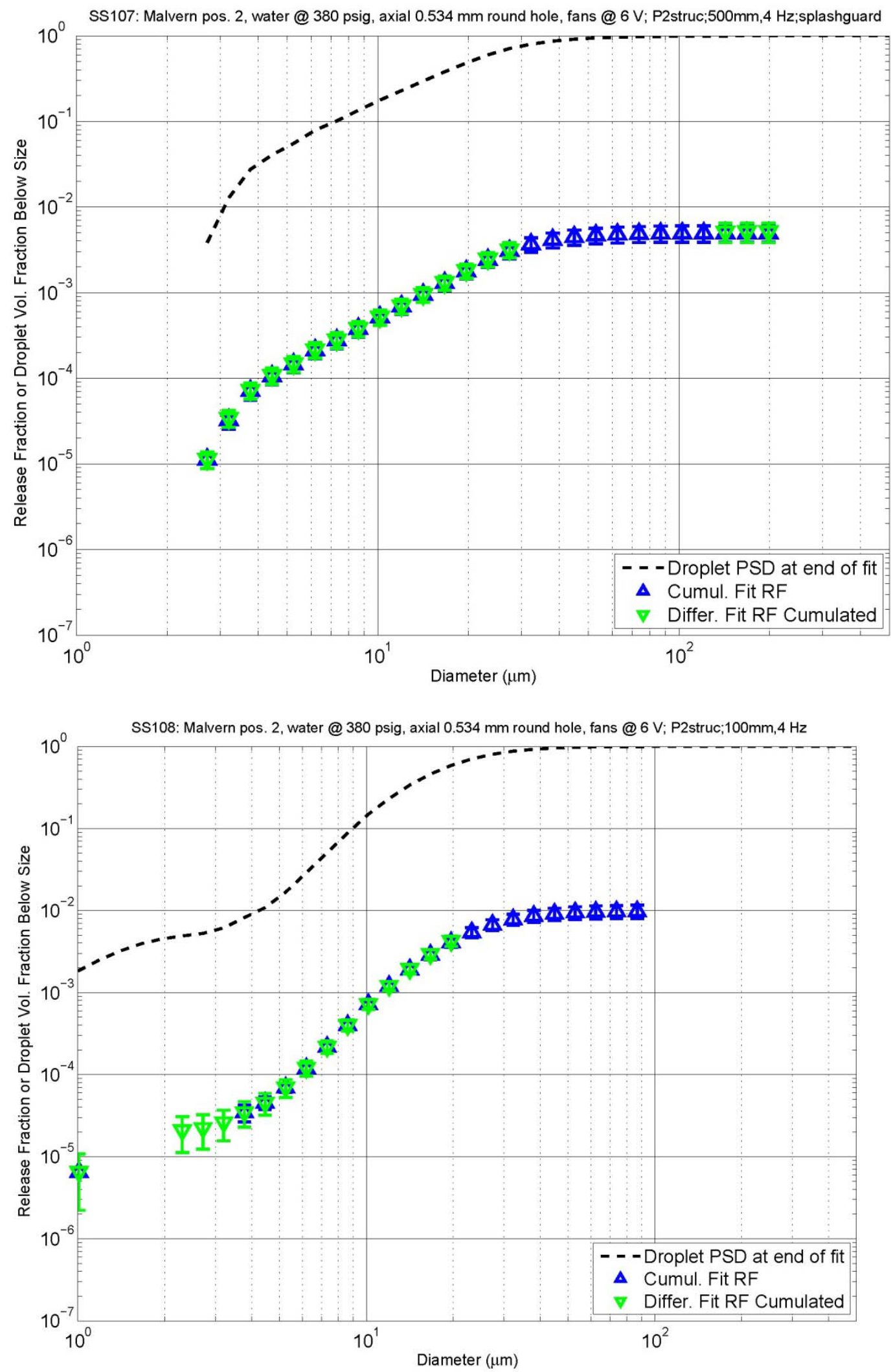

Page 11 of 204 

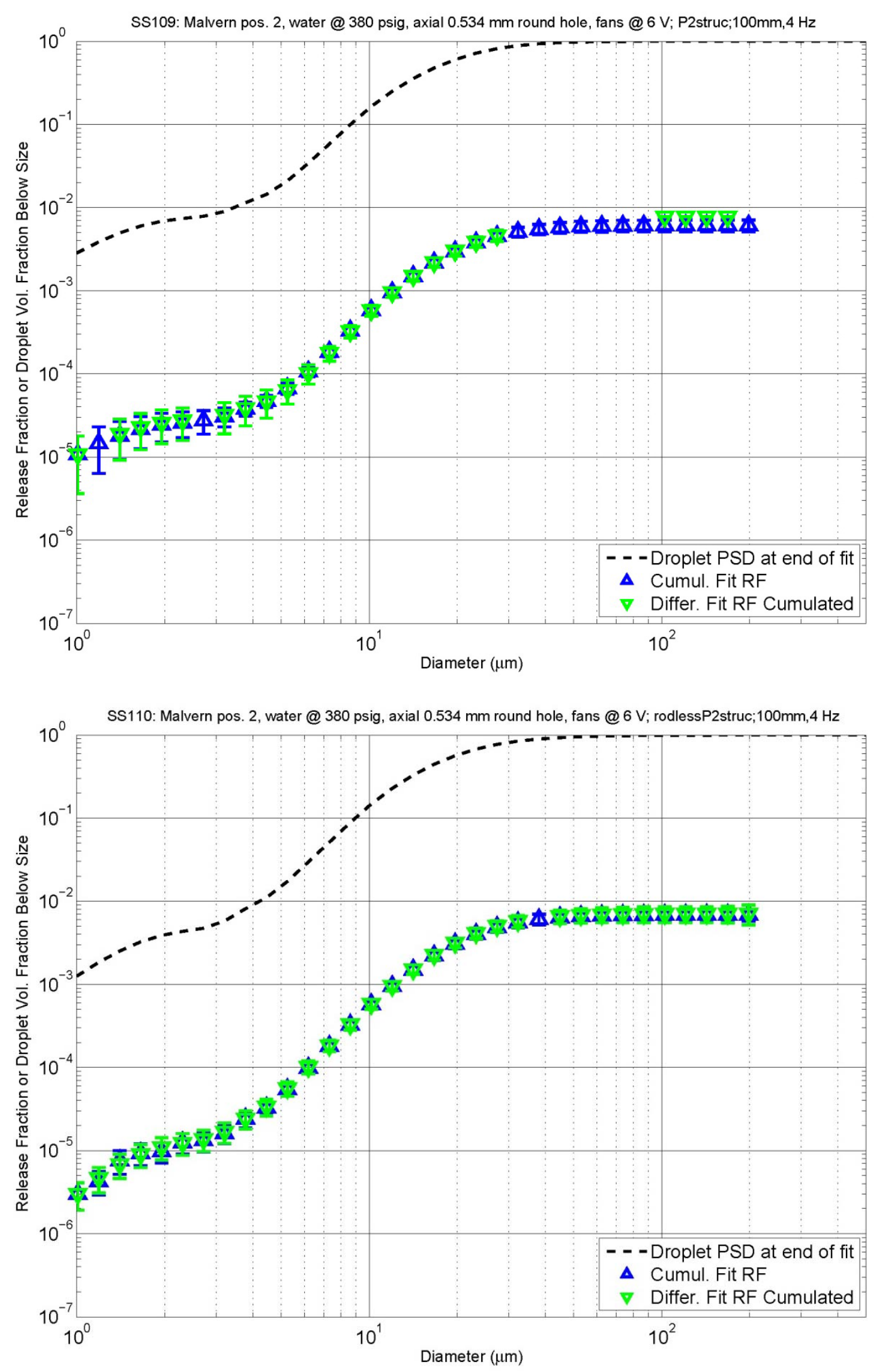

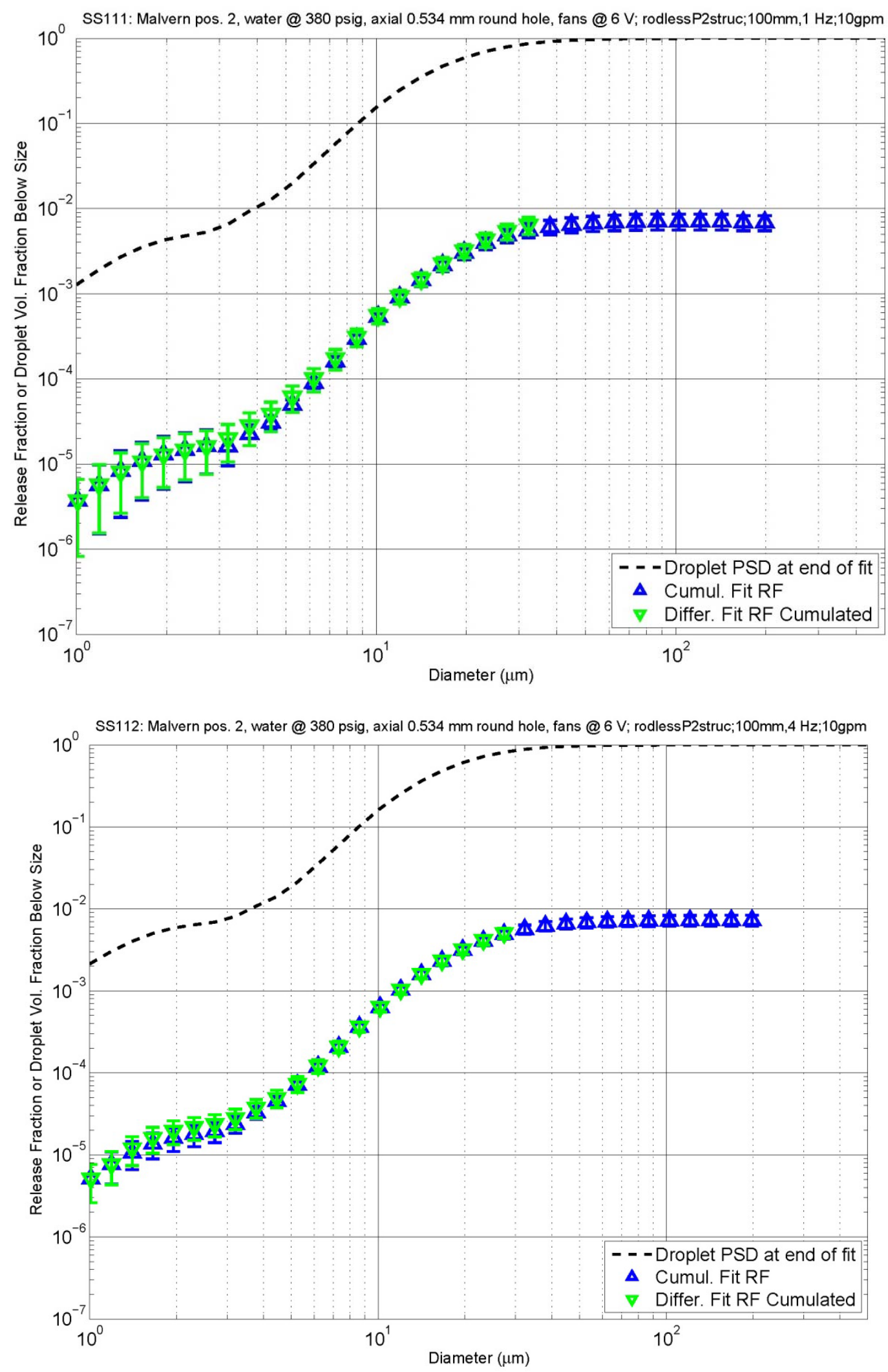

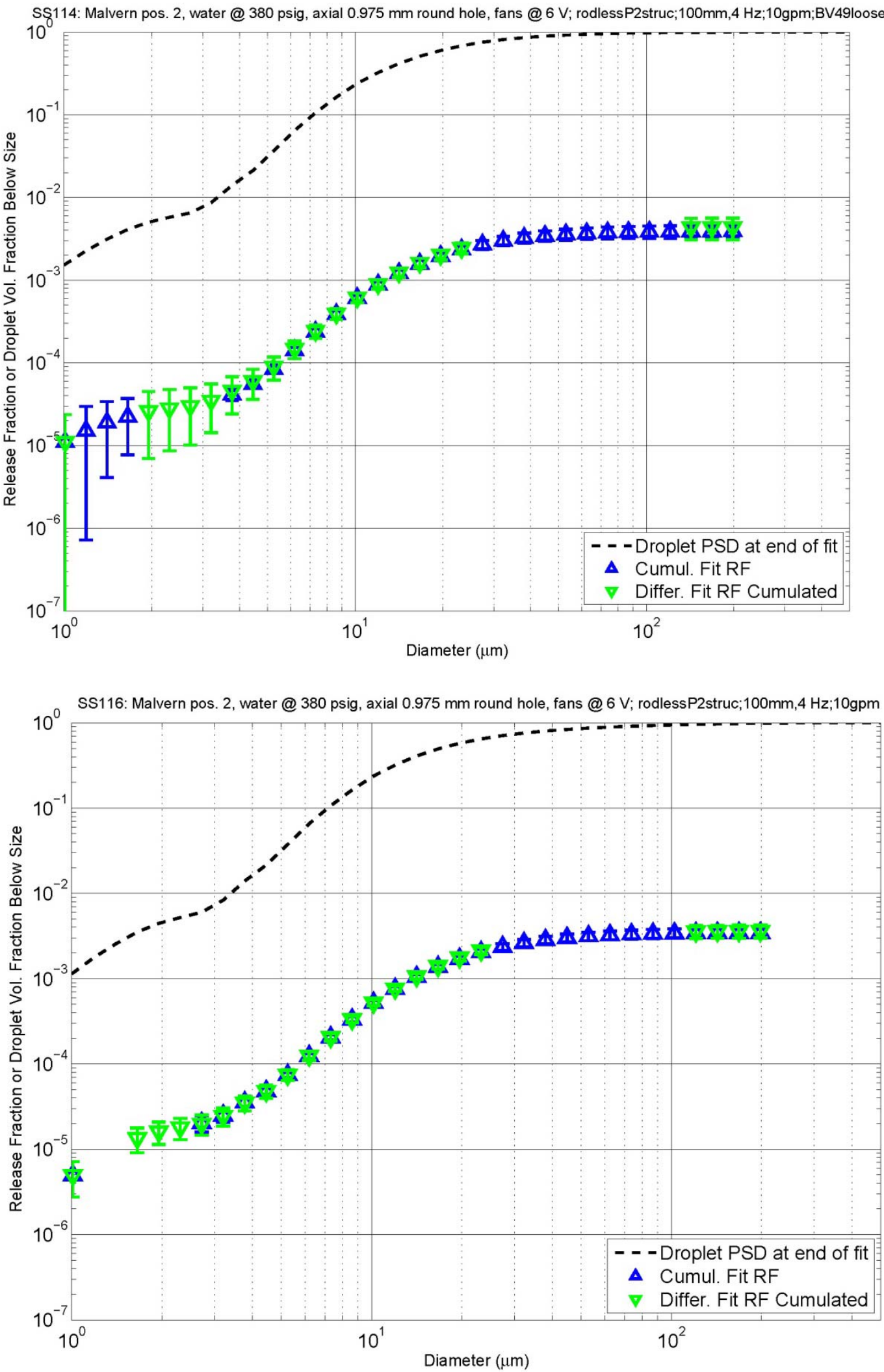

B. 7 

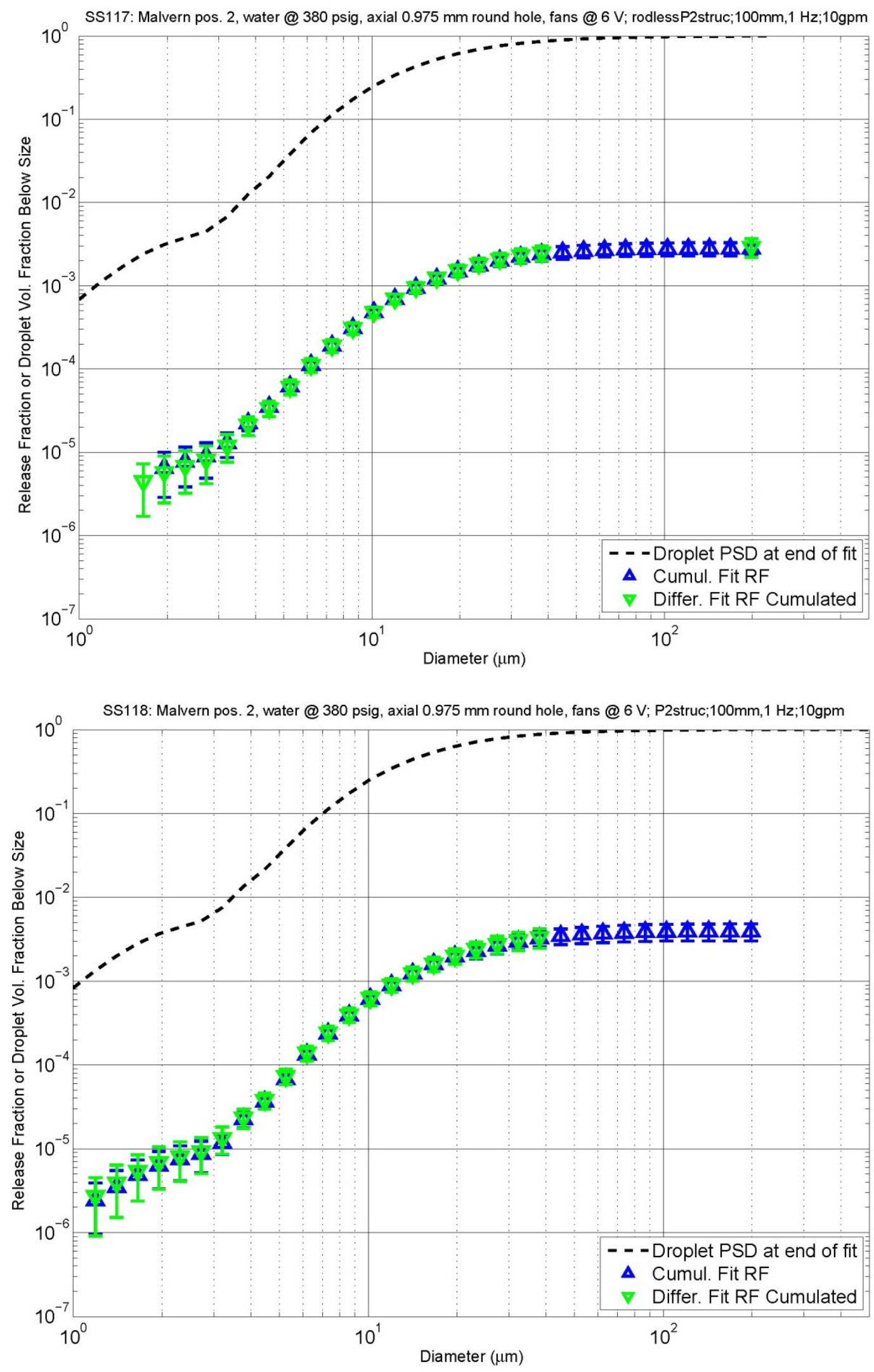

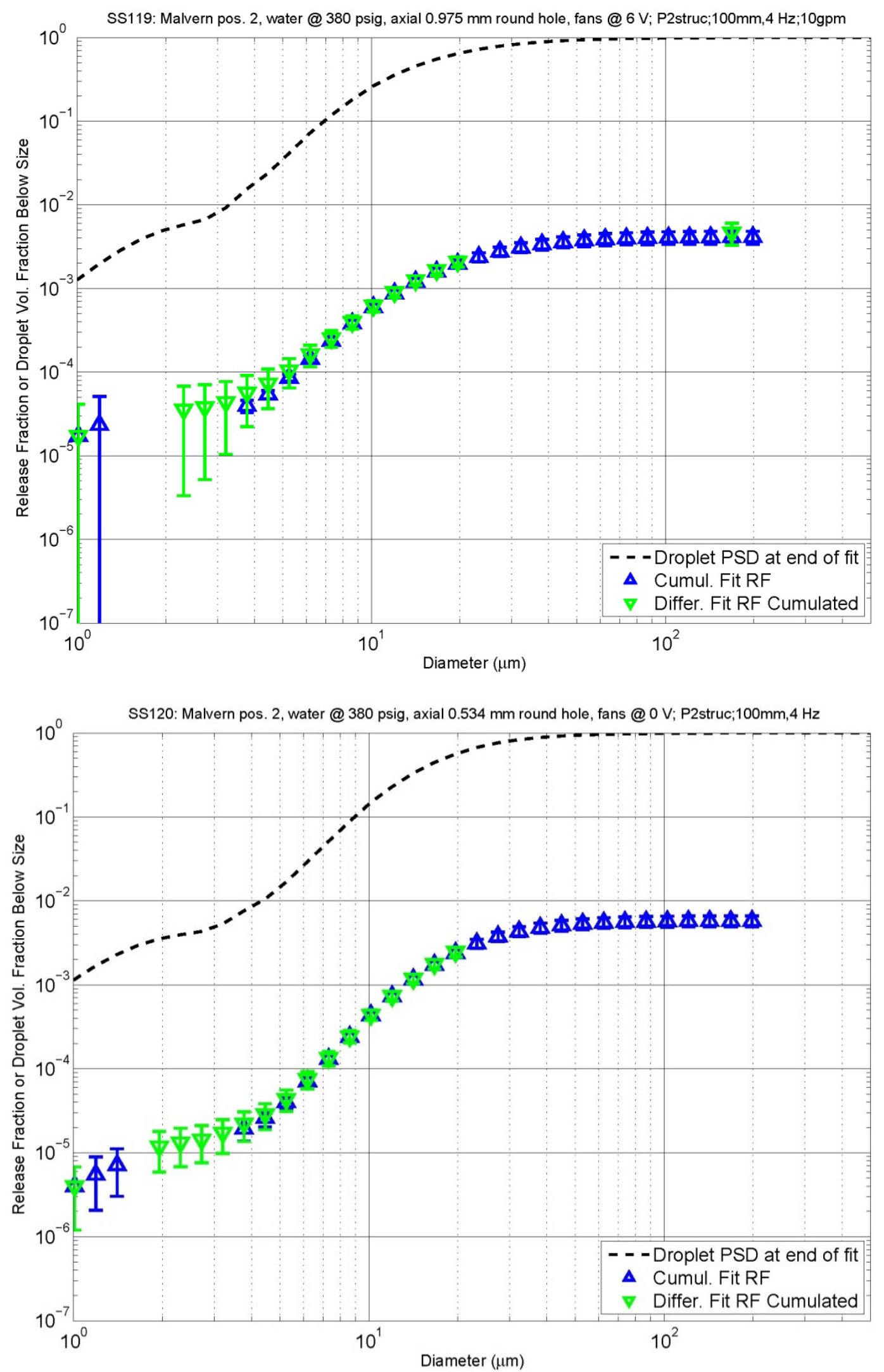

B. 9 

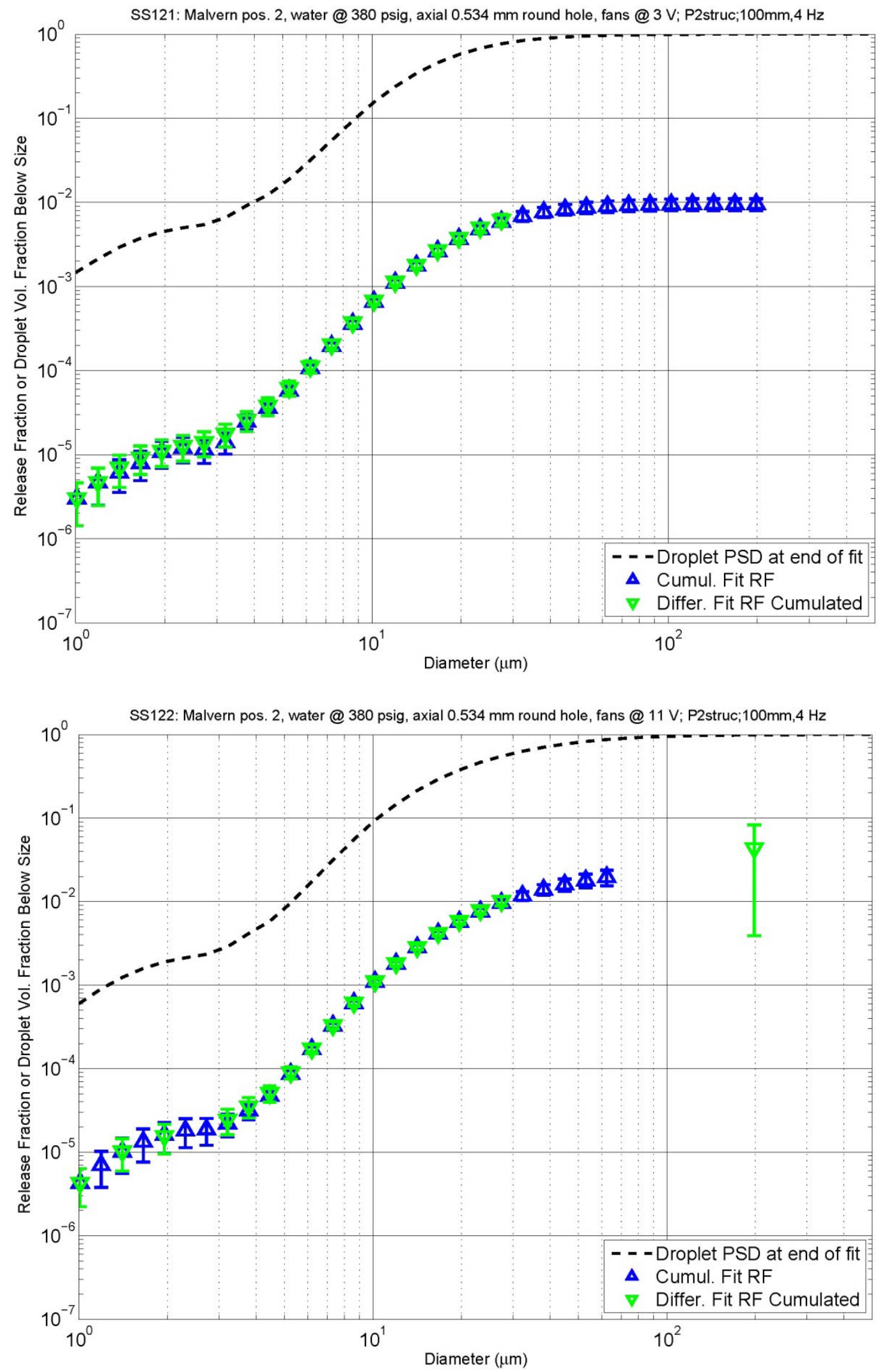

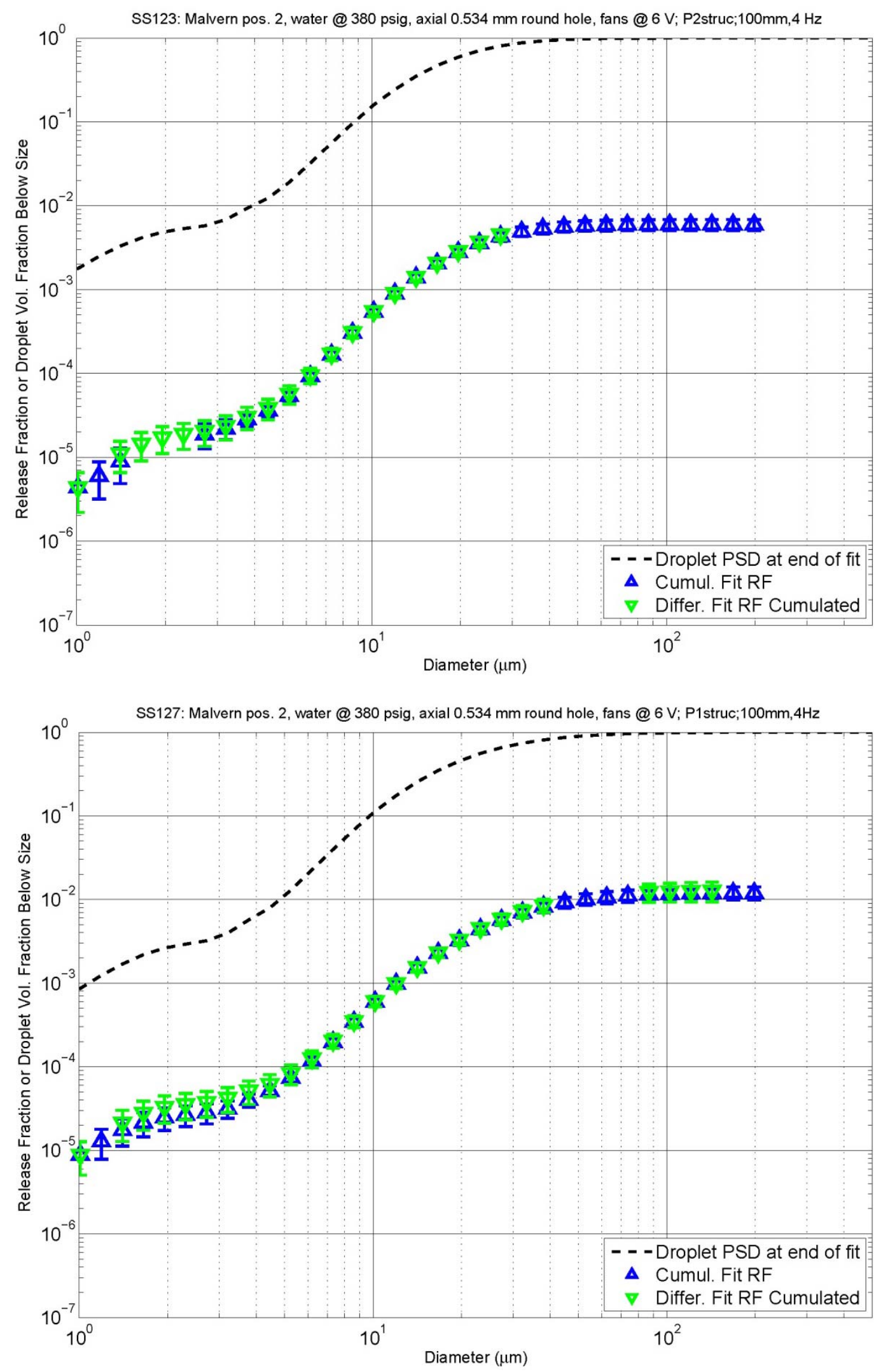

B. 11 

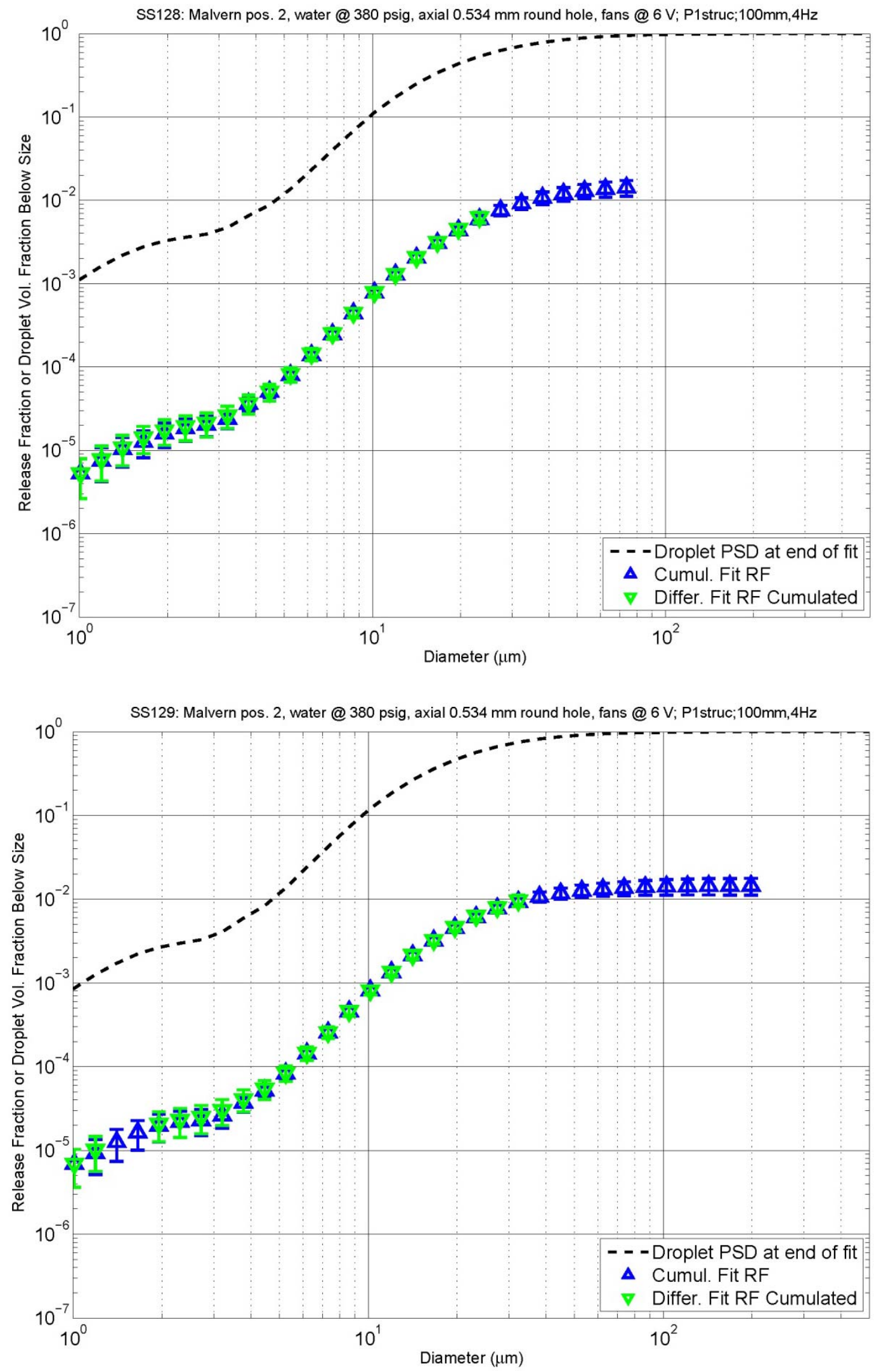

B. 12 

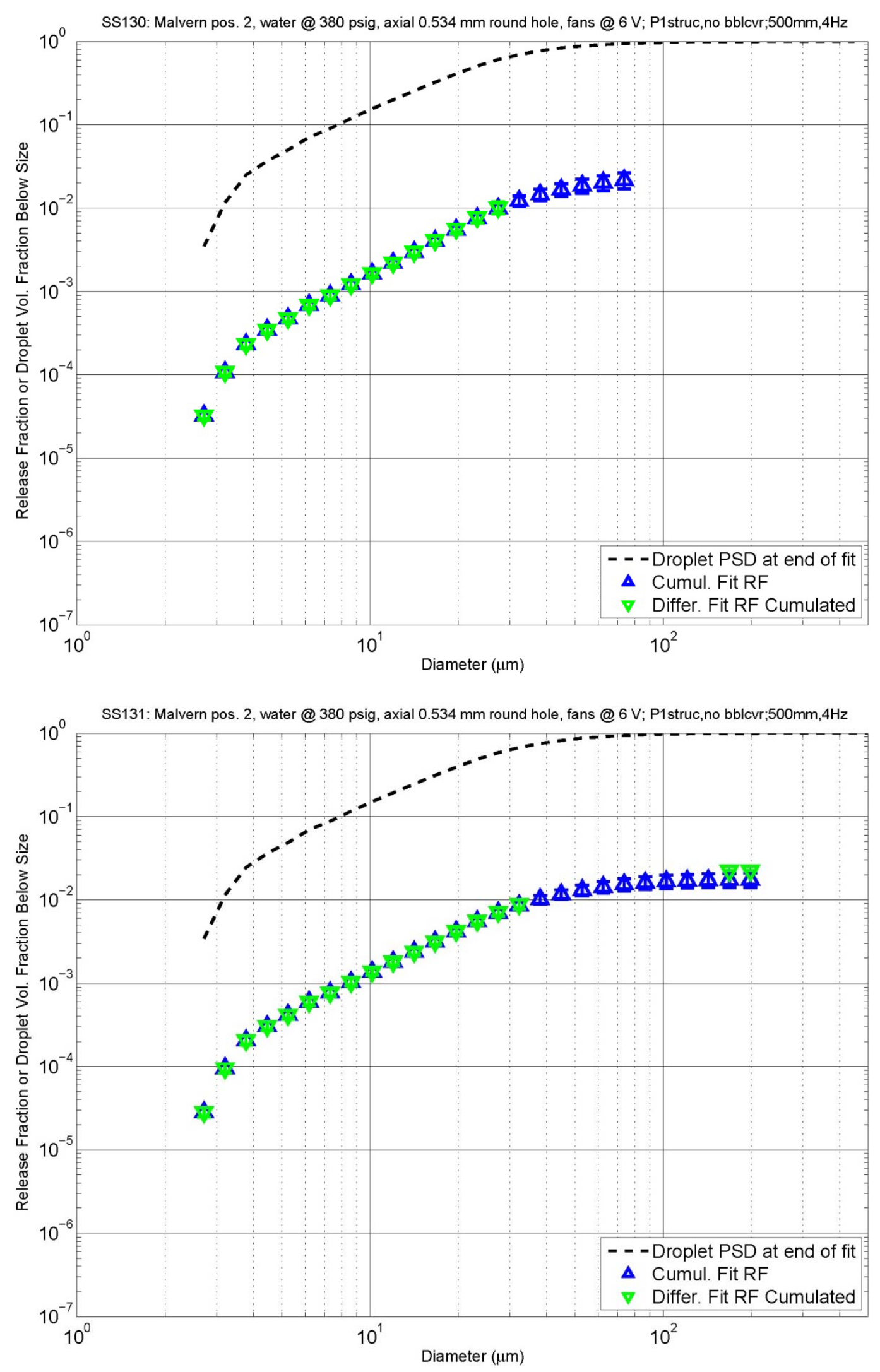

B.13 

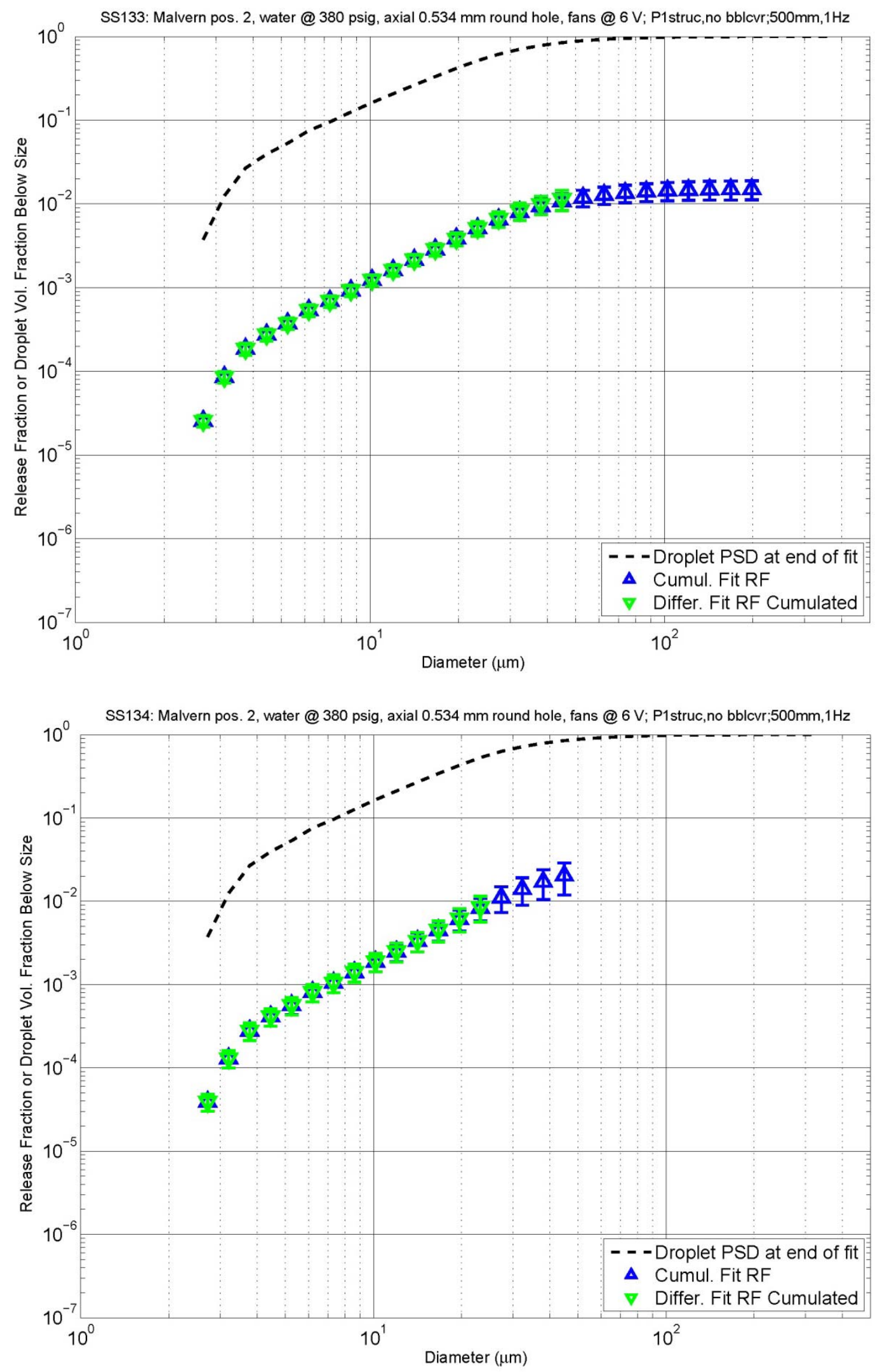

B. 14 
SS135: Malvern pos. 2, water @ 380 psig, axial 0.534 mm round hole, fans @ 6 V; P1struc,no bblcvr;500mm,1 Hz
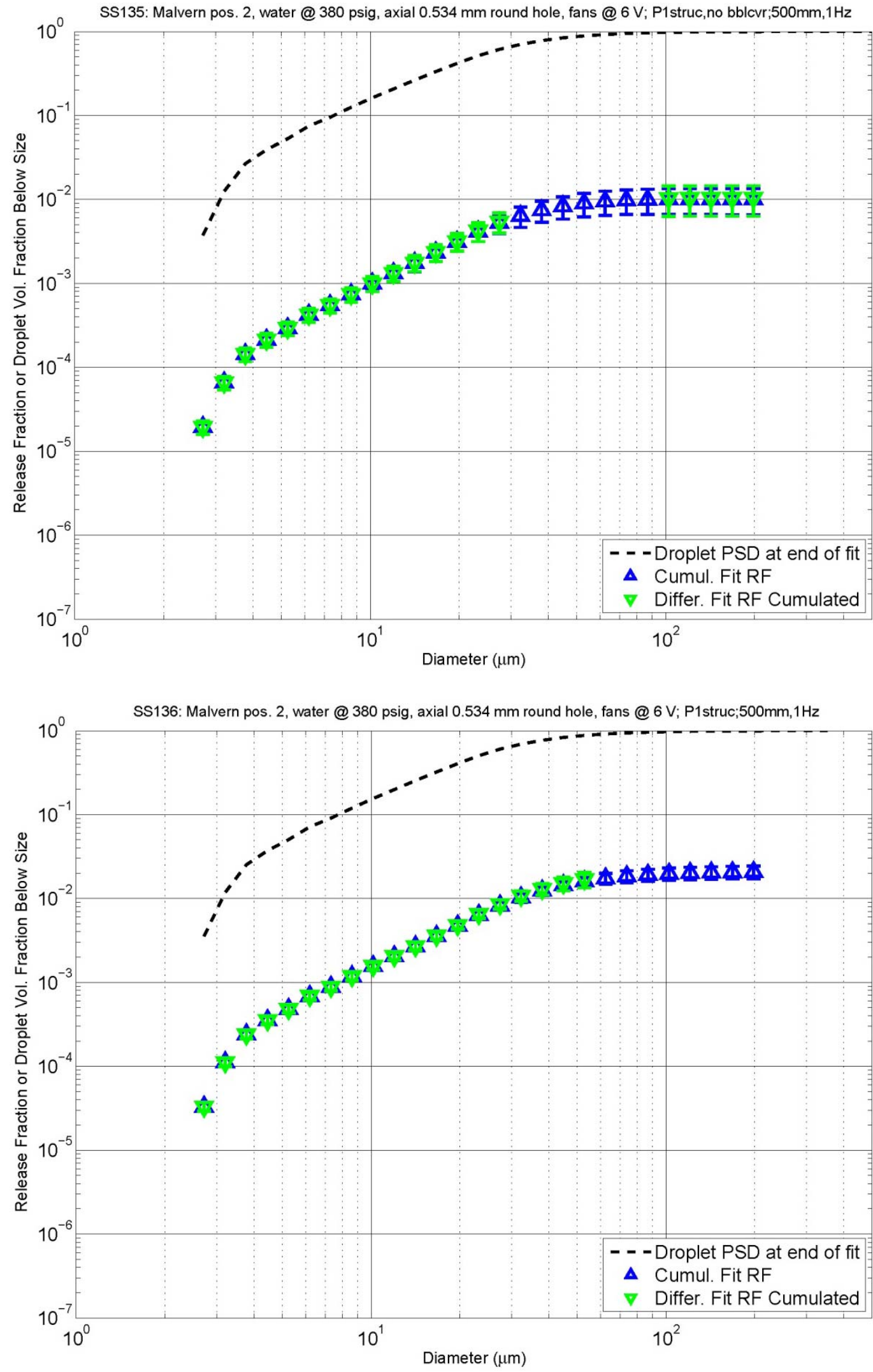

B.15 

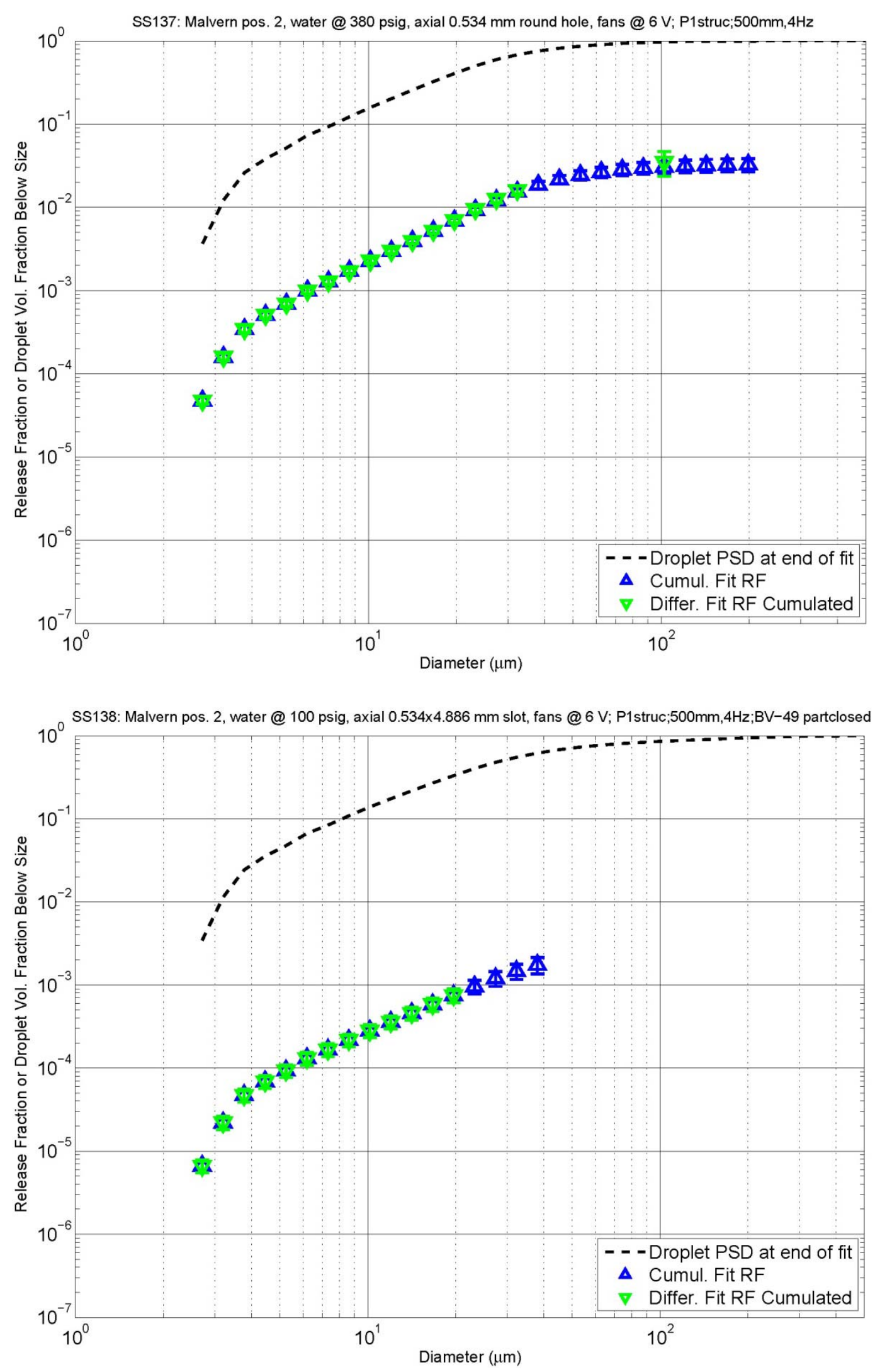

B. 16 

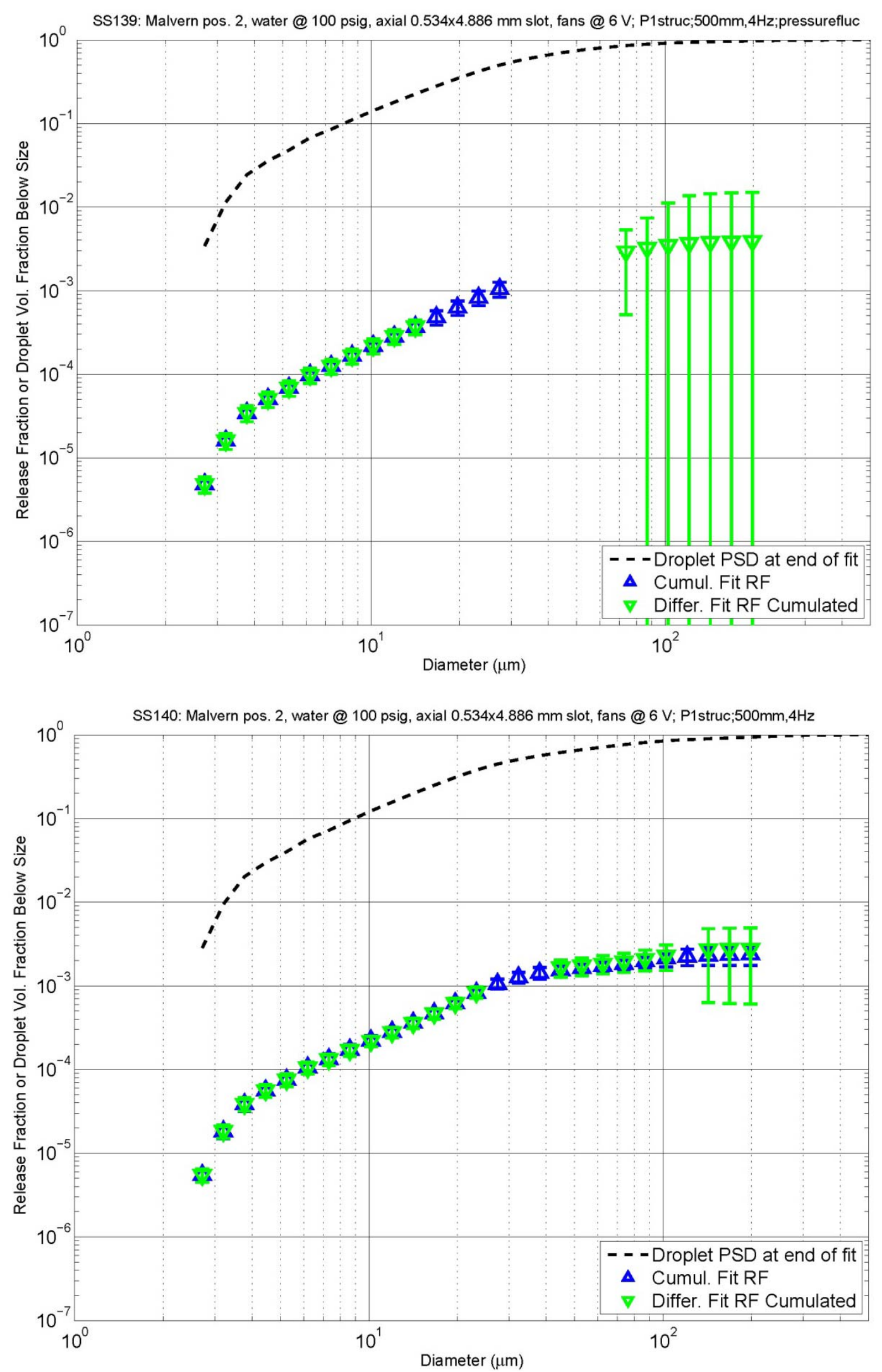

B. 17 

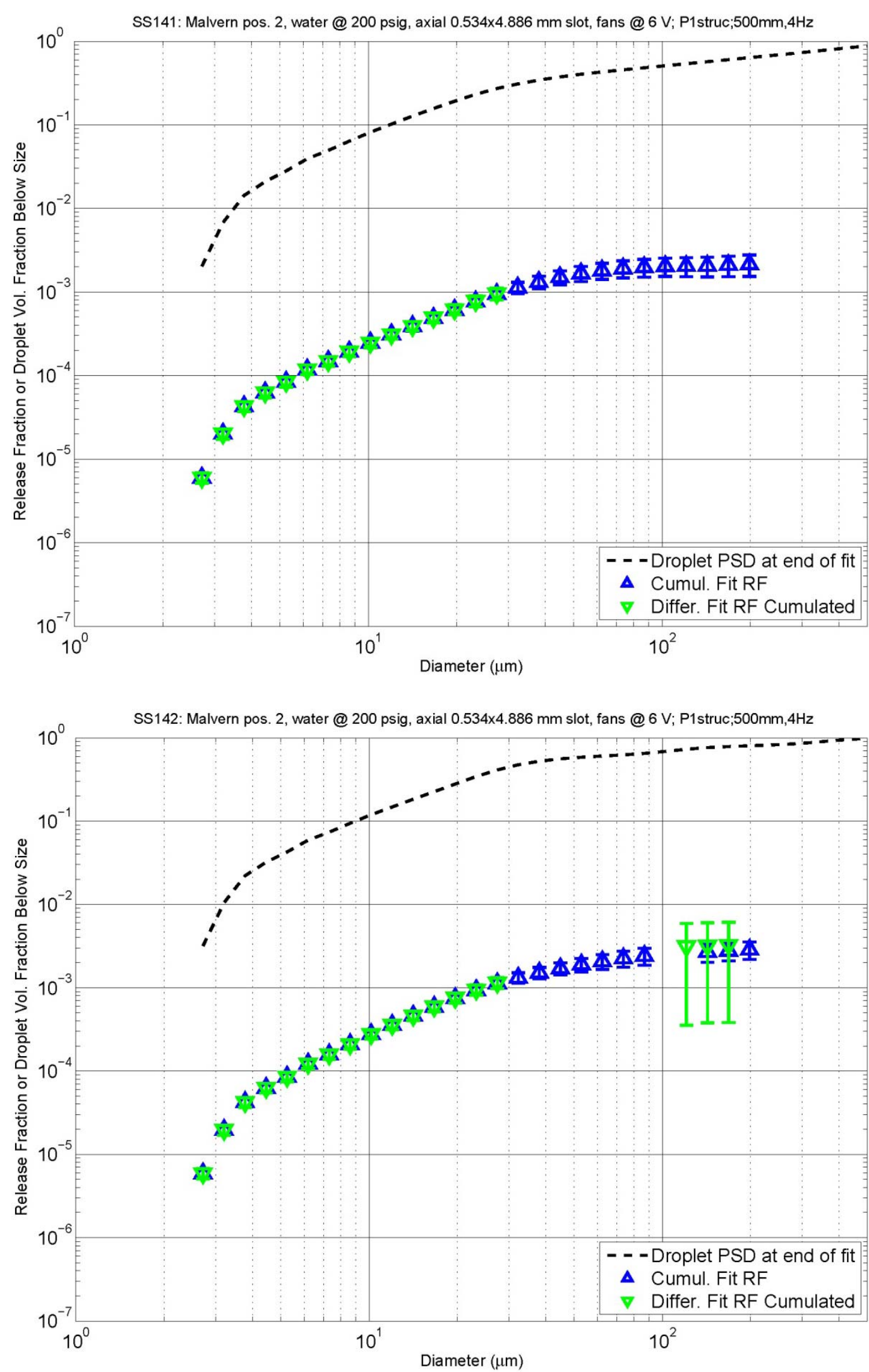

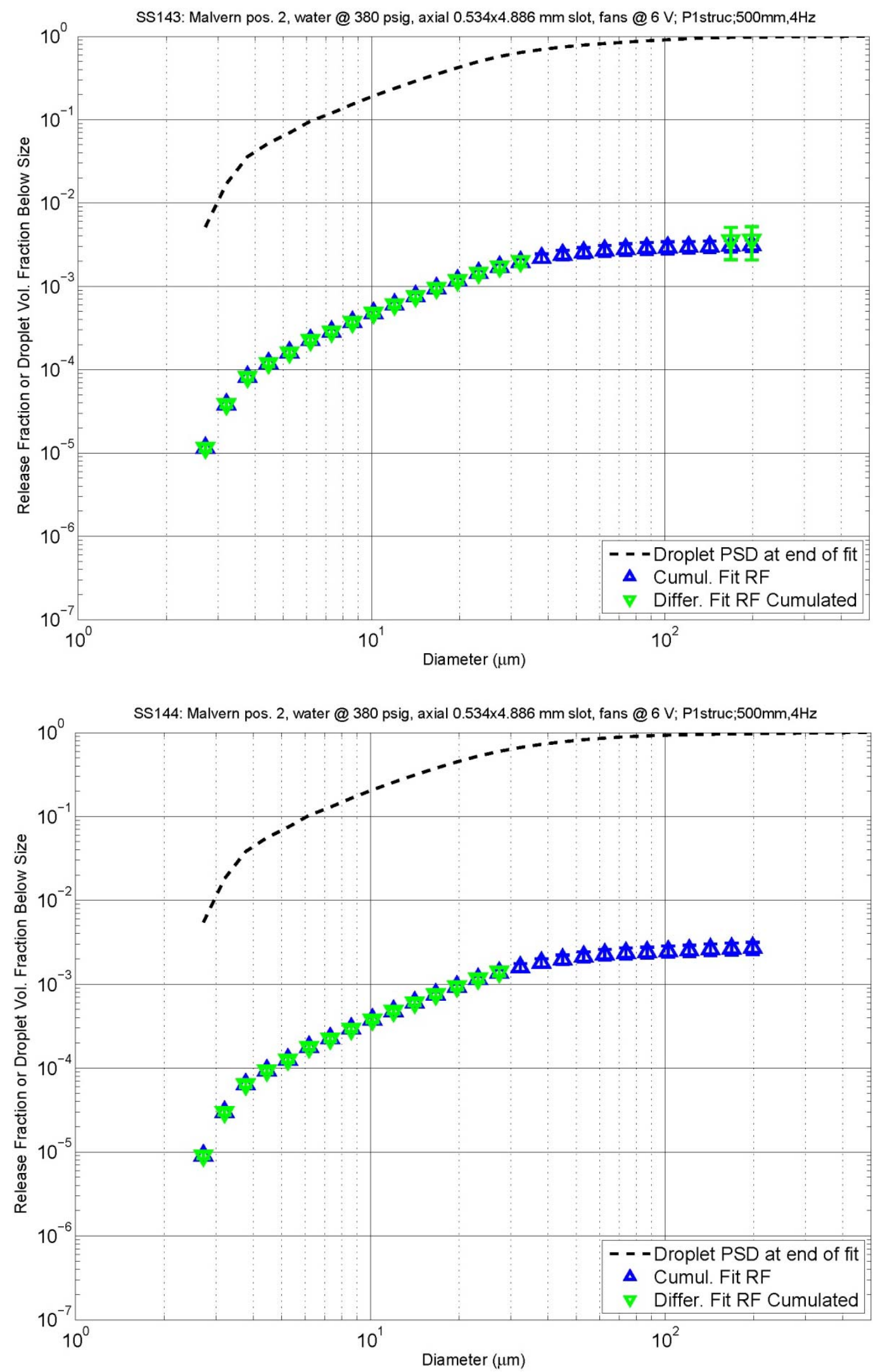

B. 19 

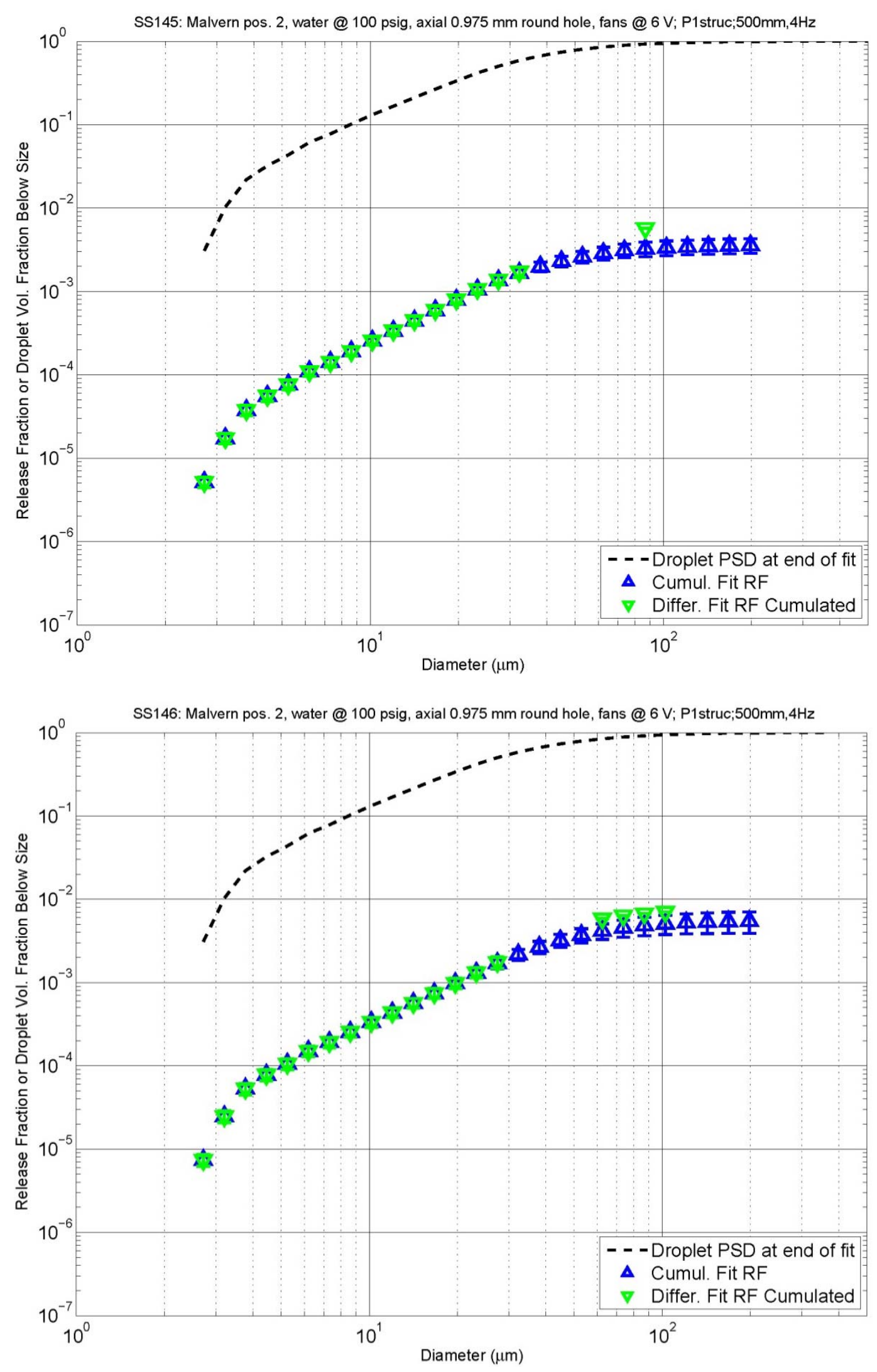

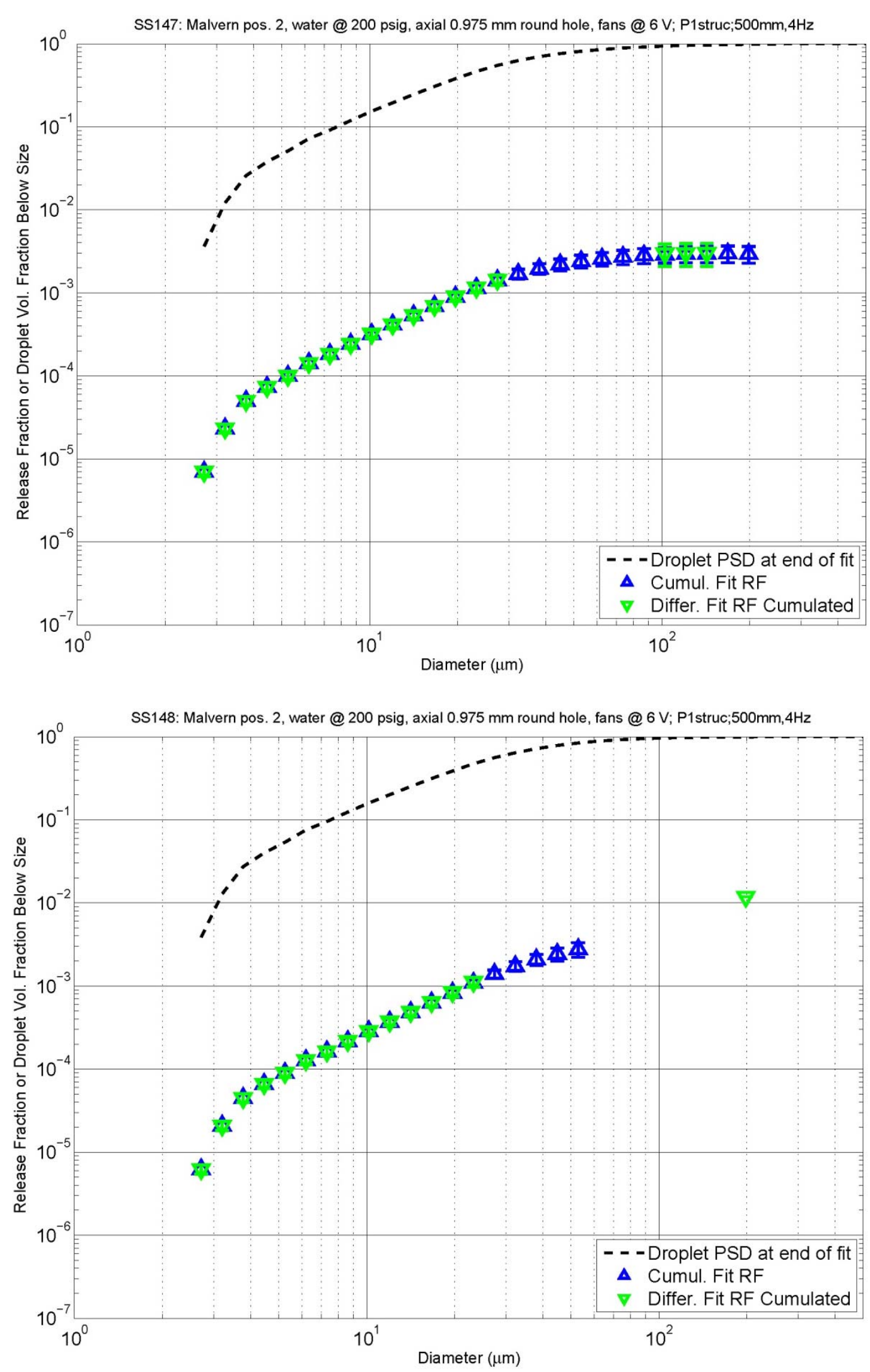

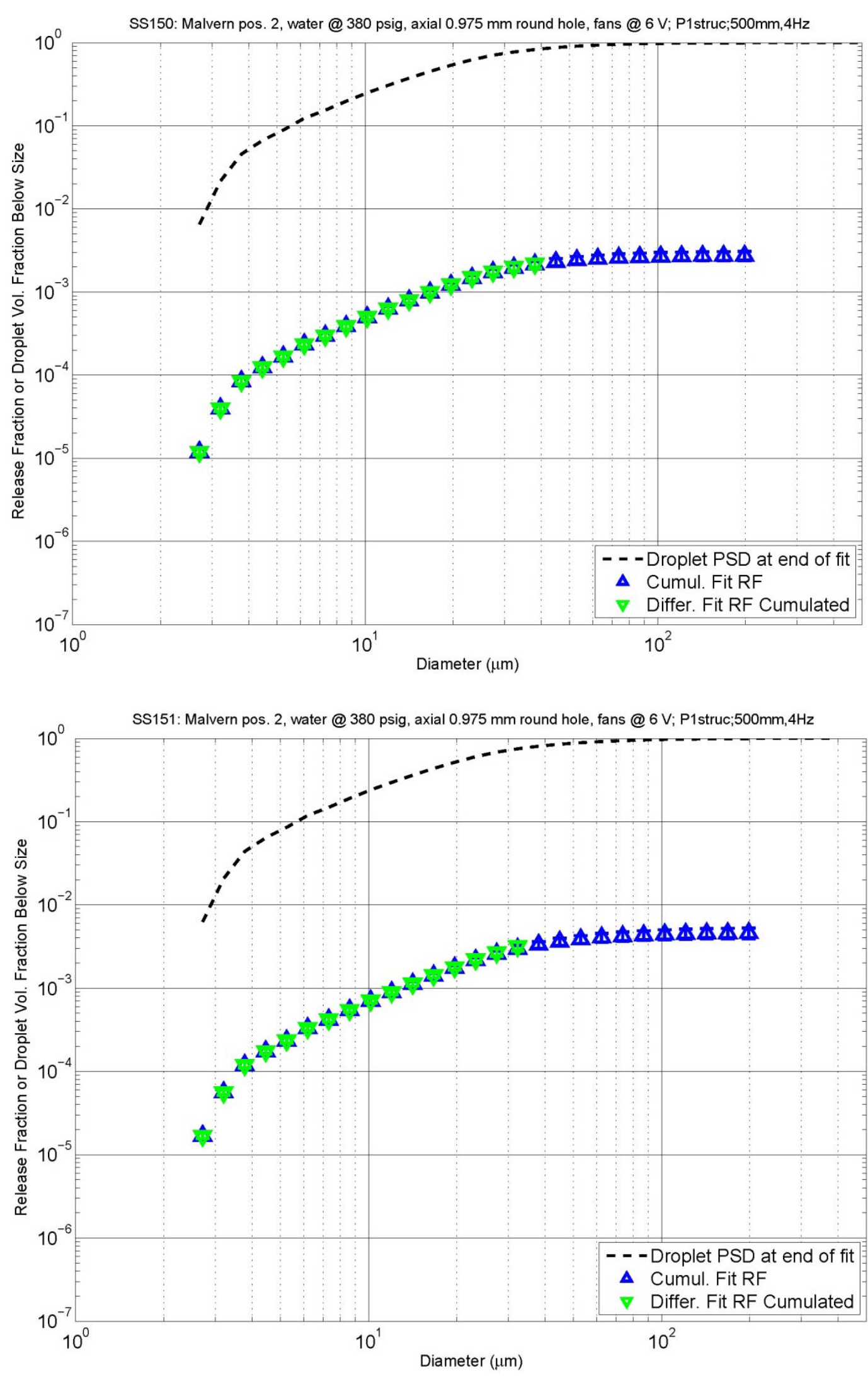

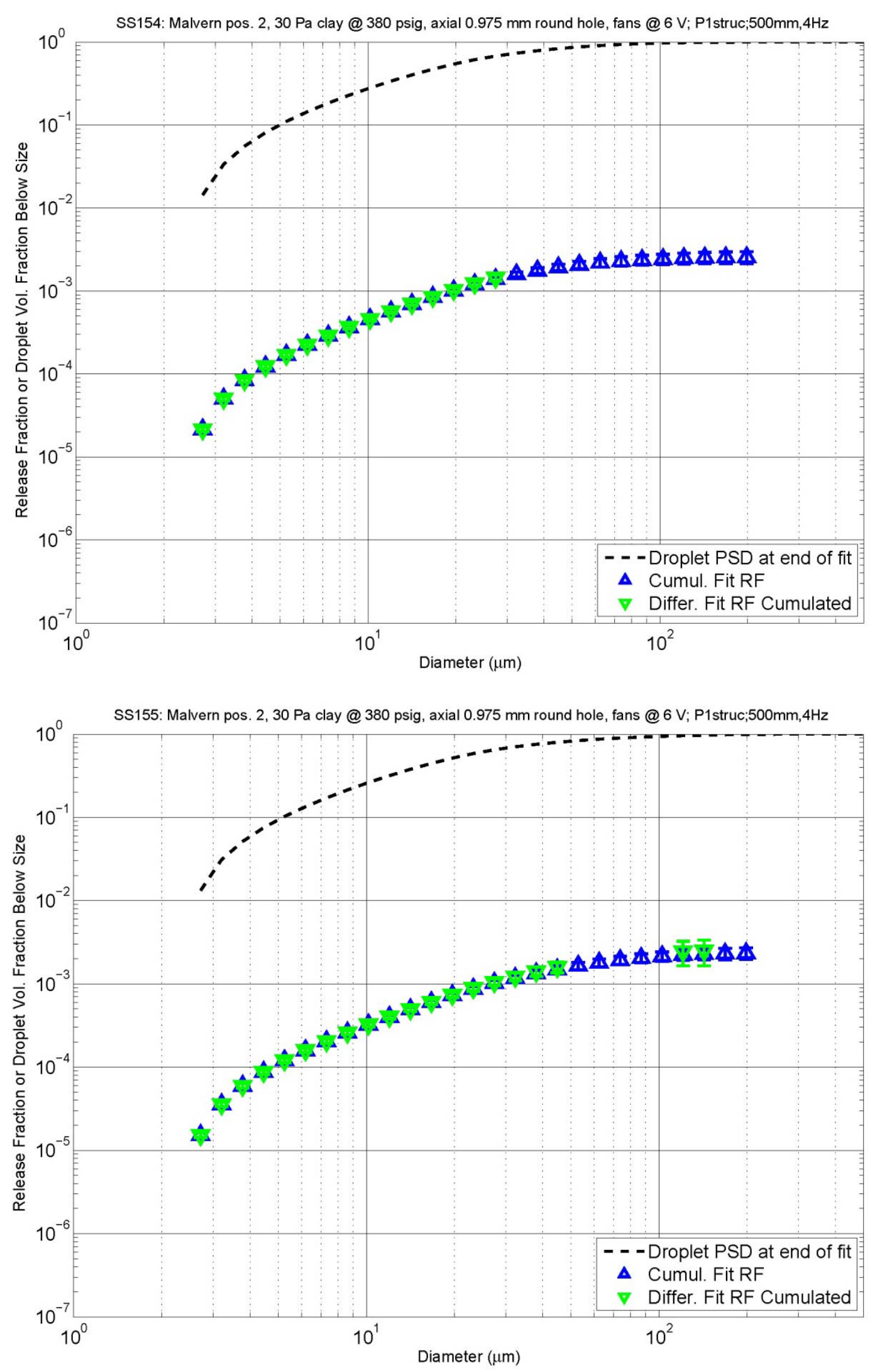

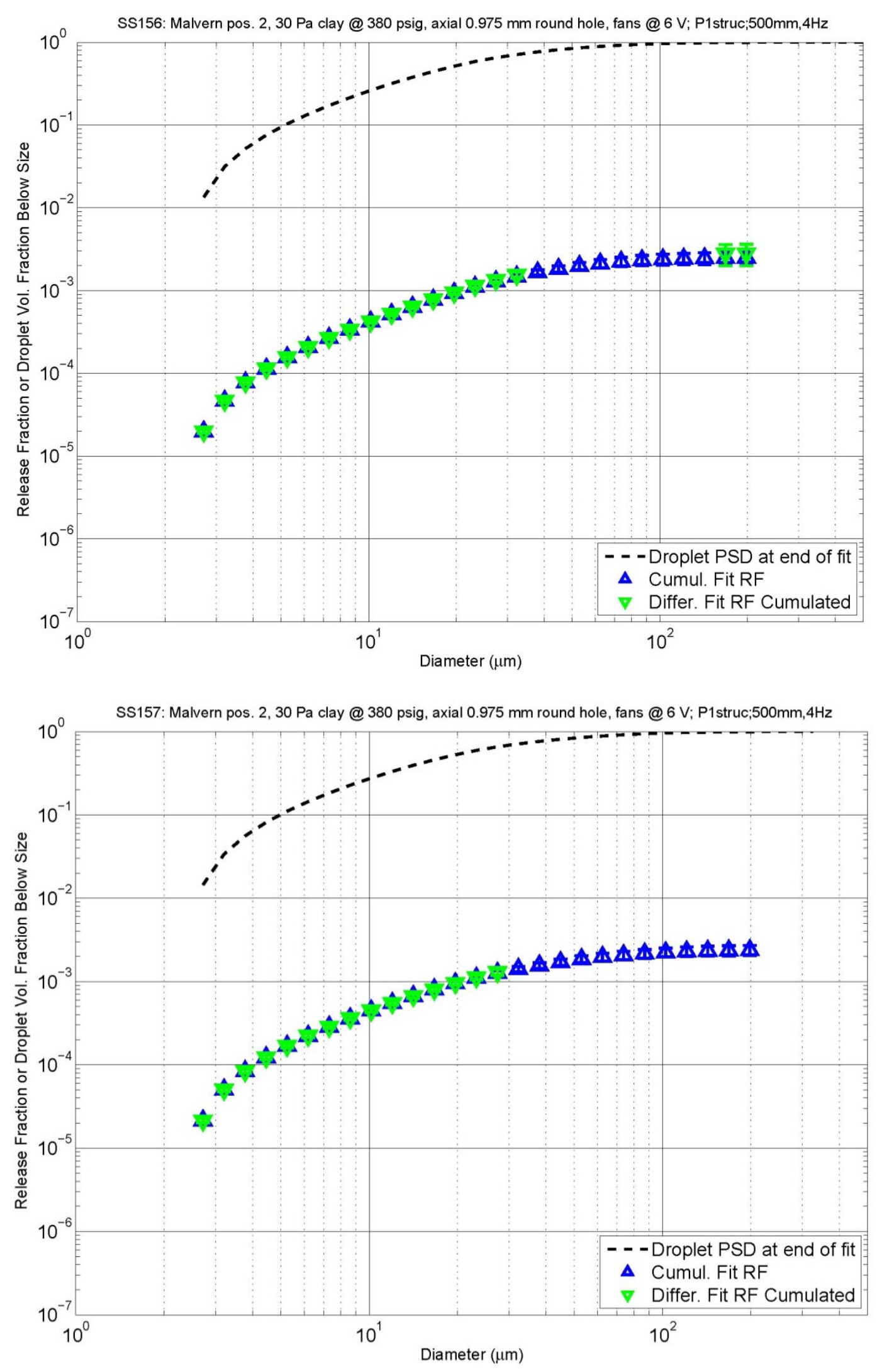

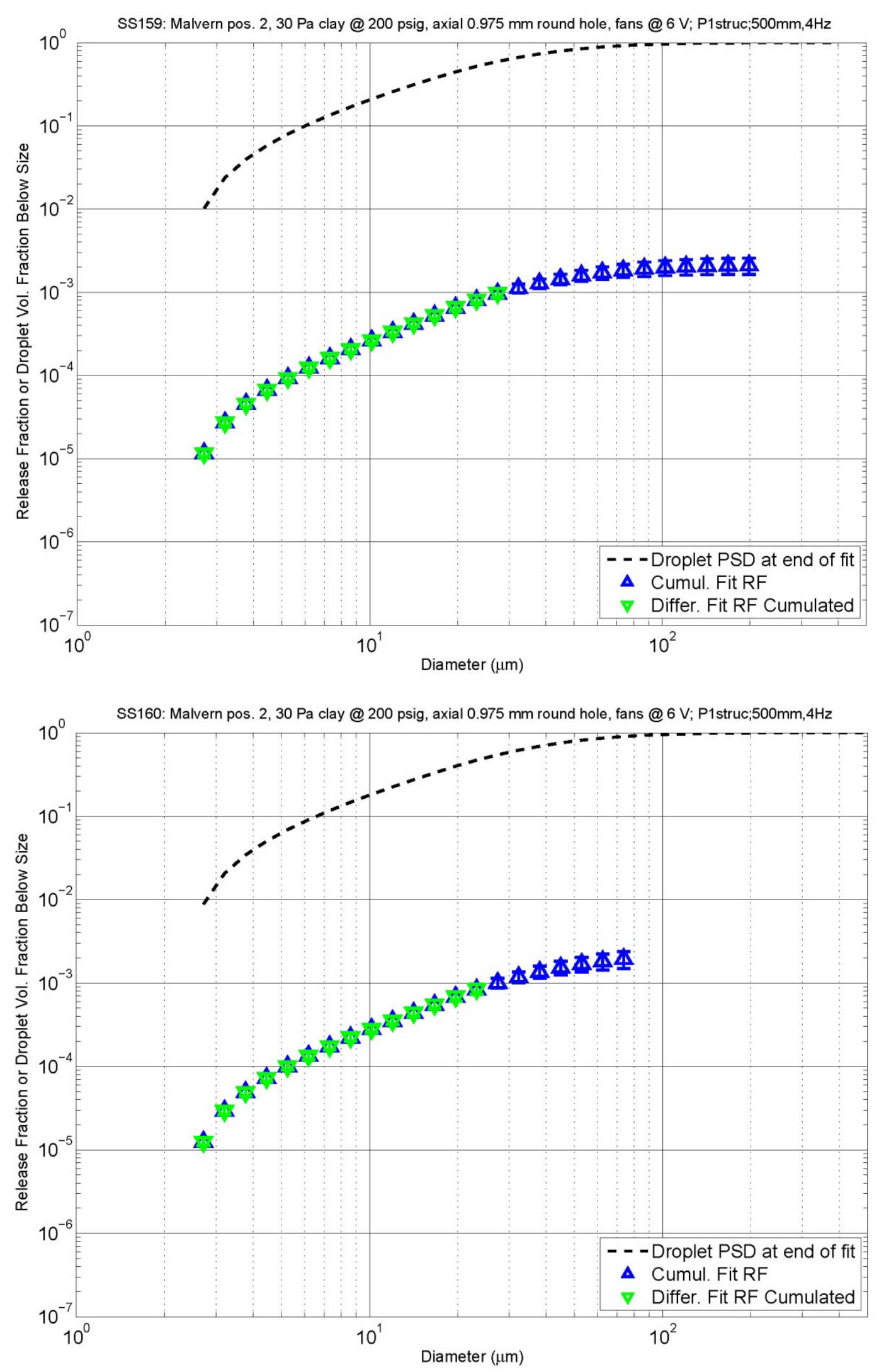

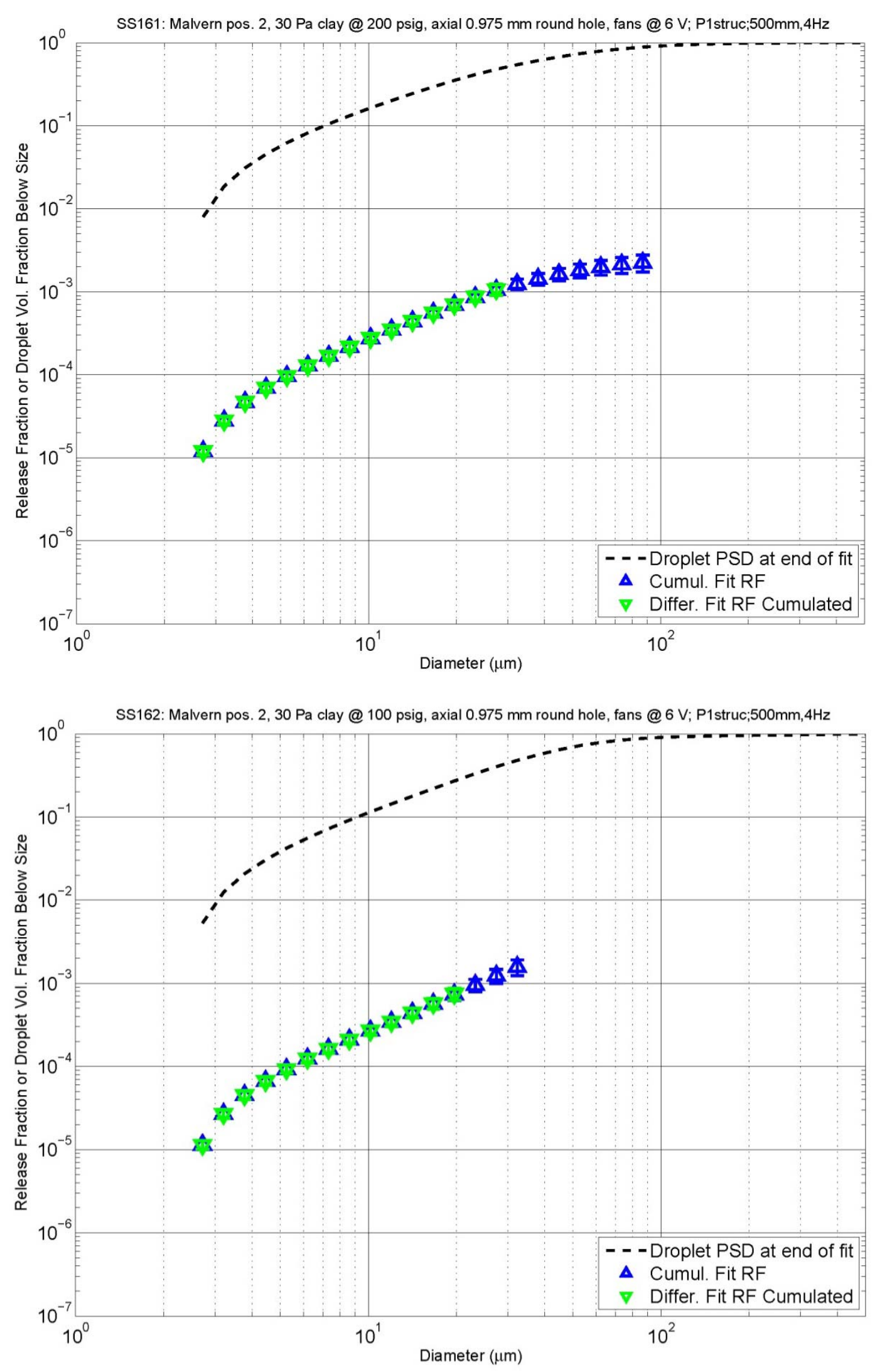

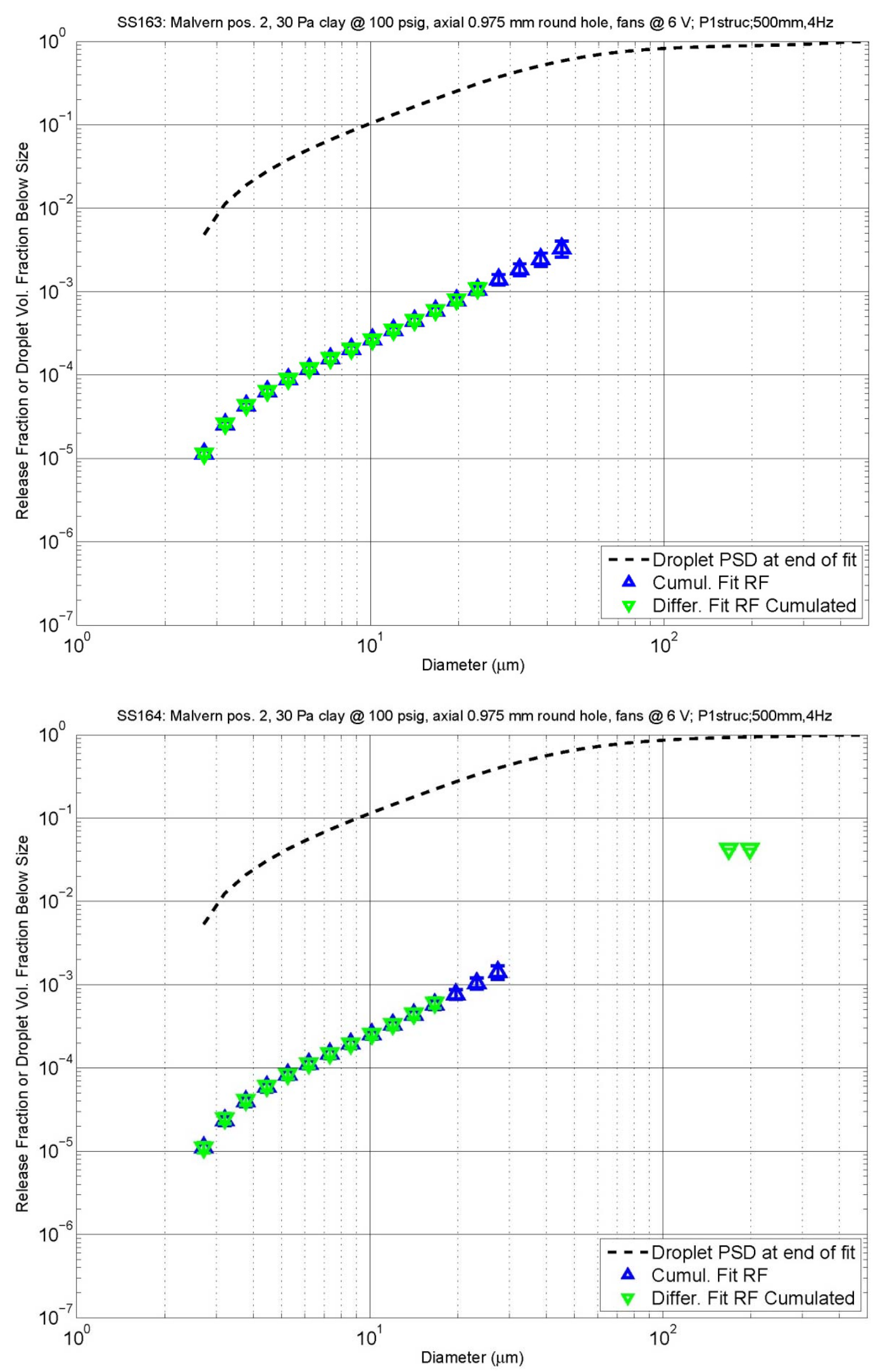

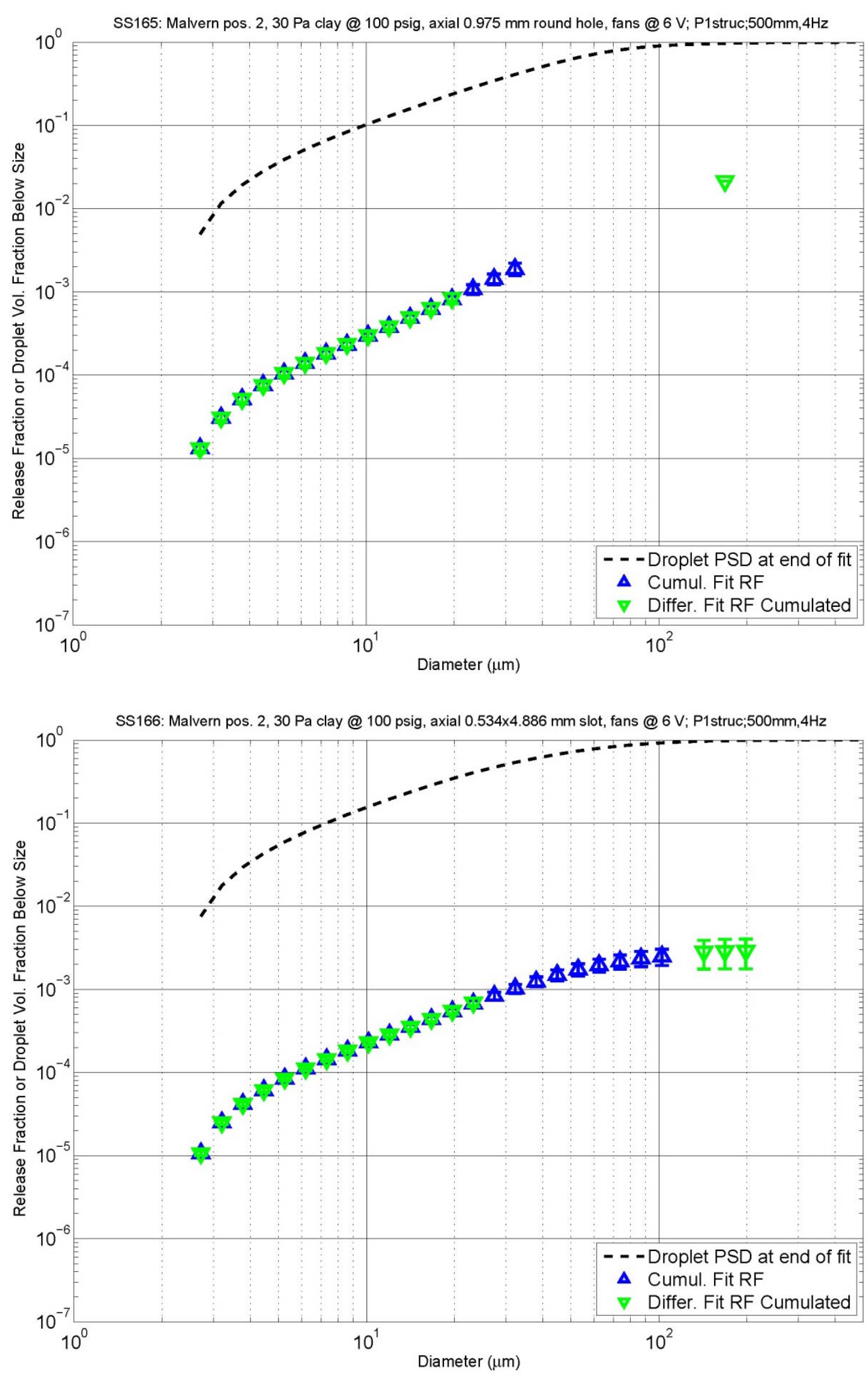

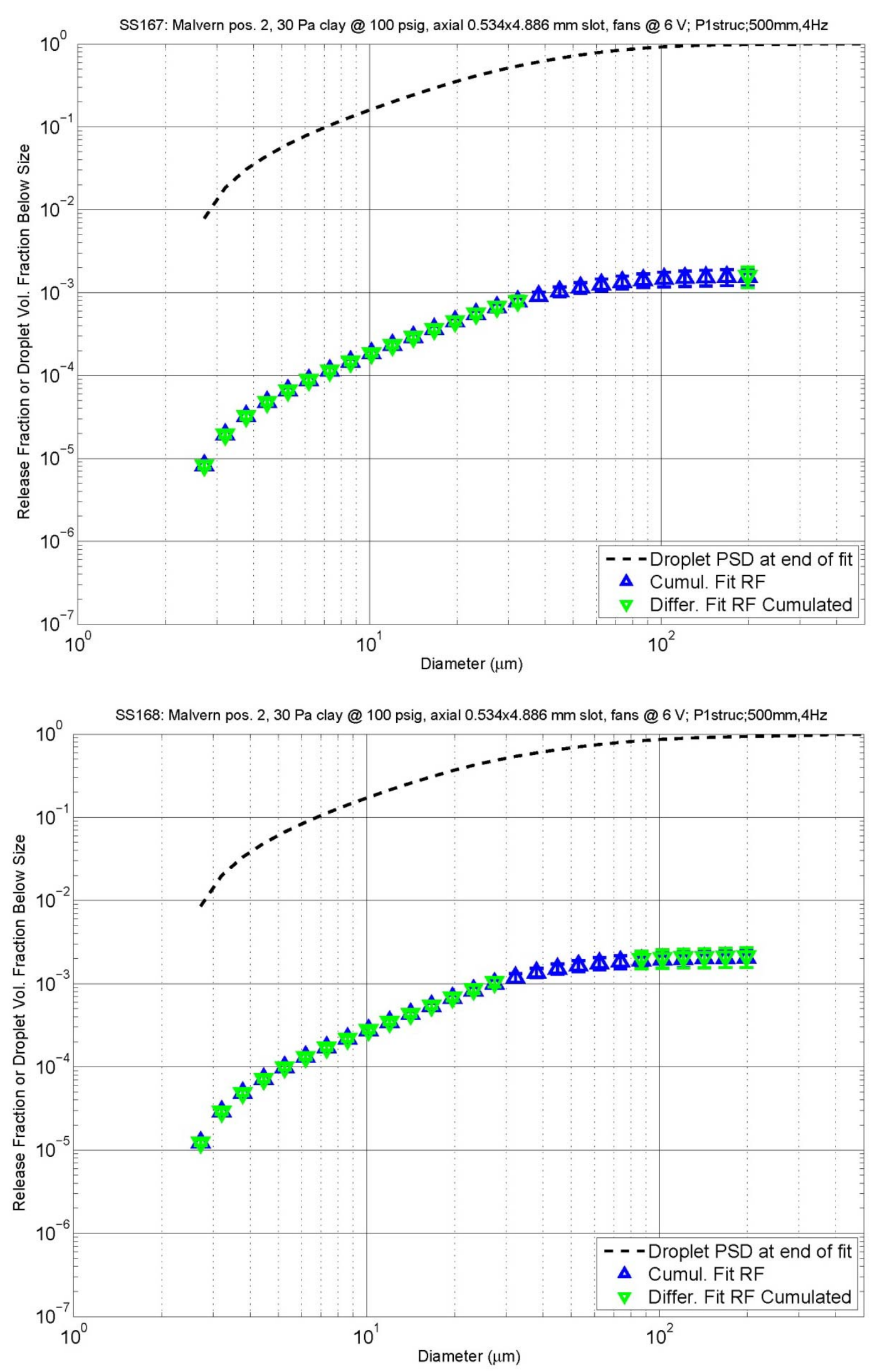

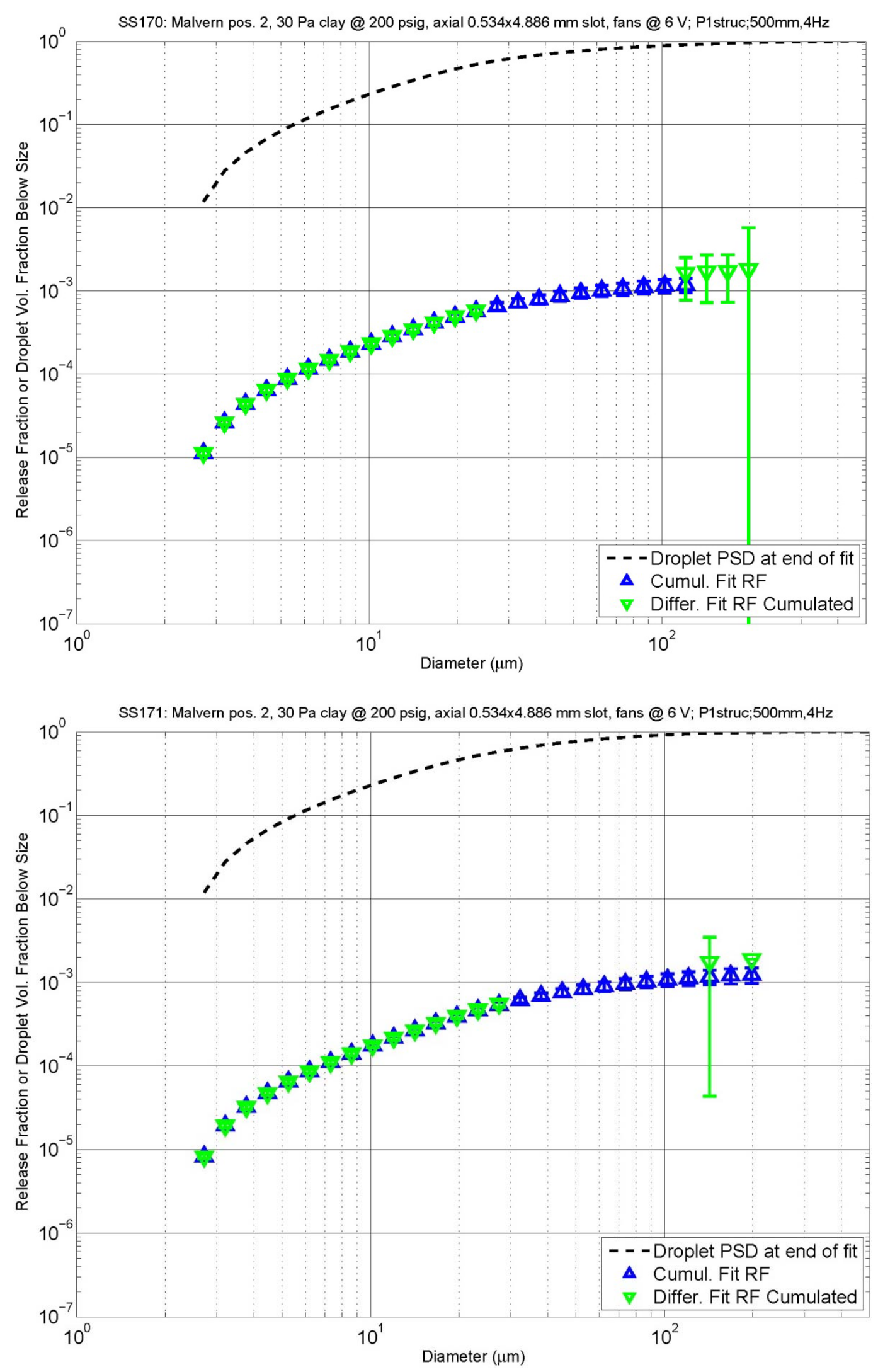

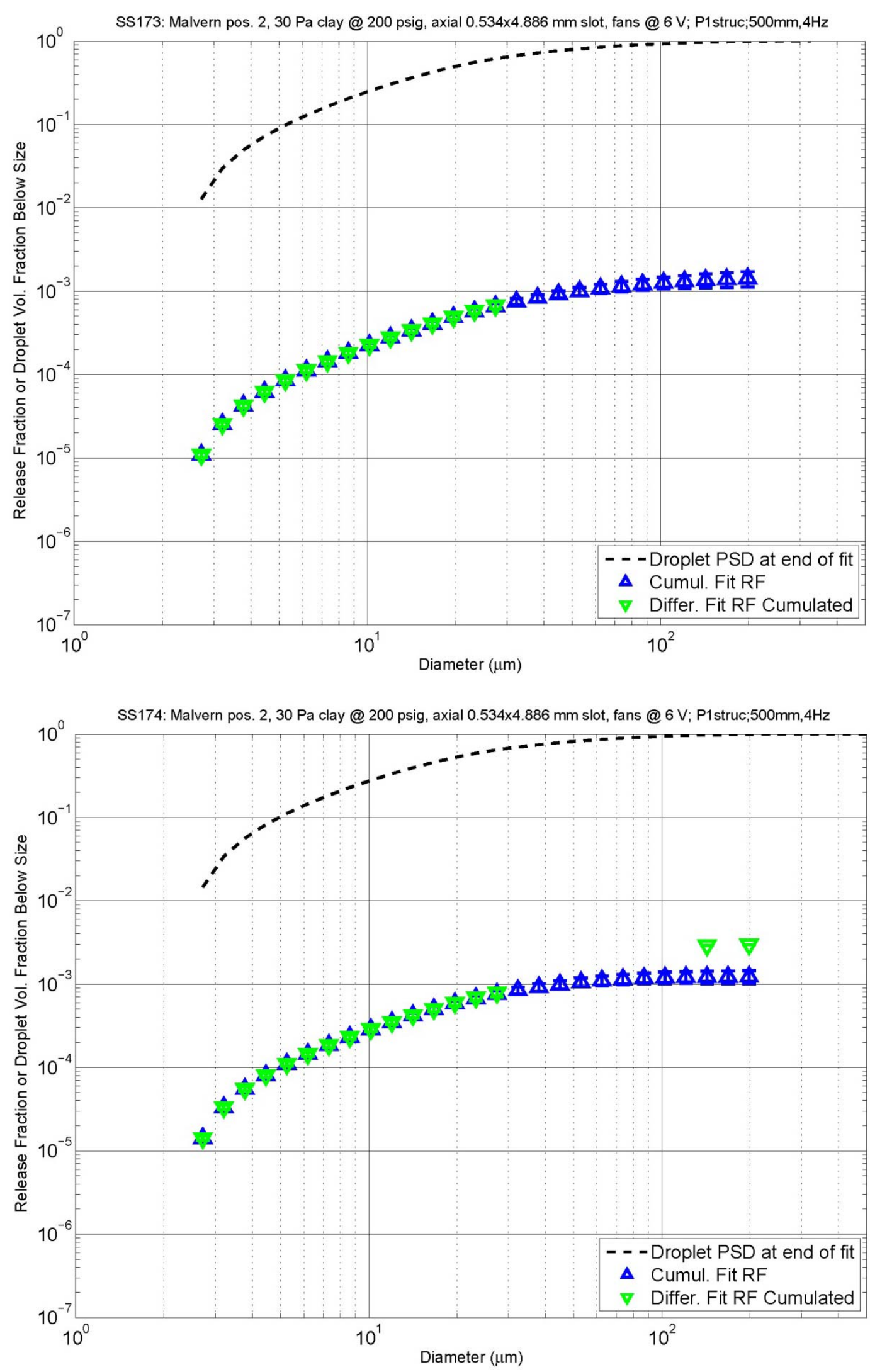

B. 31 

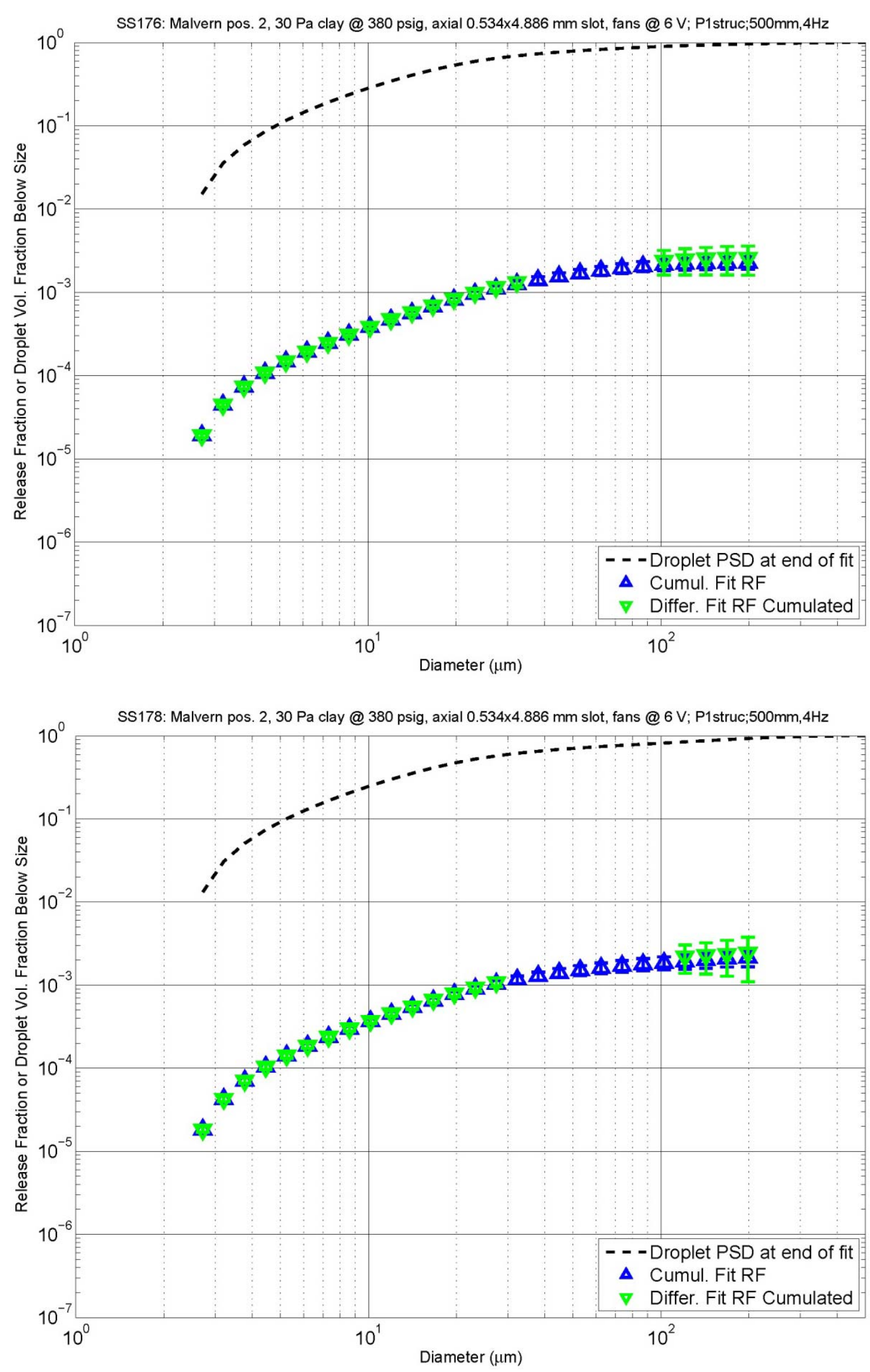

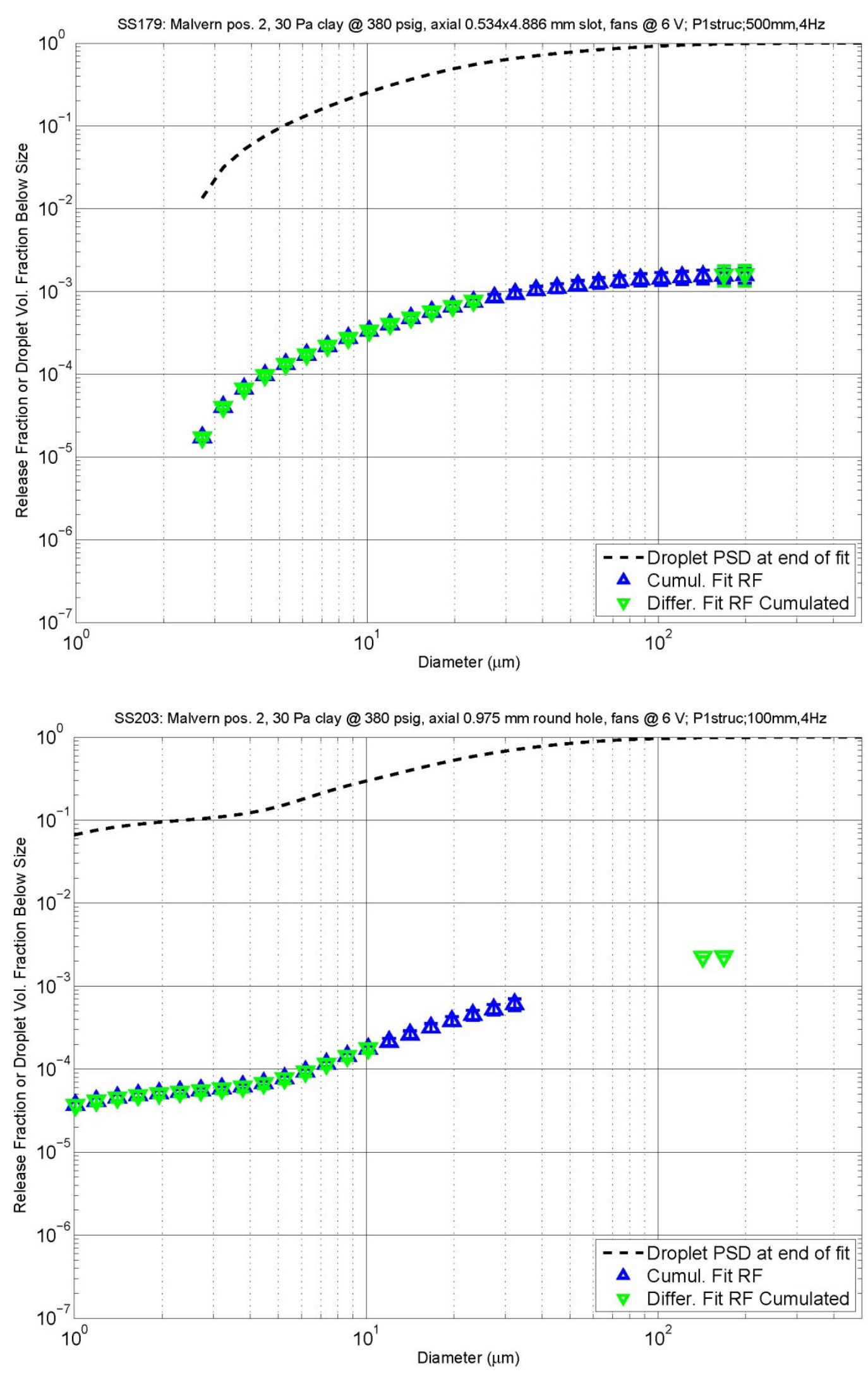

B.33 

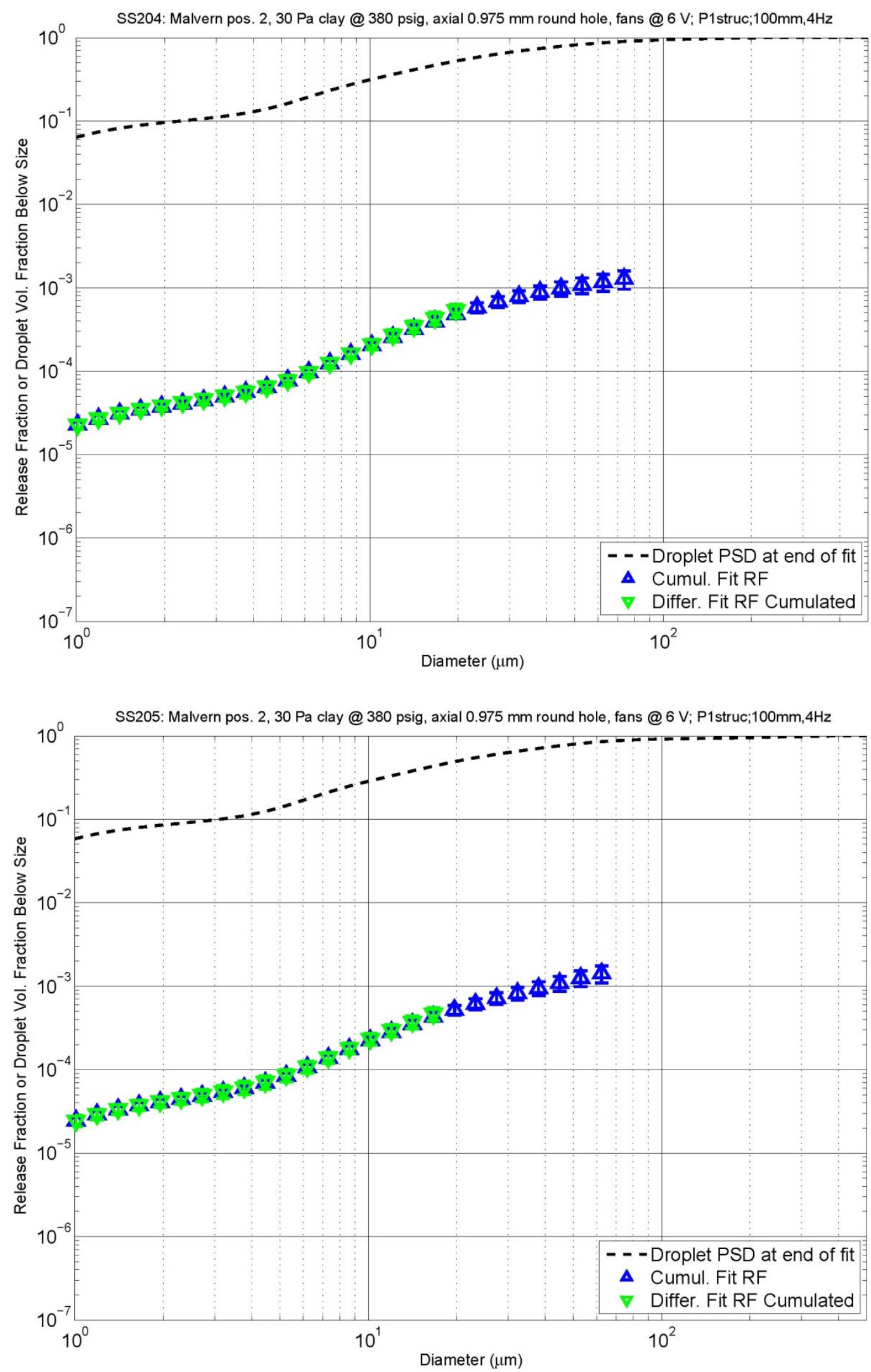

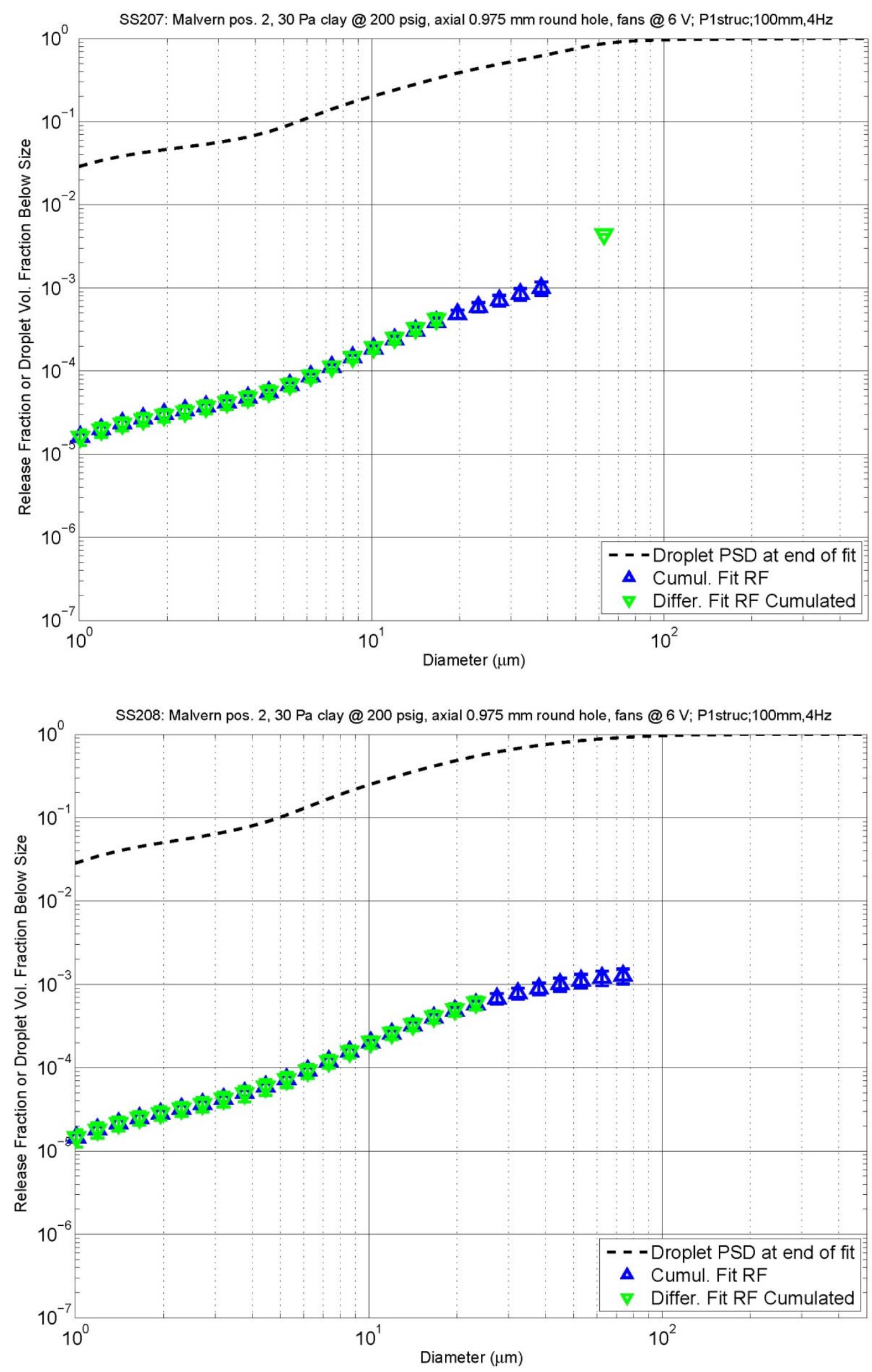

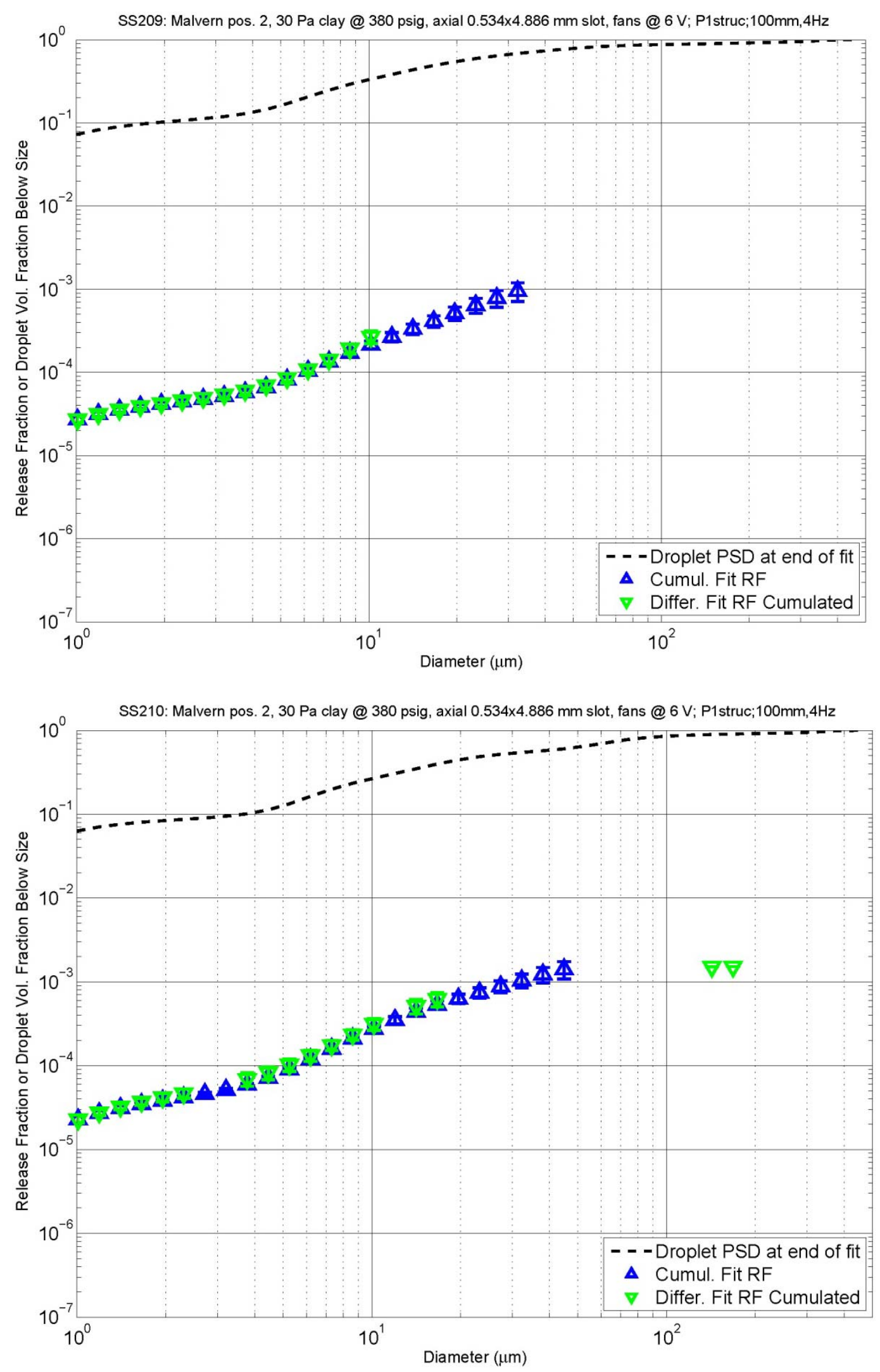

B.36 

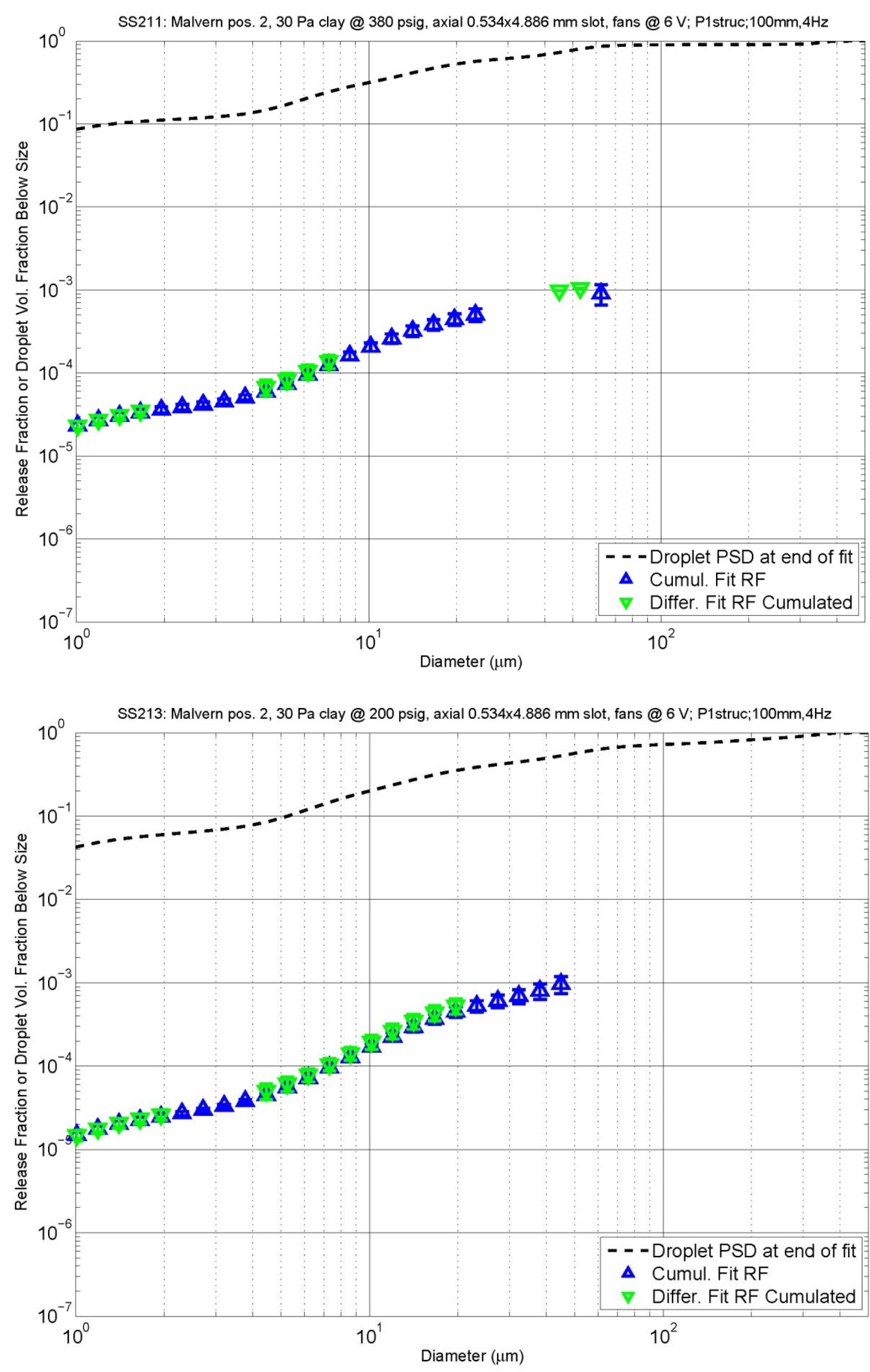

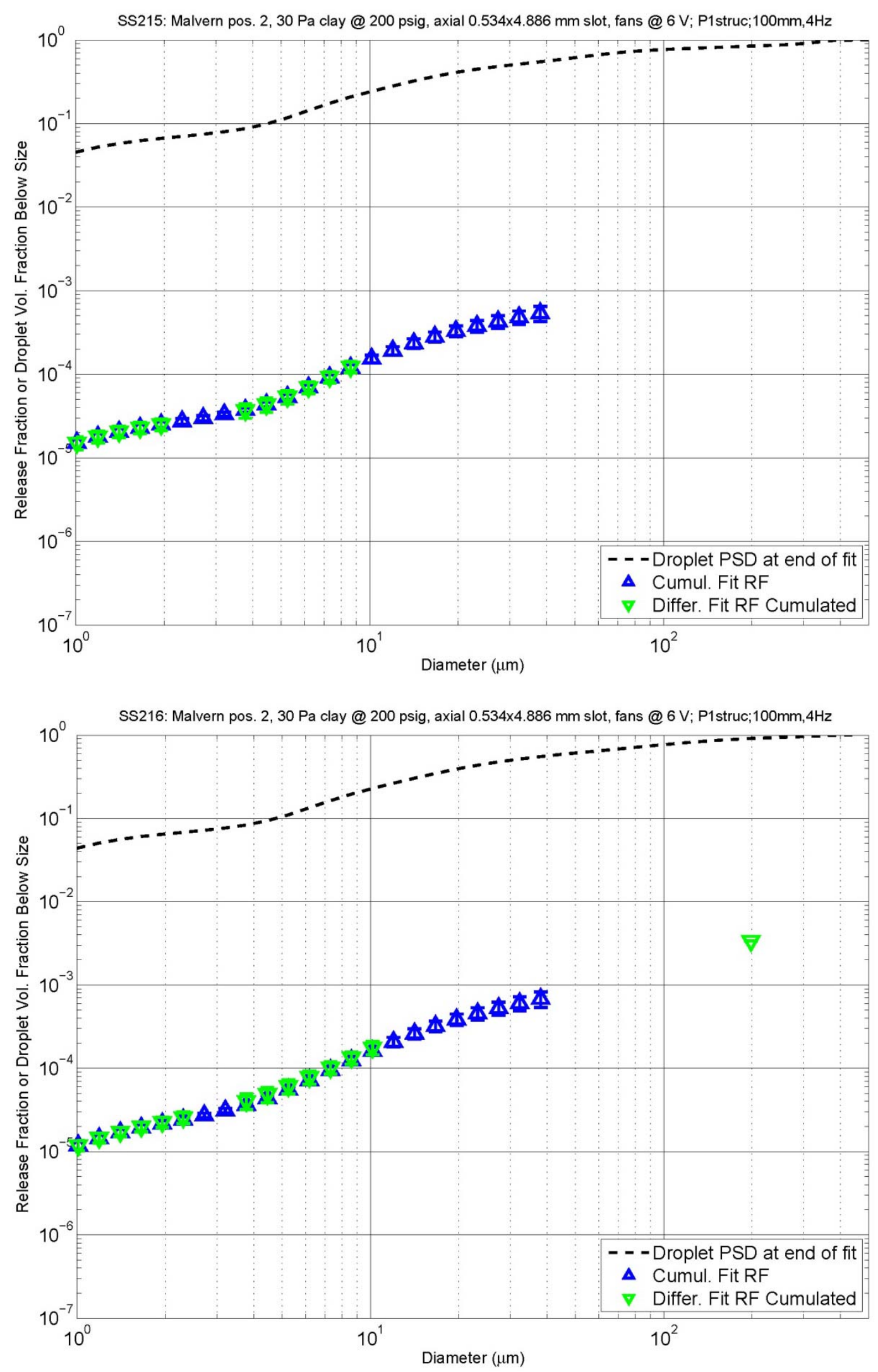

B. 38 

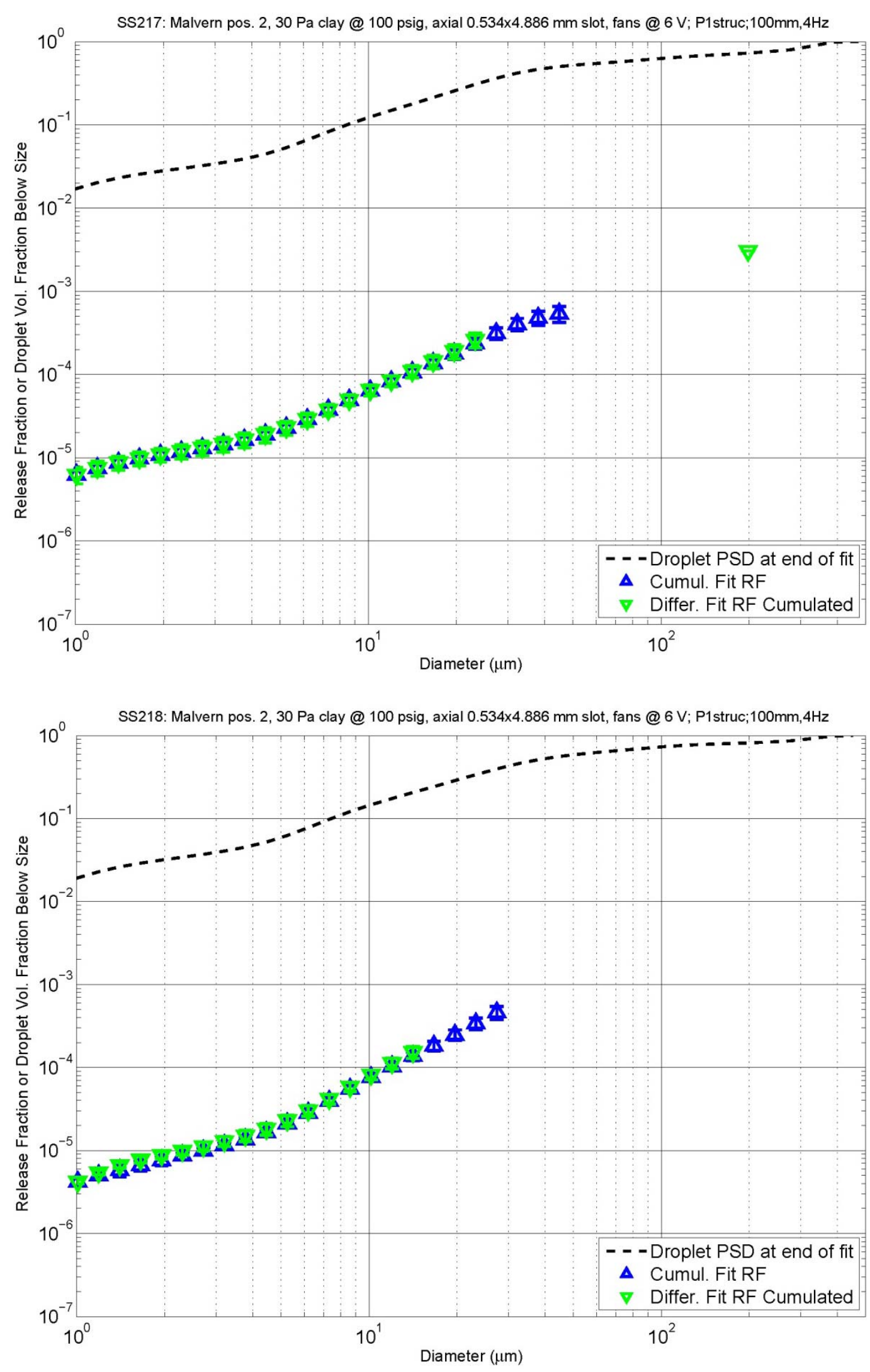

B.39 

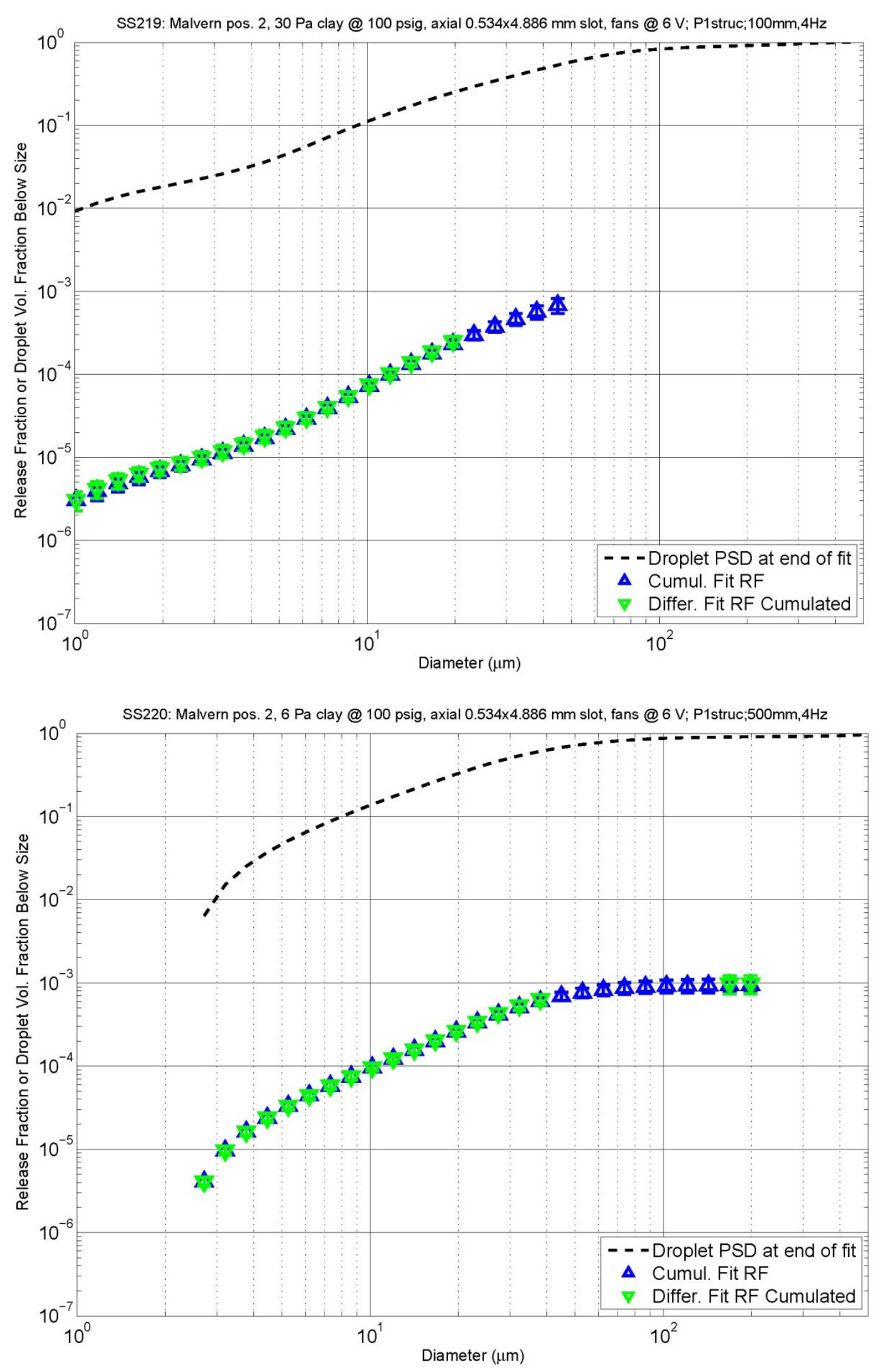

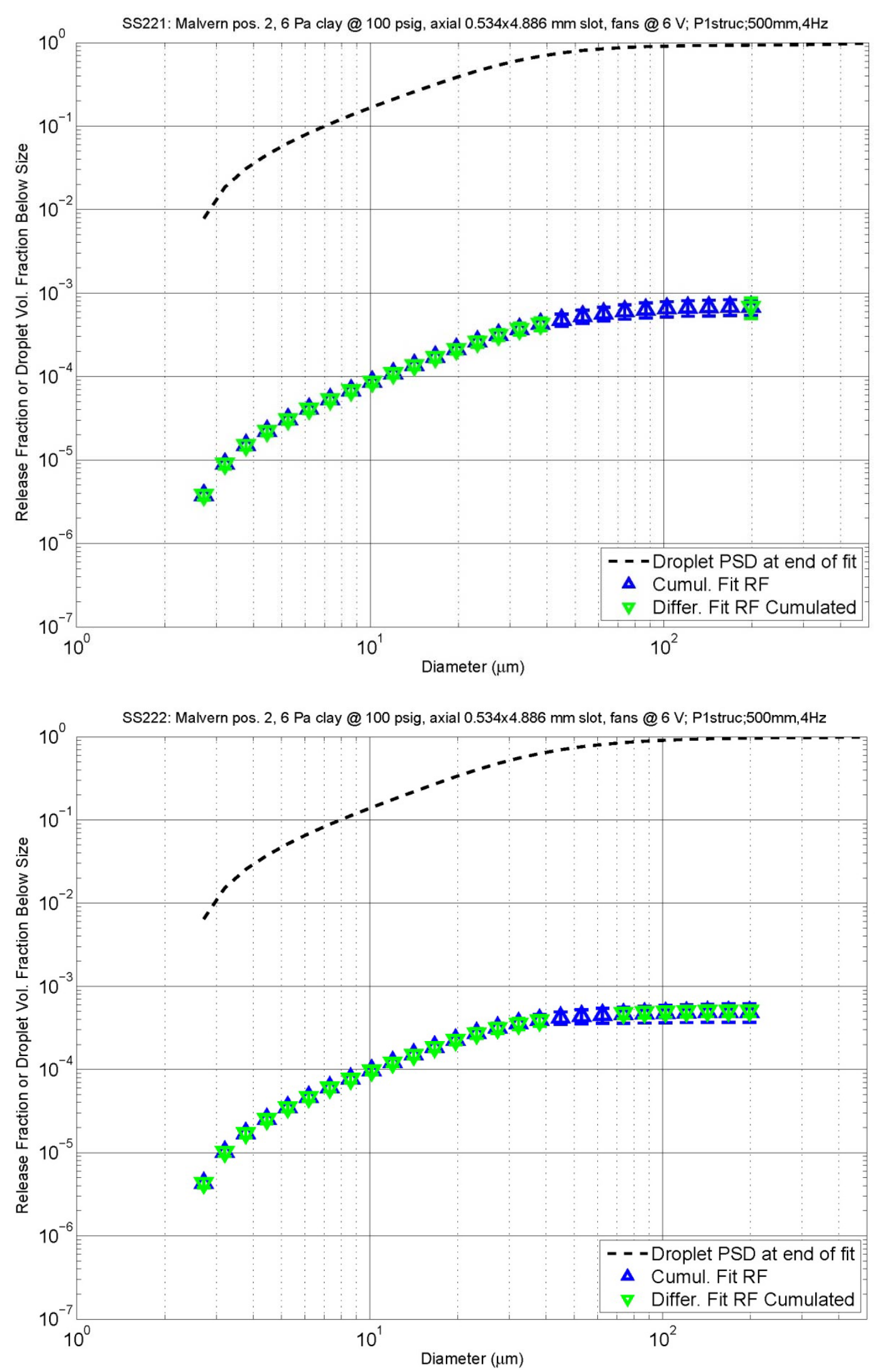

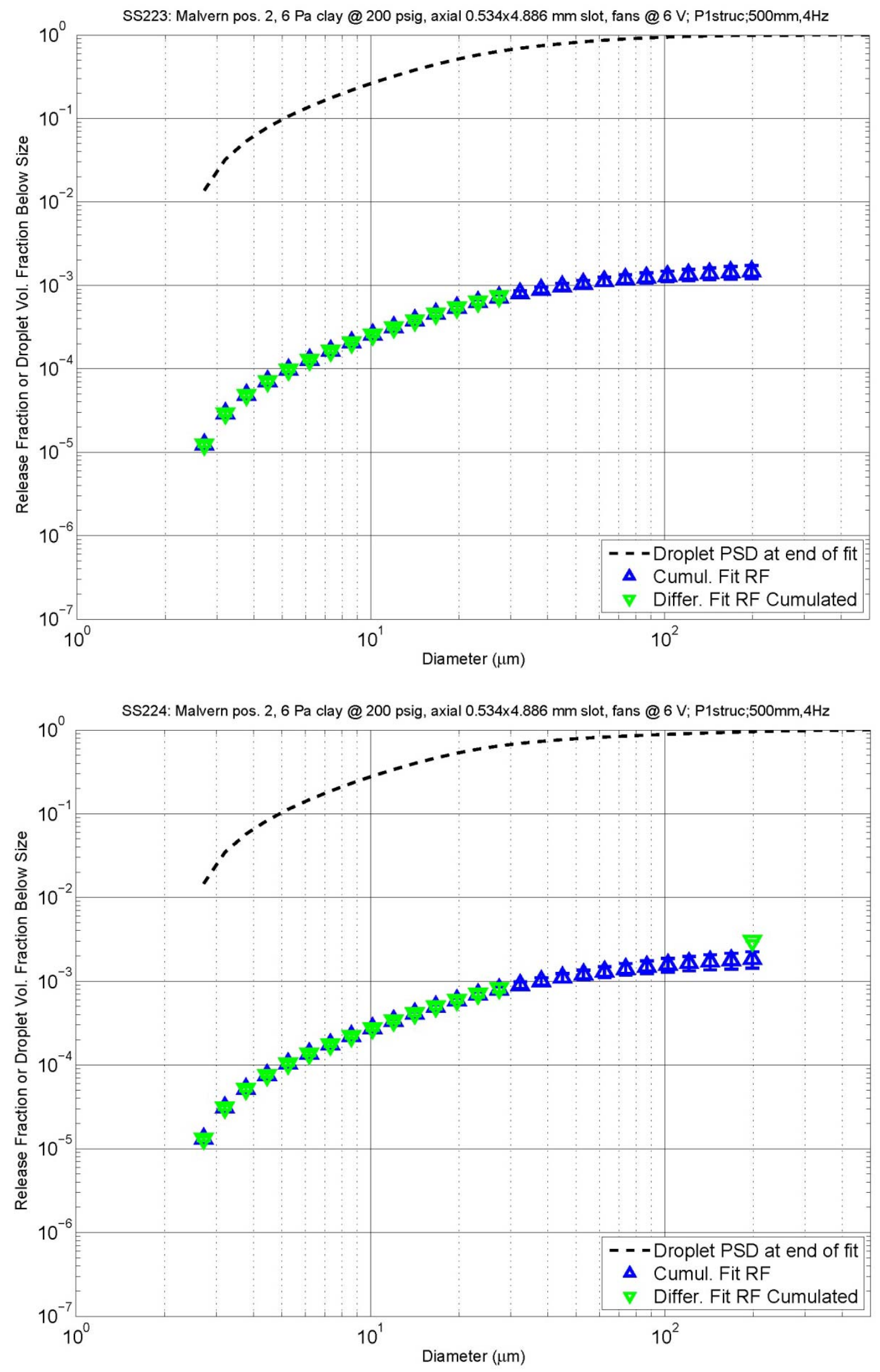

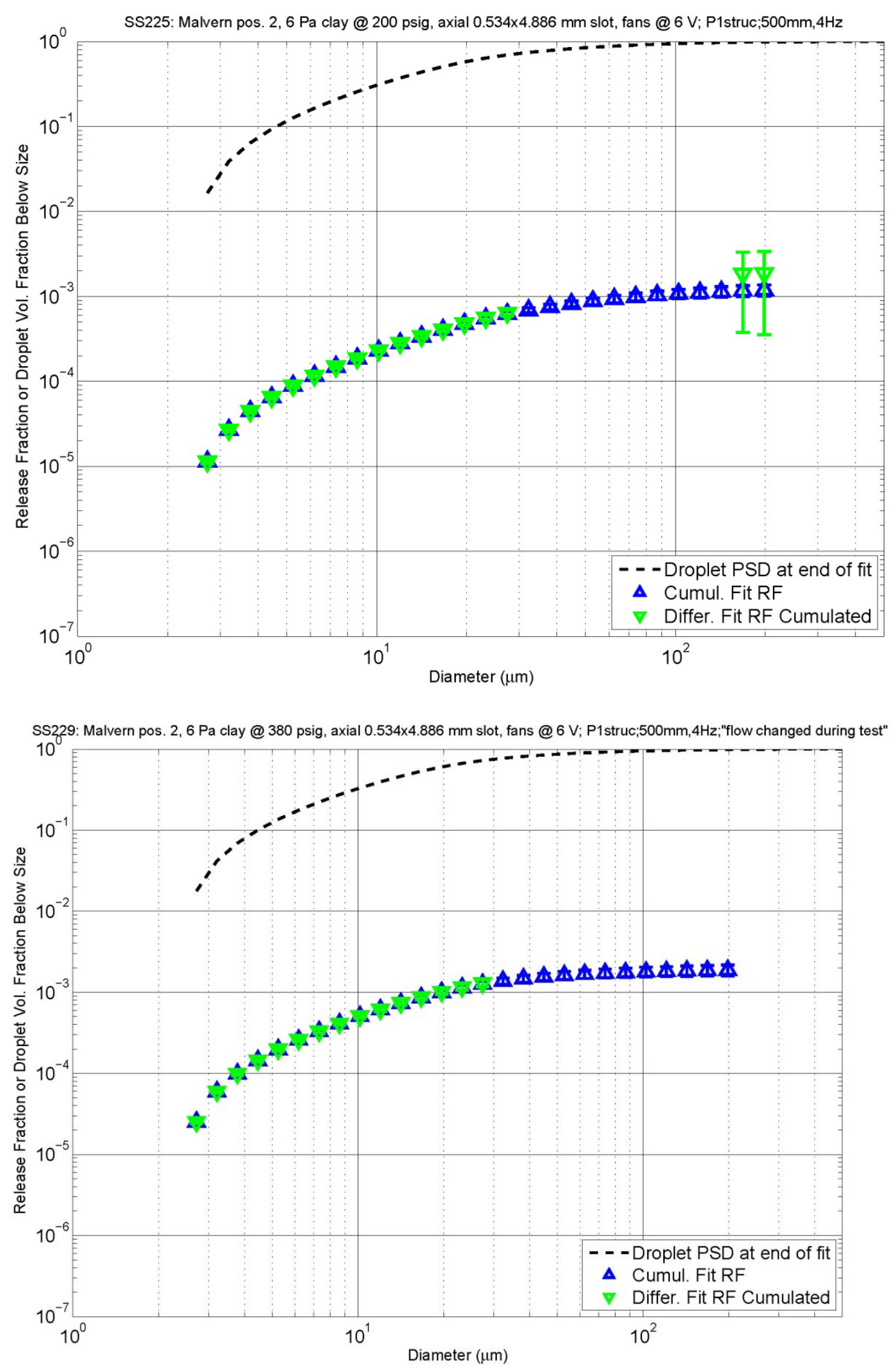

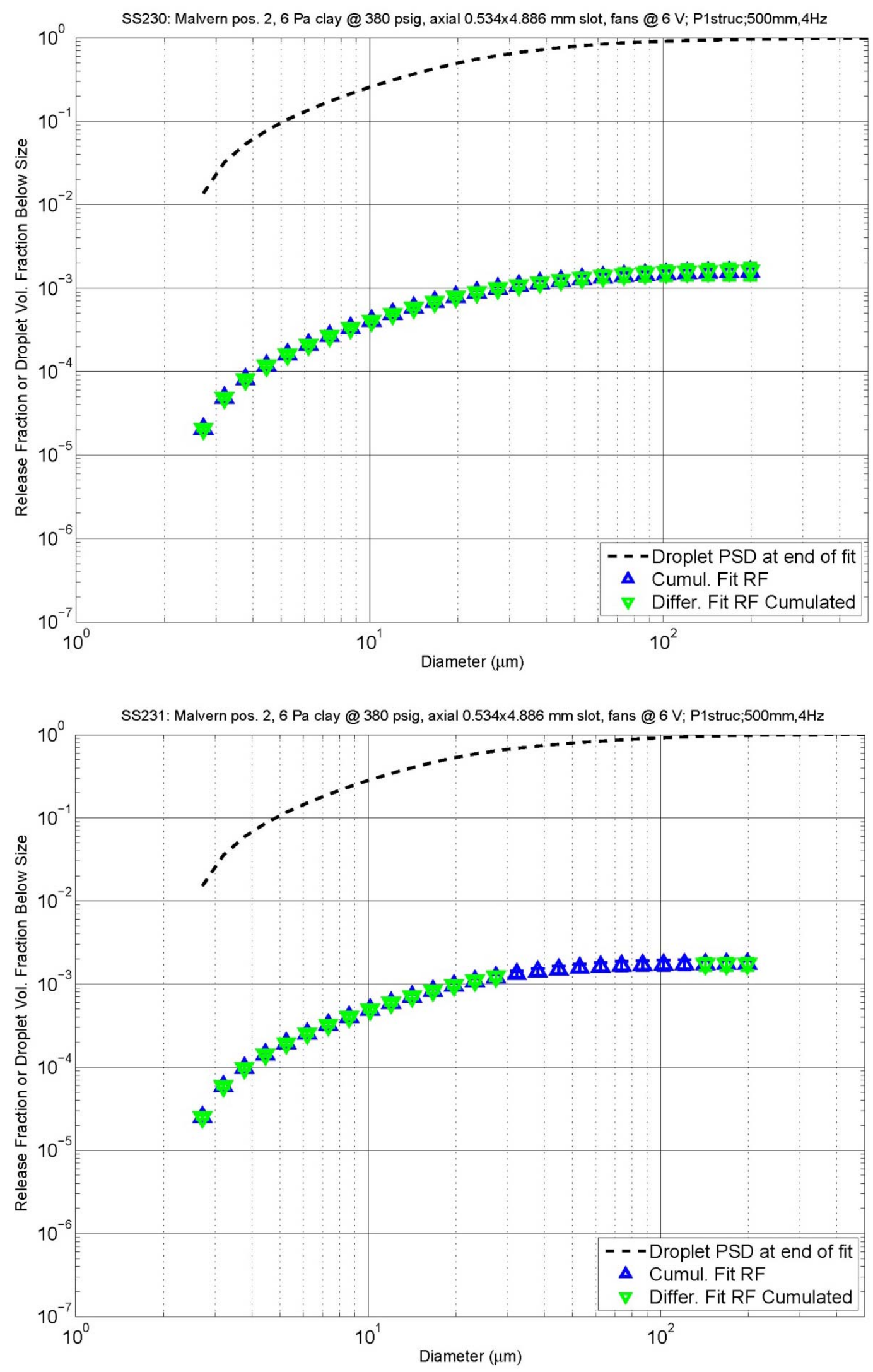

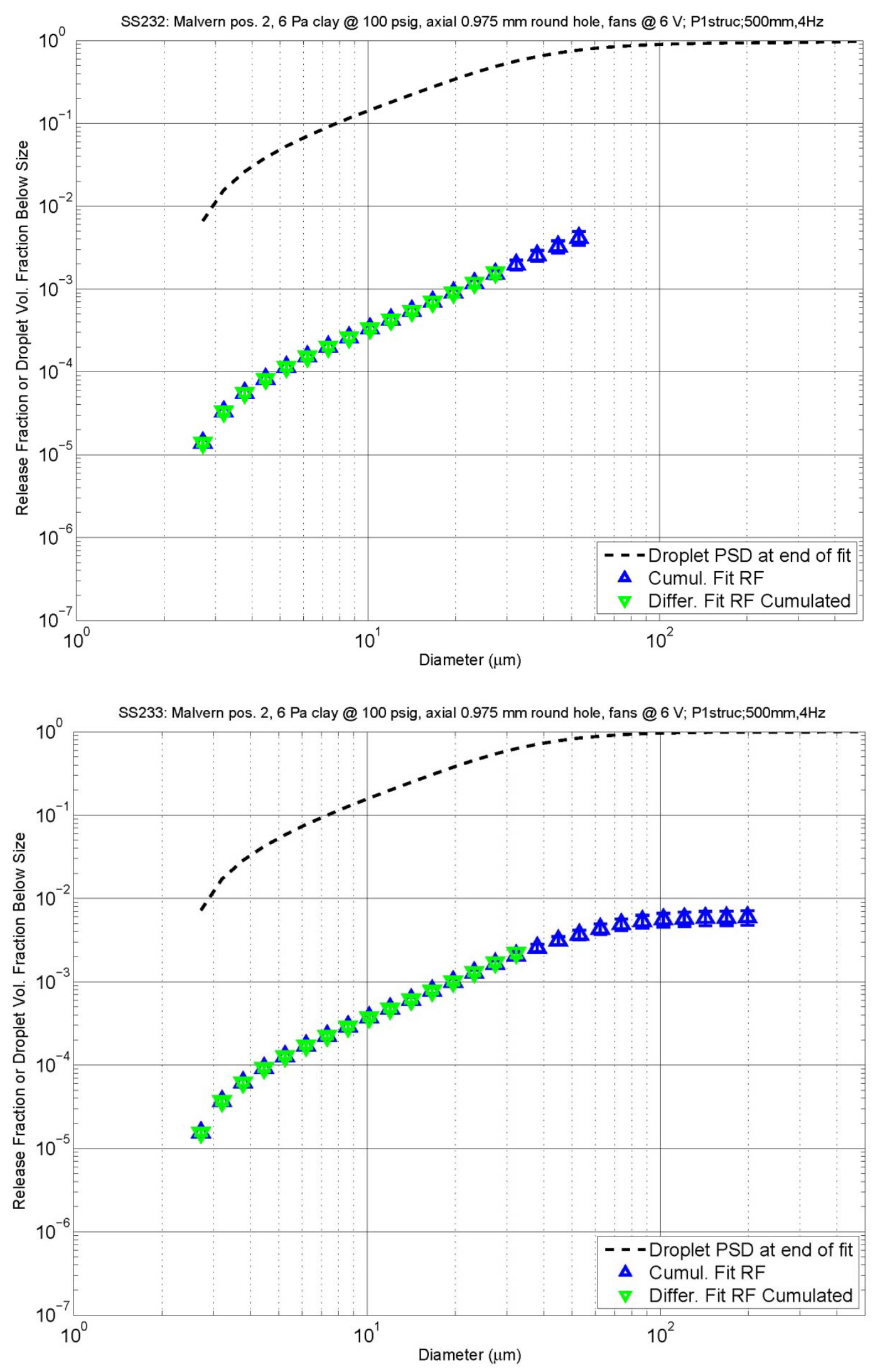

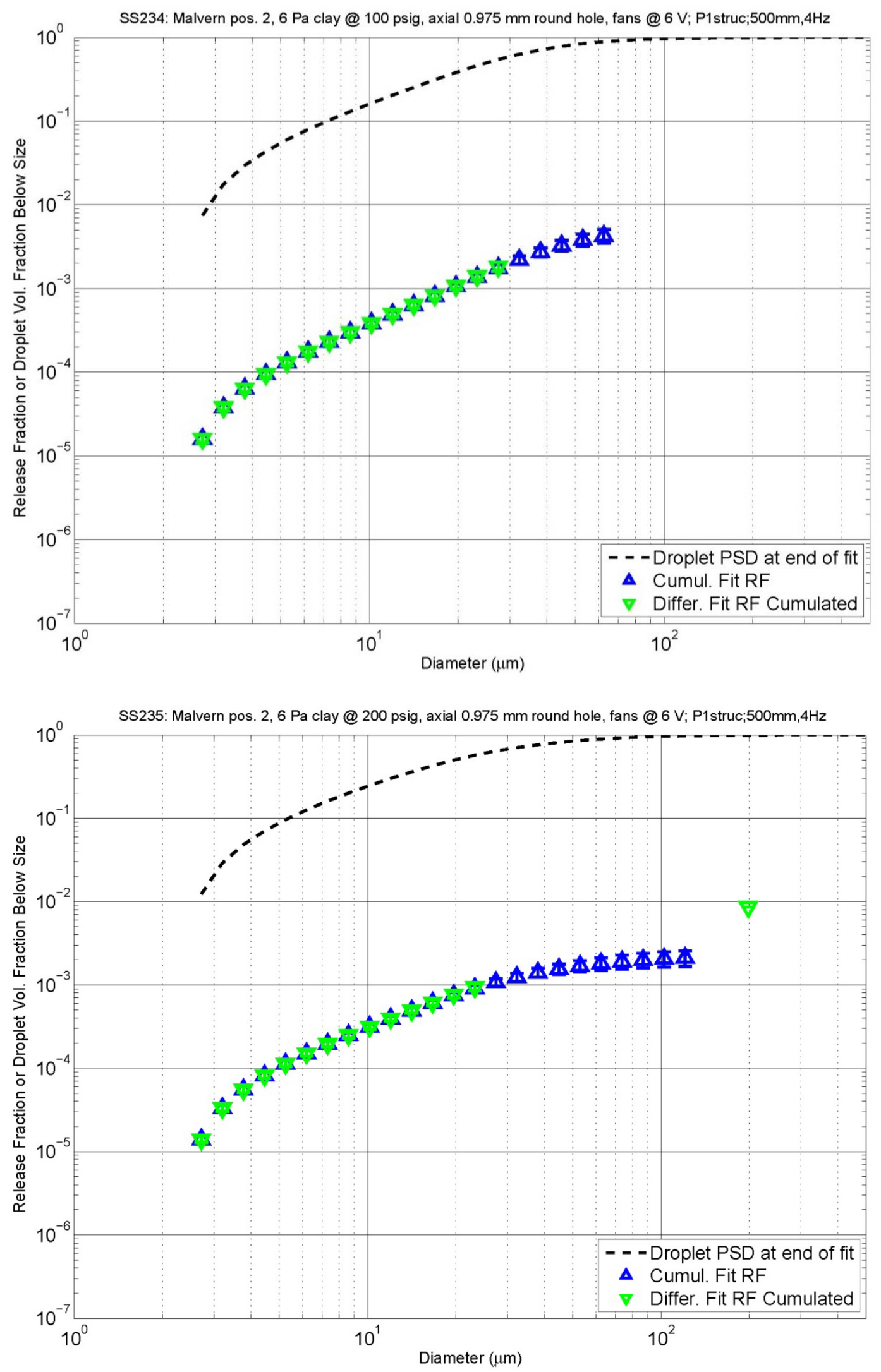

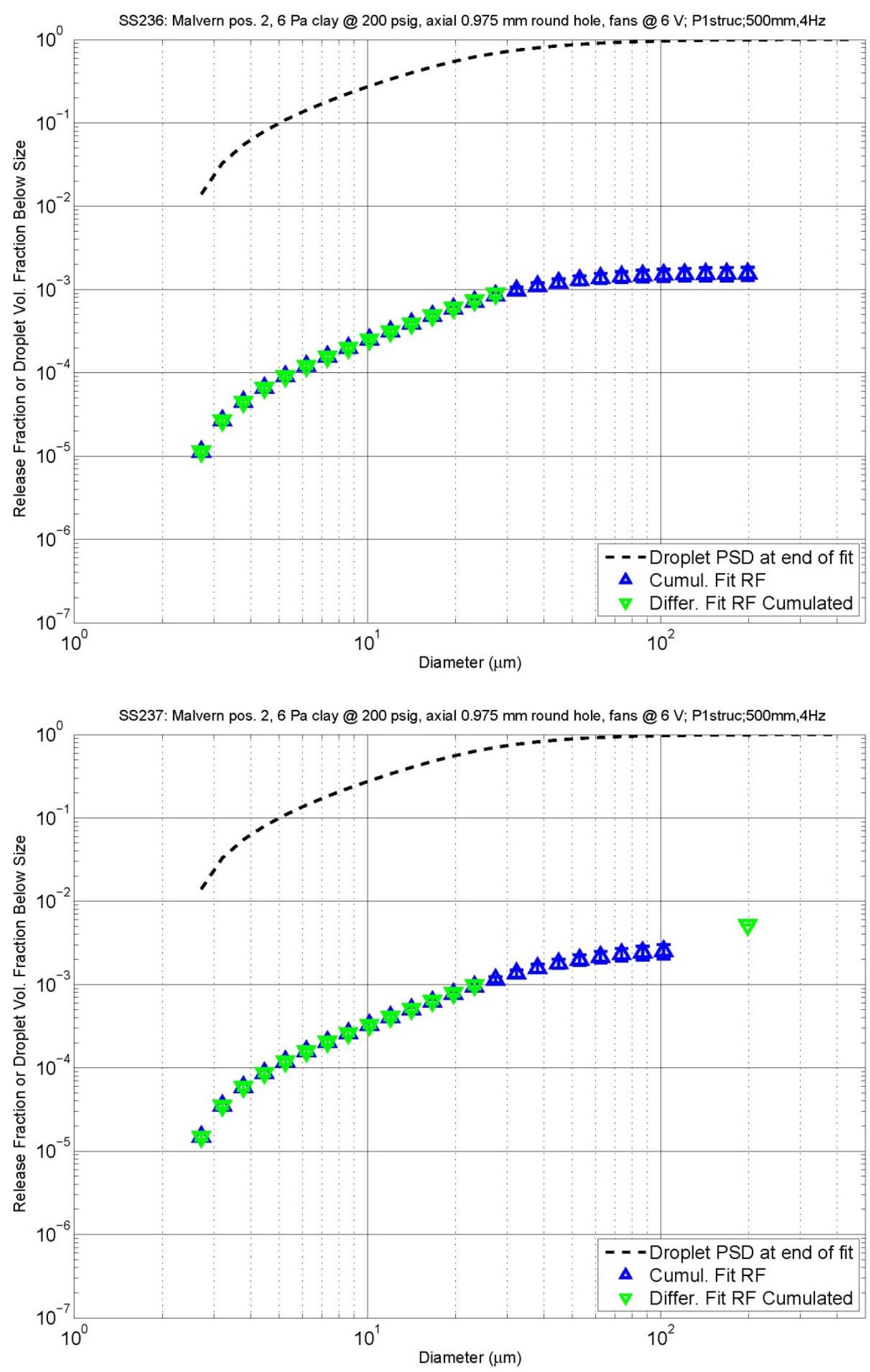

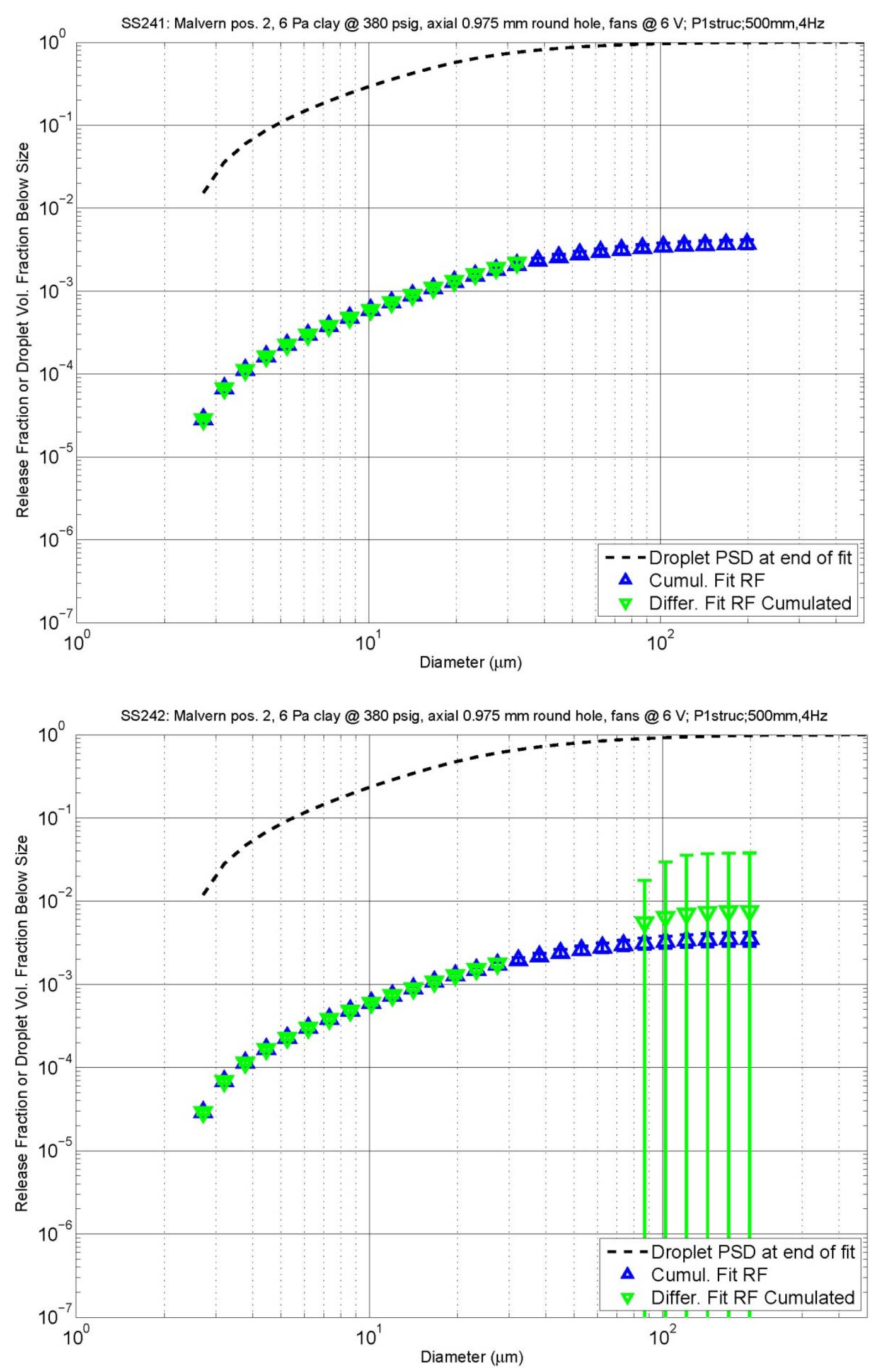

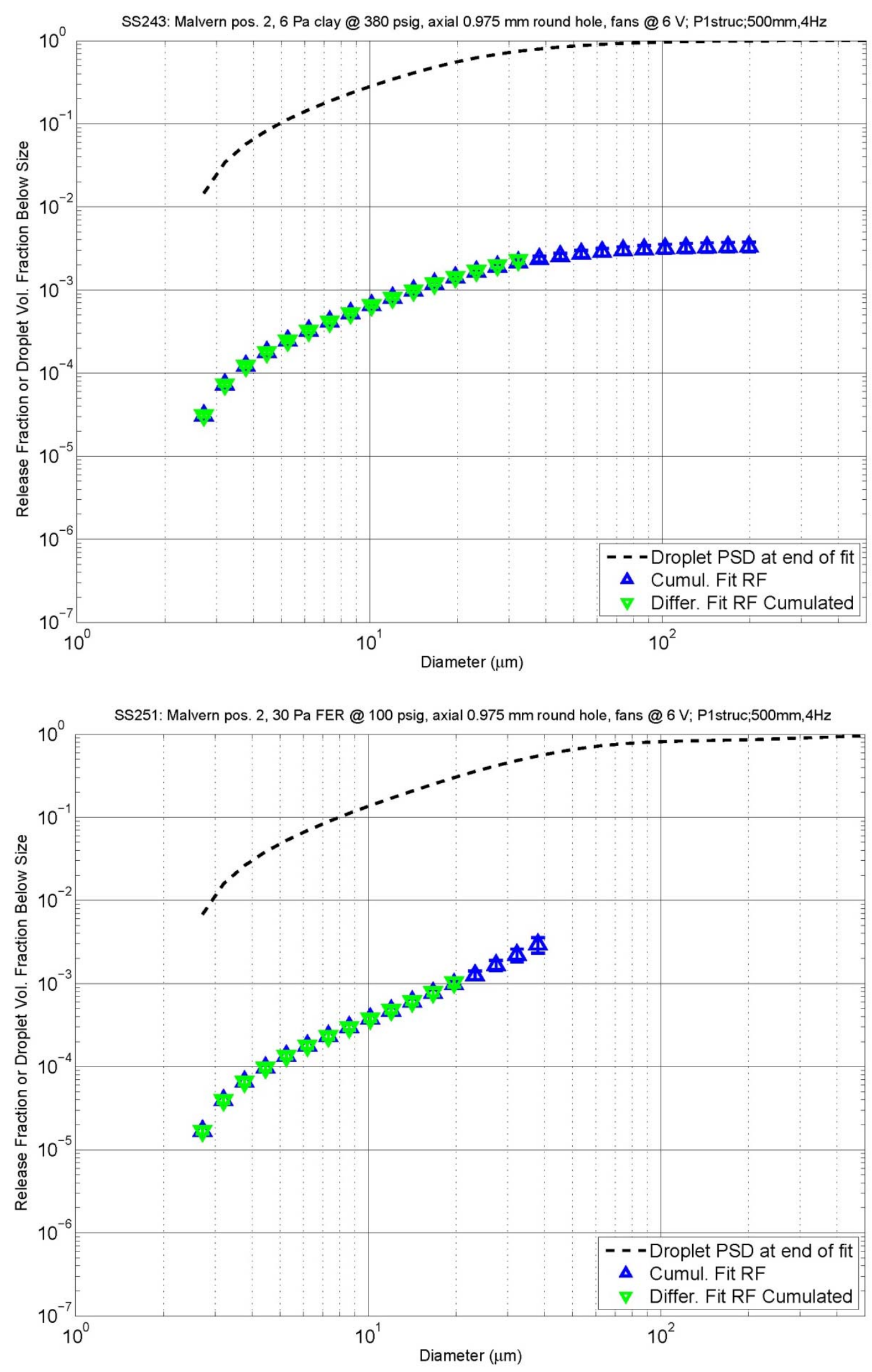

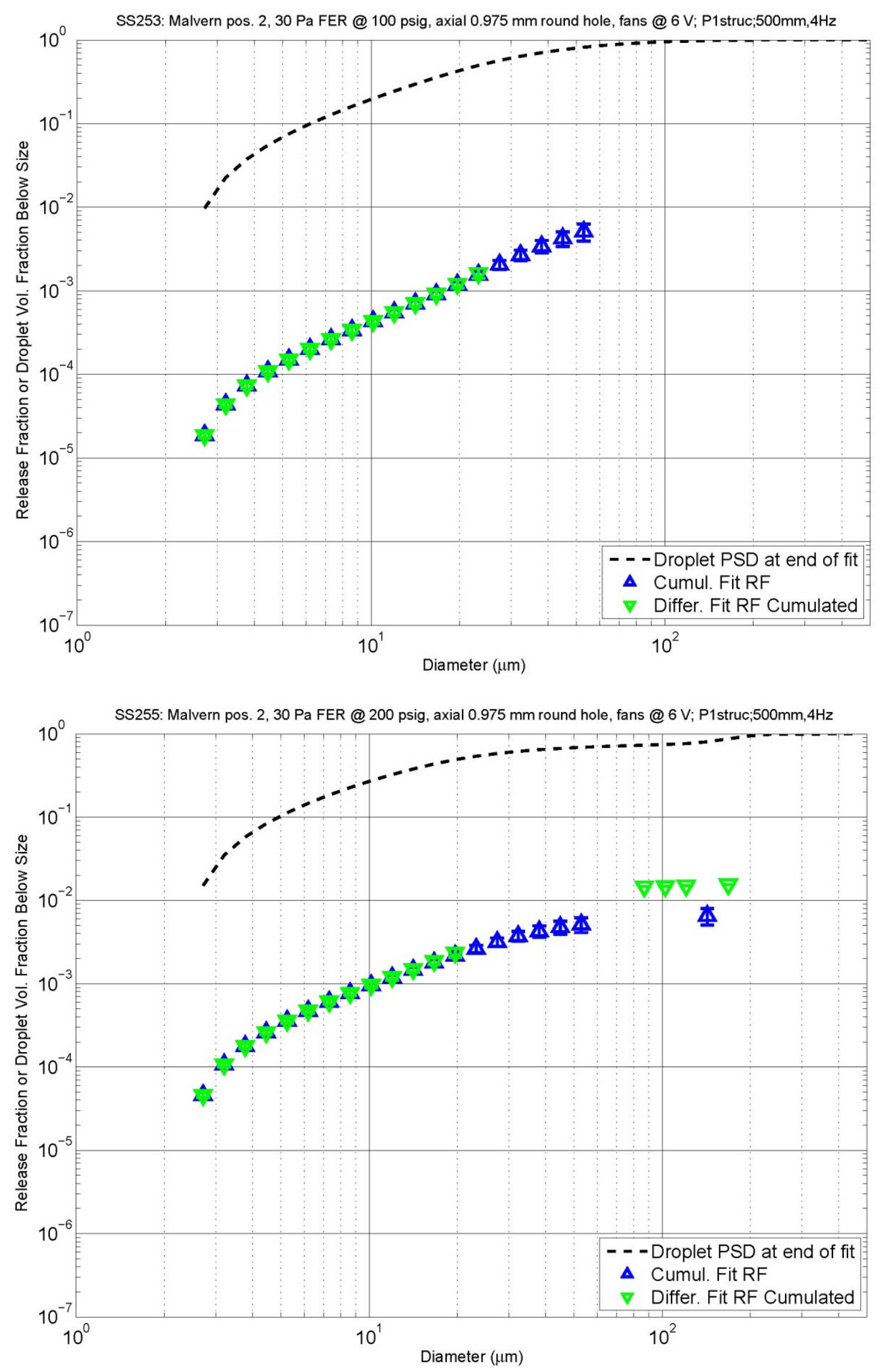

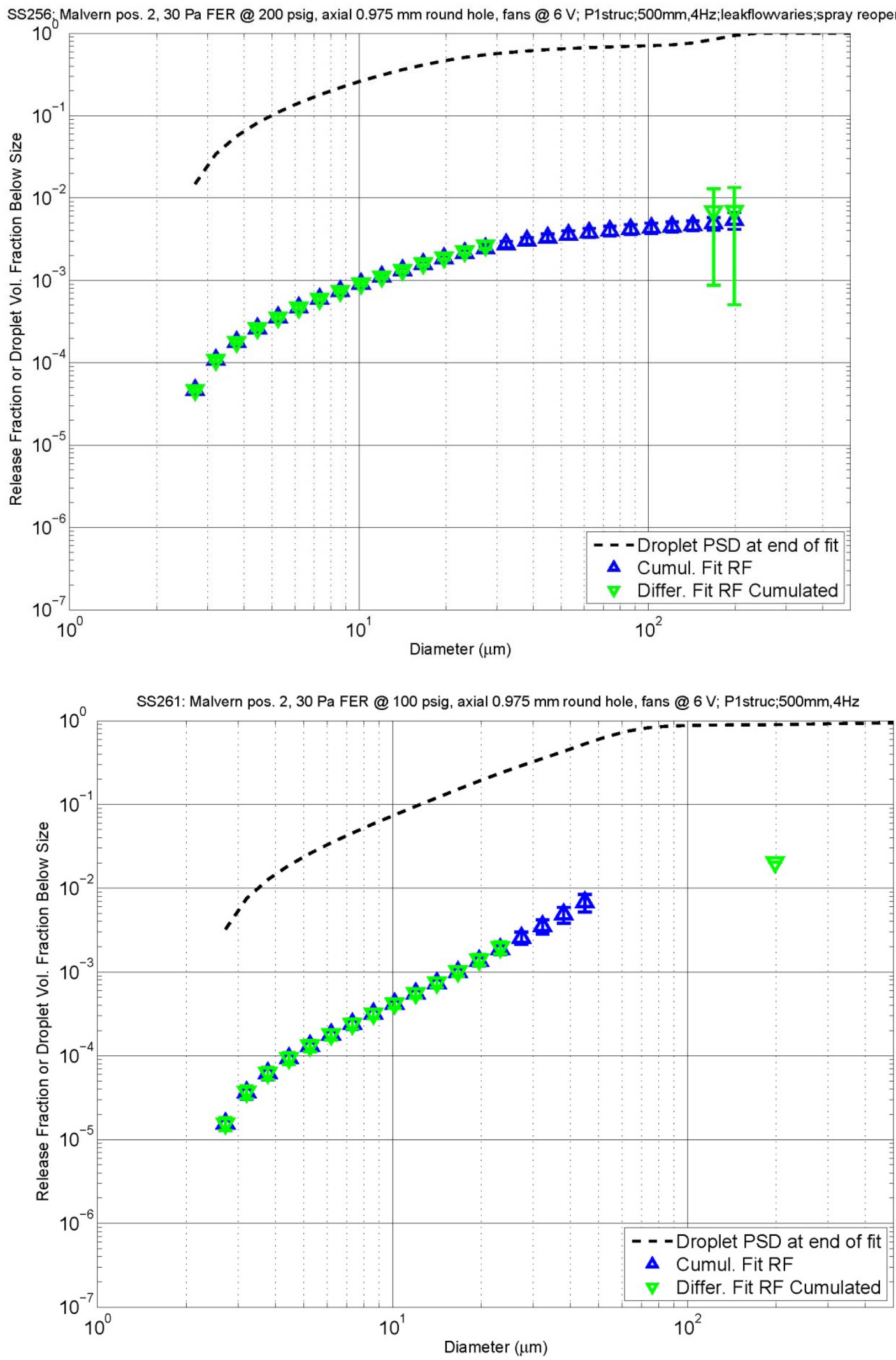

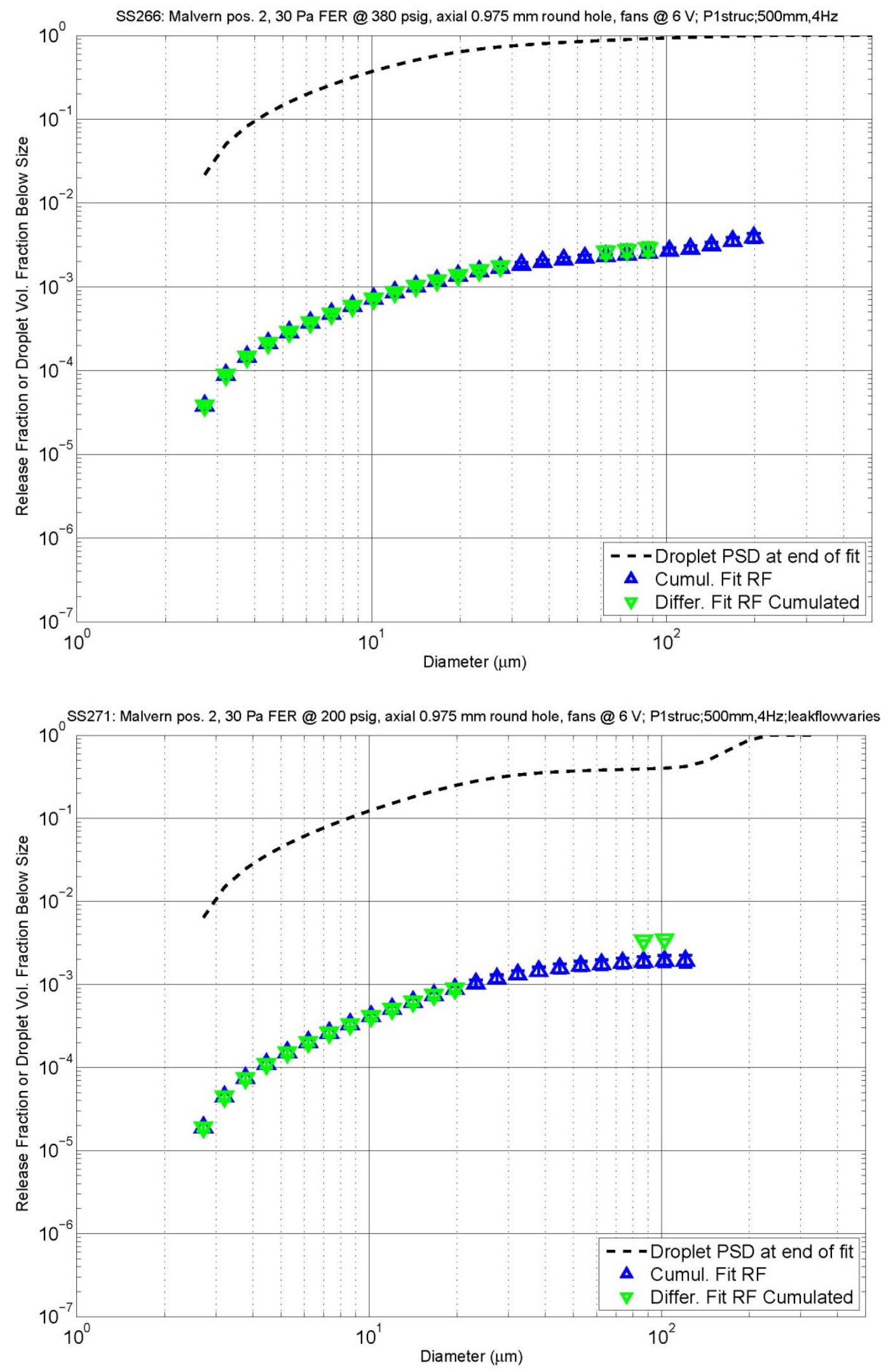

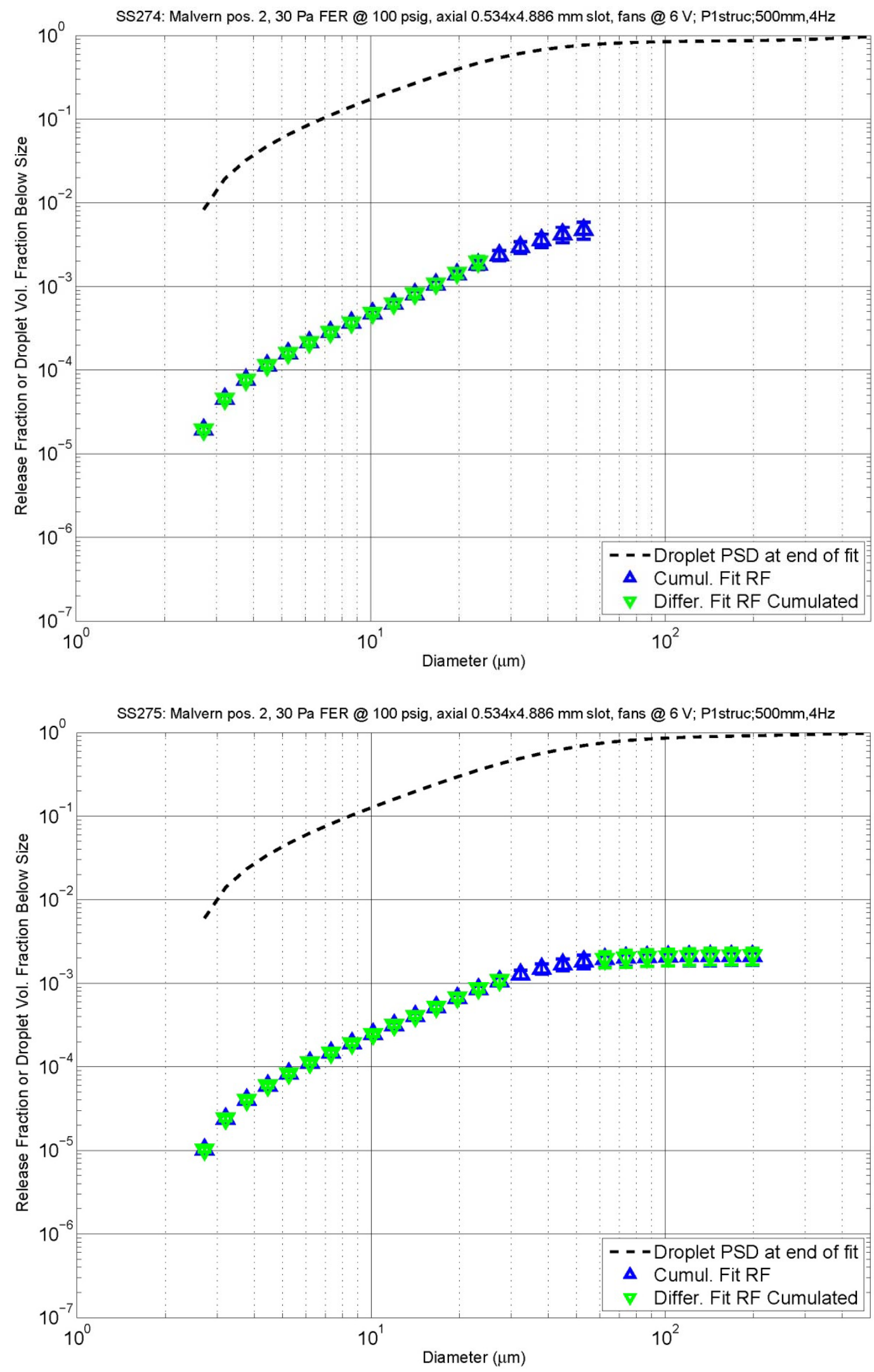

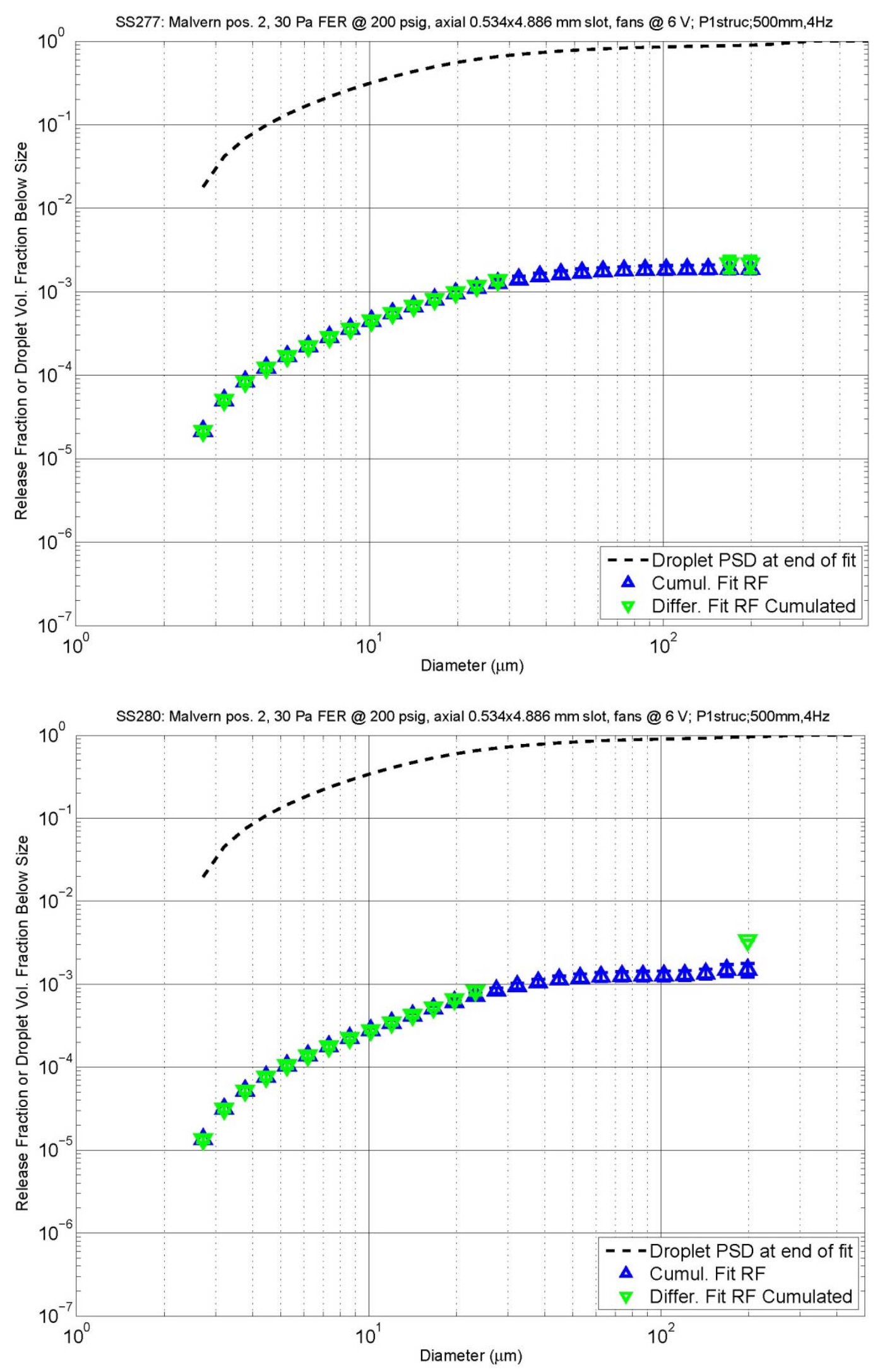

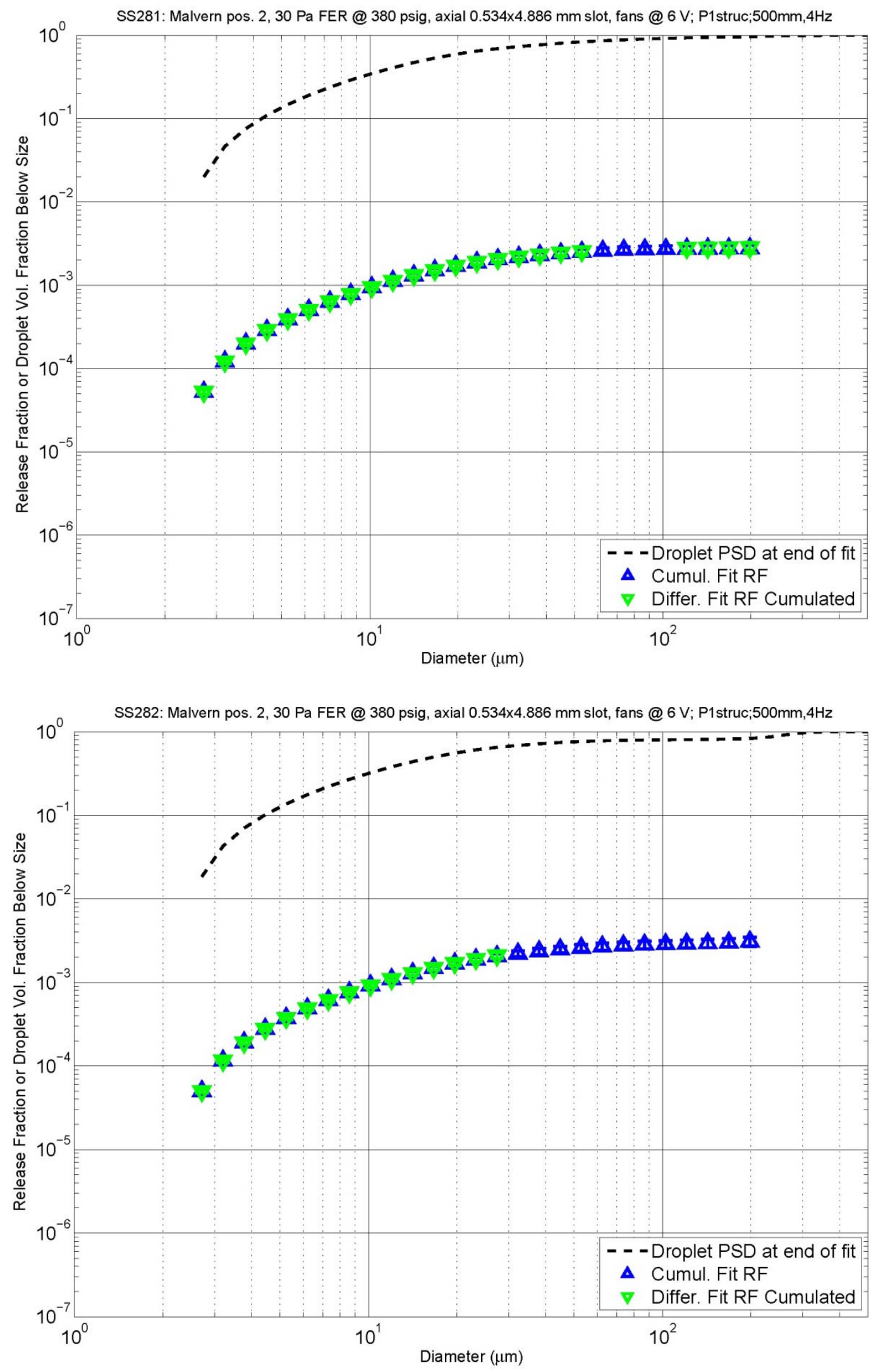

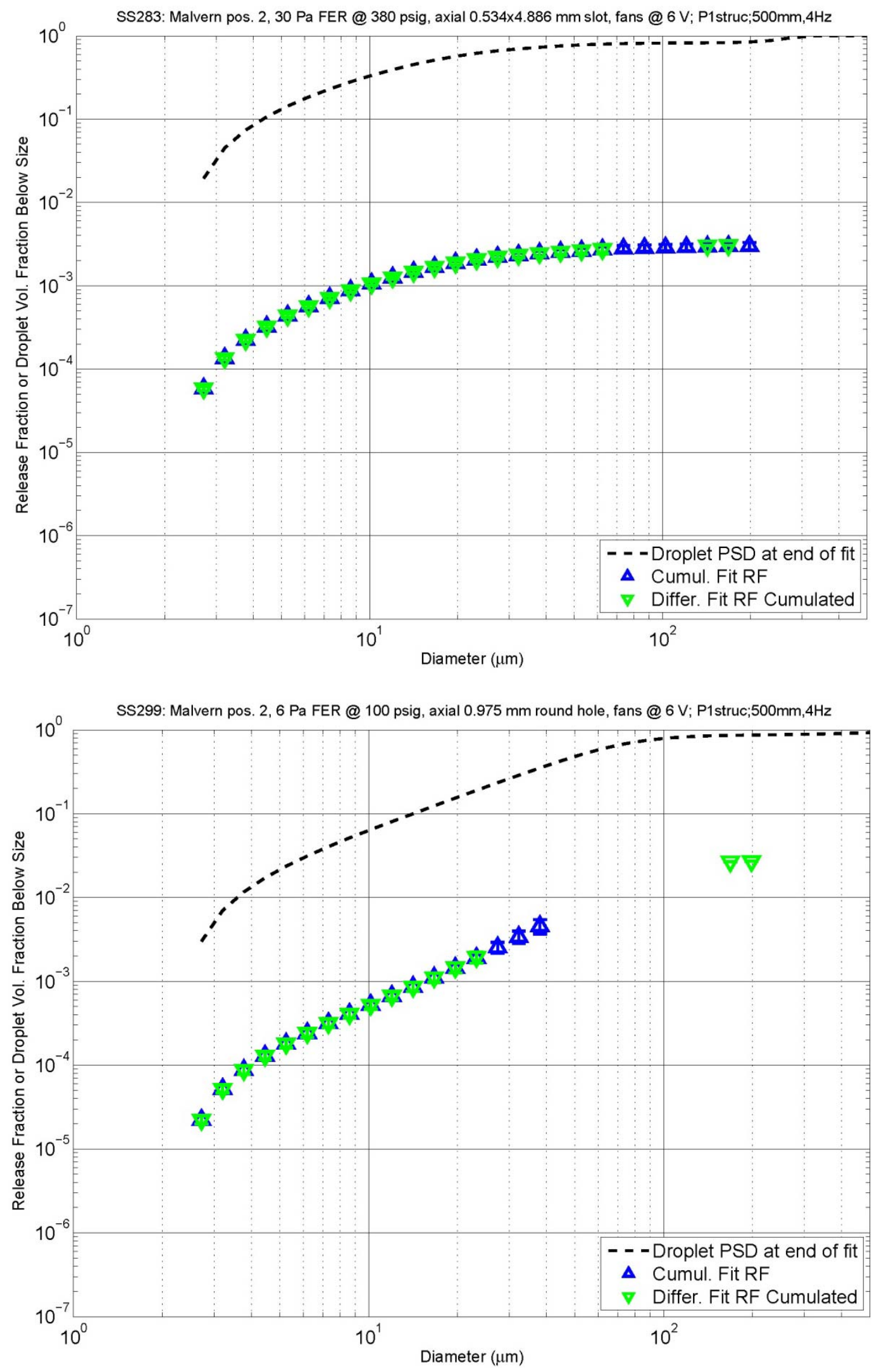

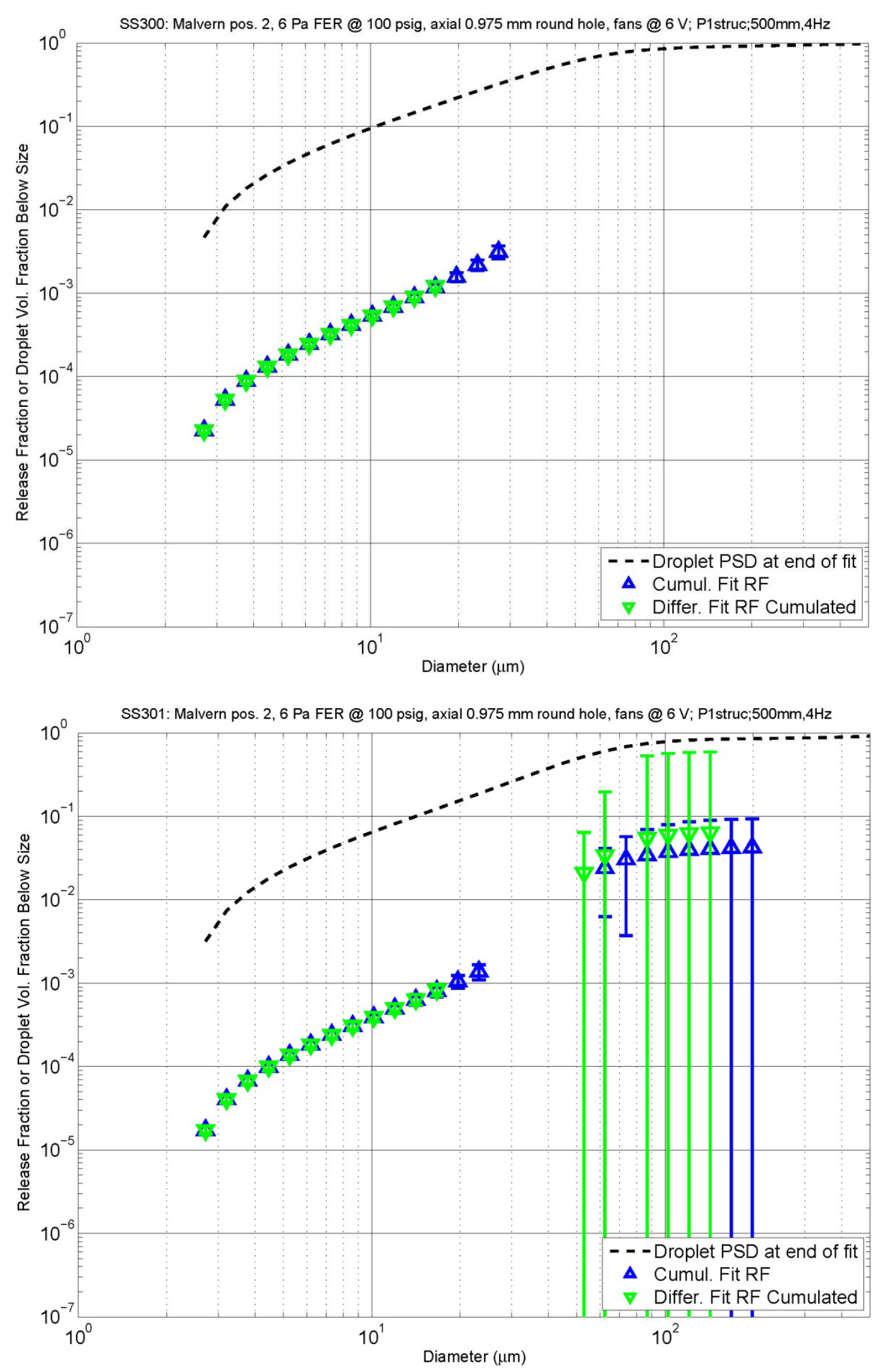

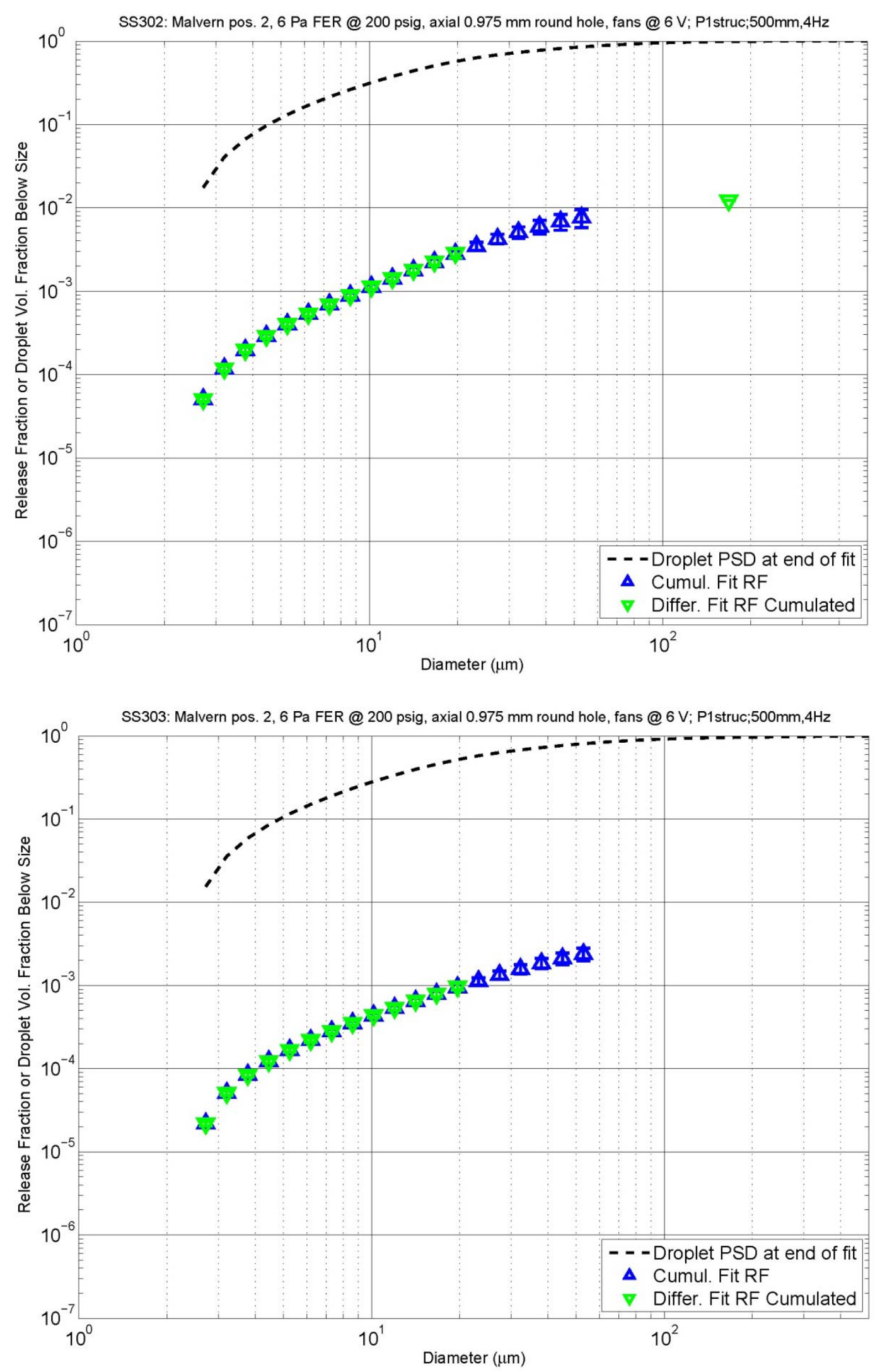

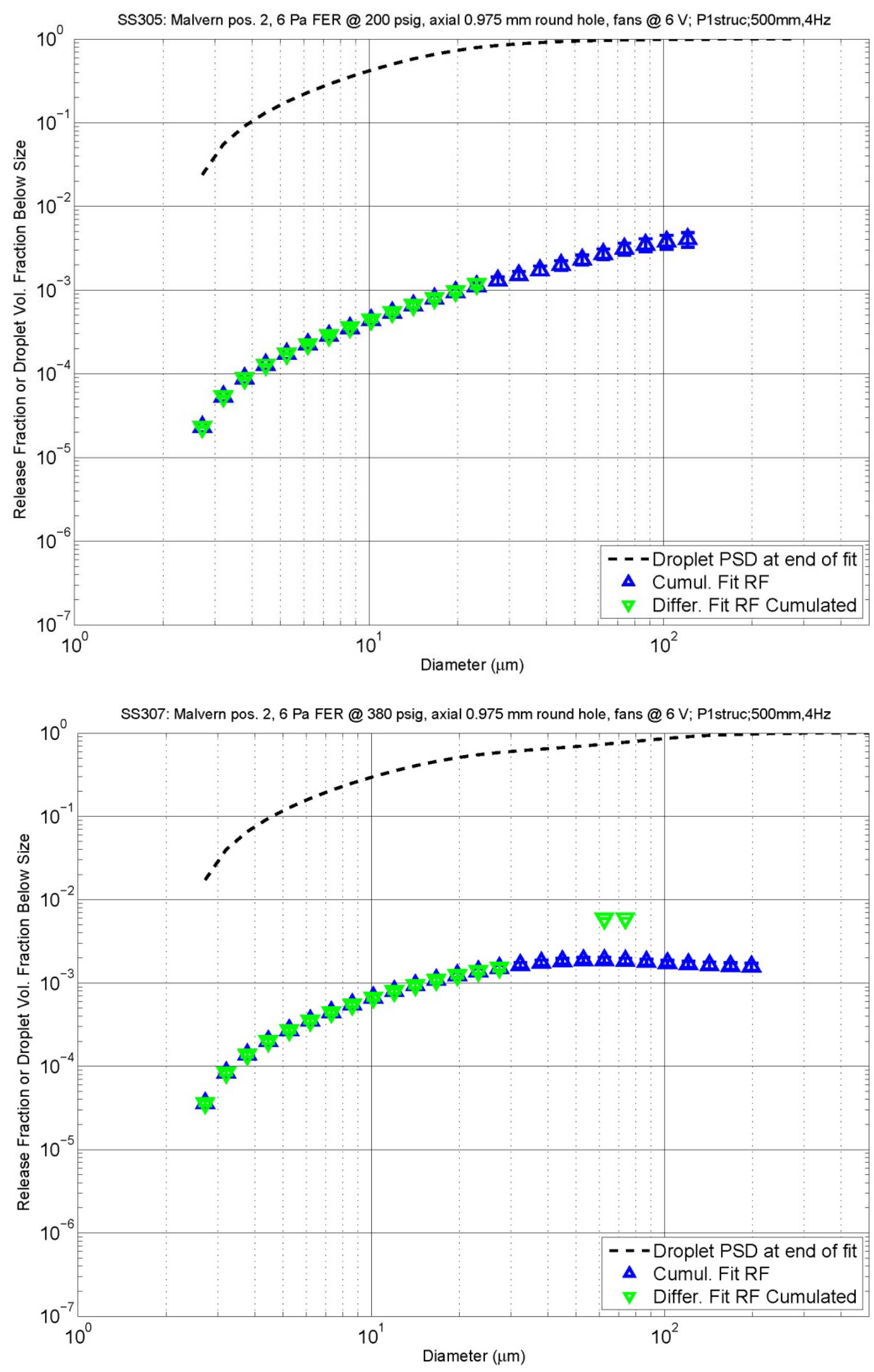

B. 59 

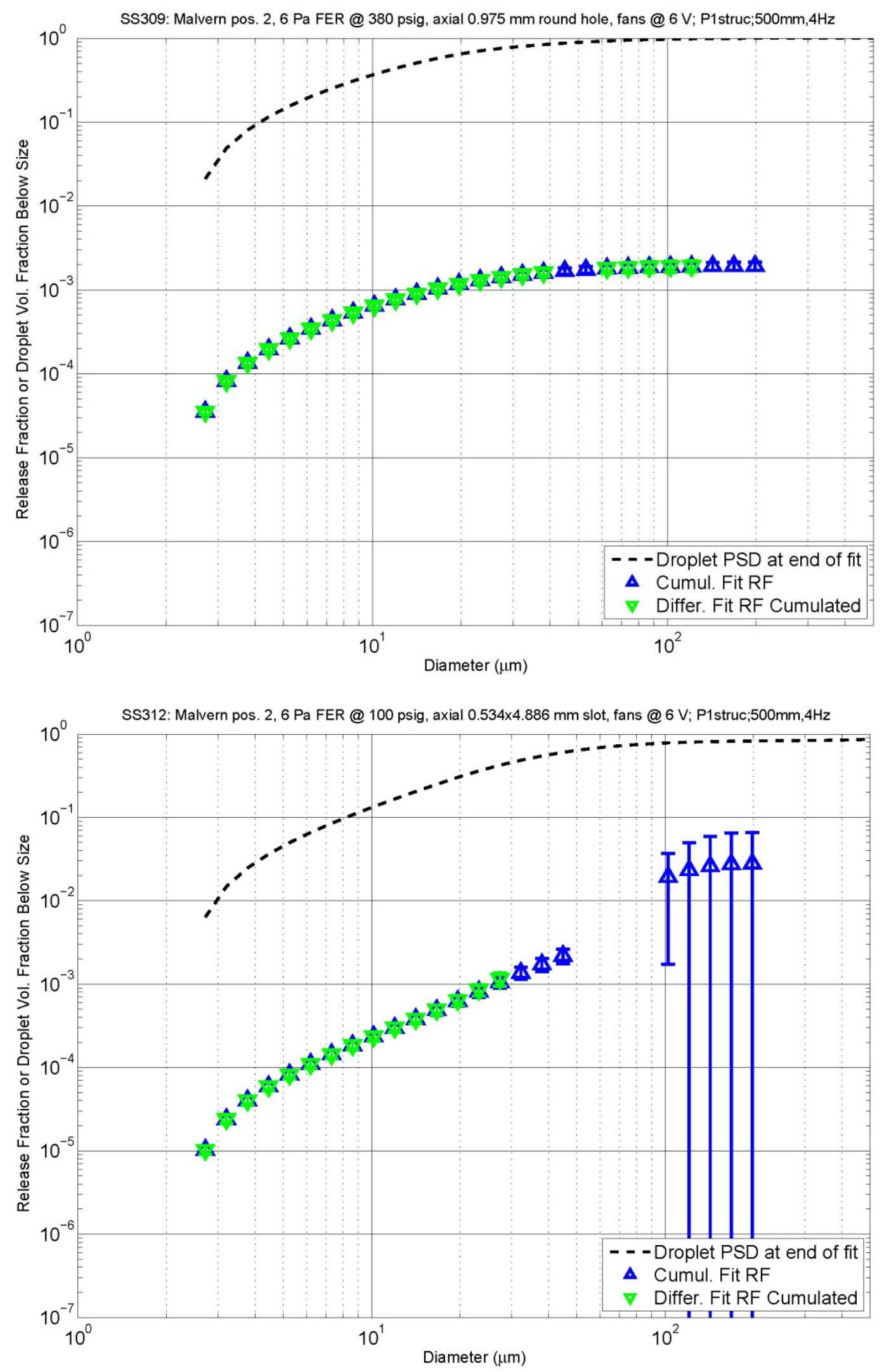

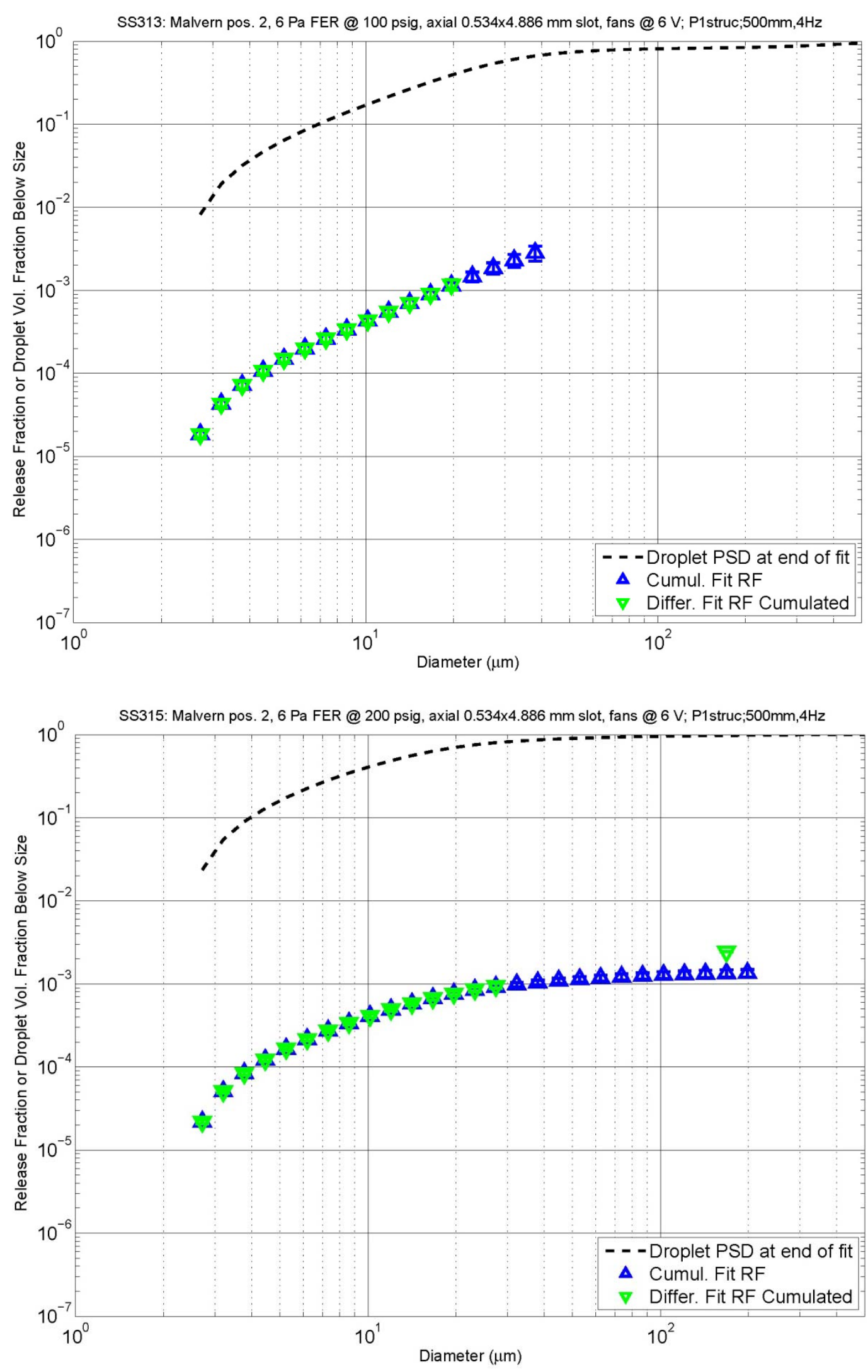

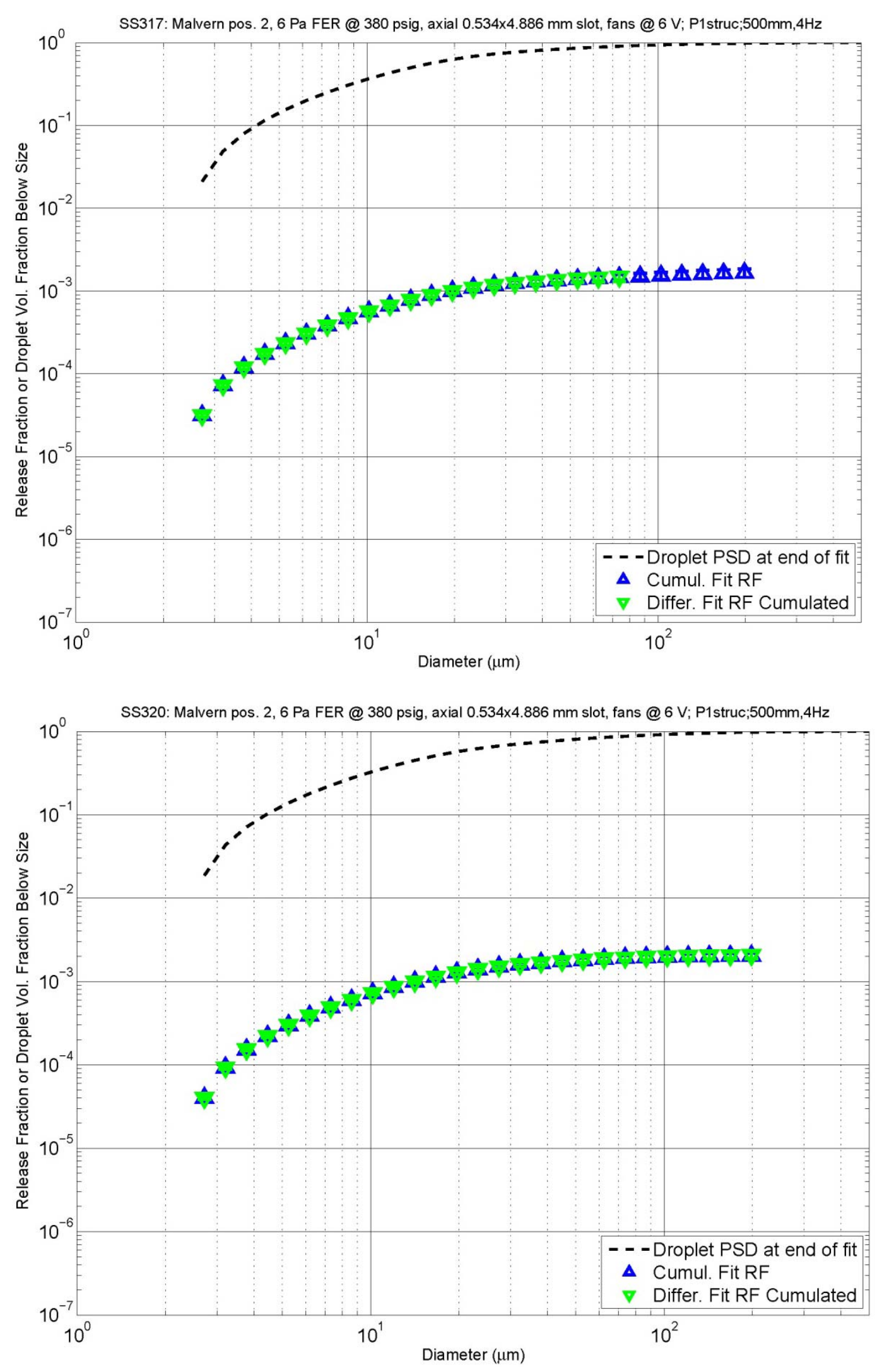

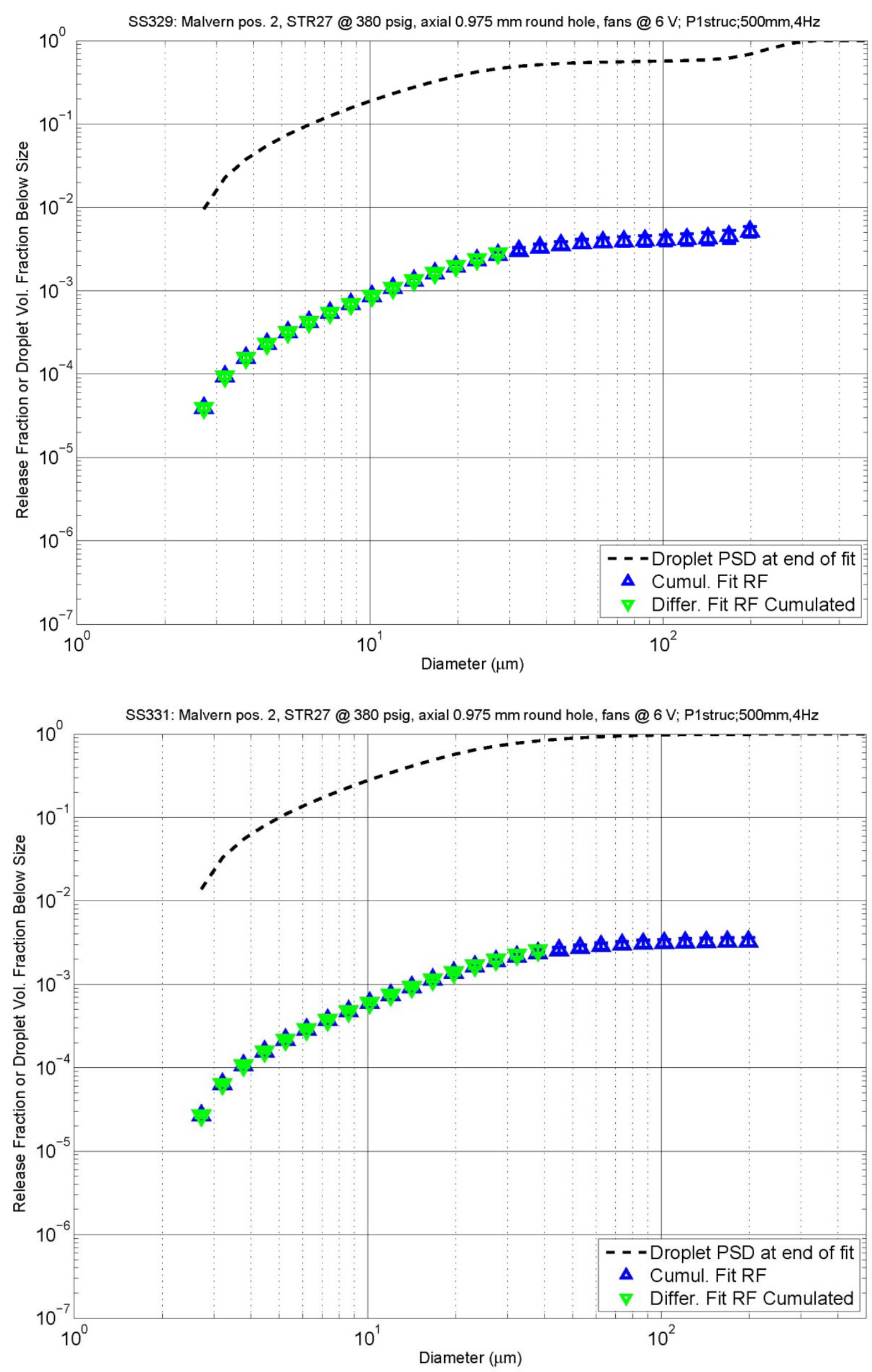

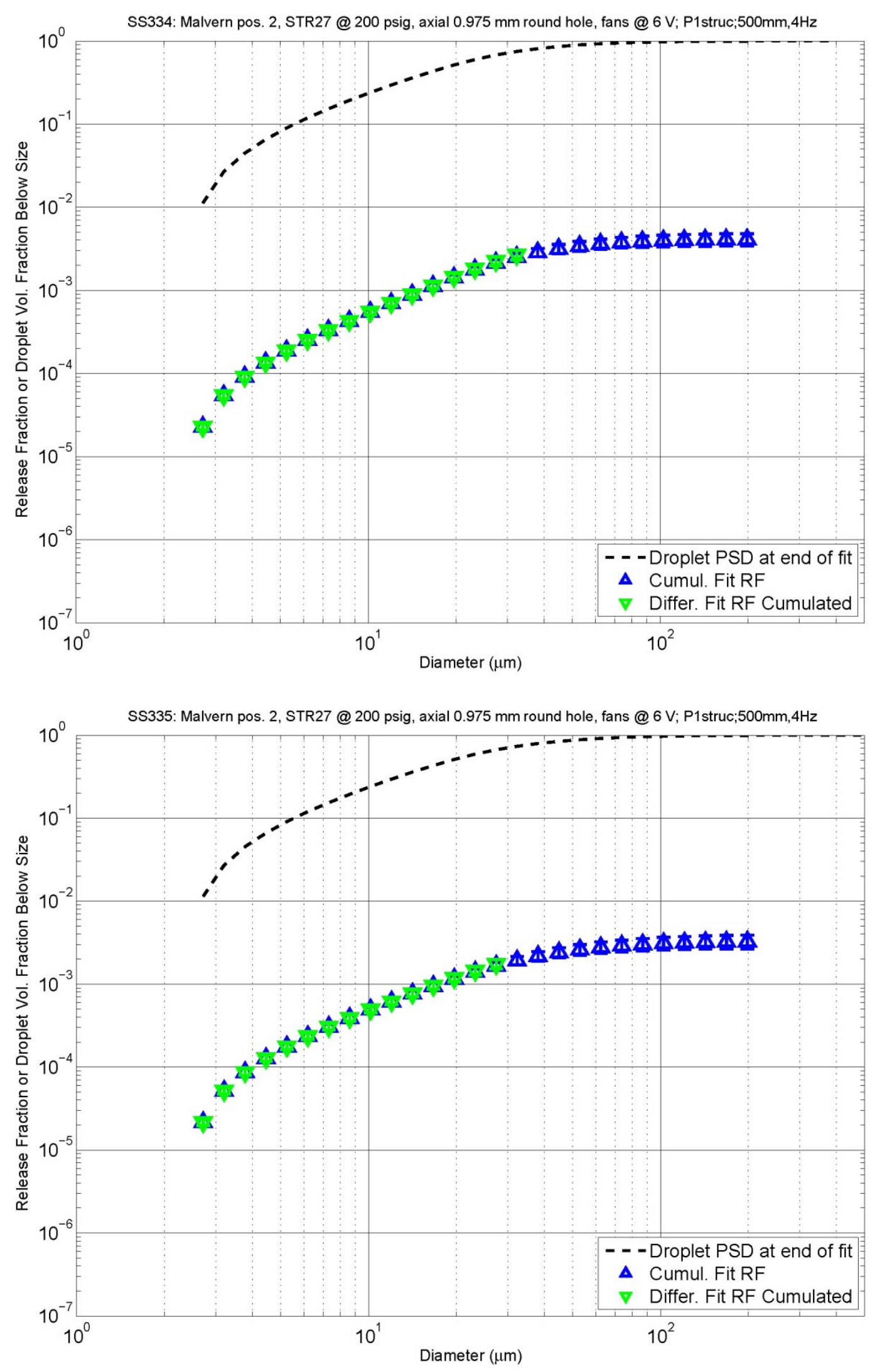

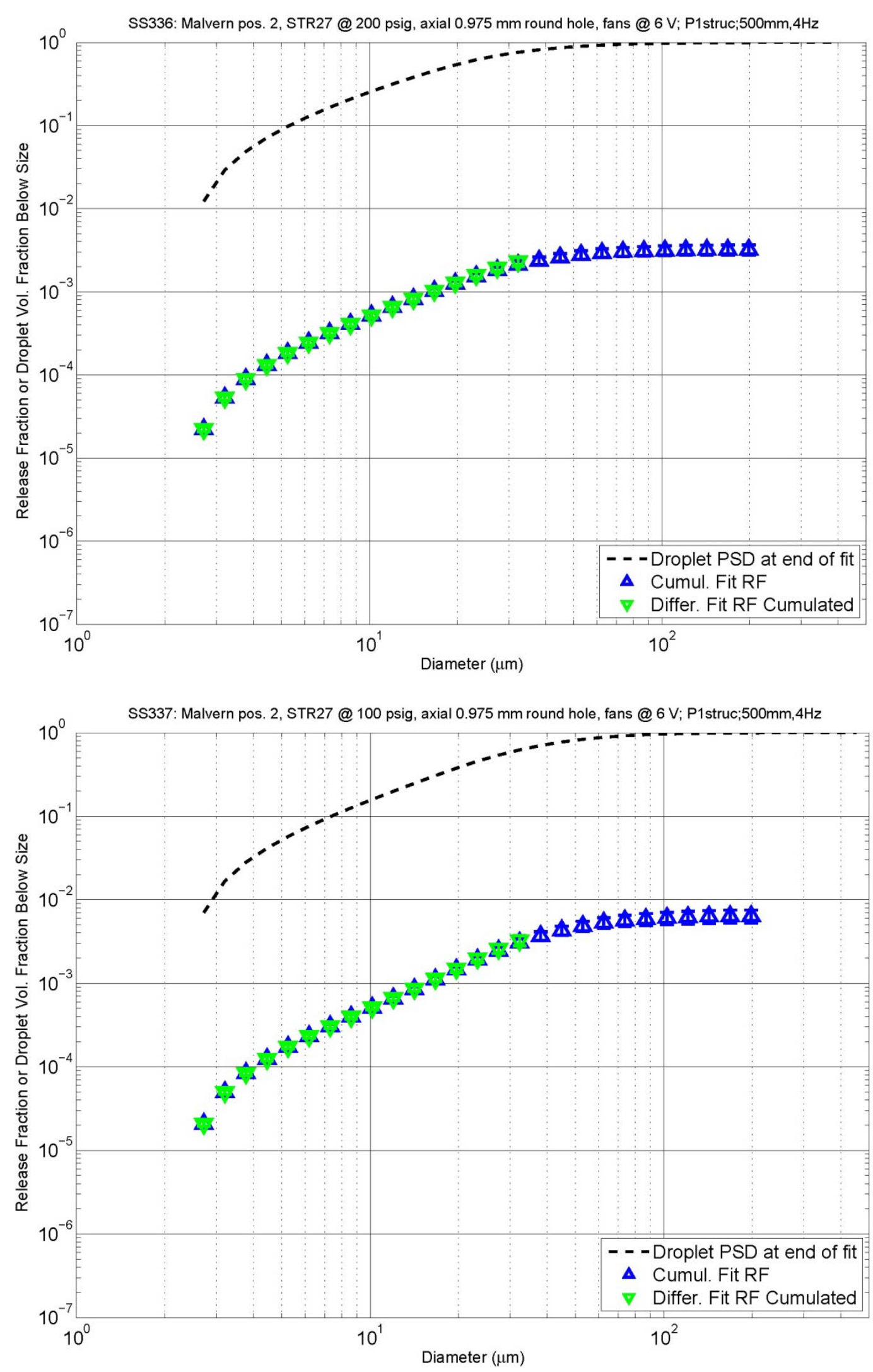

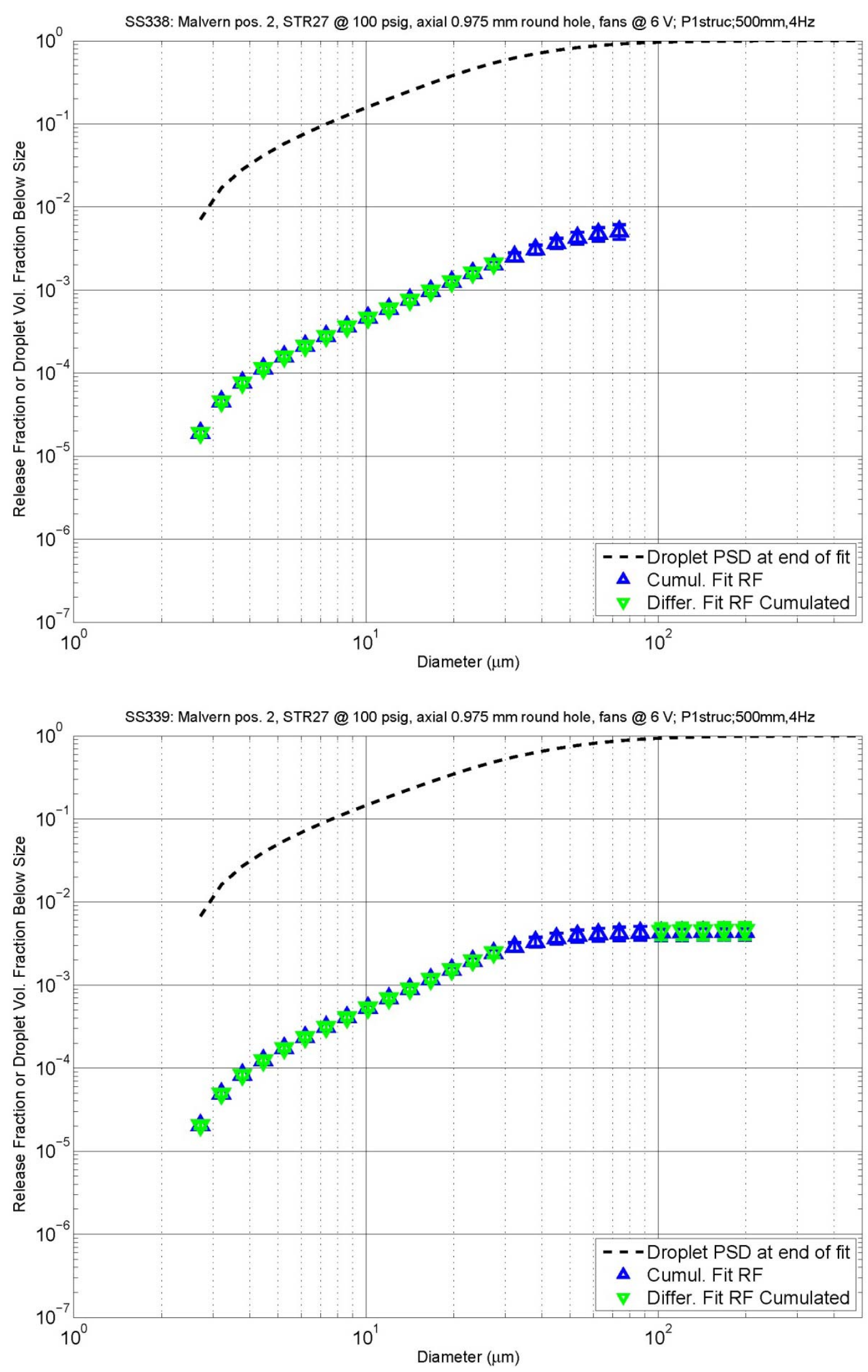

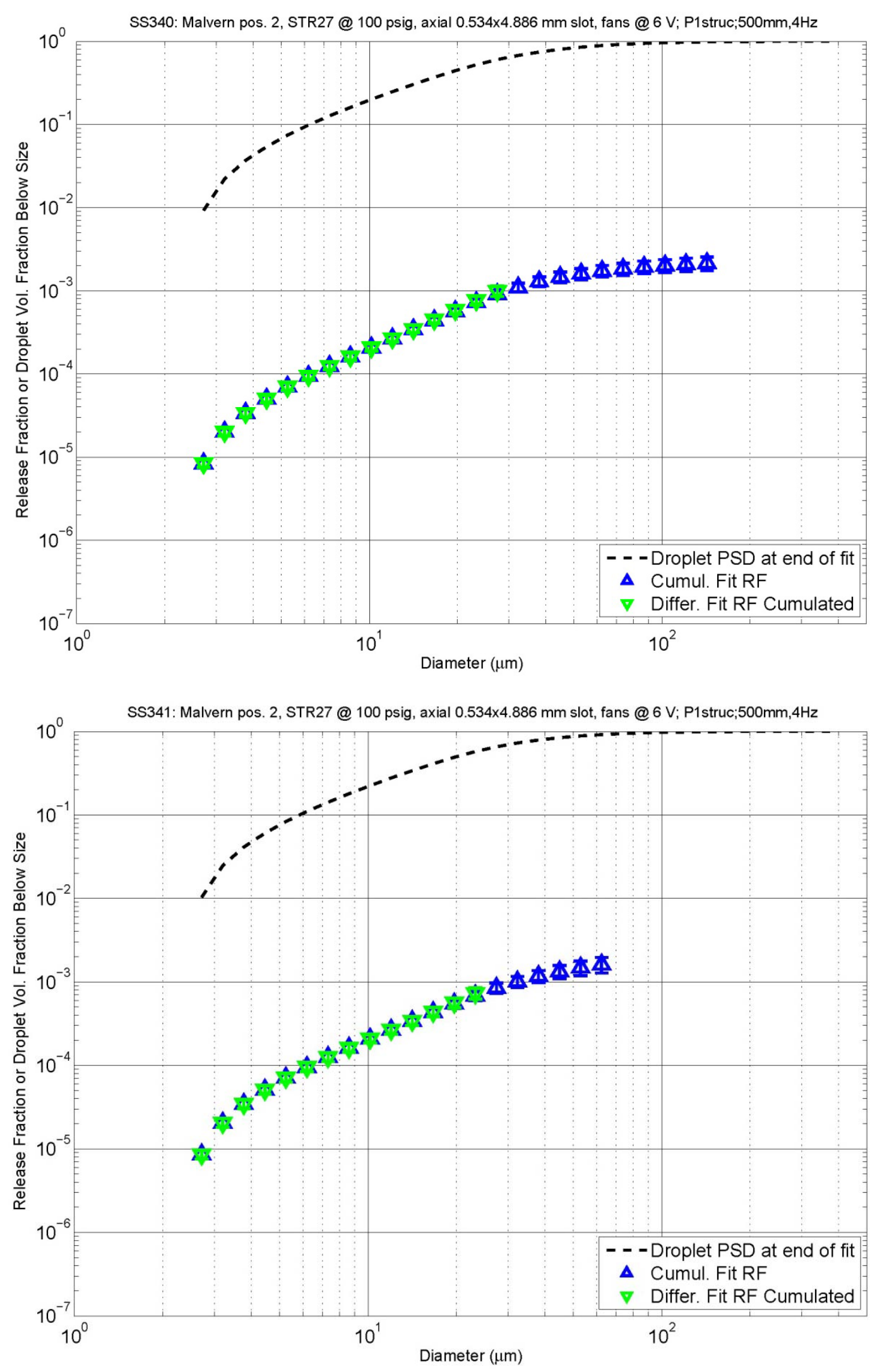

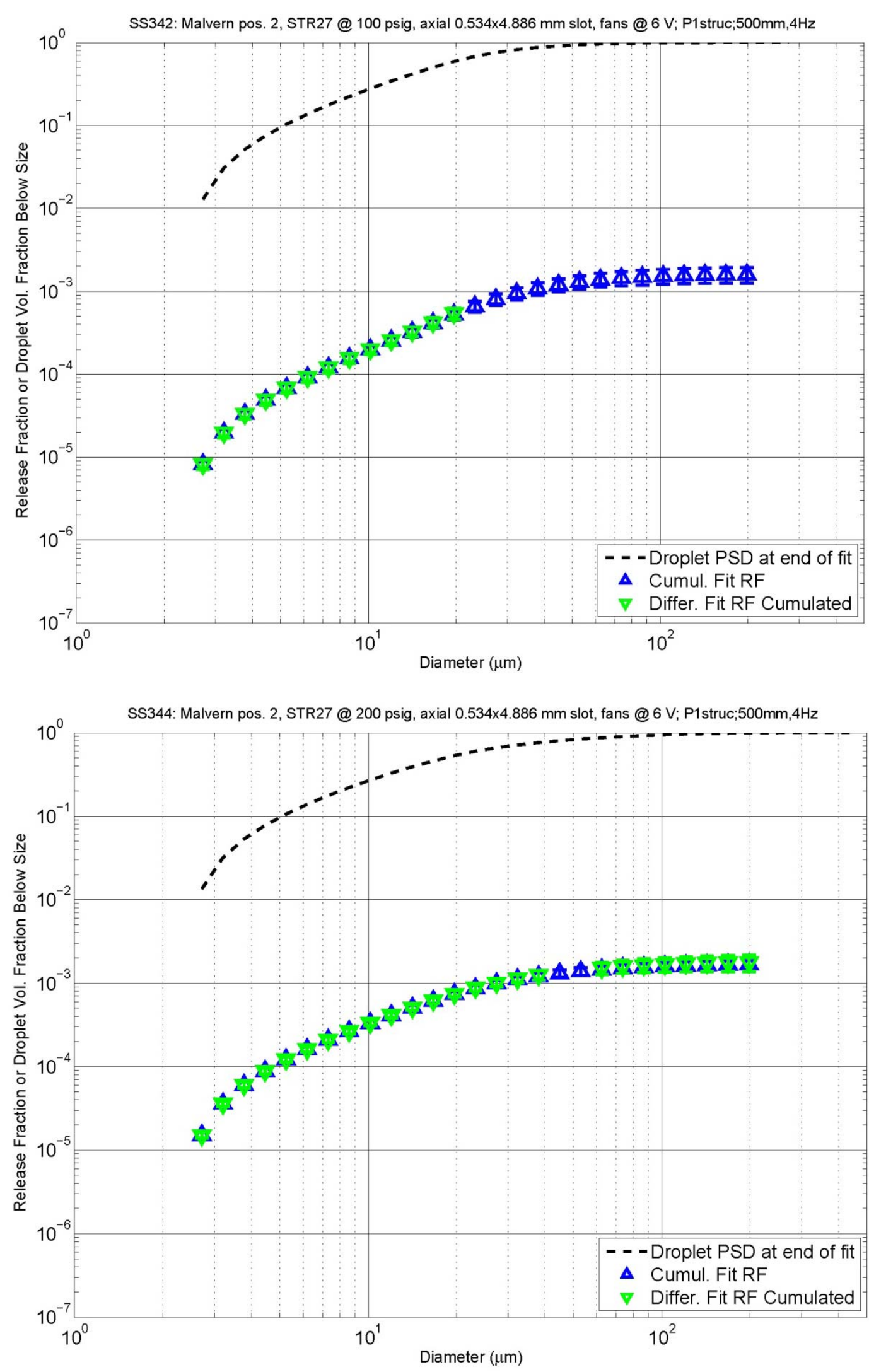

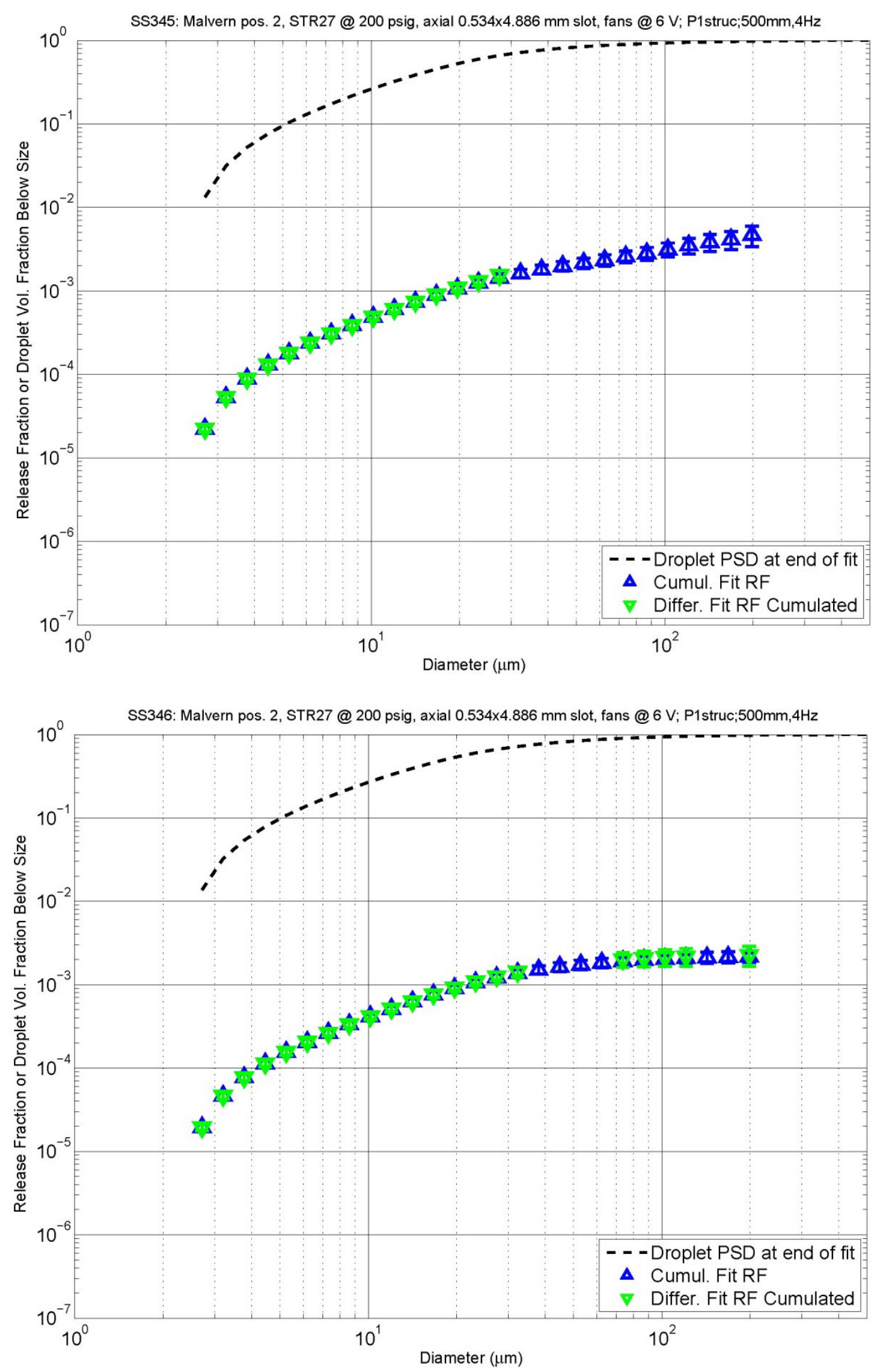

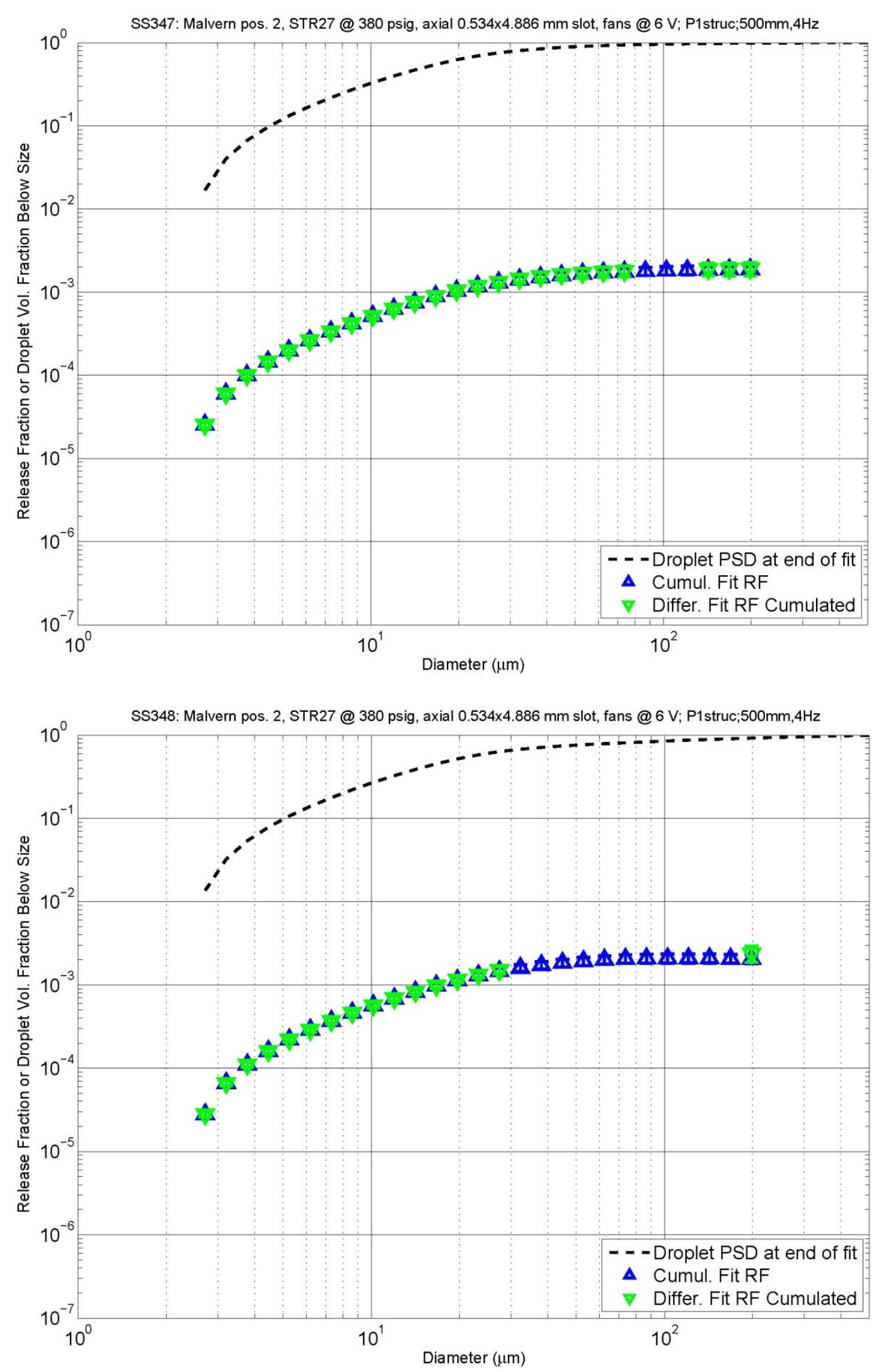

B. 70 

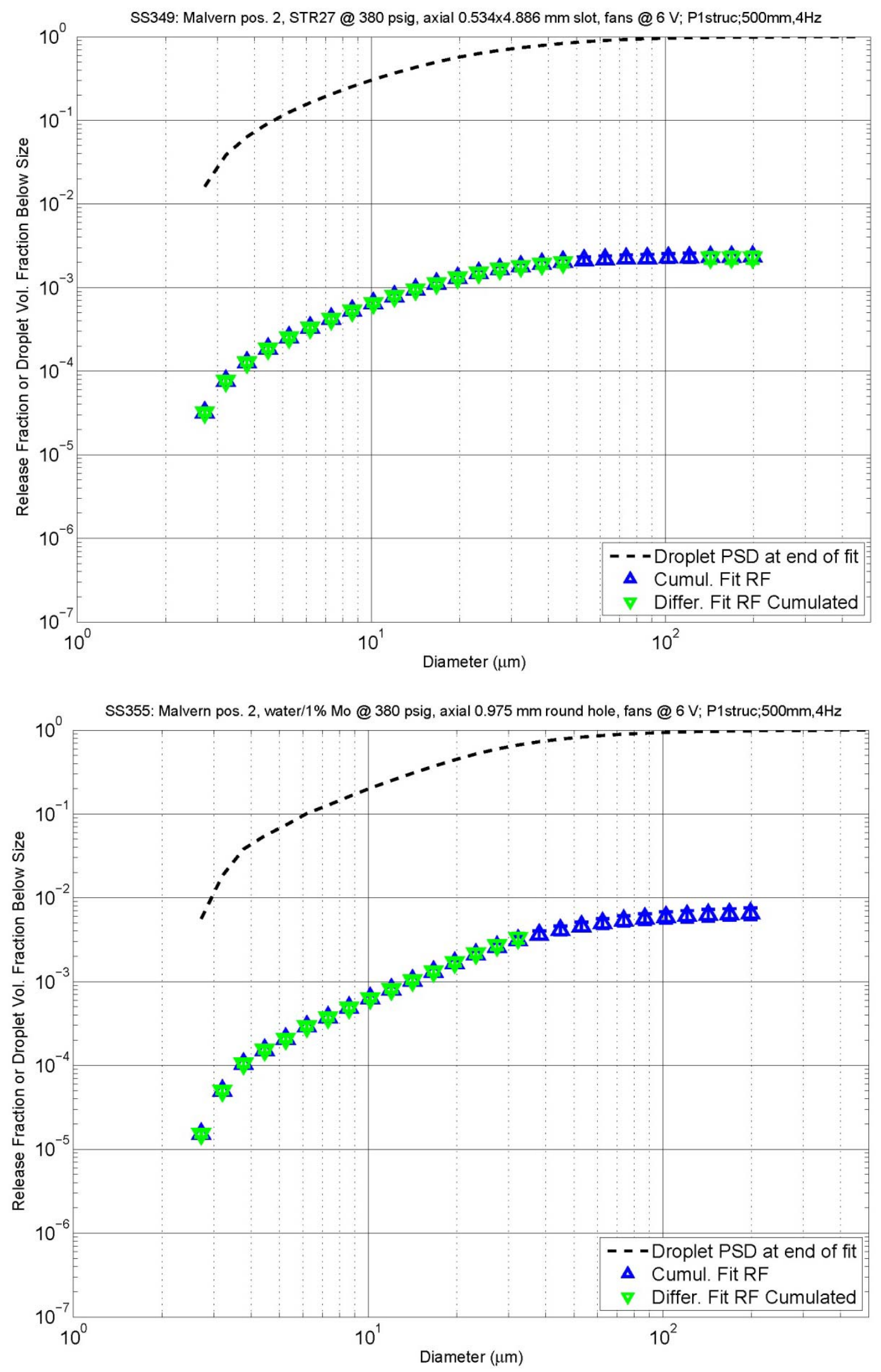

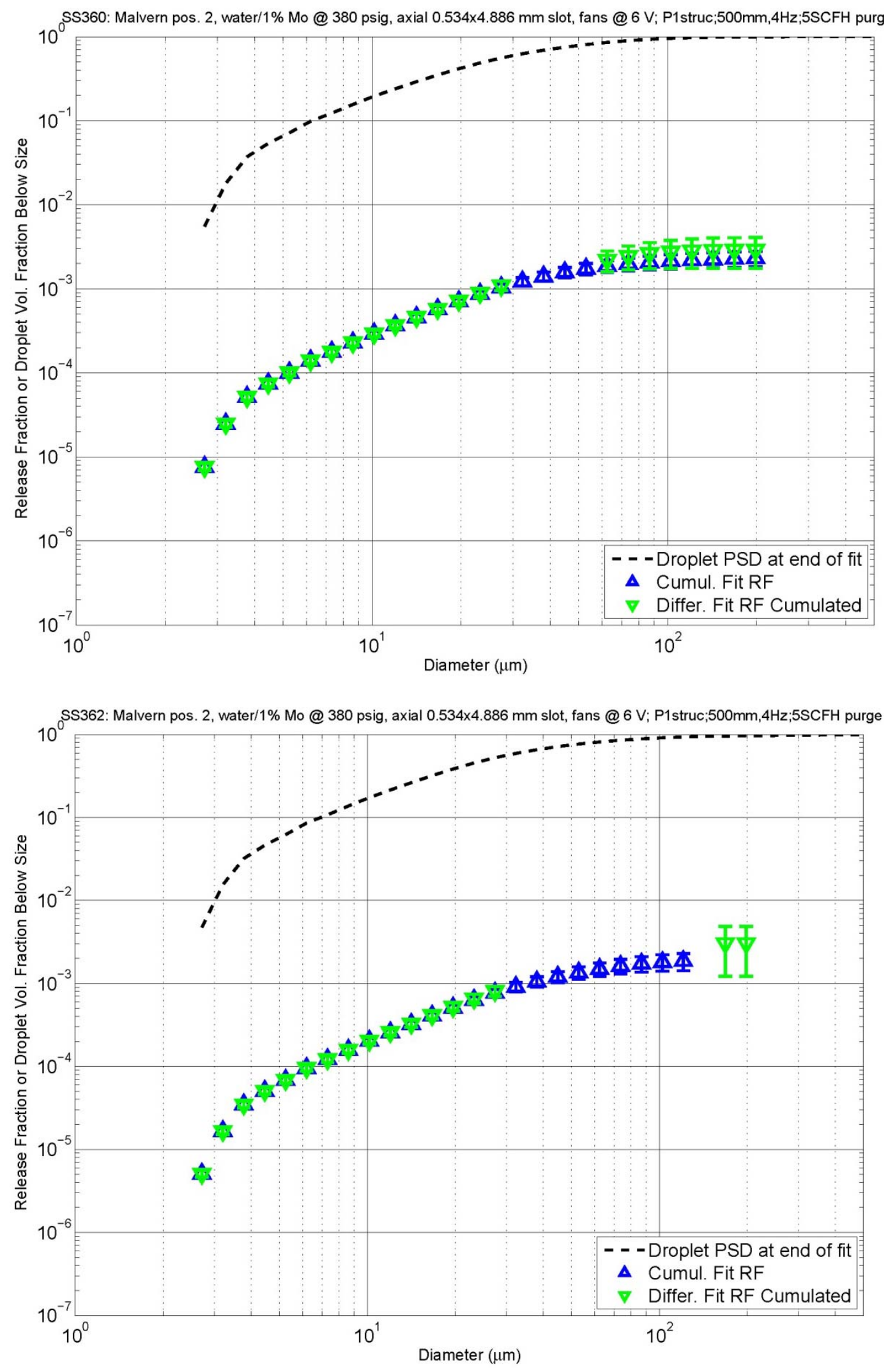

B. 72 

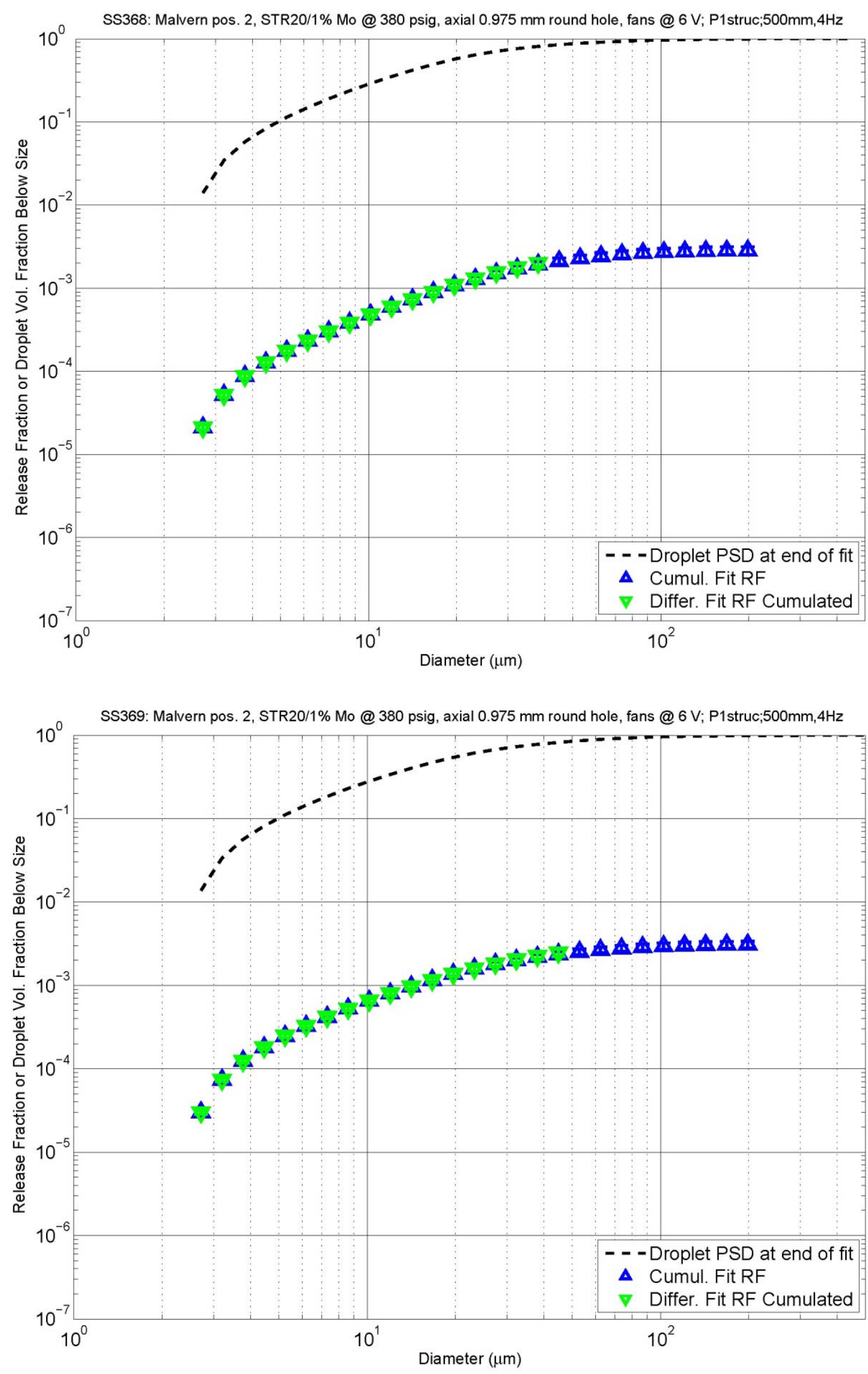

B.73 

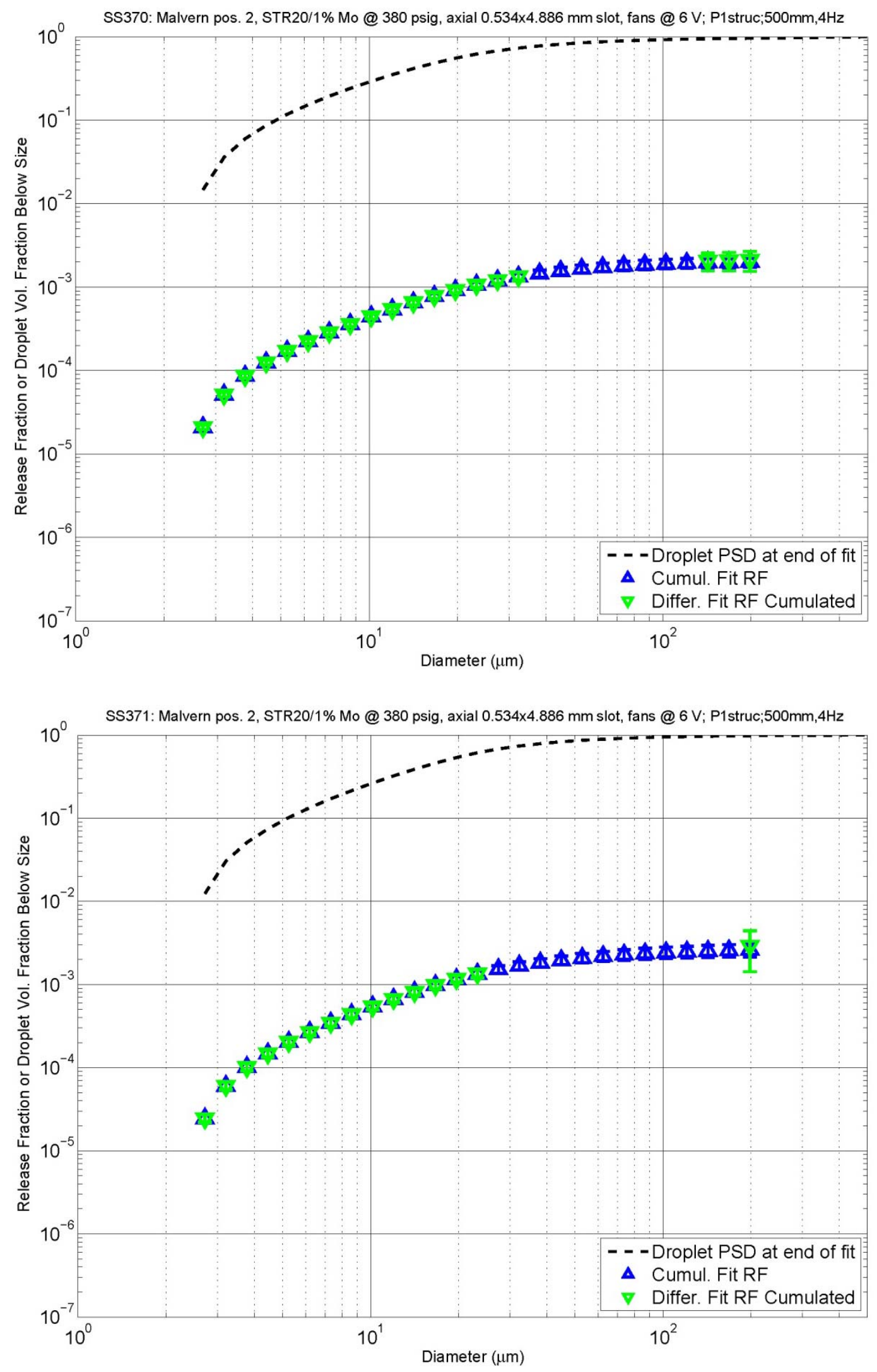

B. 74 

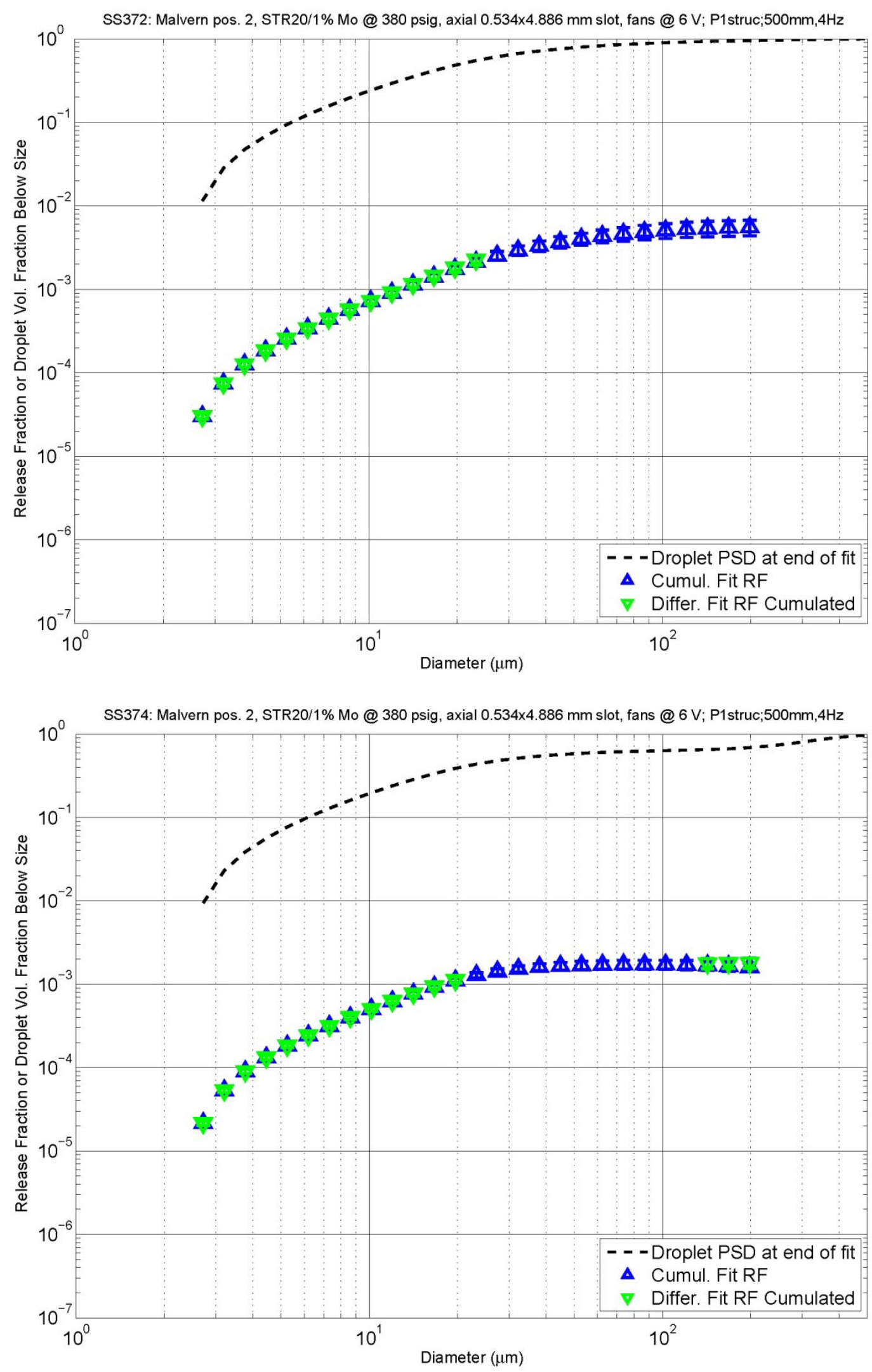

B. 75 

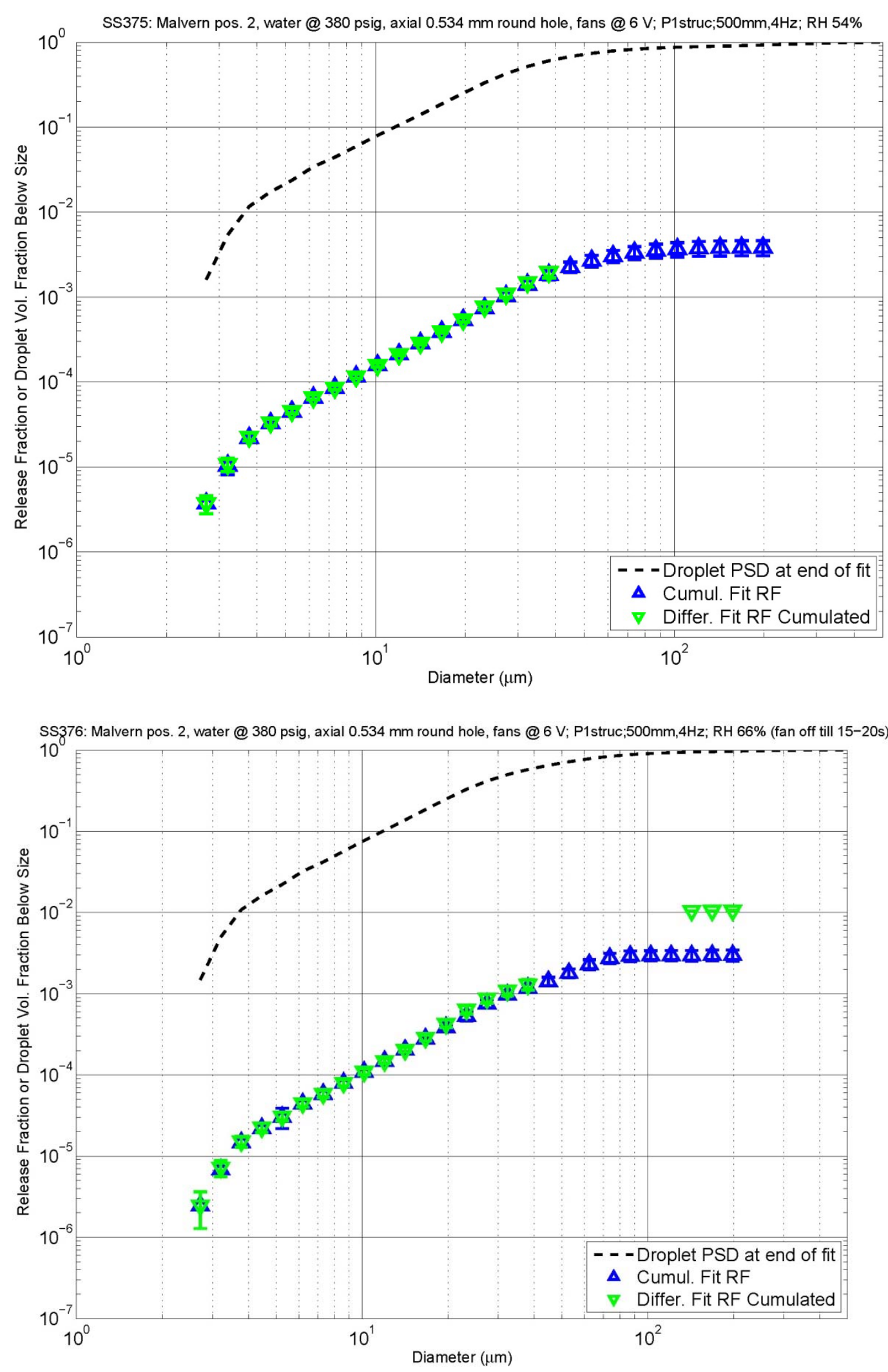

B. 76 

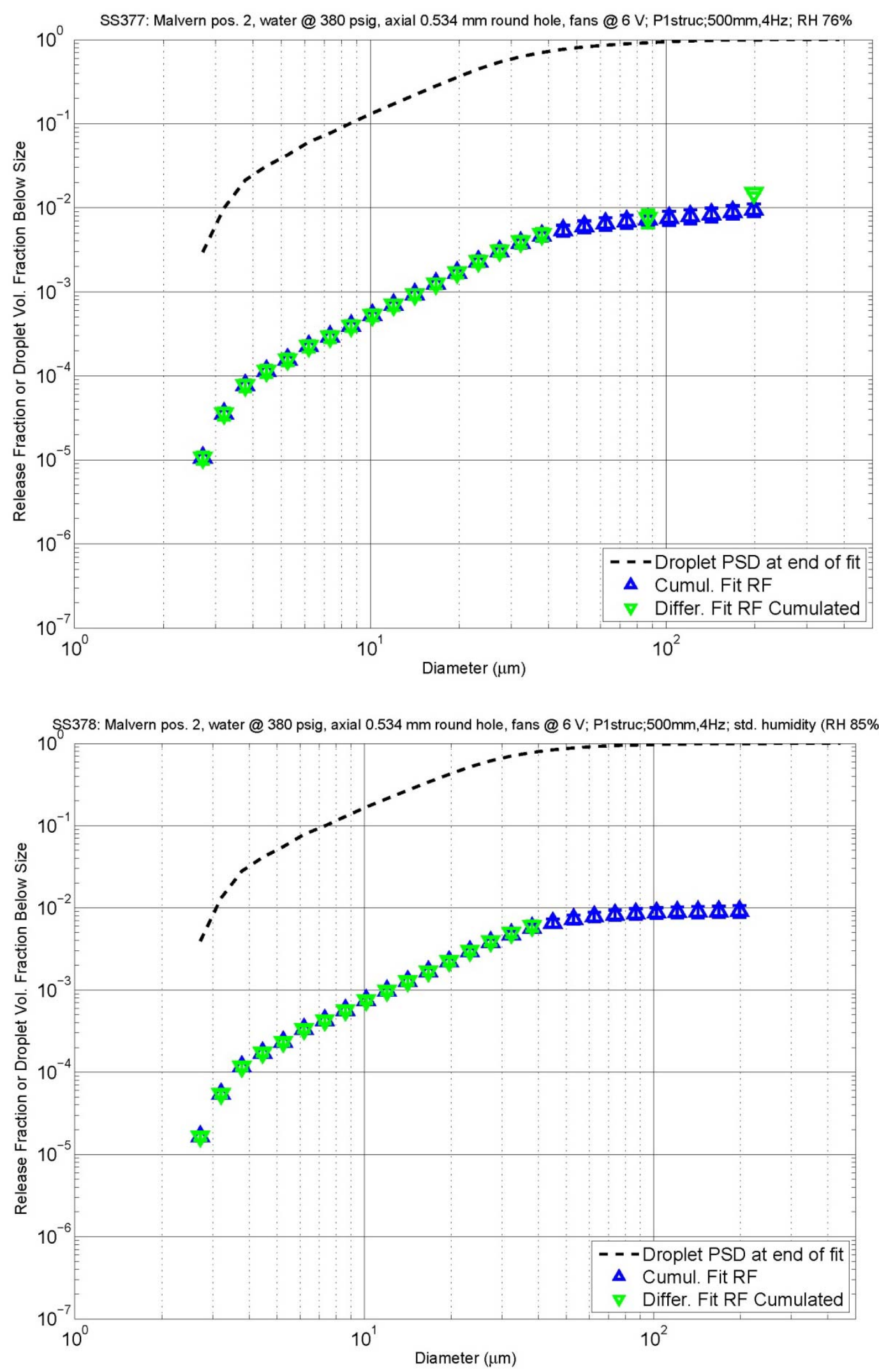

B. 77 

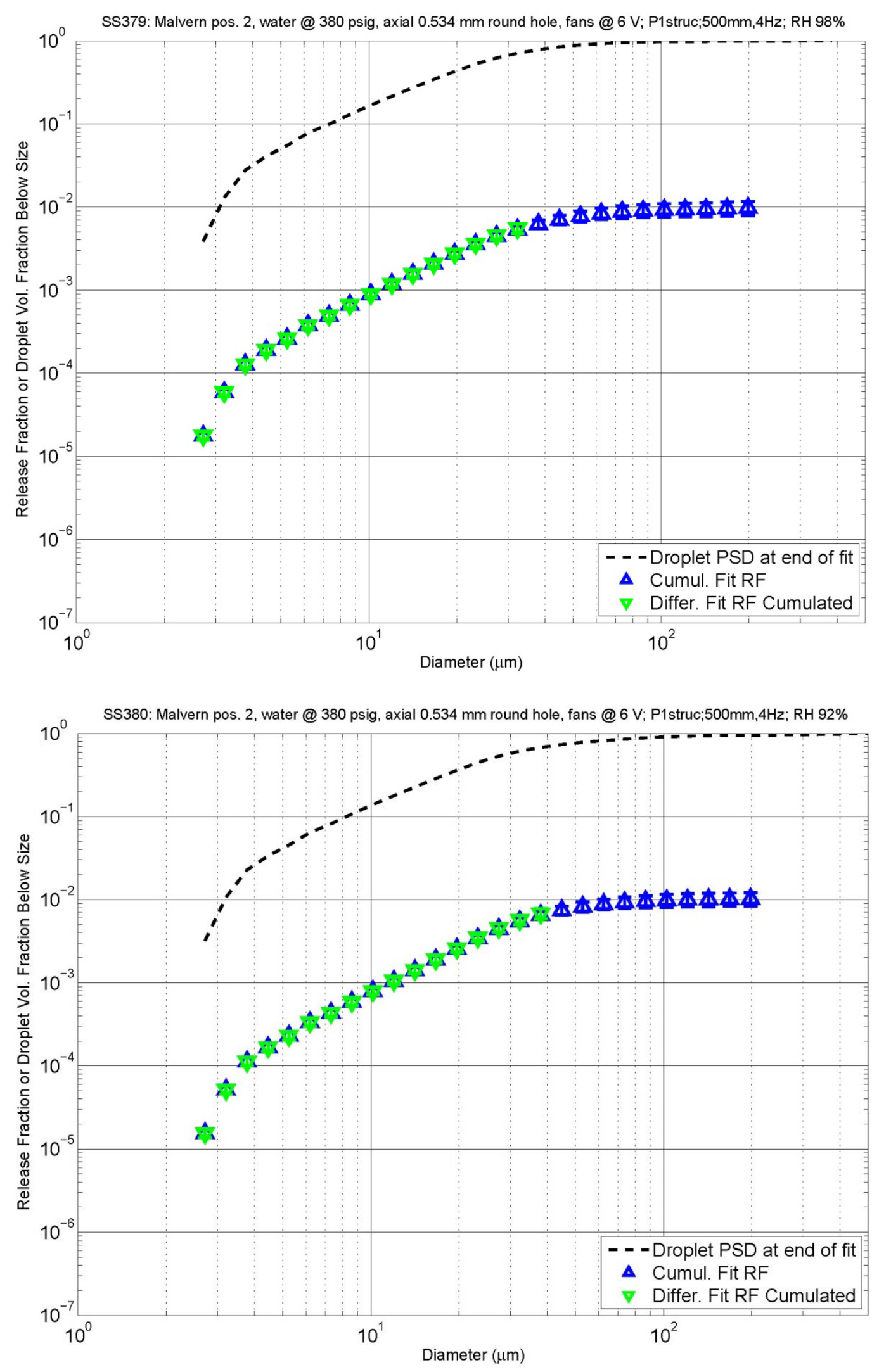

B. 78 

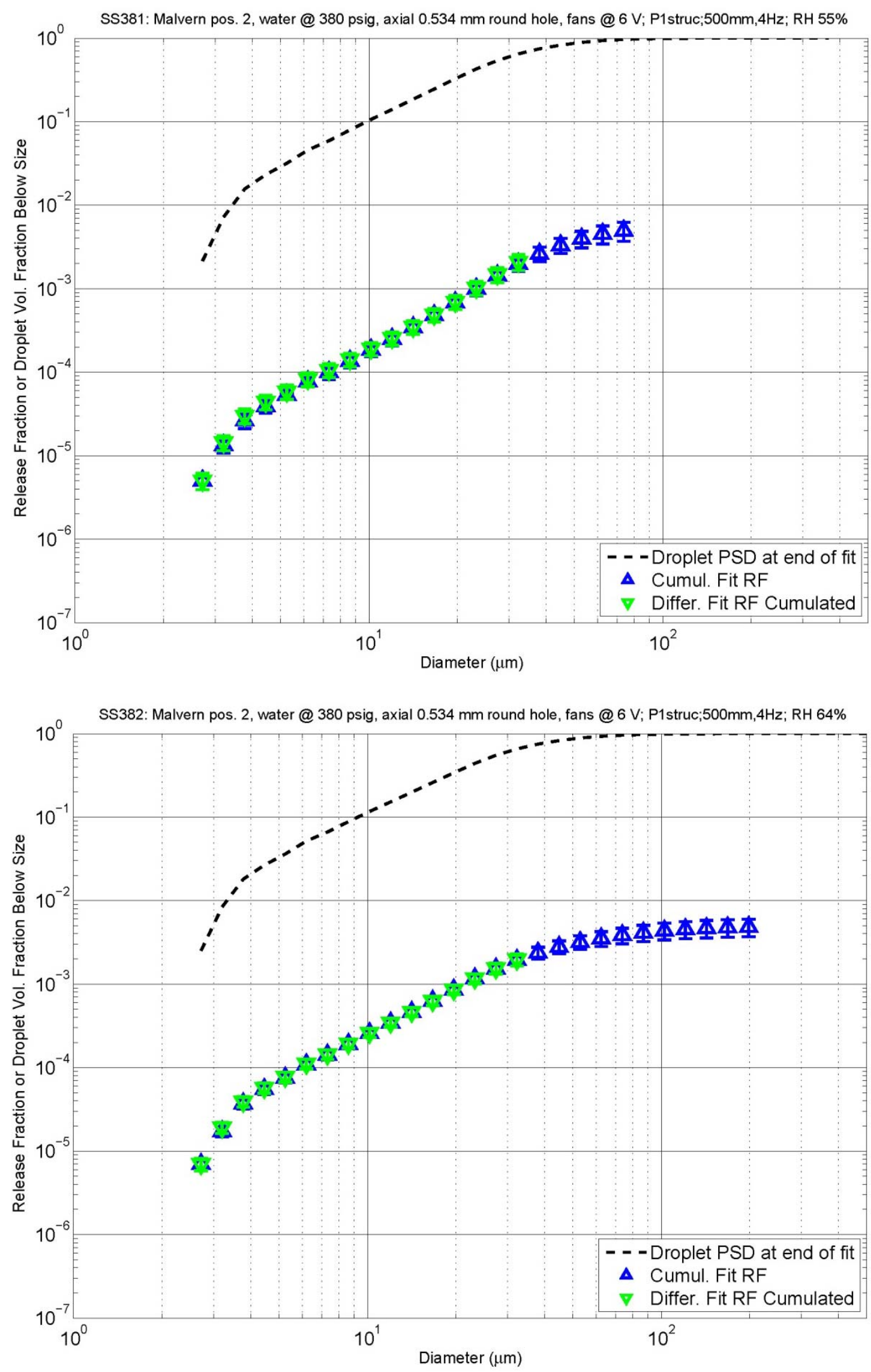

B.79 

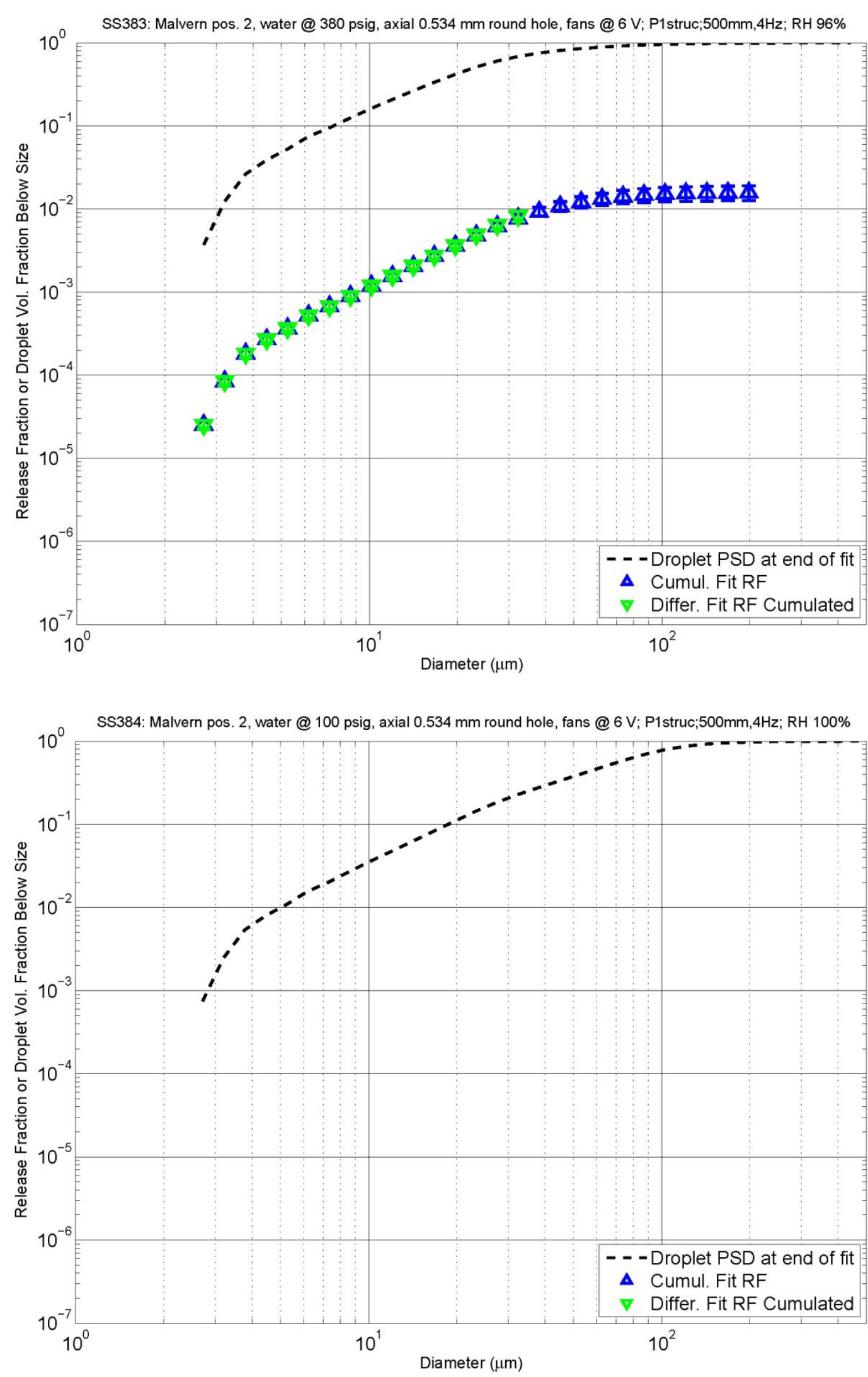

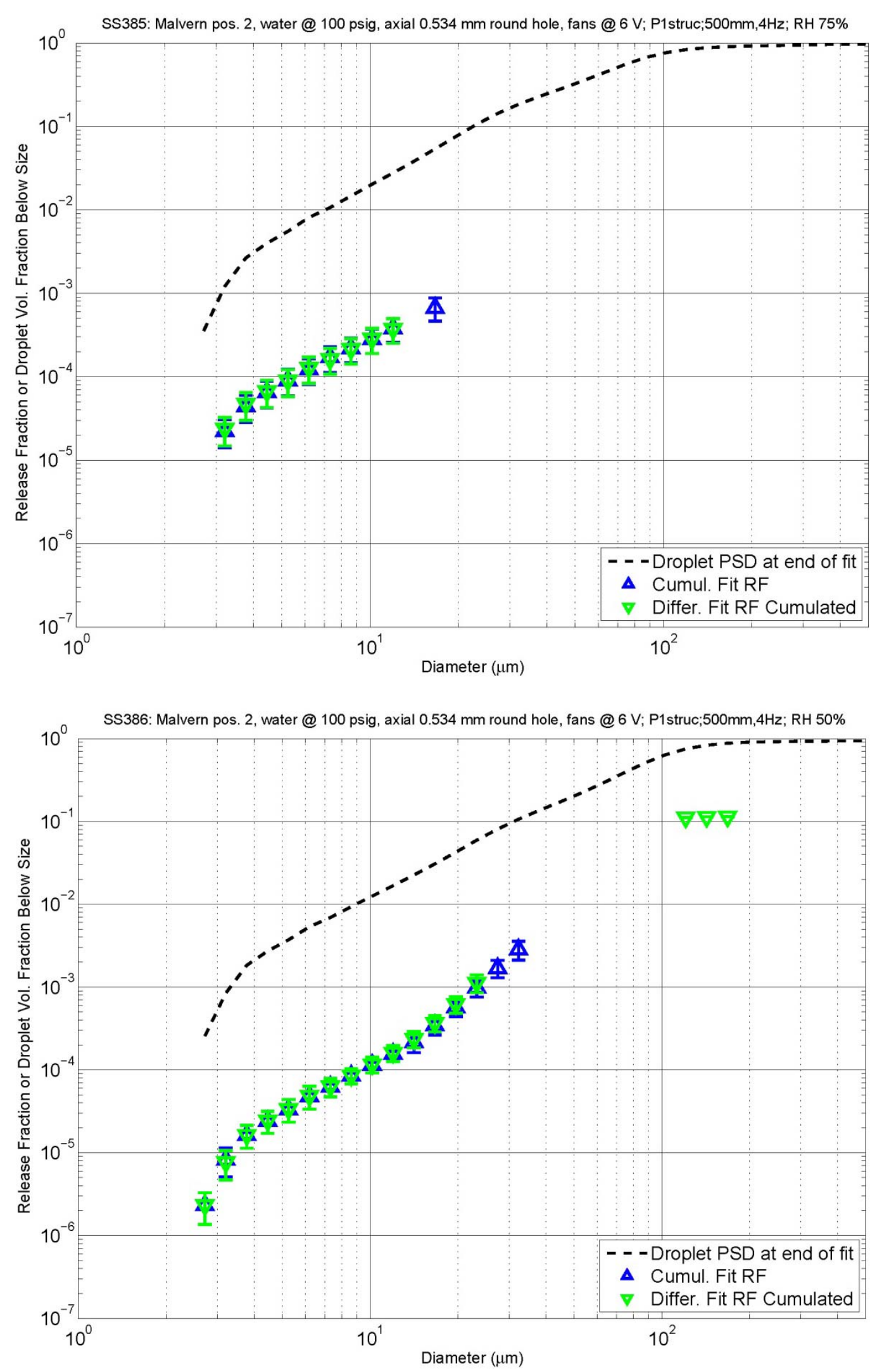

B. 81 

Appendix C

\section{List of Test Documents}





\section{Appendix C}

\section{List of Test Documents}

The test documents that define or describe the aerosol tests are listed below.

- Gauglitz PA. 2012. Test Plan for Spray Leak Quantification to Support WTP Spray Release Methodology, TP-WTPSP-031 R1.0. Appendix A of the test plan describes the basis for simulant development.

- Schonewill PP. 2012. Spray Release Methodology: Phase II Small-Scale Aerosol Release Tests Project Plan, PP-WTPSP-089 R0.0.

\section{Test Instructions}

- TI-WTPSP-090 (“Test Instruction and Procedure for Phase II Small Scale Aerosol Tests”).

- TI-WTPSP-043 (“Simulant Blending to Support Phase II Small-Scale Spray Testing”).

\section{Operating Procedures}

- OP-WTPSP-047, Rev. 2 (“Malvern Insitec-S Operating Procedure”), CA Burns.

- RPL-COLLOID-02, Rev. 2 ("Measurement of Physical and Rheological Properties of Solutions, Slurries, and Sludges"), RC Daniel.

- OP-WTPSP-035, Rev. 0 ("Measurement of Static Surface Tension of Liquids, Dispersions, and Slurries"), DN Tran.

- OP-WTPSP-004, Rev. 1 (“Operation of the Mettler Moisture Analyzer”), CA Burns.

- OP-WTPSP-003, Rev. 1 (“Size Analysis Using Malvern MS2000 (re-issuance of TPR-RPP-WTP-626)"), RC Daniel.

- OP-WTPSP-055, Rev. 0 ("Imaging Techniques for the Measurement of Spool Piece Orifice Dimensions for Large Scale Spray Release Testing”), JM Billing.

\section{Laboratory Record Book}

- BNW-61332, pages 1-101.

\section{Test Data Packages: Test Stand Operation Test Instructions}

- TDP-WTPSP-862 through -869 (water tests).

- TDP-WTPSP-870 through -879 (30 Pa/30 cP KBC [clay] tests).

- TDP-WTPSP-880 through -882 (6 Pa/6 cP KBC [clay] tests).

- TDP-WTPSP-883 through -889 (30 Pa/30 cP FEG [chemical simulant] tests).

- TDP-WTPSP-890 through -896 (6 Pa/6 cP FEG [chemical simulant] tests).

- TDP-WTPSP-897 through -900 (27 wt\% STR tests). 
- TDP-WTPSP-901 through -902 (DPW tests).

- TDP-WTPSP-903 (DST tests).

- TDP-WTPSP-910 (water tests with effect of initial humidity).

\section{Test Data Packages: Test Support}

- TDP-WTPSP-904 (“Pycnometric Density Measurement of Samples for Spray Release Project”).

- TDP-WTPSP-905 (“Surface Tension Measurement of Samples for Spray Release Project”).

- TDP-WTPSP-906 (“Rheological Measurements of Samples for Spray Release Phase II”).

- TDP-WTPSP-932 (“Gas Pycnometer Measurement of Molybdenum Metal Powder”).

- TDP-WTPSP-938 (“PSD Results for Small-Scale Spray Release Phase II Testing”).

- TDP-WTPSP-907 (“Log of Malvern Data Files Generated During Phase II Small-Scale Aerosol Testing").

- TDP-WTPSP-911 (“Log of Scale Mass (-WT) and Data Acquisition System (-DAQ) Files Generated During Phase II Small-Scale Aerosol Testing”).

- TDP-WTPSP-940 (“Images of Orifice Test Pieces (OTPs) for Small-Scale Spray Release Post-Phase II Measurement”).

\section{Computational Packages}

- CCP-WTPSP-1107, "Simulant Blending Calculations for Phase II WTP Small-Scale Spray Release." Originator: GN Brown.

- CCP-WTPSP-1145 Rev. 1, "Small-Scale Plugging-Tests Wall Shear Stress.” Originator: LA Mahoney.

- CCP-WTPSP-1163, "Volume of Small Scale Chamber.” Originator: LA Mahoney.

- CCP-WTPSP-1226, "Phase II Small-Scale Datalogger Conversions.” Originator: LA Mahoney.

- CCP-WTPSP-1247, "Compilation and Calculation of Complex Refractive Indices for use in Small-Scale Phase 2 Aerosol Tests.” Originator: RC Daniel.

- CCP-WTPSP-1251, "Particle Size Analysis and Rheograms for Small-Scale Spray Release." Originator: CA Burns.

- CCP-WTPSP-1311, "Post-Test Image Analysis of OTP for Small Scale Spray Release, Phase II." Originator: PP Schonewill.

- CCP-WTPSP-1306, "Phase 2 Small-Scale Run Timing and Conditions.” Originator: LA Mahoney.

- CCP-WTPSP-1307, "Phase 2 Small-Scale Inputs for PSD and Release Fraction Analysis." Originator: LA Mahoney.

- CCP-WTPSP-1308, “Small-Scale In-Jet PSD Analysis.” Originator: LA Mahoney.

- CCP-WTPSP-1309, "Phase 2 Small-Scale Release Fraction Analysis." Originator: LA Mahoney.

- CCP-WTPSP-1310, "Phase 2 Small-Scale Compilation and Plots." Originator: LA Mahoney. 
- CCP-WTPSP-1312, "Small-Scale Humidity Data and Calculations." Originator: LA Mahoney.

\section{General Documents}

- GD-WTPSP-005, "Run Log for Phase II Small-Scale Aerosol Tests." Originator: ML Kimura. 

Appendix D

Cross-References for Parametric Plots in Sections 6 and 7 



\section{Appendix D}

\section{Cross-References for Parametric Plots in Sections 6 and 7}

This appendix identifies the individual tests included in the parametric plots in Sections 6 and 7. The parametric plots are those that have $\mathrm{x}$ axes based on variables other than droplet diameter; in these plots, it was not practical to identify the tests in the figure captions. Instead, these tests are identified in a series of tables below, where each table contains the data sets for a single figure (except where noted). See Appendix A for more information about individual tests and Appendix B for the release fraction versus diameter plots for individual tests.

Table D.1. Tests in Figure 6.1

\begin{tabular}{ccccccc}
\hline $\begin{array}{c}\text { Target } \\
\text { Orifice } \\
\begin{array}{c}\text { Dimensions } \\
(\mathrm{mm})\end{array}\end{array}$ & $\begin{array}{c}\text { Orifice } \\
\text { Area } \\
\left(\mathrm{mm}^{2}\right)\end{array}$ & $\begin{array}{c}\text { Target } \\
\text { Pressure } \\
(\mathrm{psig})\end{array}$ & Simulant & Test ID(s) & $\begin{array}{c}\text { Discharge } \\
\text { Coefficient }\end{array}$ & Note \\
\hline 0.5 & 0.224 & 380 & Water & SS-127 & 0.65 & $100 \mathrm{~mm}$ lens \\
0.5 & 0.224 & 380 & Water & SS-128 & 0.62 & $100 \mathrm{~mm}$ lens \\
0.5 & 0.224 & 380 & Water & SS-129 & 0.59 & $100 \mathrm{~mm}$ lens \\
0.5 & 0.224 & 380 & Water & SS-136 & 0.65 & $500 \mathrm{~mm}$ lens \\
0.5 & 0.224 & 380 & Water & SS-137 & 0.54 & $500 \mathrm{~mm}$ lens \\
\hline
\end{tabular}

Table D.2. Tests in Figure 6.2

\begin{tabular}{|c|c|c|c|c|c|c|}
\hline $\begin{array}{c}\text { Target } \\
\text { Orifice } \\
\text { Dimensions } \\
(\mathrm{mm})\end{array}$ & $\begin{array}{l}\text { Orifice } \\
\text { Area } \\
\left(\mathrm{mm}^{2}\right)\end{array}$ & $\begin{array}{c}\text { Target } \\
\text { Pressure } \\
\text { (psig) }\end{array}$ & Simulant & Test ID(s) & $\begin{array}{l}\text { Discharge } \\
\text { Coefficient }\end{array}$ & Note(s) \\
\hline 1 & 0.747 & 380 & Clay-30 & SS-154 & 0.86 & $500 \mathrm{~mm}$ lens \\
\hline 1 & 0.747 & 380 & Clay-30 & SS-155 & 0.86 & $500 \mathrm{~mm}$ lens \\
\hline 1 & 0.747 & 380 & Clay-30 & SS-156 & 0.89 & $500 \mathrm{~mm}$ lens \\
\hline 1 & 0.747 & 380 & Clay-30 & SS-157 & 0.89 & $500 \mathrm{~mm}$ lens \\
\hline 1 & 0.747 & 380 & Clay-30 & SS-203 & 0.94 & $100 \mathrm{~mm}$ lens \\
\hline 1 & 0.747 & 380 & Clay-30 & SS-204 & 0.95 & $100 \mathrm{~mm}$ lens \\
\hline 1 & 0.747 & 380 & Clay-30 & SS-205 & 0.93 & $100 \mathrm{~mm}$ lens \\
\hline
\end{tabular}


Table D.3. Tests in Figure 6.3

\begin{tabular}{ccccccc}
\hline $\begin{array}{c}\text { Target } \\
\text { Orifice } \\
\begin{array}{c}\text { Dimensions } \\
(\mathrm{mm})\end{array}\end{array}$ & $\begin{array}{c}\text { Orifice } \\
\text { Area } \\
\left(\mathrm{mm}^{2}\right)\end{array}$ & $\begin{array}{c}\text { Target } \\
\text { Pressure } \\
(\mathrm{psig})\end{array}$ & Simulant & Test ID(s) & $\begin{array}{c}\text { Discharge } \\
\text { Coefficient }\end{array}$ & Note \\
\hline $0.5 \times 5$ & 2.61 & 380 & Clay-30 & SS-209 & 0.74 & $100 \mathrm{~mm}$ lens \\
$0.5 \times 5$ & 2.61 & 380 & Clay-30 & SS-210 & 0.76 & $100 \mathrm{~mm}$ lens \\
$0.5 \times 5$ & 2.61 & 380 & Clay-30 & SS-211 & 0.76 & $100 \mathrm{~mm}$ lens \\
$0.5 \times 5$ & 2.61 & 380 & Clay-30 & SS-176 & 0.73 & $500 \mathrm{~mm}$ lens \\
$0.5 \times 5$ & 2.61 & 380 & Clay-30 & SS-178 & 0.74 & $500 \mathrm{~mm}$ lens \\
$0.5 \times 5$ & 2.61 & 380 & Clay-30 & SS-179 & 0.73 & $500 \mathrm{~mm}$ lens \\
\hline
\end{tabular}

Table D.4. Tests in Figure 6.4

\begin{tabular}{ccccccc}
\hline $\begin{array}{c}\text { Target } \\
\text { Orifice } \\
\begin{array}{c}\text { Dimensions } \\
(\mathrm{mm})\end{array}\end{array}$ & $\begin{array}{c}\text { Orifice } \\
\text { Area } \\
\left(\mathrm{mm}^{2}\right)\end{array}$ & $\begin{array}{c}\text { Target } \\
\text { Pressure } \\
(\mathrm{psig})\end{array}$ & Simulant & Test ID(s) & $\begin{array}{c}\text { Discharge } \\
\text { Coefficient }\end{array}$ & Note \\
\hline 0.5 & 0.224 & 380 & Water & SS-133 & 0.69 & $1 \mathrm{~Hz}$ rate \\
0.5 & 0.224 & 380 & Water & SS-134 & 0.57 & $1 \mathrm{~Hz}$ rate \\
0.5 & 0.224 & 380 & Water & SS-135 & 0.62 & $1 \mathrm{~Hz}$ rate \\
0.5 & 0.224 & 380 & Water & SS-130 & 0.60 & $4 \mathrm{~Hz}$ rate \\
0.5 & 0.224 & 380 & Water & SS-131 & 0.64 & $4 \mathrm{~Hz} \mathrm{rate}$ \\
\hline
\end{tabular}

Table D.5. Tests in Figure 6.5

\begin{tabular}{ccccccc}
\hline $\begin{array}{c}\text { Target } \\
\text { Orifice } \\
\begin{array}{c}\text { Dimensions } \\
(\mathrm{mm})\end{array}\end{array}$ & $\begin{array}{c}\text { Orifice } \\
\text { Area } \\
\left(\mathrm{mm}^{2}\right)\end{array}$ & $\begin{array}{c}\text { Target } \\
\text { Pressure } \\
(\mathrm{psig})\end{array}$ & Simulant & Test ID(s) & $\begin{array}{c}\text { Discharge } \\
\text { Coefficient }\end{array}$ & Note \\
\hline 1 & 0.747 & 380 & Water & SS-117 & 0.78 & $1 \mathrm{~Hz}$ rate \\
1 & 0.747 & 380 & Water & SS-116 & 0.72 & $4 \mathrm{~Hz}$ rate \\
\hline
\end{tabular}


Table D.6. Tests in Figure 6.6

\begin{tabular}{ccccccc}
\hline $\begin{array}{c}\text { Target } \\
\text { Orifice } \\
\begin{array}{c}\text { Dimensions } \\
(\mathrm{mm})\end{array}\end{array}$ & $\begin{array}{c}\text { Orifice } \\
\text { Area } \\
\left(\mathrm{mm}^{2}\right)\end{array}$ & $\begin{array}{c}\text { Target } \\
\text { Pressure } \\
(\mathrm{psig})\end{array}$ & Simulant & Test ID(s) & $\begin{array}{c}\text { Discharge } \\
\text { Coefficient }\end{array}$ & Note \\
\hline 0.5 & 0.224 & 380 & Water & SS-120 & 0.60 & fan off \\
0.5 & 0.224 & 380 & Water & SS-121 & 0.61 & 3-V fan speed \\
0.5 & 0.224 & 380 & Water & SS-108 & 0.59 & 6-V fan speed \\
0.5 & 0.224 & 380 & Water & SS-109 & 0.68 & 6-V fan speed \\
0.5 & 0.224 & 380 & Water & SS-123 & 0.61 & 6-V fan speed \\
0.5 & 0.224 & 380 & Water & SS-122 & 0.60 & 11-V fan speed \\
\hline
\end{tabular}

Table D.7. Tests in Figure 6.7 and Figure 6.11

\begin{tabular}{ccccccc}
\hline $\begin{array}{c}\text { Target } \\
\text { Orifice } \\
\begin{array}{c}\text { Dimensions } \\
(\mathrm{mm})\end{array}\end{array}$ & $\begin{array}{c}\text { Orifice } \\
\text { Area } \\
\left(\mathrm{mm}^{2}\right)\end{array}$ & $\begin{array}{c}\text { Target } \\
\text { Pressure } \\
(\mathrm{psig})\end{array}$ & Simulant & Test ID(s) & $\begin{array}{c}\text { Discharge } \\
\text { Coefficient }\end{array}$ & Note \\
\hline 0.5 & 0.224 & 380 & Water & SS-379 & 0.86 & Initial rel. humidity $98 \%$ \\
0.5 & 0.224 & 380 & Water & SS-383 & 0.77 & Initial rel. humidity $96 \%$ \\
0.5 & 0.224 & 380 & Water & SS-380 & 0.67 & Initial rel. humidity $92 \%$ \\
0.5 & 0.224 & 380 & Water & SS-378 & 0.71 & Initial rel. humidity $85 \%$ \\
0.5 & 0.224 & 380 & Water & SS-377 & 0.75 & Initial rel. humidity $76 \%$ \\
0.5 & 0.224 & 380 & Water & SS-382 & 0.85 & Initial rel. humidity $64 \%$ \\
0.5 & 0.224 & 380 & Water & SS-381 & 0.73 & Initial rel. humidity $55 \%$ \\
0.5 & 0.224 & 380 & Water & SS-375 & 0.79 & Initial rel. humidity $54 \%$ \\
\hline
\end{tabular}

Table D.8. Tests in Figure 6.8 and Figure 6.12

\begin{tabular}{ccccccc}
\hline $\begin{array}{c}\text { Target } \\
\text { Orifice } \\
\begin{array}{c}\text { Dimensions } \\
(\mathrm{mm})\end{array}\end{array}$ & $\begin{array}{c}\text { Orifice } \\
\text { Area } \\
\left(\mathrm{mm}^{2}\right)\end{array}$ & $\begin{array}{c}\text { Target } \\
\text { Pressure } \\
(\mathrm{psig})\end{array}$ & Simulant & Test ID(s) & $\begin{array}{c}\text { Discharge } \\
\text { Coefficient }\end{array}$ & Note \\
\hline 0.5 & 0.224 & 100 & Water & SS-384 & 1.00 & Initial rel. humidity $100 \%$ \\
0.5 & 0.224 & 100 & Water & SS-385 & 0.85 & Initial rel. humidity $75 \%$ \\
0.5 & 0.224 & 100 & Water & SS-386 & 0.95 & Initial rel. humidity $50 \%$ \\
\hline
\end{tabular}


Table D.9. Tests in Figure 6.9 and Figure 6.10

\begin{tabular}{ccccccc}
\hline $\begin{array}{c}\text { Target } \\
\text { Orifice } \\
\begin{array}{c}\text { Dimensions } \\
(\mathrm{mm})\end{array}\end{array}$ & $\begin{array}{c}\text { Orifice } \\
\text { Area } \\
\left(\mathrm{mm}^{2}\right)\end{array}$ & $\begin{array}{c}\text { Target } \\
\text { Pressure } \\
(\mathrm{psig})\end{array}$ & Simulant & Test ID(s) & $\begin{array}{c}\text { Discharge } \\
\text { Coefficient }\end{array}$ & Note \\
\hline 0.5 & 0.224 & 380 & Water & SS-383 & 0.77 & Initial rel. humidity $96 \%$ \\
0.5 & 0.224 & 380 & Water & SS-378 & 0.71 & Initial rel. humidity $85 \%$ \\
0.5 & 0.224 & 380 & Water & SS-377 & 0.75 & Initial rel. humidity 76\% \\
0.5 & 0.224 & 380 & Water & SS-382 & 0.85 & Initial rel. humidity $64 \%$ \\
0.5 & 0.224 & 380 & Water & SS-375 & 0.79 & Initial rel. humidity $54 \%$ \\
\hline
\end{tabular}

Table D.10. Tests in Figure 6.13

\begin{tabular}{ccccccc}
\hline $\begin{array}{c}\text { Target } \\
\text { Orifice } \\
\begin{array}{c}\text { Dimensions } \\
(\mathrm{mm})\end{array}\end{array}$ & $\begin{array}{c}\text { Orifice } \\
\text { Area } \\
\left(\mathrm{mm}^{2}\right)\end{array}$ & $\begin{array}{c}\text { Target } \\
\text { Pressure } \\
(\mathrm{psig})\end{array}$ & Simulant & Test ID(s) & $\begin{array}{c}\text { Discharge } \\
\text { Coefficient }\end{array}$ & Note(s) \\
\hline 0.5 & 0.224 & 380 & Water & OS8-R1 & 0.70 & $1 \mathrm{~Hz}, 100 \mathrm{~mm}, 10 \mathrm{gpm}$, Phase I \\
0.5 & 0.224 & 380 & Water & SS-127 & 0.65 & $1 \mathrm{~Hz}, 100 \mathrm{~mm}, 11.4 \mathrm{gpm}$ \\
0.5 & 0.224 & 380 & Water & SS-128 & 0.62 & $1 \mathrm{~Hz}, 100 \mathrm{~mm}, 11.4 \mathrm{gpm}$ \\
0.5 & 0.224 & 380 & Water & SS-129 & 0.59 & $1 \mathrm{~Hz}, 100 \mathrm{~mm}, 11.4 \mathrm{gpm}$ \\
\hline
\end{tabular}

Table D.11. Tests in Figure 6.14 (top)

\begin{tabular}{cccccc}
\hline $\begin{array}{c}\text { Target } \\
\text { Orifice } \\
\begin{array}{c}\text { Dimensions } \\
(\mathrm{mm})\end{array}\end{array}$ & $\begin{array}{c}\text { Orifice } \\
\text { Area } \\
\left(\mathrm{mm}^{2}\right)\end{array}$ & $\begin{array}{c}\text { Target } \\
\text { Pressure } \\
(\mathrm{psig})\end{array}$ & Simulant & Test ID(s) & $\begin{array}{c}\text { Discharge } \\
\text { Coefficient }\end{array}$ \\
\hline $0.5 \times 5$ & 2.61 & 100 & Water & SS-138 & 0.63 \\
$0.5 \times 5$ & 2.61 & 100 & Water & SS-139 & 0.63 \\
$0.5 \times 5$ & 2.61 & 100 & Water & SS-140 & 0.62 \\
\hline
\end{tabular}

Table D.12. Tests in Figure 6.14 (bottom)

\begin{tabular}{cccccc}
\hline $\begin{array}{c}\text { Target } \\
\text { Orifice } \\
\begin{array}{c}\text { Dimensions } \\
(\mathrm{mm})\end{array}\end{array}$ & $\begin{array}{c}\text { Orifice } \\
\text { Area } \\
\left(\mathrm{mm}^{2}\right)\end{array}$ & $\begin{array}{c}\text { Target } \\
\text { Pressure } \\
(\mathrm{psig})\end{array}$ & Simulant & Test ID(s) & $\begin{array}{c}\text { Discharge } \\
\text { Coefficient }\end{array}$ \\
\hline 1 & 0.747 & 380 & Water & SS-150 & 0.75 \\
1 & 0.747 & 380 & Water & SS-151 & 0.75 \\
\hline
\end{tabular}


Table D.13. Tests in Figure 6.15 (top)

\begin{tabular}{cccccc}
\hline $\begin{array}{c}\text { Target } \\
\text { Orifice } \\
\begin{array}{c}\text { Dimensions } \\
(\mathrm{mm})\end{array}\end{array}$ & $\begin{array}{c}\text { Orifice } \\
\text { Area } \\
\left(\mathrm{mm}^{2}\right)\end{array}$ & $\begin{array}{c}\text { Target } \\
\text { Pressure } \\
(\mathrm{psig})\end{array}$ & Simulant & Test ID(s) & $\begin{array}{c}\text { Discharge } \\
\text { Coefficient }\end{array}$ \\
\hline 1 & 0.747 & 100 & Clay-6 & SS-232 & 0.98 \\
1 & 0.747 & 100 & Clay-6 & SS-233 & 0.91 \\
1 & 0.747 & 100 & Clay-6 & SS-234 & 0.84 \\
\hline
\end{tabular}

Table D.14. Tests in Figure 6.15 (bottom)

\begin{tabular}{cccccc}
\hline $\begin{array}{c}\text { Target } \\
\text { Orifice } \\
\text { Dimensions } \\
(\mathrm{mm})\end{array}$ & $\begin{array}{c}\text { Orifice } \\
\text { Area } \\
\left(\mathrm{mm}^{2}\right)\end{array}$ & $\begin{array}{c}\text { Target } \\
\text { Pressure } \\
(\mathrm{psig})\end{array}$ & Simulant & Test ID(s) & $\begin{array}{c}\text { Discharge } \\
\text { Coefficient }\end{array}$ \\
\hline $0.5 \times 5$ & 2.61 & 100 & Clay-6 & SS-220 & 0.81 \\
$0.5 \times 5$ & 2.61 & 100 & Clay-6 & SS-221 & 0.81 \\
$0.5 \times 5$ & 2.61 & 100 & Clay-6 & SS-222 & 0.79 \\
\hline
\end{tabular}

Table D.15. Tests in Figure 6.16 (top)

\begin{tabular}{cccccc}
\hline $\begin{array}{c}\text { Target } \\
\text { Orifice } \\
\text { Dimensions } \\
(\mathrm{mm})\end{array}$ & $\begin{array}{c}\text { Orifice } \\
\text { Area } \\
\left(\mathrm{mm}^{2}\right)\end{array}$ & $\begin{array}{c}\text { Target } \\
\text { Pressure } \\
(\mathrm{psig})\end{array}$ & Simulant & Test ID(s) & $\begin{array}{c}\text { Discharge } \\
\text { Coefficient }\end{array}$ \\
\hline 1 & 0.747 & 200 & Clay-30 & SS-159 & 0.98 \\
1 & 0.747 & 200 & Clay-30 & SS-160 & 0.93 \\
1 & 0.747 & 200 & Clay-30 & SS-161 & 0.95 \\
\hline
\end{tabular}

Table D.16. Tests in Figure 6.16 (bottom)

\begin{tabular}{cccccc}
\hline $\begin{array}{c}\text { Target } \\
\text { Orifice } \\
\text { Dimensions } \\
(\mathrm{mm})\end{array}$ & $\begin{array}{c}\text { Orifice } \\
\text { Area } \\
\left(\mathrm{mm}^{2}\right)\end{array}$ & $\begin{array}{c}\text { Target } \\
\text { Pressure } \\
(\mathrm{psig})\end{array}$ & Simulant & Test ID(s) & $\begin{array}{c}\text { Discharge } \\
\text { Coefficient }\end{array}$ \\
\hline $0.5 \times 5$ & 2.61 & 100 & Clay-30 & SS-166 & 0.63 \\
$0.5 \times 5$ & 2.61 & 100 & Clay-30 & SS-167 & 0.68 \\
$0.5 \times 5$ & 2.61 & 100 & Clay-30 & SS-168 & 0.70 \\
\hline
\end{tabular}


Table D.17. Tests in Figure 6.17 (top)

\begin{tabular}{cccccc}
\hline $\begin{array}{c}\text { Target } \\
\text { Orifice } \\
\begin{array}{c}\text { Dimensions } \\
(\mathrm{mm})\end{array}\end{array}$ & $\begin{array}{c}\text { Orifice } \\
\text { Area } \\
\left(\mathrm{mm}^{2}\right)\end{array}$ & $\begin{array}{c}\text { Target } \\
\text { Pressure } \\
(\mathrm{psig})\end{array}$ & Simulant & Test ID(s) & $\begin{array}{c}\text { Discharge } \\
\text { Coefficient }\end{array}$ \\
\hline 1 & 0.747 & 380 & FEG-6 & SS-307 & 0.89 \\
1 & 0.747 & 380 & FEG-6 & SS-309 & 0.94 \\
\hline
\end{tabular}

Table D.18. Tests in Figure 6.17 (bottom)

\begin{tabular}{cccccc}
\hline $\begin{array}{c}\text { Target } \\
\text { Orifice } \\
\text { Dimensions } \\
(\mathrm{mm})\end{array}$ & $\begin{array}{c}\text { Orifice } \\
\text { Area } \\
\left(\mathrm{mm}^{2}\right)\end{array}$ & $\begin{array}{c}\text { Target } \\
\text { Pressure } \\
(\mathrm{psig})\end{array}$ & Simulant & Test ID(s) & $\begin{array}{c}\text { Discharge } \\
\text { Coefficient }\end{array}$ \\
\hline 1 & 0.747 & 200 & FEG-6 & SS-302 & 0.87 \\
1 & 0.747 & 200 & FEG-6 & SS-303 & 0.96 \\
1 & 0.747 & 200 & FEG-6 & SS-305 & 0.86 \\
\hline
\end{tabular}

Table D.19. Tests in Figure 6.18 (top)

\begin{tabular}{cccccc}
\hline $\begin{array}{c}\text { Target } \\
\text { Orifice } \\
\begin{array}{c}\text { Dimensions } \\
(\mathrm{mm})\end{array}\end{array}$ & $\begin{array}{c}\text { Orifice } \\
\text { Area } \\
\left(\mathrm{mm}^{2}\right)\end{array}$ & $\begin{array}{c}\text { Target } \\
\text { Pressure } \\
(\mathrm{psig})\end{array}$ & Simulant & Test ID(s) & $\begin{array}{c}\text { Discharge } \\
\text { Coefficient }\end{array}$ \\
\hline $0.5 \times 5$ & 2.61 & 380 & FEG-30 & SS-281 & 0.81 \\
$0.5 \times 5$ & 2.61 & 380 & FEG-30 & SS-282 & 0.76 \\
$0.5 \times 5$ & 2.61 & 380 & FEG-30 & SS-283 & 0.75 \\
\hline
\end{tabular}

Table D.20. Tests in Figure 6.18 (bottom)

\begin{tabular}{cccccc}
\hline $\begin{array}{c}\text { Target } \\
\text { Orifice } \\
\begin{array}{c}\text { Dimensions } \\
(\mathrm{mm})\end{array}\end{array}$ & $\begin{array}{c}\text { Orifice } \\
\text { Area } \\
\left(\mathrm{mm}^{2}\right)\end{array}$ & $\begin{array}{c}\text { Target } \\
\text { Pressure } \\
(\mathrm{psig})\end{array}$ & Simulant & Test ID(s) & $\begin{array}{c}\text { Discharge } \\
\text { Coefficient }\end{array}$ \\
\hline 1 & 0.747 & 200 & FEG-30 & SS-255 & 0.75 \\
1 & 0.747 & 200 & FEG-30 & SS-256 & 0.80 \\
1 & 0.747 & 200 & FEG-30 & SS-271 & 0.69 \\
\hline
\end{tabular}


Table D.21. Tests in Figure 6.19 (top)

\begin{tabular}{cccccc}
\hline $\begin{array}{c}\text { Target } \\
\text { Orifice } \\
\text { Dimensions } \\
(\mathrm{mm})\end{array}$ & $\begin{array}{c}\text { Orifice } \\
\text { Area } \\
\left(\mathrm{mm}^{2}\right)\end{array}$ & $\begin{array}{c}\text { Target } \\
\text { Pressure } \\
(\mathrm{psig})\end{array}$ & Simulant & Test ID(s) & $\begin{array}{c}\text { Discharge } \\
\text { Coefficient }\end{array}$ \\
\hline $0.5 \times 5$ & 2.61 & 100 & STR-27 & SS-340 & 0.75 \\
$0.5 \times 5$ & 2.61 & 100 & STR-27 & SS-341 & 0.72 \\
$0.5 \times 5$ & 2.61 & 100 & STR-27 & SS-342 & 0.68 \\
\hline
\end{tabular}

Table D.22. Tests in Figure 6.19 (bottom)

\begin{tabular}{cccccc}
\hline $\begin{array}{c}\text { Target } \\
\text { Orifice } \\
\begin{array}{c}\text { Dimensions } \\
(\mathrm{mm})\end{array}\end{array}$ & $\begin{array}{c}\text { Orifice } \\
\text { Area } \\
\left(\mathrm{mm}^{2}\right)\end{array}$ & $\begin{array}{c}\text { Target } \\
\text { Pressure } \\
(\mathrm{psig})\end{array}$ & Simulant & Test ID(s) & $\begin{array}{c}\text { Discharge } \\
\text { Coefficient }\end{array}$ \\
\hline 1 & 0.747 & 380 & STR-27 & SS-329 & 0.85 \\
1 & 0.747 & 380 & STR-27 & SS-331 & 0.97 \\
\hline
\end{tabular}

Table D.23. Tests in Figure 6.20 (top)

\begin{tabular}{cccccc}
\hline $\begin{array}{c}\text { Target } \\
\text { Orifice } \\
\begin{array}{c}\text { imensions } \\
(\mathrm{mm})\end{array}\end{array}$ & $\begin{array}{c}\text { Orifice } \\
\text { Area } \\
\left(\mathrm{mm}^{2}\right)\end{array}$ & $\begin{array}{c}\text { Target } \\
\text { Pressure } \\
(\mathrm{psig})\end{array}$ & Simulant & Test ID(s) & $\begin{array}{c}\text { Discharge } \\
\text { Coefficient }\end{array}$ \\
\hline $0.5 \times 5$ & 2.61 & 380 & DST & SS-370 & 0.78 \\
$0.5 \times 5$ & 2.61 & 380 & DST & SS-371 & 0.74 \\
$0.5 \times 5$ & 2.61 & 380 & DST & SS-372 & 0.74 \\
$0.5 \times 5$ & 2.61 & 380 & DST & SS-374 & 0.74 \\
\hline
\end{tabular}

Table D.24. Tests in Figure 6.20 (bottom)

\begin{tabular}{cccccc}
\hline $\begin{array}{c}\text { Target } \\
\text { Orifice } \\
\begin{array}{c}\text { Dimensions } \\
(\mathrm{mm})\end{array}\end{array}$ & $\begin{array}{c}\text { Orifice } \\
\text { Area } \\
\left(\mathrm{mm}^{2}\right)\end{array}$ & $\begin{array}{c}\text { Target } \\
\text { Pressure } \\
(\mathrm{psig})\end{array}$ & Simulant & Test ID(s) & $\begin{array}{c}\text { Discharge } \\
\text { Coefficient }\end{array}$ \\
\hline 1 & 0.747 & 380 & DST & SS-368 & 0.99 \\
1 & 0.747 & 380 & DST & SS-369 & 0.90 \\
\hline
\end{tabular}


Table D.25. Tests in Figure 6.21

\begin{tabular}{cccccc}
\hline $\begin{array}{c}\text { Target } \\
\text { Orifice } \\
\text { Dimensions } \\
(\mathrm{mm})\end{array}$ & $\begin{array}{c}\text { Orifice } \\
\text { Area } \\
\left(\mathrm{mm}^{2}\right)\end{array}$ & $\begin{array}{c}\text { Target } \\
\text { Pressure } \\
(\mathrm{psig})\end{array}$ & Simulant & Test ID(s) & $\begin{array}{c}\text { Discharge } \\
\text { Coefficient }\end{array}$ \\
\hline $0.5 \times 5$ & 2.61 & 380 & DPW & SS-360 & 0.72 \\
$0.5 \times 5$ & 2.61 & 380 & DPW & SS-362 & 0.73 \\
\hline
\end{tabular}

Table D.26. Tests in Figure 7.1, Sorted in Ascending Order of Fit-Period Average Pressure

\begin{tabular}{cccccc}
\hline $\begin{array}{c}\text { Target } \\
\text { Orifice } \\
\begin{array}{c}\text { Dimensions } \\
(\mathrm{mm})\end{array}\end{array}$ & $\begin{array}{c}\text { Orifice } \\
\text { Area } \\
\left(\mathrm{mm}^{2}\right)\end{array}$ & $\begin{array}{c}\text { Target } \\
\text { Pressure } \\
(\mathrm{psig})\end{array}$ & Simulant & Test ID(s) & $\begin{array}{c}\text { Discharge } \\
\text { Coefficient }\end{array}$ \\
\hline 1 & 0.747 & 100 & Water & PV-16 & 0.79 \\
1 & 0.747 & 100 & Water & SS-146 & 0.74 \\
1 & 0.747 & 100 & Water & SS-145 & 0.75 \\
1 & 0.747 & 200 & Water & PV-14 & 0.75 \\
1 & 0.747 & 200 & Water & SS-147 & 0.74 \\
1 & 0.747 & 200 & Water & SS-148 & 0.74 \\
1 & 0.747 & 380 & Water & OS-7 & 0.74 \\
1 & 0.747 & 380 & Water & RT-19 & 0.77 \\
1 & 0.747 & 380 & Water & RT-18 & 0.71 \\
1 & 0.747 & 380 & Water & SS-151 & 0.75 \\
1 & 0.747 & 380 & Water & SS-150 & 0.75 \\
\hline
\end{tabular}

Table D.27. Tests in Figure 7.2, Sorted in Ascending Order of Fit-Period Average Pressure

\begin{tabular}{cccccc}
\hline $\begin{array}{c}\text { Target } \\
\text { Orifice } \\
\begin{array}{c}\text { Dimensions } \\
(\mathrm{mm})\end{array}\end{array}$ & $\begin{array}{c}\text { Orifice } \\
\text { Area } \\
\left(\mathrm{mm}^{2}\right)\end{array}$ & $\begin{array}{c}\text { Target } \\
\text { Pressure } \\
(\mathrm{psig})\end{array}$ & Simulant & Test ID(s) & $\begin{array}{c}\text { Discharge } \\
\text { Coefficient }\end{array}$ \\
\hline $0.5 \times 5$ & 2.61 & 100 & Water & SS-139 & 0.63 \\
$0.5 \times 5$ & 2.61 & 100 & Water & SS-138 & 0.63 \\
$0.5 \times 5$ & 2.61 & 100 & Water & SS-140 & 0.62 \\
$0.5 \times 5$ & 2.61 & 100 & Water & PV-17-R1 & 0.56 \\
$0.5 \times 5$ & 2.61 & 200 & Water & PV-15-R1 & 0.59 \\
$0.5 \times 5$ & 2.61 & 200 & Water & SS-141 & 0.62 \\
$0.5 \times 5$ & 2.61 & 200 & Water & SS-142 & 0.62 \\
$0.5 \times 5$ & 2.61 & 380 & Water & OS-12-R1-SG & 0.59 \\
$0.5 \times 5$ & 2.61 & 380 & Water & SS-144 & 0.62 \\
$0.5 \times 5$ & 2.61 & 380 & Water & SO2-R1 & 0.57 \\
$0.5 \times 5$ & 2.61 & 380 & Water & SS-143 & 0.63 \\
\hline
\end{tabular}


Table D.28. Tests in Figure 7.3, Sorted in Ascending Order of Fit-Period Average Pressure

\begin{tabular}{cccccc}
\hline $\begin{array}{c}\text { Target } \\
\text { Orifice } \\
\begin{array}{c}\text { Dimensions } \\
(\mathrm{mm})\end{array}\end{array}$ & $\begin{array}{c}\text { Orifice } \\
\text { Area } \\
\left(\mathrm{mm}^{2}\right)\end{array}$ & $\begin{array}{c}\text { Target } \\
\text { Pressure } \\
(\mathrm{psig})\end{array}$ & Simulant & Test ID(s) & $\begin{array}{c}\text { Discharge } \\
\text { Coefficient }\end{array}$ \\
\hline 1 & 0.747 & 100 & Clay-6 & SS-234 & 0.84 \\
1 & 0.747 & 100 & Clay-6 & SS-233 & 0.91 \\
1 & 0.747 & 100 & Clay-6 & SS-232 & 0.98 \\
1 & 0.747 & 200 & Clay-6 & SS-235 & 0.86 \\
1 & 0.747 & 200 & Clay-6 & SS-236 & 0.99 \\
1 & 0.747 & 200 & Clay-6 & SS-237 & 0.91 \\
1 & 0.747 & 380 & Clay-6 & SS-242 & 0.91 \\
1 & 0.747 & 380 & Clay-6 & SS-241 & 0.89 \\
1 & 0.747 & 380 & Clay-6 & SS-243 & 0.89 \\
\hline
\end{tabular}

Table D.29. Tests in Figure 7.4, Sorted in Ascending Order of Fit-Period Average Pressure

\begin{tabular}{cccccc}
\hline $\begin{array}{c}\text { Target } \\
\text { Orifice } \\
\begin{array}{c}\text { Dimensions } \\
(\mathrm{mm})\end{array}\end{array}$ & $\begin{array}{c}\text { Orifice } \\
\text { Area } \\
\left(\mathrm{mm}^{2}\right)\end{array}$ & $\begin{array}{c}\text { Target } \\
\text { Pressure } \\
(\mathrm{psig})\end{array}$ & Simulant & Test ID(s) & $\begin{array}{c}\text { Discharge } \\
\text { Coefficient }\end{array}$ \\
\hline $0.5 \times 5$ & 2.61 & 100 & Clay-6 & SS-222 & 0.79 \\
$0.5 \times 5$ & 2.61 & 100 & Clay-6 & SS-221 & 0.81 \\
$0.5 \times 5$ & 2.61 & 100 & Clay-6 & SS-220 & 0.81 \\
$0.5 \times 5$ & 2.61 & 200 & Clay-6 & SS-225 & 0.78 \\
$0.5 \times 5$ & 2.61 & 200 & Clay-6 & SS-224 & 0.77 \\
$0.5 \times 5$ & 2.61 & 200 & Clay-6 & SS-223 & 0.78 \\
$0.5 \times 5$ & 2.61 & 380 & Clay-6 & SS-231 & 0.77 \\
$0.5 \times 5$ & 2.61 & 380 & Clay-6 & SS-230 & 0.76 \\
$0.5 \times 5$ & 2.61 & 380 & Clay-6 & SS-229 & 0.77 \\
\hline
\end{tabular}

Table D.30. Tests in Figure 7.5, Sorted in Ascending Order of Fit-Period Average Pressure

\begin{tabular}{cccccc}
\hline $\begin{array}{c}\text { Target } \\
\text { Orifice } \\
\begin{array}{c}\text { Dimensions } \\
(\mathrm{mm})\end{array}\end{array}$ & $\begin{array}{c}\text { Orifice } \\
\text { Area } \\
\left(\mathrm{mm}^{2}\right)\end{array}$ & $\begin{array}{c}\text { Target } \\
\text { Pressure } \\
(\mathrm{psig})\end{array}$ & Simulant & Test ID(s) & $\begin{array}{c}\text { Discharge } \\
\text { Coefficient }\end{array}$ \\
\hline 1 & 0.747 & 100 & Clay-30 & SS-162 & 0.96 \\
1 & 0.747 & 100 & Clay-30 & SS-163 & 0.96 \\
1 & 0.747 & 100 & Clay-30 & SS-164 & 0.95 \\
1 & 0.747 & 100 & Clay-30 & SS-165 & 0.97 \\
1 & 0.747 & 200 & Clay-30 & SS-161 & 0.95 \\
1 & 0.747 & 200 & Clay-30 & SS-160 & 0.93 \\
1 & 0.747 & 200 & Clay-30 & SS-159 & 0.98 \\
1 & 0.747 & 380 & Clay-30 & SS-154 & 0.86 \\
1 & 0.747 & 380 & Clay-30 & SS-157 & 0.89 \\
1 & 0.747 & 380 & Clay-30 & SS-155 & 0.86 \\
1 & 0.747 & 380 & Clay-30 & SS-156 & 0.89 \\
\hline
\end{tabular}


Table D.31. Tests in Figure 7.6, Sorted in Ascending Order of Fit-Period Average Pressure

\begin{tabular}{cccccc}
\hline $\begin{array}{c}\text { Target } \\
\text { Orifice } \\
\begin{array}{c}\text { Dimensions } \\
(\mathrm{mm})\end{array}\end{array}$ & $\begin{array}{c}\text { Orifice } \\
\text { Area } \\
\left(\mathrm{mm}^{2}\right)\end{array}$ & $\begin{array}{c}\text { Target } \\
\text { Pressure } \\
(\mathrm{psig})\end{array}$ & Simulant & Test ID(s) & $\begin{array}{c}\text { Discharge } \\
\text { Coefficient }\end{array}$ \\
\hline $0.5 \times 5$ & 2.61 & 100 & Clay-30 & SS-166 & 0.63 \\
$0.5 \times 5$ & 2.61 & 100 & Clay-30 & SS-167 & 0.68 \\
$0.5 \times 5$ & 2.61 & 100 & Clay-30 & SS-168 & 0.70 \\
$0.5 \times 5$ & 2.61 & 200 & Clay-30 & SS-173 & 0.72 \\
$0.5 \times 5$ & 2.61 & 200 & Clay-30 & SS-171 & 0.71 \\
$0.5 \times 5$ & 2.61 & 200 & Clay-30 & SS-170 & 0.73 \\
$0.5 \times 5$ & 2.61 & 200 & Clay-30 & SS-174 & 0.70 \\
$0.5 \times 5$ & 2.61 & 380 & Clay-30 & SS-178 & 0.74 \\
$0.5 \times 5$ & 2.61 & 380 & Clay-30 & SS-176 & 0.73 \\
$0.5 \times 5$ & 2.61 & 380 & Clay-30 & SS-179 & 0.73 \\
\hline
\end{tabular}

Table D.32. Tests in Figure 7.7, Sorted in Ascending Order of Fit-Period Average Pressure

\begin{tabular}{cccccc}
\hline $\begin{array}{c}\text { Target } \\
\text { Orifice } \\
\begin{array}{c}\text { Dimensions } \\
(\mathrm{mm})\end{array}\end{array}$ & $\begin{array}{c}\text { Orifice } \\
\text { Area } \\
\left(\mathrm{mm}^{2}\right)\end{array}$ & $\begin{array}{c}\text { Target } \\
\text { Pressure } \\
(\mathrm{psig})\end{array}$ & Simulant & Test ID(s) & $\begin{array}{c}\text { Discharge } \\
\text { Coefficient }\end{array}$ \\
\hline 1 & 0.747 & 100 & FEG-6 & SS-301 & 0.92 \\
1 & 0.747 & 100 & FEG-6 & SS-299 & 0.88 \\
1 & 0.747 & 100 & FEG-6 & SS-300 & 0.90 \\
1 & 0.747 & 200 & FEG-6 & SS-302 & 0.87 \\
1 & 0.747 & 200 & FEG-6 & SS-303 & 0.96 \\
1 & 0.747 & 200 & FEG-6 & SS-305 & 0.86 \\
1 & 0.747 & 380 & FEG-6 & SS-309 & 0.94 \\
1 & 0.747 & 380 & FEG-6 & SS-307 & 0.89 \\
\hline
\end{tabular}

Table D.33. Tests in Figure 7.8, Sorted in Ascending Order of Fit-Period Average Pressure

\begin{tabular}{cccccc}
\hline $\begin{array}{c}\text { Target } \\
\text { Orifice } \\
\begin{array}{c}\text { Dimensions } \\
(\mathrm{mm})\end{array}\end{array}$ & $\begin{array}{c}\text { Orifice } \\
\text { Area } \\
\left(\mathrm{mm}^{2}\right)\end{array}$ & $\begin{array}{c}\text { Target } \\
\text { Pressure } \\
(\mathrm{psig})\end{array}$ & Simulant & Test ID(s) & $\begin{array}{c}\text { Discharge } \\
\text { Coefficient }\end{array}$ \\
\hline $0.5 \times 5$ & 2.61 & 100 & FEG-6 & SS-312 & 0.81 \\
$0.5 \times 5$ & 2.61 & 100 & FEG-6 & SS-313 & 0.76 \\
$0.5 \times 5$ & 2.61 & 200 & FEG-6 & SS-315 & 0.76 \\
$0.5 \times 5$ & 2.61 & 380 & FEG-6 & SS-320 & 0.76 \\
$0.5 \times 5$ & 2.61 & 380 & FEG-6 & SS-317 & 0.79 \\
\hline
\end{tabular}


Table D.34. Tests in Figure 7.9, Sorted in Ascending Order of Fit-Period Average Pressure

\begin{tabular}{cccccc}
\hline $\begin{array}{c}\text { Target } \\
\text { Orifice } \\
\begin{array}{c}\text { Dimensions } \\
(\mathrm{mm})\end{array}\end{array}$ & $\begin{array}{c}\text { Orifice } \\
\text { Area } \\
\left(\mathrm{mm}^{2}\right)\end{array}$ & $\begin{array}{c}\text { Target } \\
\text { Pressure } \\
(\mathrm{psig})\end{array}$ & Simulant & Test ID(s) & $\begin{array}{c}\text { Discharge } \\
\text { Coefficient }\end{array}$ \\
\hline 1 & 0.747 & 100 & FEG-30 & SS-261 & 0.91 \\
1 & 0.747 & 100 & FEG-30 & SS-251 & 0.94 \\
1 & 0.747 & 100 & FEG-30 & SS-253 & 0.98 \\
1 & 0.747 & 200 & FEG-30 & SS-271 & 0.69 \\
1 & 0.747 & 200 & FEG-30 & SS-256 & 0.80 \\
1 & 0.747 & 200 & FEG-30 & SS-255 & 0.75 \\
1 & 0.747 & 380 & FEG-30 & SS-266 & 0.97 \\
\hline
\end{tabular}

Table D.35. Tests in Figure 7.10, Sorted in Ascending Order of Fit-Period Average Pressure

\begin{tabular}{cccccc}
\hline $\begin{array}{c}\text { Target } \\
\text { Orifice } \\
\begin{array}{c}\text { Dimensions } \\
(\mathrm{mm})\end{array}\end{array}$ & $\begin{array}{c}\text { Orifice } \\
\text { Area } \\
\left(\mathrm{mm}^{2}\right)\end{array}$ & $\begin{array}{c}\text { Target } \\
\text { Pressure } \\
(\mathrm{pssig})\end{array}$ & Simulant & Test ID(s) & $\begin{array}{c}\text { Discharge } \\
\text { Coefficient }\end{array}$ \\
\hline $0.5 \times 5$ & 2.61 & 100 & FEG-30 & SS-275 & 0.76 \\
$0.5 \times 5$ & 2.61 & 100 & FEG-30 & SS-274 & 0.80 \\
$0.5 \times 5$ & 2.61 & 200 & FEG-30 & SS-277 & 0.72 \\
$0.5 \times 5$ & 2.61 & 200 & FEG-30 & SS-280 & 0.84 \\
$0.5 \times 5$ & 2.61 & 380 & FEG-30 & SS-283 & 0.75 \\
$0.5 \times 5$ & 2.61 & 380 & FEG-30 & SS-281 & 0.81 \\
$0.5 \times 5$ & 2.61 & 380 & FEG-30 & SS-282 & 0.76 \\
\hline
\end{tabular}

Table D.36. Tests in Figure 7.11, Sorted in Ascending Order of Fit-Period Average Pressure

\begin{tabular}{cccccc}
\hline $\begin{array}{c}\text { Target } \\
\text { Orifice } \\
\begin{array}{c}\text { Dimensions } \\
(\mathrm{mm})\end{array}\end{array}$ & $\begin{array}{c}\text { Orifice } \\
\text { Area } \\
\left(\mathrm{mm}^{2}\right)\end{array}$ & $\begin{array}{c}\text { Target } \\
\text { Pressure } \\
(\mathrm{psig})\end{array}$ & Simulant & Test ID(s) & $\begin{array}{c}\text { Discharge } \\
\text { Coefficient }\end{array}$ \\
\hline 1 & 0.747 & 100 & STR-27 & SS-339 & 0.88 \\
1 & 0.747 & 100 & STR-27 & SS-338 & 0.94 \\
1 & 0.747 & 100 & STR-27 & SS-337 & 0.90 \\
1 & 0.747 & 200 & STR-27 & SS-334 & 0.92 \\
1 & 0.747 & 200 & STR-27 & SS-336 & 0.88 \\
1 & 0.747 & 200 & STR-27 & SS-335 & 0.94 \\
1 & 0.747 & 380 & STR-27 & SS-331 & 0.97 \\
1 & 0.747 & 380 & STR-27 & SS-329 & 0.85 \\
\hline
\end{tabular}


Table D.37. Tests in Figure 7.12, Sorted in Ascending Order of Fit-Period Average Pressure

\begin{tabular}{cccccc}
\hline $\begin{array}{c}\text { Target } \\
\text { Orifice } \\
\text { Dimensions } \\
(\mathrm{mm})\end{array}$ & $\begin{array}{c}\text { Orifice } \\
\text { Area } \\
\left(\mathrm{mm}^{2}\right)\end{array}$ & $\begin{array}{c}\text { Target } \\
\text { Pressure } \\
(\mathrm{psig})\end{array}$ & Simulant & Test ID(s) & $\begin{array}{c}\text { Discharge } \\
\text { Coefficient }\end{array}$ \\
\hline $0.5 \times 5$ & 2.61 & 100 & STR-27 & SS-341 & 0.72 \\
$0.5 \times 5$ & 2.61 & 100 & STR-27 & SS-342 & 0.68 \\
$0.5 \times 5$ & 2.61 & 100 & STR-27 & SS-340 & 0.75 \\
$0.5 \times 5$ & 2.61 & 200 & STR-27 & SS-346 & 0.74 \\
$0.5 \times 5$ & 2.61 & 200 & STR-27 & SS-344 & 0.72 \\
$0.5 \times 5$ & 2.61 & 200 & STR-27 & SS-345 & 0.76 \\
$0.5 \times 5$ & 2.61 & 380 & STR-27 & SS-347 & 0.75 \\
$0.5 \times 5$ & 2.61 & 380 & STR-27 & SS-349 & 0.77 \\
$0.5 \times 5$ & 2.61 & 380 & STR-27 & SS-348 & 0.77 \\
\hline
\end{tabular}

Table D.38. Tests in Figure 7.13

\begin{tabular}{cccccc}
\hline $\begin{array}{c}\text { Target } \\
\text { Orifice } \\
\begin{array}{c}\text { Dimensions } \\
(\mathrm{mm})\end{array}\end{array}$ & $\begin{array}{c}\text { Orifice } \\
\text { Area } \\
\left(\mathrm{mm}^{2}\right)\end{array}$ & $\begin{array}{c}\text { Target } \\
\text { Pressure } \\
(\mathrm{psig})\end{array}$ & Simulant & Test ID(s) & $\begin{array}{c}\text { Discharge } \\
\text { Coefficient }\end{array}$ \\
\hline 1 & 0.747 & 380 & Water & SS-150 & 0.75 \\
1 & 0.747 & 380 & Water & SS-151 & 0.75 \\
1 & 0.747 & 380 & STR-8 & SV-34 & 0.82 \\
1 & 0.747 & 380 & STR-20 & SV-36 & 0.76 \\
1 & 0.747 & 380 & STR-27 & SS-331 & 0.97 \\
\hline
\end{tabular}

Table D.39. Tests in Figure 7.14

\begin{tabular}{rrrllc}
\hline $\begin{array}{r}\text { Target } \\
\text { Orifice } \\
\text { Dimensions } \\
(\mathrm{mm})\end{array}$ & $\begin{array}{r}\text { Orifice } \\
\text { Area } \\
\left(\mathrm{mm}^{2}\right)\end{array}$ & $\begin{array}{r}\text { Target } \\
\text { Pressure } \\
(\mathrm{psig})\end{array}$ & Simulant & Test ID(s) & $\begin{array}{c}\text { Discharge } \\
\text { Coefficient }\end{array}$ \\
\hline $0.5 \times 5$ & 2.61 & 380 & Water & SS-143 & 0.63 \\
$0.5 \times 5$ & 2.61 & 380 & Water & SS-144 & 0.62 \\
$0.5 \times 5$ & 2.61 & 380 & STR-8 & SV-35A & 0.62 \\
$0.5 \times 5$ & 2.61 & 380 & STR-8 & SV-35B & 0.62 \\
$0.5 \times 5$ & 2.61 & 380 & STR-8 & SV-35C & 0.58 \\
$0.5 \times 5$ & 2.61 & 380 & STR-20 & SV-37C & 0.60 \\
$0.5 \times 5$ & 2.61 & 380 & STR-27 & SS-347 & 0.75 \\
$0.5 \times 5$ & 2.61 & 380 & STR-27 & SS-348 & 0.77 \\
$0.5 \times 5$ & 2.61 & 380 & STR-27 & SS-349 & 0.77 \\
\hline
\end{tabular}


Table D.40. Tests in Figure 7.15

\begin{tabular}{cccccc}
\hline $\begin{array}{c}\text { Target } \\
\text { Orifice } \\
\text { Dimensions } \\
(\mathrm{mm})\end{array}$ & $\begin{array}{c}\text { Orifice } \\
\text { Area } \\
\left(\mathrm{mm}^{2}\right)\end{array}$ & $\begin{array}{c}\text { Target } \\
\text { Pressure } \\
(\mathrm{psig})\end{array}$ & Simulant & Test ID(s) & $\begin{array}{c}\text { Discharge } \\
\text { Coefficient }\end{array}$ \\
\hline 1 & 0.747 & 380 & Water & SS-150 & 0.75 \\
1 & 0.747 & 380 & Water & SS-151 & 0.75 \\
1 & 0.747 & 380 & DPW & SS-355 & 0.86 \\
\hline
\end{tabular}

Table D.41. Tests in Figure 7.16

\begin{tabular}{cccccc}
\hline $\begin{array}{c}\text { Target } \\
\text { Orifice } \\
\begin{array}{c}\text { Dimensions } \\
(\mathrm{mm})\end{array}\end{array}$ & $\begin{array}{c}\text { Orifice } \\
\text { Area } \\
\left(\mathrm{mm}^{2}\right)\end{array}$ & $\begin{array}{c}\text { Target } \\
\text { Pressure } \\
(\mathrm{psig})\end{array}$ & Simulant & Test ID(s) & $\begin{array}{c}\text { Discharge } \\
\text { Coefficient }\end{array}$ \\
\hline $0.5 \times 5$ & 2.61 & 380 & Water & SS-143 & 0.63 \\
$0.5 \times 5$ & 2.61 & 380 & Water & SS-144 & 0.62 \\
$0.5 \times 5$ & 2.61 & 380 & DPW & SS-360 & 0.72 \\
$0.5 \times 5$ & 2.61 & 380 & DPW & SS-362 & 0.73 \\
\hline
\end{tabular}

Table D.42. Tests in Figure 7.17

\begin{tabular}{cccccc}
\hline $\begin{array}{c}\text { Target } \\
\text { Orifice } \\
\begin{array}{c}\text { Dimensions } \\
(\mathrm{mm})\end{array}\end{array}$ & $\begin{array}{c}\text { Orifice } \\
\text { Area } \\
\left(\mathrm{mm}^{2}\right)\end{array}$ & $\begin{array}{c}\text { Target } \\
\text { Pressure } \\
(\mathrm{psig})\end{array}$ & Simulant & Test ID(s) & $\begin{array}{c}\text { Discharge } \\
\text { Coefficient }\end{array}$ \\
\hline 1 & 0.747 & 380 & STR-20 & SV-36 & 0.76 \\
1 & 0.747 & 380 & DST & SS-368 & 0.99 \\
1 & 0.747 & 380 & DST & SS-369 & 0.90 \\
\hline
\end{tabular}

Table D.43. Tests in Figure 7.18

\begin{tabular}{cccccc}
\hline $\begin{array}{c}\text { Target } \\
\text { Orifice } \\
\begin{array}{c}\text { Dimensions } \\
(\mathrm{mm})\end{array}\end{array}$ & $\begin{array}{c}\text { Orifice } \\
\text { Area } \\
\left(\mathrm{mm}^{2}\right)\end{array}$ & $\begin{array}{c}\text { Target } \\
\text { Pressure } \\
(\mathrm{psig})\end{array}$ & Simulant & Test ID(s) & $\begin{array}{c}\text { Discharge } \\
\text { Coefficient }\end{array}$ \\
\hline $0.5 \times 5$ & 2.61 & 380 & STR-20 & SV-37C & 0.60 \\
$0.5 \times 5$ & 2.61 & 380 & DST & SS-370 & 0.78 \\
$0.5 \times 5$ & 2.61 & 380 & DST & SS-371 & 0.74 \\
$0.5 \times 5$ & 2.61 & 380 & DST & SS-372 & 0.74 \\
$0.5 \times 5$ & 2.61 & 380 & DST & SS-374 & 0.74 \\
\hline
\end{tabular}


Table D.44. Tests in Figure 7.19

\begin{tabular}{cccccc}
\hline $\begin{array}{c}\text { Target } \\
\text { Orifice } \\
\begin{array}{c}\text { Dimensions } \\
(\mathrm{mm})\end{array}\end{array}$ & $\begin{array}{c}\text { Orifice } \\
\text { Area } \\
\left(\mathrm{mm}^{2}\right)\end{array}$ & $\begin{array}{c}\text { Target } \\
\text { Pressure } \\
(\mathrm{psig})\end{array}$ & Simulant & Test ID(s) & $\begin{array}{c}\text { Discharge } \\
\text { Coefficient }\end{array}$ \\
\hline 1 & 0.747 & 100 & Water & SS-145 & 0.75 \\
1 & 0.747 & 100 & Water & SS-146 & 0.74 \\
1 & 0.747 & 100 & Clay-6 & SS-232 & 0.98 \\
1 & 0.747 & 100 & Clay-6 & SS-233 & 0.91 \\
1 & 0.747 & 100 & Clay-6 & SS-234 & 0.84 \\
1 & 0.747 & 100 & Clay-30 & SS-162 & 0.96 \\
1 & 0.747 & 100 & Clay-30 & SS-163 & 0.96 \\
1 & 0.747 & 100 & Clay-30 & SS-164 & 0.95 \\
1 & 0.747 & 100 & Clay-30 & SS-165 & 0.97 \\
\hline
\end{tabular}

Table D.45. Tests in Figure 7.20

\begin{tabular}{cccccc}
\hline $\begin{array}{c}\text { Target } \\
\text { Orifice } \\
\begin{array}{c}\text { Dimensions } \\
(\mathrm{mm})\end{array}\end{array}$ & $\begin{array}{c}\text { Orifice } \\
\text { Area } \\
\left(\mathrm{mm}^{2}\right)\end{array}$ & $\begin{array}{c}\text { Target } \\
\text { Pressure } \\
(\mathrm{psig})\end{array}$ & Simulant & Test ID(s) & $\begin{array}{c}\text { Discharge } \\
\text { Coefficient }\end{array}$ \\
\hline 1 & 0.747 & 200 & Water & SS-147 & 0.74 \\
1 & 0.747 & 200 & Water & SS-148 & 0.74 \\
1 & 0.747 & 200 & Clay-6 & SS-235 & 0.86 \\
1 & 0.747 & 200 & Clay-6 & SS-236 & 0.99 \\
1 & 0.747 & 200 & Clay-6 & SS-237 & 0.91 \\
1 & 0.747 & 200 & Clay-30 & SS-159 & 0.98 \\
1 & 0.747 & 200 & Clay-30 & SS-160 & 0.93 \\
1 & 0.747 & 200 & Clay-30 & SS-161 & 0.95 \\
\hline
\end{tabular}

Table D.46. Tests in Figure 7.21

\begin{tabular}{cccccc}
\hline $\begin{array}{c}\text { Target } \\
\text { Orifice } \\
\begin{array}{c}\text { Dimensions } \\
(\mathrm{mm})\end{array}\end{array}$ & $\begin{array}{c}\text { Orifice } \\
\text { Area } \\
\left(\mathrm{mm}^{2}\right)\end{array}$ & $\begin{array}{c}\text { Target } \\
\text { Pressure } \\
(\mathrm{psig})\end{array}$ & Simulant & Test ID(s) & $\begin{array}{c}\text { Discharge } \\
\text { Coefficient }\end{array}$ \\
\hline 1 & 0.747 & 380 & Water & SS-150 & 0.75 \\
1 & 0.747 & 380 & Water & SS-151 & 0.75 \\
1 & 0.747 & 380 & Clay-6 & SS-241 & 0.89 \\
1 & 0.747 & 380 & Clay-6 & SS-242 & 0.91 \\
1 & 0.747 & 380 & Clay-6 & SS-243 & 0.89 \\
1 & 0.747 & 380 & Clay-30 & SS-154 & 0.86 \\
1 & 0.747 & 380 & Clay-30 & SS-155 & 0.86 \\
1 & 0.747 & 380 & Clay-30 & SS-156 & 0.89 \\
1 & 0.747 & 380 & Clay-30 & SS-157 & 0.89 \\
\hline
\end{tabular}


Table D.47. Tests in Figure 7.22

\begin{tabular}{cccccc}
\hline $\begin{array}{c}\text { Target } \\
\text { Orifice } \\
\begin{array}{c}\text { Dimensions } \\
(\mathrm{mm})\end{array}\end{array}$ & $\begin{array}{c}\text { Orifice } \\
\text { Area } \\
\left(\mathrm{mm}^{2}\right)\end{array}$ & $\begin{array}{c}\text { Target } \\
\text { Pressure } \\
(\mathrm{psig})\end{array}$ & Simulant & Test ID(s) & $\begin{array}{c}\text { Discharge } \\
\text { Coefficient }\end{array}$ \\
\hline $0.5 \times 5$ & 2.61 & 100 & Water & SS-138 & 0.63 \\
$0.5 \times 5$ & 2.61 & 100 & Water & SS-139 & 0.63 \\
$0.5 \times 5$ & 2.61 & 100 & Water & SS-140 & 0.62 \\
$0.5 \times 5$ & 2.61 & 100 & Clay-6 & SS-220 & 0.81 \\
$0.5 \times 5$ & 2.61 & 100 & Clay-6 & SS-221 & 0.81 \\
$0.5 \times 5$ & 2.61 & 100 & Clay-6 & SS-222 & 0.79 \\
$0.5 \times 5$ & 2.61 & 100 & Clay-30 & SS-166 & 0.63 \\
$0.5 \times 5$ & 2.61 & 100 & Clay-30 & SS-167 & 0.68 \\
$0.5 \times 5$ & 2.61 & 100 & Clay-30 & SS-168 & 0.70 \\
\hline
\end{tabular}

Table D.48. Tests in Figure 7.23

\begin{tabular}{cccccc}
\hline $\begin{array}{c}\text { Target } \\
\text { Orifice } \\
\begin{array}{c}\text { Dimensions } \\
(\mathrm{mm})\end{array}\end{array}$ & $\begin{array}{c}\text { Orifice } \\
\text { Area } \\
\left(\mathrm{mm}^{2}\right)\end{array}$ & $\begin{array}{c}\text { Target } \\
\text { Pressure } \\
(\mathrm{psig})\end{array}$ & Simulant & Test ID(s) & $\begin{array}{c}\text { Discharge } \\
\text { Coefficient }\end{array}$ \\
\hline $0.5 \times 5$ & 2.61 & 200 & Water & SS-141 & 0.62 \\
$0.5 \times 5$ & 2.61 & 200 & Water & SS-142 & 0.62 \\
$0.5 \times 5$ & 2.61 & 200 & Clay-6 & SS-223 & 0.78 \\
$0.5 \times 5$ & 2.61 & 200 & Clay-6 & SS-224 & 0.77 \\
$0.5 \times 5$ & 2.61 & 200 & Clay-6 & SS-225 & 0.78 \\
$0.5 \times 5$ & 2.61 & 200 & Clay-30 & SS-170 & 0.73 \\
$0.5 \times 5$ & 2.61 & 200 & Clay-30 & SS-171 & 0.71 \\
$0.5 \times 5$ & 2.61 & 200 & Clay-30 & SS-173 & 0.72 \\
$0.5 \times 5$ & 2.61 & 200 & Clay-30 & SS-174 & 0.70 \\
\hline
\end{tabular}

Table D.49. Tests in Figure 7.24

\begin{tabular}{cccccc}
\hline $\begin{array}{c}\text { Target } \\
\text { Orifice } \\
\begin{array}{c}\text { Dimensions } \\
(\mathrm{mm})\end{array}\end{array}$ & $\begin{array}{c}\text { Orifice } \\
\text { Area } \\
\left(\mathrm{mm}^{2}\right)\end{array}$ & $\begin{array}{c}\text { Target } \\
\text { Pressure } \\
(\mathrm{psig})\end{array}$ & Simulant & Test ID(s) & $\begin{array}{c}\text { Discharge } \\
\text { Coefficient }\end{array}$ \\
\hline $0.5 \times 5$ & 2.61 & 380 & Water & SS-143 & 0.63 \\
$0.5 \times 5$ & 2.61 & 380 & Water & SS-144 & 0.62 \\
$0.5 \times 5$ & 2.61 & 380 & Clay-6 & SS-229 & 0.77 \\
$0.5 \times 5$ & 2.61 & 380 & Clay-6 & SS-230 & 0.76 \\
$0.5 \times 5$ & 2.61 & 380 & Clay-6 & SS-231 & 0.77 \\
$0.5 \times 5$ & 2.61 & 380 & Clay-30 & SS-176 & 0.73 \\
$0.5 \times 5$ & 2.61 & 380 & Clay-30 & SS-178 & 0.74 \\
$0.5 \times 5$ & 2.61 & 380 & Clay-30 & SS-179 & 0.73 \\
\hline
\end{tabular}


Table D.50. Tests in Figure 7.25

\begin{tabular}{cccccc}
\hline $\begin{array}{c}\text { Target } \\
\text { Orifice } \\
\begin{array}{c}\text { Dimensions } \\
(\mathrm{mm})\end{array}\end{array}$ & $\begin{array}{c}\text { Orifice } \\
\text { Area } \\
\left(\mathrm{mm}^{2}\right)\end{array}$ & $\begin{array}{c}\text { Target } \\
\text { Pressure } \\
(\mathrm{psig})\end{array}$ & Simulant & Test ID(s) & $\begin{array}{c}\text { Discharge } \\
\text { Coefficient }\end{array}$ \\
\hline 1 & 0.747 & 100 & Water & SS-145 & 0.75 \\
1 & 0.747 & 100 & Water & SS-146 & 0.74 \\
1 & 0.747 & 100 & FEG-6 & SS-299 & 0.88 \\
1 & 0.747 & 100 & FEG-6 & SS-300 & 0.90 \\
1 & 0.747 & 100 & FEG-6 & SS-301 & 0.92 \\
1 & 0.747 & 100 & FEG-30 & SS-251 & 0.94 \\
1 & 0.747 & 100 & FEG-30 & SS-253 & 0.98 \\
1 & 0.747 & 100 & FEG-30 & SS-261 & 0.91 \\
\hline
\end{tabular}

Table D.51. Tests in Figure 7.26

\begin{tabular}{cccccc}
\hline $\begin{array}{c}\text { Target } \\
\text { Orifice } \\
\begin{array}{c}\text { Dimensions } \\
(\mathrm{mm})\end{array}\end{array}$ & $\begin{array}{c}\text { Orifice } \\
\text { Area } \\
\left(\mathrm{mm}^{2}\right)\end{array}$ & $\begin{array}{c}\text { Target } \\
\text { Pressure } \\
(\mathrm{psig})\end{array}$ & Simulant & Test ID(s) & $\begin{array}{c}\text { Discharge } \\
\text { Coefficient }\end{array}$ \\
\hline 1 & 0.747 & 200 & Water & SS-147 & 0.74 \\
1 & 0.747 & 200 & Water & SS-148 & 0.74 \\
1 & 0.747 & 200 & FEG-6 & SS-302 & 0.87 \\
1 & 0.747 & 200 & FEG-6 & SS-303 & 0.96 \\
1 & 0.747 & 200 & FEG-6 & SS-305 & 0.86 \\
1 & 0.747 & 200 & FEG-30 & SS-255 & 0.75 \\
1 & 0.747 & 200 & FEG-30 & SS-256 & 0.80 \\
1 & 0.747 & 200 & FEG-30 & SS-271 & 0.69 \\
\hline
\end{tabular}

Table D.52. Tests in Figure 7.27

\begin{tabular}{cccccc}
\hline $\begin{array}{c}\text { Target } \\
\text { Orifice } \\
\begin{array}{c}\text { Dimensions } \\
(\mathrm{mm})\end{array}\end{array}$ & $\begin{array}{c}\text { Orifice } \\
\text { Area } \\
\left(\mathrm{mm}^{2}\right)\end{array}$ & $\begin{array}{c}\text { Target } \\
\text { Pressure } \\
(\mathrm{psig})\end{array}$ & Simulant & Test ID(s) & $\begin{array}{c}\text { Discharge } \\
\text { Coefficient }\end{array}$ \\
\hline 1 & 0.747 & 380 & Water & SS-150 & 0.75 \\
1 & 0.747 & 380 & Water & SS-151 & 0.75 \\
1 & 0.747 & 380 & FEG-6 & SS-307 & 0.89 \\
1 & 0.747 & 380 & FEG-6 & SS-309 & 0.94 \\
1 & 0.747 & 380 & FEG-30 & SS-266 & 0.97 \\
\hline
\end{tabular}


Table D.53. Tests in Figure 7.28

\begin{tabular}{cccccc}
\hline $\begin{array}{c}\text { Target } \\
\text { Orifice } \\
\text { Dimensions } \\
(\mathrm{mm})\end{array}$ & $\begin{array}{c}\text { Orifice } \\
\text { Area } \\
\left(\mathrm{mm}^{2}\right)\end{array}$ & $\begin{array}{c}\text { Target } \\
\text { Pressure } \\
(\mathrm{psig})\end{array}$ & Simulant & Test ID(s) & $\begin{array}{c}\text { Discharge } \\
\text { Coefficient }\end{array}$ \\
\hline $0.5 \times 5$ & 2.61 & 100 & Water & SS-138 & 0.63 \\
$0.5 \times 5$ & 2.61 & 100 & Water & SS-139 & 0.63 \\
$0.5 \times 5$ & 2.61 & 100 & Water & SS-140 & 0.62 \\
$0.5 \times 5$ & 2.61 & 100 & FEG-6 & SS-312 & 0.81 \\
$0.5 \times 5$ & 2.61 & 100 & FEG-6 & SS-313 & 0.76 \\
$0.5 \times 5$ & 2.61 & 100 & FEG-30 & SS-274 & 0.80 \\
$0.5 \times 5$ & 2.61 & 100 & FEG-30 & SS-275 & 0.76 \\
\hline
\end{tabular}

Table D.54. Tests in Figure 7.29

\begin{tabular}{cccccc}
\hline $\begin{array}{c}\text { Target } \\
\text { Orifice } \\
\begin{array}{c}\text { Dimensions } \\
(\mathrm{mm})\end{array}\end{array}$ & $\begin{array}{c}\text { Orifice } \\
\text { Area } \\
\left(\mathrm{mm}^{2}\right)\end{array}$ & $\begin{array}{c}\text { Target } \\
\text { Pressure } \\
(\mathrm{psig})\end{array}$ & Simulant & Test ID(s) & $\begin{array}{c}\text { Discharge } \\
\text { Coefficient }\end{array}$ \\
\hline $0.5 \times 5$ & 2.61 & 200 & Water & SS-141 & 0.62 \\
$0.5 \times 5$ & 2.61 & 200 & Water & SS-142 & 0.62 \\
$0.5 \times 5$ & 2.61 & 200 & FEG-6 & SS-315 & 0.76 \\
$0.5 \times 5$ & 2.61 & 200 & FEG-30 & SS-277 & 0.72 \\
$0.5 \times 5$ & 2.61 & 200 & FEG-30 & SS-280 & 0.84 \\
\hline
\end{tabular}

Table D.55. Tests in Figure 7.30

\begin{tabular}{cccccc}
\hline $\begin{array}{c}\text { Target } \\
\text { Orifice } \\
\begin{array}{c}\text { Dimensions } \\
(\mathrm{mm})\end{array}\end{array}$ & $\begin{array}{c}\text { Orifice } \\
\text { Area } \\
\left(\mathrm{mm}^{2}\right)\end{array}$ & $\begin{array}{c}\text { Target } \\
\text { Pressure } \\
(\mathrm{psig})\end{array}$ & Simulant & Test ID(s) & $\begin{array}{c}\text { Discharge } \\
\text { Coefficient }\end{array}$ \\
\hline $0.5 \times 5$ & 2.61 & 380 & Water & SS-143 & 0.63 \\
$0.5 \times 5$ & 2.61 & 380 & Water & SS-144 & 0.62 \\
$0.5 \times 5$ & 2.61 & 380 & FEG-6 & SS-317 & 0.79 \\
$0.5 \times 5$ & 2.61 & 380 & FEG-6 & SS-320 & 0.76 \\
$0.5 \times 5$ & 2.61 & 380 & FEG-30 & SS-281 & 0.81 \\
$0.5 \times 5$ & 2.61 & 380 & FEG-30 & SS-282 & 0.76 \\
$0.5 \times 5$ & 2.61 & 380 & FEG-30 & SS-283 & 0.75 \\
\hline
\end{tabular}


Table D.56. Tests in Figure 7.31

\begin{tabular}{cccccc}
\hline $\begin{array}{c}\text { Target } \\
\text { Orifice } \\
\begin{array}{c}\text { Dimensions } \\
(\mathrm{mm})\end{array}\end{array}$ & $\begin{array}{c}\text { Orifice } \\
\text { Area } \\
\left(\mathrm{mm}^{2}\right)\end{array}$ & $\begin{array}{c}\text { Target } \\
\text { Pressure } \\
(\mathrm{psig})\end{array}$ & Simulant & Test ID(s) & $\begin{array}{c}\text { Discharge } \\
\text { Coefficient }\end{array}$ \\
\hline 1 & 0.747 & 100 & FEG-30 & SS-251 & 0.94 \\
1 & 0.747 & 100 & FEG-30 & SS-253 & 0.98 \\
1 & 0.747 & 100 & FEG-30 & SS-261 & 0.91 \\
1 & 0.747 & 100 & Clay-30 & SS-162 & 0.96 \\
1 & 0.747 & 100 & Clay-30 & SS-163 & 0.96 \\
1 & 0.747 & 100 & Clay-30 & SS-164 & 0.95 \\
1 & 0.747 & 100 & Clay-30 & SS-165 & 0.97 \\
\hline
\end{tabular}

Table D.57. Tests in Figure 7.32

\begin{tabular}{cccccc}
\hline $\begin{array}{c}\text { Target } \\
\text { Orifice } \\
\text { Dimensions } \\
(\mathrm{mm})\end{array}$ & $\begin{array}{c}\text { Orifice } \\
\text { Area } \\
\left(\mathrm{mm}^{2}\right)\end{array}$ & $\begin{array}{c}\text { Target } \\
\text { Pressure } \\
(\mathrm{psig})\end{array}$ & Simulant & Test ID(s) & $\begin{array}{c}\text { Discharge } \\
\text { Coefficient }\end{array}$ \\
\hline 1 & 0.747 & 200 & FEG-30 & SS-255 & 0.75 \\
1 & 0.747 & 200 & FEG-30 & SS-256 & 0.80 \\
1 & 0.747 & 200 & FEG-30 & SS-271 & 0.69 \\
1 & 0.747 & 200 & Clay-30 & SS-159 & 0.98 \\
1 & 0.747 & 200 & Clay-30 & SS-160 & 0.93 \\
1 & 0.747 & 200 & Clay-30 & SS-161 & 0.95 \\
\hline
\end{tabular}

Table D.58. Tests in Figure 7.33

\begin{tabular}{cccccc}
\hline $\begin{array}{c}\text { Target } \\
\text { Orifice } \\
\begin{array}{c}\text { Dimensions } \\
(\mathrm{mm})\end{array}\end{array}$ & $\begin{array}{c}\text { Orifice } \\
\text { Area } \\
\left(\mathrm{mm}^{2}\right)\end{array}$ & $\begin{array}{c}\text { Target } \\
\text { Pressure } \\
(\mathrm{psig})\end{array}$ & Simulant & Test ID(s) & $\begin{array}{c}\text { Discharge } \\
\text { Coefficient }\end{array}$ \\
\hline 1 & 0.747 & 380 & FEG-30 & SS-266 & 0.97 \\
1 & 0.747 & 380 & Clay-30 & SS-154 & 0.86 \\
1 & 0.747 & 380 & Clay-30 & SS-155 & 0.86 \\
1 & 0.747 & 380 & Clay-30 & SS-156 & 0.89 \\
1 & 0.747 & 380 & Clay-30 & SS-157 & 0.89 \\
\hline
\end{tabular}


Table D.59. Tests in Figure 7.34

\begin{tabular}{cccccc}
\hline $\begin{array}{c}\text { Target } \\
\text { Orifice } \\
\text { Dimensions } \\
(\mathrm{mm})\end{array}$ & $\begin{array}{c}\text { Orifice } \\
\text { Area } \\
\left(\mathrm{mm}^{2}\right)\end{array}$ & $\begin{array}{c}\text { Target } \\
\text { Pressure } \\
(\mathrm{psig})\end{array}$ & Simulant & Test ID(s) & $\begin{array}{c}\text { Discharge } \\
\text { Coefficient }\end{array}$ \\
\hline $0.5 \times 5$ & 2.61 & 100 & FEG-30 & SS-274 & 0.80 \\
$0.5 \times 5$ & 2.61 & 100 & FEG-30 & SS-275 & 0.76 \\
$0.5 \times 5$ & 2.61 & 100 & Clay-30 & SS-166 & 0.63 \\
$0.5 \times 5$ & 2.61 & 100 & Clay-30 & SS-167 & 0.68 \\
$0.5 \times 5$ & 2.61 & 100 & Clay-30 & SS-168 & 0.70 \\
\hline
\end{tabular}

Table D.60. Tests in Figure 7.35

\begin{tabular}{cccccc}
\hline $\begin{array}{c}\text { Target } \\
\text { Orifice } \\
\text { Dimensions } \\
(\mathrm{mm})\end{array}$ & $\begin{array}{c}\text { Orifice } \\
\text { Area } \\
\left(\mathrm{mm}^{2}\right)\end{array}$ & $\begin{array}{c}\text { Target } \\
\text { Pressure } \\
(\mathrm{psig})\end{array}$ & Simulant & Test ID(s) & $\begin{array}{c}\text { Discharge } \\
\text { Coefficient }\end{array}$ \\
\hline $0.5 \times 5$ & 2.61 & 200 & FEG-30 & SS-277 & 0.72 \\
$0.5 \times 5$ & 2.61 & 200 & FEG-30 & SS-280 & 0.84 \\
$0.5 \times 5$ & 2.61 & 200 & Clay-30 & SS-170 & 0.73 \\
$0.5 \times 5$ & 2.61 & 200 & Clay-30 & SS-171 & 0.71 \\
$0.5 \times 5$ & 2.61 & 200 & Clay-30 & SS-173 & 0.72 \\
$0.5 \times 5$ & 2.61 & 200 & Clay-30 & SS-174 & 0.70 \\
\hline
\end{tabular}

Table D.61. Tests in Figure 7.36

\begin{tabular}{cccccc}
\hline $\begin{array}{c}\text { Target } \\
\text { Orifice } \\
\begin{array}{c}\text { Dimensions } \\
(\mathrm{mm})\end{array}\end{array}$ & $\begin{array}{c}\text { Orifice } \\
\text { Area } \\
\left(\mathrm{mm}^{2}\right)\end{array}$ & $\begin{array}{c}\text { Target } \\
\text { Pressure } \\
(\mathrm{psig})\end{array}$ & Simulant & Test ID(s) & $\begin{array}{c}\text { Discharge } \\
\text { Coefficient }\end{array}$ \\
\hline $0.5 \times 5$ & 2.61 & 380 & FEG-30 & SS-281 & 0.81 \\
$0.5 \times 5$ & 2.61 & 380 & FEG-30 & SS-282 & 0.76 \\
$0.5 \times 5$ & 2.61 & 380 & FEG-30 & SS-283 & 0.75 \\
$0.5 \times 5$ & 2.61 & 380 & Clay-30 & SS-176 & 0.73 \\
$0.5 \times 5$ & 2.61 & 380 & Clay-30 & SS-178 & 0.74 \\
$0.5 \times 5$ & 2.61 & 380 & Clay-30 & SS-179 & 0.73 \\
\hline
\end{tabular}


Table D.62. Tests in Figure 7.37

\begin{tabular}{cccccc}
\hline $\begin{array}{c}\text { Target } \\
\text { Orifice } \\
\begin{array}{c}\text { Dimensions } \\
(\mathrm{mm})\end{array}\end{array}$ & $\begin{array}{c}\text { Orifice } \\
\text { Area } \\
\left(\mathrm{mm}^{2}\right)\end{array}$ & $\begin{array}{c}\text { Target } \\
\text { Pressure } \\
(\mathrm{psig})\end{array}$ & Simulant & Test ID(s) & $\begin{array}{c}\text { Discharge } \\
\text { Coefficient }\end{array}$ \\
\hline 1 & 0.747 & 100 & FEG-6 & SS-299 & 0.88 \\
1 & 0.747 & 100 & FEG-6 & SS-300 & 0.90 \\
1 & 0.747 & 100 & FEG-6 & SS-301 & 0.92 \\
1 & 0.747 & 100 & Clay-6 & SS-232 & 0.98 \\
1 & 0.747 & 100 & Clay-6 & SS-233 & 0.91 \\
1 & 0.747 & 100 & Clay-6 & SS-234 & 0.84 \\
\hline
\end{tabular}

Table D.63. Tests in Figure 7.38

\begin{tabular}{cccccc}
\hline $\begin{array}{c}\text { Target } \\
\text { Orifice } \\
\begin{array}{c}\text { Dimensions } \\
(\mathrm{mm})\end{array}\end{array}$ & $\begin{array}{c}\text { Orifice } \\
\text { Area } \\
\left(\mathrm{mm}^{2}\right)\end{array}$ & $\begin{array}{c}\text { Target } \\
\text { Pressure } \\
(\mathrm{psig})\end{array}$ & Simulant & Test ID(s) & $\begin{array}{c}\text { Discharge } \\
\text { Coefficient }\end{array}$ \\
\hline 1 & 0.747 & 200 & FEG-6 & SS-302 & 0.87 \\
1 & 0.747 & 200 & FEG-6 & SS-303 & 0.96 \\
1 & 0.747 & 200 & FEG-6 & SS-305 & 0.86 \\
1 & 0.747 & 200 & Clay-6 & SS-235 & 0.86 \\
1 & 0.747 & 200 & Clay-6 & SS-236 & 0.99 \\
1 & 0.747 & 200 & Clay-6 & SS-237 & 0.91 \\
\hline
\end{tabular}

Table D.64. Tests in Figure 7.39

\begin{tabular}{cccccc}
\hline $\begin{array}{c}\text { Target } \\
\text { Orifice } \\
\begin{array}{c}\text { Dimensions } \\
(\mathrm{mm})\end{array}\end{array}$ & $\begin{array}{c}\text { Orifice } \\
\text { Area } \\
\left(\mathrm{mm}^{2}\right)\end{array}$ & $\begin{array}{c}\text { Target } \\
\text { Pressure } \\
(\mathrm{psig})\end{array}$ & Simulant & Test ID(s) & $\begin{array}{c}\text { Discharge } \\
\text { Coefficient }\end{array}$ \\
\hline 1 & 0.747 & 380 & FEG-6 & SS-307 & 0.89 \\
1 & 0.747 & 380 & FEG-6 & SS-309 & 0.94 \\
1 & 0.747 & 380 & Clay-6 & SS-241 & 0.89 \\
1 & 0.747 & 380 & Clay-6 & SS-242 & 0.91 \\
1 & 0.747 & 380 & Clay-6 & SS-243 & 0.89 \\
\hline
\end{tabular}


Table D.65. Tests in Figure 7.40

\begin{tabular}{cccccc}
\hline $\begin{array}{c}\text { Target } \\
\text { Orifice } \\
\begin{array}{c}\text { Dimensions } \\
(\mathrm{mm})\end{array}\end{array}$ & $\begin{array}{c}\text { Orifice } \\
\text { Area } \\
\left(\mathrm{mm}^{2}\right)\end{array}$ & $\begin{array}{c}\text { Target } \\
\text { Pressure } \\
(\mathrm{psig})\end{array}$ & Simulant & Test ID(s) & $\begin{array}{c}\text { Discharge } \\
\text { Coefficient }\end{array}$ \\
\hline $0.5 \times 5$ & 2.61 & 100 & FEG-6 & SS-312 & 0.81 \\
$0.5 \times 5$ & 2.61 & 100 & FEG-6 & SS-313 & 0.76 \\
$0.5 \times 5$ & 2.61 & 100 & Clay-6 & SS-220 & 0.81 \\
$0.5 \times 5$ & 2.61 & 100 & Clay-6 & SS-221 & 0.81 \\
$0.5 \times 5$ & 2.61 & 100 & Clay-6 & SS-222 & 0.79 \\
\hline
\end{tabular}

Table D.66. Tests in Figure 7.41

\begin{tabular}{cccccc}
\hline $\begin{array}{c}\text { Target } \\
\text { Orifice } \\
\begin{array}{c}\text { Dimensions } \\
(\mathrm{mm})\end{array}\end{array}$ & $\begin{array}{c}\text { Orifice } \\
\text { Area } \\
\left(\mathrm{mm}^{2}\right)\end{array}$ & $\begin{array}{c}\text { Target } \\
\text { Pressure } \\
(\mathrm{psig})\end{array}$ & Simulant & Test ID(s) & $\begin{array}{c}\text { Discharge } \\
\text { Coefficient }\end{array}$ \\
\hline $0.5 \times 5$ & 2.61 & 200 & FEG-6 & SS-315 & 0.76 \\
$0.5 \times 5$ & 2.61 & 200 & Clay-6 & SS-223 & 0.78 \\
$0.5 \times 5$ & 2.61 & 200 & Clay-6 & SS-224 & 0.77 \\
$0.5 \times 5$ & 2.61 & 200 & Clay-6 & SS-225 & 0.78 \\
\hline
\end{tabular}

Table D.67. Tests in Figure 7.42

\begin{tabular}{cccccc}
\hline $\begin{array}{c}\text { Target } \\
\text { Orifice } \\
\begin{array}{c}\text { Dimensions } \\
(\mathrm{mm})\end{array}\end{array}$ & $\begin{array}{c}\text { Orifice } \\
\text { Area } \\
\left(\mathrm{mm}^{2}\right)\end{array}$ & $\begin{array}{c}\text { Target } \\
\text { Pressure } \\
(\mathrm{psig})\end{array}$ & Simulant & Test ID(s) & $\begin{array}{c}\text { Discharge } \\
\text { Coefficient }\end{array}$ \\
\hline $0.5 \times 5$ & 2.61 & 380 & FEG-6 & SS-317 & 0.79 \\
$0.5 \times 5$ & 2.61 & 380 & FEG-6 & SS-320 & 0.76 \\
$0.5 \times 5$ & 2.61 & 380 & Clay-6 & SS-229 & 0.77 \\
$0.5 \times 5$ & 2.61 & 380 & Clay-6 & SS-230 & 0.76 \\
$0.5 \times 5$ & 2.61 & 380 & Clay-6 & SS-231 & 0.77 \\
\hline
\end{tabular}



Appendix E

Selected Rheograms from Phase II Simulants 



\section{Appendix E}

\section{Selected Rheograms from Phase II Simulants}

This appendix presents additional rheograms for two Newtonian (27 wt $\%$ STR and $20 \mathrm{wt} \%$ DST) simulants and two non-Newtonian (chemical slurry and clay slurry) simulants. The rheology measurements were conducted as described in Section 3.3.2. In each figure, the range of data fit using the Bingham model to determine the yield stress and consistency is indicated by the red line, and in cases where two data fit ranges were used, a green line. When separate curves are distinguishable, the up and down ramps for shear rate are indicated by arrows superimposed on the figures. Note that in all figures, the $\mathrm{y}$-axis is shear stress $(\tau)$ with units of $\mathrm{Pa}$, and the $\mathrm{x}$-axis is shear rate $(\dot{\gamma})$ with units of $\mathrm{s}^{-1}$.

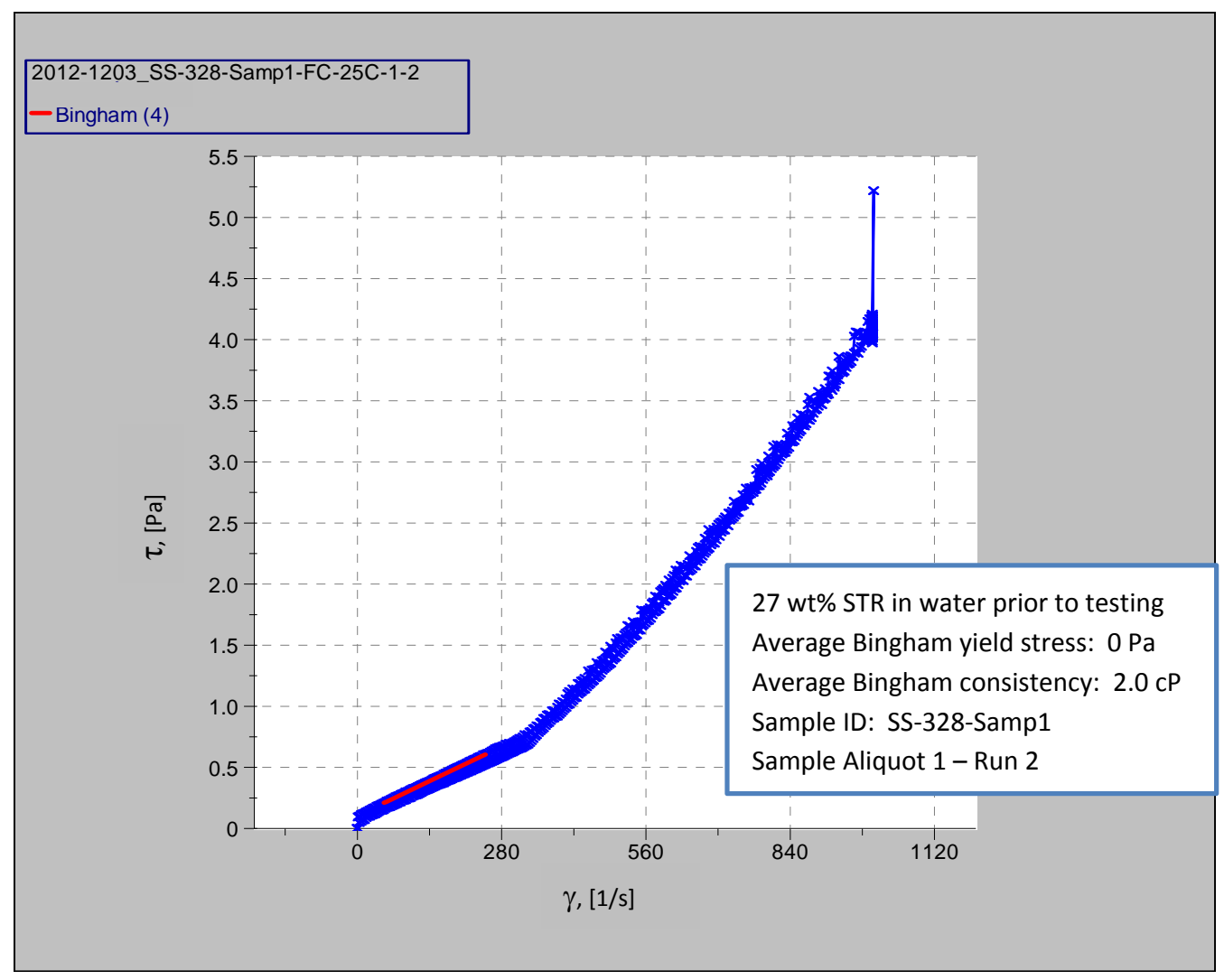

Figure E.1. Rheogram ( $\tau$ vs. $\dot{\gamma})$ for $27 \mathrm{wt} \%$ STR Simulant 


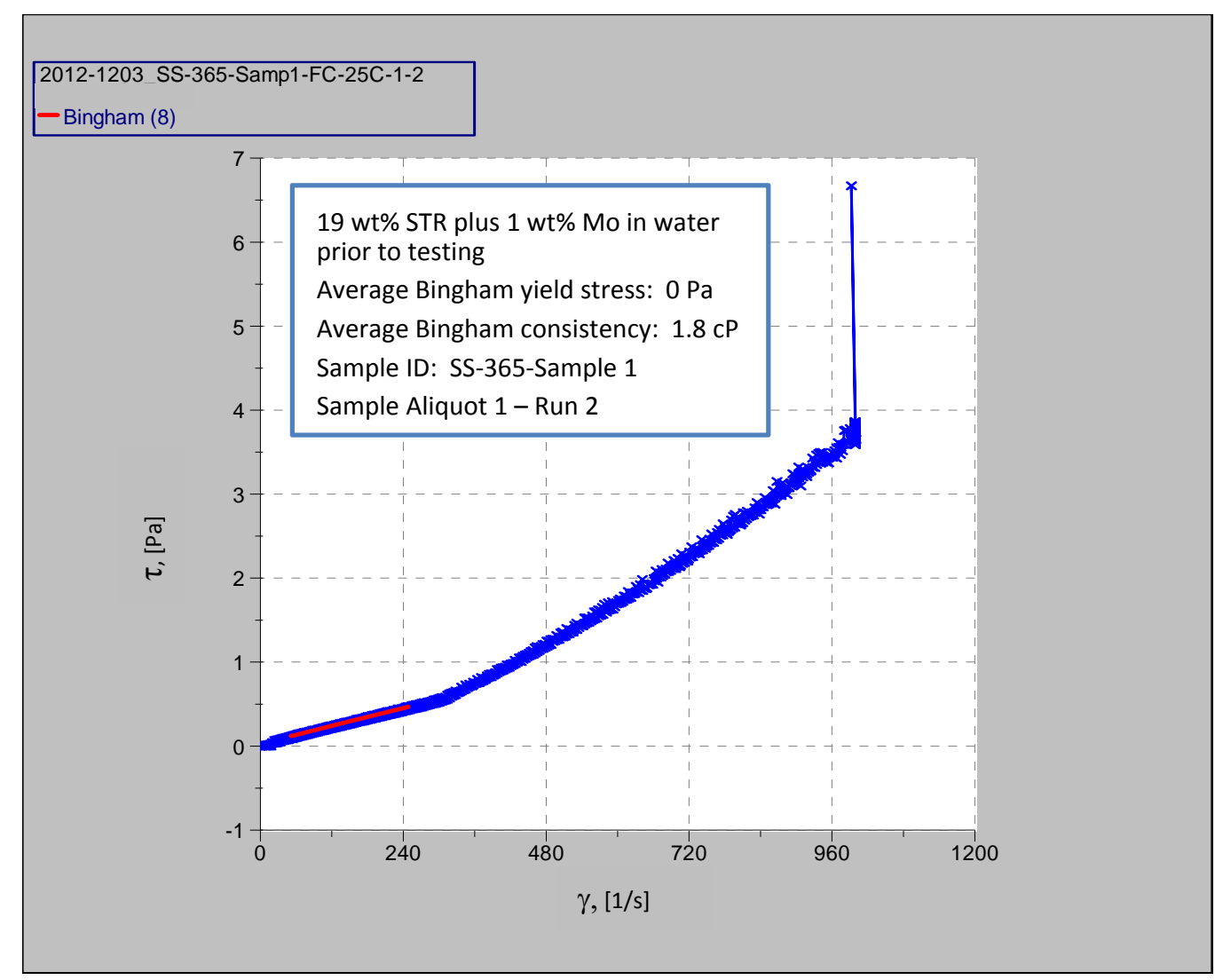

Figure E.2. Rheogram ( $\tau$ vs. $\dot{\gamma}$ ) for $20 \mathrm{wt} \%$ DST (19 wt\% STR plus $1 \mathrm{wt} \%$ Mo) Simulant 


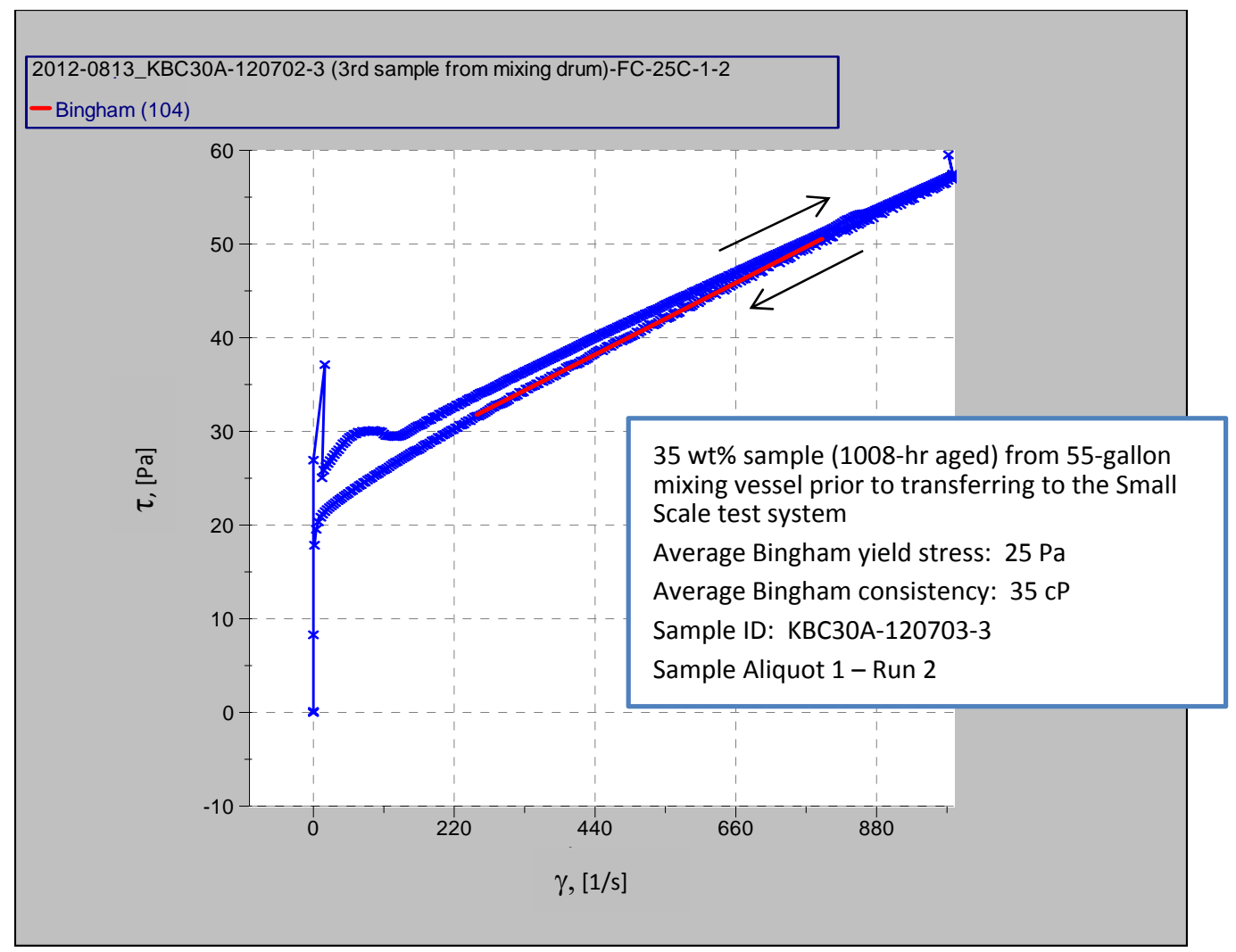

Figure E.3. Rheogram ( $\tau$ vs. $\dot{\gamma})$ for $35 \mathrm{wt} \%$ Clay Simulant (1008 $\mathrm{hr}$ aged) from the 55-Gallon Mixing Vessel Prior to Transferring to the Small-Scale Test System 


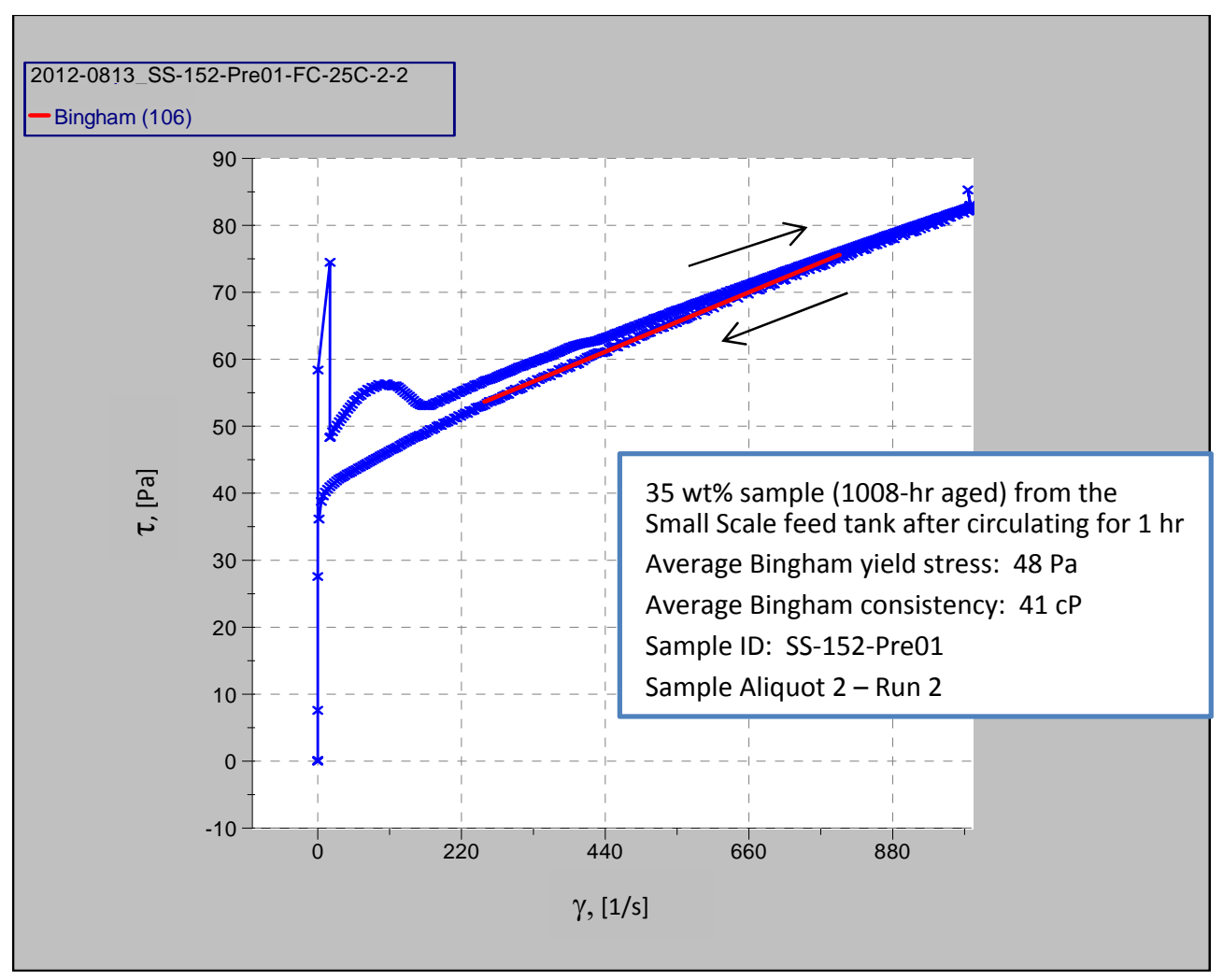

Figure E.4. Rheogram ( $\tau$ vs. $\dot{\gamma})$ for $35 \mathrm{wt} \%$ Clay Simulant (1008 hr aged) from the Small-Scale Feed Tank After Circulating for $1 \mathrm{hr}$ 


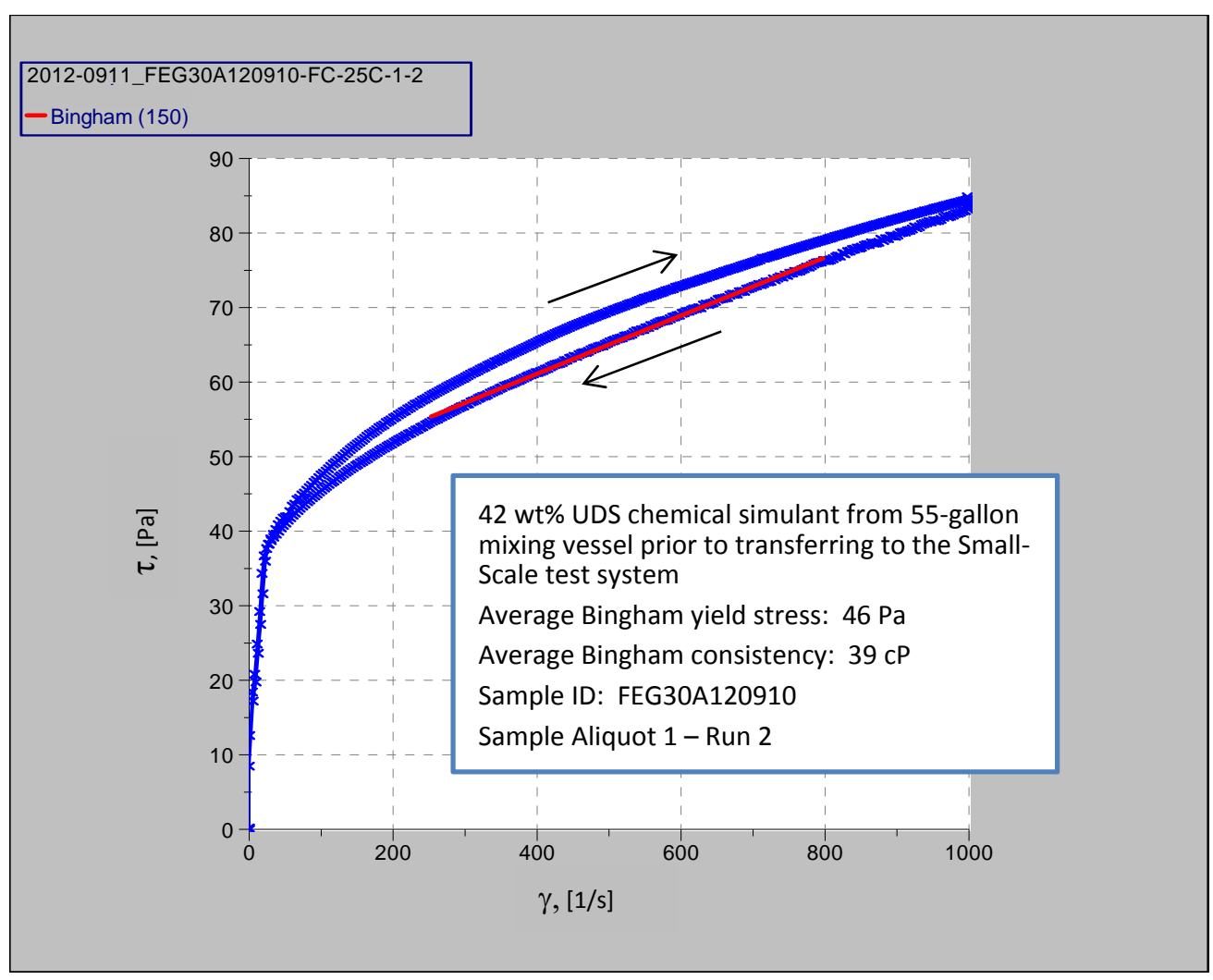

Figure E.5. Rheogram ( $\tau$ vs. $\dot{\gamma}$ ) for Chemical Simulant from the 55-Gallon Mixing Vessel Prior to Transferring to the Small-Scale Test System 


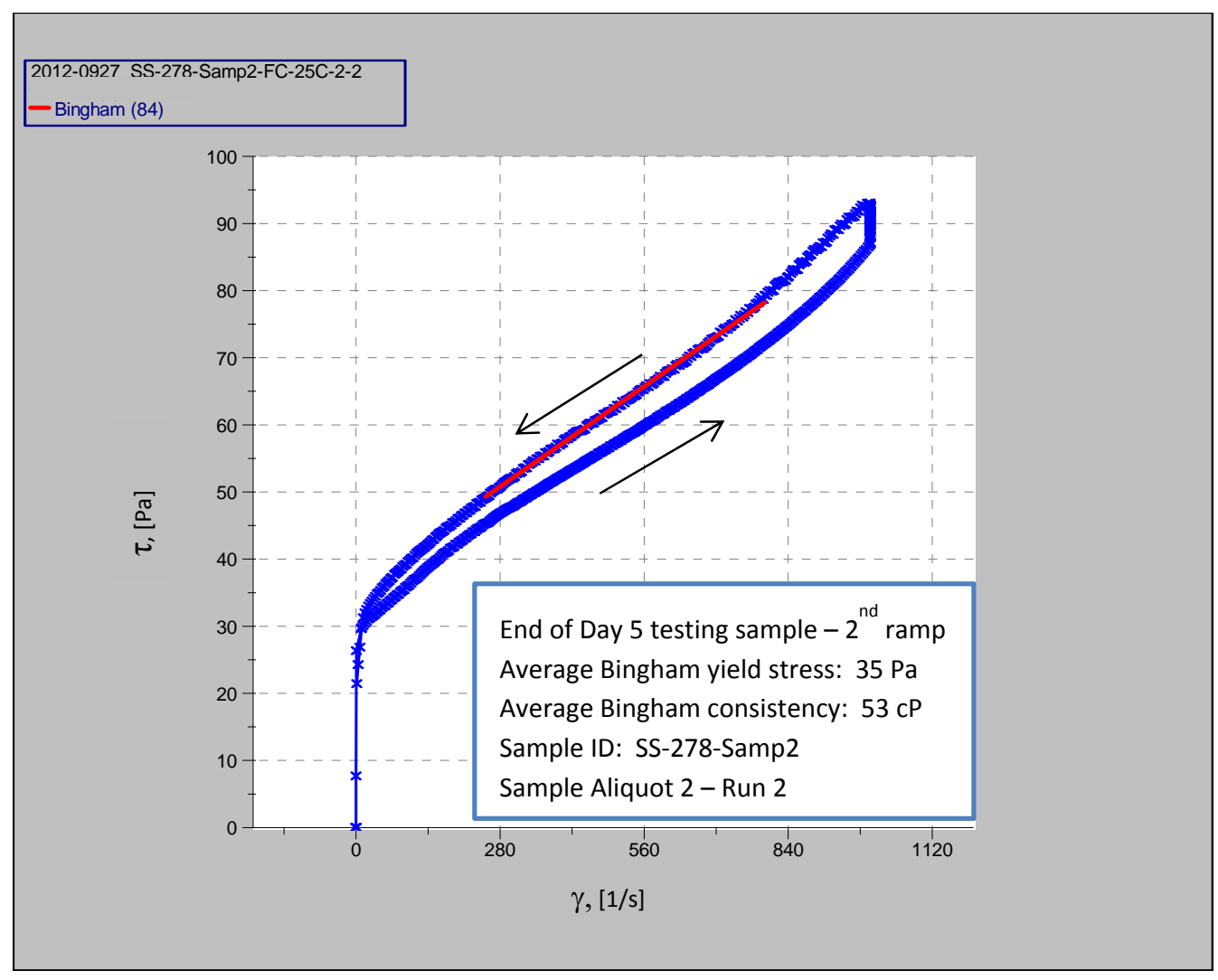

Figure E.6. Rheogram ( $\tau$ vs. $\dot{\gamma}$ ) for Chemical Simulant at the End of Day 5 of the 7 -day $30 \mathrm{~Pa} / 30 \mathrm{cP}$ Testing 


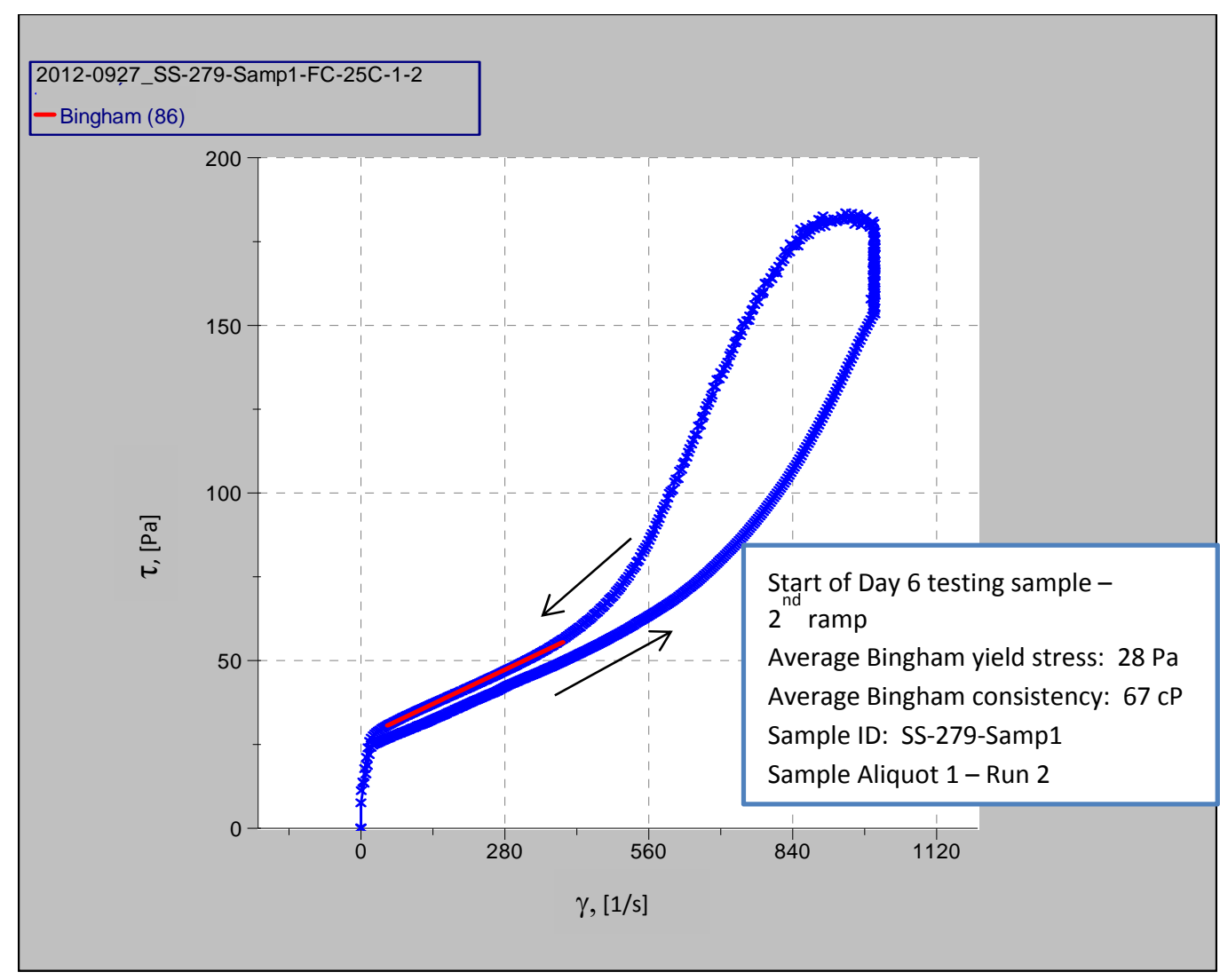

Figure E.7. Rheogram ( $\tau$ vs. $\dot{\gamma}$ ) for Chemical Simulant at the Beginning of Day 6 of the 7-day $30 \mathrm{~Pa} / 30 \mathrm{cP}$ Testing; Measured Shear Rate Range of $0-1000 \mathrm{~s}^{-1}$ 


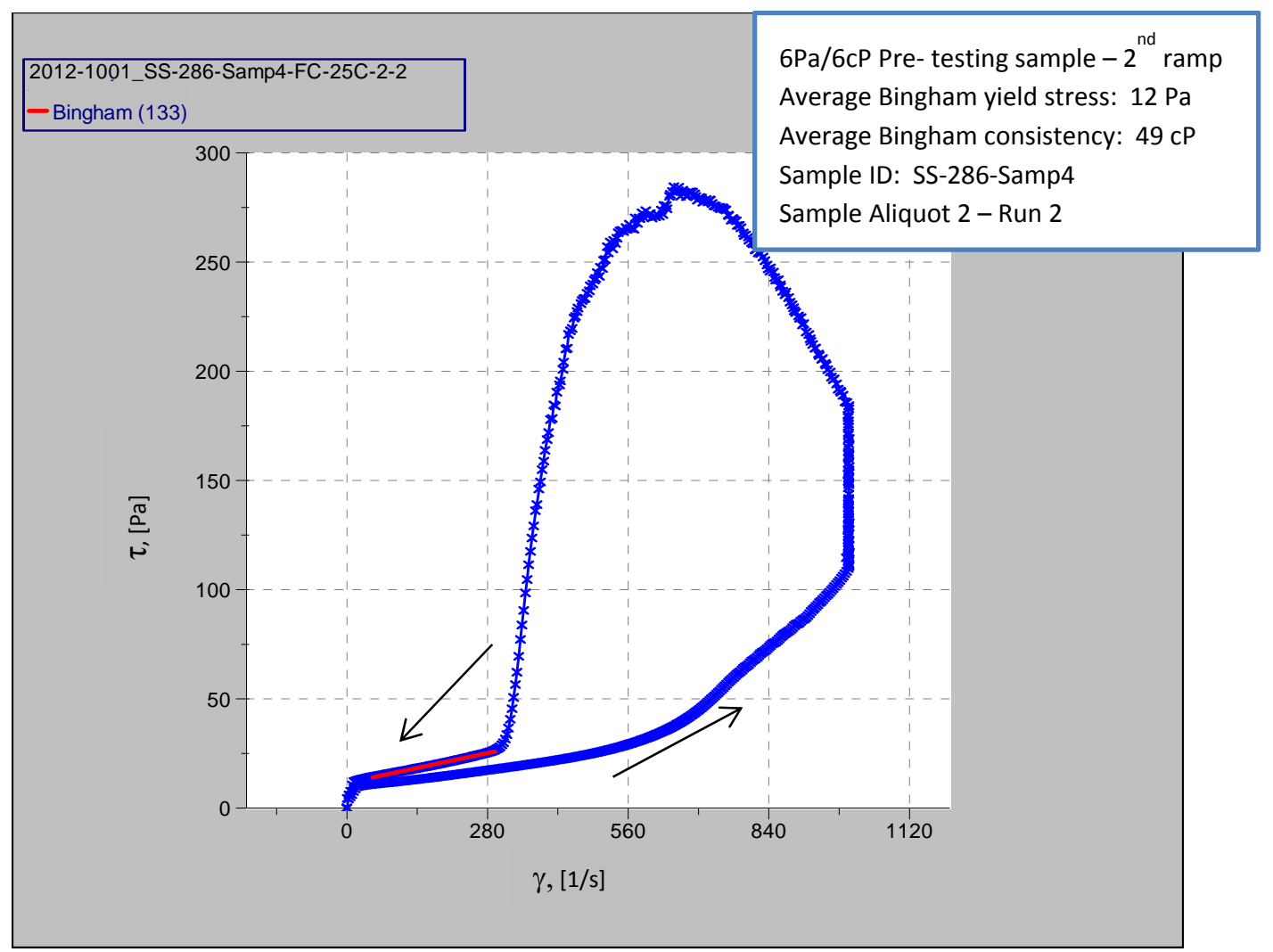

Figure E.8. Rheogram ( $\tau$ vs. $\dot{\gamma})$ for Pre-Testing Sample of Chemical Simulant After In Situ Dilution of the $30 \mathrm{~Pa} / 30 \mathrm{cP}$ Simulant; Measured Shear Rate Range of $0-1000 \mathrm{~s}^{-1}$ 


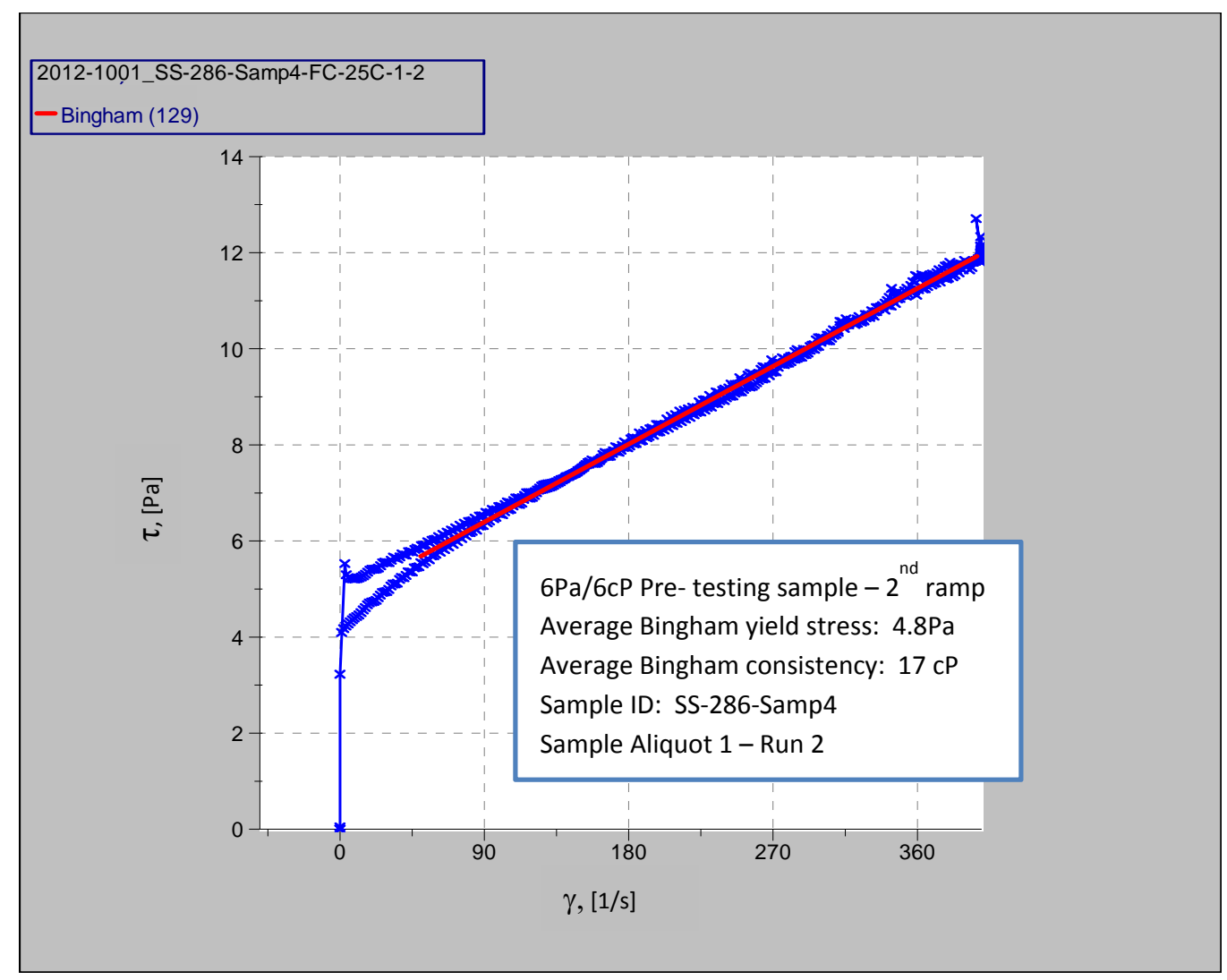

Figure E.9. Rheogram ( $\tau$ vs. $\dot{\gamma}$ ) for Pre-Testing Sample of Chemical Simulant After In Situ Dilution of the $30 \mathrm{~Pa} / 30 \mathrm{cP}$ Simulant; Measured Shear Rate Range of $0-400 \mathrm{~s}^{-1}$ 


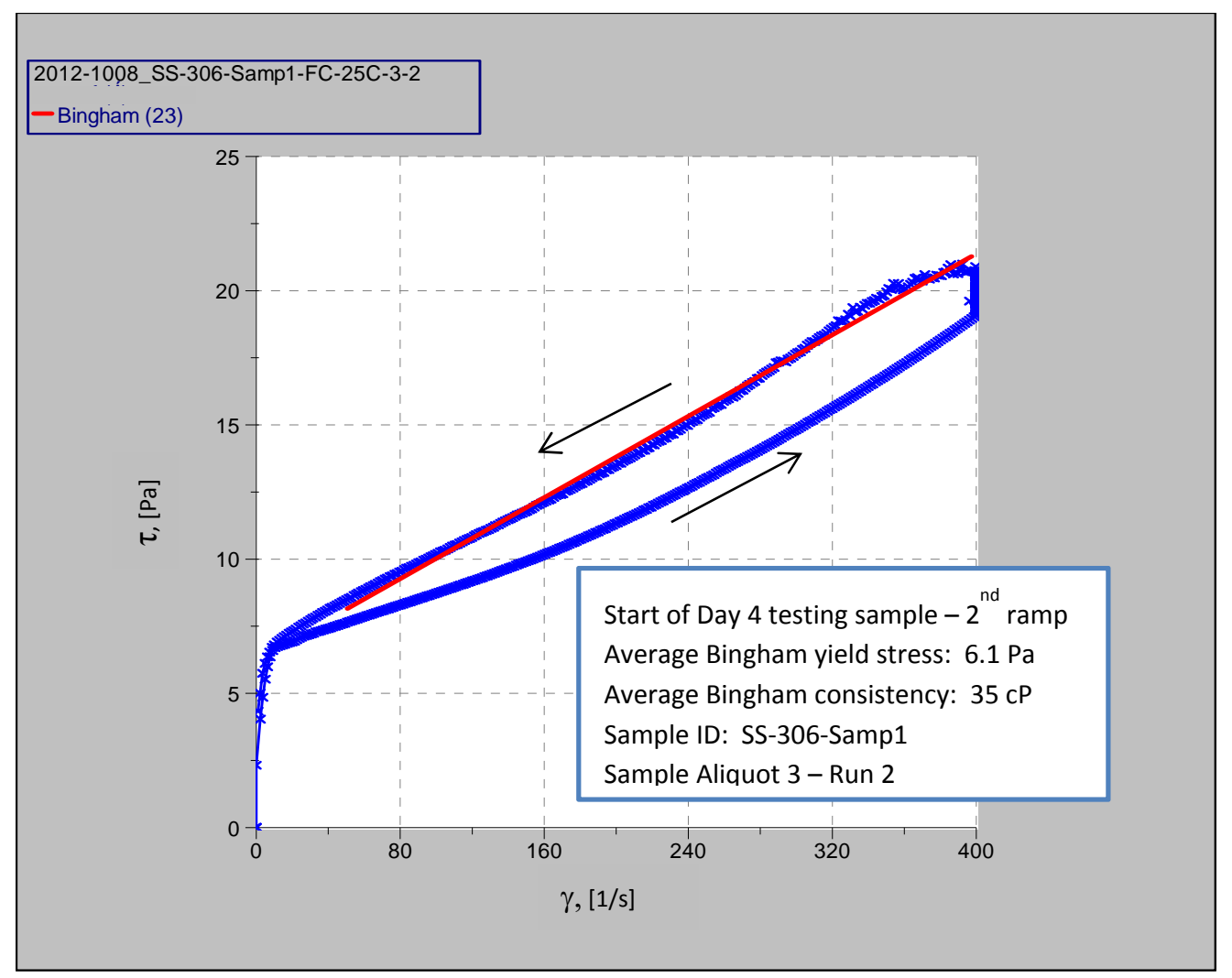

Figure E.10. Rheogram ( $\tau$ vs. $\dot{\gamma}$ ) for Chemical Simulant at the Beginning of Day 4 of the 7-day $6 \mathrm{~Pa} / 6 \mathrm{cP}$ Testing; Measured Shear Rate Range of $0-400 \mathrm{~s}^{-1}$ 


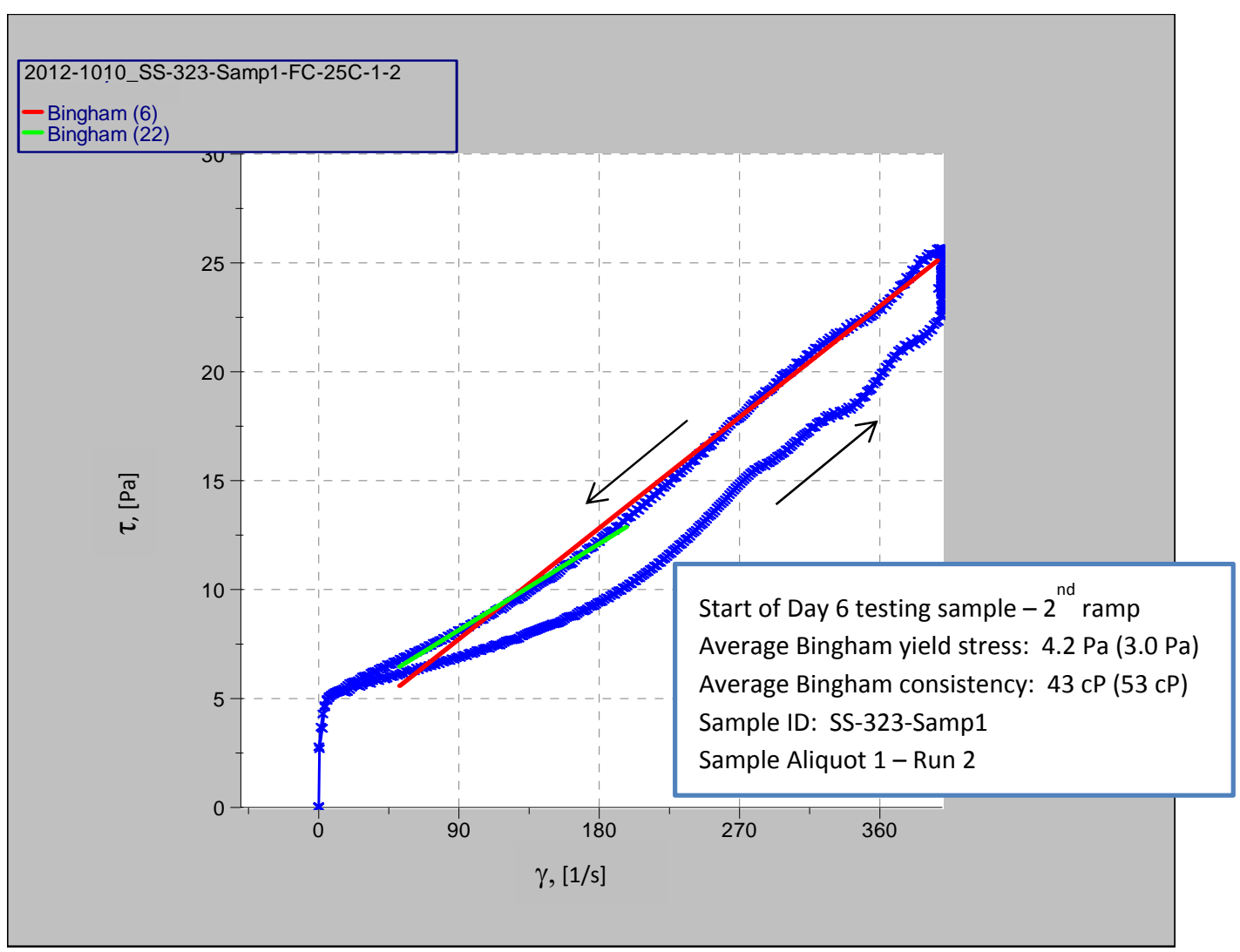

Figure E.11. Rheogram ( $\tau$ vs. $\dot{\gamma}$ ) for Chemical Simulant at the Beginning of Day 6 of the 7-day $6 \mathrm{~Pa} / 6 \mathrm{cP}$ Testing; Measured Shear Rate Range of $0-400 \mathrm{~s}^{-1}$ 

PNNL-22402

WTP-RPT-222, Rev. 0

\section{Distribution*}

No. of

Copies

ONSITE

3 DOE Office of River Protection

JS Fox

H6-60

CC Harrington

DL Noyes

6 Bechtel National Inc.

A Hassan

HR Hazen

S Omberg-Carro

RJ VanVleet

JL Weamer

WTP PETD Docs

H6-60

H6-60

H4-02

H4-02

$\mathrm{H} 4-02$

H4-02

$\mathrm{H} 4-02$

H4-02
No. of

Copies

10 Pacific Northwest National Laboratory
JR Bontha

GN Brown

CA Burns

RC Daniel

PA Gauglitz

ML Kimura

DE Kurath

LA Mahoney

PP Schonewill

DN Tran

Project File

Information Release (pdf)
P7-25

P7-25

P7-25

P7-22

K7-15

K6-28

K3-52

K7-15

P7-25

K6-24

$\mathrm{K} 3-52$

*All distribution will be made electronically.

Dist. 1 




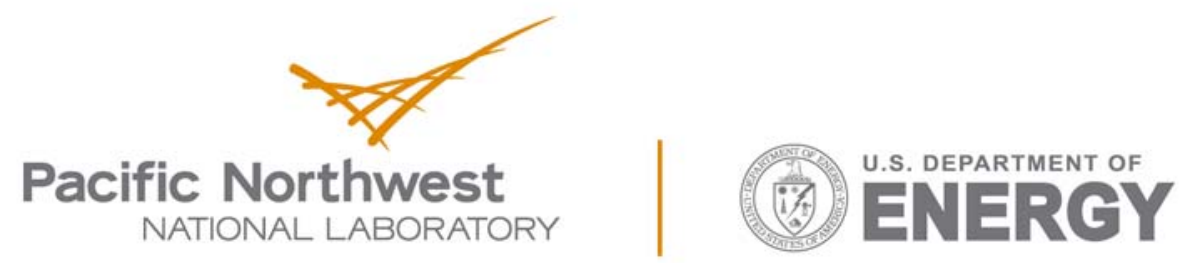

Proudly Operated by Battelle Since 1965

902 Battelle Boulevard

P.O. Box 999

Richland, WA 99352

1-888-375-PNNL (7665)

www.pnnl.gov 